DEPARTAMENTO DE INGENIERÍA ENERGÉTICA

ESCUELA TÉCNICA SUPERIOR DE INGENIEROS INDUSTRIALES

UNIVERSIDAD POLITÉCNICA DE MADRID
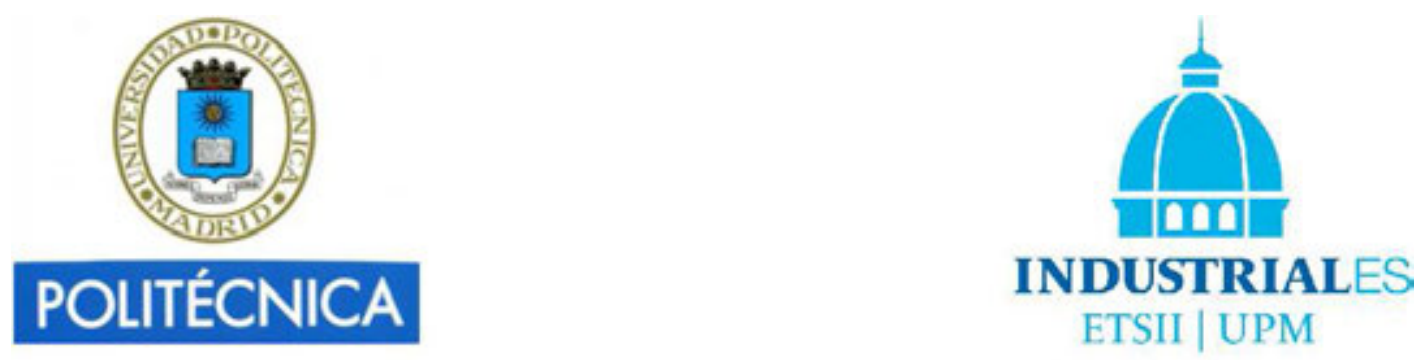

\title{
DISEÑO Y ANÁLISIS MULTIDISCIPLINAR DE LA FUENTE DE NEUTRONES DE MEDIA INTENSIDAD PROPUESTA PARA ESS-BILBAO
}

Tesis Doctoral

Autor:

Santiago Terrón Fraile

Ingeniero Industrial (UPM)

Directores:

Fernando Sordo Balbín

Dr. Ingeniero Industrial (UPM)

Alberto Abánades Velasco

Dr. Ingeniero Industrial (UPM) 

A mi abuela Herminia, por enseñarnos a estudiar a todos.

A Mercedes, por ayudarme a ser quien quiero ser.

A Tiago, por ser mi verdadera contribución original. 



\section{Agradecimientos}

Quisiera dar las gracias a todos aquellos que, con su apoyo, han hecho posible esta tesis.

En primer lugar, quisiera agradecer su colaboración a mis directores, Fernando Sordo y Alberto Abánades, sin cuyo soporte y guía este trabajo no habría llegado a buen puerto.

A Javier Bermejo y a todo el equipo de ESS-Bilbao. Gracias por vuestro empeño en hacer avanzar la ciencia española, aunque el viento no siempre sople a favor.

A Manolo Perlado, por acogernos en el Instituto de Fusión Nuclear. Gracias por animarnos y ayudarnos en toda ocasión; y por ser, en última instancia, la alma mater de tantos.

Gracias a todos. Ha sido un placer y un honor trabajar con vosotros.

En el plano personal, quisiera agradecer el ánimo que mis amigos y mi familia me han transmitido durante este viaje.

No puedo sino agradecer aquí a mis padres todos los sacrificios que han hecho por mi hermano y por mí. Todo os lo debo. Todo aquello que de bueno pueda hacer, es vuestro. Gracias por todo, siempre.

A Mercedes, mi esposa y compañera. Gracias por tu infinita paciencia. Gracias por tu amor y tu apoyo incondicional. Gracias por el esfuerzo que has puesto en este trabajo, a tantos niveles. Siempre estaré en deuda contigo. Esta tesis es más tuya que mía. Gracias.

Y por último, a mi hijo, Santiago. Gracias por iluminar mi vida con tu alegría, y por hacer que el teclado me ardiera en las manos.

Barcelona, julio de 2018. 



\section{Índice general}

1. Introducción 1

1.1. Los neutrones en la ciencia . . . . . . . . . . . . . . . . . . 2

1.1.1. Transferencia . . . . . . . . . . . . . . . . . 2

1.1.2. Captura neutrónica . . . . . . . . . . . . . . . 4

1.1.3. Dispersión neutrónica . . . . . . . . . . . . . . . 5

1.2. Reacciones de producción de neutrones . . . . . . . . . . . . . . 9

1.2.1. Procesos de desintegración radiactiva . . . . . . . . . . . . 9

1.2.2. Reacciones de fusión . . . . . . . . . . . . . . . . . 10

1.2.3. Reacciones de fisión . . . . . . . . . . . . . . . . . . . 12

1.2.4. Fuentes de bremsstrahlung . . . . . . . . . . . . . . . 13

1.2.5. Reacciones de espalación . . . . . . . . . . . . . . . 15

1.2.6. Reacciones de extracción (Stripping) . . . . . . . . . . . 16

1.3. Elementos de una fuente de neutrones . . . . . . . . . . . . . . . . . . . . 19

1.3.1. Acelerador . . . . . . . . . . . . . . . . . . . . 20

1.3.2. Blanco . . . . . . . . . . . . . . . . . . . . 22

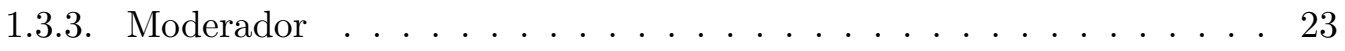

1.3.4. Reflector ..................... . . 23

1.3.5. Blindajes radiológicos . . . . . . . . . . . . . . . . . 24

1.4. Estado del arte de las fuentes de neutrones guiadas por acelerador . . . . . 26

1.4.1. Fuentes de alta potencia . . . . . . . . . . . . . . 26

1.4.1.1. SNS, Laboratorio Nacional de Oak Ridge . . . . . . . . . 26

1.4.1.2. JSNS, Complejo japonés de investigación en aceleradores de protones . . . . . . . . . . . . . . 27

1.4.1.3. SINQ, Paul Scherrer Institute . . . . . . . . . . . . . 27 
1.4.2. Fuentes de media potencia . . . . . . . . . . . . . 28

1.4.2.1. ISIS, Rutherford Appleton Laboratory . . . . . . . . . . 29

1.4.2.2. CSNS, Academia china de ciencias . . . . . . . . . . 29

1.4.3. Fuentes cctas . . . . . . . . . . . . . . . . 30

1.4.3.1. LENS, Universidad de Indiana . . . . . . . . . . . . . 30

1.4.3.2. RANS, Centro de investigación RIKEN . . . . . . . . . . 31

1.4.3.3. PKUNIFTY, Universidad de Pekín . . . . . . . . . . . . 31

1.4.3.4. CPHS, Universidad de Qinghua . . . . . . . . . . . 32

1.5. El proyecto de la fuente europea de espalación (ESS) . . . . . . . . . . . 32

1.6. Motivación: La propuesta de ESS-Bilbao . . . . . . . . . . . . . . . . . . 34

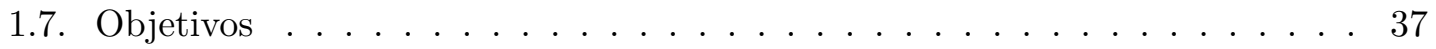

1.8. Métodos . . . . . . . . . . . . . . . . . . . . 38

2. Diseño y optimización del blanco de la fuente de ESS-Bilbao 41

2.1. Parámetros base de diseño de la fuente de ESS-Bilbao . . . . . . . . . . . . 41

2.2. Elección del material del blanco . . . . . . . . . . . . . . . . . . . . 44

2.3. Optimización del blanco . . . . . . . . . . . . . . . . . . 46

2.3.1. Elección del método de refrigeración . . . . . . . . . . . . . 46

2.3.2. Cálculo de la deposición energética del haz de ESS-Bilbao . . . . . . 56

2.3.3. Análisis termomecánico . . . . . . . . . . . . . . . 60

2.3.3.1. Optimización del espesor del blanco . . . . . . . . . . . 66

2.3.3.2. Determinación del número de placas en el blanco rotatorio 74

2.3.4. Estudio de vida útil . . . . . . . . . . . . . . . . . . . 79

2.3.4.1. Implantación de hidrógeno y generación de gases . . . . . 79

2.3.4.2. Evolución de las propiedades mecánicas . . . . . . . . . 85

2.3.4.3. Daño por fatiga . . . . . . . . . . . . . . 88

2.3.4.4. Corrosión y control químico . . . . . . . . . . . . . 89

3. Diseño conceptual de la estructura del blanco 91

3.1. Descripción general . . . . . . . . . . . . . . . . . . . . . . 92

3.2. Cálculo termohidráulico . . . . . . . . . . . . . . . . . . . . . . 99

3.2.1. Dominio de cálculo: Geometría, materiales y mallado . . . . . . . . . 99

3.2.2. Condiciones de contorno y cargas . . . . . . . . . . . . . 101 
3.2.3. Parámetros de cálculo . . . . . . . . . . . . . . . . . . . . . . 103

3.2.4. Resultados . . . . . . . . . . . . . . . . . . . 104

3.3. Cálculo termomecánico . . . . . . . . . . . . . . . . . . . 106

3.3.1. Dominio de cálculo: Geometría, materiales y mallado . . . . . . . . 106

3.3.2. Condiciones de contorno y cargas . . . . . . . . . . . . . 107

3.3.3. Parámetros de cálculo . . . . . . . . . . . . . . . . . . . . 108

3.3.4. Resultados . . . . . . . . . . . . . . . . 108

3.4. Evaluación de los márgenes de seguridad frente a accidente . . . . . . . . 113

3.4.1. Concentración excesiva del haz de protones . . . . . . . . . . . . 114

3.4.2. Bloqueo del giro del blanco . . . . . . . . . . . . . . . . 117

3.4.3. Desfase haz - blanco . . . . . . . . . . . . . . . . . . 123

3.4.4. Accidente de pérdida de flujo . . . . . . . . . . . . . . . 125

3.4.5. Accidente de pérdida de vacío . . . . . . . . . . . . . 129

3.4.6. Reacciones químicas a alta temperatura . . . . . . . . . . . 131

3.4.7. Energía de los protones anormalmente baja . . . . . . . . . . 132

4. Análisis de producción neutrónica $\quad 135$

4.1. Caracterización del término fuente neutrónico . . . . . . . . . . . . . 135

4.2. Configuración de referencia de la fuente de ESS-Bilbao . . . . . . . . . . . 138

4.2.1. Moderador . . . . . . . . . . . . . . . . . . 139

4.2.1.1. Elección de material, configuración y dinámica . . . . . . . 139

4.2.1.2. Optimización de espesor . . . . . . . . . . . . . . . 143

4.2.1.3. Efecto de la temperatura del moderador . . . . . . . . . . 144

4.2.2. Reflector . . . . . . . . . . . . . . . . 145

4.2.3. Blindajes y estación del blanco . . . . . . . . . . . . . 147

4.3. Producción neutrónica de referencia . . . . . . . . . . . . . . 150

4.4. Configuraciones alternativas y avanzadas . . . . . . . . . . . . . . 154

4.4.1. Filtro de berilio . . . . . . . . . . . . . . . . . . . . . . . 154

4.4.2. Dos moderadores . . . . . . . . . . . . . . . 157

4.4.3. Producción de neutrones térmicos . . . . . . . . . . . 159

4.5. Evaluación preliminar de aplicaciones . . . . . . . . . . . . . . . . 161

$\begin{array}{lr}\text { 5. Futuros trabajos } & 167\end{array}$ 
5.1. Una nueva generación de fuentes: fuentes de alto brillo . . . . . . . . . . . 167

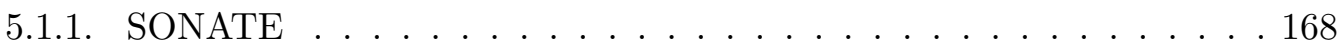

5.1.2. HBS, High Brilliance Source . . . . . . . . . . . . . . . . 169

5.2. Prototipo termohidráulico . . . . . . . . . . . . . . . . 171

$\begin{array}{lr}\text { 6. Conclusiones } & 177\end{array}$

$\begin{array}{ll}\text { A. Evolución del diseño de la estructura del blanco } & 181\end{array}$

A.1. Generación del concepto de estructura del blanco . . . . . . . . . . . . . 181

A.1.1. Concepto inicial . . . . . . . . . . . . . . . 181

A.1.2. Posibles soluciones constructivas . . . . . . . . . . . . . 182

A.1.2.1. Soluciones modulares . . . . . . . . . . . . . 183

A.1.2.2. Solución integral . . . . . . . . . . . . . . . 185

A.1.3. Selección de la solución constructiva . . . . . . . . . . . . . 185

A.2. Análisis prospectivos del blanco integral . . . . . . . . . . . . . 187

A.2.1. Análisis y mejora del comportamiento hidráulico . . . . . . . . 189

A.2.2. Análisis y mejora del comportamiento mecánico . . . . . . . . . . 193

B. Análisis paramétrico del efecto de la duración del pulso 207

$\begin{array}{ll}\text { C. Planos del prototipo termohidráulico } & 211\end{array}$ 


\section{Índice de figuras}

1-1. Longitud de dispersión de neutrones y rayos $\mathrm{X}$ con diferentes elementos en función de su número atómico $[3] \ldots \ldots \ldots$. . . . . . . . . . . . 3

1-2. Neutrografía (izq.) y Radiografía (der.) de un surtidor de gasolina [2]. . . . 4

1-3. Esquema de un experimento de dispersión de neutrones [5]. . . . . . . . 5

1-4. Triángulo de dispersión $[6] \ldots \ldots \ldots \ldots \ldots$

1-5. Sección eficaz (a) y reactividad (b) de las reacciones de fusión de mayor probabilidad a bajas energías, en función de la energía del proyectil y de la energía cinética relativa respectivamente $[12] \ldots \ldots \ldots 11$

1-6. Espectro energético de la reacción de fisión del ${ }_{92}^{235} \mathrm{U}$ comparado con el de espalación producida por protones de $800 \mathrm{MeV}$ impactando en W [33]. . . 17

1-7. Producción de neutrones por $\mu C$ de haz impactando sobre blancos espesos. 18

1-8. Conjunto blanco - moderador - reflector de la fuente JSNS [64] . . . . . . . 25

1-9. Conjunto blanco - moderador - reflector de la fuente SNS (izq.) [65] y blanco de SNS en celda caliente (der.) [66]. . . . . . . . . . . . . 26

1-10. Blanco de la fuente SINQ $[71] . \ldots \ldots \ldots \ldots$

1-11. Fuente de neutrones RANS del centro japonés RIKEN [82] . . . . . . . . . 31

2-1. Curva de Nukiyama para agua saturada a presión atmosférica [121] . . . . 50

2-2. Flujo crítico de ebullición en agua (Ecuación 2.4 [123], [124]) . . . . . . . 53

2-3. Blanco rotatorio de grafito para la producción de muones en JPARC [127] . 55

2-4. Dimensiones de la sección transversal del haz y del área de impacto $[\mathrm{cm}] . \quad$. 57

2-5. Distribución de flujo en el haz de ESS-Bilbao $x, y[m] ; z\left[M W / m^{2}\right] \ldots$. . 58

2-6. Deposición energética del haz de ESS-Bilbao en berilio $\left[\mathrm{W} / \mathrm{m}^{3}\right] \ldots \ldots$. . . 59

2-7. Ejemplo de pico de Bragg: poder de frenado de partículas $\alpha$ en aire [132] . . 59

2-8. Esquema de cálculo termomecánico. . . . . . . . . . . . . . . . . . . 60

2-9. Mallado ANSYS de una placa de $12 \mathrm{~mm}$ de espesor. . . . . . . . . . . . . . 61

2-10. Potencia implementada en ANSYS en una placa de Be de $12 \mathrm{~mm}\left[\mathrm{~W} / \mathrm{m}^{3}\right]$. . 62 
2-11. Evolución de la temperatura máxima en una placa de Be de $12 \mathrm{~mm}$ con una frecuencia de impacto de $1 \mathrm{~Hz} . \ldots \ldots$. . . . . . . . . . 64

2-12. Distribución de temperatura $[\mathrm{K}]$ en una placa de Be de $12 \mathrm{~mm}$ de espesor, en el momento en el que se alcanza el máximo del pseudo-estacionario. . . . 65

2-13. Evolución de la tensión de von Mises máxima en una placa de Be de $12 \mathrm{~mm}$ con una frecuencia de impacto de $1 \mathrm{~Hz} \ldots \ldots$. . . . . . . . . . 66

2-14. Distribución de tensiones $[\mathrm{Pa}]$ en una placa de Be de $12 \mathrm{~mm}$ de espesor, en el momento en el que se alcanza el máximo del pseudo-estacionario.

2-15. Evolución de la potencia volumétrica máxima implementada durante el pulso en la placa de Be en función de su espesor. . . . . . . . . . . . . 6 68

2-16. Evolución de la potencia implementada durante el pulso en la placa de Be en función de su espesor. . . . . . . . . . . . . . . . . . . . . 69

2-17. Evolución de producción neutrónica (neutrones por protón incidente) en función del espesor de la placa de Be. . . . . . . . . . . . . . 70

2-18. Evolución de la temperatura máxima en la placa de Be en función de su

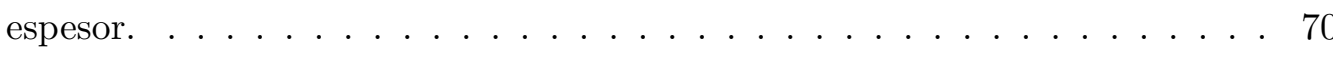

2-19. Evolución de la tensión de von Mises máxima en la placa de Be en función de su espesor. . . . . . . . . . . . . . . . . . . 71

2-20. Comparativa de la evolución temporal de la temperatura máxima en la placa de Be para diferentes espesores. . . . . . . . . . . . . 72

2-21. Comparativa de la evolución temporal de la tensión equivalente máxima en la placa de Be para diferentes espesores. . . . . . . . . . . . . 73

2-22. Potencia implementada en ANSYS en una placa de Be de $9 \mathrm{~mm}\left[\mathrm{~W} / \mathrm{m}^{3}\right]$. $\quad 74$

2-23. Distribución de temperatura $[\mathrm{K}]$ en una placa de Be de $9 \mathrm{~mm}$ de espesor en el momento en el que se alcanza el máximo del pseudo-estacionario. . . . 75

2-24. Distribución de tensiones $[\mathrm{Pa}]$ en una placa de Be de $9 \mathrm{~mm}$ de espesor en el momento en el que se alcanza el máximo del pseudo-estacionario. . . . . 75

2-25. Evolución de la temperatura máxima en una placa de Be de $9 \mathrm{~mm}$ con una frecuencia de impacto de $1 \mathrm{~Hz} \ldots \ldots \ldots \ldots . \ldots . \ldots 76$

2-26. Evolución de la tensión equivalente máxima en una placa de Be de $9 \mathrm{~mm}$ con una frecuencia de impacto de $1 \mathrm{~Hz} \ldots \ldots \ldots . . \ldots 76$

2-27. Evolución de la tensión equivalente máxima en una placa de Be de $9 \mathrm{~mm}$ para distintas frecuencias de impacto. . . . . . . . . . . . . 77

2-28. Evolución de la tensión de von Mises máxima en una placa de Be de $9 \mathrm{~mm}$ para distintas frecuencias de impacto. . . . . . . . . . . 78

2-29. Fallo del blanco de la fuente de neutrones LENS (Indiana University) por implantación de hidrógeno $[139] . \ldots \ldots . \ldots . \ldots . \ldots 80$

2-30. Perfil de concentración de H en la placa de Be tras 2000 horas de operación 81 
2-31. Perfil de concentración máxima de ${ }^{4} \mathrm{He}$ generado en la placa de berilio, tras 2000 horas de operación, calculados con diferentes modelos nucleares. . . . . 83

2-32. Porcentaje de hinchamiento en función del contenido de $H e$ y la temperatura de irradiación del berilio. . . . . . . . . . . . . . . 84

2-33. Evolución de las propiedades mecánicas de diversos tipos de berilio comercial bajo irradiación de neutrones [155]: a) Límite elástico. b) Tensión de

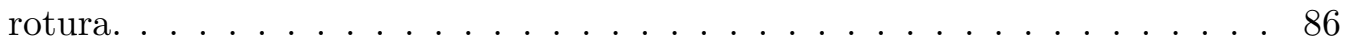

3-1. Vista general de la estructura del blanco . . . . . . . . . . . . . . . 93

3-2. Vistas explosionadas, superior en inferior, de la estructura del blanco. . . . 93

3-3. Flujo de refrigerante dentro de la estructura del blanco. . . . . . . . . . . . 94

3-4. Sección de la estructura del blanco. Flujo de refrigerante y posición relativa del haz de protones. . . . . . . . . . . . . . . . . . . . 94

3-5. Dimensiones principales de la estructura $[\mathrm{mm}]$ y cortes a ambos niveles de

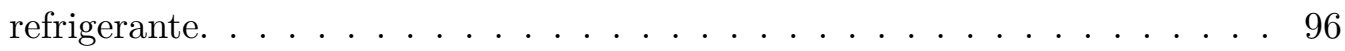

3-6. Vista explosionada de un sector de $18^{\circ}$ de la estructura del disco. . . . . . . 97

3-7. Sector de $18^{\circ}$ del dominio fluido. . . . . . . . . . . . . . 100

3-8. Malla del dominio fluido del cálculo termohidráulico. . . . . . . . . . . . 100

3-9. Detalle de la malla del dominio fluido del cálculo termohidráulico. . . . . . 101

3-10. Velocidades del refrigerante dentro del disco. . . . . . . . . . . . . . . . 105

3-11. Temperaturas medias $\left[{ }^{\circ} \mathrm{C}\right]$ en operación del disco. . . . . . . . . . . . 106

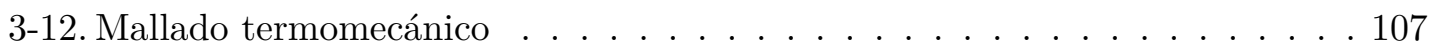

3-13. Cálculo termomecánico. Tensiones equivalentes de von Mises $[P a]$. . . . . 109

3-14. Cálculo termomecánico. Tensiones equivalentes de von Mises $[\mathrm{Pa}]$ en el submodelo de la zona de tensión máxima. Detalle de la malla refinada. . . . 110

3-15. Cálculo termomecánico. Desplazamiento total $[m]$. . . . . . . . . . . . 112

3-16. Cálculo termomecánico. Desplazamiento en la dirección del eje del disco $[m] .112$

3-17. Cálculo termomecánico. Distribución de presión en los contactos $[P a]$. . . . 113

3-18. Evolución de la tensión de von Mises máxima $[M P a]$ en la placa de berilio para distintos radios de haz. . . . . . . . . . . . . . . . . 115

3-19. Evolución de la temperatura máxima $\left[{ }^{\circ} \mathrm{C}\right]$ en la placa de berilio para distintos radios de haz.. . . . . . . . . . . . . . . . . 116

3-20. Evolución de la tensión de von Mises máxima $[M P a]$ en la placa de berilio durante el accidente de bloqueo. . . . . . . . . . . . . . . 118

3-21. Evolución de la temperatura máxima $\left[{ }^{\circ} \mathrm{C}\right]$ en la placa de berilio durante el accidente de bloqueo. . . . . . . . . . . . . . . . . . . . 119

3-22. Deposición energética del haz de ESS-Bilbao en aluminio 6061-T6 $\left[\mathrm{W} / \mathrm{m}^{3}\right]$. 120 
3-23. Evolución de la tensión de von Mises máxima [MPa] en el aluminio 6061-T6 durante el accidente de bloqueo.

3-24. Evolución de la temperatura máxima $\left[{ }^{\circ} \mathrm{C}\right]$ en el aluminio 6061-T6 durante el accidente de bloqueo. . . . . . . . . . . . . . . . . . 122

3-25. Evolución de la temperatura máxima $\left[{ }^{\circ} \mathrm{C}\right]$ en el aluminio 6061-T6 por el impacto del haz. . . . . . . . . . . . . . . . . . . 124

3-26. Evolución de la tensión de von Mises máxima $[M P a]$ en el aluminio 6061-T6 por el impacto del haz. . . . . . . . . . . . . . . . . . . . . . . . . 124

3-27. Evolución de la temperatura máxima en el Be en función de la velocidad del refrigerante. . . . . . . . . . . . . . . . . . . . 128

3-28. Evolución de la tensión máxima de von Mises en el Be en función de la velocidad del refrigerante. . . . . . . . . . . . . . . . . 128

3-29. Tasa de reacción del berilio macizo en contacto con vapor de agua en función de la temperatura $[183] \ldots 132$

4-1. Espectro de flujo neutrónico en función de la posición angular con respecto al haz de protones, normalizado por protón incidente. . . . . . . . . . . 137

4-2. Razón entre el flujo en la dirección colineal con el haz de protones y el flujo neutrónico a $45^{\circ}$ en función de la energía de los neutrones. . . . . . . . . . . 138

4-3. Disposición del blanco, el haz de protones y las líneas experimentales. . . . 139

4-4. Esquema de las tres configuraciones blanco-moderador básicas; slab (a), wing (b) and flux-trap (c) [201]. . . . . . . . . . . . . . . 141

4-5. Brillo neutrónico de $E<5 \mathrm{meV}$ en la superficie del moderador de ESSBilbao en función de su espesor, normalizado por protón incidente. . . . . . 145

4-6. Espectro de brillo neutrónico en la superficie del moderador para distintas temperaturas de este, normalizado por $M W$ de haz. . . . . . . . . . 146

4-7. Distribución temporal de brillo de neutrones de $4,4<E<5,3 \mathrm{meV}$ en la superficie del moderador para distintos materiales reflectores, normalizado por $M W$ de haz. . . . . . . . . . . . . . . . . . . . 147

4-8. Estación del blanco. . . . . . . . . . . . . . . . . . . . . . . . . . 149

4-9. Dimensiones principales de la vasija de contención $[\mathrm{mm}] \ldots$. . . . . . . 150

4-10. Inserto del moderador ( $i z q$.$) y unidad de transporte del blanco (der.) . . . . 150$

4-11. Modelo MCNPX de referencia. Vista global y área del moderador . . . . . . 151

4-12. Sección eficaz del berilio a $77 \mathrm{~K}$ : Medida experimental y valor teórico ajustado para incluir el efecto de la presencia impurezas y de óxido de berilio

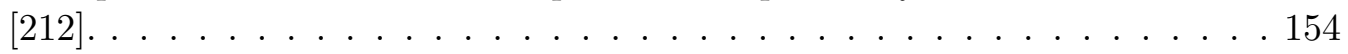

4-13. Modelo de MCNPX incluyendo filtro de berilio . . . . . . . . . . . 155

4-14. Espectro de brillo neutrónico en la superficie del moderador, con y sin filtro de berilio, normalizado por $M W$ de haz. . . . . . . . . . . . 156 
4-15. Distribución temporal de brillo de neutrones de 4,4<E<5,3 $\mathrm{meV}$ en la superficie del moderador con y sin filtro de berilio, normalizado por $M W$ de haz. . . . . . . . . . . . . . . . . . . . . 156

4-16. Modelo MCNPX con dos moderadores de metano sólido. . . . . . . . . . . 158

4-17. Brillo de neutrones de $E<600 \mathrm{meV}$ en la superficie del moderador de ESS-Bilbao en función de su espesor, normalizado por protón incidente. . . 160

5-1. Evolución de la disponibilidad de tiempo de instrumento para usuarios franceses [236] a partir de datos de [237] . . . . . . . . . . . . . . . 169

5-2. Concepto de fuente de alto brillo con blancos múltiples $[241]$. . . . . . . 170

5-3. Vista general del prototipo del blanco. . . . . . . . . . . . . . . . 171

5-4. Comparativa entre un sector del blanco de ESS-Bilbao y el prototipo. . . . 172

5-5. Prototipo ensamblado. . . . . . . . . . . . . . . . . . . 173

5-6. Vista explosionada del prototipo. . . . . . . . . . . . . . . . . . 174

5-7. Componentes del prototipo fabricados. . . . . . . . . . . . . . 175

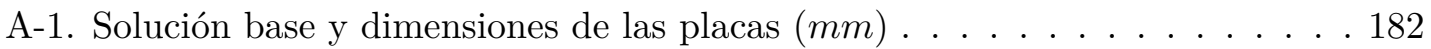

A-2. Ejemplo de solución modular atornillada . . . . . . . . . . . . . . . . 183

A-3. Esquema de unión de una solución atornillada. . . . . . . . . . . . . . . 184

A-4. Blanco, solución atornillada (conductos semi-descubiertos). . . . . . . . . . 184

A-5. Ejemplo de solución modular embridada. . . . . . . . . . . . . . . 185

A-6. Velocidad de refrigerante en la opción atornillada. . . . . . . . . . . . 186

A-7. Diseño inicial del blanco integral (haz de protones en amarillo). . . . . . . 188

A-8. Vista explosionada del concepto inicial de blanco integral. . . . . . . . . . . 189

A-9. Vista seccionada de la configuración inicial del blanco. Haz de protones representado, incluyendo margen de seguridad a su alrededor. . . . . . . . . 190

A-10.Volumen interno de refrigerante en la configuración inicial del blanco. . . . 190

A-11.Dominio de cálculo fluido inicial. . . . . . . . . . . . . . . . . . . . . 191

A-12.Distribución de velocidades del refrigerante en la configuración inicial. . . . 191

A-13.Distribución de velocidades del refrigerante en una configuración con 24 canales distribuidores y colectores y canales de regreso ampliados. . . . . . 193

A-14.Distribución de velocidades del refrigerante en el dominio fluido de referencia.194

A-15.Vista explosionada de un sector de $18^{\circ}$ del diseño inicial. . . . . . . . . . . 195

A-16.Mallado mecánico inicial. . . . . . . . . . . . . . . . . 196 
A-17.Distribución de desplazamientos en la dirección axial del disco en modelo mecánico lineal inicial $[\mathrm{m}] \ldots$. . . . . . . . . . . . . . . . 196

A-18.Distribución de tensión de von Mises en modelo mecánico lineal inicial [Pa]. 197

A-19.Vista explosionada de un sector de $18^{\circ}$ de la configuración modificada. . . . 199

A-20.Distribución de desplazamientos en la dirección axial del disco en modelo mecánico lineal modificado $[\mathrm{m}]$. . . . . . . . . . . . . . . 200

A-21.Distribución de tensión de von Mises en modelo mecánico lineal modificado

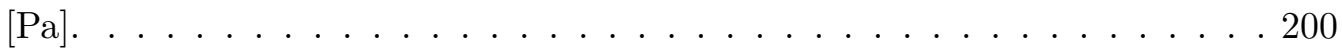

A-22.Sector de $18^{\circ}$ de la geometría del disco rigidizado. . . . . . . . . . . . 201

A-23.Vista explosionada del disco rigidizado. . . . . . . . . . . . . . . . . 201

A-24.Vista explosionada de un sector de $18^{\circ}$ de la configuración rigidizada. . . . 202

A-25.Detalle de las uniones atornilladas de la estructura del blanco. . . . . . . . . 203

A-26.Distribución de desplazamientos en la dirección axial del disco en modelo mecánico lineal rigidizado $[\mathrm{m}] \ldots$. . . . . . . . . . . . . . . . . 204

A-27.Distribución de tensión de von Mises en modelo mecánico lineal rigidizado [Pa] . . . . . . . . . . . . . . . . . . . . . 205

B-1. Evolución de la temperatura máxima en una placa de Be de $9 \mathrm{~mm}$ para distintas combinaciones de longitud de pulso y frecuencia de impacto con

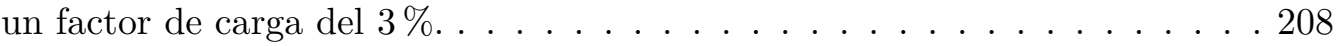

B-2. Evolución de la tensión de von Mises máxima en una placa de Be de $9 \mathrm{~mm}$ para distintas combinaciones de longitud de pulso y frecuencia de impacto con un factor de carga del $3 \%$. . . . . . . . . . . . . . . 208 


\section{Índice de tablas}

1.1. Tipos de neutrones en función de su energía [10] . . . . . . . . . . . . . . . 24

1.2. Requisitos de fuente en función de las aplicaciones . . . . . . . . . . 36

1.3. Rangos típicos de término fuente de las instalaciones existentes en función de la reacción productora de neutrones. . . . . . . . . . . . . . 37

2.1. Parámetros principales del acelerador de ESS-Bilbao [113] . . . . . . . . . 42

2.2. Parámetros del acelerador de fuentes de neutrones ordenados por potencia. 43

2.3. Proporción de conversión neutrónica y energía media de los neutrones producidos por protones de $50 \mathrm{MeV}$. Valores calculados usando la librería de secciones eficaces ENDF/B-VII.0 [114] . . . . . . . . . . . . . . . 44

2.4. Valores tipicos del coeficiente de transmisión de calor, $h$ [121] . . . . . . . . 48

2.5. Estudio paramétrico de los efectos del espesor del blanco. Porcentajes calculados con respecto a la placa de $12 \mathrm{~mm}$ de espesor . . . . . . . . . . 72

2.6. Estudio paramétrico de los efectos de la frecuencia de impacto en el blanco. Porcentajes calculados con respecto a los resultados para $1 \mathrm{~Hz} \ldots$. . . . 79

2.7. Valores máximos de concentración en appm de isótopos de $\mathrm{H}$ y $\mathrm{He}$ en la placa de Be de $9 \mathrm{~mm}$, tras 2000 horas de operación, calculados con diferentes modelos nucleares. . . . . . . . . . . . . . . . . . 82

3.1. Números de Nusselt para flujo laminar en tubos de diversa sección transversal $[179] \ldots \ldots \ldots 127$

3.2. Análisis de temperaturas y tensiones en caso de pérdida de flujo de refrigerante127

4.1. Razón de conversión neutrónica de la reacción ${ }^{9} B e(p, x n)$. Resultados numéricos comparados en el trabajo experimental. [184] . . . . . . . . . . 136

4.2. Comparativa de moderadores hidrogenados [194] . . . . . . . . . . . 140

4.3. Término fuente y brillo de neutrones $E<0,4 \mathrm{eV}$ de distintas fuentes de espalación [209] y relaciones respecto a los valores esperables para ESS-Bilbao.153

4.4. Comparativa de las características temporales del pulso de neutrones de $4,4<E<5,3 \mathrm{meV}$ sin filtro de berilio y con él. . . . . . . . . . . . . 157 
4.5. Brillo de neutrones de energía inferior a $5 \mathrm{meV}$ en la superficie de los moderadores $\left[n / \mathrm{cm}^{2} \cdot s \cdot s r\right] \ldots \ldots \ldots \ldots \ldots$

4.6. Comparación entre flujo en instrumento para diversas técnicas neutrónicas y flujo esperable en ESS-Bilbao estimado en base a [214] . . . . . . . . . 165 


\section{Resumen}

Desde su descubrimiento en 1930, los neutrones se han constituido como una herramienta fundamental en la ciencia y la tecnología para la exploración de la materia. Desafortunadamente, su producción para experimentación requiere de procesos nucleares, y el desarrollo de fuentes de neutrones presenta relevantes desafíos de ingeniería.

En la actualidad, la Unión Europea construye la que está llamada a ser la fuente de neutrones guiada por acelerador más potente del mundo, la European Spallation Source (ESS). En 2013, la participación española al proyecto, aglutinada en el consorcio ESSBilbao, planteó la posibilidad de canalizar la contribución en especie a ESS a través de la construcción de una fuente de neutrones compacta de media intensidad. Esta fuente, que se ubicaría en el campus de Vizcaya de la Universidad del País Vasco (UPV/EHU), serviría como estación de desarrollo y banco de pruebas de los componentes de ESS, así como instalación con interés científico propio para la comunidad de técnicas neutrónicas.

El presente trabajo tiene por objeto el diseño de los componentes principales de la propuesta de fuente de neutrones para ESS-Bilbao, evaluando su viabilidad técnica y rango de aplicación.

Para llevar esto a cabo, el grueso del trabajo se concentra en el diseño de un blanco de producción de neutrones. En una fuente de neutrones guiada por acelerador, este componente recibe el impacto de partículas aceleradas, induciéndose en él las reacciones de producción de neutrones. En su diseño, la eficiencia de la producción neutrónica es la característica clave a optimizar. No obstante, la intensa deposición energética originada por el impacto del haz de partículas, conlleva requisitos en términos de refrigerabilidad, tensiones termomecánicas, fatiga y daño por irradiación, que el diseño del blanco debe satisfacer. 
Una vez se ha definido el blanco, y por tanto la producción neutrónica de la fuente, se deben especificar y optimizar los componentes relevantes para hacer los neutrones útiles para experimentación. Hecho esto, se concluye el estudio con una evaluación preliminar del potencial rango de aplicación de la fuente.

Este proceso de diseño y análisis se ha dividido en las siguientes fases:

El Capítulo 1 introduce brevemente las técnicas de experimentación con neutrones, junto con los diferentes procesos nucleares que pueden dar lugar a su producción. Se desglosan con detalle los elementos de una fuente guiada por acelerador, y se hace un breve repaso del estado del arte de estos dispositivos. Por último, se describe el contexto histórico del proyecto ESS, y la motivación e interés de la propuesta de fuente de ESSBilbao.

En el Capítulo 2, se deciden las principales opciones de diseño para la definición del blanco de producción de neutrones. El acelerador de protones propuesto para la instalación, acelera las partículas hasta los $50 \mathrm{MeV}$ en pulsos de 1,5 $\mathrm{ms}$, con una corriente media de 2,25 mA y una potencia total de $112,5 \mathrm{~kW}$. La fuente propuesta será, por tanto, una fuente de media intensidad y pulso largo. Partiendo de este acelerador, se selecciona el material del blanco en base a la optimización de la producción neutrónica. A continuación, se definen los parámetros básicos del sistema de refrigeración necesario, y se estudia el efecto en términos de tensión y temperatura del impacto de las partículas, evaluando la influencia del espesor del blanco y de la frecuencia de impacto del haz en estas magnitudes. Por último, se analizan los aspectos relacionados con la vida útil en operación del blanco.

Como resultado del estudio realizado, se concluye que la solución base de diseño de la fuente propuesta para ESS-Bilbao, es un blanco rotatorio de berilio refrigerado por agua en régimen forzado monofásico, en el que los neutrones se producen mediante la reacción de extracción (o stripping). Los análisis de optimización termomecánica efectuados definen un blanco formado por 20 placas de berilio de $9 \mathrm{~mm}$ de espesor, sobre las que impacta el haz de protones con una frecuencia de $1 \mathrm{~Hz}$ en cada una. Con estos parámetros de diseño, se configura una fuente con una intensidad de $8,0 \cdot 10^{14}$ neutrones por segundo, alcanzándose valores aceptables de temperatura y tensión en el berilio. El estudio de las consecuencias de la irradiación estima la vida útil de las placas en un mínimo de 4 años, de acuerdo a los cálculos más conservadores. 
En el Capítulo 3, se diseña una estructura que materializa el concepto de blanco rotatorio previamente definido. Los cálculos termomecánicos y termohidráulicos llevados a cabo definen un diseño capaz de albergar las placas de berilio, proporcionarles la refrigeración necesaria mediante canales internos; y soportar tanto las cargas de operación normal, como aquellas derivadas de las secuencias accidentales previsibles. El diseño obtenido es el resultado de un proceso iterativo de análisis y mejora, cuyo histórico se recoge en el Apéndice A.

En el Capítulo 4, se deciden los principales parámetros de diseño del moderador y el reflector de la instalación, estableciendo una configuración de referencia para el conjunto blanco-moderador-reflector, definitorio de comportamiento neutrónico de la fuente. El proceso de diseño se ha orientado a optimizar la producción de neutrones fríos, buscando aumentar la capacidad de la fuente para la aplicación de técnicas experimentales basadas en dispersión de neutrones. La configuración de referencia resultante cuenta con un moderador de metano sólido en configuración "slab", rodeado de un reflector de berilio y del que parten tres líneas experimentales. Con estas decisiones de diseño, la configuración de referencia propuesta para la fuente de ESS-Bilbao produciría un brillo de neutrones de 5 $m e V$ de $3,6 \cdot 10^{10} \mathrm{n} / \mathrm{cm}^{2} \cdot \mathrm{sr} \cdot \mathrm{s}$, y de $1,3 \cdot 10^{11} \mathrm{n} / \mathrm{cm}^{2} \cdot \mathrm{sr} \cdot \mathrm{s}$ para neutrones de energía inferior a los $0,4 \mathrm{eV}$.

Sobre esta configuración de referencia, diversas configuraciones alternativas y avanzadas se han analizado, como la inclusión de un segundo moderador, o de filtros de berilio. También se ha evaluado la capacidad de la fuente para producir neutrones térmicos. Los análisis muestran que empleando un moderador de agua ligera, la producción de neutrones térmicos alcanzaría los $1,4 \cdot 10^{11} \mathrm{n} / \mathrm{cm}^{2} \cdot \mathrm{sr} \cdot \mathrm{s}$.

Estos valores de brillo neutrónico son, grosso modo, un orden de magnitud inferiores a los de las grandes fuentes; instalaciones éstas con aceleradores en el rango del megavatio de potencia, basadas en reacciones de espalación. No obstante, dichas fuentes cuentan con una producción neutrónica aproximadamente dos órdenes de magnitud superior a la propuesta para ESS-Bilbao, por lo que este diseño presenta una alta eficiencia. Esta eficiencia es especialmente patente cuando se consideran la complejidad y el coste de las estructuras aceleradoras necesarias en cada caso.

La evaluación preliminar de las potenciales aplicaciones de la fuente muestra que, con 
los flujos neutrónicos generados, ésta podría dar servicio a técnicas de experimentales basadas en activación, transferencia y a gran parte de las técnicas basadas en dispersión de neutrones; además de a otras actividades como irradiación de componentes, calibración de detectores, prueba de moderadores, etc.

En el Capítulo 5, a modo de enlace con futuros trabajos, se referencian dos proyectos europeos que en la actualidad buscan construir sendas fuentes de características similares a la aquí propuesta, y se describe un prototipo representativo del diseño desarrollado en este trabajo, fabricado para validar los aspectos termohidráulicos del blanco.

Como conclusiones, recogidas en el Capítulo 6, cabe destacar que este diseño define por primera vez una fuente de media intensidad y alta eficiencia basada en la reacción de extracción, que con costes mucho menores que las grandes instalaciones basadas en la reacción de espalación, o los reactores experimentales de fisión, habilita técnicas neutrónicas normalmente sólo disponibles en estos. Estas características han hecho que, en los últimos tiempos, el interés por este tipo de fuentes haya crecido, de tal manera que se plantean como candidatas a constituir una nueva generación de fuentes de neutrones. Esta generación está llamada a sustituir a los reactores experimentales próximos a su fin de vida, y a crear una red de instalaciones complementaria a las grandes fuentes de espalación, que expanda el acceso a las técnicas de experimentación neutrónica. 


\section{Abstract}

Since its discovery in 1930, neutrons have become a fundamental tool in science and technology for the exploration of matter. Unfortunately, its production for experimental purposes requires nuclear processes, and the development of neutron sources presents significant engineering challenges.

Nowadays, the European Union is building what is set to become the world's most powerful accelerator-driven neutron source, the European Spallation Source (ESS). In 2013, Spain's participation in the project, brought together in the ESS-Bilbao consortium, raised the possibility of channelling the in-kind contribution to ESS through the construction of a medium intensity compact neutron source. This source, which would be located in the Vizcaya campus of the University of the Basque Country (UPV/EHU), would serve as a development station and test bench for ESS components, as well as an installation with its own scientific interest for the neutron techniques community.

This thesis aims to design the main components of the neutron source proposed for ESS-Bilbao, evaluating its technical feasibility and range of application.

To do this, the bulk of the work focuses on the design of a neutron production target. In an accelerator-driven neutron source, this component is impacted by accelerated particles, inducing neutron production reactions. In its design, neutron production efficiency is the key feature to optimize. However, the intense energy deposition caused by the impact of the particle beam leads to requirements in terms of refrigerability, thermomechanical stresses, fatigue and irradiation damage, which the target design must meet.

Once the target has been defined, and therefore the neutron output of the source, the relevant components to make the neutrons useful for rearch activities must be specified and optimized. After this, the study concludes with a preliminary assessment of the potential 
range of application of the source.

This design and analysis process has been divided into the following phases:

Chapter 1 briefly introduces the neutronic experimental techniques, together with the different nuclear processes that can lead to neutron production. The elements of an accelerator-driven source are broken down in detail, and a brief review of the state of the art of these devices is presented. Finally, this chapter describes the historical context of the ESS project, and the motivation and interest of the ESS-Bilbao source proposal.

The main design options for the definition of the neutron production target are decided in Chapter 2. The proton accelerator proposed for the ESS-Bilbao facility accelerates the particles up to $50 \mathrm{MeV}$ in 1,5 $\mathrm{ms}$ long pulses, with an average current of 2,25 $\mathrm{mA}$ and a total power of $112,5 \mathrm{~kW}$. The proposed source will therefore be a medium intensity, long pulse source. With this accelerator as starting point, the target material is selected based on the optimization of neutron production. The basic parameters of the required cooling system are then defined, and the effect in terms of stress and temperature of the impact of the particles is studied, evaluating the influence of the thickness of the target and the frequency of beam impact on these magnitudes. Finally, the aspects related to the operational lifetime of the target are analysed.

As a result of the study carried out, it was concluded that the base design solution of the source proposed for ESS-Bilbao is a rotating beryllium target cooled by water in a monophasic forced regime, in which the neutrons are produced by means of stripping reactions. The thermomechanical optimization analyses performed define a target made of 20 beryllium plates of $9 \mathrm{~mm}$ thickness, on which the proton beam impacts with a frequency of $1 \mathrm{~Hz}$ each. With these design parameters, a source with an intensity of $8,0 \cdot 10^{14}$ neutrons per second is configured, achieving acceptable beryllium temperature and stress values. The study of the consequences of the proton irradiation estimates the lifetime of the plates in a minimum of 4 years, according to the most conservative analyses.

In Chapter 3, a structure that embodies the concept of the previously defined rotating target is designed. The thermo-mechanical and thermo-hydraulic calculations carried out define a design capable of housing the beryllium plates, providing them with the necessary cooling through internal channels; and able to withstand both normal operation and accidental loads. The design here presented is the result of an iterative process of analysis 
and improvement, the history of which is described in Appendix A.

In Chapter 4, the main design parameters of the moderator and the reflector of the facility are defined, establishing a reference configuration for the target-moderator-reflector assembly, which is key in the neutronic behaviour of the source. The design process has been oriented to optimize the production of cold neutrons, seeking to increase the capacity of the source for the application of experimental techniques based on neutron scattering. The resulting reference configuration has a solid methane moderator in "slab" configuration, surrounded by a beryllium reflector and three experimental lines. With these design choices, the proposed reference configuration for the ESS-Bilbao source would produce a $5 \mathrm{meV}$ neutron brightness of $3,6 \cdot 10^{10} \mathrm{n} / \mathrm{cm}^{2} \cdot \mathrm{sr} \cdot \mathrm{s}$, and of $1,3 \cdot 10^{11} \mathrm{n} / \mathrm{cm}^{2} \cdot \mathrm{sr} \cdot \mathrm{s}$ for neutrons below $0,4 \mathrm{eV}$. From this reference configuration, several alternative and advanced configurations have been analyzed, such as the inclusion of a second moderator or beryllium filters. The ability of the source to produce thermal neutrons has also been evaluated. Analyses show that using a light water moderator, thermal neutron production would reach $1,4 \cdot 10^{11} \mathrm{n} / \mathrm{cm}^{2} \cdot \mathrm{sr} \cdot \mathrm{s}$.

These neutron brightness values are, approximately, an order of magnitude lower than those of large sources equiped with accelerators in the megawatt power range, based on spallation reactions. However, these sources have a neutron production approximately two orders of magnitude higher than ESS-Bilbao's proposal, so this design presents a high efficiency. This efficiency is particularly evident when considering the complexity and cost of the accelerating structures required in each case.

The preliminary evaluation of the potential applications of the source shows that, with the calculated neutron fluxes, the neutron source proposed for ESS-Bilbao could serve experimental techniques based on activation, neutron transfer and most of the techniques based on neutron scttaring; in addition to other activities such as component irradiation, detector calibration, moderator testing, etc.

In Chapter 5, and as a link to future works, two European projects currently seeking to build neutron sources similar to the one proposed here are referred to. This chapter also describes the prototype manufactured to validate the thermohydraulic aspects of ESS-Bilbao target design.

As a matter of conclusions, gathered in Chapter 6, it can be pointed out that this work 
defines for the first time a medium intensity and high efficiency neutron source based on the stripping reaction, which enables, at much lower costs, neutronic experimental techniques normally only available in large spallation reaction-based installations or experimental fission reactors. These characteristics have recently led to an increase in interest in this type of source, so that they are now considered as candidates for a new generation of neutron sources. This generation is called upon to replace the experimental reactors nearing the end of their operational lifetime and to create a network of facilities complementary to the major spallation sources, expanding the access to neutron experimentation techniques. 


\section{Capítulo 1}

\section{Introducción}

\section{Resumen}

Desde su descubrimiento en 1930, los neutrones se han constituido como una herramienta fundamental en la ciencia y la tecnología para la exploración de la materia. Desafortunadamente, su producción para experimentación requiere de procesos nucleares, y el desarrollo de fuentes de neutrones presenta relevantes desafíos de ingeniería.

En la actualidad, la Unión Europea construye la que está llamada a ser la fuente de neutrones guiada por acelerador más potente del mundo, la European Spallation Source (ESS). En 2013, la participación española al proyecto, aglutinada en el consorcio ESSBilbao, planteó la posibilidad de canalizar la contribución en especie a ESS a través de la construcción, en el campus de Vizcaya de la Universidad del País Vasco (UPV/EHU), de una fuente de neutrones compacta de media intensidad. Esta fuente serviría como estación de desarrollo y banco de pruebas de los componentes de ESS, así como instalación con interés científico propio para la comunidad de técnicas neutrónicas.

En este capítulo se introducen brevemente las técnicas de experimentación con neutrones, además de los diferentes procesos nucleares que pueden dar lugar a su producción. De entre éstos, las reacciones inducidas por partículas aceleradas son las elegidas para configurar las fuentes de ESS y de la propuesta de ESS-Bilbao, por lo que los elementos de una fuente guiada por acelerador se desglosan con detalle, y se hace un breve repaso del estado del arte de estos dispositivos. Para finalizar, se describe el contexto histórico del 
proyecto ESS, la motivación e interés de la propuesta de fuente de ESS-Bilbao y, radicados en éstos, los objetivos del presente trabajo.

\subsection{Los neutrones en la ciencia}

El neutrón es una partícula subatómica constituyente de los núcleos atómicos. Como partícula libre fuera del átomo, los neutrones son inestables y decaen mediante una desintegración $\beta^{-}$con una vida media de $\sim 15$ minutos; por lo que para su aplicación en las distintas técnicas experimentales, es necesario disponer de métodos de producción que permitan obtenerlos en tiempo real con las intensidades adecuadas.

Los neutrones no tienen carga eléctrica, poseen una masa de $m=1,0087$ unidades de masa atómica, spin $1 / 2$, y un momento magnético $\mu=-1,913 \cdot e \hbar 2^{-1} m_{p}^{-1}$; donde $\hbar=h / 2 \pi$ es la constante de Planck reducida, $e$ es la carga eléctrica elemental y $m_{p}$ es la masa del protón. La ausencia de carga eléctrica implica que los neutrones sólo pueden interaccionar con la materia mediante interacciones nucleares y magnéticas. Estas interacciones son de corto alcance y tienen secciones eficaces relativamente bajas, especialmente si se comparan con la interacción de partículas con carga eléctrica, por tanto pueden atravesar espesores significativos de material sin interaccionar. Por otro lado, la interacción directa con el núcleo atómico permite que los neutrones sean sensibles, no sólo al número atómico, sino también al número másico del material.

Estas particularidades convierten a los neutrones en una sonda excepcional para el estudio de la materia, impulsando durante los últimos 60 años el desarrollo de técnicas experimentales basadas en la interacción de los neutrones con la materia. Las diferentes técnicas neutrónicas se pueden agrupar en función del tipo de interacción en el que se basen: transferencia, captura neutrónica o dispersión.

\subsubsection{Transferencia}

Las técnicas de transferencia se basan en la capacidad de los neutrones para atravesar el material, sin discriminar el tipo de interacción ejercida a su paso. Estas técnicas se utilizan típicamente para medir secciones eficaces totales de los materiales [1], magnitud representativa de la probabilidad total de interacción con el material, o bien para producir 
imágenes mediante técnicas de neutrografía [2].

La gran ventaja de los neutrones para la obtención de imágenes mediante técnicas de transferencia, es su sensibilidad no sólo al número atómico del elemento sino también a su número másico. La Figura 1-1 muestra la longitud de dispersión de diferentes elementos ante rayos $\mathrm{X}$ y neutrones, como medida de la probabilidad de interacción en función del número másico del elemento. La probabilidad de interacción de los rayos X es proporcional al cuadrado del número atómico, por lo que los isótopos de un mismo elemento son indistinguibles y los elementos más pesados de la tabla periódica son, en la práctica, opacos a esta radiación.

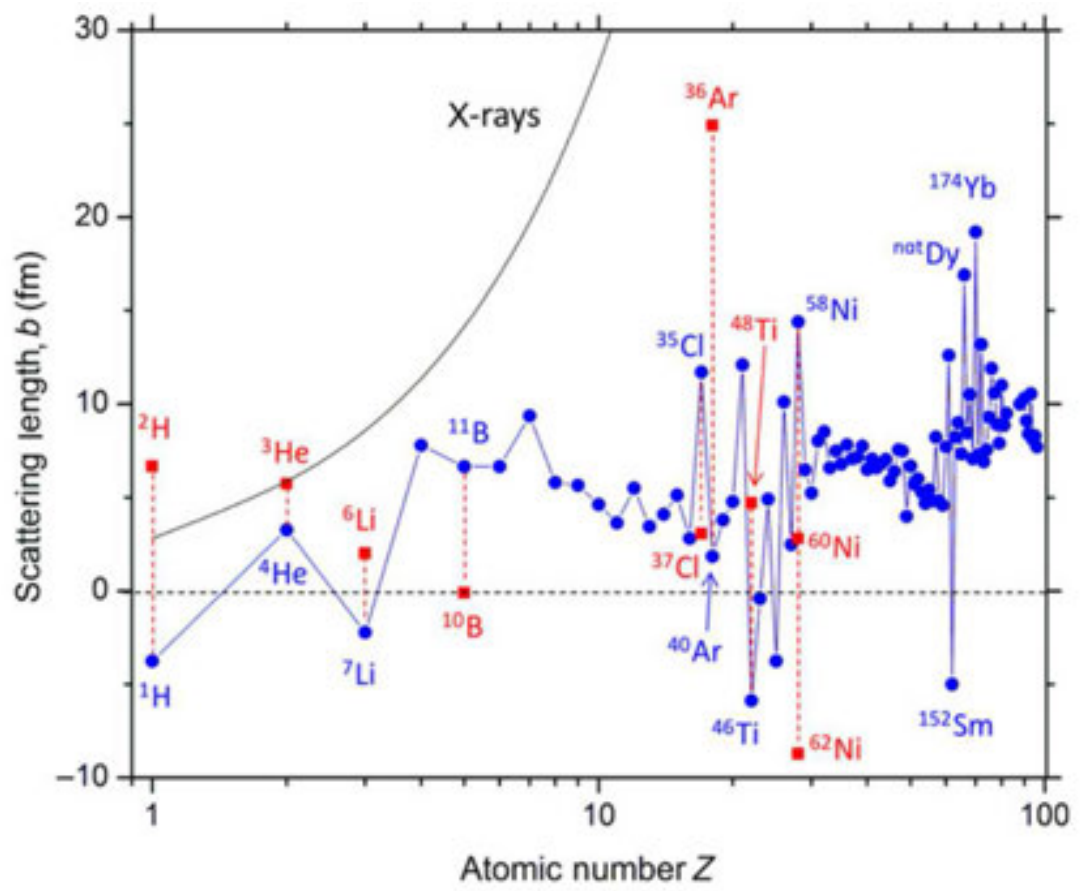

Figura 1-1: Longitud de dispersión de neutrones y rayos $X$ con diferentes elementos en función de su número atómico [3].

En contraposición, la probabilidad de interacción de los neutrones no aumenta con el número másico y es fuertemente dependiente de cada caso concreto, lo que permite distinguir no sólo entre isótopos, sino también entre elementos próximos como el ${ }^{35} \mathrm{Cl}$ y el ${ }^{36}$ Ar. Además no muestra un crecimiento significativo con el número atómico, por tanto los neutrones pueden atravesar también elementos pesados. Estas dos propiedades, alta penetración y sensibilidad al número másico, son la base de las técnicas de imagen por neutrones. 
La Figura 1-2 compara la radiografía y neutrografía de un surtidor de gasolina. En ella se puede observar como los neutrones penetran mejor las partes metálicas y proporcionan un mayor contraste en los materiales hidrogenados. En cambio, los rayos X penetran los elementos ligeros, permitiendo por ejemplo observar los componentes metálicos internos del mango. Ambas técnicas, radiografía y neutrografía, son por tanto complementarias.
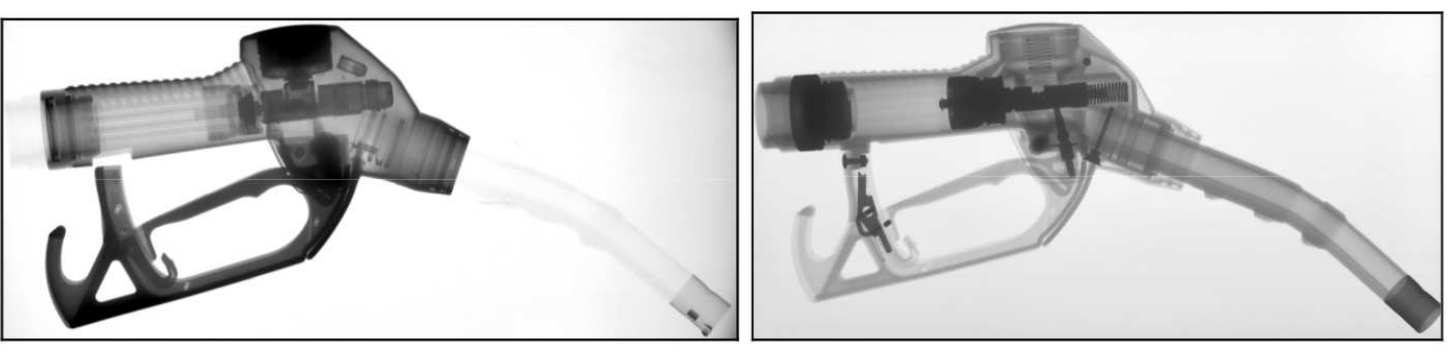

Figura 1-2: Neutrografía (izq.) y Radiografía (der.) de un surtidor de gasolina [2].

\subsubsection{Captura neutrónica}

Tal como se comentó en apartados anteriores, los neutrones interaccionan directamente con los núcleos atómicos, dando lugar a reacciones nucleares. Una de estas reacciones es la captura. En este tipo de reacción el neutrón es absorbido por el núcleo atómico dando lugar a un nuevo isótopo del mismo elemento (Ecuación 1.1).

$$
{ }_{A}^{M} N+n={ }_{A}^{M+1} N
$$

En la mayoría de los casos, este nuevo isótopo es inestable y por tanto emitirá radiación hasta alcanzar la estabilidad. La medida de esta radiación de decaimiento permite identificar el isótopo emisor y, en base a ello, la composición original del material. Este fenómeno da lugar a las técnicas de análisis por activación [4].

El análisis por activación es una técnica que permite estimar de forma muy precisa la composición de un material, detectando concentraciones del orden de $\mu g g^{-1}$. La sensibilidad del método está asociada a la sección eficaz de captura del material, y a la radiación de decaimiento de los isótopos generados por captura.

Estas técnicas de análisis por activación se extendieron durante los años setenta y ochenta con el desarrollo de reactores experimentales de baja potencia, y aún hoy continúan 
representando un papel destacado en estudios de alta precisión.

\subsubsection{Dispersión neutrónica}

Las técnicas de dispersión neutrónica (neutron scattering, en inglés) se basan en colisiones de neutrones de baja energía (i.e. 0.1-100 meV ) con los átomos de la muestra.

Al sufrir dispersión, el neutrón experimenta un cambio en su momento, lo cual se traduce en un cambio en su dirección y/o velocidad. La Figura 1-3 presenta un esquema simplificado del proceso de dispersión neutrónica. En él, un neutrón de baja energía colisiona sin ser absorbido con el material de la muestra, cambiando su momento.

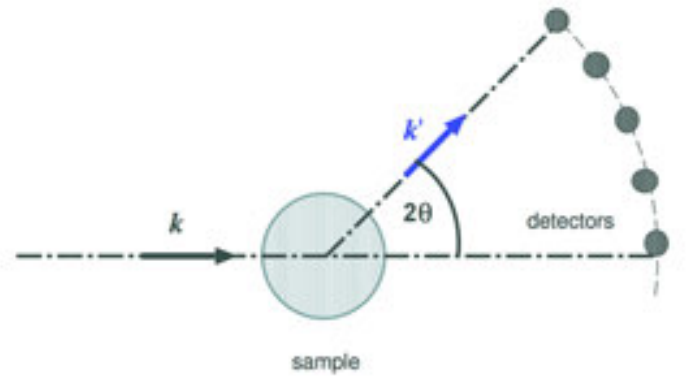

FIGURA 1-3: Esquema de un experimento de dispersión de neutrones [5].

El momento de un neutrón se describe como $\mathbf{p}=\hbar \mathbf{k}$, donde $\mathbf{k}$ es el vector de onda con $|\mathbf{k}|=k=2 \pi / \lambda$, siendo $\lambda$ la longitud de onda asociada. La energía del neutrón viene dada por la Ecuación 1.2

$$
E_{n}=\frac{h^{2}}{2 m \lambda^{2}}=\frac{\hbar^{2} k^{2}}{2 m}
$$

El intercambio de momento sufrido durante la interacción puede describirse mediante el vector intercambio de momento o vector de dispersión, $\mathbf{Q}$, definido como la diferencia entre el vector de onda incidente y dispersado (Ecuación 1.3), siendo $\mathbf{k}^{\prime}$ el vector de onda dispersado. El cambio de momento en el neutrón es pues $\hbar \mathbf{Q}$.

$$
\mathbf{Q}=\mathbf{k}-\mathbf{k}^{\prime}
$$

La relación entre estos vectores queda descrita gráficamente en la Figura 1-4, mediante 
el denominado triángulo de dispersión. Esta construcción pone de manifiesto como la magnitud y dirección del vector dispersión, $\mathbf{Q}$, dependen de las magnitudes de los vectores de onda de los neutrones incidente y dispersado, y del ángulo $2 \theta$ con el que el neutrón es deflectado en el proceso de dispersión, también llamado ángulo de dispersión.

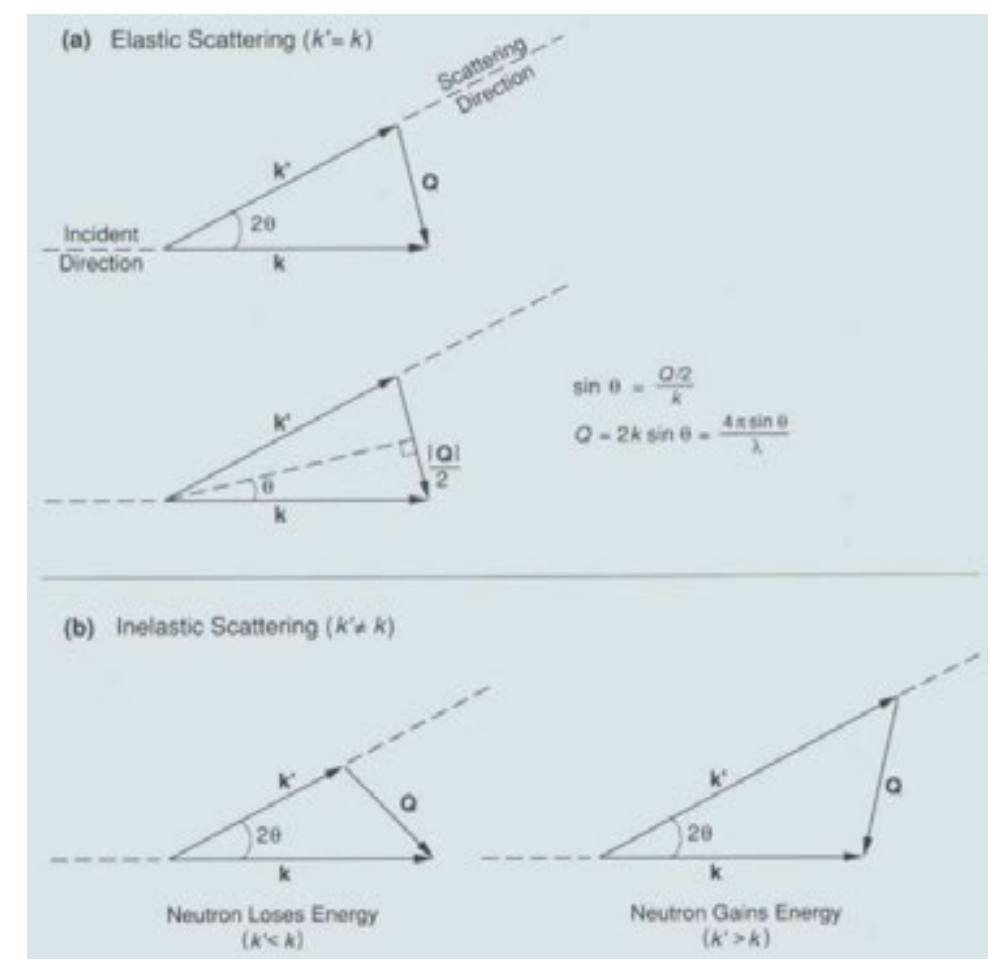

Figura 1-4: Triángulo de dispersión [6]

Podemos notar la variación de energía sufrida por el neutrón como $\epsilon=E-E^{\prime}$. Si durante la dispersión el neutrón cambia de dirección pero mantiene la misma energía, $\epsilon=$ 0 , la dispersión se califica como elástica. En este caso $k=k^{\prime}$ y $Q$ puede calcularse mediante la Ecuación 1.4.

$$
Q=2 k \sin \theta=4 \pi \sin (\theta) \lambda^{-1}
$$

En todos los experimentos de dispersión se mide la intensidad de los neutrones dispersados como una función de las variables $\mathbf{Q}$ y $\epsilon$. Esta intensidad de dispersión, $I(\mathbf{Q}, \epsilon)$, es normalmente conocida como la ley de dispersión neutrónica de la muestra.

Van Hove en [7] demostró que la ley de dispersión de un conjunto de núcleos, puede ser descrita con exactitud en términos de correlaciones temporales entre las posiciones 
de los pares de átomos de la muestra. Su resultado hace uso de la observación de Fermi, que indica que la interacción entre un neutrón y un núcleo puede ser remplazada por un potencial efectivo mucho más débil que la interacción real [8]. Este pseudo-potencial genera la misma dispersión que la interacción real, pero es suficientemente débil como para que se le pueda aplicar la teoría de expansión de perturbaciones de Born [9]. Este resultado implica que $I(\mathbf{Q}, \epsilon)$ es simplemente proporcional a la transformada de Fourier de una función, que da la probabilidad de encontrar dos átomos a una cierta distancia. Así pues, midiendo la intensidad de los neutrones dispersados en función del vector dispersión y de la energía intercambiada, podemos obtener información sobre la posición y el movimiento relativos de los átomos.

El formalismo de Van Hove puede ser manipulado para estudiar efectos de dos tipos. El primer tipo es la dispersión coherente, en la cual el vector de onda del neutrón interacciona con toda la muestra como una unidad, de tal manera que los vectores de onda dispersados por distintos núcleos interfieren entre sí. Este tipo de dispersión depende de la distancia relativa entre los átomos y, por tanto, da información sobre la estructura de la muestra. La dispersión coherente elástica da información sobre la estructura en equilibrio, mientras que la inelástica, en la que el neutrón ve variada su energía, proporciona información sobre el movimiento colectivo de los átomos, como por ejemplo el desplazamiento de ondas vibracionales en una estructura cristalina.

En el segundo tipo de dispersión, la dispersión incoherente, el vector de onda del neutrón interacciona independientemente con cada núcleo de la muestra, y por tanto los vectores de onda dispersados en distintos núcleos no interfieren, sino que las intensidades de dispersión de cada núcleo se suman. Este fenómeno puede darse, por ejemplo, como resultado de la interacción con el mismo átomo en distintos tiempos y posiciones, por lo que puede emplearse en el estudio de la difusión atómica.

La dispersión coherente elástica puede ser empleada para el estudio de la estructura atómica, empleando neutrones de una longitud de onda en el rango de las distancias entre los planos atómicos del material. La relación que liga la longitud de onda de los neutrones empleados como sonda, las distancias entre planos atómicos, $d$, y el ángulo de dispersión, es conocida como Ley de Bragg (Ecuación 1.5) y es la misma que la descubierta en 1912 para los rayos X; por tanto la distancia entre planos se puede calcular a través de la medida 
de $Q$. Esta técnica también es conocida como difracción.

$$
n \lambda=2 d \sin \theta
$$

Para emplear la dispersión coherente elástica en el estudio de estructuras mayores que las distancias interatómicas, es necesario conseguir que la longitud de onda de la sonda sea mayor, ya sea disminuyendo el ángulo de dispersión o aumentando la longitud de onda de los neutrones. En la práctica, para estudiar estructuras de un tamaño en el rango del de los virus o los polímeros, se necesitan longitudes de onda mayores que $5 \AA^{\mathrm{I}}$ (i.e. neutrones con una energía menor que $\approx 3,3 \mathrm{meV}$ ) y ángulos de dispersión menores a un grado. Debido a esta característica, esta técnica es conocida como dispersión neutrónica de ángulo pequeño (SANS Small Angle Neutron Scattering).

Por otro lado, los neutrones también son capaces de interaccionar con la materia en virtud de su momento magnético, más concretamente con otras partículas magnéticas como los electrones. La mayoría de los átomos en la materia están emparejados de tal manera que el momento magnético de uno de ellos, cancela el del otro miembro del par. En algunos compuestos, no todos los electrones de la capa de valencia de los átomos están emparejados, y los neutrones pueden sufrir dispersión por interacción con los momentos magnéticos resultantes. Así pues, se pueden emplear experimentos de difracción para medir la densidad de electrones desemparejados.

Las técnicas de dispersión neutrónica engloban a una gran familia de técnicas experimentales, aplicables al análisis de la materia condensada. A modo indicativo se listan las más significativas a continuación:

- Dispersión elástica.

- Dispersión de ángulos pequeño (SANS).

- Difracción de polvos y cristales.

- Reflectometría.

- Disepersión inelástica.

- Espectroscopía de tres ejes.

\footnotetext{
${ }^{\mathrm{I}} \mathrm{Un}$ angstrom $(\AA)=10^{-10} \mathrm{~m}$
} 
- Dispersión de geometría inversa (Backscattering).

- Neutron spin echo.

No es objetivo de este documento abordar esta temática en profundidad, por lo que se recomienda al lector interesado consultar bibliografía especializada [6, 3]. Baste remarcar que las técnicas de dispersión de neutrones son una herramienta imprescindible para el estudio de la materia desde hace más de 60 años, y que continúan inmersas en un intenso desarrollo. Este amplio rango de aplicación, y los avances técnicos y científicos en este campo, demandan la creación de fuentes de neutrones de baja energía, para explorar nuevos rangos de transferencia de momento y aplicar nuevas técnicas de análisis.

\subsection{Reacciones de producción de neutrones}

Como ya se ha mencionado, los neutrones fuera del átomo son partículas inestables con una vida media relativamente corta, y por tanto es necesario producirlos en el momento de su utilización. La producción de neutrones se apoya en ciertas familias de reacciones nucleares, en base a las cuales nacen los diferentes conceptos de fuente. Dichos procesos son:

- Procesos de desintegración radiactiva.

- Reacciones de fusión.

- Reacciones de fisión.

- Reacciones de radiación de frenado (Bremhsstrahlung).

- Reacciones de extracción (Stripping).

- Reacciones de espalación.

A lo largo de los siguientes apartados se realizará una breve descripción de los diferentes tipos de reacción y los conceptos de fuente asociados. Información más detallada puede encontrarse en [10].

\subsubsection{Procesos de desintegración radiactiva}

La producción de neutrones mediante procesos de desintegración se basa en utilizar isótopos inestables que, o bien emiten directamente neutrones, o bien producen partículas 
de alta energía que generan procesos de producción de neutrones. Este tipo de fuentes suelen ser conocidas como fuentes isotópicas.

Para el primer proceso se utilizan elementos transuránidos inestables, como ${ }_{94}^{240} \mathrm{Pu}$, ${ }_{96}^{250} \mathrm{Cm} \mathrm{o}{ }_{98}^{252} \mathrm{Cf}$, que sufren procesos de fisión espontánea. Estos procesos de fisión producen una cierta cantidad de neutrones, de forma análoga a los procesos de fisión inducidos por capturas neutrónicas que se describirán más adelante. La vida media de estos isótopos va desde los 2,64 años del ${ }_{98}^{252} \mathrm{Cf}$ hasta los 8300 años del ${ }_{96}^{250} \mathrm{Cm}[11]$.

El segundo concepto de fuente isotópica se basa en la combinación de un elemento de alto número másico, emisor de partículas $\alpha$ de alta energía, y un elemento ligero que produce neutrones por reacción con las primeras. Las más comunes son las constituidas por ${ }_{95}^{241} \mathrm{Am}-{ }_{4}^{9} \mathrm{Be}$ (el proceso se describe como ejemplo en las Ecuaciones 1.6 y 1.7 ), ${ }_{84}^{210} \mathrm{Po}-{ }_{4}^{9} \mathrm{Be}$ o ${ }_{95}^{241} \mathrm{Am}-{ }_{3}^{7} \mathrm{Li}$. La evolución temporal de estas fuentes depende del periodo de semidesintegración del emisor alfa, que varían desde los 138 días del ${ }_{84}^{210} \mathrm{Po}$, hasta 458 años en el caso del ${ }_{95}^{241} \mathrm{Am}[11]$.

$$
\begin{aligned}
& { }_{95}^{241} \mathrm{Am} \longrightarrow{ }_{93}^{237} \mathrm{~Np}+{ }_{2}^{4} \mathrm{He} \\
& { }_{4}^{9} \mathrm{Be}+{ }_{2}^{4} \mathrm{He} \longrightarrow{ }_{6}^{12} \mathrm{C}+n
\end{aligned}
$$

El número de neutrones emitido por estas fuentes isotópicas se sitúa en torno a los $10^{6}-10^{9} \mathrm{n} / \mathrm{s}$ [11], y puesto que utilizan isótopos que no están presentes en la naturaleza, es necesario inducir reacciones nucleares previas con las que generar los isótopos inestables.

\subsubsection{Reacciones de fusión}

En una reacción de fusión dos átomos ligeros se combinan para producir elementos más pesados liberando energía. En algunos casos, este proceso emite neutrones tal como muestran las siguientes reacciones entre isótopos del hidrógeno.

$$
\begin{aligned}
& { }_{1}^{2} \mathrm{D}+{ }_{1}^{3} \mathrm{~T} \longrightarrow{ }_{2}^{4} \mathrm{He}+n \\
& { }_{1}^{2} \mathrm{D}+{ }_{1}^{2} \mathrm{D} \longrightarrow{ }_{2}^{3} \mathrm{He}+n
\end{aligned}
$$

Para producir una reacción de fusión entre dos isótopos ligeros es necesario vencer la 
barrera de potencial electrostático coulombiano. Por lo tanto, estas reacciones necesitan una energía umbral de las partículas situada en el rango de los cientos de $k e V$. La Figura 15 muestra la sección eficaz y la reactividad de las distintas reacciones de fusión de átomos ligeros, en función de la energía de la colisión. Se puede apreciar que la reacción más probable, y por tanto la que permitirá producir neutrones más fácilmente, es la fusión entre deuterio y tritio ${ }^{\mathrm{II}}$.

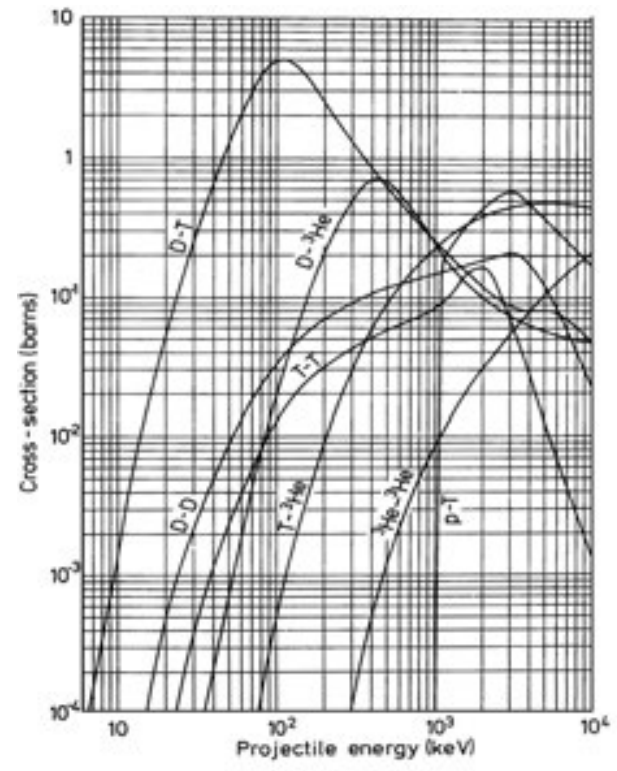

(a)

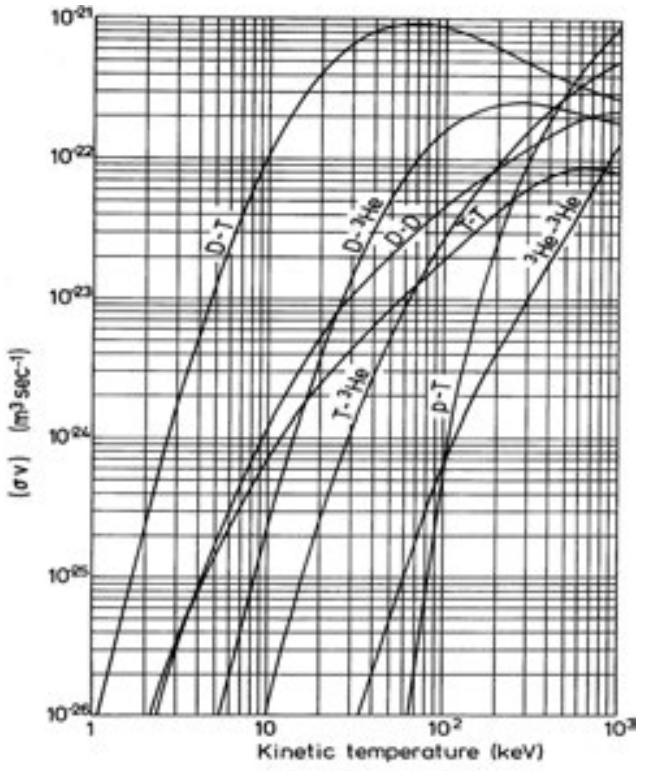

(b)

Figura 1-5: Sección eficaz (a) y reactividad (b) de las reacciones de fusión de mayor probabilidad a bajas energías, en función de la energía del proyectil y de la energía cinética relativa respectivamente [12].

Para conseguir colisiones entre núcleos ligeros con la energía necesaria para dar lugar a reacciones de fusión, se plantean dos métodos:

- Sistema en equilibrio termodinámico: Se configura un plasma en el cual los átomos se encuentran en una situación de equilibrio termodinámico. Es decir, la energía cinética media de los átomos del sistema se encuentra en el rango de los cientos de keV y por tanto, al producirse colisiones entre ellos, se generan reacciones de fusión. Este método se postula como candidato para los reactores de fusión para generación de energía mediante el confinamiento inercial [13] o el magnético [14].

- Acelerador de partículas: Se utiliza un acelerador de iones ligeros para producir un

\footnotetext{
${ }^{\mathrm{II}}$ Obviando la problemática de la obtención del tritio.
} 
haz de partículas con una energía cinética elevada, que inciden sobre una matriz de átomos en reposo. Este proceso, a pesar de ser menos eficiente que el primero desde el punto de vista energético, permite configurar sistemas compactos de producción de neutrones, mucho más sencillos que los reactores necesarios para conseguir confinamiento del plasma.

El primer método se encuentra en desarrollo con el objetivo de construir centrales de potencia para la producción eléctrica, y su utilización como fuente de neutrones no parece posible a corto y medio plazo. Por el contrario, el segundo método se lleva utilizando desde los años sesenta para producir fuentes compactas de neutrones. Estas fuentes de neutrones utilizan típicamente aceleradores de baja energía $(\sim 100-500 \mathrm{keV})$ para producir un haz de iones de deuterio o de tritio, que se hace impactar sobre un material blanco, generalmente un compuesto químico deuterado, dando lugar a las reacciones de fusión.

El concepto de fuente basada en reacciones de fusión inducidas por acelerador se encuentra, en la práctica, limitado a niveles de producción neutrónica entre $10^{8}(\mathrm{DD})-10^{10}$ (DT) $\mathrm{n} / s[15]$.

\subsubsection{Reacciones de fisión}

La reacción de fisión es un proceso conocido desde 1939 por el cual un átomo pesado, generalmente uranio, se escinde produciendo dos o más fragmentos. Este proceso puede venir desencadenado por la absorción de un neutrón o ser un proceso espontáneo. En el caso del uranio, la fisión genera la emisión de uno o dos neutrones (e.g. Ecuación 1.10), lo cual permite mantener una reacción en cadena. Desde su descubrimiento, esta reacción ha sido la base de la tecnología nuclear, permitiendo el desarrollo tanto de los reactores de producción energética, como de los reactores de alto flujo destinados a investigación.

$$
n+{ }_{92}^{235} U \rightarrow{ }_{92}^{236} U^{*} \rightarrow n+{ }_{39}^{96} Y^{*}+{ }_{53}^{139} I^{*}
$$

Los sistemas basados en reacciones de fisión típicamente se configuran como sistemas autosostenidos, en los cuales los neutrones generados en una reacción de fisión son absorbidos por núcleos de uranio, dando lugar a nuevas reacciones de fisión. Este proceso 
ha demostrado ser la forma más económica de producir neutrones a gran escala para investigación. Prueba de ello son los reactores del Centro de Investigación Neutrónica del National Institute of Standards and Technology (NIST) [16] en EE.UU y el High-Flux Reactor (HFR) del Institut Laue-Langevin (ILL) [17] en Francia, en operación desde los años sesenta. Ambas son, a día de hoy, las fuentes de neutrones para investigación más potentes del mundo en cuanto a cantidad de neutrones producida.

A pesar de la eficiencia de los sistemas basados en reacciones de fisión, el proceso de reacción autosostenida incorpora sus propias limitaciones ya que, al igual que las fuentes isotópicas, se trata de sistemas que emiten continuamente neutrones ${ }^{\mathrm{III}}$, lo cual condiciona sus aplicaciones.

Sin ánimo de entrar en detalle, las fuentes basadas en reacciones de fisión pueden configurar fuentes del orden $10^{21}-10^{22} \mathrm{n} / \mathrm{s}$. No obstante, debe tenerse en cuenta que estas fuentes aparecen distribuidas en grandes volúmenes, lo que limita los valores de flujo a niveles $10^{14}$ y $10^{15} \mathrm{n} / \mathrm{cm}^{2} s$ [15]. En los años ochenta, EE.UU planteó el proyecto Advanced Neutron Source para la construcción de una fuente de neutrones basada en un reactor de alto flujo. El nuevo reactor estaría refrigerado por agua pesada y presentaría una potencia de $350 M W$ térmicos [19], significativamente mayor que la del HFR de ILL con $58 M W$. Sin embargo, el estudio de diseño demostró que los desafíos técnicos, junto con los crecientes requisitos de seguridad, reducían el interés del concepto; optándose finalmente por una fuente controlada por acelerador [20].

\subsubsection{Fuentes de bremsstrahlung}

Las fuentes de neutrones basadas en radiación de frenado, o Bremhsstrahlung, proliferaron durante los años setenta y ochenta siguiendo el desarrollo de los aceleradores de electrones, y alcanzaron su máximo desarrollo con el acelerador lineal Helios, en Harwell (U.K) [21], ahora en desmantelamiento. A pesar de que la tecnología alcanzó su madurez en los años ochenta, existen instalaciones dedicadas a ciertos nichos científicos que continúan utilizando este concepto, como el Oak Ridge Electron Linear Accelerator (ORELA)

\footnotetext{
${ }^{\mathrm{III}} \mathrm{La}$ única excepción es el reactor ruso IBR-2 [18], de $2 \mathrm{MW}$ de potencia y $\mathrm{PuO}_{2}$ como combustible, cuyo funcionamiento pulsado se logra haciendo rotatorio una parte de su reflector, lo cual genera pulsos periódicos de criticidad.
} 
[22] en EE.UU, la fuente del Centro Atómico de Bariloche en Argentina [23] o el acelerador lineal de Geel (GELINA) [24] en Bélgica.

Estos dispositivos utilizan un proceso en dos etapas partiendo de un haz de electrones. Los electrones, con energías de 10-45 MeV inciden sobre un blanco de material de alto número atómico, lo que produce un perdida brusca de energía. Los electrones al frenarse emiten radiación $\gamma$ muy intensa. Estos rayos $\gamma$ son capaces de interaccionar con el material del blanco, produciendo reacciones $(\gamma, \mathrm{n})$. La eficiencia global del proceso se sitúa en 0,05 $\mathrm{n} / e^{-}[25]$.

La producción de neutrones mediante la reacción $(\gamma, \mathrm{n})$ aumenta rápidamente con la energía de los electrones, hasta los $30-45 \mathrm{MeV}$. Por encima de este umbral la producción de neutrones satura, de tal manera que ésta puede ser descrita para la mayoría de materiales pesados mediante la Ecuación 1.11; donde la producción neutrónica, $N$, se obtiene en $\mathrm{n} / s$ por $k W$ de potencia del haz de electrones, en función del número atómico del blanco, $Z$. Esta correlación es cierta excepto para el caso del $U$, donde la producción neutrónica es aproximadamente el doble debido a los procesos de fisión que se inducen en el material [25].

$$
N(Z)=9,3 \cdot 10^{10} Z^{0,73}
$$

Este tipo de fuentes presentan dos grandes ventajas. Por una parte, los aceleradores de electrones son relativamente simples comparados con sus homólogos para otras partículas cargadas, lo que permite manejar corrientes muy elevadas de electrones a un coste reducido. Por otra, los aceleradores de electrones pueden generar pulsos de electrones con duraciones del orden de $\mu s$, permitiendo obtener fuentes de neutrones con tiempos característicos de entre 1-10 $\mu s$, lo cual es de utilidad en ciertas áreas científicas.

Como contrapartida, este tipo de fuentes de neutrones nacen acompañadas de campos de radiación $\gamma$ muy intensos asociados al frenado de los electrones, lo que aumenta considerablemente los requisitos de blindaje, el daño por radiación y la carga térmica a disipar. La producción máxima de este tipo de fuentes basadas en radiación de frenado se ve limitada por la deposición energética en el blanco (2000 MeV por neutrón generado), lo que a su vez limita la corriente de electrones y, por extensión, la fuente de neutrones a 
valores por debajo de $10^{14} \mathrm{n} / \mathrm{s}$ [21].

\subsubsection{Reacciones de espalación}

La reacción de espalación fue descrita por primera vez por Seaborg en 1937 [26]. Esta reacción comenzó a utilizarse para configurar fuentes intensas de neutrones en los años ochenta, con la construcción de la fuente KENS en el Laboratorio Nacional de Física de Altas Energías (KEK) de Japón [27], la Intense Pulsed Neutron Source (IPNS), primera fuente pulsada abierta a usuarios externos, en el Laboratorio Nacional de Argonne (EE.UU), y la fuente ISIS [28] en el Rutherford Appleton Laboratory (UK).

La espalación se trata de una reacción nuclear en dos etapas, inducida por un hadrón de alta energía (generalmente un protón) al incidir sobre el núcleo de un elemento pesado. Para que el hadrón dé lugar al proceso de espalación, debe tener una longitud de onda asociada, $\lambda_{p}$ (Ecuación 1.12), del orden de $1 \mathrm{fm}^{\mathrm{IV}}$, de modo que pueda interaccionar de forma directa con los nucleones del núcleo atómico. En el caso de los protones, el umbral de la reacción se sitúa entre 70 y $80 \mathrm{MeV}$.

$$
\lambda_{p}=\frac{h}{\sqrt{2 m_{p} E_{p}}}
$$

La primera fase de la reacción se conoce como cascada intranuclear [29, 30]. En ella el hadrón incidente colisiona de forma directa con los nucleones, transmitiéndoles una parte de su energía cinética. Si estos nucleones se encuentran próximos a la superficie del núcleo y tienen energía suficiente, lo abandonan. Los procesos de colisión de esta primera fase conservan la cantidad de movimiento del proyectil inicial, por lo que dan lugar a una emisión de partículas de alta energía con una fuerte direccionalidad.

Finalizada la primera fase de la reacción, el núcleo atómico se encuentra en una configuración inestable y sufre un proceso conocido como evaporación/fisión [31]. Mediante este proceso, el núcleo busca la estabilidad emitiendo nucleones de su superficie o bien fisionándose. Este proceso emite partículas de menor energía que la cascada intranuclear y lo hace de forma isótropa.

La reacción de espalación es el proceso de producción de neutrones basado en iones

\footnotetext{
${ }^{\mathrm{IV}}$ Un femtómetro $(\mathrm{fm})=10^{-15} \mathrm{~m}$
} 
acelerados con mayor razón de neutrones producidos por partícula incidente, $\approx 10-30$ $\mathrm{n} /$ partícula. La gran energía de las partículas incidentes empleada en este tipo de fuentes, por lo general superior a los $500 \mathrm{MeV}$, hace que el rango de alcance de éstas en el material blanco sea elevado, lo que contribuye a distribuir la deposición energética en un volumen amplio. Este hecho ha permitiendo configurar fuentes con potencias de haz por encima del $M W$, algunas de las cuales se describirán en la Sección 1.4.1. El máximo exponente de esta tecnología es el proyecto de la European Spallation Source (ESS), actualmente en fase de construcción en Lund (Suecia), con una potencia de haz de $5 M W$ y una producción neutrónica estimada en $\sim 10^{18} \mathrm{n} / s$ [32]. El histórico de este proyecto se describirá en la Sección 1.5.

La ventaja de este tipo de reacciones como fuente intensa de neutrones es que, a diferencia de los reactores de fisión, puede constituir una fuente pulsada. Esto permite que los neutrones emitidos puedan ser seleccionados mediante técnicas de tiempo de vuelo. La gran contrapartida es el elevado coste de construcción y operación de las estructuras de aceleración necesarias para producir partículas de alta energía.

Otro gran condicionante de este tipo de fuentes es el espectro energético de los neutrones producidos. La Figura 1-6 muestra el espectro de los neutrones producidos en una reacción típica de espalación, respecto al espectro de fisión. Ambos espectros presentan su valor máximo en torno a $1 \mathrm{MeV}$, sin embargo la primera fase de la reacción de espalación genera una cola de neutrones de muy alta energía, alcanzando prácticamente la energía de la partícula incidente. Estos neutrones son muy difíciles de blindar y por tanto tienen un importante efecto en los costes de construcción de la instalación.

Por último, los productos resultantes de las reacciones de espalación de la mayoría de los núcleos pesados constituyen un amplio rango de isótopos inestables, que originan un importante nivel de radiación y calor residual tras el apagado del acelerador. Aunque esta potencia residual es sensiblemente inferior a la de los productos de fisión, debe ser cuidadosamente considerada en el diseño de los sistemas de refrigeración y seguridad de las fuentes de neutrones por espalación.

\subsubsection{Reacciones de extracción (Stripping)}

Las reacciones nucleares pueden clasificarse, en función de su tiempo característico, en reacciones directas o de núcleo compuesto. Las reacciones directas tienen lugar en escalas 


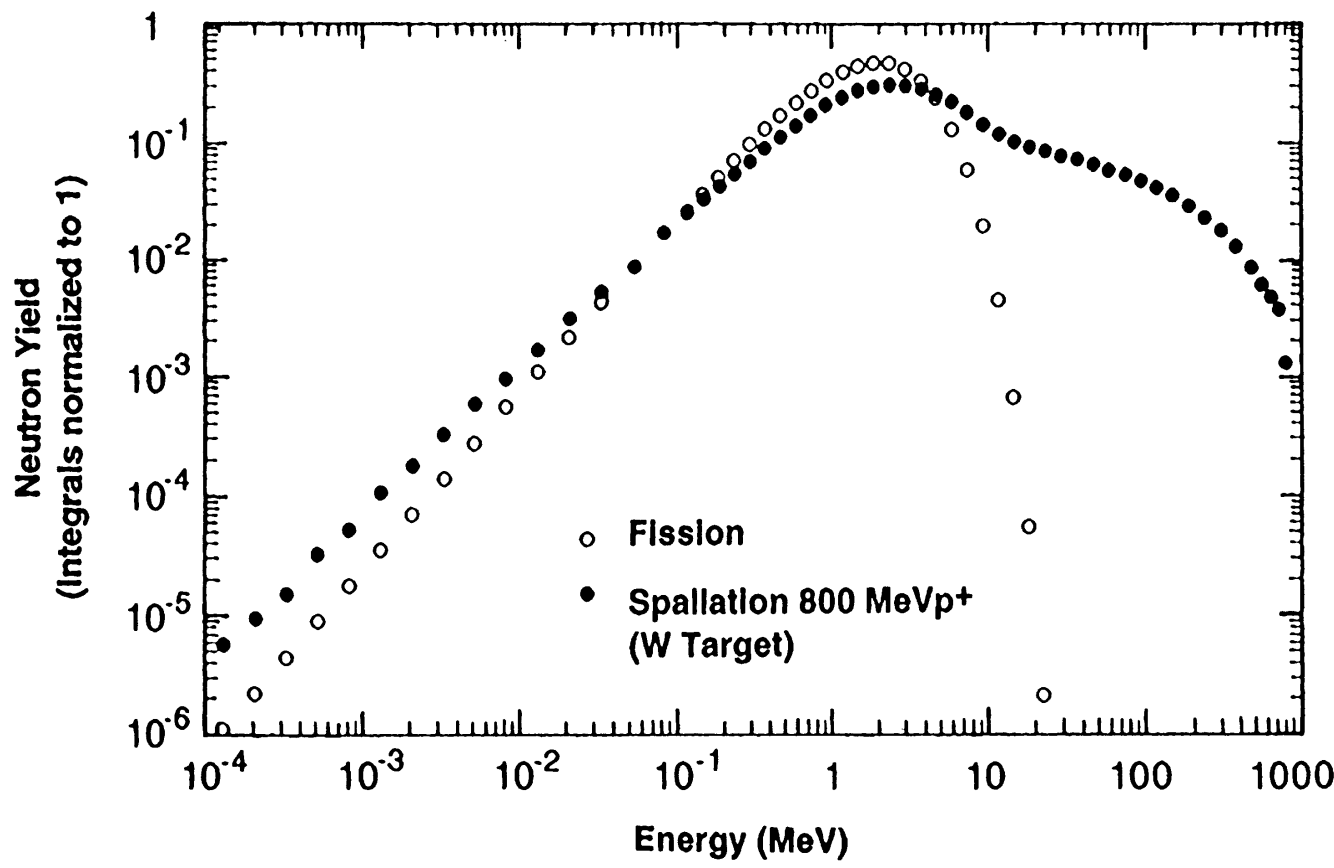

Figura 1-6: Espectro energético de la reacción de fisión del ${ }_{92}^{235} \mathrm{U}$ comparado con el de espalación producida por protones de $800 \mathrm{MeV}$ impactando en $\mathrm{W}$ [33].

de tiempo comparables al que necesita la partícula incidente para atravesar el núcleo $(\sim$ $10^{-22} s$ ). Dado que la partícula incidente interacciona directamente con los nucleones, la conservación de la cantidad movimiento hace que este tipo de reacción presente una fuerte direccionalidad en la emisión de las partículas emergentes. Un ejemplo de este tipo de reacción sería la fase de cascada intranuclear de la reacción de espalación antes mencionada.

Por el contrario, en las reacciones de núcleo compuesto, la partícula incidente se integra en el núcleo generando un estado inestable, que decae en tiempos considerablemente superiores al de las reacciones directas $\left(>10^{-16} s\right)$. La formación del núcleo compuesto y su correspondiente decaimiento implica que la reacción pierde la direccionalidad, ya que el decaimiento es un proceso isótropo. La fase evaporación/fisión de la reacción de espalación es, por tanto, un ejemplo de reacción de núcleo compuesto.

La reacciones de extracción son procesos de interacción directa producidos cuando un ion ligero, generalmente hidrógeno o deuterio, impacta sobre un blanco de bajo número másico ( $\mathrm{Li}, \mathrm{Be}, \mathrm{C}$ ) emitiendo neutrones. Conforme a lo anteriormente referido, estas reacciones presentan una dirección preferencial para la emisión de neutrones. La Figu- 
ra 1-7 muestra la sección eficaz de producción de neutrones para diferentes procesos de extracción.

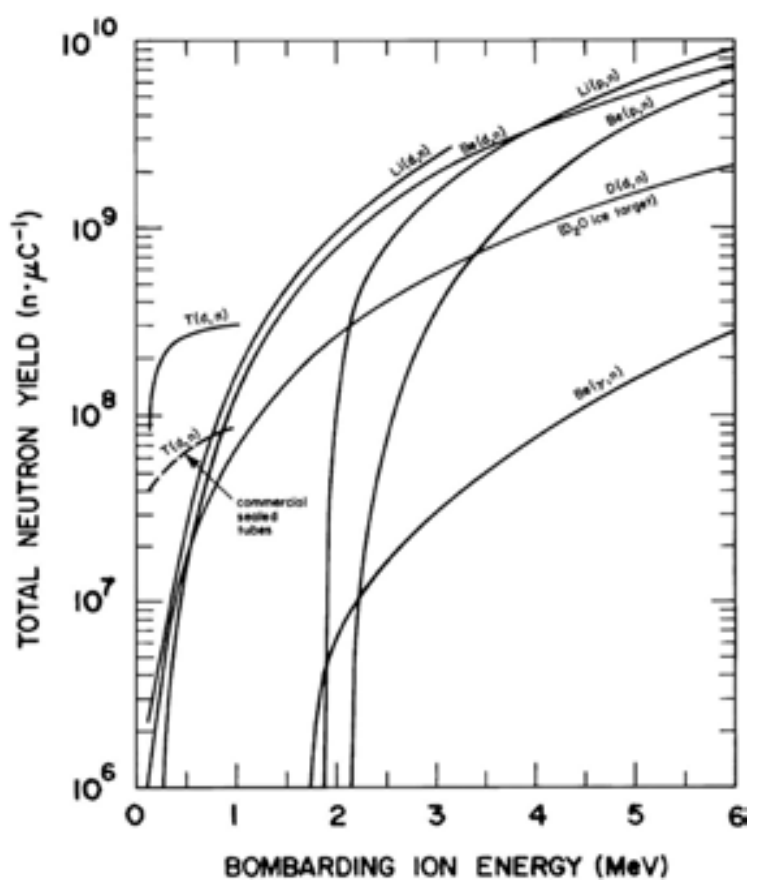

Figura 1-7: Producción de neutrones por $\mu C$ de haz impactando sobre blancos espe$\operatorname{sos}^{V}$ ([34] con datos provenientes de [35]).

La eficiencia de este tipo de reacciones, en términos de neutrones producidos por protón incidente, es considerablemente inferior al de las reacciones de espalación. Esto se debe, fundamentalmente, a que la energía de la partícula incidente es órdenes de magnitud inferior y el núcleo blanco es relativamente pequeño, lo cual limita las posibilidades de extracción de neutrones. Los valores típicos de producción de neutrones están comprendidos entre $10^{-3}$ y $10^{-1} \mathrm{n} /$ partícula, por lo que históricamente estas reacciones han sido empleadas en fuentes de baja intensidad con niveles de producción similares a las instalaciones de Bremhsstrahlung. Sin embargo, las reacciones de extracción presentan varias ventajas importantes que han despertado el interés de la comunidad científica a lo largo de los últimos años:

- Baja energía de la partícula incidente. Las reacciones de interacción directa comienzan a aparecer para energías del proyectil entre 1-30 $\mathrm{MeV}$, muy por debajo de los

\footnotetext{
${ }^{\mathrm{V}}$ i.e. con espesor suficiente para detener todas las partículas incidentes.
} 
niveles necesarios para inducir reacciones de espalación, lo que reduce de forma considerable el coste de los equipos de aceleración.

- La energía media de los neutrones producidos es considerablemente inferior al de las reacciones de espalación, lo cual reduce los requisitos de blindaje.

- Los residuos de la reacción son elementos relativamente estables, lo que da lugar a una producción muy baja de residuos radiactivos, y por tanto su gestión presenta una problemática mucho menor que la asociada a reacciones de fisión o espalación.

Estas ventajas, unidas al incremento de las corrientes admisibles en aceleradores lineales, han permitido la utilización de este tipo de reacciones para configurar fuentes de neutrones compactas con términos fuente $<10^{14} \mathrm{n} / \mathrm{s}$, como la Low Energy Neutron Source (LENS) [36] de la Universidad de Indiana (EE.UU), o la fuente RIKEN Accelerator-driven compact neutron source (RANS) [37] en el centro de investigación RIKEN (Japón). Estos y otros ejemplos de fuente compacta se describen en la Sección 1.4.3.

Como un caso excepcional dentro de las fuentes de neutrones basadas en reacciones de extracción, aparece el proyecto de la International Fusion Materials Irradiation Facility (IFMIF) [38]. IFMIF es un proyecto de instalación destinada a la irradiación de materiales para tecnología de fusión nuclear, que plantea utilizar reacciones de extracción $(\mathrm{D}, \mathrm{Li})$ para generar un espectro neutrónico similar al esperable en un futuro reactor de fusión basado en la reacción $(\mathrm{D}, \mathrm{T})$. El diseño de IFMIF está orientado a la creación de una fuente de neutrones de gran intensidad con un término fuente de $\sim 10^{17} \mathrm{n} / \mathrm{s}$.

\subsection{Elementos constitutivos de una fuente de neutrones guiada por acelerador}

Dado que el presente trabajo está encaminado al diseño de una fuente guiada por acelerador, introducimos a continuación los elementos constitutivos básicos de este tipo de fuentes. Información más completa puede ser consultada en las referencias empleadas $[10,39]$. 


\subsubsection{Acelerador}

El acelerador es el encargado de dotar a las partículas incidentes de la energía necesaria para inducir las reacciones productoras de neutrones. La generación del haz de partículas se produce gracias a la acción secuencial de distintos dispositivos que configuran la cadena de aceleración. Los elementos generales de dicha cadena son:

- Fuente de iones/electrones: Este dispositivo produce las partículas a acelerar. El tipo de fuente depende del tipo de partícula. Los electrones son generados por un cátodo. Este puede ser un cátodo caliente (i.e. la emisión de electrones se produce al calentar un material), un cátodo frío, un fotocátodo o un cátodo de radiofrecuencia. Los protones en cambio pueden producirse en distintos tipos de fuentes [40]. El proceso de producción implica la generación de un plasma de hidrógeno, que es extraído y transportado a las estructuras aceleradoras. Dicho plasma, compuesto por los núcleos y los electrones del hidrógeno, es confinado mediante resonancia electrónica de ciclotrón (ECR) [41], una trampa Penning [42] o algún otro tipo de campo electromagnético en el que las trayectorias cerradas de las partículas realimentan la ionización. La extracción de las partículas cargadas se realiza mediante una plataforma de alto voltaje. La energía de las partículas a la salida de la fuente es normalmente de algunas decenas de $\mathrm{keV}$.

- Sección de baja energía o Low Energy Beam Transport (LEBT): Esta sección, formada normalmente por solenoides, transporta y condiciona el haz hasta la primera estructura aceleradora.

- Cuadrupolo de radiofrecuencia (RFQ): Teorizado en 1970 [43], el cuadrupolo de radiofrecuencia es la sección que enfoca, divide en pulsos y acelera ligeramente el haz continuo proveniente de la fuente. Este dispositivo permite la preparación y transferencia eficiente de haces de alta corriente y baja energía para su aceleración posterior. La energía típica de las partículas a la salida del RFQ es de unos pocos $\mathrm{MeV}$.

- Sección de media energía o Medium Energy Beam Transport (MEBT): La función de esta sección de la cadena de aceleración, es condicionar el haz resultante del RFQ de modo que sea adecuado para las subsiguientes estructuras aceleradoras. 
Entre otras funciones, el MEBT puede tener como misión modificar el tamaño y la distribución temporal de los pulsos, así como eliminar el halo o las colas de éstos mediante elementos interceptores.

- Estructuras aceleradoras: El tipo y longitud de las estructuras aceleradoras varía desde la decena de metros para aceleradores de electrones, hasta los cientos de metros para los aceleradores de protones de las grandes instalaciones. Energías de miles de $M e V$ pueden alcanzarse con estructuras como los Drift-Tube Linac (DTL) [44, 45], los Coupled Cavity Linac (CCL) [46] o los Side Coupled Drift-Tube Linac [47] (SDTL). Lo común en las instalaciones recientes es emplear estructuras superconductoras a partir de los $50-80 \mathrm{MeV}$, no obstante, existen casos como el del acelerador lineal del Los Alamos Neutron Science Center (LANSCE. Los Álamos, EE.UU) [48], construido a principios de los setenta, en el que los protones alcanzan $800 \mathrm{MeV}$ con estructuras convencionales.

- Elementos ópticos: La finalidad de estos elementos, fundamentalmente cuadrupolos, es enfocar o desenfocar el haz para ayudar a su transporte.

- Estructuras acumuladoras: Estas estructuras, sincrotrones o anillos compresores, son las encargadas de acumular el haz inyectado por el acelerador lineal uniendo varios pulsos en uno, comprimir la longitud de este desde típicamente cientos de $\mu s$ o algunos $m s$ hasta duraciones inferiores al $\mu s$, y también de incrementar la energía del haz por medio de trayectorias circulares sucesivas. Dichas estructuras son necesarias para fuentes de pulso corto $(\lesssim 1 \mu s)$ pero no así para fuentes continuas o de pulso largo $(\sim 1 \mathrm{~ms})$. Una vez los pulsos tienen las características deseadas, estos pueden ser extraídos de la trayectoria circular mediante dipolos de conmutación rápida o kickers [49].

- Sección de alta energía o High Energy Beam Transport (HEBT): Este conjunto de elementos, generalmente cuadrupolos y octupolos, tiene como finalizad condicionar el haz para su incidencia en el blanco, proporcionando el tamaño y forma necesarios a la huella de impacto. 


\subsubsection{Blanco}

El blanco es el componente que recibe el impacto de las partículas aceleradas, induciéndose en él las reacciones de producción de neutrones. En su diseño, la eficiencia de la producción neutrónica es la característica clave a optimizar. No obstante, la intensa deposición energética originada por el impacto del haz de partículas, conlleva restricciones en términos de refrigerabilidad, tensiones termomecánicas, fatiga y daño por irradiación, que el diseño del blanco debe satisfacer.

Para hacer frente a estos aspectos, distintas opciones constructivas se han considerado. La opción que podríamos considerar básica es el empleo de un blanco sólido estacionario, que reciba el impacto completo de las partículas aceleradas. Sin embargo, en caso necesario, se puede plantear repartir la energía del haz en un volumen mayor haciendo el blanco rotatorio. Esto aumenta el volumen efectivo del blanco, sin diluir la zona en la que se producen los neutrones. La primera vez que se planteó el empleo de esta solución para el diseño de una fuente de gran potencia, fue en el proyecto de la fuente alemana de espalación SNQ [50] en los años ochenta.

Otra posible solución es el empleo de un blanco líquido, de tal manera que éste circule hasta un intercambiador de calor donde se lleve a cabo la refrigeración. Con este concepto se consigue el mismo reparto de energía que en con el blanco rotatorio, y además se evitan los problemas asociados al daño del material por fatiga e irradiación; si no en toda la estructura, al menos sí en el mismo material del blanco. Sin embargo, este concepto presenta también desventajas. Los materiales empleados como blancos líquidos, mercurio en el caso de la fuente SNS [51] en el Laboratorio Nacional de Oak Ridge (Tennessee, EE.UU) y de la fuente japonesa de espalación JSNS [52] (Tokai), o la mezcla eutéctica de plomo y bismuto empleada en el blanco experimental MEGAPIE [53], en el Paul Scherrer Institute (Villigen, Suiza), son materiales corrosivos, especialmente a alta temperatura, cuyo manejo y circuitería presentan requisitos poco convencionales. Además, la deposición energética súbita y concentrada en el seno del fluido puede originar cavitación, con el consecuente daño a las estructuras contenedoras. Este fenómeno ha sido reportado en SNS $[54,55]$ y JSNS [56], y los intentos de mitigarlo mediante la inclusión de burbujas de helio en el flujo del blanco han obtenido un éxito parcial [57].

Así pues, el diseño de un blanco para la producción de neutrones constituye un desafío 
de ingeniería importante y es clave en la definición de la potencia de la instalación y su rango de operación.

\subsubsection{Moderador}

Como se muestra en la Figura 1-6, la mayoría de los neutrones producidos por fisión o espalación presentan una energía media entre los 1 y los $6 \mathrm{MeV}$. Sin embargo, los neutrones empleados en ciencia de materia condensada necesitan tener típicamente energías entre los 0,1 meV y los $100 \mathrm{meV}$. Especialmente útiles son aquellos cuya energía es inferior a los $10 \mathrm{meV}$, denominados neutrones fríos. Por tanto, la energía de los neutrones producidos por los métodos anteriormente mencionados debe ser disminuida en múltiples órdenes de magnitud.

El proceso de disminución de energía es comúnmente conocido como moderación. El medio material en el que los neutrones pierden su energía es conocido como moderador y, por extensión, el componente que contiene dicho medio también recibe ese nombre. Los neutrones disminuyen su energía cediéndola a los átomos del moderador a través de múltiples colisiones con éstos. Una vez este proceso se ha completado, los neutrones terminan en equilibrio térmico con el moderador y, por tanto, su energía viene determinada por la temperatura del moderador empleado. La eficiencia del proceso de moderación depende del material moderador y de su estructura molecular. En general, materiales que contengan átomos ligeros, especialmente el hidrógeno, y moléculas con modos de vibración que puedan ser excitados por las interacciones con los neutrones, presentan mejores propiedades moderadoras. No obstante, también es necesario que dicho material no presente secciones eficaces de captura neutrónica elevadas, ya que esto disminuiría los neutrones disponibles. Desde un punto de vista de la ingeniería de su diseño, el moderador debe ser capaz, además, de afrontar la irradiación neutrónica consustancial a su función, así como, en muchos casos, operar a las temperaturas criogénicas necesarias para la producción de neutrones de muy baja energía. La Tabla 1.1 muestra los rangos de energía en los que los neutrones suelen ser clasificados.

\subsubsection{Reflector}

Con objetivo de maximizar el flujo de neutrones útiles que alcanzan los dispositivos experimentales, el moderador se encuentra rodeado, allí donde sea posible, por material 


\begin{tabular}{|cccc|}
\hline $\begin{array}{c}\text { Rango de } \\
\text { energía }\end{array}$ & $\begin{array}{c}\text { Energía típica } \\
{[\mathbf{m e} \boldsymbol{V}]}\end{array}$ & $\begin{array}{c}\text { Longitud de } \\
\text { onda }[\AA]\end{array}$ & Aplicaciones típicas \\
\hline Ultra fríos & $<3 \cdot 10^{-4}$ & $\geq 500$ & $\begin{array}{c}\text { Propiedades fundamentales del } \\
\text { neutrón, Dispersión de ángulo } \\
\text { ultra-pequeño (USANS) }\end{array}$ \\
\hline Muy fríos & $310^{-4}-0.1$ & $500-30$ & $\begin{array}{c}\text { Espectroscopía, Estructuras } \\
\text { supramoleculares }\end{array}$ \\
\hline Fríos & $0.1-10$ & $30-3$ & Espectroscopía de alta resolución \\
\hline Térmicos & $10-100$ & $3-1$ & Espectroscopía, Difracción \\
\hline Epitérmicos & $100-600$ & $1-0.37$ & Espectroscopía de alta energía \\
\hline Resonantes & $600-10^{5}$ & $0.37-0.03$ & Dispersión inelástica profunda \\
\hline Intermedios & $10^{5}-10^{8}$ & $0.03-9 \cdot 10^{-4}$ & Física atómica, Irradiación de \\
componentes
\end{tabular}

TABLA 1.1: Tipos de neutrones en función de su energía [10].

reflector. El propósito de este componente es reflejar los neutrones que escapan del moderador a una energía mayor que la adecuada, y darles así otra oportunidad de moderación.

Los materiales empleados como reflector deben tener una alta sección eficaz de dispersión y una baja absorción neutrónica. En algunos casos, como en el del berilio, el reflector puede contribuir a la producción neutrónica mediante reacciones tipo $(n, 2 n)$.

La inclusión del moderador tiene efectos sobre la distribución temporal de la producción de los neutrones útiles que deben ser considerados en el diseño. En un sentido más amplio, la configuración del conjunto blanco - moderador - reflector (TMR, por sus siglas en inglés) constituye un complejo problema de optimización en el que debe tenerse en cuenta las características de cada sistema de modo integral.

En los últimos 30 años, importantes esfuerzos se han dedicado a entender el comportamiento del conjunto TMR y su optimización [58, 59, 60], especialmente en el campo del diseño de moderadores [61, 62, 63], obteniendo impactos sustanciales sobre la eficacia global de las instalaciones. A modo de ejemplo de uno de estos dispositivos, la Figura 1-8 muestra el conjunto TMR de la fuente JSNS.

\subsubsection{Blindajes radiológicos}

Los neutrones que no abandonan la fuente en las direcciones adecuadas para alcanzar los instrumentos experimentales, deben ser detenidos para evitar que generen dosis biológica y daño a los materiales circundantes. Además, las propias reacciones de generación de 


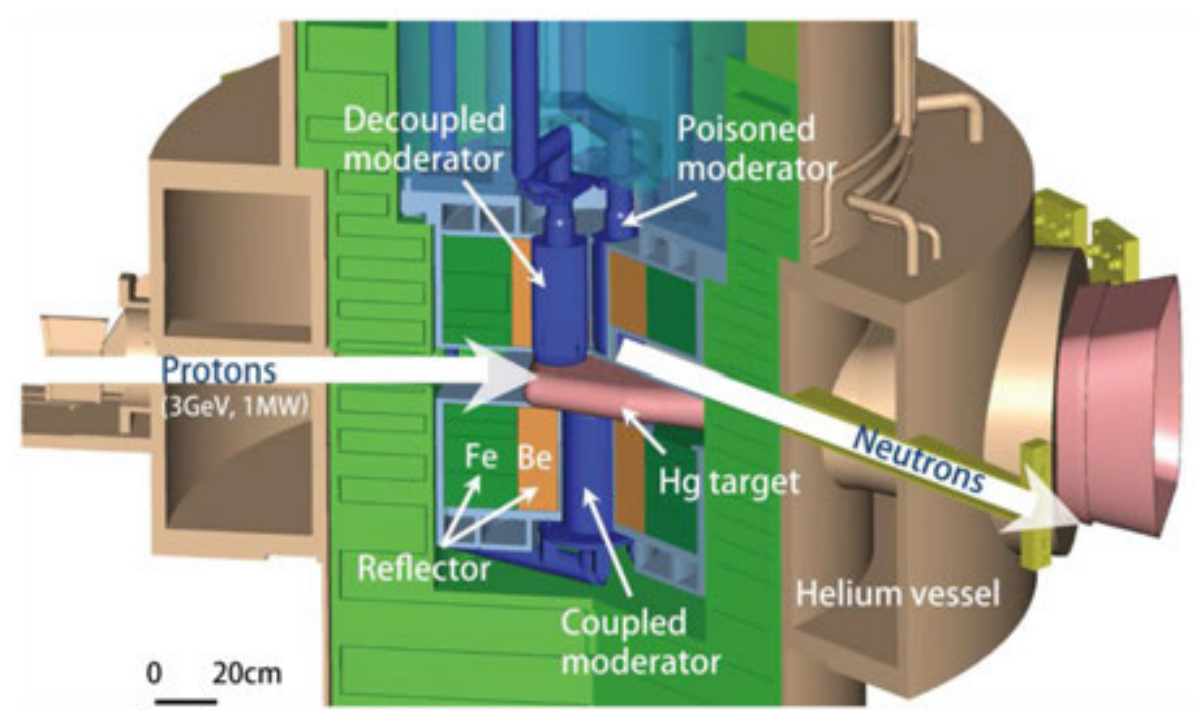

FiguRA 1-8: Conjunto blanco - moderador - reflector de la fuente JSNS [64].

neutrones provocan la emisión de radiación de otros tipos, notablemente radiación $\gamma$, que también deben ser blindadas. Por último, la interacción de los neutrones con los materiales circundantes puede activarlos, i.e. convertirlos en elementos inestables, y por tanto en emisores de radiación.

Buscando detener las radiaciones emitidas por los medios antes mencionados, el conjunto TMR debe ser rodeado de materiales que constituyan un blindaje radiológico eficaz. Los materiales para el blindaje de neutrones suelen estar constituidos por elementos ligeros (como hidrógeno, carbono y oxigeno) con altas secciones eficaces de dispersión, capaces de moderarlos eficazmente. Los blindajes de fuentes pequeñas suelen estar compuestos de polietileno o parafina, mientras que los de las grandes instalaciones suelen estar formados por grandes volúmenes de agua o cemento. Elementos con alta sección eficaz de captura neutrónica, como el boro, el cadmio o el gadolinio, suelen incluirse dispersos en el material de blindaje para absorber los neutrones. El polietileno borado, el carburo de boro $\left(\mathrm{B}_{4} \mathrm{C}\right)$, las aleaciones aluminio-boro y el ácido bórico $\left(\mathrm{H}_{3} \mathrm{BO}_{3}\right)$ disuelto en el agua, son ejemplos de materiales de blindaje que incorporan boro en su composición. El blindaje de neutrones, especialmente de neutrones rápidos, puede estar también constituido por elementos de alto número atómico y alta densidad como el plomo, el bismuto o el tungsteno. Estos elementos también se emplean para blindar la radiación $\gamma$. Este tipo de radiación puede generarse, además, por las reacciones de captura neutrónica en el propio blindaje. 


\subsection{Estado del arte de las fuentes de neutrones guiadas por acelerador}

Con el objetivo de dar contexto a este trabajo, se presenta a continuación una pequeña recopilación de las principales instalaciones para la producción de neutrones mediante el empleo de un haz de partículas, agrupadas en función de sus características. Información más detallada sobre estos sistemas, así como sobre instalaciones basadas en reactores de fisión puede encontrarse en [10].

\subsubsection{Fuentes de alta potencia}

\subsubsection{SNS, Laboratorio Nacional de Oak Ridge}

La fuente de neutrones de espalación (SNS [51]) del Laboratorio Nacional de Oak Ridge (ORNL. Tennessee, EE.UU), consta de un acelerador lineal de 1,0 $\mathrm{GeV}$ y un anillo acumulador que hace impactar protones sobre un blanco de mercurio (Figura 1-9).
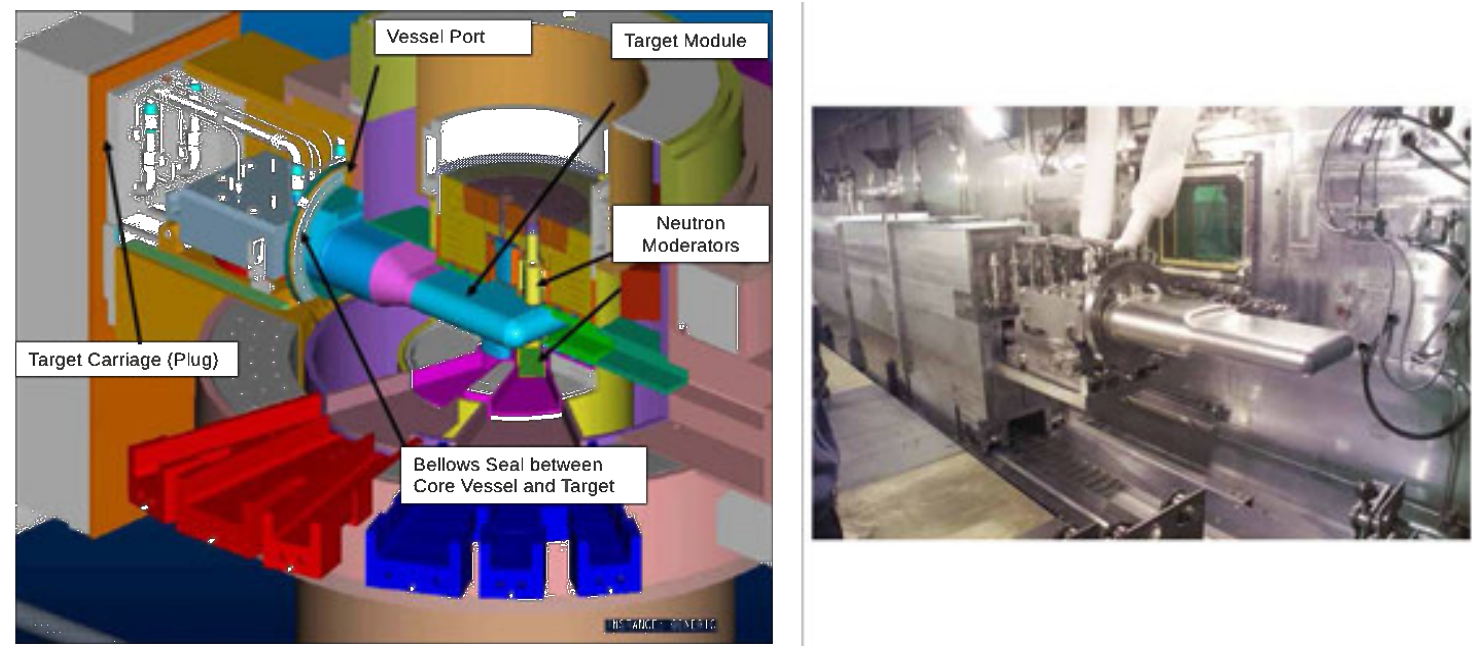

FiguRA 1-9: Conjunto blanco - moderador - reflector de la fuente SNS (izq.) [65] y blanco de SNS en celda caliente (der.) [66].

El acelerador lineal tiene $340 \mathrm{~m}$ de longitud y el anillo acumulador $250 \mathrm{~m}$ de perímetro. Los pulsos de $\approx 1 \mathrm{~ms}$ del acelerador se comprimen a 0,695 $\mu \mathrm{s}$ impactando con un frecuencia de $60 \mathrm{~Hz}$ en el blanco. La instalación está concebida para alcanzar los $2 \mathrm{MW}$ de potencia, no obstante, la potencia máxima de operación alcanzada hasta la fecha es de 1,4 $M W$. 
En la actualidad se está llevando a cabo un programa de mejora orientado a alcanzar la potencia de diseño.

La instalación cuenta con un moderador de agua a temperatura ambiente para la producción de neutrones térmicos, y tres moderadores de hidrógeno criogénico a $20 \mathrm{~K}$ para la producción de neutrones fríos, localizados encima y debajo del blanco, y rodeados por un reflector de berilio refrigerado por agua.

Desde su fase de diseño se ha contemplado una ampliación de potencia hasta los 3 $M W$, mediante el incremento de la energía de las partículas hasta los 1,3 $\mathrm{GeV}$ y de la corriente del acelerador hasta los 2,3 $\mathrm{mA}$. Dicha ampliación contaría con la instalación de un segundo blanco [67] que recibiría un tercio de los pulsos del acelerador sin pasar por el anillo acumulador, por lo que operaría como fuente de pulso largo (i.e. del orden de $m s$ ).

\subsubsection{JSNS, Complejo japonés de investigación en aceleradores de protones}

La fuente japonesa de espalación (JSNS) [52] acelera iones $\mathrm{H}^{-}$hasta los $3 \mathrm{GeV}$ alcanzando una potencia de haz de $1 M W$. En una primera fase, los iones son acelerados hasta los $400 \mathrm{MeV}$ por un acelerador lineal de $250 \mathrm{~m}$, con una corriente pico de $50 \mathrm{~mA}$ y una tasa de $50 \mathrm{~Hz}$. El haz es inyectado en un anillo acumulador de $\approx 350 \mathrm{~m}$ de perímetro, alcanzando los $3 \mathrm{GeV}$ en pulsos de $1 \mu \mathrm{s}$. Parte de los pulsos del acelerador lineal son enviados a una instalación de transmutación de residuos, mientras que parte de los pulsos de $3 \mathrm{GeV}$ es dirigida a una instalación para la investigación en astrofísica y física de hadrones $[68]$.

El resto de los iones de $3 \mathrm{GeV}$ impactan en un blanco de mercurio similar al de SNS. El conjunto TMR de esta fuente (Figura 1-8) incluye tres moderadores de hidrógeno criogénico a $20 \mathrm{~K}$, rodeados por un reflector de berilio refrigerado por agua.

\subsubsection{SINQ, Paul Scherrer Institute}

Ubicada en el Paul Scherrer Institute (Villigen, Suiza), la fuente de neutrones por espalación de Suiza (SINQ) [69, 70] es una fuente continua de 1,3 $M W$ de potencia, operada por un ciclotrón que proporciona una corriente de 2,3 $\mathrm{mA}$ de protones a 590 $\mathrm{MeV}$. 
Una pequeña parte del haz $(20 \mu A)$ se deriva a un laboratorio de irradiación con protones y a una instalación de terapia contra el cáncer. El haz principal pasa a través de dos blancos de grafito para la producción de piones, reduciendo su corriente a 1,3 mA y la energía de los protones a $570 \mathrm{MeV}$, por lo que la potencia que recibe el blanco de la fuente de neutrones es $0,75 \mathrm{MW}$.

Dicho blanco está constituido por un conjunto de barras de zircaloy rellenas de plomo y refrigeradas por agua pesada en flujo cruzado. Las barras se encuentran agrupadas en una estructura que se inserta dentro de una vasija de aluminio 5754, que guía el flujo de refrigerante (Figura 1-10).

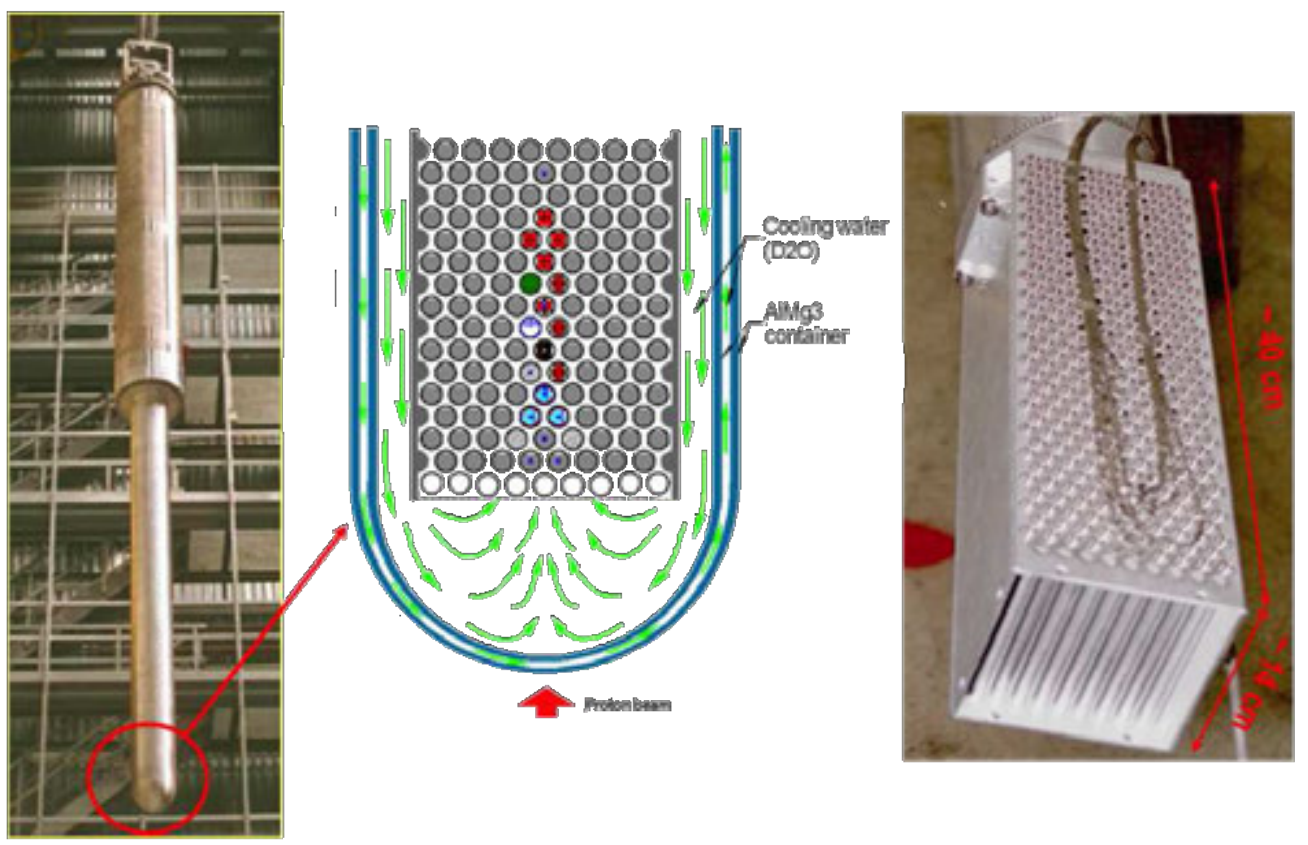

Figura 1-10: Blanco de la fuente SINQ [71].

El blanco se encuentra rodeado de un tanque moderador de agua pesada, en el que se incluye un moderador frío de deuterio líquido a $25 \mathrm{~K}$. Todo el conjunto se encuentra, a su vez, rodeado de un reflector de agua ligera.

\subsubsection{Fuentes de media potencia}

De modo general, pueden considerarse fuentes de potencia media aquellas cuyo haz transporta una potencia en el rango de los centenares de $k W$. A continuación se describen los casos más destacados de esta tipología de instalación. 


\subsubsection{ISIS, Rutherford Appleton Laboratory}

La fuente ISIS cuenta con un sincrotrón que acelera una corriente de protones de 240 $\mu \mathrm{A}$ hasta los $800 \mathrm{MeV}$, con una tasa de repetición de $50 \mathrm{~Hz}$. Dicho acelerador sirve a dos estaciones de blanco, ambas equipadas con sendos blancos de tungsteno recubierto de tántalo, refrigerados por agua pesada, y rodeados de reflectores de berilio también refrigerados por agua pesada.

La estación de blanco número uno comenzó a operar en 1984 [72]. En este blanco impactan 4 de cada 5 pulsos del acelerador, lo que constituye una potencia de haz de 128 $k W$. Esta estación cuenta con cuatro moderadores: dos de agua a temperatura ambiente, uno de metano líquido a $100 \mathrm{~K}$, y un cuarto de hidrógeno líquido a $20 \mathrm{~K}$. A finales de 2015, la junta directiva de la instalación autorizó la actualización de la estación de blanco número uno. El objetivo de la actualización, que tiene como horizonte temporal 2021, es optimizar el diseño del conjunto TMR. Los análisis del nuevo diseño muestran una mejora de un factor 2 en el flujo de neutrones de algunos instrumentos, para el mismo haz de protones incidentes [58].

La estación del blanco número dos comenzó a operar en 2009 [73], como consecuencia de las excelentes perspectivas para la mejora y el desarrollo de las técnicas experimentales neutrónicas, creadas por la estación de blanco inicial [74]. El blanco de esta estación recibe la parte restante de los pulsos del acelerador, lo cual implica una potencia recibida de 32 $k W$. Dicha estación está optimizada para la generación de neutrones fríos y cuenta con dos moderadores, uno de metano sólido a $35 \mathrm{~K}$ y otro de hidrógeno líquido a $20 \mathrm{~K}$.

\subsubsection{CSNS, Academia china de ciencias}

La fuente china de neutrones por espalación (CSNS), perteneciente al instituto de física de altas energías de la academia china de ciencias, ha finalizado recientemente su construcción en la provincia de Guangdong, al sur de China.

Esta instalación cuenta con un sincrotrón que acelera un haz de protones hasta los 1,6 GeV, con una potencia de $100 \mathrm{~kW}$ y una tasa de repetición de $25 \mathrm{~Hz}$. El blanco está constituido por placas de tungsteno recubiertas de tántalo y refrigeradas por agua pesada. La producción de neutrones útiles se consigue mediante el empleo de tres moderadores, 
uno térmico de agua ligera y dos criogénicos de hidrógeno a $20 \mathrm{~K}$. Todo el conjunto se encuentra rodeado por un reflector de berilio y acero refrigerado por agua pesada [75].

El diseño de la fuente está planteado para poder efectuar varias fases de mejora hasta alcanzar una potencia de $500 \mathrm{~kW}$ [76]. Actualmente la instalación se encuentra en fase de puesta en marcha, habiendo conseguido operar la fuente con un haz de $10 \mathrm{~kW}$ de potencia [77].

\subsubsection{Fuentes cctas}

Se entiende por fuente compacta, aquellas fuentes en las que debido a la baja energía final de las partículas aceleradas, la producción de neutrones está muy concentrada en el volumen del blanco. Esto, unido al comparativamente bajo nivel de activación y blindajes requeridos, hace que el volumen total del blanco y los componentes anexos, conjunto TMR y blindajes, sea de un tamaño mucho menor al de las fuentes de espalación.

Varias decenas de fuentes compactas se encuentran en operación o construcción en el mundo actualmente. En esta sección destacaremos algunas de las más relevantes, no obstante, una excelente recopilación de este tipo de fuentes puede consultarse en [78].

\subsubsection{LENS, Universidad de Indiana}

La Low Energy Neutron Source (LENS) de la Universidad de Indiana (EE.UU) cuenta con un acelerador lineal de protones de $4 \mathrm{~kW}$, en el que las partículas alcanzan los 13 $\mathrm{MeV}$. Su corriente pico es de $25 \mathrm{~mA}$, con una tasa de repetición de $20 \mathrm{~Hz}$ y una longitud de pulso de referencia de 0,6 ms. Este haz puede impactar sobre uno de los dos blancos idénticos de berilio refrigerados por agua. Con esta configuración la fuente LENS produce $\approx 10^{13} \mathrm{n} / \mathrm{s}$. Uno de los blancos cuenta con un moderador de metano sólido a $4 \mathrm{~K}$, mientras que el otro cuenta con un moderador de polietileno a temperatura ambiente [79].

La baja activación producida en el conjunto de la instalación permite una gran flexibilidad de operación. Uno de los blancos de LENS cuenta con tres líneas experimentales, dos de ellas con instrumentos, y la tercera está dedicada a la experimentación y caracterización de moderadores y detectores de neutrones. El segundo blanco cuenta con una línea experimental para irradiación con neutrones y neutrografía. 


\subsubsection{RANS, Centro de investigación RIKEN}

La RIKEN Accelerator-driven compact neutron source (RANS) [80], en el centro de investigación RIKEN (Japón), se orienta fundamentalmente hacia la investigación en materiales y las técnicas de inspección no destructivas.

Para ello, está equipada con un acelerador de protones de $0,7 \mathrm{~kW}$ de potencia máxima, en el que las partículas alcanzan los $7 \mathrm{MeV}$. La corriente media máxima del haz de protones es $100 \mu \mathrm{A}$, con una tasa de repetición variable desde los 20 a los $200 \mathrm{~Hz}$. Este haz impacta en un blanco de berilio reforzado mecánicamente con vanadio y refrigerado por agua [81]. Con esta configuración la fuente RANS produce $\approx 10^{12} \mathrm{n} / \mathrm{s}$. La instalación mide aproximadamente $15 \mathrm{~m}$ en su dirección principal (Figura 1-11) y puede operar con un moderador de polietileno a temperatura ambiente, o con un moderador de mesitileno criogénico.

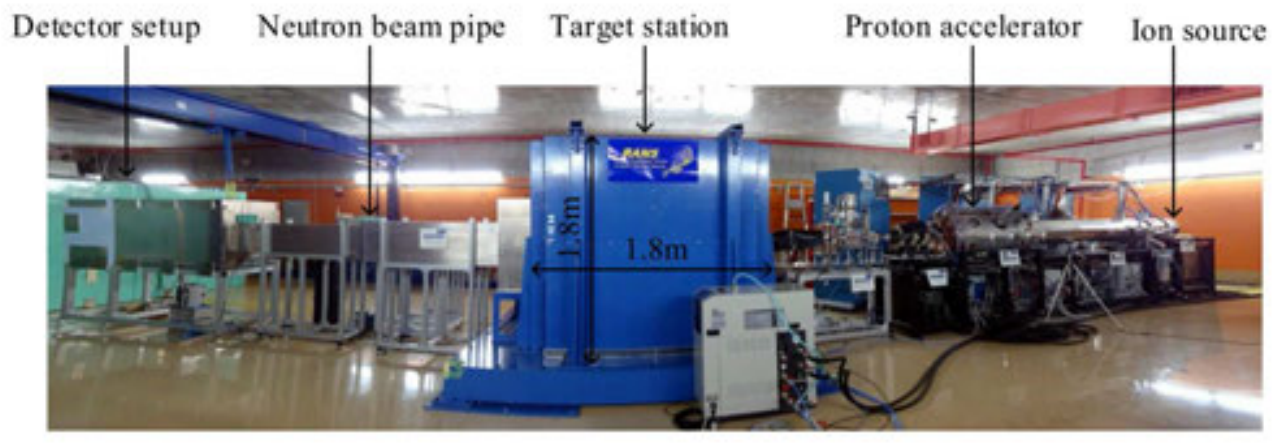

Figura 1-11: Fuente de neutrones RANS del centro japonés RIKEN [82].

\subsubsection{PKUNIFTY, Universidad de Pekín}

La instalación de neutrografía de la Universidad de Pekín, cuyo nombre abreviado en inglés es PKUNIFTY [83], dispone de un acelerador RFQ de deuterones capaz de acelerar una corriente pico de $40 \mathrm{~mA}$ hasta los $2 \mathrm{MeV}$, con una tasa de repetición de $100 \mathrm{~Hz}$, configurando una fuente $8 \mathrm{~kW}$ de potencia.

El blanco de esta fuente es de berilio refrigerado por agua, en el cual se producen $\approx 3 \cdot 10^{12} \mathrm{n} / \mathrm{s}$. El moderador es de polietileno a temperatura ambiente y el reflector de agua ligera. Todo el conjunto TMR se encuentra encapsulado en una vasija de aluminio, 
recubierta de blindaje radiológico compuesto por polietileno borado. Incluido el acelerador, la fuente mide aproximadamente $10 \mathrm{~m}$ en su dirección principal.

Con este dispositivo, la instalación provee servicios de neutrografía [84] y ha realizado medidas de secciones eficaces. Además se emplea en experimentación y docencia de física nuclear.

\subsubsection{CPHS, Universidad de Qinghua}

La Compact Pulsed Hadron Source (CPHS) de la Universidad Qinghua de Pekín, cuenta con un RFQ de protones de $3 \mathrm{MeV}$ y tiene prevista la instalación de una sección DTL, con el objetivo de aumentar la energía de partículas hasta los $13 \mathrm{MeV}$. Tras esa ampliación el haz transportará $16 \mathrm{~kW}$ de potencia con una corriente media de 1,25 $\mathrm{mA}$ y pico de $50 \mathrm{~mA}$, para una tasa de repetición de $50 \mathrm{~Hz}$; con lo que se busca configurar una fuente de $\approx 10^{13} \mathrm{n} / \mathrm{s}$. La producción de neutrones se da en un blanco de berilio refrigerado por agua. Dos moderadores, uno de metano sólido y otro de agua a temperatura ambiente, rodeados por un reflector de agua, completan el conjunto TMR [85].

En 2013 se obtuvieron los primeros resultados de neutrografía empleando el haz de $3 \mathrm{MeV}$ y el moderador térmico. Una segunda línea experimental de este moderador se emplea para el desarrollo de detectores de neutrones. Además, la instalación se emplea en docencia y, tras la ampliación, espera instalar un instrumento de dispersión de pequeño ángulo y aumentar su rango de aplicaciones industriales [86].

\subsection{El proyecto de la fuente europea de espalación (ESS)}

La experimentación con neutrones se consolidó en Europa con la construcción de la primera gran fuente de neutrones en Grenoble (Francia), el High-Flux Reactor (HFR) del Institut Laue-Langevin (ILL) [17], que comenzó a producir neutrones en 1971 con el propósito de aplicar técnicas neutrográficas. La fuente ISIS, considerada como la primera fuente de espalación de potencia elevada, comenzó a operar en 1984. Esta nueva aproximación permitió el desarrollo de un gran número de instrumentos y técnicas experimentales. El éxito de la fuente ISIS hizo que en 1993 la Asociación Europea de Dispersión Neutrónica [87] comenzara a apoyar la idea de la creación de la mayor fuente de espalación del mundo, 
la European Spallation Source (ESS).

La ciencia de neutrones fue estableciéndose como una herramienta estratégica en el desarrollo técnico y científico a nivel mundial. En 1999, la Organización para la Cooperación y el Desarrollo Económicos (OCDE) recomendó la creación de una nueva generación de grandes fuentes de neutrones, una en Europa, otra en América y una tercera en Asia [88]. EE.UU y Japón respondieron rápidamente y fueron capaces de poner en marcha sus instalaciones, SNS y JSNS, en la primera década del nuevo milenio. Sin embargo, la estructura de toma de decisión en Europa, que para entonces contaba con una red de fuentes de neutrones de pequeño y medio tamaño, así como con las mayores instalaciones hasta la fecha, hizo que el proyecto ESS arrancara más lentamente.

En 2002 un grupo de trabajo europeo se reunió en Bonn para estudiar la creación de la gran fuente europea desde un punto de vista técnico, científico y económico [89, 90, 91, 92]. El consenso positivo del grupo de trabajo cristalizó, en 2003, en un nuevo concepto de fuente de $5 M W$ y blanco de mercurio, que tenía 2019 como horizonte temporal para el comienzo de su operación [93].

En los siguientes cinco años, se llevó a cabo un proceso competitivo para la selección de la sede de la futura ESS. Los candidatos fueron tres: Bilbao, en España; Debrecen, en Hungría; y Lund, en Suecia. La discusión se desarrolló a través del Foro Estratégico Europeo para las Infraestructuras de Investigación (ESFRI), que finalmente se decantó por la propuesta sueca. En mayo de 2009, la presidencia de la Unión Europea, que entonces recaía en la República Checa, ratificaba en Bruselas la elección de Lund como sede de la fuente europea de espalación. En 2010, la iniciativa de la fuente europea de espalación toma cuerpo mediante la fundación de la organización ESS AB, bajo la forma de una sociedad publica participada al $75 \%$ por Suecia y al $25 \%$ por Dinamarca.

Tras un intenso periodo de desarrollo, en 2013 ESS presenta su Informe Técnico de Diseño [32]. Éste contempla la construcción de un blanco de tungsteno refrigerado por helio a alta velocidad. La potencia objetivo de la fuente se mantiene en $5 \mathrm{MW}$. Para ello se diseña un acelerador lineal de $\approx 500 \mathrm{~m}$, que proporciona pulsos de protones de $2,5 \mathrm{GeV}$ con una longitud de 2,86 ms y una tasa de repetición de $14 \mathrm{~Hz}$, generando una corriente media de $2 m A$. Con estas características se busca configurar una fuente de neutrones de pulso largo con un término fuente de $\sim 10^{18} \mathrm{n} / \mathrm{s}$. La producción de neutrones útiles 
se consigue mediante el empleo de moderadores de hidrógeno líquido rodeados por un reflector de berilio.

La fase de construcción comienza en Septiembre de 2014 con la ceremonia de colocación de la primera piedra. En Octubre de 2015, ESS se convierte en un Consorcio Europeo de Infraestructura Científica (ERIC), cuyos miembros fundadores son: República Checa, Dinamarca, Estonia, Francia, Alemania, Hungría, Italia, Noruega, Polonia, Suecia y Suiza. Como observadores fundadores del consorcio se incluyen Bélgica, Holanda, Reino Unido y España. A fecha de escritura de este trabajo, España se perfila para ser miembro de pleno derecho en los próximos meses. El proyecto, actualmente, tiene prevista la producción de los primeros neutrones para 2019 y la llegada de los primeros usuarios para 2023.

\subsection{Motivación: La propuesta de ESS-Bilbao}

Tras la selección de Lund como sede de ESS, la candidatura española acordó apoyar el proyecto como entidad independiente mediante la creación del consorcio ESS-Bilbao [94], compuesto al $50 \%$ por el gobierno central español y la administración autonómica vasca. El acuerdo con la candidatura sueca concedía a ESS-Bilbao el estatus de subsede del proyecto ESS, e incluía el compromiso por parte de España de aportar un $10 \%$ del presupuesto total del proyecto, es decir, 180 millones de euros de un total estimado de 1800.

La dirección de ESS-Bilbao, en aquellas fechas, tomó la decisión de materializar la aportación al proyecto ESS mediante la construcción de una fuente de neutrones compacta de media intensidad, en el campus de Vizcaya de la Universidad del País Vasco (UPV/EHU). Esta fuente serviría como banco de pruebas y estación de desarrollo para los componentes de ESS, así como instalación independiente con interés científico propio para la comunidad local e internacional de técnicas neutrónicas.

La motivación para esta decisión tiene una doble vertiente: técnica y estratégica. Desde un punto de vista técnico, las fuentes compactas permiten un mayor grado de flexibilidad en su operación y configuración. Dado su menor nivel de activación radiológica, éstas pueden albergar cambios de componentes mucho más sencilla y rápidamente que las grandes instalaciones de espalación en el rango del $M W$ de potencia. Por otro lado, dada su 
orientación experimental, estas fuentes pueden acomodar en su planificación actividades de tipo exploratorio más fácilmente que las grandes fuentes, cuya función principal es proporcionar tiempo de instrumento a los usuarios. De entre las actividades de interés para una gran instalación que una fuente compacta puede desarrollar, destacan:

- Caracterización de medios moderadores.

- Prueba y validación de conceptos de moderador completo.

- Desarrollo y validación de herramientas numéricas para la simulación de la interacción neutrónica.

- Prueba y validación de diseños de blanco.

- Medidas de activación de materiales.

- Prueba y validación de guías e instrumentos neutrónicos.

- Desarrollo y prueba de detectores de neutrones.

Esta complementariedad entre grandes instalaciones y fuentes compactas se ha hecho patente en múltiples ocasiones, entre las que destacan los trabajos llevados a cabo en la fuente LENS de la Universidad de Indiana para la fuente de espalación SNS [95], o la fuente de neutrones de la Universidad de Hokkaido (HUNS [96], Japón) para la fuente de espalación JSNS [97, 98].

La motivación estratégica de la propuesta de la fuente de ESS-Bilbao, viene dada por la necesidad de crear un centro de referencia nacional con proyección internacional de técnicas neutrónicas. La comunidad nacional de experimentación neutrónica, reunida alrededor de la Sociedad Española de Técnicas Neutrónicas [99], lleva a cabo la mayor parte de su actividad en el ILL (Francia) y en la fuente ISIS (UK), instalaciones de las que España participa con un $6 \%$ y un $2.5 \%$ respectivamente [100]. Se trata de una comunidad consolidada, que cuenta con más de 200 científicos distribuidos en diferentes universidades y centros de investigación nacionales e internacionales [101]. Por tanto, España cuenta con capacidades para sacar el máximo partido de una instalación propia, instalación que a su vez nuclearía y potenciaría esta comunidad.

Estas dos vertientes de la motivación de la propuesta de fuente para ESS-Bilbao, vienen refrendadas por el informe de la Agencia Internacional de la Energía Atómica (IAEA, 
[102]), "Oportunidades de desarrollo para fuentes de neutrones pequeñas y medianas guiadas por acelerador" [103]. En este informe se señala que "Existe una clara sinergia entre la operación de fuentes de alta y baja a media potencia, lo que es beneficioso para ambos tipos."..."Las fuentes de pequeña y mediana potencia constituyen una red de instalaciones que proporciona un valor incalculable para la experimentación, que también sirve para el desarrollo de la tecnología y para el formación de la comunidad. No todas las mediciones o experimentos requieren la intensidad del haz que ofrecen las fuentes de alta potencia, y excelentes programas de ciencia se pueden llevar a cabo en instalaciones más pequeñas."

Además de por su complementariedad con ESS, el interés técnico de la propuesta de ESS-Bilbao se ve incrementado por las técnicas de experimentación neutrónica que esta instalación podría desarrollar como capacidades científicas propias.

Las técnicas neutrónicas disponibles en cada instalación están ligadas, a través del flujo neutrónico requerido, al término fuente de neutrones producidos por segundo. Para dar una estimación orientativa del término fuente requerido por cada técnica, se propone la Tabla 1.2, donde se recoge un rango aproximado de término fuente necesario para la aplicación de las distintas técnicas neutrónicas.

\begin{tabular}{|c|c|}
\hline Aplicación & $\mathbf{n} / \boldsymbol{s}$ \\
\hline Calibración de detectores & $10^{7}-10^{10}$ \\
Análisis por activación & $10^{9}-10^{12}$ \\
Transferencia y neutrografía & $10^{12}-10^{14}$ \\
Medida de secciones eficaces & $10^{13}-10^{14}$ \\
Dispersión de neutrones & $>10^{14}$ \\
\hline
\end{tabular}

TABLA 1.2: Requisitos de fuente en función de las aplicaciones

El nivel de fuente requerido, permite asociar a cada rango una solución tecnológica adecuada conforme a criterios de simplicidad y optimización de coste. En base a lo expuesto en la Sección 1.2, se recopila la Tabla 1.3 a modo de resumen.

De forma general, las fuentes isotópicas y de fusión se utilizan como fuentes portátiles a nivel de laboratorio o para inspecciones in situ, en áreas como el análisis por activación o la calibración de detectores de neutrones.

Numerosas fuentes compactas como las descritas en la Sección 1.4.3, basadas en reac- 


\begin{tabular}{|c|c|c|}
\hline Tipo de fuente & Reacción & $\mathbf{n} / \mathbf{s}$ \\
\hline Fuentes portátiles & Desintegración/fisión espontánea & $10^{6}-10^{9}$ \\
& Fusión & $10^{8}-10^{10}$ \\
\hline Fuentes compactas & Bremsstrahlung & $<10^{14}$ \\
& Extracción (Stripping) & $<10^{14}$ \\
\hline Grandes instalaciones continuas & Fisión & $10^{21}-10^{22}$ \\
\hline Grandes instalaciones pulsadas & Espalación & $10^{17}-10^{18}$ \\
\hline IFMIF (en desarrollo) & Extracción (Stripping) & $10^{17}$ \\
\hline
\end{tabular}

TABla 1.3: Rangos típicos de término fuente de las instalaciones existentes en función de la reacción productora de neutrones.

ciones de bremsstrahlung y extracción, operan en la actualidad, realizando contribuciones científicas en campos tan diversos como [78]: Técnicas de inspección no destructivas, irradiación de componentes electrónicos, generación de datos nucleares, aplicaciones médicas de terapia de captura de neutrones, producción de isótopos y experimentación en astrofísica nuclear.

Las técnicas de dispersión de neutrones son las técnicas más demandantes en cuanto a producción neutrónica requerida, por lo que están disponibles fundamentalmente en grandes instalaciones científicas que emplean reactores nucleares o fuentes de espalación.

La fuente propuesta para ESS-Bilbao, postulada como una fuente de media intensidad, daría pues acceso a múltiples técnicas neutrónicas, incluyendo potencialmente técnicas de dispersión de neutrones. Esto supondría un gran avance para la experimentación neutrónica en el marco nacional, y una contribución relevante en el panorama internacional.

\subsection{Objetivos}

Conforme a lo anteriormente expuesto, el objetivo de este trabajo es diseñar la propuesta de fuente de neutrones para ESS-Bilbao, definiendo sus componentes principales para obtener una evaluación de su viabilidad y rango de aplicación.

Para materializar esta propuesta, el grueso del trabajo se concentra en el diseño de un blanco de producción de neutrones, elemento clave de la instalación que define su término fuente. La definición de este componente requiere del desarrollo de las siguientes tareas: 
- Elección del material de blanco.

- Elección del método de refrigeración.

- Análisis y optimización del comportamiento termomecánico del blanco.

- Diseño y análisis de la estructura de soporte y refrigeración.

- Análisis de vida útil del blanco.

- Evaluación de los márgenes de seguridad del diseño.

Las actividades de análisis y diseño se orientarán a la búsqueda de la robustez y a la detección temprana de potenciales características que pudieran comprometer su viabilidad.

Una vez definido el término fuente, se optimizarán y especificarán las características principales de los componentes relevantes para hacer la producción de neutrones útil para experimentación, evaluando el compartimento neutrónico de la instalación propuesta. El proceso de optimización se enfocará en maximizar la producción de neutrones fríos, estimando la capacidad de la fuente para la aplicación de técnicas experimentales basadas en dispersión de neutrones.

\subsection{Métodos}

El diseño de una fuente de neutrones requiere tener en cuenta fenomenologías muy diversas. A grandes rasgos, los estudios que han de llevarse a cabo pueden agruparse en aquellos que requieren del análisis de fenómenos nucleares y aquellos que implican el estudio de variables macroscópicas.

La producción y moderación de neutrones, y en general la interacción de partículas individuales con la materia, son procesos cuya simulación requiere de herramientas específicas. En el presente trabajo estos procesos se han estudiado empleando el código Monte Carlo N-Particle eXtended (MCNPX) v2.7e [104]. Este código ha sido desarrollado y actualizado por el Laboratorio Nacional de Los Álamos (EE.UU) [105] desde 1957 [106]. MCNPX permite simular, mediante técnicas estocásticas, el resultado de un gran abanico de interacciones entre partículas. A nivel internacional, se trata del código de referencia para este tipo de cálculos y entre sus aplicaciones se encuentran la protección radiológica, el diseño de reactores, la física médica y el diseño de blindajes radiológicos, entre otras. 
A lo largo de este trabajo, distintos datos y modelos nucleares han sido empleados en MCNPX para evaluar el resultado de las interacciones simuladas. Las particularidades, rango de aplicación y referencias de cada uno de ellos se detallan en los subsiguientes capítulos.

Las reacciones a nivel microscópico tienen implicaciones macroscópicas, fundamentalmente en forma de generación de calor y de evolución en la composición de los materiales implicados. Ambos procesos pueden calcularse mediante el código MCNPX, no obstante sus consecuencias requieren de análisis específicos.

Las consecuencias en cuanto a propiedades mecánicas de la evolución de la composición y estructura de los materiales irradiados, se analizan en la Sección 2.3.4 por comparación con la evidencia experimental disponible.

Los efectos de la deposición de potencia térmica de origen nuclear se han estudiando empleando el software ANSYS [107]. Este software resuelve las ecuaciones que modelan distintos fenómenos físicos mediante aproximaciones matemáticas discretas. En concreto, en este trabajo se emplean el módulo mecánico de ANSYS y el módulo ANSYS-CFX [108].

El módulo mecánico de ANSYS calcula una solución aproximada de las ecuaciones que describen el comportamiento mecánico de los sólidos (ecuaciones de equilibrio y compatibilidad), así como la ecuación del calor mediante el método de los elementos finitos. Este módulo se emplea para obtener el campo de temperaturas, desplazamientos y tensiones en los componentes mecánicos diseñados.

El módulo ANSYS-CFX calcula una solución aproximada de las ecuaciones de NavierStokes mediante el método de los volúmenes finitos. Este módulo se emplea para el análisis del comportamiento de los fluidos, obteniendo el campo de velocidades, presión y temperaturas en su volumen, y permitiendo así evaluar la eficacia de los sistemas de refrigeración diseñados.

El software ANSYS cuenta con un extenso programa de validación, que garantiza la correcta representación de los fenómenos físicos incluidos en su rango de aplicación ([109], [110], [111]). Más detalle sobre los modelos matemáticos concretos simulados con el software ANSYS, y sobre el flujo de información entre sus distintos módulos y MCNPX, se proporciona en los siguientes capítulos. 
Por último, para la generación de geometría 3D se ha empleado el software de diseño asistido por ordenador (CAD) Catia V5 [112]. Este software tiene su origen en la industria aeronáutica y, a día de hoy, cuenta con una muy importante posición en el mercado de las herramientas para el diseño mecánico y la integración de sistemas. 


\section{Capítulo 2}

\section{Diseño y optimización del blanco de la fuente de ESS-Bilbao}

\section{Resumen}

En este capítulo se escogen las principales opciones de diseño para la definición del blanco de ESS-Bilbao. Partiendo de la configuración del acelerador propuesto para la instalación, se selecciona el material del blanco en base a la optimización de la producción neutrónica. Una vez elegido el material, se calcula la potencia térmica implementada por el haz en él, para a continuación definir los parámetros básicos del sistema de refrigeración necesario. Se estudia también el efecto en términos de tensión y temperatura del impacto de las partículas, y se analiza la influencia del espesor del blanco y de la frecuencia de impacto del haz sobre estas magnitudes, eligiendo así unos valores adecuados para ambos parámetros. Por último, se analizan los aspectos relacionados con la vida útil en operación del blanco, como son el daño por irradiación, la fatiga y la corrosión.

\subsection{Parámetros base de diseño de la fuente de ESS-Bilbao}

Las características del acelerador de ESS-Bilbao [113] son el principal dato de entrada para el diseño de esta fuente de neutrones. Dicho acelerador ha sido concebido para reproducir las primeras etapas de aceleración de la fuente de ESS. Con esto, se pretende 
habilitar a ESS-Bilbao para la realización de pruebas de componentes del acelerador de ESS y obtener dinámicas de producción de neutrones similares, aunque con menor flujo, para el desarrollo y prueba de moderadores, instrumentos, etc. Así pues, los parámetros del haz de partículas que impacta en el blanco diseñado en este trabajo quedan fijados de partida por las características del acelerador de ESS-Bilbao. Dichos parámetros quedan resumidos en la Tabla 2.1 .

\begin{tabular}{|c|c|}
\hline Partículas aceleradas & protones \\
Energía & $50 \mathrm{MeV}$ \\
Tasa de repetición & $20 \mathrm{~Hz}$ \\
Longitud del pulso & $1,5 \mathrm{~ms}$ \\
Corriente pico & $75 \mathrm{~mA}$ \\
Corriente media & $2,25 \mathrm{~mA}$ \\
Potencia pico & $3,74 \mathrm{MW}$ \\
Potencia media & $112,52 \mathrm{~kW}$ \\
\hline
\end{tabular}

TABLA 2.1: Parámetros principales del acelerador de ESS-Bilbao [113]

El acelerador de ESS-Bilbao, al igual que el acelerador de ESS, es un acelerador de protones pulsado. El acelerador propuesto para ESS-Bilbao tiene una tasa de repetición de $20 \mathrm{~Hz}$. La longitud del puso es de $1,5 \mathrm{~ms}$, lo cual convierte a esta fuente en una fuente de pulso largo, al igual que ESS. Durante cada pulso la corriente de partículas que atraviesa el acelerador, es decir, la corriente pico, es de $75 \mathrm{~mA}$. Considerando la longitud del pulso y la tasa de repetición, el factor de carga del acelerador, i.e. tiempo de pulso sobre tiempo total, es del $3 \%$, generando una corriente media de $2,25 \mathrm{~mA}$, o lo que es lo mismo, $1,4 \cdot 10^{16}$ partículas por segundo. Este valor de corriente se sitúa en rango alto para los valores usuales de este parámetro y se debe, fundamentalmente, a la búsqueda de similitud de este acelerador con el de ESS.

El diseño del acelerador de ESS-Bilbao cuenta con un RFQ y cuatro módulos DTL, que junto con la fuente de iones y las secciones LEBT y MEBT, suman una longitud total de 23,6 $m$ [113]. Estas estructuras aceleran los protones hasta los $50 \mathrm{MeV}$, energía sustancialmente mayor que el rango típico de las fuentes compactas $(2-15 \mathrm{MeV})$, pero 
varios órdenes de magnitud inferior a la de las grandes fuentes de espalación (800 $\mathrm{MeV}$ $3 \mathrm{GeV})$.

La combinación de la energía de las partículas y la corriente determina la potencia transportada por el haz (Ecuación 2.1, donde $E_{p}$ es la energía de los protones en $e V$ y $C$ es la corriente en $A$ ). Con estos parámetros, la potencia media del haz es de $112,5 \mathrm{~kW}$ y la potencia pico es de $3,74 M W$. Por poner en contexto estos valores de potencia, cada pulso deposita $5625 \mathrm{~J}$, por lo que el haz sería capaz de llevar un litro de agua completamente a ebullición desde temperatura ambiente en menos de $3 \mathrm{~s}$.

$$
P[W]=1,6022 \cdot 10^{-19} E_{p} 6,2415 \cdot 10^{18} C
$$

La Tabla 2.2 recoge los parámetros del acelerador de diversas fuentes de neutrones en operación o construcción ordenados por potencia de haz. Como podemos observar, la fuente propuesta para ESS-Bilbao ocupa un lugar singular en la tabla. Su potencia está en el rango medio $(\approx 100 \mathrm{~kW})$, junto a fuentes como ISIS o CSNS, siendo esta muy superior al de otras fuentes compactas. No obstante, esta potencia media se consigue por una combinación de corriente elevada y energía de los protones baja en comparación con las de otras fuentes en este rango, normalmente basadas en reacciones de espalación.

\begin{tabular}{|l|c|c|c|c|c|}
\hline Fuente & $\begin{array}{c}\mathbf{E}_{p} \\
{[\mathbf{M e} \boldsymbol{V}]}\end{array}$ & $\begin{array}{c}\text { Tasa } \\
{[\boldsymbol{H} \boldsymbol{z}]}\end{array}$ & $\begin{array}{c}\text { Longitud } \\
\text { Pulso }[\boldsymbol{m s}]\end{array}$ & $\begin{array}{c}\text { Corriente } \\
\text { media }[\boldsymbol{m A}]\end{array}$ & $\begin{array}{c}\text { Potencia } \\
{[\boldsymbol{k} \boldsymbol{W}]}\end{array}$ \\
\hline ESS [32] & 2500 & 14 & 2.86 & 2 & 5000 \\
\hline SNS [51] & 1000 & 60 & $6.95 \mathrm{E}-04$ & 2 & 2000 \\
\hline SINQ [69] & 590 & Cont. & Cont. & 2.3 & 1360 \\
\hline JSNS [68] & 3000 & 25 & $1 \mathrm{E}-03$ & 0.33 & 1000 \\
\hline ISIS-TS1 [73] & 800 & 40 & $4 \mathrm{E}-04$ & 0.16 & 128 \\
\hline ISIS-TS2 [73] & 800 & 10 & $4 \mathrm{E}-04$ & 0.04 & 32 \\
\hline CSNS [76] & 1600 & 25 & $5 \mathrm{E}-04$ & 0.0625 & 100 \\
\hline CSNS-Upgrade [76] & 1600 & 25 & $5 \mathrm{E}-04$ & 0.312 & 500 \\
\hline ESS-Bilbao [113] & 50 & 20 & 1.5 & 2.25 & 112.5 \\
\hline CPHS [85] & 13 & 50 & 0.5 & 1.25 & 16 \\
\hline PKUNIFTY[83] & 2 & 100 & 1 & 4 & 8 \\
\hline LENS [79] & 13 & 20 & 0.6 & 0.3 & 4 \\
\hline RANS [81] & 7 & 20 & 0.5 & 0.1 & 0.7 \\
\hline
\end{tabular}

TABla 2.2: Parámetros del acelerador de fuentes de neutrones ordenados por potencia. 


\subsection{Elección del material del blanco}

La energía final de los protones en el acelerador propuesto para ESS-Bilbao no es suficiente como para producir reacciones de espalación. En este rango de energías, las reacciones de extracción (Sección 1.2.6) son las más adecuadas para producir neutrones.

Como blancos para este tipo de reacciones, tres materiales son los normalmente usados por sus conocidas propiedades para la producción de neutrones en este rango de energías: litio, carbono y berilio. Con el fin de elegir el material que maximiza la producción neutrónica para protones de $50 \mathrm{MeV}$, se analiza la proporción de conversión neutrónica, es decir, el número de neutrones producido por cada protón incidente, así como la energía media de los neutrones producidos por los tres materiales. Para ello se simula el impacto del haz de ESS-Bilbao en un espesor suficiente de cada material como para detener totalmente los protones. Para este cálculo se emplea el código Monte Carlo MCNPX v2.7e [104] y la librería de secciones eficaces ENDF/B-VII.0 [114] . La selección de este método de cálculo se justificará más detalladamente en el Capítulo 4. Los resultados se presentan en la Tabla 2.3 .

\begin{tabular}{|c|c|c|}
\hline Material & $\mathbf{n} / \mathbf{p}$ & Energía media $[\mathbf{M e V}]$ \\
\hline Carbono & $7,5 \cdot 10^{-3}$ & 8,0 \\
\hline Litio & $4,3 \cdot 10^{-2}$ & 13,3 \\
\hline Berilio & $6,5 \cdot 10^{-2}$ & 7,8 \\
\hline
\end{tabular}

TABla 2.3: Proporción de conversión neutrónica y energía media de los neutrones producidos por protones de $50 \mathrm{MeV}$. Valores calculados usando la librería de secciones eficaces ENDF/B-VII.0 [114].

La mayor razón de conversión neutrónica se obtiene para el berilio, con un valor de $6,5 \cdot 10^{-2}$ neutrones por protón incidente. La proporción de conversión neutrónica del carbono es prácticamente un orden de magnitud inferior al de las otras dos opciones, no obstante, este material es en ocasiones empleado en blancos bajo la forma de grafito, debido a sus excepcionales propiedades mecánicas a alta temperatura. Para flujos de energía de haz moderados, este material puede permitir el diseño de un blanco sin refrigeración activa que evacue la potencia térmica por radiación a alta temperatura contra el ambiente. Esta aproximación se utiliza, por ejemplo, en el diseño de la instalación SPIRAL 2 [115] del 
Gran Acelerador Nacional de Iones Pesados (GANIL [116]) situado en Caen, Francia. En esta instalación se busca producir haces de isótopos exóticos mediante reacciones de fisión inducidas por los neutrones producidos en el grafito. Sin embargo, la evacuación de calor por radiación desde el blanco a alta temperatura puede ser empleada únicamente cuando no se contempla la presencia de un moderador criogénico en las cercanías del blanco, puesto que la irradiación de calor al moderador impediría que éste mantuviese la temperatura deseada.

Las proporciones de conversión de lito y berilio están en el mismo rango, aunque la del berilio es aproximadamente un $50 \%$ superior con respecto a la del litio. Además el litio puro es químicamente muy inestable, siendo altamente inflamable y ligeramente explosivo en contacto con el aire y el agua. Esto último dificulta su utilización, especialmente si se precisa de refrigeración activa. Así mismo el litio puro tiene propiedades mecánicas muy pobres, con un límite elástico inferior a $15 M P a$ y un modulo de Young inferior a 5 GPa [117], [118], [119], lo que no permite emplearlo como material estructural.

Cabe mencionar que en caso de que la generación de neutrones rápidos fuera un objetivo relevante para el diseño del blanco, el litio podría ser una opción de interés, ya que de los tres materiales es el que produce neutrones con una energía media superior. No obstante, las malas propiedades mecánicas de este elemento obligarían a usarlo bien aleado, bien reforzado (lo cual reduciría la eficacia de la producción neutrónica); o fundido en estado líquido, solución planteada en el proyecto IFMIF [38], lo que acarrea importantes complicaciones en el diseño y agudiza los problemas relacionados con su reactividad.

El berilio, en cambio, presenta una capa de óxido superficial que lo hace químicamente muy estable. Además posee buenas propiedades mecánicas, como se detallará más adelante. Por tanto, la combinación de alta razón de conversión neutrónica y buenas propiedades mecánicas convierten al berilio en el material elegido para el blanco de ESS-Bilbao.

La principal desventaja reseñable del berilio, dejando a parte consideraciones económicas, es su elevada toxicidad. El berilio es carcinógeno y su inhalación, y la de sus óxidos, provoca una grave afección pulmonar, por lo que los procesos de sinterizado y mecanizado de berilio requieren de precauciones especiales. Sin embargo, su toxicidad no imposibilita su uso en el marco de una instalación nuclear. 


\subsection{Optimización del blanco}

\subsubsection{Elección del método de refrigeración}

Como se describió en la Sección 2.1, la potencia media del haz del acelerador de ESSBilbao es de 112,5 kW. El diámetro del haz puede variar a lo largo de la cadena de aceleración, no obstante, al final de ésta se plantea el empleo de tres cuadrupolos para desenfocar el haz y dotarlo de una forma y diámetros adecuados [113]. El haz de ESS-Bilbao al impactar con el blanco tiene una sección transversal circular de $10 \mathrm{~cm}$ de diámetro, por lo que el flujo térmico medio que transporta es de $14,32 \mathrm{MW} / \mathrm{m}^{2}$.

Dada la baja capacidad de penetración de los protones de $50 \mathrm{MeV}$ en el berilio, la potencia del haz no se distribuirá en un gran volumen, por tanto el flujo de calor que el refrigerante debe ser capaz de extraer, ha de ser similar al que introduce el haz. Con el objetivo de seleccionar un método de refrigeración para el blanco, se emplea el valor de flujo medio del haz como valor objetivo del flujo de calor a evacuar, para evitar el calentamiento incontrolado y el consecuente daño al blanco. Para hacer esta evaluación, se emplea la temperatura superficial necesaria para evacuar dicho flujo mediante distintos métodos de transmisión de calor. Esta temperatura es una cota minorante de la temperatura en el interior del blanco, por lo que es un valor adecuado para estudiar la viabilidad de cada método de refrigeración. Más adelante se analizará la capacidad del sistema de refrigeración escogido para hacer frente a la naturaleza pulsada del haz, los valores de flujo pico y su distribución espacial.

De acuerdo a lo mencionado en la Sección 2.2, la radiación térmica es un método empleado en blancos de grafito para la evacuación del calor de forma pasiva. Aunque su empleo no está indicado cuando se pretende emplear moderadores criogénicos en la proximidad del blanco, podemos estudiar la viabilidad de este método de evacuación del calor estimando la temperatura superficial media a la que el berilio tendría que operar para evacuar el flujo térmico objetivo.

Asumiendo que el blanco estaría rodeado por un entorno radiativamente grande a temperatura suficientemente homogénea, la temperatura de trabajo del blanco puede ser calculada mediante la Ecuación 2.2. 


$$
\overline{q^{\prime \prime}}=\epsilon \sigma\left(T_{\text {blanco }}^{4}-T_{\text {ambiente }}^{4}\right)
$$

donde $\overline{q^{\prime \prime}}$ es el flujo medio a refrigerar $\left(W / m^{2}\right), \epsilon$ es la emisividad del material, $\sigma=$ $5,67 \cdot 10^{-8} \mathrm{~W} / \mathrm{m}^{4} \mathrm{~K}$ es la constante de Stefan-Boltzmann y $T_{\text {ambiente }}$ la temperatura del ambiente contra el que irradia el blanco en $K$.

Para tener una indicación de la temperatura mínima que el berilio tendría que alcanzar para evacuar por radiación térmica el flujo incidente medio de $14,32 \mathrm{MW} / \mathrm{m}^{2}$, podemos asumir una temperatura ambiente baja, $T_{\text {ambiente }}=25{ }^{\circ} \mathrm{C}$, y una emisividad máxima, $\epsilon=1$. Este último parámetro es muy dependiente del estado superficial del material y de la temperatura del mismo. El rango de emisividad esperable del berilio varía entre 0,04 y 0,81 [120], por lo que asumir $\epsilon=1$ es una hipótesis conservadora. Con estos valores, la temperatura superficial media del berilio alcanzaría $3713,30{ }^{\circ} \mathrm{C}$, la cual está muy por encima de la temperatura de fusión de este material, $1287^{\circ} C$ [120], por lo que el berilio se fundiría antes de alcanzar el equilibrio térmico con el entorno.

Para que la temperatura media superficial del berilio en equilibrio quedara justo en su temperatura de fusión, el flujo del haz debería ser $\approx 40$ veces menor, o lo que es lo mismo, repartirse en una superficie $\approx 40$ veces mayor. No obstante, aun así el blanco no podría operar a esta temperatura por la consecuente degradación de propiedades mecánicas. Por todo lo anteriormente expuesto, y por lo previamente referido sobre la necesidad de moderadores criogénicos en las cercanías del blanco, la refrigeración pasiva por radiación térmica no sería viable.

Excluida la radiación térmica, pasamos ahora a estudiar la convección como posible método de refrigeración. El modo de transmisión de calor por convección es aquel que se da entre un sólido y un fluido en contacto. El flujo de calor transmitido entre ambos por convección viene determinado por la Ecuación 2.3, donde $h$ es el coeficiente de transmisión de calor, parámetro que caracteriza la eficacia de cada configuración convectiva particular. Cuanto más eficaz es la convección, mayor es $h$ y por tanto menor diferencia de temperatura se da entre el sólido y el fluido para transmitir el mismo flujo de calor. La Tabla 2.4 recoge valores representativos del rango típico de $h$ para distintos tipos de procesos convectivos.

$$
q^{\prime \prime}=h\left(T_{\text {sólido }}-T_{\text {fluido }}\right)
$$




\begin{tabular}{|l|c|}
\hline Proceso & $\mathbf{h}\left[\boldsymbol{W} / \boldsymbol{m}^{\mathbf{2}} \boldsymbol{K}\right]$ \\
\hline Convección libre & \\
Gases & $2-25$ \\
Líquidos & $50-1000$ \\
\hline $\begin{array}{c}\text { Convección forzada } \\
\text { Gases } \\
\text { Líquidos }\end{array}$ & $25-250$ \\
Convección con cambio de fase & $100-2 \cdot 10^{4}$ \\
Ebullición o condensación & $2500-10^{5}$ \\
\hline
\end{tabular}

TABla 2.4: Valores tipicos del coeficiente de transmisión de calor, h [121].

Como podemos observar, los coeficientes de transmisión de calor menores para convección sin cambio de fase se dan cuando el fluido en juego es un gas; tanto para convección libre como forzada. Por el término convección libre nos referimos a aquella en la que el movimiento del fluido se debe únicamente a las fuerzas de flotación provocadas por las variaciones de densidad generadas en el fluido por el gradiente de temperatura que se establece en su interior. Por convección forzada nos referimos a aquella en la que el movimiento de fluido se debe a la acción de un elemento impulsor, como un compresor o una bomba. Para analizar si la refrigeración del blanco con gas es factible, calculamos una estimación de qué temperatura media alcanzaría la superficie del blanco de berilio, si se optase por este método para evacuar el flujo medio incidente. Tomando valores optimistas de los coeficientes de transmisión de calor por convección monofásica libre y forzada con gas, i.e 25 y $250 \mathrm{~W} / \mathrm{m}^{2} \mathrm{~K}$, y asumiendo una temperatura del gas de $25 \mathrm{C}^{\circ}$, el berilio tendría que alcanzar una temperatura de varias decenas de miles de grados en ambos casos, por tanto este régimen de trabajo nunca se podría dar, ya que se fundiría mucho antes.

Es necesario mencionar que la convección monofásica con gases puede mejorar por encima del rango característico descrito en la Tabla 2.4 empleando gases a muy alta velocidad. Por ejemplo, con helio a velocidades en el rango de las centenas de metros por segundo, pueden alcanzarse coeficientes de transmisión de calor del orden de pocos miles de $W / m^{2} K$. Estos valores $h$ siguen siendo insuficientes para extraer el flujo medio del haz de ESS-Bilbao, si éste no se reparte en una superficie aproximadamente un factor 10 mayor que su sección transversal.

El helio a alta velocidad es la opción escogida para la refrigeración del blanco de ESS 
[32]. Dicho blanco presenta una distribución de potencia repartida en un gran volumen de tungsteno, que precisa de refrigeración mediante canales internos. Este volumen de deposición de potencia se debe al alto alcance de los protones de 2,5 $\mathrm{GeV}$ de esta fuente. El helio se escogió buscando reducir la absorción neutrónica que generaría el empleo de un volumen significativo de agua en los canales internos del blanco. Sin embargo, en una fuente compacta, donde la producción neutrónica es local, la reducida absorción neutrónica del helio supone una ventaja menos relevante.

En cualquier caso, el empleo de helio a alta velocidad requiere de potentes compresores, así como de sofisticados sistemas de filtrado para eliminar las partículas derivadas de la erosión generada por las altas velocidades. Estos sistemas encarecerían considerablemente la instalación. Como veremos a continuación, existen soluciones eficaces tecnológicamente menos demandantes. Por lo tanto, el empleo de gases a alta velocidad no está justificado. Así pues, la refrigeración monofásica con gas queda en principio descartada para la refrigeración del blanco de ESS-Bilbao.

Volviendo a la Tabla 2.4 podemos observar también como la convección con cambio de fase es el modo convectivo más eficaz. En estos modos el calor latente de cambio de fase entra en juego, por lo que el intercambio de energía térmica se puede dar sin aumentar la temperatura del fluido. Esto permite transmitir grandes flujos de calor sin que sea necesaria una gran diferencia de temperatura entre el sólido y el fluido, o lo que es lo mismo, esto genera coeficientes de transmisión de calor muy altos.

Dado que buscamos formas de refrigerar un solido mediante un fluido, nos centraremos en el estudio de la ebullición, ya que en este modo convectivo el cambio de fase de líquido a gas se sostiene mediante el calor cedido por el sólido al fluido. La ebullición se produce cuando la temperatura de la superficie sobrepasa la temperatura de saturación del líquido a la presión a la que se encuentre, lo cual genera burbujas de la fase gaseosa del fluido que crecen y se separan de la superficie. La formación y comportamiento de las burbujas depende de forma intrincada de la diferencia entre la temperatura del sólido y la temperatura de saturación, así como de otros parámetros de la superficie y del fluido e.g. tensión superficial, densidades de las fases líquida y sólida, velocidad del fluido etc. La dinámica de las burbujas condiciona el movimiento del fluido en las cercanías de la superficie, hasta el punto de que se observan distintos regímenes de transmisión de calor 
caracterizados por coeficientes de transmisión de calor muy diferentes.

El primero en identificar los diferentes regímenes de ebullición fue Nukiyama en 1934 [122], quien los describió mediante la gráfica presente en la Figura 2-1. Dicha curva relaciona los valores de flujo de calor transmitido por ebullición con la diferencia entre la temperatura de la superficie del sólido y la temperatura de saturación del líquido, magnitud conocida como exceso de temperatura $\Delta T_{e} \equiv\left(T_{e}-T_{\text {sat }}\right)$.

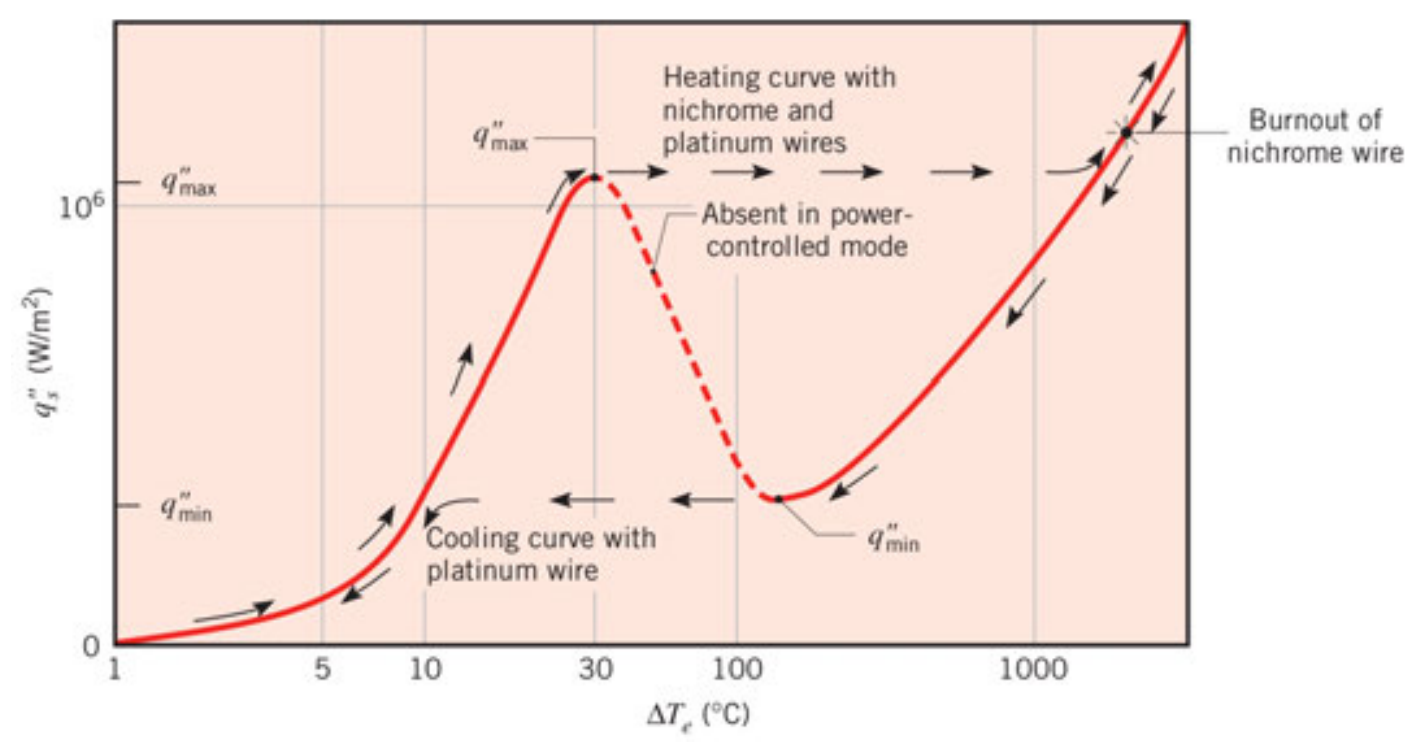

FIgURA 2-1: Curva de Nukiyama para agua saturada a presión atmosférica [121]

La curva fue obtenida mediante un dispositivo experimental que permitía variar el flujo de calor disipado por una superficie sumergida, por lo que $\Delta T_{e} \equiv\left(T_{e}-T_{\text {sat }}\right)$ es la variable dependiente en la gráfica. En concreto empleó un filamento calentado eléctricamente, sumergido en agua en reposo (ebullición en piscina) a presión atmosférica y temperatura de saturación. No obstante, la fenomenología encontrada es extrapolable como descripción general de la convección por ebullición. El filamento empleado inicialmente era de Nicromo, una aleación $80 \% \mathrm{Ni}, 20 \% \mathrm{Cr}$, con una temperatura de fusión cercana a los $1400{ }^{\circ} \mathrm{C}$, empleado por su resistencia a la oxidación a altas temperaturas.

Nukiyama observó cómo a partir de un $\Delta T_{e} \equiv\left(T_{e}-T_{\text {sat }}\right)$ de $5{ }^{\circ} \mathrm{C}$ la ebullición es evidente. A medida que aumenta el flujo disipado, la ebullición y el $\Delta T_{e}$ iban en aumento, pero este último aumentaba de modo muy inferior al flujo disipado. Esta fase inicial es conocida como ebullición nucleada y es un mecanismo convectivo de transmisión de calor 
muy eficaz. En ella el vapor se forma en la superficie del filamento y la abandona como burbujas individuales, siendo éstas sustituidas por fluido frío que moja la superficie y vuelve a evaporarse.

Aumentando el flujo disipado, Nukiyama observó cómo a partir de cierto valor, para un $\Delta T_{e} \approx 30^{\circ} \mathrm{C}$, el filamento se calentaba súbitamente llegando a fallar por sobrecalentamiento. Una vez sustituido el filamento por uno de platino, comprobó cómo por encima de ese valor de flujo, conocido como flujo crítico, la eficacia de la transmisión de calor por ebullición decrecía abruptamente, siendo necesario un $\Delta T_{e}$ mayor de $1000{ }^{\circ} C$ para disipar dichos niveles de flujo. Dicho comportamiento se observa en la Figura 2-1 siguiendo la curva de calentamiento, una vez alcanzado el flujo crítico, $q_{\text {max }}^{\prime \prime}$.

A partir de este valor de flujo, el régimen de ebullición que se da es conocido como ebullición en película. En él, la cantidad de vapor producida es tal que éste forma una película gaseosa alrededor de la superficie. A través de esta capa, el calor se transmite por conducción en el vapor y por radiación entre la superficie y el fluido, lo cual en cierto modo aísla térmicamente la superficie e induce un fuerte aumento de temperatura en esta última. Este fenómeno de degradación súbita de la transmisión de calor es conocido como crisis de ebullición.

Nukiyama también observó cómo, una vez sobrepasado el flujo crítico, aunque se reduzca el nivel de flujo disipado, la ebullición en película permanece, como se observa en la curva de enfriamiento de la Figura 2-1. Para alcanzar el régimen de ebullición nucleada de nuevo, es necesario reducir el flujo por debajo de un cierto valor de flujo, $\left(q_{\min }^{\prime \prime}\right)$, conocido como punto de Leindenfrost.

Para analizar la idoneidad de la convección en ebullición como método de refrigeración para el blanco de ESS-Bilbao, estudiaremos el valor de flujo crítico como indicativo del valor de flujo máximo refrigerable de modo seguro. Como fluido para este estudio consideraremos únicamente el agua, dadas sus excelentes propiedades refrigerantes, el buen conocimiento existente de las mismas, la disponibilidad de correlaciones, y la facilidad para dimensionar y elegir los elementos del circuito de refrigeración de entre componentes comerciales.

Para obtener una buena estimación del valor de flujo crítico, emplearemos la correlación descrita en la Ecuación 2.4 obtenida por Zubber [123] y Kutateladze [124], para ebullición 
saturada en piscina.

$$
q_{\text {max }}^{\prime \prime}=C h_{f g} \rho_{v}\left[\frac{\sigma g\left(\rho_{l}-\rho_{v}\right)}{\rho_{v}^{2}}\right]^{1 / 4}
$$

donde $h_{f g}$ es el calor latente de ebullición, $\sigma$ es la tensión superficial del fluido, $g$ la aceleración de la gravedad, $\rho_{l}, \rho_{v}$ las densidades de las fases líquida y gaseosa respectivamente, y $C$ un coeficiente dependiente de la geometría de la superficie caliente $(C=0,149$ para placas horizontales grandes; $C=\pi / 24 \approx 0,131$ para cilindros horizontales grandes, esferas y grandes superficies en general). Todas las propiedades se evalúan a temperatura de saturación.

Aplicando la Ecuación 2.4 para agua a presión atmosférica se obtiene un flujo crítico $q_{\max }^{\prime \prime}=1,25 \mathrm{MW} / \mathrm{m}^{2}$, aproximadamente un orden de magnitud inferior al flujo medio del haz de ESS-Bilbao, 14,32 $\mathrm{MW} / \mathrm{m}^{2}$, por lo que no se podría refrigerar el haz sin que se diera la crisis de ebullición.

El flujo crítico es fuertemente dependiente de la presión, fundamentalmente a través de la tensión superficial y el calor latente, por ello una forma de aumentar este valor es presurizando el refrigerante. No obstante, presurizar el refrigerante implica nuevos requerimientos mecánicos sobre el blanco, por lo que sólo consideramos aumentos de presión moderados. Aplicando de nuevo la correlación descrita en la Ecuación 2.4, el flujo crítico para agua a 5 y $10 \mathrm{bar}$ es, respectivamente, $2,34 \mathrm{MW} / \mathrm{m}^{2}$ y $2,98 \mathrm{MW} / \mathrm{m}^{2}$; por tanto, a pesar del aumento significativo, tampoco sería suficiente para refrigerar el haz.

Incluso suponiendo que la estructura del blanco pudiera soportar un incremento de presión indefinido, dicho aumento no induciría un aumento indefinido de flujo crítico. Como indica la correlación descrita en la Ecuación 2.4 y demostraron experimentalmente Cichelli y Bonilla [125], el flujo crítico crece para valores crecientes de presión inferiores a aproximadamente un tercio de la presión crítica del fluido. Para presiones mayores, éste desciende hasta cero a la presión crítica. En el caso del agua, la presión crítica es 220 bar y el flujo crítico máximo alcanza $4,5 \mathrm{MW} / \mathrm{m}^{2}$ alrededor de los 70 bar (Figura 2-2), por lo que a priori es imposible evitar la crisis de ebullición si se pretende evacuar el flujo de calor del haz de ESS-Bilbao en esta configuración.

Si el fluido se encuentra a una temperatura inferior a la de saturación, las burbujas 


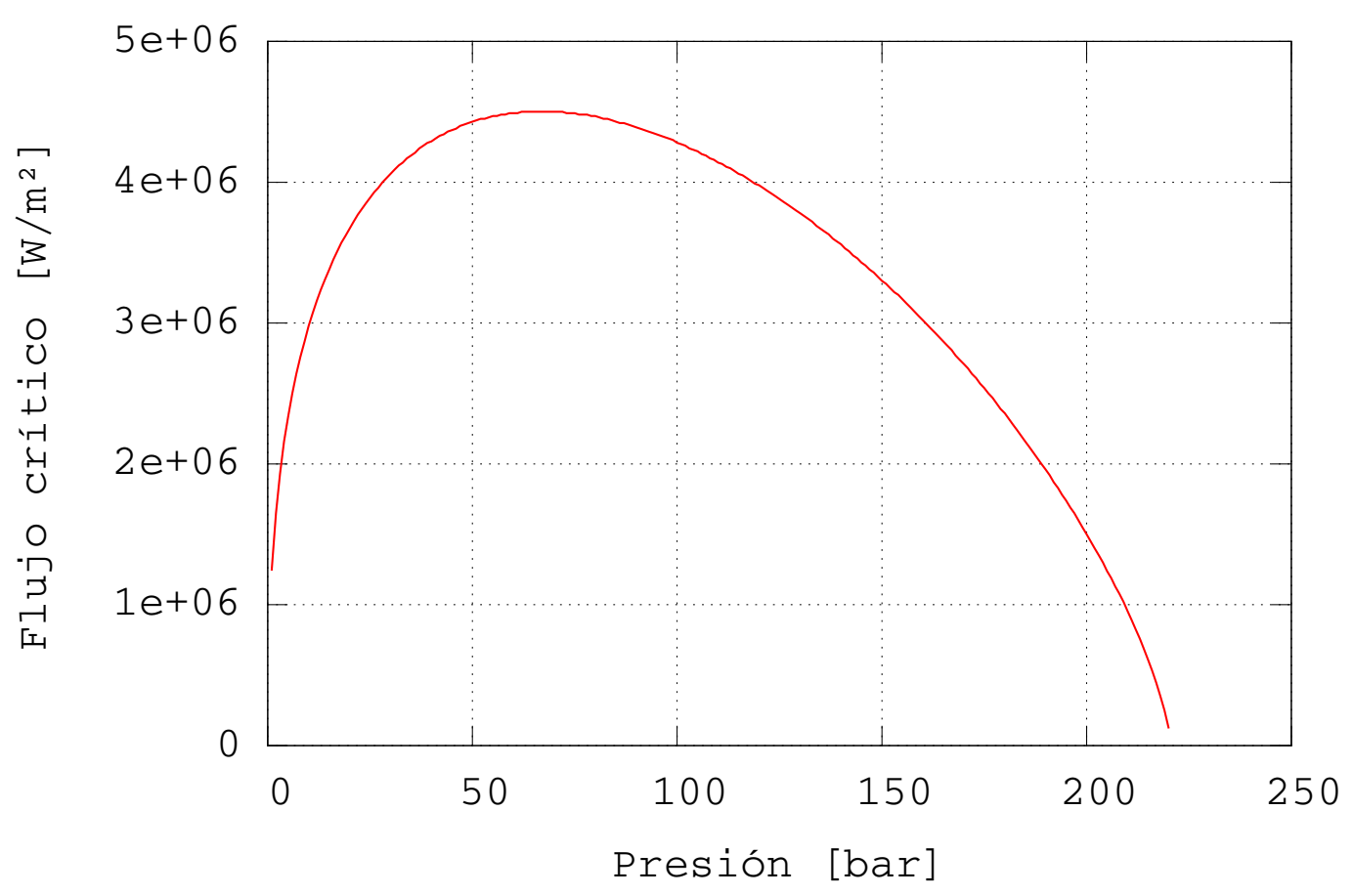

Figura 2-2: Flujo crítico de ebullición en agua (Ecuación 2.4 [123], [124])

colapsan en el fluido al separarse de la superficie caliente. Este tipo de refrigeración es conocida como ebullición subenfriada. Sin embargo, este fenómeno por si solo tiene un efecto despreciable sobre el valor de flujo crítico [121], por tanto las conclusiones trazadas son válidas para el caso de un refrigerante subenfriado.

Durante la ebullición, el movimiento de las fases líquida y gaseosa del fluido está gobernado por las fuerzas de flotación debido a las diferencias de densidad. No obstante, es posible aumentar el flujo crítico si en vez de sumergir el sólido caliente en un fluido en reposo, se hace circular el fluido sobre su superficie impulsándolo mediante un elemento mecánico externo. Este régimen convectivo se denomina ebullición forzada. En él se han descrito valores experimentales de flujo crítico de hasta $35 \mathrm{MW} / \mathrm{m}^{2}$ [126] en modo estacionario, es decir, mayores al flujo medio del haz de $14,32 \mathrm{MW} / \mathrm{m}^{2}$.

Sería por tanto concebible la refrigeración del blanco por medio de un flujo en ebullición forzada subenfriada. Sin embargo, es importante recordar que aunque estamos empleando el valor de flujo medio del haz como medida de la capacidad de refrigeración necesaria, la naturaleza pulsada del haz hace que nuestro problema sea transitorio y que el flujo pico del haz sea mucho mayor que el flujo medio, del orden de $477,5 \mathrm{MW} / \mathrm{m}^{2}$. Si se diseñase 
la fuente para operar en ebullición nucleada subenfriada, las variaciones tan acusadas de flujo instantáneo aplicadas en periodos tan cortos, 1,5 $\mathrm{ms}$, provocarían una fenomenología compleja con pulsos de ebullición, posiblemente en el rango de la ebullición en película. La dinámica y coalescencia de las burbujas en el canal dominarían la temperatura del blanco, pero éstas serían difíciles de prever. En cualquier caso, los valores de flujo instantáneo entregados por el haz de ESS-Bilbao están muy por encima de los valores de flujo que se han conseguido extraer por ebullición forzada.

Por otra parte, la ebullición subenfriada tiene como inconveniente añadido los posibles fenómenos de cavitación generados por el colapso de las burbujas. Si las burbujas colapsan sobre la superficie del blanco u otros componentes, este colapso acarrea picos muy fuertes de presión que erosionan el material.

Así pues, al uso de convección en ebullición conlleva elevadas incertidumbres en cuanto a su eficacia y efectos a largo plazo. Por todo ello, se decide no considerar esta opción como solución base de diseño para la refrigeración del blanco de ESS-Bilbao.

Descartadas por tanto la radiación, la convección libre, la convección forzada de gases y la convección en ebullición, queda por analizar la convección monofásica forzada de líquidos. De acuerdo con [121], el coeficiente de transmisión de calor máximo esperable para este modo convectivo está en el rango de los $10^{4} \mathrm{~W} / \mathrm{m}^{2} \mathrm{~K}$ (Tabla 2.4). Considerando una vez más el agua como fluido refrigerante, la diferencia máxima de temperatura entre el sólido y el fluido para que no aparezca ebullición es del orden de $\operatorname{los} 100{ }^{\circ} \mathrm{C}$. Por tanto, aplicando la Ecuacion 2.3, el flujo máximo refrigerable por convección forzada monofásica con agua puede estimarse en el rango de $1 \mathrm{MW} / \mathrm{m}^{2}$. Este rango es aproximadamente un orden de magnitud inferior al flujo medio del haz, por lo que se hace necesario repartir la energía del mismo en un área $\approx 10$ veces mayor.

Una posible forma de lograr esto sería extender el área de impacto del haz desenfocándolo. No obstante, esto ampliaría la zona de producción de neutrones diluyendo el flujo neutrónico, lo cual es desaconsejable de cara a la experimentación.

Otro posible método para repartir el haz en una superficie de impacto mayor sin modificar su huella, es hacer el blanco rotatorio. De esta manera, cada pulso de haz impacta en una superficie distinta, lo que reparte su energía y reduce el flujo medio. Esta solución ya ha sido puesta en práctica en blancos pequeños de grafito como, por ejemplo, el de 
SPIRAL 2 en GANIL [115], el de la fuente de muones de JPARC [127] (Figura 2-3) o el de la fuente de isótopos TRI $\mu \mathrm{P}$ [128] de la Universidad de Groninga (Países Bajos). El empleo de un blanco rotatorio se plantea también como opción para la futura segunda estación de blanco de SNS [129] y es la solución base de diseño de ESS [130].

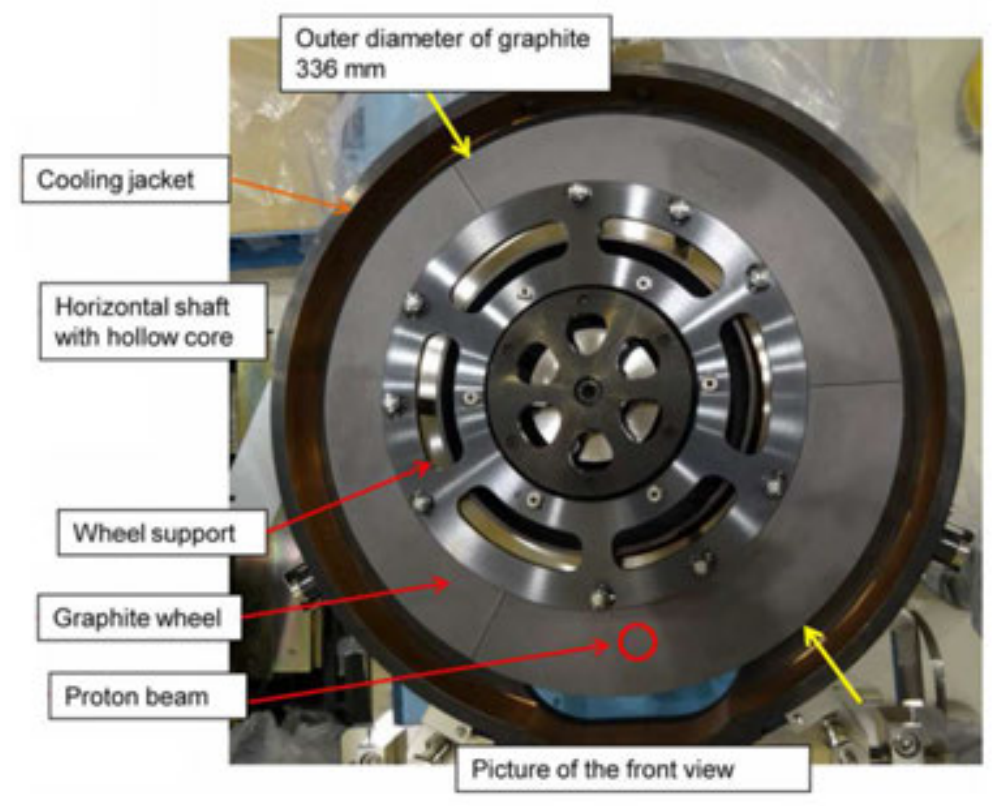

FIGURA 2-3: Blanco rotatorio de grafito para la producción de muones en JPARC [127]

Para el caso que nos ocupa, aumentar el área de impacto del haz un factor 10 se considera factible. La estrategia de refrigeración, por tanto, consistiría en repartir el haz en un blanco rotatorio suficientemente grande, haciendo circular agua en contacto con éste a una velocidad tal que proporcione un coeficiente de transmisión de calor del orden de $10^{4} \mathrm{~W} / \mathrm{m}^{2} \mathrm{~K}$. Si el haz se reparte en una superficie suficientemente grande y el coeficiente de transmisión de calor es el adecuado, la temperatura del blanco en contacto con el agua nunca superaría la temperatura de saturación del líquido, lo que proporcionaría una refrigeración monofásica estable y una temperatura de operación del blanco baja.

Con estas consideraciones, la solución base de diseño que se selecciona para la fuente de ESS-Bilbao es un blanco rotatorio de berilio refrigerado por un flujo forzado monofásico de agua. En las siguientes secciones se desarrollará esta solución conceptual.

Cabe destacar que otras fuentes de potencia similar a la propuesta para ESS-Bilbao, 
como pueden ser ISIS o CSNS, no necesitan implementar un blanco rotatorio. Esto se debe a que la energía de las partículas de sus haces es muy superior, en el rango de los $G e V$, lo cual hace que éstas penetren más y que la potencia térmica se reparta en un volumen mucho mayor, lo que reduce el flujo superficial a extraer. Las grandes fuentes pulsadas en operación actualmente, SNS y JSNS, con una potencia en el rango de los $M W$, además de contar también con energías de partículas muy elevadas, emplean blancos líquidos en circulación, lo cual también reparte la energía del haz en más material del blanco.

\subsubsection{Cálculo de la deposición energética del haz de ESS-Bilbao}

Para analizar el comportamiento termomecánico del blanco, es necesario conocer con precisión la distribución de deposición energética generada por el haz al impactar sobre el berilio. Para ello se emplea el código Monte Carlo para el cálculo del transporte de partículas MCNPX v2,7e [104], con el que simularemos el efecto del impacto del haz de protones del acelerador de ESS-Bilbao en una lámina de berilio lo suficientemente espesa como para detener completamente el haz. Para realizar este cálculo es necesario definir dos parámetros de diseño de la fuente: el ángulo de incidencia entre el haz y el blanco y la distribución de flujo de partículas dentro del haz.

El ángulo de incidencia entre el haz y el blanco es un parámetro de diseño que afecta al área de impacto del haz, y a la configuración geométrica de la fuente. Cuanto menor es el ángulo de incidencia del haz, mayor es el área en la que se reparte la energía y, por tanto, menor es el flujo; lo cual facilita la refrigeración. Sin embargo, dicho ángulo no puede ser indefinidamente pequeño, pues aumentaría indefinidamente el área de impacto, diluyendo la zona donde se producen los neutrones. Además dificultaría la integración del resto de elementos de la fuente, como moderadores, guías de neutrones e instrumentos. En base a la configuración prevista para la propuesta de ESS-Bilbao, se elige un ángulo de incidencia de $45^{\circ}$. Esta elección se argumentará más detalladamente en el Capítulo 4. Con este ángulo, el ratio entre el área de impacto y la sección transversal del haz es de $1 / \sin 45^{\circ}=\sqrt{2}$ (Ecuación 2.5), lo cual supone un incremento de área del $\approx 41,4$ \% (Figura 2-4).

$$
\frac{A_{\text {impacto }}}{A_{\text {haz }}}=\frac{\pi a b}{\pi R^{2}}=\frac{\pi\left(R / \sin 45^{\circ}\right) R}{\pi R^{2}}=\sqrt{2}
$$




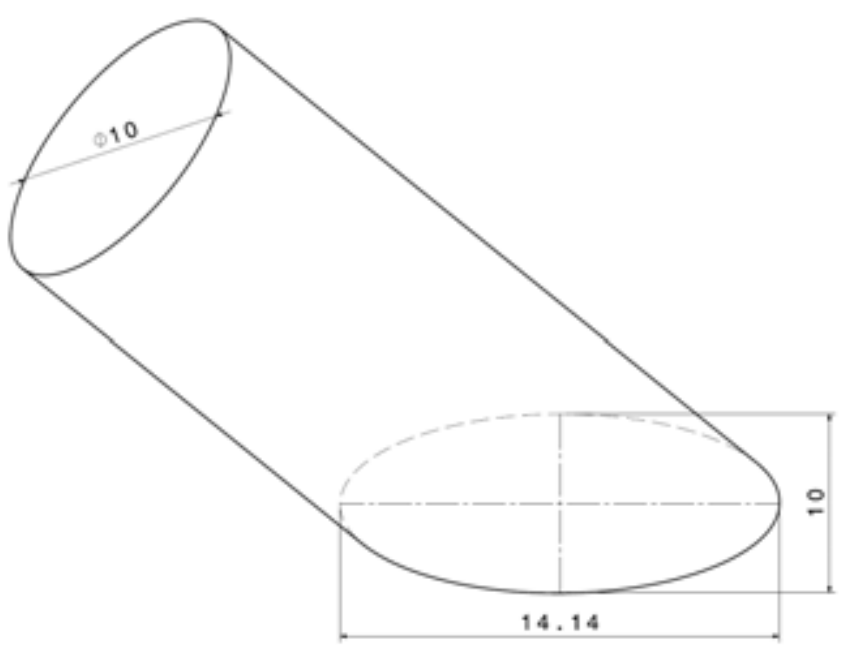

Figura 2-4: Dimensiones de la sección transversal del haz y del área de impacto $[\mathrm{cm}]$.

El segundo parámetro de diseño a definir es la distribución de flujo dentro del haz. Debido a la dinámica de aceleración de las partículas, los pulsos del acelerador no transportan la misma cantidad de partículas por unidad de superficie en toda la sección transversal del haz, sino que el flujo tiene un perfil espacial. El haz generado por el acelerador de ESS-Bilbao tiene un perfil de flujo bidimensional gaussiano axisimétrico (Ecuación 2.6) con unos valores de desviación típica en ambos ejes de $\sigma_{x}=\sigma_{y}=2,5 \mathrm{~cm}$.

$$
\phi(x, y)\left[W / m^{2}\right]=A \exp \left(-\left(\frac{x^{2}}{2 \sigma_{x}^{2}}+\frac{y^{2}}{2 \sigma_{y}^{2}}\right)\right)
$$

La integral de superficie del perfil de flujo es igual a la potencia total del haz. Integrando la Ecuación 2.6 podemos calcular el parámetro $A$ de la distribución gaussiana, que describe el valor del flujo en el centro del haz.

$$
P[W]=\int_{-\infty}^{\infty} \int_{-\infty}^{\infty} \phi(x, y) d x d y=2 \pi A \sigma_{x} \sigma_{y}
$$

De acuerdo a la Ecuación 2.7, para una potencia media de haz de $P=112,5 \mathrm{~kW}$, el valor de flujo en el centro del haz es $A=28,64 \mathrm{MW} / \mathrm{m}^{2}$. Teniendo en cuenta este valor, la Figura 2-5 muestra la distribución de flujo en el haz de ESS-Bilbao.

La concentración del flujo provocada por la distribución gaussiana hace que el valor de 


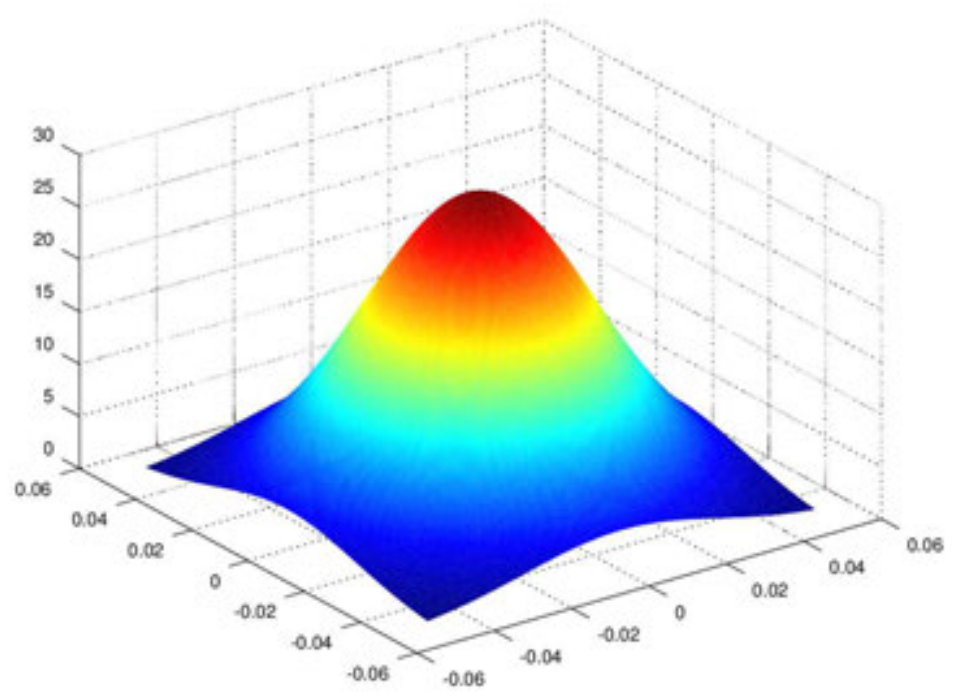

Figura 2-5: Distribución de flujo en el haz de ESS-Bilbao $x, y[m] ; z\left[M W / m^{2}\right]$

flujo en el centro del haz duplique el valor de flujo medio, 14,12 $\mathrm{MW} / \mathrm{m}^{2}$. Esta concentración es un importante factor a tener en cuenta en el diseño del sistema de refrigeración.

Así pues, con un ángulo de incidencia igual a $45^{\circ}$ y el perfil de flujo en el haz anteriormente descrito, la deposición de potencia generada en el berilio durante un pulso del acelerador es la mostrada en la Figura 2-6.

En dicha imagen, podemos observar claramente como los protones se detienen a $\approx 11$ $m m$ de la superficie, lo cual, dado el ángulo de incidencia, implica que los protones tienen un alcance en el berilio de $\approx 15 \mathrm{~mm}$.

También podemos observar como la distribución de potencia se concentra abruptamente alrededor del final de la trayectoria de los protones. Esto se debe al modo en que la energía disipada por unidad de longitud recorrida varía con la energía de las partículas. Dicha magnitud es conocida como poder de frenado. En el caso de partículas cargadas a altas energías, éste aumenta a medida que las partículas pierden energía [131]. Esto provoca que la disipación energética se concentre en la parte final de su trayectoria, fenómeno conocido como pico de Bragg (Figura 2-7).

En el presente caso, la deposición de energía alcanza durante el pulso un valor máximo de $152 \mathrm{~kW} / \mathrm{cm}^{3}$, lo cual es un valor extremadamente alto. Por poner en contexto este 


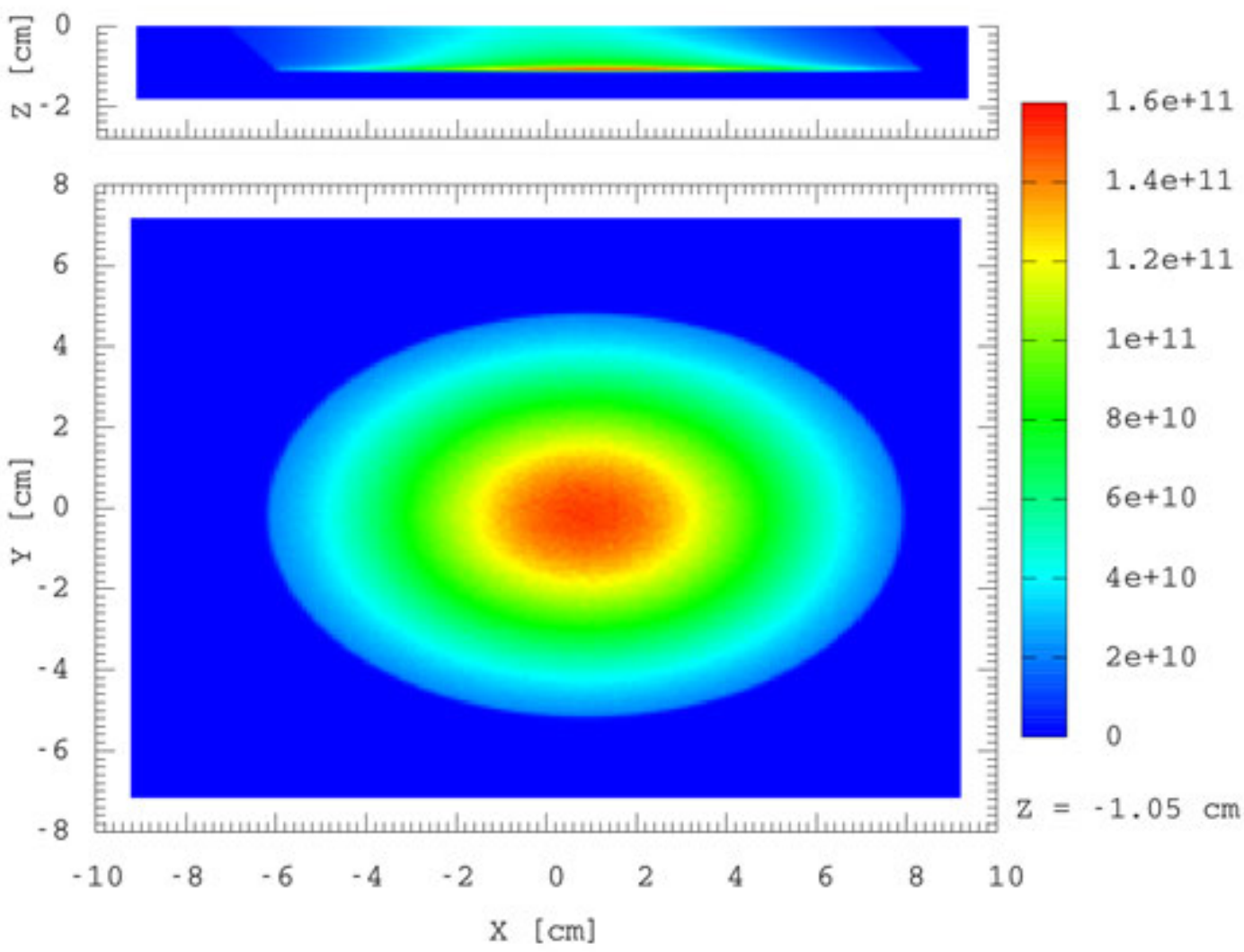

Figura 2-6: Deposición energética del haz de ESS-Bilbao en berilio [ $\left.\mathrm{W} / \mathrm{m}^{3}\right]$

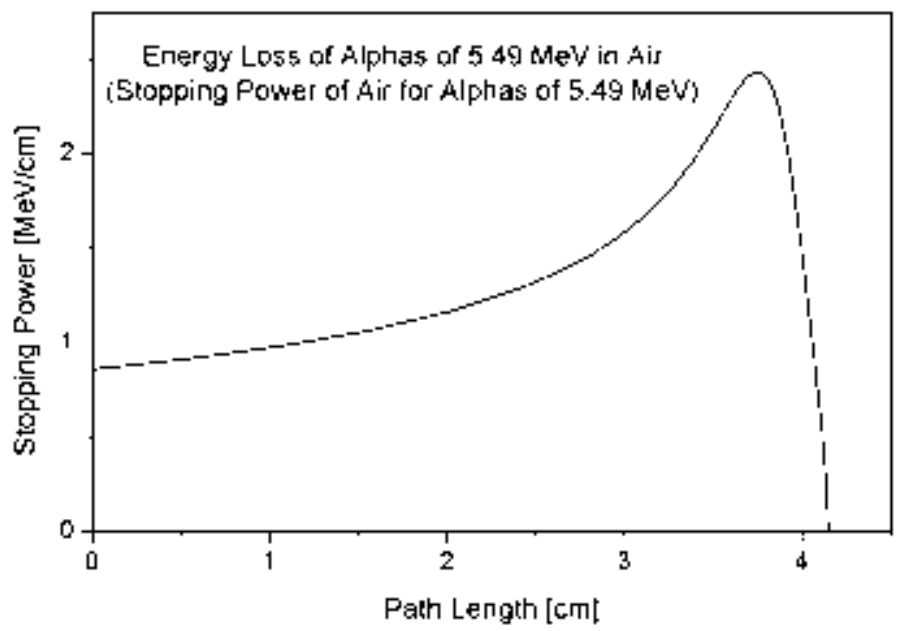

Figura 2-7: Ejemplo de pico de Bragg: poder de frenado de partículas $\alpha$ en aire [132] 
valor, la densidad de potencia en el combustible de un reactor nuclear de tipo PWR es de $\approx 0,35 \mathrm{~kW} / \mathrm{cm}^{3}$ [133]. La integral de volumen de la distribución de potencia calculada por MCNPX es de 3,58 $\mathrm{MW}$, menor que la potencia transportada por durante el pulso como energía cinética de las partículas del haz, 3,74 $\mathrm{MW}$. La energía restante, entorno al $\approx 5 \%$, abandona el berilio en forma de radiación $\gamma$ y de energía cinética de los neutrones producidos.

\subsubsection{Análisis termomecánico}

Una vez conocida la deposición energética inducida por el haz, pasamos a analizar qué efectos tiene en el blanco en términos de temperatura y tensiones mecánicas.

Para este estudio emplearemos el software comercial de elementos finitos ANSYS [107]. En él desarrollaremos el cálculo termomecánico transitorio en tres fases (Figura 2-8).

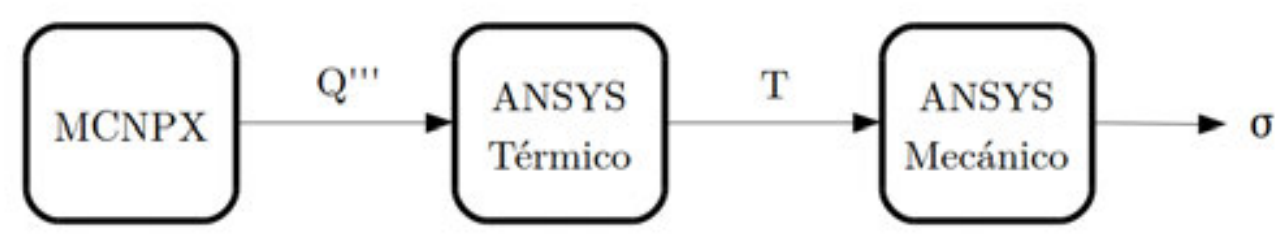

Figura 2-8: Esquema de cálculo termomecánico.

Primero implementamos la deposición energética calculada por MCNPX en el mallado de elementos finitos de ANSYS. Dado que la malla de ANSYS y los puntos de cálculo de MCNPX no son los mismos, es necesario interpolar la deposición energética en los elementos de ANSYS. Para ello se ha desarrollado una rutina ad hoc en el lenguaje de programación APDL (ANSYS Parametric Design Language) capaz de procesar el archivo de texto generado por MCNPX con el mapa tridimensional de deposición, interpolarlo en los elementos de ANSYS y calcular la integral de volumen de ambas deposiciones, original e interpolada, para comprobar que la interpolación ha sido exitosa.

La Figura 2-9 muestra el mallado generado en ANSYS. Se trata de una placa de $140 \mathrm{x}$ $182 \times 12 \mathrm{~mm}^{3}$, cuyas dimensiones se han calculado para acomodar el haz con un margen de $20 \mathrm{~mm}$ de distancia entre éste y sus extremos, en previsión de espacio para fijarla mediante una brida. La longitud de uno de sus lados es mayor para acomodar el haz incidente con $45^{\circ}$ 
grados de inclinación. El espesor de $12 \mathrm{~mm}$ se ha seleccionado para que el blanco detenga completamente los protones, lo que maximiza la producción neutrónica. Las aristas más cortas de la placa se han redondeado con un radio de $20 \mathrm{~mm}$ para evitar concentración de tensiones, no obstante, es una opción de diseño que no afecta a los resultados a calcular. El mallado es más fino en la dirección perpendicular a las caras mayores, y se refina cerca de la profundidad a la que se da el Pico de Bragg para capturar el fuerte gradiente de deposición de potencia de manera adecuada. El modelo aprovecha la simetría del problema para simular sólo una mitad del dominio sólido, lo cual hace el cálculo más eficiente.

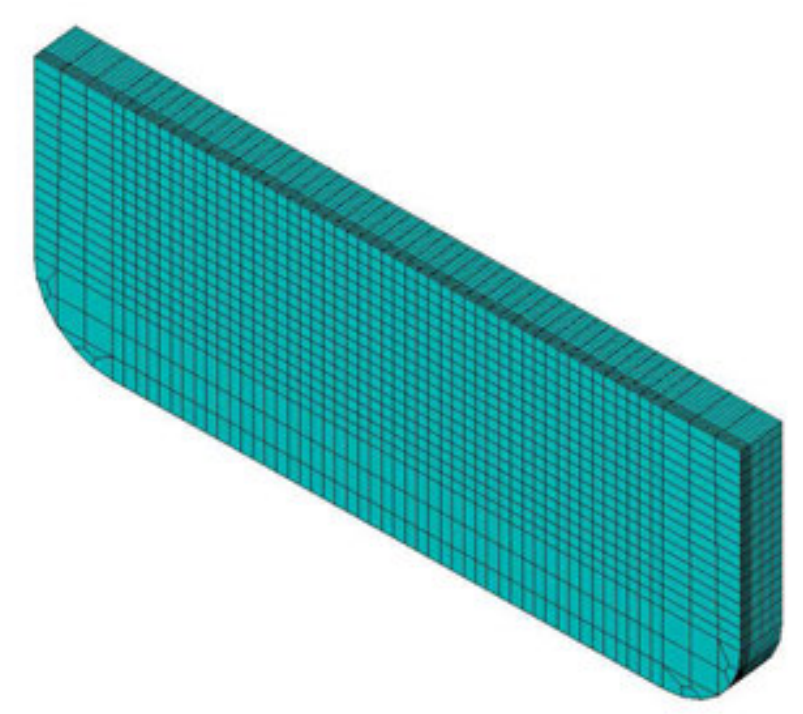

Figura 2-9: Mallado ANSYS de una placa de $12 \mathrm{~mm}$ de espesor.

La Figura 2-10 muestra la distribución de potencia una vez interpolada en ANSYS. Podemos observar como la interpolación es fiel a la distribución por MCNPX, reproduciendo el valor máximo con menos de un $2 \%$ de error. Esta pequeña discrepancia se debe a que el pico de Bragg es tan abrupto y su máximo tan local que, salvo que un punto de interpolación coincida justamente con el valor del máximo, cualquier interpolación tiende a suavizarlo. No obstante, la integral de volumen de la distribución de potencia interpolada en la malla de ANSYS es 3,59 $M W$, es decir, menos de un 0,3\% de diferencia con la potencia calculada por MCNPX, lo que garantiza que la malla de ANSYS es suficientemente fina como para replicar la distribución de potencia.

La segunda fase del proceso de análisis es el cálculo transitorio térmico. Para este 


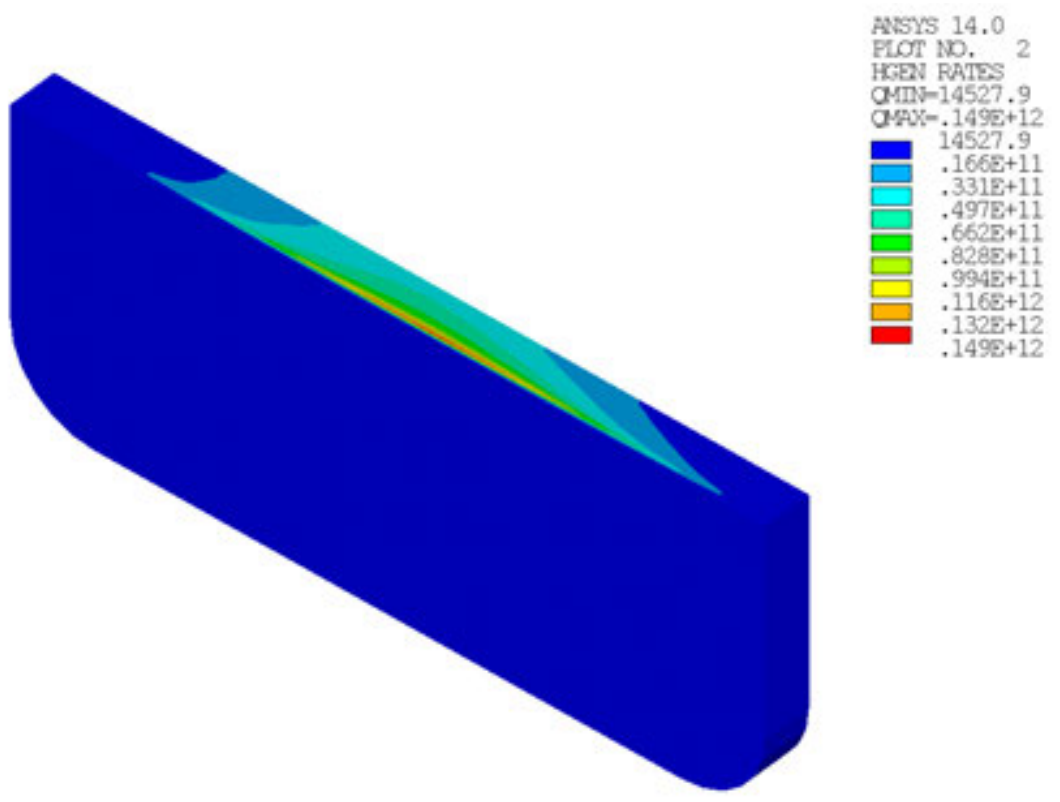

Figura 2-10: Potencia implementada en ANSYS en una placa de Be de $\left.12 \mathrm{~mm} / \mathrm{W} / \mathrm{m}^{3}\right]$.

cálculo se ha desarrollado un modelo térmico con las condiciones de refrigeración como condiciones de contorno y la distribución de potencia antes mostrada como carga térmica.

En base a lo discutido en la Sección 2.3.1, la placa de berilio será refrigerada por convección forzada con agua a $300 K\left(\approx 27^{\circ} \mathrm{C}\right)$. Como coeficiente de transmisión de calor representativo de dicha condición convectiva se elige $10^{4} \mathrm{~W} / \mathrm{m}^{2} \mathrm{~K}$. Para ganar margen con respecto a la ebullición se considera que el agua se va a presurizar a 5 bar, lo cual mantiene el fluido monofásico hasta $\operatorname{los} 152^{\circ} \mathrm{C}$. Como método de refrigeración se prevé que el agua esté en contacto directo con el berilio, por lo que este coeficiente se aplica como condición de contorno térmica en la cara de la placa opuesta a la que recibe el haz. Este método de refrigeración, poniendo el agua en contacto directo con el berilio, es el empleado en fuentes como LENS y otras fuentes compactas que emplean berilio como material de blanco. Al no interponer ningún material entre el agua y el berilio se reduce la absorción de los neutrones producidos y, por tanto, se mejora el rendimiento de la fuente.

Para simular el impacto del haz, la distribución de potencia descrita en la Figura 2-6 se implementa como carga térmica durante la duración del pulso, 1,5 ms, y se hace nula entre pulsos, ya que la potencia residual en el berilio es despreciable.

De acuerdo a lo discutido en la Sección 2.3.1, para poder refrigerar el haz se estima 
necesario reducir el flujo medio en, al menos, un orden de magnitud haciendo el blanco rotatorio. En nuestro caso, hacer el blanco rotatorio implica distribuir angularmente varias placas en un disco, de tal manera que al rotar sincronizadamente con el acelerador, el haz impacte en una placa distinta, repartiendo el flujo medio del haz en más superficie. Así pues, la frecuencia de impacto sobre una misma placa viene determinada por la frecuencia del haz y el número de placas. Para este cálculo, como referencia, adoptamos una frecuencia de impacto sobre cada placa de $1 \mathrm{~Hz}$. Esto equivale a disponer en el blanco 20 placas, de forma que los 20 pulsos que el acelerador entrega en cada segundo impacten en una placa distinta, recibiendo cada una de ellas un pulso por segundo. El efecto del número de placas del blanco, es decir, de la frecuencia de impacto, se analizará en la Sección 2.3.3.2.

De entre los distintos grados de berilio comercialmente disponible se selecciona por sus buenas propiedades mecánicas el berilio S-200 F [134], fabricado mediante sinterizado de alta presión en caliente (también referido como S-200 VHP). Las propiedades del berilio empleadas en el modelo térmico son la conductividad, $\lambda$, la densidad, $\rho$, y el calor específico, c. A temperatura ambiente dichas propiedades toman los siguientes valores: $\lambda=210$ $W / m K, \rho=1850 \mathrm{~kg} / \mathrm{m}^{3}, c=1925 \mathrm{~J} / \mathrm{KgK}$ [134]. Cabe resaltar que la conductividad y el calor especifico del berilio son muy elevados, mientras que su densidad es muy reducida, lo cual le convierte en el mejor disipador térmico por unidad de masa [135].

Aplicando este modelo térmico y partiendo de una temperatura inicial de $27^{\circ} \mathrm{C}$, la Figura 2-11 muestra la evolución de la temperatura máxima en la placa. En ella podemos observar el súbito calentamiento de la placa con cada pulso. El salto de temperatura máxima inducido por el primer pulso es de $55^{\circ} \mathrm{C}$. Este valor está en el rango de lo que experimentaría un elemento diferencial de berilio, sometido a una potencia volumétrica igual a la máxima calculada por MCNPX, durante la longitud de un pulso en condiciones adiabáticas.

También observamos cómo tras varios impactos, la oscilación de la temperatura máxima de la placa se estabiliza, repitiéndose cíclicamente la evolución de temperaturas con cada pulso. La Figura 2-11 muestra el efecto de los 12 primeros pulsos, momento tras el cual la diferencia entre los picos de temperatura entre pulsos es menor de $1^{\circ} C$, y se puede considerar que un estado pseudo-estacionario cíclico se ha alcanzado.

La Figura 2-12 muestra la distribución de temperaturas en la placa al final del pulso, 


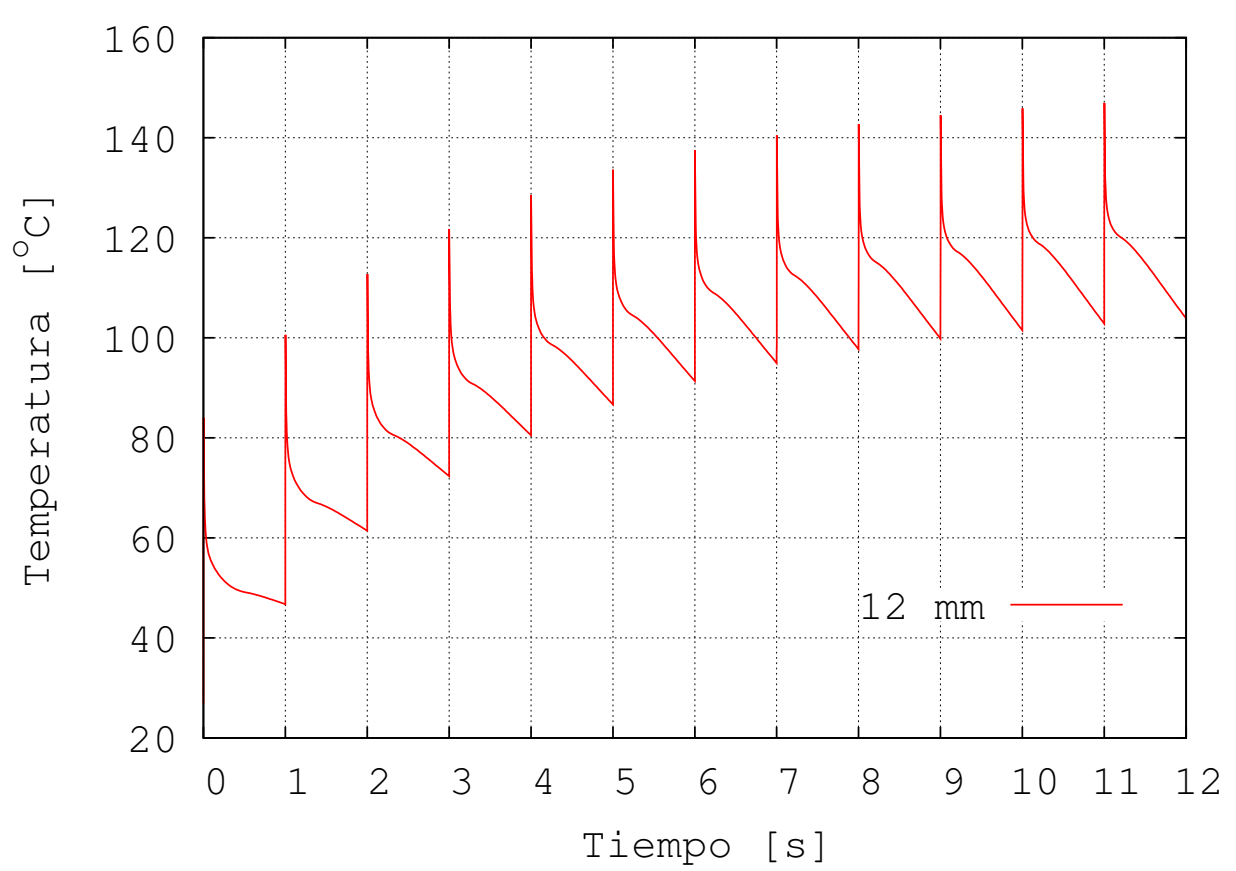

Figura 2-11: Evolución de la temperatura máxima en una placa de Be de $12 \mathrm{~mm}$ con una frecuencia de impacto de $1 \mathrm{~Hz}$.

momento en el que se alcanzan $147,3{ }^{\circ} \mathrm{C}$, siendo éste el máximo del pseudo-estacionario. La temperatura máxima de la cara en contacto con el agua se sitúa en torno a los 92 ${ }^{\circ} \mathrm{C}$. El margen con respecto a la temperatura de ebullición del refrigerante, $152{ }^{\circ} \mathrm{C}$, es suficiente. Comprobamos, por tanto, que las estimaciones efectuadas en la Sección 2.3.1 para predimensionar la refrigeración han sido correctas.

Una vez calculada la evolución de temperaturas en la placa, pasamos a la tercera fase del cálculo, el cálculo mecánico. Para ello implementamos la evolución de temperaturas en un modelo mecánico de ANSYS y calculamos las tensiones térmicas asociadas a la dilatación diferencial de la placa. Se ha usado el mismo mallado, esto es, la misma posición de nodos en ambos modelos, ya que el refinamiento del modelo térmico permite capturar los gradientes de tensiones del modelo mecánico.

Como condición de contorno mecánica se permite dilatación libre en toda la placa. De este modo hacemos independientes los resultados de tensiones que obtendremos del sistema de sujeción de la placa, estudiando únicamente aquellos valores de tensión que son consustanciales al impacto del haz y no provocados por el sistema de sujeción. Las propiedades del berilio empleadas en el modelo mecánico son el módulo de Young, E, el 


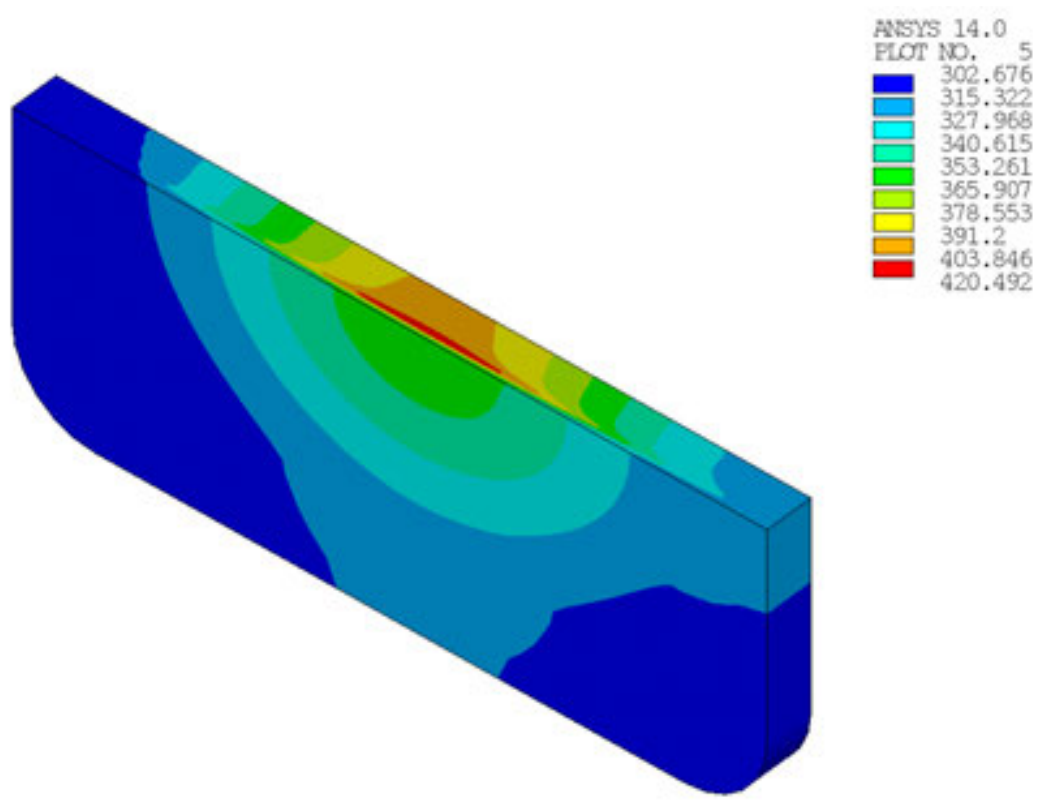

Figura 2-12: Distribución de temperatura [K] en una placa de Be de $12 \mathrm{~mm}$ de espesor, en el momento en el que se alcanza el máximo del pseudo-estacionario.

coeficiente de Poisson, $\nu$, y el coeficiente de dilatación térmica, $\alpha$. A temperatura ambiente dichas propiedades toman los siguientes valores: $E=287 G P a, \nu=0,032$ y $\alpha=11,5 \cdot 10^{-} 6$ $K^{-1}$ [134]. Como puede verse, el módulo de Young del berilio es muy elevado. Por ponerlo en contexto, este parámetro en los aceros de construcción habituales alrededor de 200 $G P a$ [136]. Esto, unido a su baja densidad, le conceden una elevada rigidez específica, por lo que este material es frecuentemente empleado para la fabricación de lentes de grandes telescopios.

La Figura 2-13 muestra la evolución de la tensión equivalente de Von-Mises máxima en la placa de berilio de $12 \mathrm{~mm}$ de espesor, provocada por el impacto del haz. El impacto del primer pulso de protones generará un calentamiento local que induce un brusco incremento de tensión equivalente de $123 \mathrm{MPa}$. A medida que los pulsos del acelerador se suceden, la distribución de temperaturas se hace un poco más homogénea y el estado tensional de la placa se estabiliza. Cuando la placa ha alcanzado el pseudo-estacionario térmico, cada pulso del acelerador induce un salto de tensión equivalente máxima de, aproximadamente, $80 \mathrm{MPa}$ alcanzando un valor máximo de $190 \mathrm{MPa}$. Estos valores de tensión son inferiores al límite elástico del berilio S-200 F, $250 \mathrm{MPa}$ [137], y a su tensión de rotura, 324 $M P a$ [137]; por tanto el material trabajaría en régimen elástico y el fallo estático de la 
placa quedaría descartado. Cabe destacar que el material seleccionado mantiene buenas propiedades mecánicas a elevadas temperaturas. Con una temperatura de fusión de 1287 ${ }^{\circ} \mathrm{C}$, mantiene su límite elástico prácticamente inalterado hasta $\operatorname{los} 150{ }^{\circ} \mathrm{C}$, y superior a los $200 \mathrm{MPa}$ hasta los $315^{\circ} \mathrm{C}$. A partir de dicha temperatura, esta propiedad comienza a degradarse aunque manteniéndose por encima de los $100 \mathrm{MPa}$ hasta los $600{ }^{\circ} \mathrm{C}$ [138].

La Figura 2-14 muestra la distribución de tensiones equivalentes al final del pulso una vez alcanzado el estado pseudo-estacionario. Podemos observar como la zona de máxima tensión se encuentra en la zona de máximo gradiente térmico, alrededor del centro del pico de Bragg.

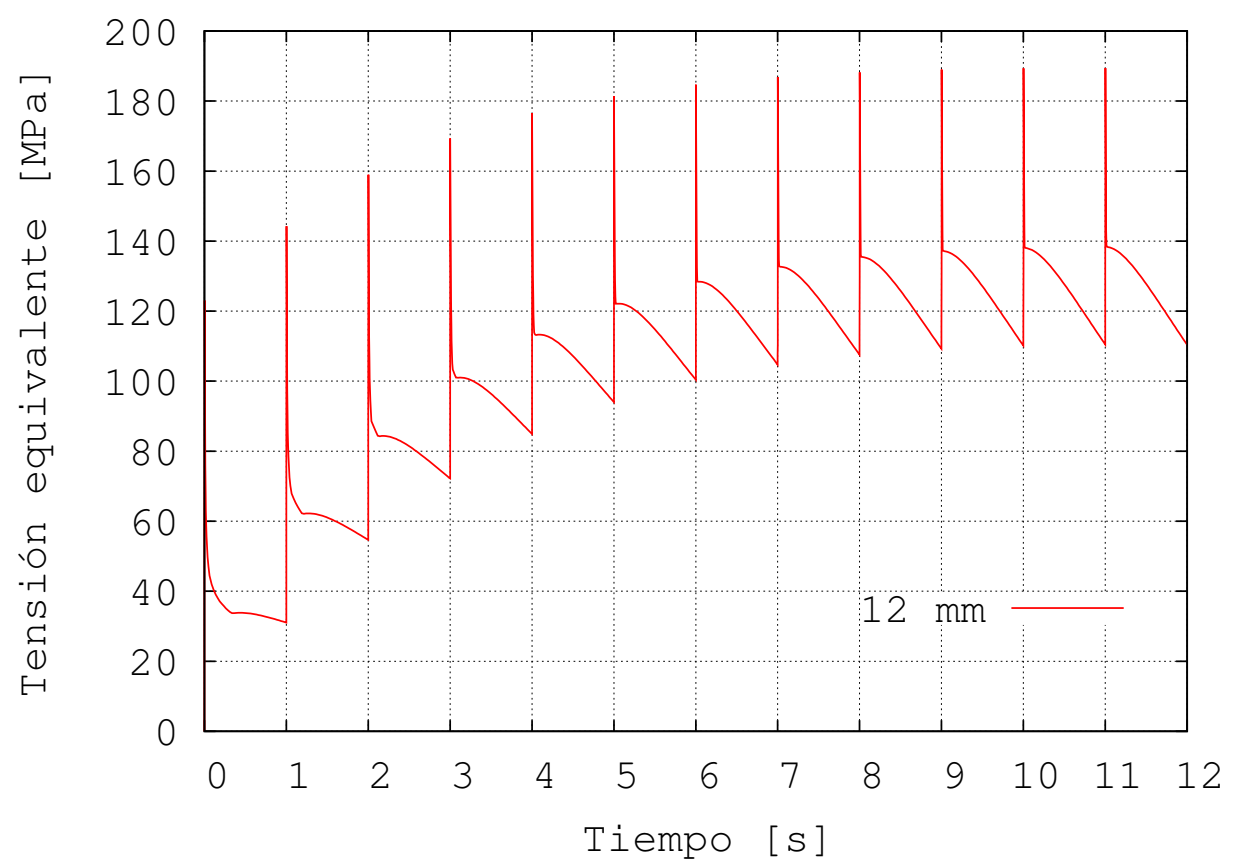

Figura 2-13: Evolución de la tensión de von Mises máxima en una placa de Be de 12 mm con una frecuencia de impacto de $1 \mathrm{~Hz}$.

\subsubsection{Optimización del espesor del blanco}

En el análisis anterior, se observa como la concentración de la deposición energética alrededor del pico de Bragg es la contribución dominante a los valores de tensión y temperatura máximos. Es pues evidente que si se pudiera evitar esta concentración de deposición energética, las condiciones de operación del blanco serían más favorables. Para ello se haría necesario reducir el espesor del blanco, permitiendo que los protones lo atravesaran, y 


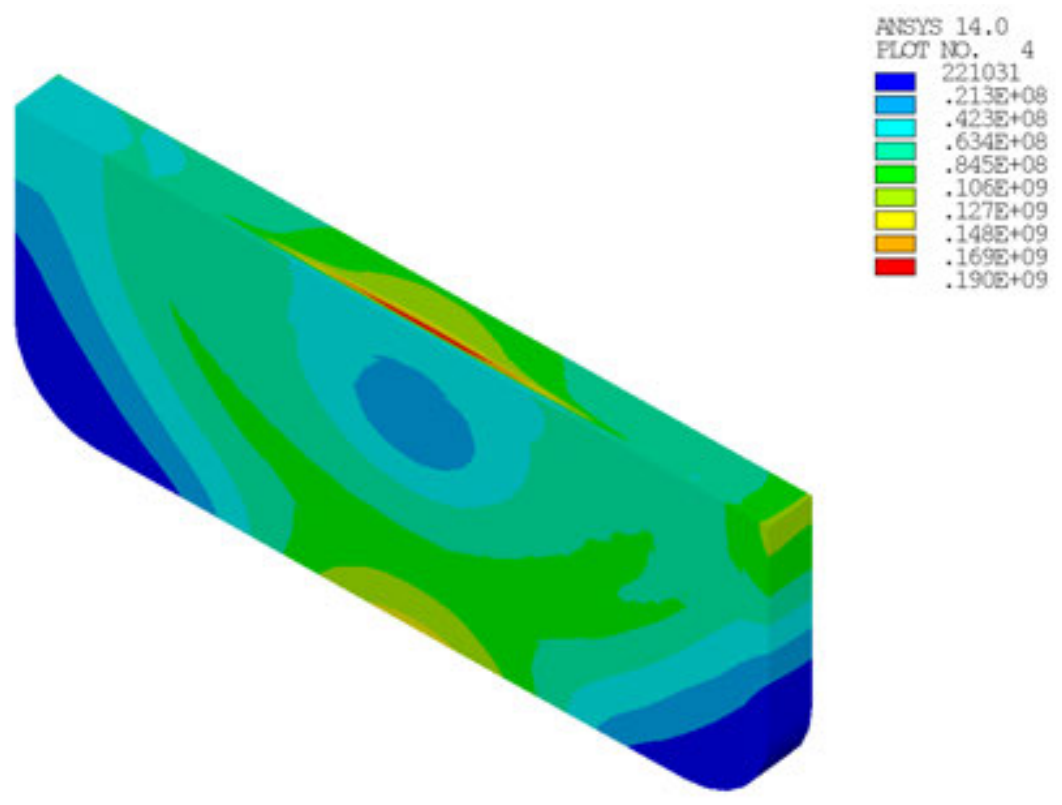

Figura 2-14: Distribución de tensiones [Pa] en una placa de Be de $12 \mathrm{~mm}$ de espesor, en el momento en el que se alcanza el máximo del pseudo-estacionario.

evitando así la deposición concentrada al final de su trayectoria y por tanto la aparición del pico de Bragg. Esta solución reduce el volumen de interacción entre los protones y el berilio, y consecuentemente la producción neutrónica; pero dado que la mayoría de los neutrones son producidos por protones rápidos, es decir, al comienzo de su trayectoria en el berilio, y que la concentración de la deposición energética es producida por protones de baja energía, esto es, al final de su trayectoria, reducir el espesor para dejar fuera el pico de Bragg puede generar una fuerte reducción de la deposición energética sin inducir un deterioro proporcional en la producción neutrónica.

Con el fin de analizar esta posible mejora, efectuamos un análisis paramétrico repitiendo la secuencia de cálculo descrita en la Sección 2.3.3 para distintos espesores de placa decrecientes.

La Figura 2-15 muestra el máximo de potencia volumétrica en la placa en función de su espesor. En ella podemos observar como este valor crece de forma casi exponencial hasta la aparición del pico de Bragg alrededor de los $11 \mathrm{~mm}$ de profundidad, alcanzando la deposición volumétrica el valor máximo de $152 \mathrm{~kW} / \mathrm{cm}^{3}$ calculado en la Sección 2.3.2. Para espesores mayores el máximo ya se ha alcanzado y por tanto este valor permanece 
constante. Para espesores menores, se observa que a partir de los $9 \mathrm{~mm}$ este valor se dispara por la cercanía del pico de Bragg y el comienzo de la concentración de la deposición energética.

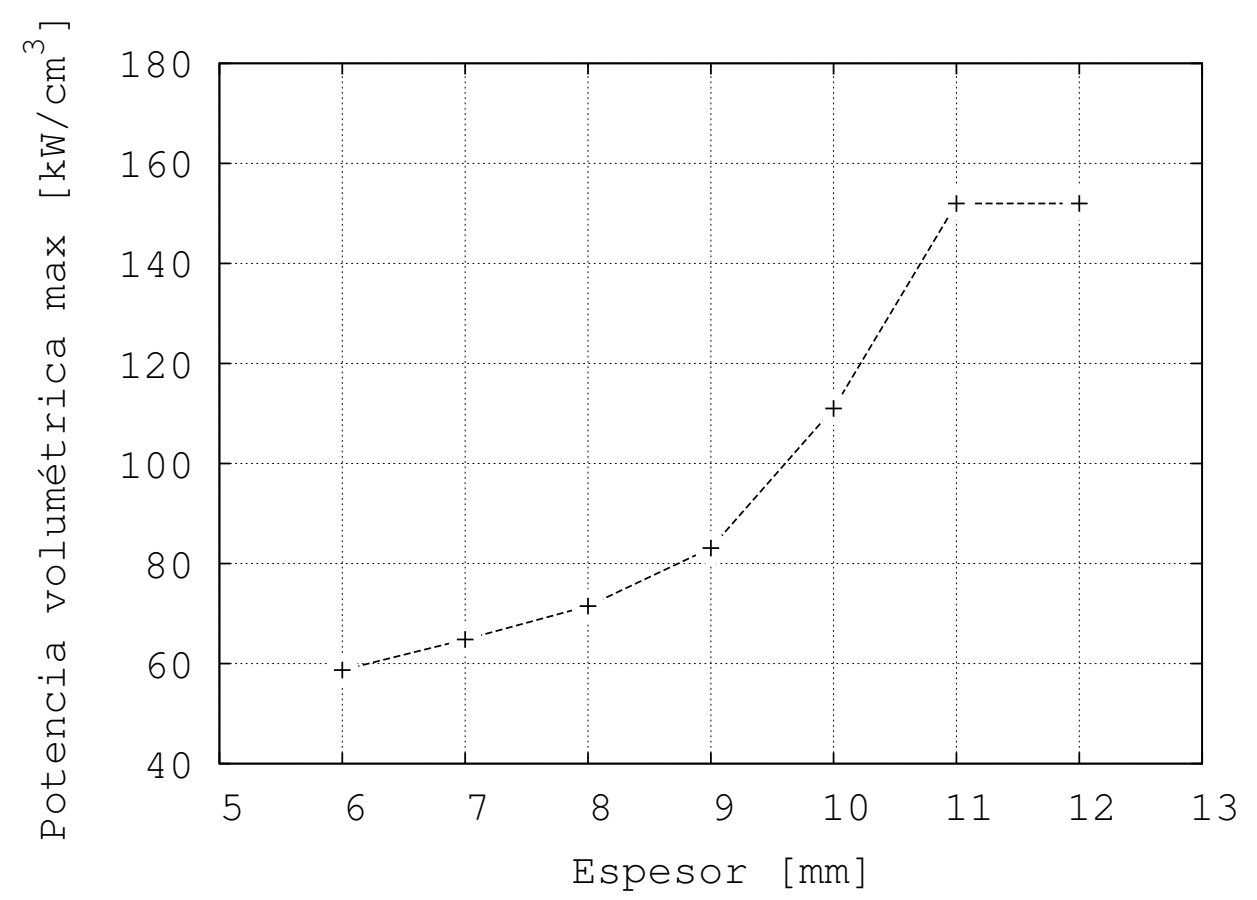

Figura 2-15: Evolución de la potencia volumétrica máxima implementada durante el pulso en la placa de Be en función de su espesor.

La Figura 2-16 muestra la evolución de la potencia total implementada en la placa durante el pulso en función de su espesor. En ella podemos observar como la potencia total implementada en la placa crece de forma más lineal que la potencia volumétrica máxima, hasta la aparición del pico de Bragg a los $11 \mathrm{~mm}$. El ligero crecimiento de este valor para espesores mayores se debe a la dispersión en el alcance máximo de los protones y a la energía absorbida debida a fotones. La potencia total implementada máxima alcanza el valor de 3,58 $M W$ calculado en la Sección 2.3.2.

Se estudia a continuación cuál es el efecto del espesor sobre la producción neutrónica. La Figura 2-17 muestra la razón de conversión neutrón/protón en función de este parámetro. Como se calculó en la Sección 2.2, la tasa máxima una vez todos los protones se han detenido es $6,5 \cdot 10^{-2} \mathrm{n} / \mathrm{p}$. En dicha gráfica podemos observar como la contribución incremental de cada milímetro de espesor a la tasa de producción neutrónica se reduce según se acerca el pico de Bragg; de tal manera que, por ejemplo, los protones que sobrepasan los 


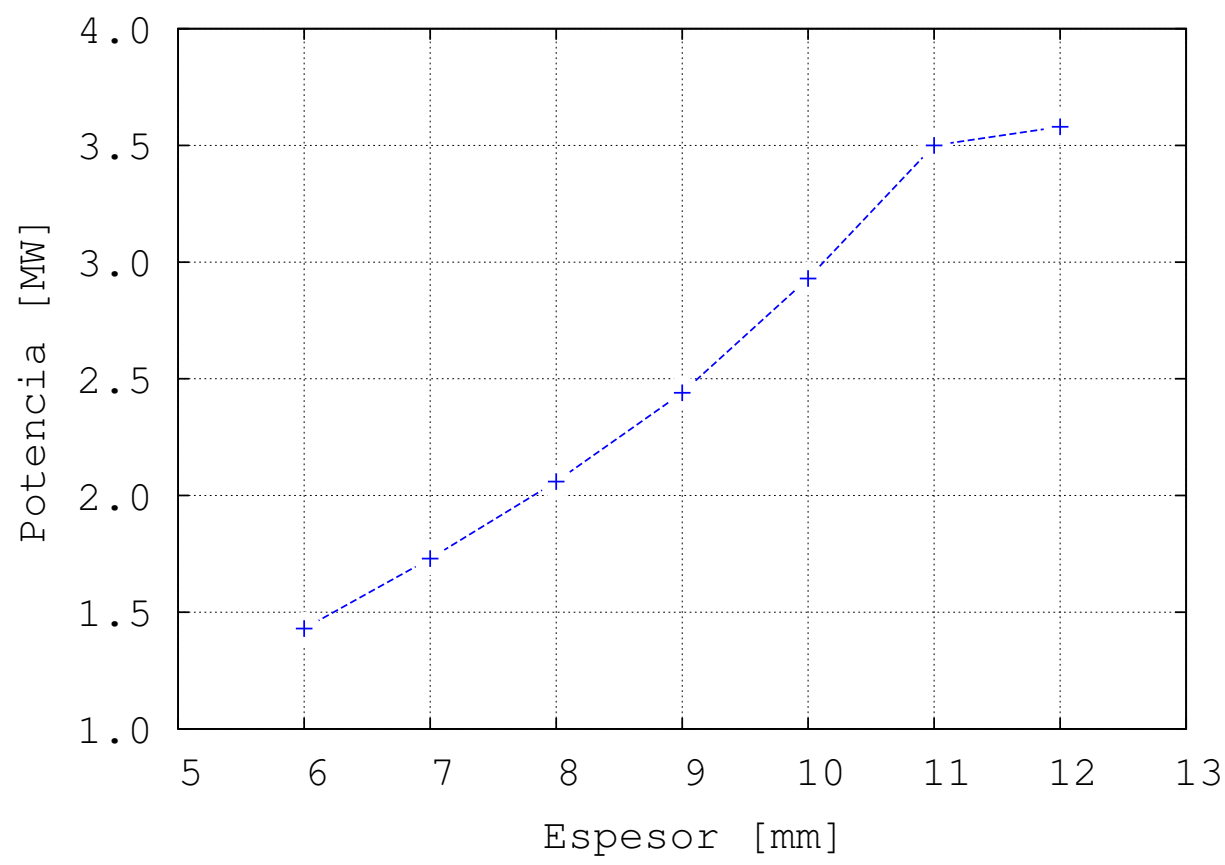

Figura 2-16: Evolución de la potencia implementada durante el pulso en la placa de Be en función de su espesor.

$10 \mathrm{~mm}$ de profundidad solo contribuyen con aproximadamente un $4,5 \%$ a la producción neutrónica.

Con el estudio precedente se comprueba y cuantifica como la mayor contribución a la deposición energética es realizada por los protones al final de su trayectoria, mientras que la principal contribución a la producción neutrónica es aportada por los protones al principio de la misma.

Analicemos ahora los efectos termomecánicos de esta fenomenología. Las Figuras 2-18 y 2-19 representan la evolución de la temperatura y tensión equivalente máximas en la placa en función de su espesor. En ellas, podemos observar como ambas magnitudes sufren un importante incremento para espesores mayores a los $9 \mathrm{~mm}$. Este comportamiento se debe al súbito aumento de la potencia volumétrica descrito en la Figura 2-15 por el comienzo del pico de Bragg, lo cual genera fuertes gradientes térmicos y, consecuentemente, tensiones por dilatación diferencial.

Las Figuras 2-20 y 2-21 muestran la evolución temporal de la temperatura y tensión equivalente máximas para distintos espesores. En ellas, podemos observar como el salto 


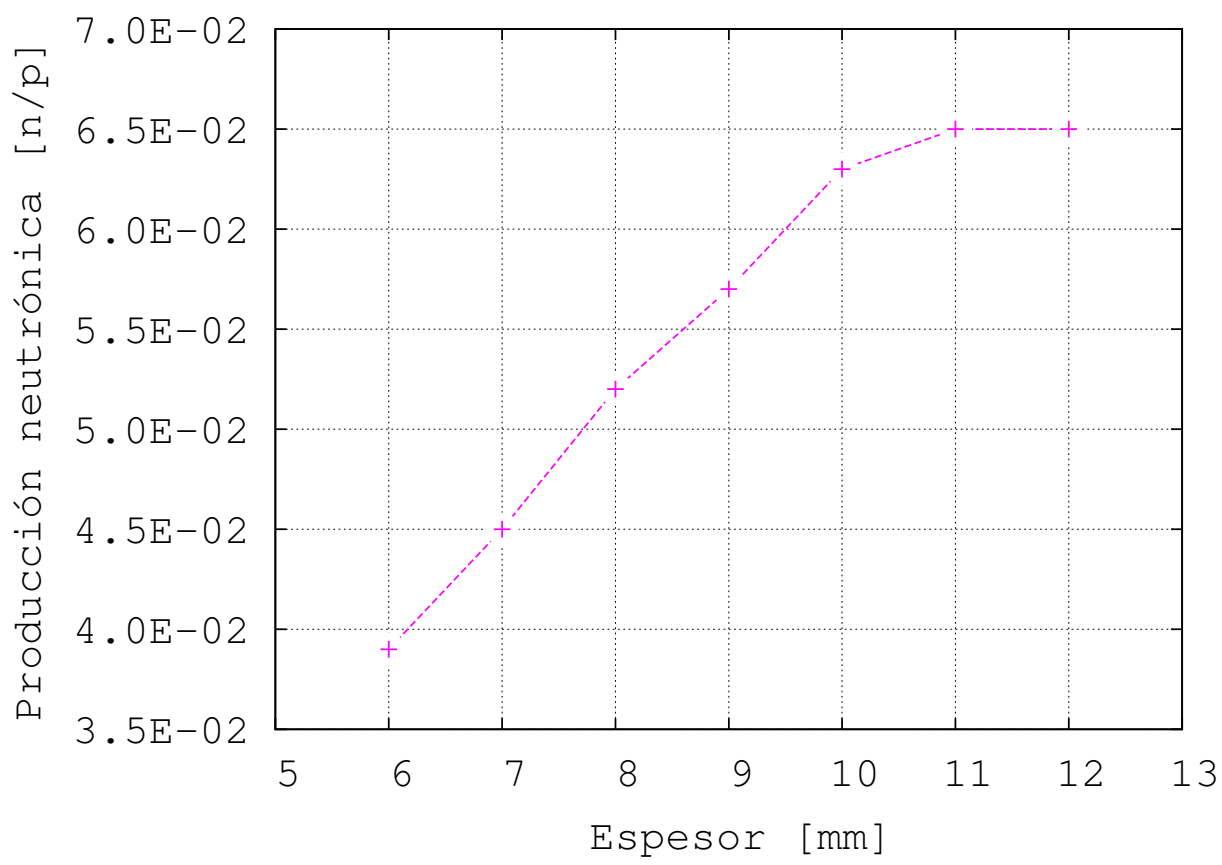

FigURA 2-17: Evolución de producción neutrónica (neutrones por protón incidente) en función del espesor de la placa de Be.

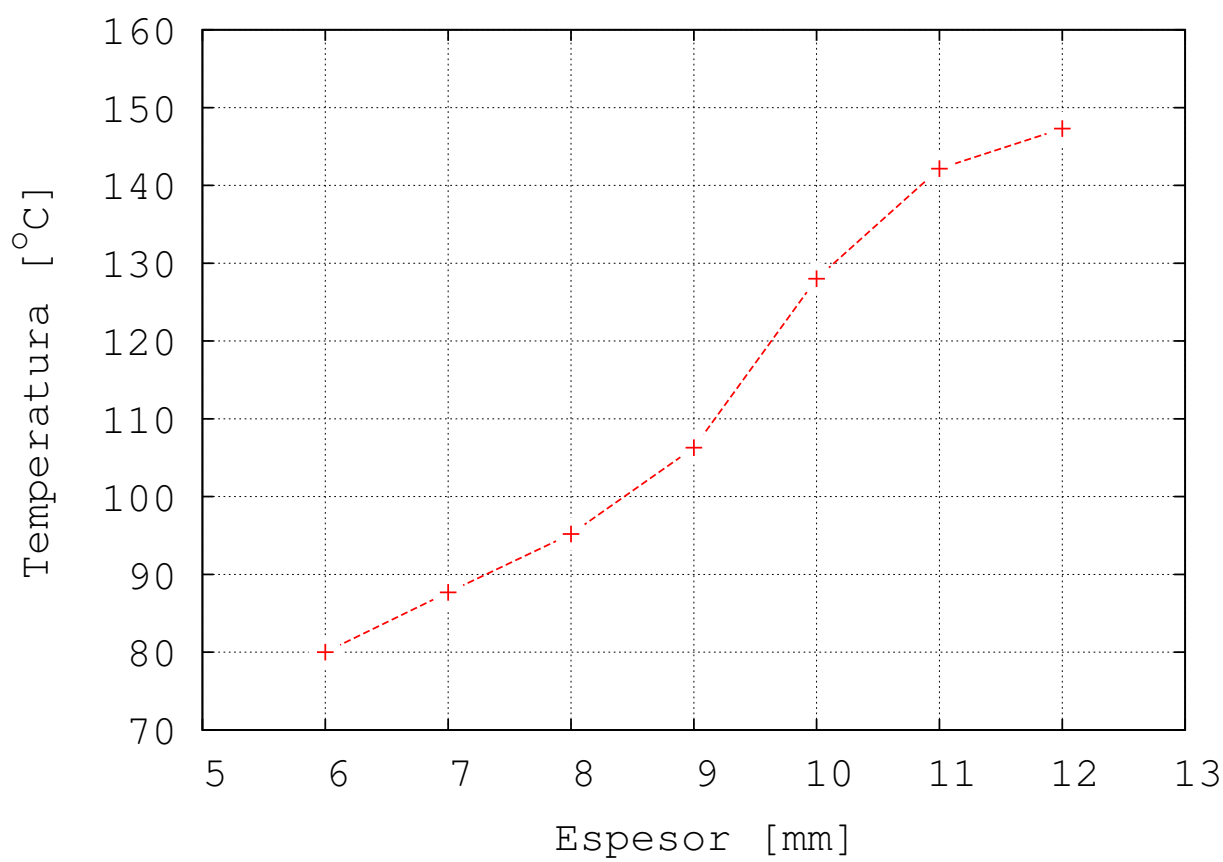

Figura 2-18: Evolución de la temperatura máxima en la placa de Be en función de su espesor. 


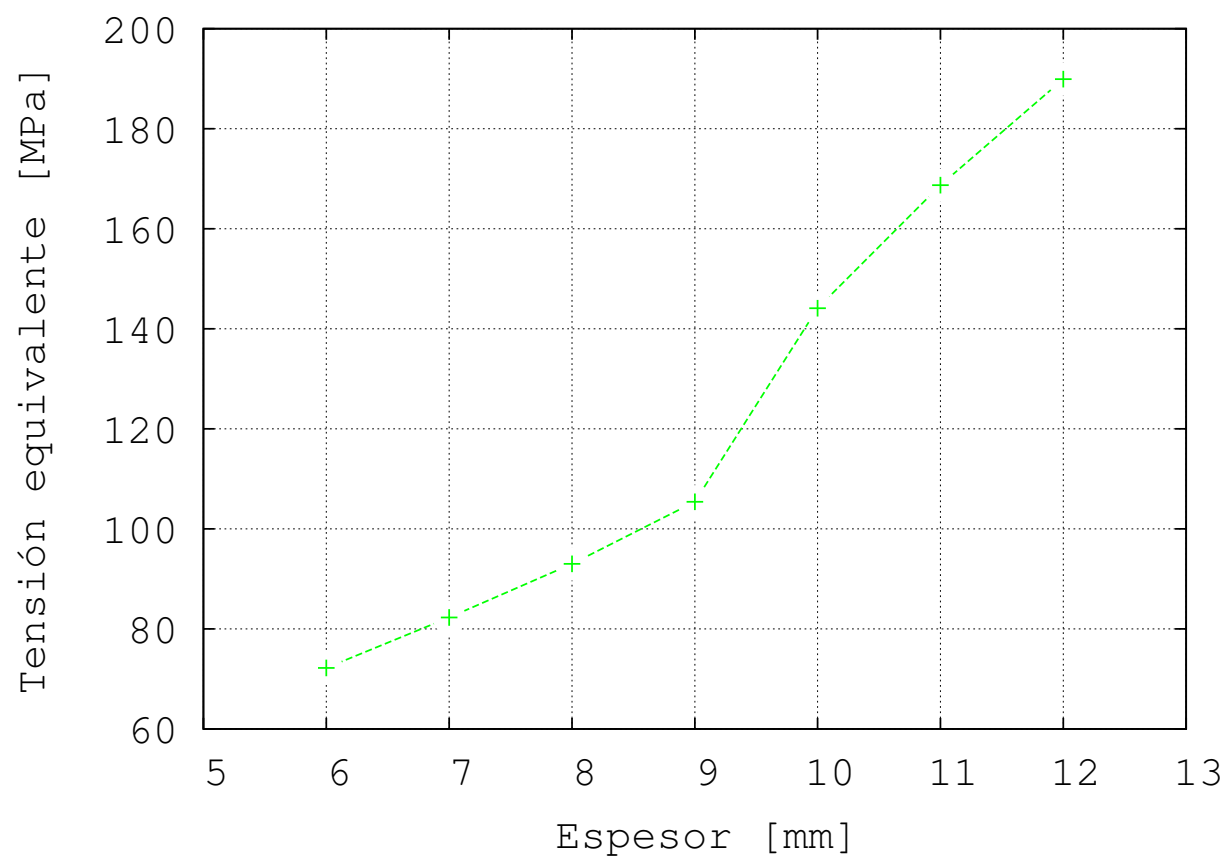

Figura 2-19: Evolución de la tensión de von Mises máxima en la placa de Be en función de su espesor.

en temperatura y tensión inducidos por el pulso es mucho mayor si se produce el pico de Bragg en la placa. Por claridad en dichas figuras el número de espesores representados es menor que en las gráficas anteriores.

La Tabla 2.5 resume la información presentada en las gráficas anteriores. En ella se establece la comparación con el máximo obtenido para la placa de $12 \mathrm{~mm}$, incluyendo la reducción porcentual de cada magnitud con respecto a esta configuración en función del espesor de la placa. En dicha tabla podemos observar como la reducción en potencia volumétrica máxima y la reducción en tensión equivalente máxima son parejas para todos los espesores, con lo que se confirma la relación causa efecto entre ambas magnitudes.

Observamos también como una reducción del espesor del blanco a $9 \mathrm{~mm}$ induce una reducción de la tensión equivalente máxima del 44,5\%, y una reducción de temperatura máxima del 27,8\% con respecto a la placa de $12 \mathrm{~mm}$; mientras que la producción neutrónica solo cae un 12,3\%. Mayores reducciones del espesor continúan reduciendo la tensión y temperaturas máximas, pero a un ritmo menor e induciendo pérdidas de producción neutrónica mayores del $20 \%$. Por tanto, se toma $9 \mathrm{~mm}$ como espesor de referencia para el blanco de ESS-Bilbao. Con esta configuración, la producción neutrónica total alcanzaría 


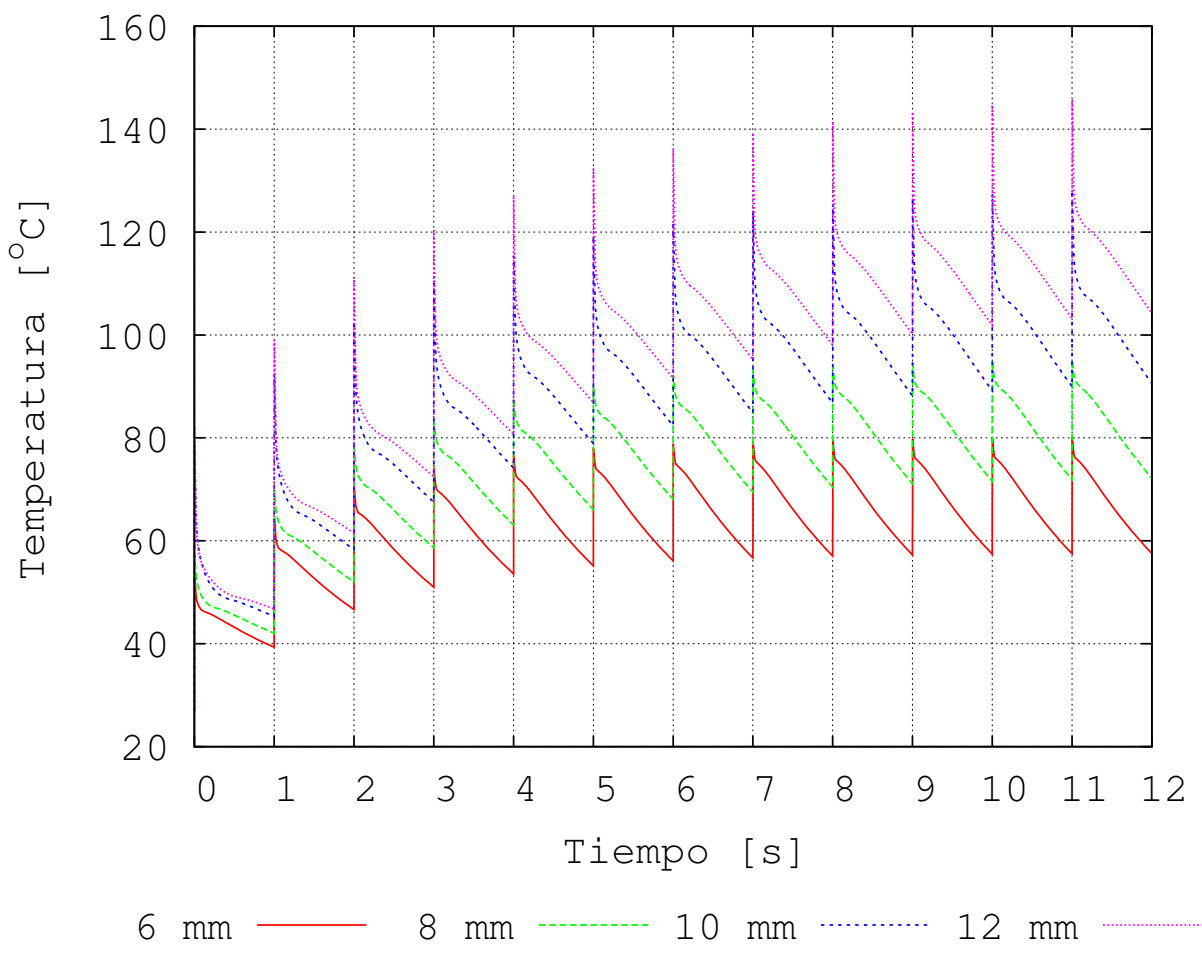

Figura 2-20: Comparativa de la evolución temporal de la temperatura máxima en la placa de Be para diferentes espesores.

\begin{tabular}{|c|cc|cc|cc|cc|cc|}
\hline Esp & \multicolumn{2}{|c|}{$\mathbf{P}$} & \multicolumn{2}{|c|}{$\mathbf{P}$ vol max } & \multicolumn{2}{c|}{$\mathbf{n} / \mathbf{p}$} & \multicolumn{2}{c|}{$\mathbf{T} \max$} & \multicolumn{2}{c|}{$\sigma \max$} \\
$\mathbf{m m}$ & $\boldsymbol{M} \boldsymbol{W}$ & $\boldsymbol{\Delta} \%$ & $\boldsymbol{k} \boldsymbol{W} / \mathbf{c m}^{\mathbf{3}}$ & $\boldsymbol{\Delta} \%$ & $\mathbf{n} / \mathbf{p}$ & $\boldsymbol{\Delta} \%$ & ${ }^{o} \boldsymbol{C}$ & $\boldsymbol{\Delta} \%$ & $\boldsymbol{M} \boldsymbol{P a}$ & $\boldsymbol{\Delta} \%$ \\
\hline $\mathbf{6}$ & 1.43 & -60.1 & 58.7 & -61.4 & $3,2 \cdot 10^{-2}$ & -50.8 & 80.0 & -45.7 & 72.2 & -62.0 \\
$\mathbf{7}$ & 1.73 & -51.7 & 64.8 & -57.4 & $4,5 \cdot 10^{-2}$ & -30.8 & 87.7 & -40.5 & 82.3 & -56.7 \\
$\mathbf{8}$ & 2.06 & -42.5 & 71.5 & -53.0 & $5,2 \cdot 10^{-2}$ & -20.0 & 95.2 & -35.4 & 93.0 & -51.0 \\
$\mathbf{9}$ & 2.44 & -31.8 & 83.1 & -45.4 & $5,7 \cdot 10^{-2}$ & -12.3 & 106.3 & -27.8 & 105.4 & -44.5 \\
$\mathbf{1 0}$ & 2.93 & -18.2 & 111.5 & -26.7 & $6,3 \cdot 10^{-2}$ & -3.1 & 128.0 & -13.1 & 144.1 & -24.1 \\
$\mathbf{1 1}$ & 3.50 & -2.2 & 152.1 & 0.0 & $6,5 \cdot 10^{-2}$ & 0.0 & 142.1 & -3.5 & 168.7 & -11.2 \\
$\mathbf{1 2}$ & 3.58 & - & 152.1 & - & $6,5 \cdot 10^{-2}$ & - & 147.3 & - & 189.9 & - \\
\hline
\end{tabular}

Tabla 2.5: Estudio paramétrico de los efectos del espesor del blanco. Porcentajes calculados con respecto a la placa de $12 \mathrm{~mm}$ de espesor 


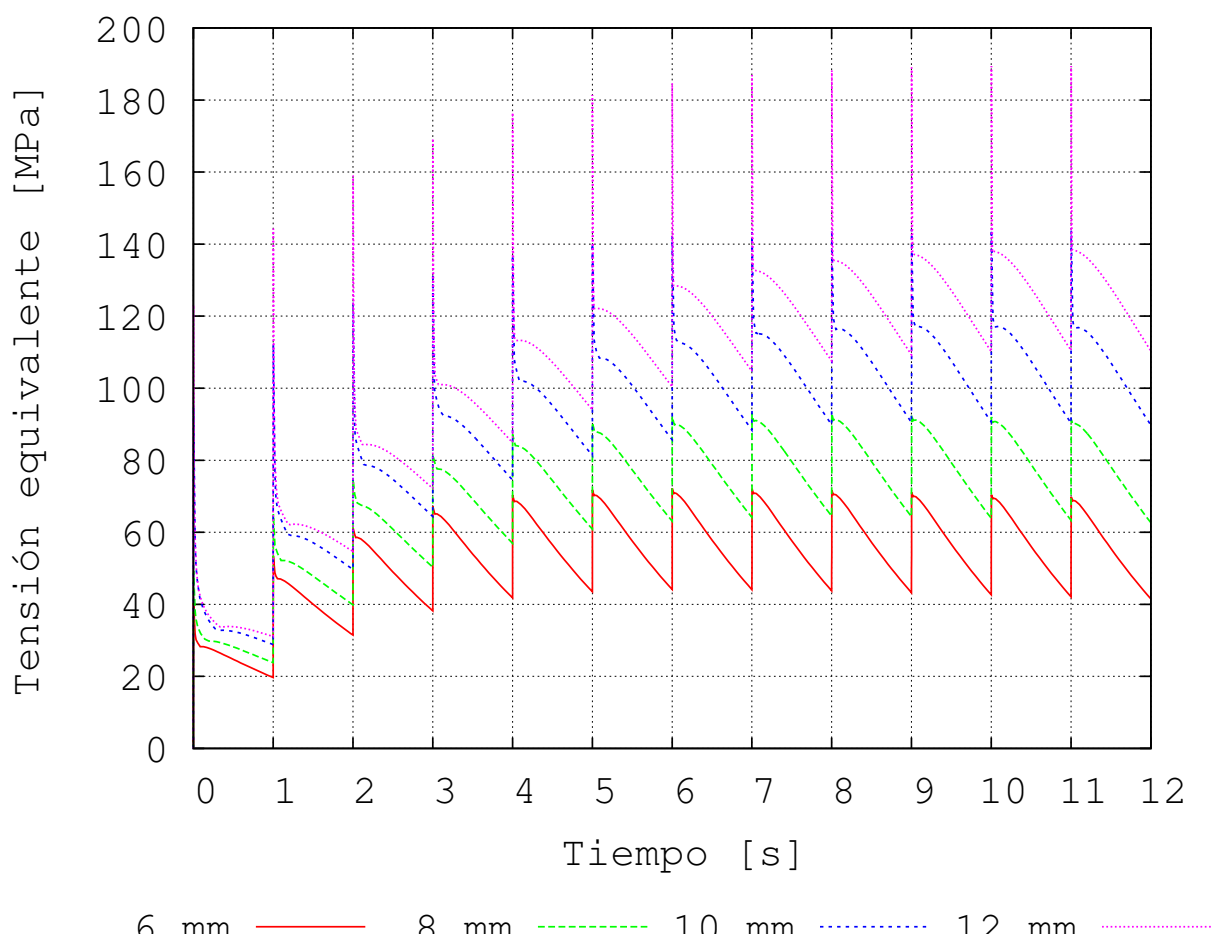

Figura 2-21: Comparativa de la evolución temporal de la tensión equivalente máxima en la placa de Be para diferentes espesores. 
$\operatorname{los} 8,0 \cdot 10^{14} \mathrm{n} / s$.

Las Figuras 2-22, 2-23 y 2-24 muestran la distribución de potencia, temperatura y tensión en la placa de $9 \mathrm{~mm}$; estas dos últimas para el momento en el que se alcanza el máximo del pseudo-estacionario. Con espesores inferiores a la profundidad del pico de Bragg, la temperatura máxima en la placa al final de cada pulso se da en la cara en contacto con el refrigerante, ya que en esta zona es donde se encuentra la máxima deposición energética. Las Figuras 2-25 y 2-26 representan la evolución temporal de la tensión y temperatura máximas para la placa de referencia.

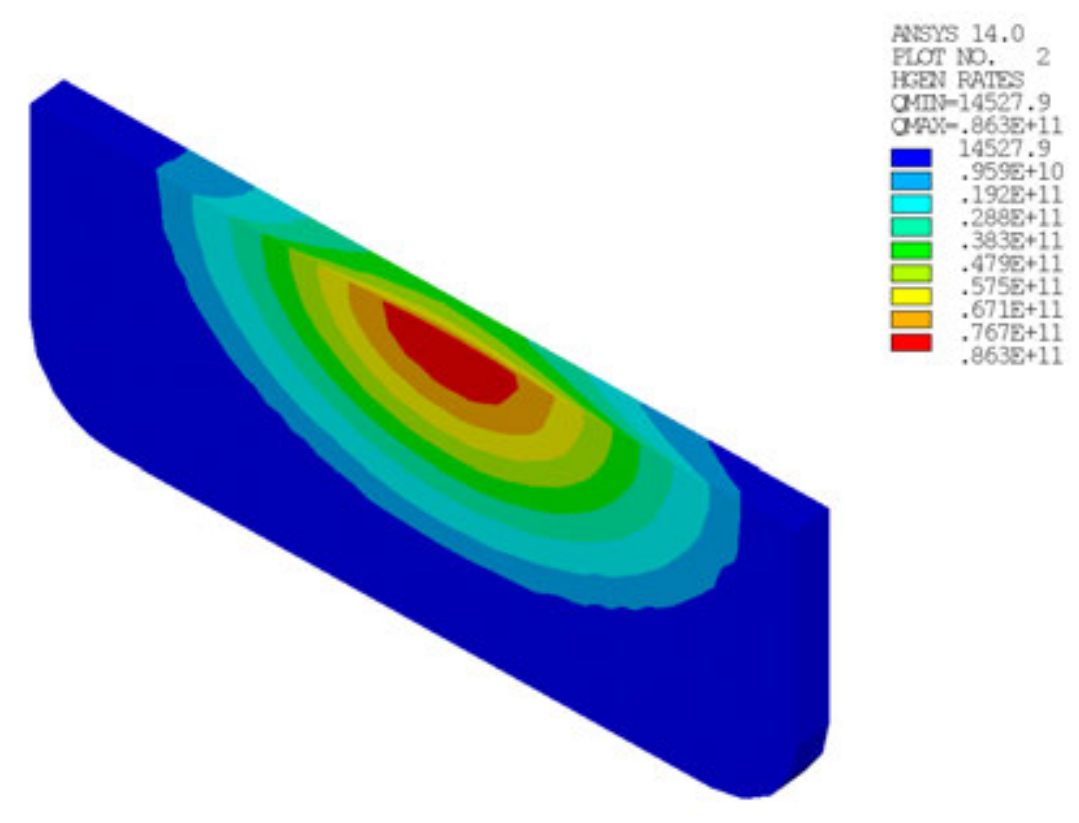

Figura 2-22: Potencia implementada en ANSYS en una placa de Be de $9 \mathrm{~mm}\left[\mathrm{~W} / \mathrm{m}^{3}\right]$.

\subsubsection{Determinación del número de placas en el blanco rotatorio}

Una vez que se ha determinado el espesor de referencia del blanco, se procede a estudiar el efecto del número de placas entre las que se reparten los pulsos del acelerador. Como se ha mencionado anteriormente, el número de placas condiciona la frecuencia de impacto en cada placa y, por tanto, la potencia media que cada una de ellas recibe.

Con el objetivo de llevar a cabo este estudio, se realiza un análisis paramétrico del efecto del número de placas sobre la tensión y temperatura máximas en el berilio. Empleando 


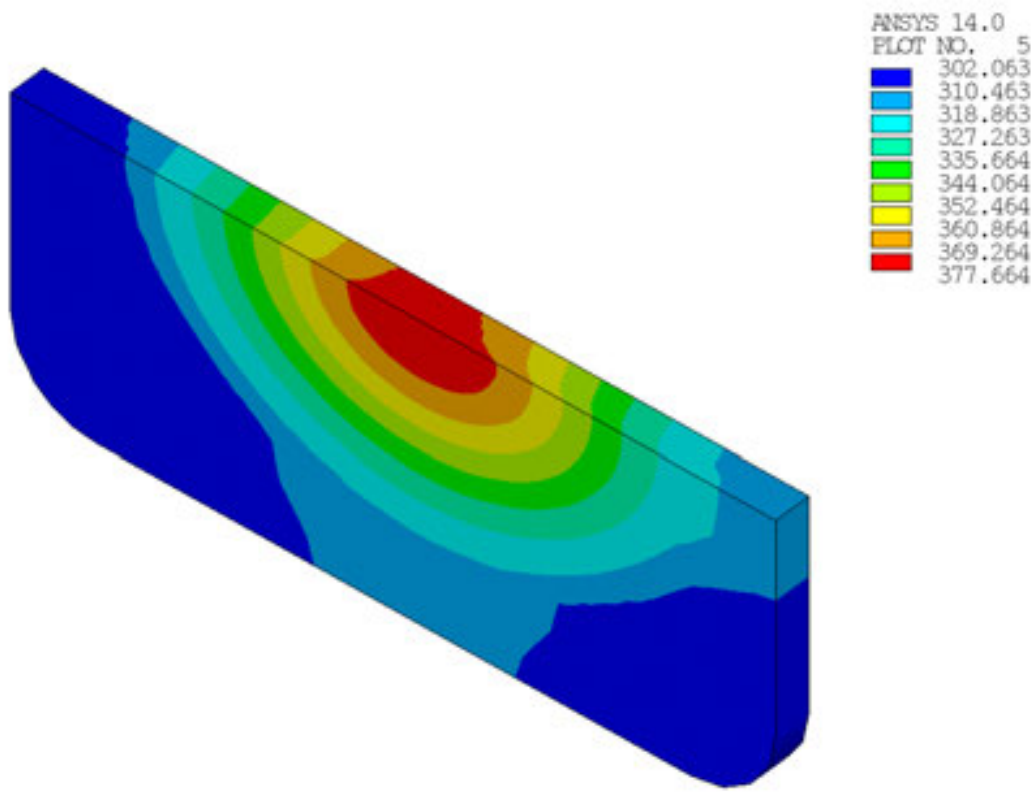

$T$ Max $=378.020191 \mathrm{~K}(\mathrm{t}=11.0015)$

Figura 2-23: Distribución de temperatura [K] en una placa de Be de $9 \mathrm{~mm}$ de espesor en el momento en el que se alcanza el máximo del pseudo-estacionario.

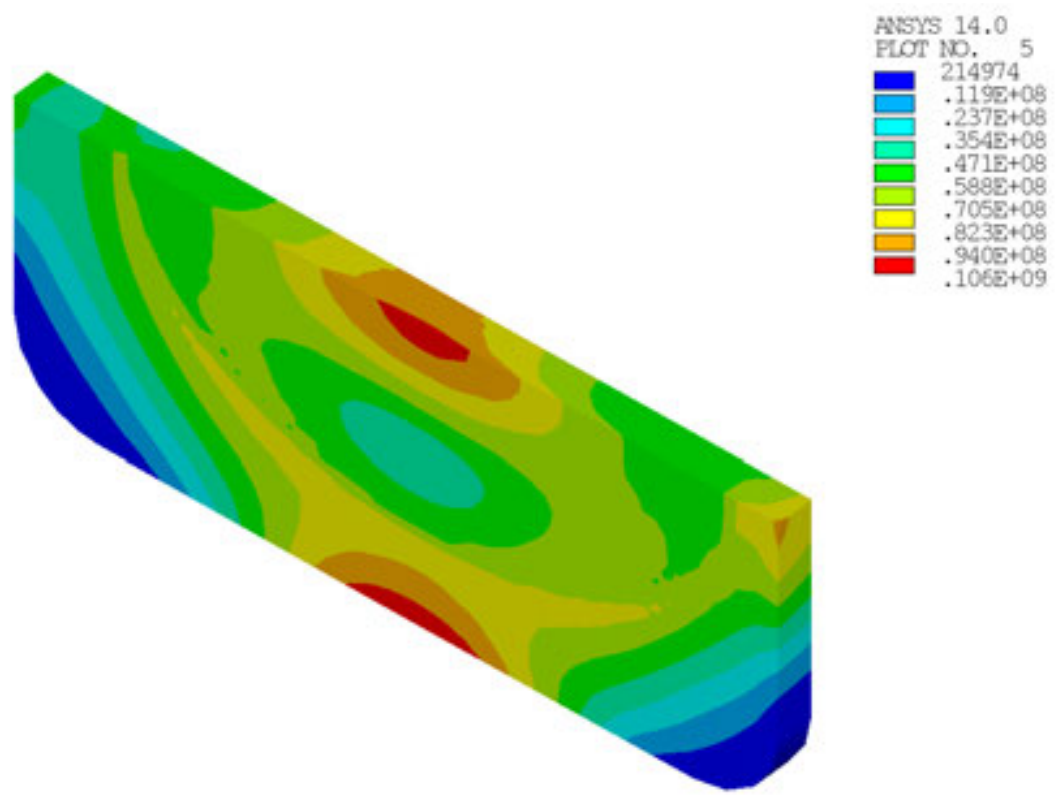

Max stress $=105705918 \mathrm{~Pa}(t=8.0015)$

Figura 2-24: Distribución de tensiones [Pa] en una placa de Be de $9 \mathrm{~mm}$ de espesor en el momento en el que se alcanza el máximo del pseudo-estacionario. 


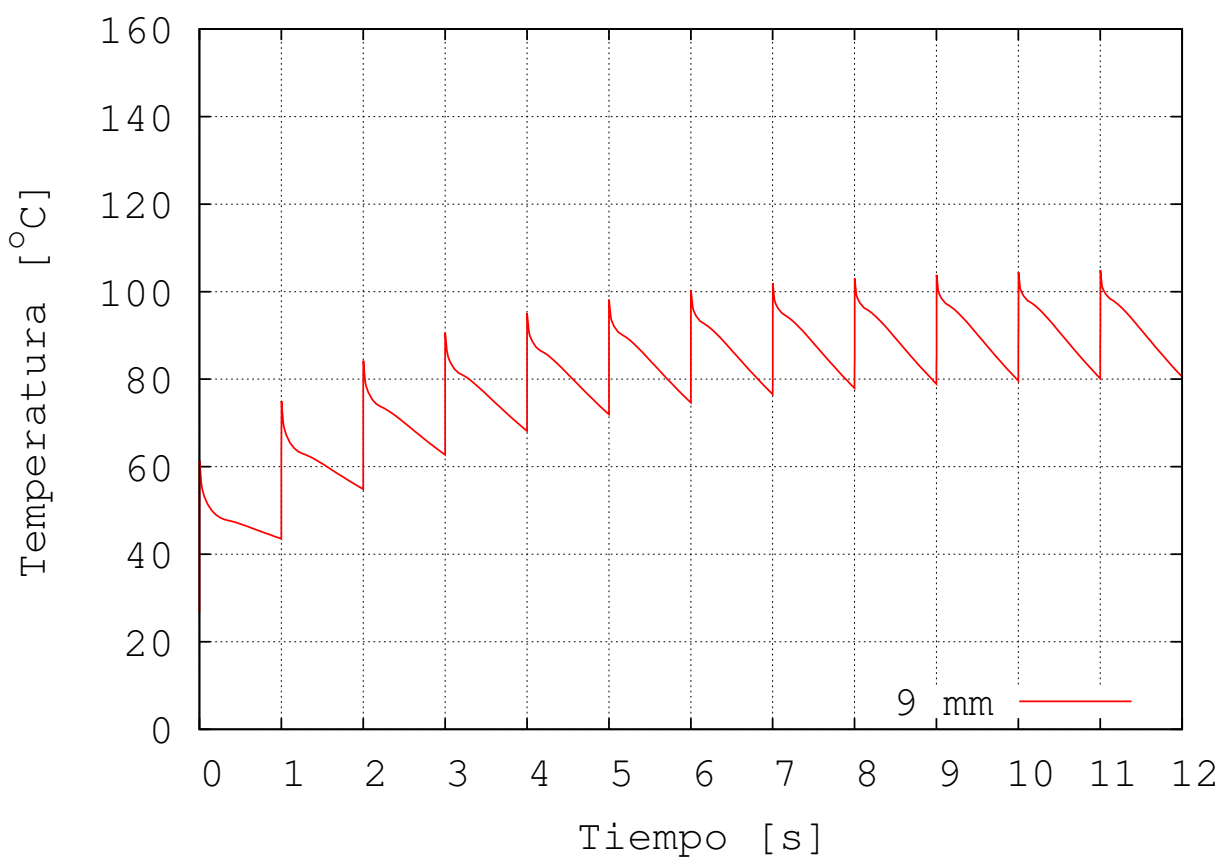

Figura 2-25: Evolución de la temperatura máxima en una placa de Be de $9 \mathrm{~mm}$ con una frecuencia de impacto de $1 \mathrm{~Hz}$.

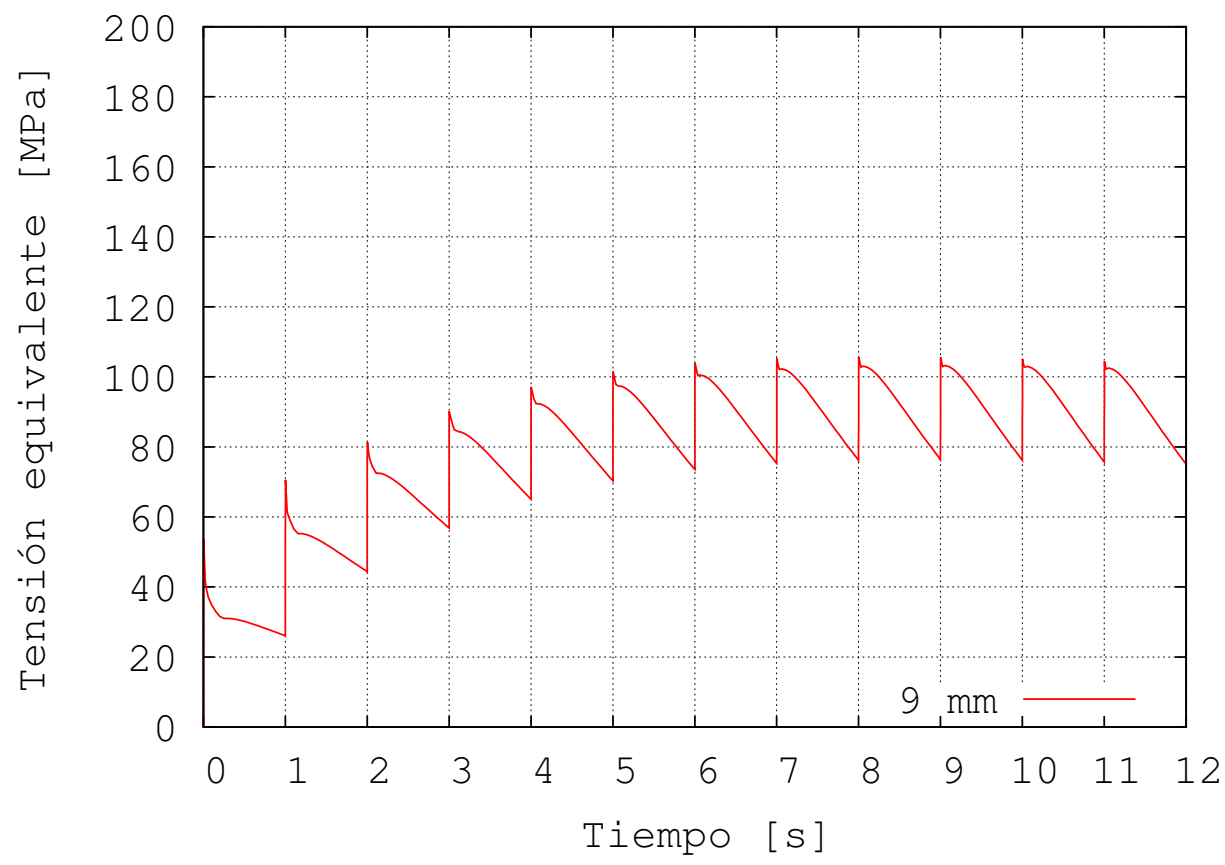

Figura 2-26: Evolución de la tensión equivalente máxima en una placa de Be de $9 \mathrm{~mm}$ con una frecuencia de impacto de $1 \mathrm{~Hz}$. 
las mismas hipótesis de cálculo y modelado, estas magnitudes se han calculado para las siguientes frecuencias de impacto: 0.5, 1.0, 1.5 y $2.0 \mathrm{~Hz}$; lo cual implicaría el empleo de 40, 20, $\approx 13$ y 10 placas. Las Figuras 2-27 y 2-28 presentan los resultados obtenidos.

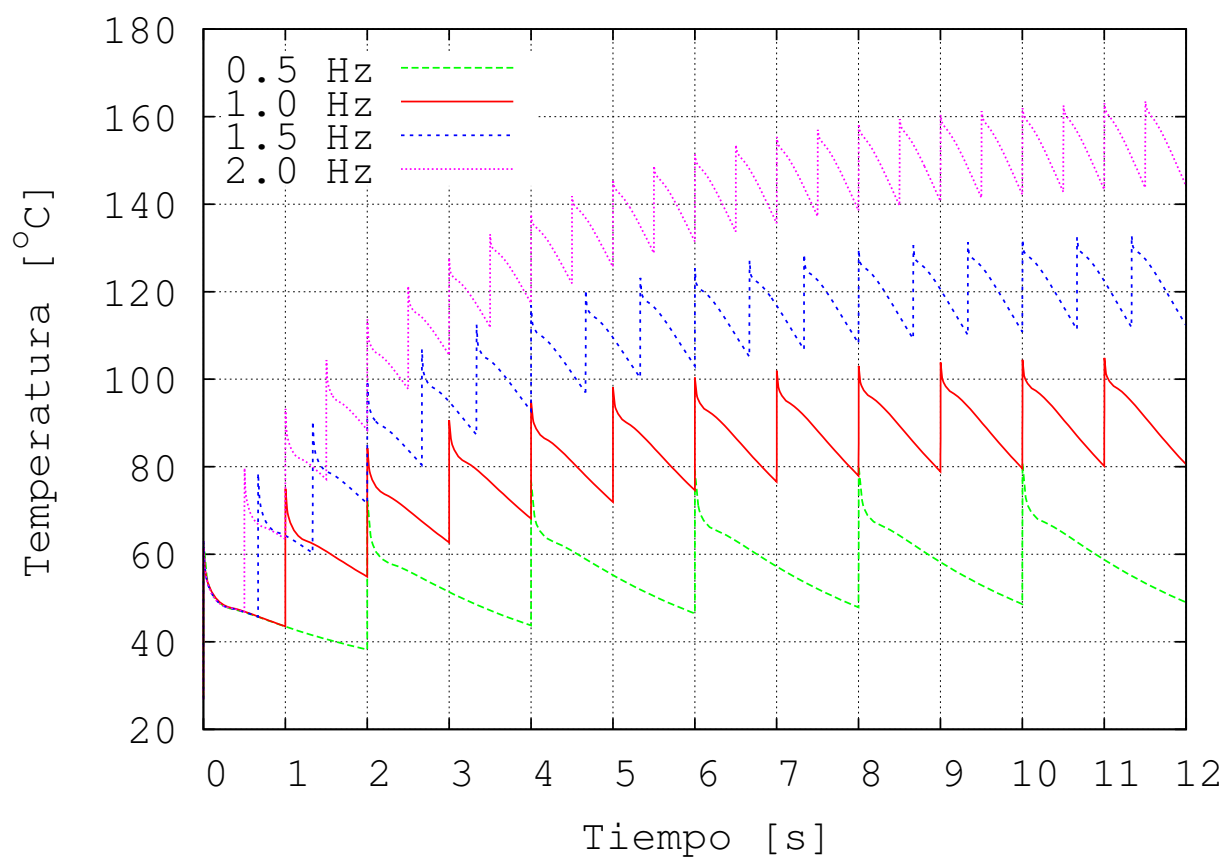

Figura 2-27: Evolución de la tensión equivalente máxima en una placa de Be de $9 \mathrm{~mm}$ para distintas frecuencias de impacto.

Podemos observar como la frecuencia de impacto juega un importante papel sobre el comportamiento termomecánico de las placas. A mayor frecuencia, mayores son los valores de temperatura y tensión máxima alcanzados durante el pseudo-estacionario, puesto que la refrigeración no tiene tiempo de relajar estos valores entre pulso y pulso. La Tabla 2.6 resume los resultados y establece la diferencia porcentual con la configuración de referencia de 20 placas con una frecuencia de impacto de $1 \mathrm{~Hz}$.

Se observa como la temperatura máxima, que para el espesor de $9 \mathrm{~mm}$ se da en la cara en contacto con el refrigerante, excede la temperatura de ebullición de éste para la configuración de 10 placas $(2,0 \mathrm{~Hz})$. Esto impediría mantener el flujo monofásico, por lo que se descartan configuraciones con menos de 10 placas ${ }^{\mathrm{I}}$.

\footnotetext{
${ }^{\text {I }}$ Debe mencionarse que el modelo no tiene en cuenta la variación de coeficiente de transmisión de calor debida a la ebullición, por lo que los valores por encima de $152{ }^{\circ} \mathrm{C}$ no deben considerarse como representativos de la temperatura máxima del agua.
} 


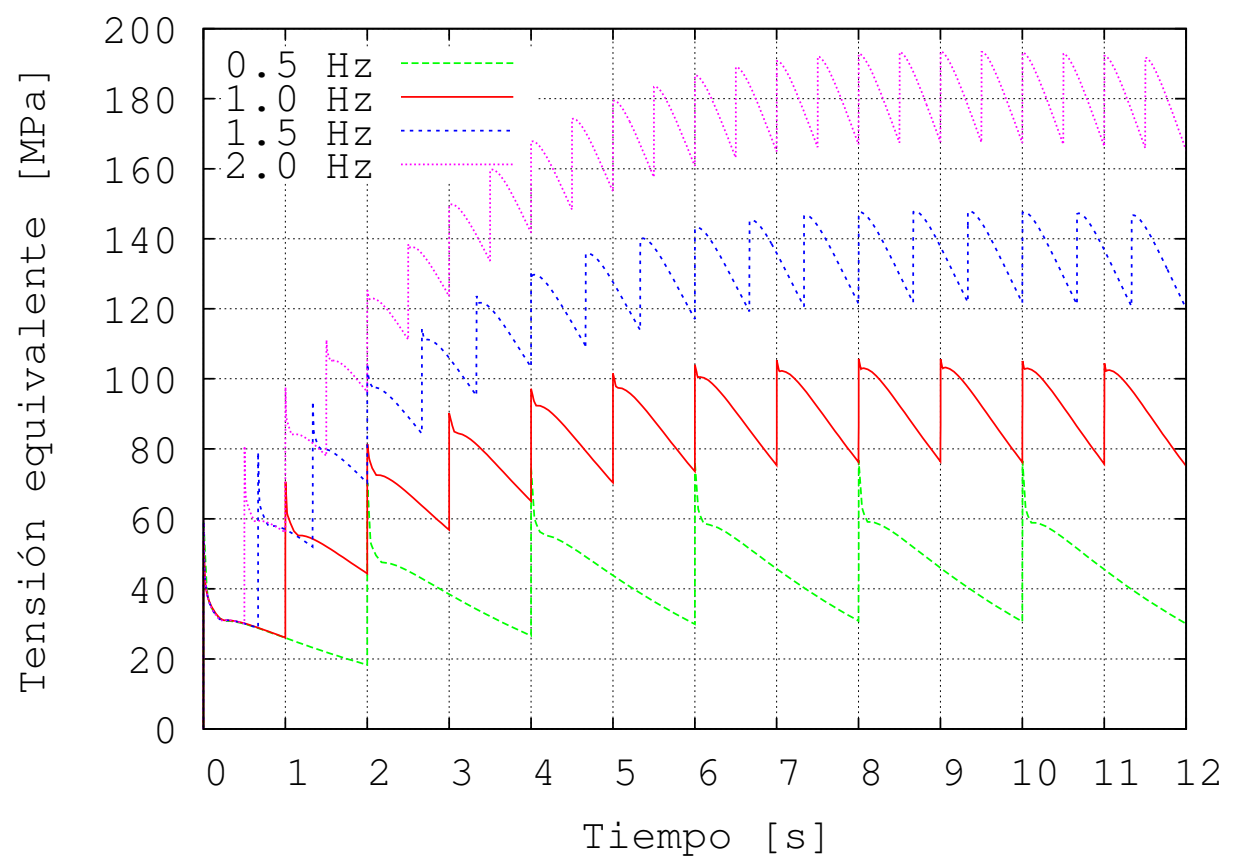

Figura 2-28: Evolución de la tensión de von Mises máxima en una placa de Be de $9 \mathrm{~mm}$ para distintas frecuencias de impacto.

De modo general, reducir el número de placas con respecto a la configuración de referencia conlleva una reducción proporcional del radio de la estructura que las alberga II, lo cual ayudaría a diseñar un blanco rotatorio más compacto. No obstante, esta disminución de tamaño se produciría a costa de reducir los márgenes de diseño con respecto a los límites máximos de temperatura y tensión, establecidos como criterio de referencia en la temperatura de ebullición del refrigerante y el límite elástico del berilio. Dado que no existen requisitos de ingeniería fuertes que exijan minimizar el tamaño del blanco, el número de placas es pues un parámetro con el que se puede jugar para aumentar los mencionados márgenes de diseño, siempre y cuando el tamaño final sea realizable.

Como se verá en el Capítulo 3, la estructura de referencia con 20 placas tiene un radio aproximado de $1 \mathrm{~m}$, y dados los valores de tensión y temperatura obtenidos, esta configuración se considera un buen compromiso entre el tamaño total y los márgenes de diseño. Por ejemplo, la configuración con 40 placas, $0,5 \mathrm{~Hz}$, rebaja los valores de tensión y temperaturas máximas un $\approx 25 \%$, pero requeriría un radio de $\approx 2 \mathrm{~m}$. Por todo lo

\footnotetext{
${ }^{\mathrm{II}} \mathrm{El}$ área de una corona circular de radio mucho mayor que su anchura es aproximadamente proporcional a su radio medio.
} 
anteriormente expuesto, se fija como solución base de diseño para el blanco rotatorio la opción con 20 placas.

\begin{tabular}{|c|cc|cc|}
\hline Frecuencia & \multicolumn{2}{|c|}{ T max } & \multicolumn{2}{c|}{$\sigma \max$} \\
$\boldsymbol{H z}$ & ${ }^{\boldsymbol{o}} \boldsymbol{C}$ & $\boldsymbol{\Delta} \%$ & $\boldsymbol{M P a}$ & $\boldsymbol{\Delta} \%$ \\
\hline 0.5 & 80.8 & -24.0 & 76.2 & -27.7 \\
1 & 106.3 & - & 105.4 & - \\
1.5 & 132.8 & 24.9 & 146.7 & 39.2 \\
2 & $>152$ & $>43.0$ & 192.8 & 82.9 \\
\hline
\end{tabular}

TABla 2.6: Estudio paramétrico de los efectos de la frecuencia de impacto en el blanco. Porcentajes calculados con respecto a los resultados para $1 \mathrm{~Hz}$.

\subsubsection{Estudio de vida útil}

\subsubsection{Implantación de hidrógeno y generación de gases}

El bombardeo del blanco con protones tiene otros efectos además de los puramente termomecánicos. Si el espesor de blanco es mayor que el alcance de los protones, estos no atraviesan el blanco y quedan implantados en el material. Los protones implantados en bajas concentraciones pueden permanecer disueltos en los espacios intersticiales de la red metálica o, en concentraciones mayores, pueden formar burbujas de hidrógeno molecular. El hidrógeno intersticial es muy móvil y difunde más rápidamente que el resto de elementos, debido a su pequeño tamaño y baja carga eléctrica. No obstante, puesto que la implantación producida por un haz de protones monoenergéticos es muy localizada, la concentración de hidrógeno implantado puede crecer más rápidamente de lo que éste puede difundir; saturando el material y llegando a formar ampollas de tamaño macroscópico, que fragilizan el material y deterioran su refrigeración. Este fenómeno ha sido observado en la fuente de neutrones LENS, donde ha originado múltiples fallos del blanco [139]. La Figura 2-29 muestra una imagen de uno de estos fallos donde la ampolla formada en el berilio es claramente visible.

Para estimar la concentración de hidrógeno implantado por el acelerador, procedemos a calcular con MCNPX la última posición de los protones en el material, como indicativa de su lugar de implantación. Simulando el número de partículas equivalente a un año de 

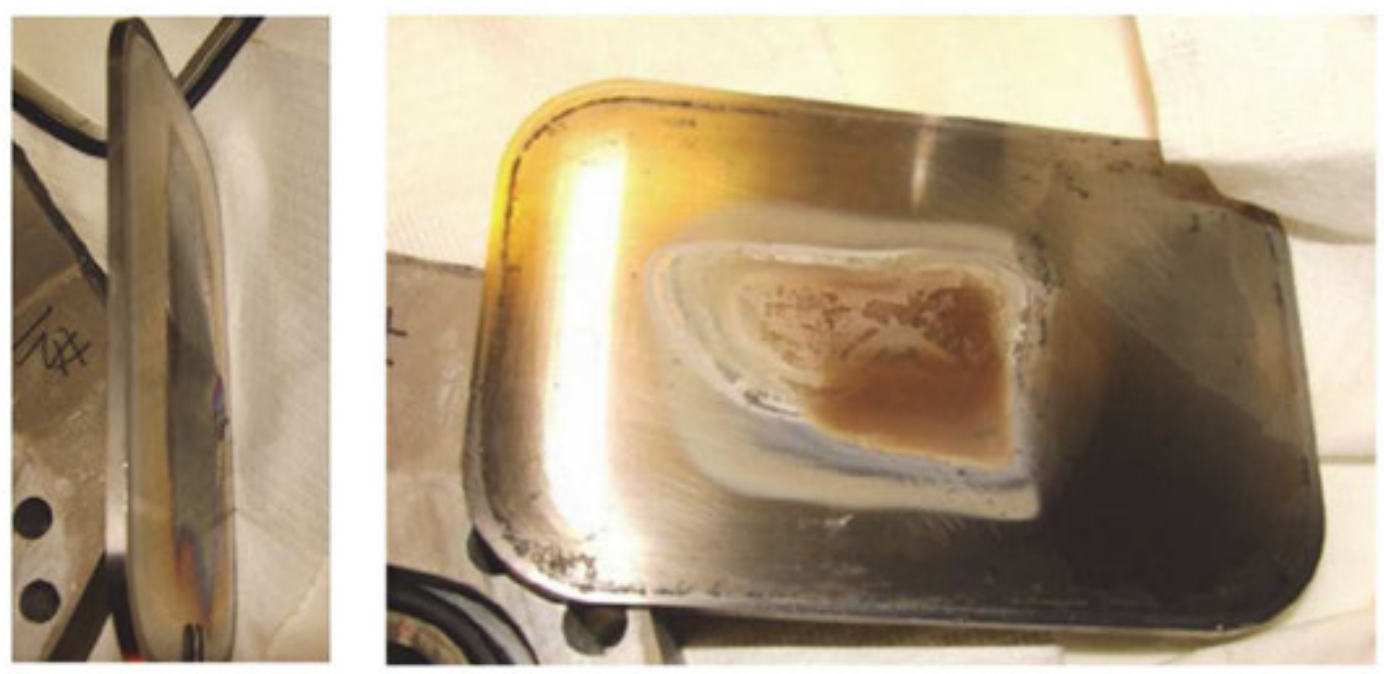

Figura 2-29: Fallo del blanco de la fuente de neutrones LENS (Indiana University) por implantación de hidrógeno [139].

operación del acelerador, obtenemos el perfil de concentración de hidrógeno acumulado en el espesor de la placa durante ese tiempo. Se han tomado 2000 horas de operación continua del acelerador a su máxima potencia por año. Teniendo en cuenta el carácter experimental de la instalación y los tiempos de mantenimiento, esta estimación se considera conservadora.

La Figura 2-30 muestra el perfil de concentración máxima de hidrógeno expresada en partes atómicas por millón, appm, en el espesor de una placa espesa. Como podemos observar, los protones tienden a acumularse mayoritariamente una vez han cedido toda su energía cinética, alcanzándose un máximo de 2200 appm alrededor del pico de Bragg. Una placa de $9 \mathrm{~mm}$, como la escogida para el blanco del ESS-Bilbao, acumularía sin embargo $\approx 150$ appm por año como máximo a lo largo de su espesor. Por tanto, reducir el espesor del blanco no es sólo conveniente desde el punto de vista termomecánico, sino también desde el de la prevención de la degradación de la placa.

Esta solución para evitar la aparición del pico de Bragg y la implantación de hidrógeno en el material, dejando que los protones atraviesen el blanco, ha demostrado su eficacia en LENS, donde gracias a una reducción del espesor del blanco, se ha conseguido hacer desaparecer los fallos inducidos por burbujas de hidrógeno antes referidos [140].

El hidrógeno, junto con otros isótopos, también puede ser producido por reacciones 


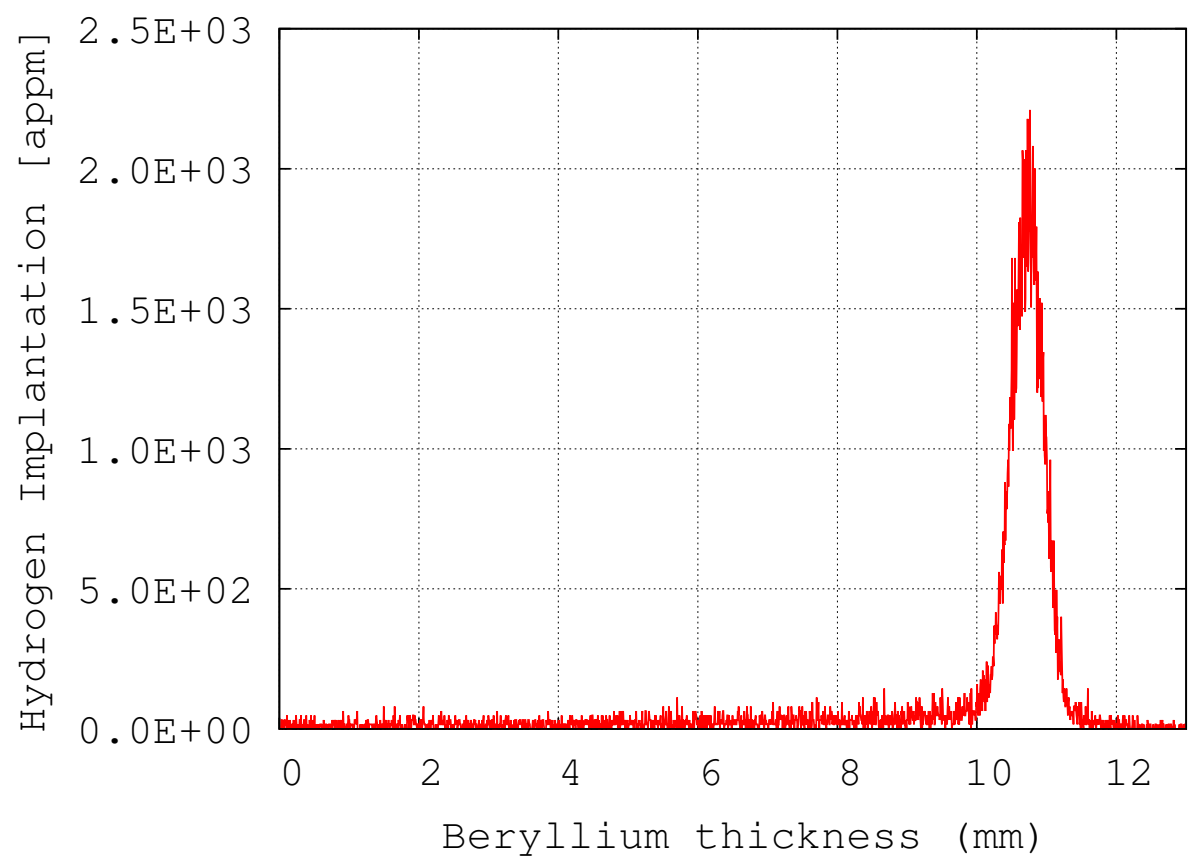

Figura 2-30: Perfil de concentración de H en la placa de Be tras 2000 horas de operación

nucleares inducidas por los protones en el seno del blanco. De entre los elementos que pueden producirse, el helio es el que domina la fragilización del material.

Para analizar la contribución de las reacciones nucleares a la concentración de hidrógeno y a la producción de helio, empleamos una vez más el código MCNPX. Dado que no se disponen de secciones eficaces para las reacciones $B e(n, x)^{z} \mathrm{H}$ y $\mathrm{Be}(n, x)^{z} \mathrm{He}$, emplearemos distintos modelos de cascada intranuclear (Bertini [141], INCL4 [142, 143], ISABEL [144], CEM03 [145, 146]), junto con distintos modelos de desexcitación (ABLA [147], Dresner [148]), para obtener una estimación del orden de magnitud de la concentración de isótopos de estos elementos, esperable tras un año de operación.

La Tabla 2.7 muestra los valores máximos de concentración en appm de los isótopos de hidrógeno y helio en la placa de Be de $9 \mathrm{~mm}$, tras 2000 horas de operación, calculados con las distintas combinaciones de modelos. La Figura 2-31 presenta los perfiles de concentración máxima de ${ }^{4} \mathrm{He}$ obtenidos. En los resultados se observa como el modelo que simula la fase de cascada intranuclear domina el valor obtenido, percibiéndose poca diferencia en los resultados fruto de la fase de desexcitación. Todas las simulaciones arrojan unos valores bastante concordantes excepto el modelo CEM03 que proporciona unos resultados 
sistemáticamente menores.

\begin{tabular}{|c|ccc|c||cc|c|}
\hline Modelo & ${ }^{\mathbf{1}} \boldsymbol{H}$ & ${ }^{\mathbf{2}} \boldsymbol{H}$ & ${ }^{\mathbf{3}} \boldsymbol{H}$ & $\boldsymbol{H}$ total & ${ }^{\mathbf{3}} \boldsymbol{H} \boldsymbol{~}$ & ${ }^{\mathbf{4}} \boldsymbol{H} \boldsymbol{e}$ & $\boldsymbol{H} \boldsymbol{e}$ total \\
\hline Bertini-ABLA & 147 & 25 & 5 & 177 & 3 & 139 & 142 \\
Bertini-Dresner & 147 & 25 & 5 & 177 & 3 & 139 & 142 \\
INCL4-ABLA & 130 & 27 & 16 & 173 & 13 & 119 & 132 \\
INCL4-Dresner & 132 & 28 & 16 & 176 & 13 & 120 & 133 \\
ISABEL-ABLA & 154 & 18 & 3 & 176 & 2 & 139 & 141 \\
ISABEL-Dresner & 155 & 18 & 3 & 176 & 2 & 140 & 142 \\
CEM03 & 128 & 15 & 12 & 155 & 13 & 84 & 97 \\
\hline
\end{tabular}

TABLA 2.7: Valores máximos de concentración en appm de isótopos de $\mathrm{H}$ y He en la placa de Be de $9 \mathrm{~mm}$, tras 2000 horas de operación, calculados con diferentes modelos nucleares.

La contribución de las reacciones nucleares a la concentración de hidrógeno es inferior a $\approx 180$ appm, teniendo en cuenta todos los isótopos y considerando los resultados más pesimistas. Este valor está en el mismo rango que la concentración producida por los protones implantados. De acuerdo con [149], la concentración total debida a ambas contribuciones no conlleva riesgo para la integridad de la placa, y supone un ritmo de generación que permite al hidrógeno difundir en el berilio hasta ser desorbido en su superficie.

El helio en cambio apenas difunde y las $\approx 150$ appm generadas anualmente, según los resultados más pesimistas, se acumularían con el tiempo de operación, provocando hinchamiento del material y deterioro de las propiedades mecánicas.

De acuerdo con [150], la concentración de helio es la causa dominante del hinchamiento del berilio irradiado. En dicho estudio se recopilan distintas experiencias de irradiación de berilio con neutrones, en un rango de temperaturas que va desde $\operatorname{los} 40^{\circ} \mathrm{C}$ y $\operatorname{los} 350{ }^{\circ} \mathrm{C}$, y se describe la relación entre concentración de helio, temperatura de irradiación e incremento de volumen del material medido. No se cuenta con datos experimentales específicos para irradiación con protones, pero se considera que las conclusiones son razonablemente extrapolables independientemente del origen de helio en el material.

La Figura 2-32 presenta la recopilación de datos experimentales antes mencionada [150], indicando la temperatura de irradiación y el reactor donde se llevó a cabo el experimento. En esta figura se incluyen también los valores de hinchamiento predichos por 


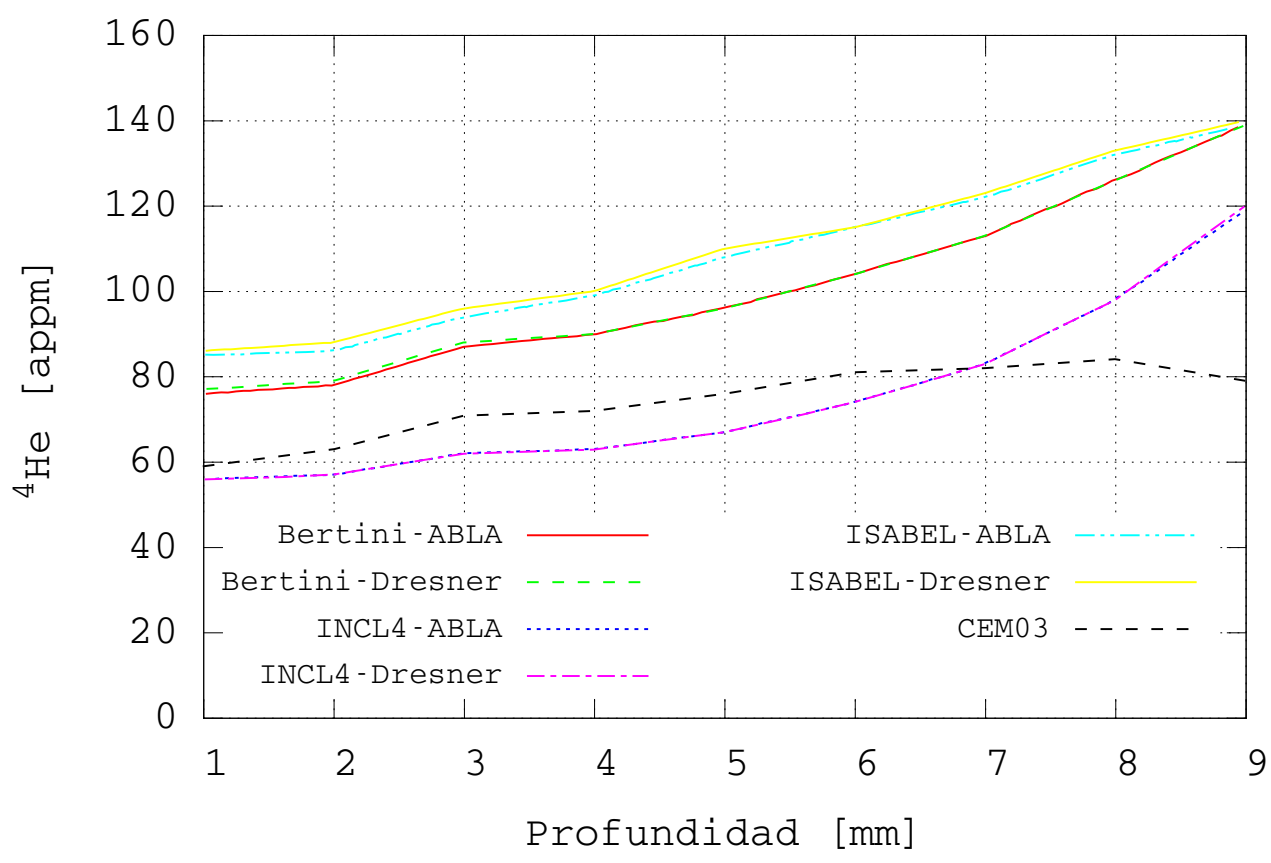

Figura 2-31: Perfil de concentración máxima de ${ }^{4}$ He generado en la placa de berilio, tras 2000 horas de operación, calculados con diferentes modelos nucleares.

el código de simulación ANFIBE (ANalysis of Fusion Irradiated BEryllium) [151], desarrollado por el Forschungszentrum Karlsruhe (FZK), y los predichos por la Ecuación 2.8, desarrollada por M.C. Billone [152], donde $C_{H e}$ es la concentración de helio en appm y $T$ la temperatura en $K$.

$$
\Delta V / V[\%]=1,15 \cdot 10^{-4} C_{H e}\left[1+9,49 \cdot 10^{-5} C_{H e}^{0,5} T^{1,5} \exp (-3940 / T)\right]
$$

Para evaluar la vida útil de la placa de berilio a partir de los datos de [150], se establece de manera conservadora un valor admisible máximo de hinchamiento del 0,5\%. Empleando dichos datos, evaluamos la concentración de helio necesaria para inducir ese incremento de volumen y, teniendo en cuenta la tasa de creación de helio calculada $(\approx 150$ appm/año), obtenemos una estimación del tiempo de operación necesario para alcanzar dicha concentración. Dada la dispersión de los datos experimentales, evaluaremos las distintas concentraciones de helio a las que el valor máximo de hinchamiento se ha dado experimentalmente, obteniendo de esta manera un rango esperable de vida útil en lo que respecta a hinchamiento. 


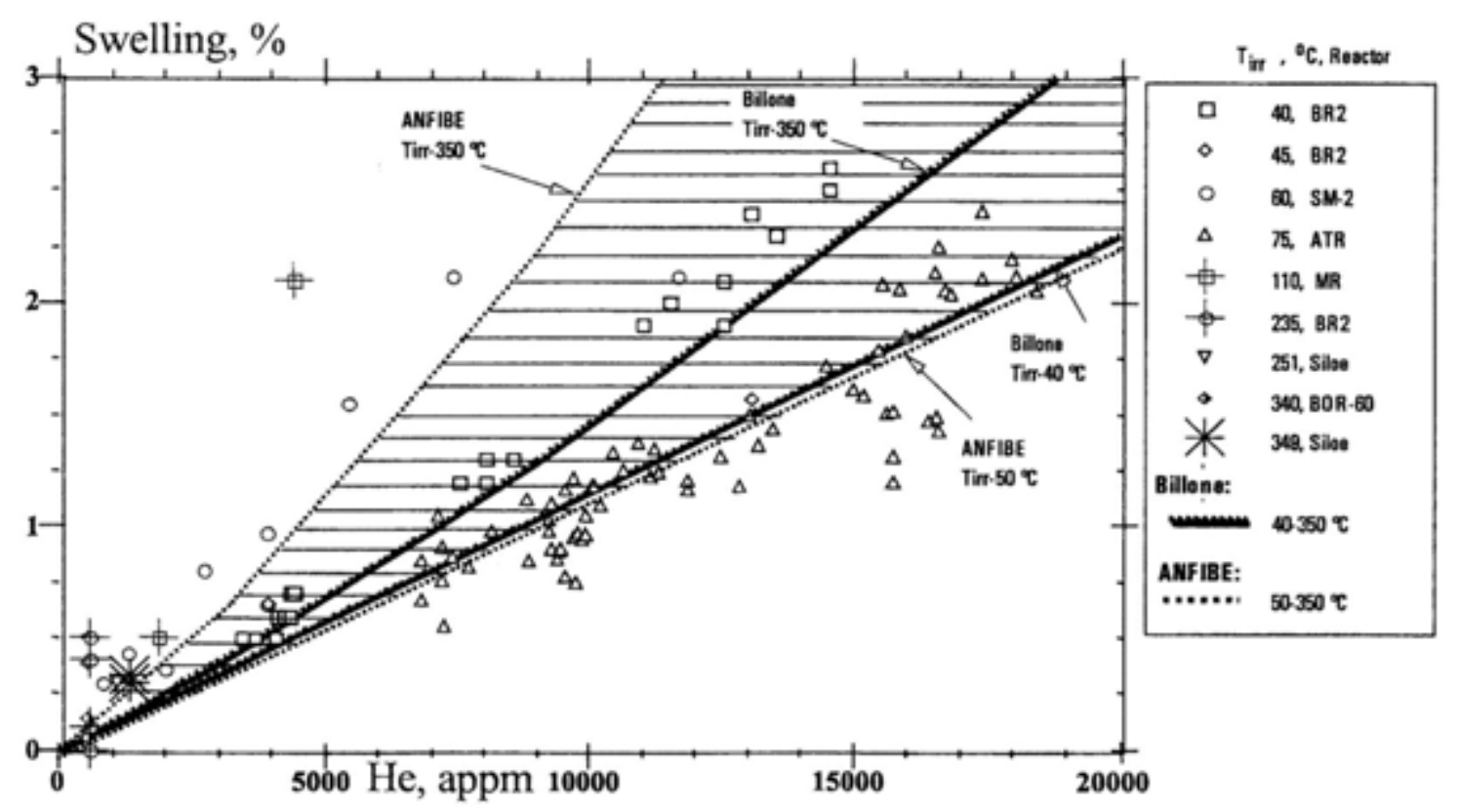

Figura 2-32: Porcentaje de hinchamiento en función del contenido de He y la temperatura de irradiación del berilio.

Así pues, analizando la Figura 2-32, el 0,5\% de hinchamiento se alcanzaría para $\approx 600$ appm de helio según el punto experimental más penalizante para cualquier temperatura de irradiación, obtenido en el reactor ruso MR del Instituto Kurchatov [153] a $235{ }^{\circ} \mathrm{C}$. Para una temperatura representativa de la temperatura máxima de operación de la placa, $106,3{ }^{\circ} \mathrm{C}$, la irradiación llevada a cabo en el reactor BR2 del Centro de Estudios Nucleares belga (SCK-CEN) [154], a $110{ }^{\circ} \mathrm{C}$, muestra que el límite de hinchamiento se alcanzaría para $\approx 2000$ appm. Aplicando la Ecuación 2.8, el 0,5\% de incremento de volumen a la temperatura máxima de la placa se daría cuando esta acumule $\approx 4350$ appm. Por tanto, la vida útil de la placa, dada la tasa de generación de $\approx 150$ appm/año, se estimaría en cada caso aproximadamente en 4, 13 y 29 años.

Aceptando la peor de las estimaciones, una vida útil de 4 años se considera suficiente, antes de proceder a la sustitución de las placas, por lo que la vida útil del blanco sería adecuada. Es necesario puntualizar que esta estimación sería doblemente conservadora, ya que el 0,5\% de hinchamiento sólo se alcanzaría en la zona donde la tasa de creación de helio es máxima, no en toda la placa. Por ello, el incremento global del tamaño sería mucho menor. En cualquier caso, una monitorización regular del estado del blanco y de sus sistemas de sujeción durante los periodos de mantenimiento sería aconsejable. 


\subsubsection{Evolución de las propiedades mecánicas}

Puesto que no se encuentran disponibles datos de irradiación de berilio con protones de la energía del acelerador de ESS-Bilbao, usaremos como referencia los resultados experimentales presentados en [155] para estimar la evolución de las propiedades mecánicas del blanco. En dicho trabajo, se irradiaron probetas de berilio de distintos grados comerciales en el reactor belga $\mathrm{BR} 2$, hasta una fluencia de neutrones rápidos $\left(E_{n}>1 \mathrm{MeV}\right)$ de $\approx 1,6 \cdot 10^{21} \mathrm{n} / \mathrm{cm}^{2}$. El efecto de esta irradiación en el berilio escogido como referencia para el blanco, S-200 VHP, se tradujo en una rigidización del material, con un incremento del límite elástico de aproximadamente el $65 \%$ (Figura 2-33a) y de la tensión de rotura del $20 \%$ (Figura 2-33b). Estos resultados son característicos del efecto de la radiación en metales, ya que la inclusión de defectos en su estructura dificulta el desplazamiento de las dislocaciones y por tanto su deformación. Los resultados presentados en [155] también reportan que todos los tipos de berilio sufren ruptura frágil a las temperaturas características de operación del blanco, cayendo su elongación a rotura a valores muy bajos después de la irradiación. Este cambio en la temperatura de transición dúctil-frágil es también característico del daño por irradiación.

Si se acepta la hipótesis arbitraria de que los efectos de la irradiación con neutrones rápidos reportada en [155] son equiparables, en cuanto a evolución de las propiedades mecánicas, a la irradiación con los protones de ESS-Bilbao, podemos comparar los valores de fluencia en ambos casos para estimar groseramente si los efectos allí descritos son esperables en nuestro blanco.

Teniendo en cuenta la corriente del acelerador de ESS-Bilbao, $2,25 m A=1,4 \cdot 10^{16}$ $\mathrm{p} / \mathrm{s}$, el radio del haz, $5 \mathrm{~cm}$, el factor 2 de concentración en el centro del haz gaussiano, el ángulo de incidencia de $45^{\circ}$ y el reparto del haz entre las 20 placas, se deduce que el flujo de protones máximo en cada placa es de $1,26 \cdot 10^{13} \mathrm{p} / \mathrm{cm}^{2} \mathrm{~s}$. Con este valor de flujo, la fluencia experimentada en [155], 1,6 $\cdot 10^{21}$ partículas $/ \mathrm{cm}^{2}$, se alcanzaría a los $\approx 17,6$ años de irradiación ${ }^{\mathrm{III}}$.

No obstante, la hipótesis de que ambas irradiaciones son equivalentes en cuanto a los efectos en el material, puede ser no conservadora. Buscando refinar esta estimación, empleamos los resultados presentados en [156]. En este trabajo se estudia la producción de 

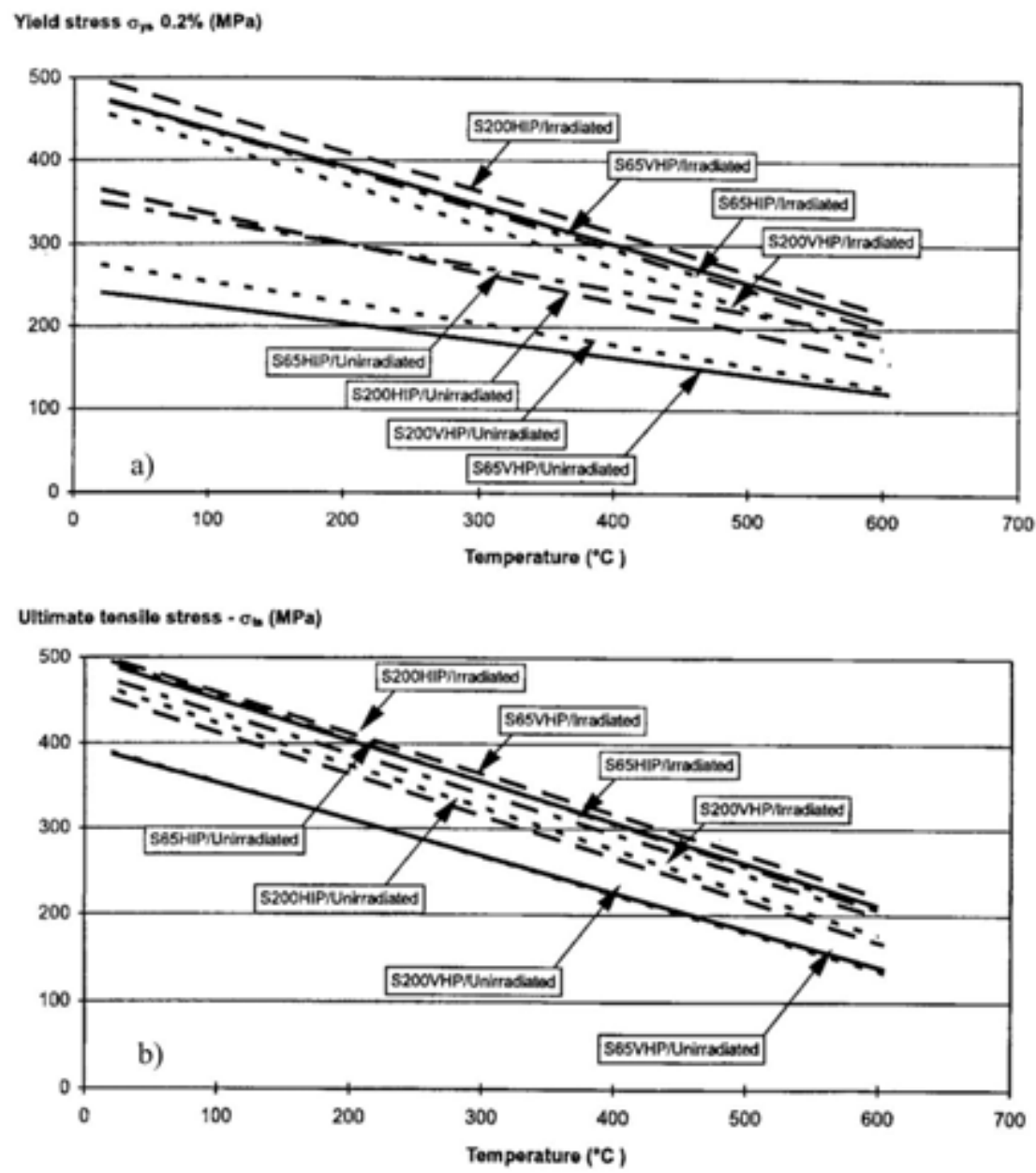

Figura 2-33: Evolución de las propiedades mecánicas de diversos tipos de berilio comercial bajo irradiación de neutrones [155]: a) Limite elástico. b) Tensión de rotura. 
helio en berilio a $\approx 50{ }^{\circ} \mathrm{C}$ debido a la irradiación con neutrones en el reactor belga BR2. En ese estudio, se concluye que la concentración de helio crece linealmente con la fluencia de neutrones de $E_{n}>1 \mathrm{MeV}$ según la Ecuación 2.9 [156], donde $C_{H e}$ es la concentración de helio en appm y $\phi$ es la fluencia en $\mathrm{n} / \mathrm{cm}^{2}$.

$$
C_{H e}=(4880 \pm 90) \cdot 10^{-22} \phi
$$

Así pues, empleando la correlación experimental descrita en la Ecuación 2.9, podemos deducir que la concentración de helio generada por la fluencia de neutrones alcanzada en [155] sería de $\approx 780$ appm. Si aceptamos la hipótesis de que la evolución de propiedades mecánicas está correlacionada linealmente con la concentración de helio, independientemente de cuál sea el origen de éste, podemos estimar cuánto tiempo tardarían en aparecer en el blanco de ESS-Bilbao los efectos descritos en [155], calculando cuánto se tardaría en alcanzar esta concentración de helio.

Para validar esta hipótesis haría falta contar con resultados experimentales específicos, sin embargo, parece una hipótesis razonable, dado que la concentración de helio domina la evolución de las propiedades en otros materiales como los aceros [157].

Teniendo en cuenta la tasa de producción de helio calculada en la sección 2.3.4.1 para nuestra sistema, $\approx 150 \mathrm{appm} / \mathrm{año}$, alcanzaríamos condiciones de irradiación equivalentes a las de [155], y por tanto una evolución de propiedades análoga, después de 5,2 años de operación ${ }^{\mathrm{III}}$.

Independientemente de los tiempos estimados, resulta evidente que se debe contar con cierto grado de rigidización del material debido a la irradiación. No obstante, con estos datos en la mano, es razonable pensar que la degradación de propiedades mecánicas no será un factor limitante de la vida del blanco en el corto plazo. Dados los niveles de tensión esperables en el blanco, la rigidización prevista del material no tiene consecuencias relevantes. En todo caso, aumenta el margen de tensión que el blanco puede soportar sin presentar deformación permanente. La reducción de la elongación a ruptura no es un problema añadido, ya que el grado de berilio escogido presenta antes de irradiación una elongación a ruptura de sólo el $2 \%$ a temperatura ambiente [137], por lo que de ningún

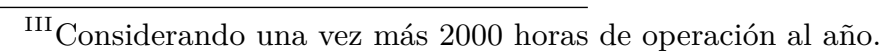


modo puede contarse con observar una evidente deformación plástica antes de rotura, y debe esperarse que en caso de fallo éste se dé por fractura frágil. Sin embargo, el gran margen entre la tensión de operación prevista y la tensión de rotura permitiría operar el blanco con confianza.

\subsubsection{Daño por fatiga}

Otro posible factor limitante para la vida de las placas es el daño por fatiga ante la aplicación repetida de cargas. Dada la naturaleza pulsada del estado tensional del blanco, este mecanismo de fallo debe ser considerado.

Afortunadamente el berilio cuenta con una resistencia destacadamente alta ante este fenómeno. El grado de berilio comercial S-200 F elegido como material de referencia tiene un límite de fatiga, definido como el nivel de tensión que genera fallo a los $10^{7}$ ciclos $^{\mathrm{IV}}$, de $262 \mathrm{MPa}[134]$ y supera en test de fatiga rotatorio los $10^{7}$ ciclos para una carga oscilando entre su límite elástico aplicado a tracción y compresión [158].

Así pues, incluso considerando de forma conservadora el valor máximo de tensión de von Mises en el pseudo-estacionario, 105,4 MPa, como valor de la amplitud de tensión oscilante, al permanecer sustancialmente por debajo de estos valores, no se espera la aparición de fatiga hasta mucho después de los $10^{7}$ pulsos de haz (i.e $\approx 2780$ horas de operación). Considerando la pequeña amplitud de la tensión oscilante real en cada pulso, $\approx 30 \mathrm{MPa}$, es concebible que, de aparecer fatiga,ésta ocurriera en el rango de ciclos considerado ultra alto (i.e.> $10^{8}$ ciclos) [159].

Es necesario mencionar que el nivel de tensión considerado para esta estimación de la vida a fatiga, no tiene en cuenta la tensión resultante de la restricción mecánica de la placa. No obstante, se ha preferido esta aproximación dado que las tensiones aquí consideradas son ineludibles, al menos para el espesor seleccionado de las placas, y por tanto podrían constituir un problema mayor para la viabilidad del blanco. Por otro lado, las tensiones derivadas de la restricción mecánica son fuertemente dependientes del diseño de detalle del sistema de sujeción. En cualquier caso, es posible considerar que un diseño adecuado pueda mantener estas tensiones derivadas de la sujeción mecánica por debajo del nivel

\footnotetext{
${ }^{\mathrm{IV}}$ Valor conocido como endurance limit
} 
de las tensiones termomecánicas consideradas en esta sección, con lo que las conclusiones aquí extraídas seguirían siendo válidas.

A modo de comentario final, cabe destacar como un mayor número de placas en el blanco no sólo es beneficioso por la reducción del nivel de tensión oscilante, sino también por la disminución de la frecuencia de los ciclos de carga, lo cual permite operar más horas con menos ciclos por placa. Análogamente, el reparto del impacto del haz entre varias placas es beneficioso con respecto a los efectos de la irradiación estudiados en las Secciones 2.3.4.1 y 2.3.4.2.

\subsubsection{Corrosión y control químico}

La experiencia operativa de la fuente LENS de la Universidad de Indiana, ha demostrado que los procesos radiolíticos que tienen lugar en el refrigerante detrás de la zona de impacto del haz, tienden a aumentar la adherencia de los productos de corrosión en la superficie del blanco. Este fenómeno degrada las condiciones de refrigeración del berilio, hasta el punto de haber sido el origen de al menos dos fallos del blanco en la mencionada instalación [139]. Dicho comportamiento se ve acentuado por la implantación de los protones en el agua.

Con el objetivo de combatir este peligro potencial para la integridad del blanco, se recomiendan aplicar las tres medidas de control químico que han corregido la aparición de este tipo de fallos en LENS [139]. La primera de ellas es reducir la corrosión galvánica. Para ello es recomendable diseñar toda circuitería en aluminio, controlar el $\mathrm{pH}$ del refrigerante empleando aditivos básicos e incluir un ánodo de sacrificio. La segunda medida consistiría en la limitación del contenido de partículas en suspensión. Para ello LENS emplea filtros de carbón activo y dos filtros mecánicos consecutivos de $20 \mu m$ y $5 \mu m$, hasta mantener el contenido de partículas sólidas disueltas por debajo de los $0,5 \mathrm{mg} / \mathrm{l}$. Y por último, el control de la composición iónica del agua mediante la inclusión de un tanque de recombinación y decaimiento y de un filtro des-ionizante. Con estas medidas, adaptadas al caso de ESSBilbao, parece factible evitar también este tipo de fallos. 


\section{Conclusiones del capítulo}

Tras los estudios aquí presentados, se concluye que la solución base de diseño de la fuente propuesta para ESS-Bilbao es un blanco rotatorio de berilio refrigerado por agua en régimen forzado monofásico. Los análisis termomecánicos efectuados definen un blanco con 20 placas de berilio de $9 \mathrm{~mm}$ de espesor, sobre las que impacta el haz de protones con una frecuencia de $1 \mathrm{~Hz}$ en cada una, y un ángulo de incidencia de $45^{\circ}$. Con estos parámetros de diseño se alcanzan valores aceptables de temperatura y tensión en el berilio, manteniendo el valor máximo de tensión por debajo del límite de fatiga del material. Se han analizado las consecuencias de la implantación de hidrógeno y de la producción de gases, y se han dado indicaciones sobre cómo evitar la corrosión. Las estimaciones más conservadoras sitúan la vida útil de las placas en un mínimo de 4 años. Por tanto, con estas opciones de diseño, es posible configurar una fuente con una intensidad de $8,0 \cdot 10^{14} \mathrm{n} / \mathrm{s}$. 


\section{Capítulo 3}

\section{Diseño conceptual de la estructura del blanco}

\section{Resumen}

Una vez definidos los parámetros básicos del blanco de ESS-Bilbao, es necesario diseñar una estructura capaz de materializar el concepto propuesto de blanco rotatorio de berilio refrigerado por agua. Para ello la estructura debe ser capaz de cumplir con las siguientes funciones:

- Refrigeración: La estructura debe proporcionar un flujo de agua presurizada suficiente para garantizar las condiciones de refrigeración asumidas en el proceso de diseño de los elementos del blanco.

- Soporte mecánico: La estructurara debe albergar las placas de berilio y proporcionar el movimiento rotatorio necesario para distribuir la energía del haz.

Este capítulo describe el diseño conceptual de una estructura concebida para cumplir ambas funciones, así como los análisis termohidráulicos y termomecánicos que lo soportan.

Por último, se evalúa el comportamiento del blanco frente a posibles sucesos accidentales, con el objetivo de estimar los márgenes de seguridad del diseño y detectar posibles puntos débiles en su concepción. 
El diseño descrito en este capítulo es el resultado de un proceso iterativo de análisis y mejora, cuyo histórico se recoge en el Apéndice A.

\subsection{Descripción general}

Habiendo definido en el Capítulo 2 los parámetros base del blanco, es necesario diseñar ahora una estructura capaz de materializar las decisiones de diseño tomadas. Así pues, dicha estructura debe ser capaz de albergar 20 placas de berilio de unas dimensiones adecuadas para acomodar la huella del haz, así como de proporcionarles el flujo de agua a 5 bar de presión a una velocidad suficiente como para generar un coeficiente de transmisión de calor de $10^{4} \mathrm{~W} / \mathrm{m}^{2} \mathrm{~K}$, todo ello con suficiente robustez y estabilidad mecánica.

Con estos objetivos en mente, se ha concebido la estructura propuesta para el blanco de ESS-Bilbao presentada en la Figura 3-1. Esta consiste en un disco que sujeta las 20 placas de berilio y alberga en su interior los canales por los que circula el refrigerante. El disco consta de distintas piezas unidas entre sí por uniones atornilladas. Los canales de refrigeración quedan definidos por el espacio existente entre las piezas de la estructura. Esta solución evita la necesidad de fabricar canales internos de formas complejas y hace todas las piezas fabricables por procesos de mecanizado estándar, fundamentalmente fresado 2.5D [160] y taladrado, a partir de preformas sencillas. La Figura 3-2 muestra una vista explosionada de los componentes del disco.

Las Figuras 3-3 y 3-4 muestran el flujo de refrigerante en el disco en una imagen tridimensional y un plano de corte respectivamente. El refrigerante entra en el disco axialmente por el interior de la rueda central. Se distribuye por los conductos radiales superiores hacia el espacio anular distribuidor definido entre el disco superior y el disco inferior. De éste parten los canales que pasan bajo las placas de berilio, refrigerándolas. Una vez que el fluido ha pasado bajo las placas, alcanza el borde externo del disco, donde es deflectado volviendo radialmente hacia el interior del mismo. Después, el refrigerante pasa al espacio anular colector a través de los orificios del disco inferior. Una vez allí, avanza por los conductos radiales inferiores de la rueda central, abandonando la estructura del blanco coaxialmente al fluido entrante.

Las distintas piezas se mantienen solidarias entre sí mediante uniones atornilladas. En 


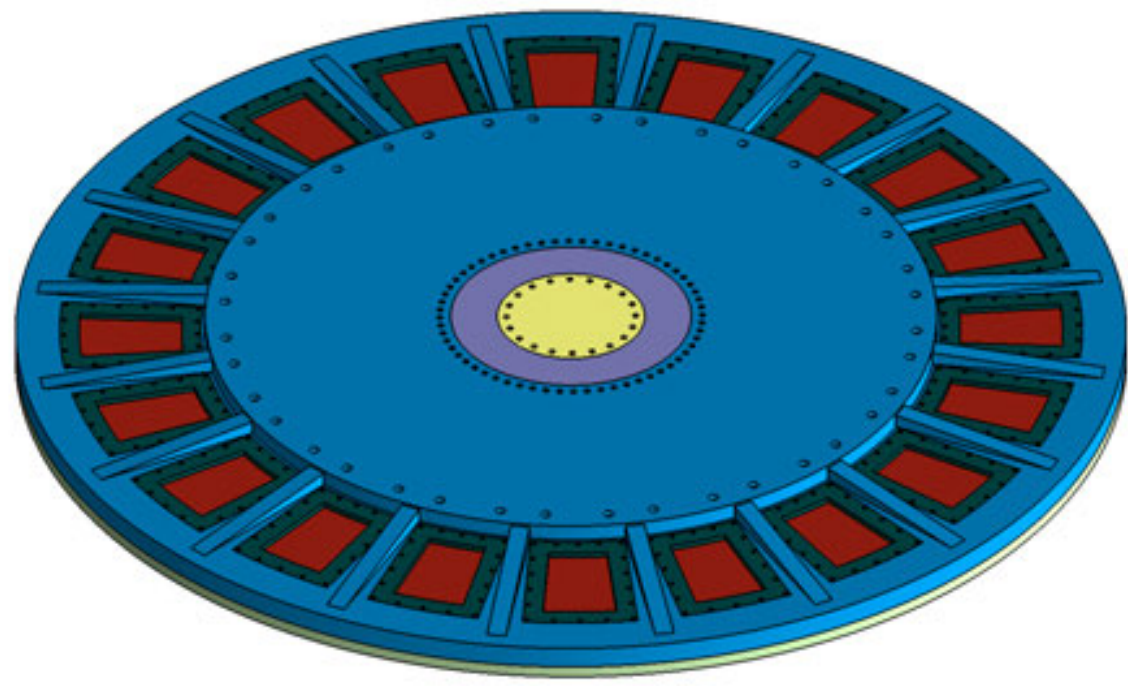

FiguRA 3-1: Vista general de la estructura del blanco

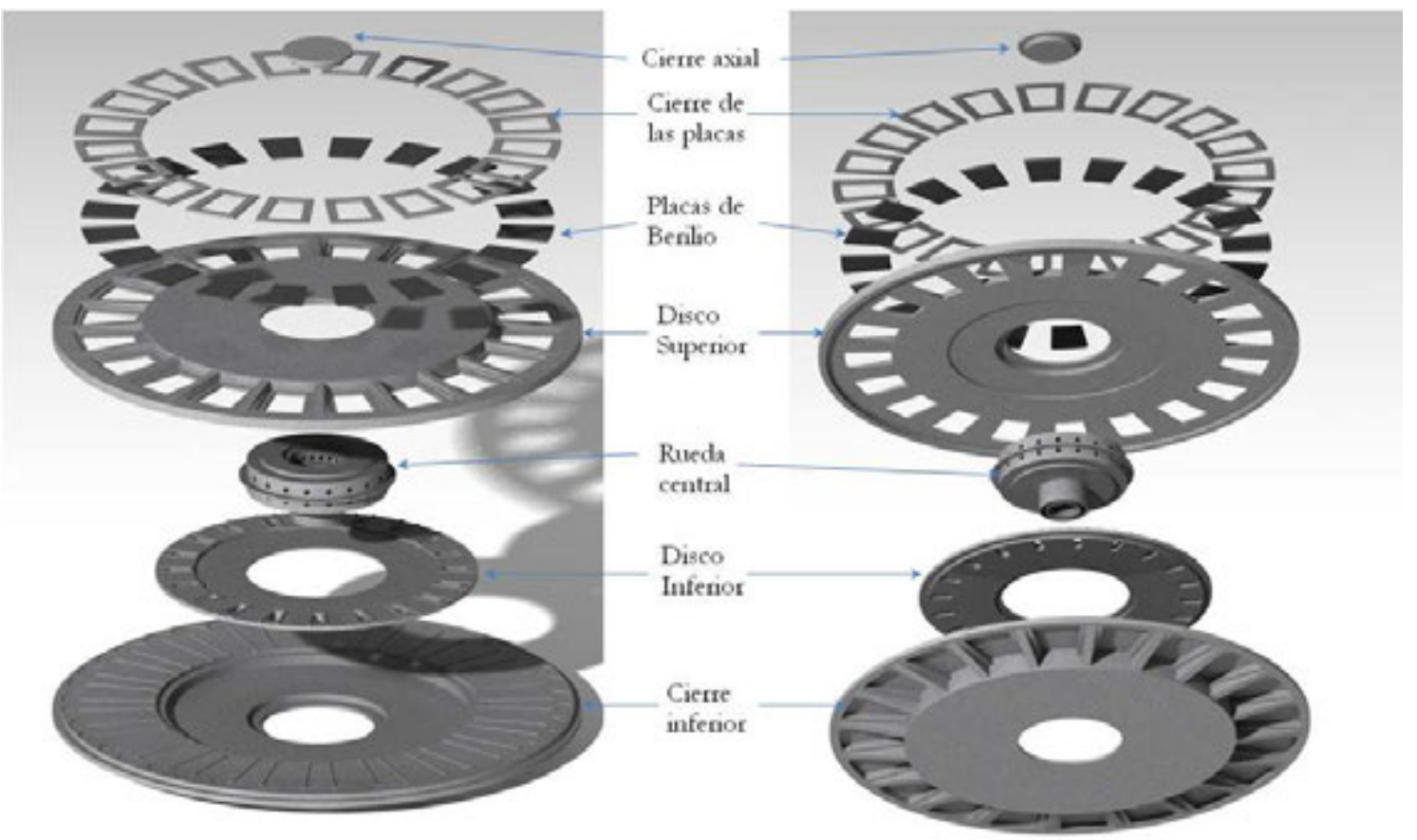

Figura 3-2: Vistas explosionadas, superior en inferior, de la estructura del blanco. 


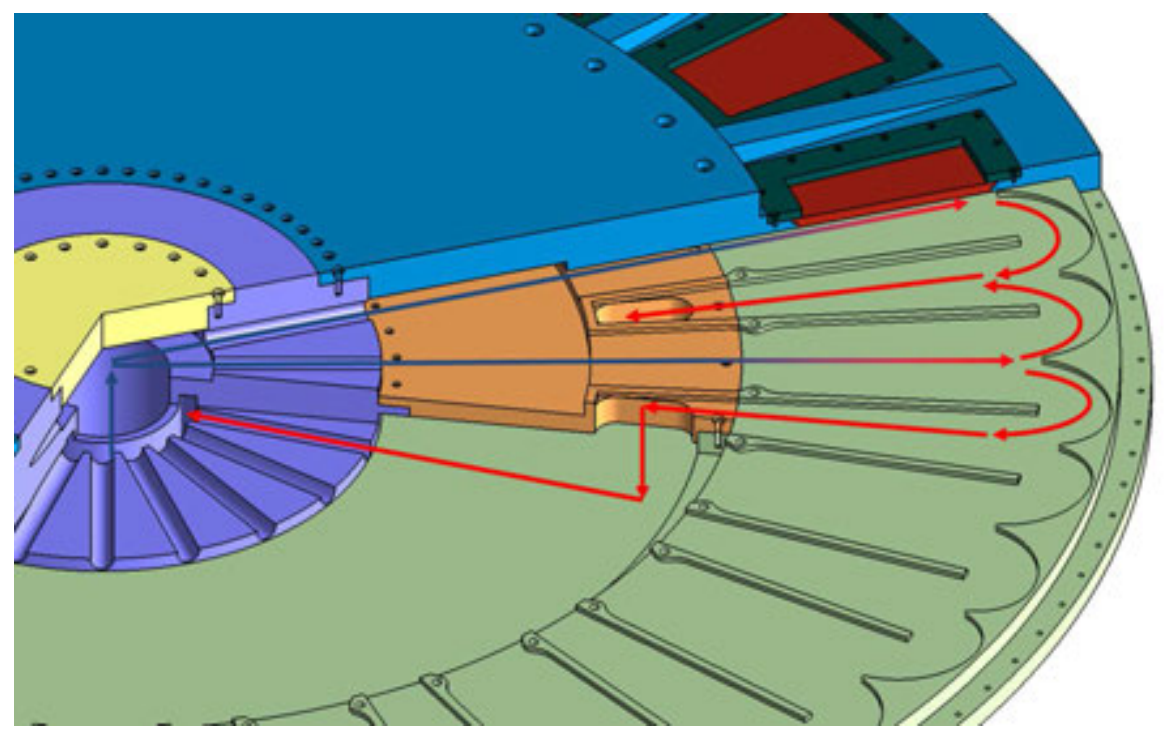

Figura 3-3: Flujo de refrigerante dentro de la estructura del blanco.

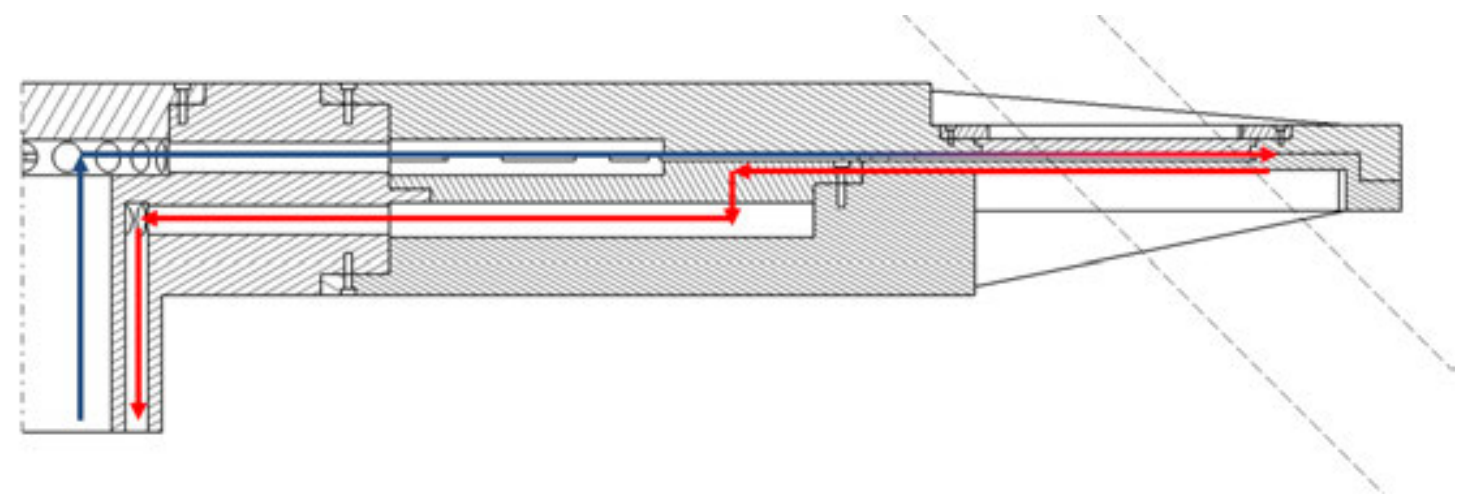

Figura 3-4: Sección de la estructura del blanco. Flujo de refrigerante y posición relativa del haz de protones. 
las uniones donde la estanqueidad del circuito es necesaria se ha previsto espacio para la inclusión de juntas y arandelas de estanqueidad en sus correspondientes ranurados. No obstante, dichas geometrías no se han incluido para simplificar el modelo a analizar por elementos finitos. En cualquier caso, el diseño de dichas juntas no supone un desafío para la fabricación del conjunto y se considera parte de la ingeniería de detalle.

Las dimensiones principales de las estructura se describen en la Figura 3-5. Su radio principal es de aproximadamente $1 \mathrm{~m}$ y su espesor 15,4 $\mathrm{cm}$. El peso total, considerando el refrigerante es ligeramente inferior a una tonelada. La Figura 3-6 describe el encaje entre las diferentes piezas mediante una vista explosionada de uno de los 20 sectores iguales de $18^{\circ}$ de los que consta el disco. Información más precisa sobre las uniones atornilladas puede consultarse en el Apéndice A.

El material seleccionado para la estructura del disco es el aluminio 6061-T6. Se trata de una aleación con base de aluminio $(\approx 96 \%)$ ampliamente utilizada en la industria aeronáutica, cuyos principales aleantes son el magnesio y el silicio. Este material se escoge por su combinación de buenas propiedades mecánicas y neutrónicas. Sus límites elástico y de rotura son 250 y $310 \mathrm{MPa}$ respectivamente [161], valores que se sitúan en el rango de los aceros. Sin embargo, la absorción de neutrones y la vida media de los isótopos generados por la activación de esta aleación son significativamente menores que las del acero, lo cual maximiza la producción de neutrones útiles y simplifica el manejo y mantenimiento de la fuente. Esta aleación presenta además alta resistencia a la corrosión y buena mecanizabilidad [161] [162]. En función del esquema de mantenimiento y desmantelamiento del disco, y de las herramientas disponibles para estas operaciones, se podría plantear la unión soldada entre las piezas de aleación de aluminio. Esta opción sería especialmente interesante para reforzar la estanqueidad entre el disco superior y el cierre inferior, pero debe tenerse en cuenta que este procedimiento normalmente requiere de tratamiento térmico posterior.

Las únicas desventajas de esta aleación, comparada con aceros convencionales, son su inferior temperatura de fusión y su menor módulo de Young. La aleación 6061-T6 comienza a fundirse a $582{ }^{\circ} \mathrm{C}$ [161] aunque sus propiedades mecánicas se degradan a temperaturas inferiores. Este material mantiene el límite elástico alrededor de $250 \mathrm{MPa}$ hasta los 100 ${ }^{\circ} \mathrm{C}$, descendiendo hasta $215 \mathrm{MPa}$ a los $150{ }^{\circ} \mathrm{C}$, y hasta $100 \mathrm{MPa}$ a los $200{ }^{\circ} \mathrm{C}$ [161]. Esta degradación se debe a la pérdida del estado templado y precipitado característico del 

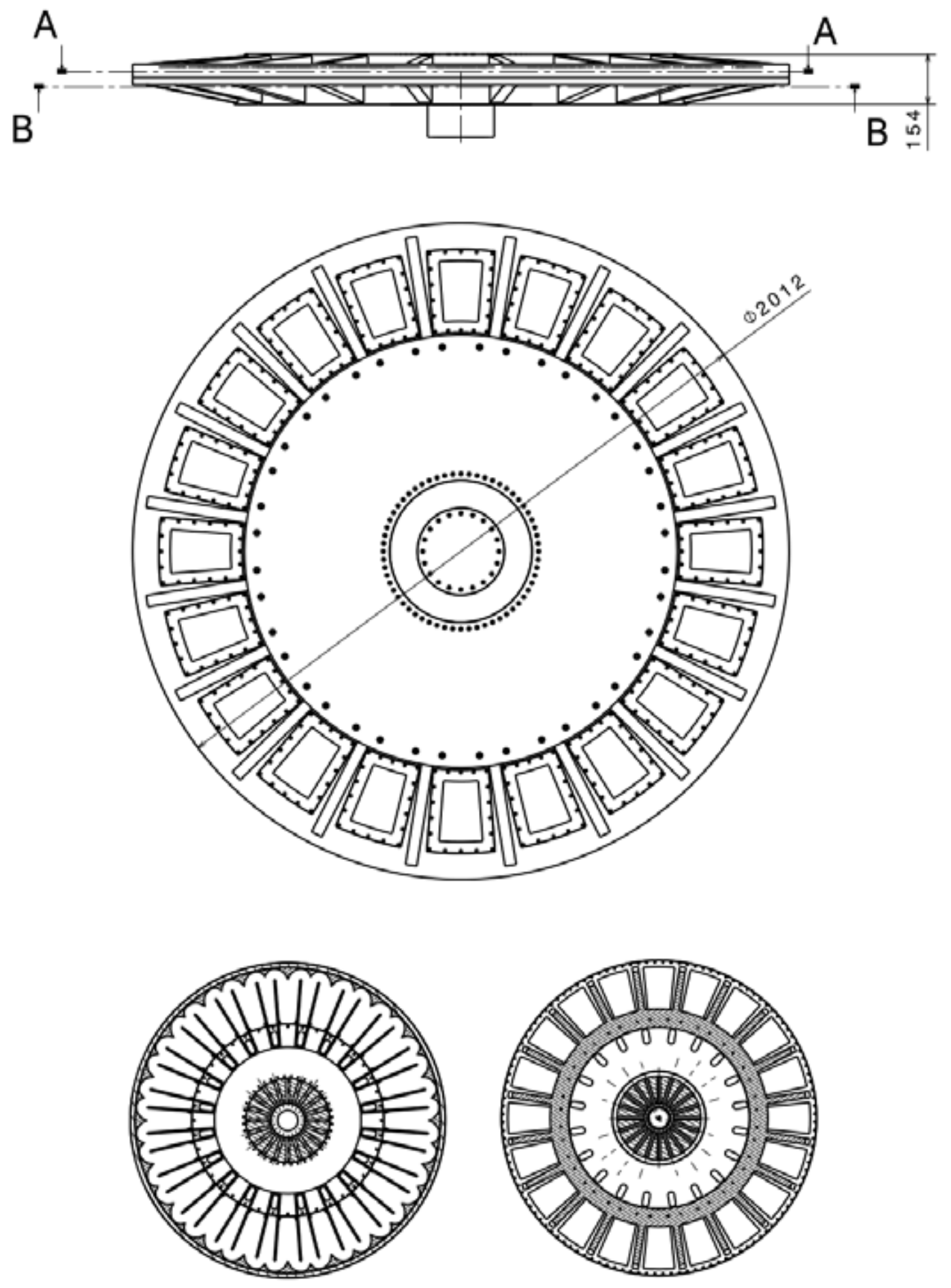

A.A

B-B

Figura 3-5: Dimensiones principales de la estructura [mm] y cortes a ambos niveles de refrigerante. 


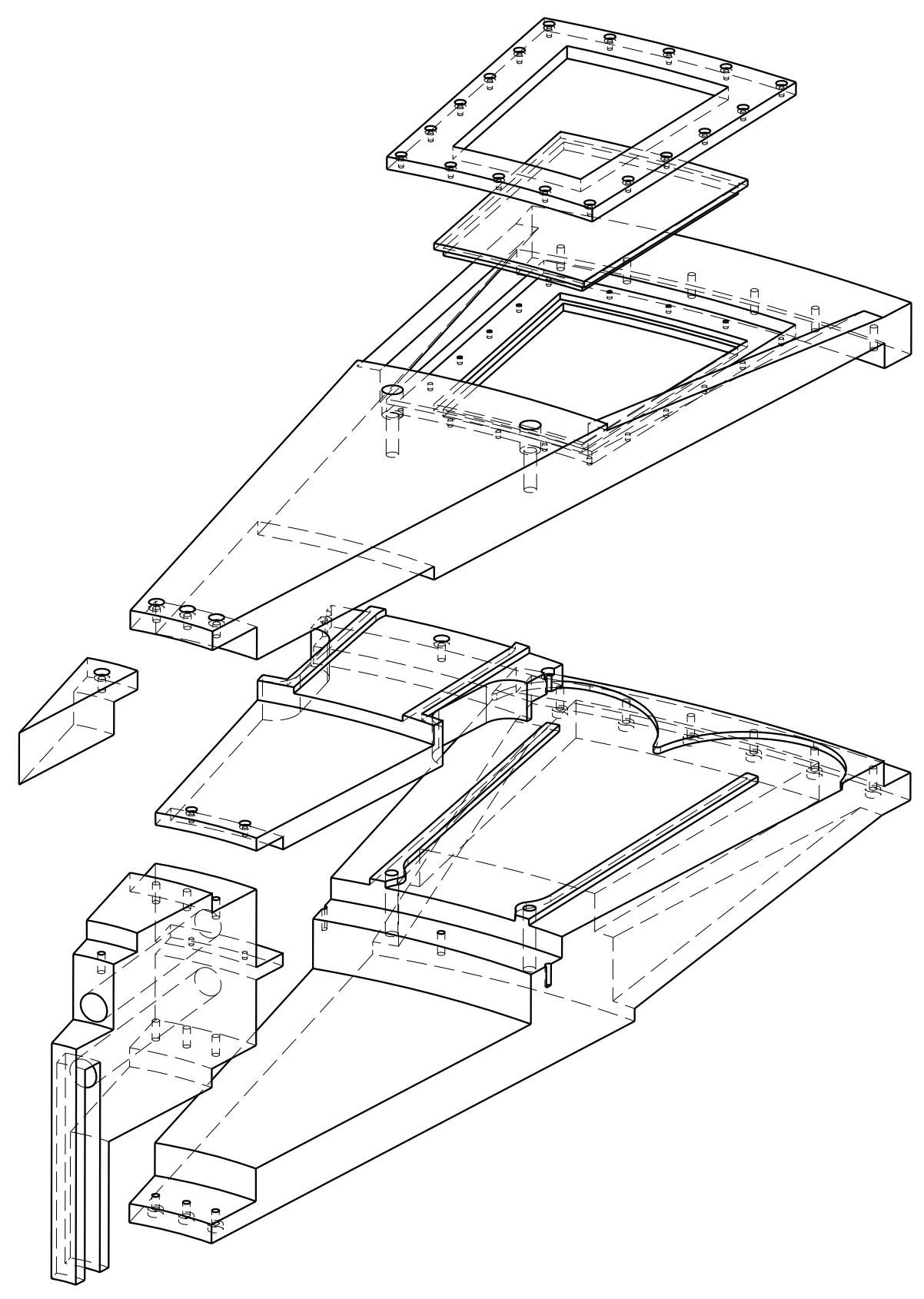

FigurA 3-6: Vista explosionada de un sector de $18^{\circ}$ de la estructura del disco. 
tratamiento térmico T6. Los aceros funden al rededor de $1350{ }^{\circ} \mathrm{C}$, y su temperatura de trabajo máxima se sitúa entre los 500 y $\operatorname{los} 800{ }^{\circ} C^{\mathrm{I}}[163]$. No obstante, las temperaturas de trabajo del disco se prevén inferiores a $100{ }^{\circ} C$, por lo que se cuenta con un margen suficiente. Por otro lado, el módulo de Young de la aleación seleccionada es 69 GPa [161] frente a $\approx 200 \mathrm{GPa}$ de los aceros, por lo que a igualdad de tensión, la deformación de la aleación de aluminio es $\approx 3$ veces mayor. En cambio, dada la menor densidad del aluminio, ambos materiales poseen rigideces específicas próximas $\left(\approx 25 \cdot 10^{6} \mathrm{~m}^{2} \mathrm{~s}^{-2}\right)$, lo cual permite incrementar las secciones resistentes de 6061-T6 para reducir las tensiones y la deformación manteniendo el peso. Respecto a sus propiedades térmicas, el coeficiente de dilatación de esta aleación es superior al de los aceros, 23,5 $10^{-6} K^{-1}[161]$ frente a $\sim 1510^{-6} K^{-1}$, no obstante su conductividad térmica, $167 \mathrm{~W} / \mathrm{Km}$ [161], es aproximadamente 10 veces mayor que la de éstos, lo cual globalmente contribuye a reducir las tensiones de origen termomecánico. En cualquier caso, si el uso de la aleación de aluminio generara en la fase de ingeniería de detalle algún obstáculo insalvable por diseño, el uso de un acero inoxidable austenítico convencional (AISI 304 o AISI 316) es concebible como opción secundaria.

Una vez instaladas en el disco, las placas de berilio son refrigeradas por un canal de agua a presión de $5 \mathrm{~mm}$ de espesor situado bajo éstas. El agua está en contacto directo con las placas, cerrando ellas mismas el circuito de refrigerante. El espesor de aleación de aluminio tras la placa de berilio es de $7 \mathrm{~mm}$. Ambos espesores buscan reducir la absorción de neutrónica. Entre las placas, las piezas de aluminio están reforzadas por nervios rigidizadores en ángulo, de forma que aporten resistencia mecánica sin interponerse en la trayectoria mayoritaria de los neutrones.

Las placas de berilio son fijadas a la estructura del blanco mediante bridas de sujeción. Como solución conceptual se ha optado por soportar las placas en un rebaje del disco superior de la estructura, en el que éstas encajan mediante un escalón en sus caras laterales. De este modo se consigue que el refrigerante entre en contacto con la placa tangentemente a su superficie, evitando ensanchamientos bruscos que pudieran producir zonas de recirculación y remanso pobremente refrigeradas bajo la placa. La solución de sujeción cuenta con espacio para albergar las juntas de estanqueidad necesarias. A priori, dado la previsible irradiación de los materiales en la zona, se considera como solución más probable emplear juntas metálicas elasto-plásticas tipo Helicoflex ${ }^{\circledR}$ [164], no obstante el uso de

\footnotetext{
${ }^{\mathrm{I}}$ Los aceros específicos para aplicaciones de alta temperatura pueden operar hasta $1100{ }^{\circ} \mathrm{C}$.
} 
juntas tóricas convencionales debe considerarse junto con el esquema de mantenimiento y desmantelamiento global de la instalación.

Las placas y las bridas que las mantienen en posición están dimensionadas para dejar una distancia mínima con respecto a la trayectoria del haz de $2 \mathrm{~cm}$. Esta distancia busca dar un margen de seguridad para absorber pequeños desfases o desenfoques del haz. Con el objetivo de acomodar mejor posibles variaciones en la forma del haz se ha optado por un diseño de placa de ancho creciente en la dirección radial. De esta manera se maximiza la superficie de berilio. No obstante, la forma de la placa puede modificarse por motivos de fabricación y ensamblaje si fuera necesario, siempre que se respete la huella del haz.

\subsection{Cálculo termohidráulico}

\subsubsection{Dominio de cálculo: Geometría, materiales y mallado}

El objetivo del cálculo termohidráulico es doble: por un lado, se busca evaluar la capacidad del diseño hidráulico de los canales para proporcionar la refrigeración asumida en el diseño de las placas; y por otro, se busca obtener una distribución de temperaturas en el disco representativa de las condiciones de operación, que permita la evaluación termomecánica de la estructura.

Dada la simetría axial del conjunto, toda la geometría del disco puede simularse empleando uno de los 20 sectores iguales de $18^{\circ}$ de los que se compone, aplicando condiciones de simetría cíclica a las caras laterales del dominio.

El cálculo termohidráulico resuelve la velocidad, presión y temperatura del dominio fluido y la temperatura del dominio sólido asociado, resolviendo la transmisión de calor conjugada entre ambos dominios. La Figura 3-7 muestra el dominio fluido del cálculo termohidráulico. El dominio sólido es el resultado de la unión de las piezas descritas en la Figura 3-6.

Las propiedades del refrigerante empleadas se han obtenido de la asociación internacional para las propiedades del agua y el vapor (IAPWS) [165]. Las propiedades de la aleación 6061-T6 empleadas se han obtenido de la asociación americana de metalurgia (ASM) [161]. Las propiedades del berilio implementadas en la placa son las mismas que 


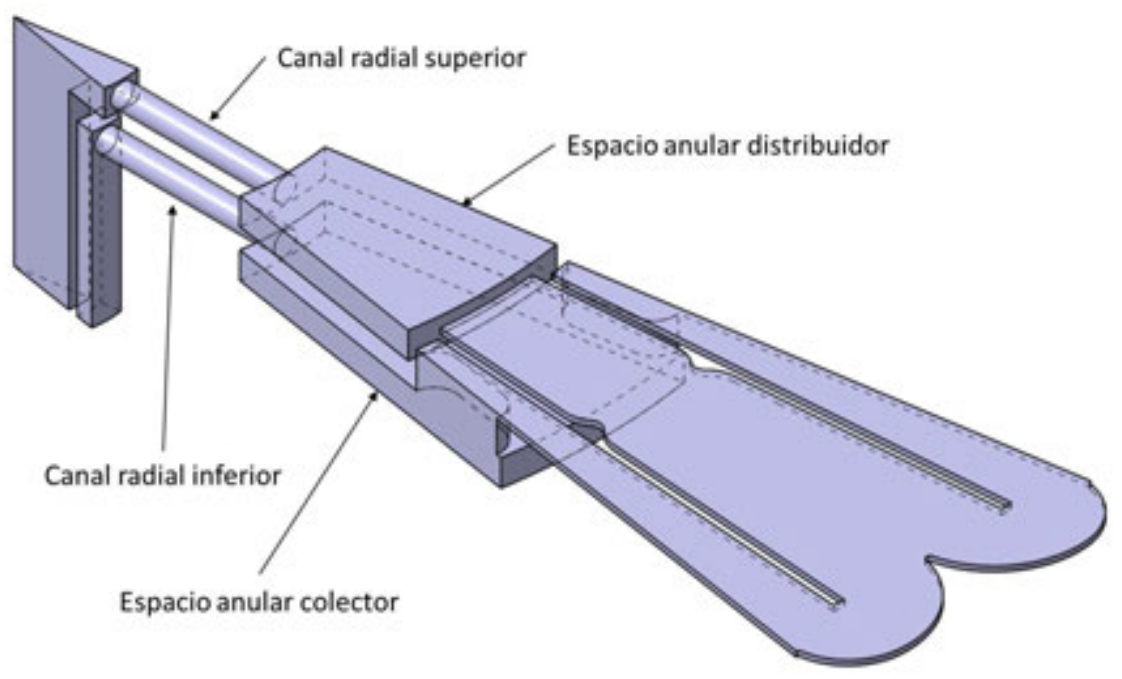

Figura 3-7: Sector de $18^{\circ}$ del dominio fluido.

las empleadas en el Capítulo 2.

El domino fluido es mallado con una malla tetraédrica en el seno del fluido y una malla de elementos tipo cuña en la capa límite, con elementos tipo pirámide en la zona de transición (Figura 3-8). El dominio solido es mallado con una malla predominantemente hexaédrica mediante la opción "Hex dominant" del mallador de ANSYS, la cual permite crear un mallado fundamentalmente hexaédrico sin necesidad de preparar la geometría, empleando minoritariamente tetraedros y pirámides en las transiciones entre zonas de hexaedros.

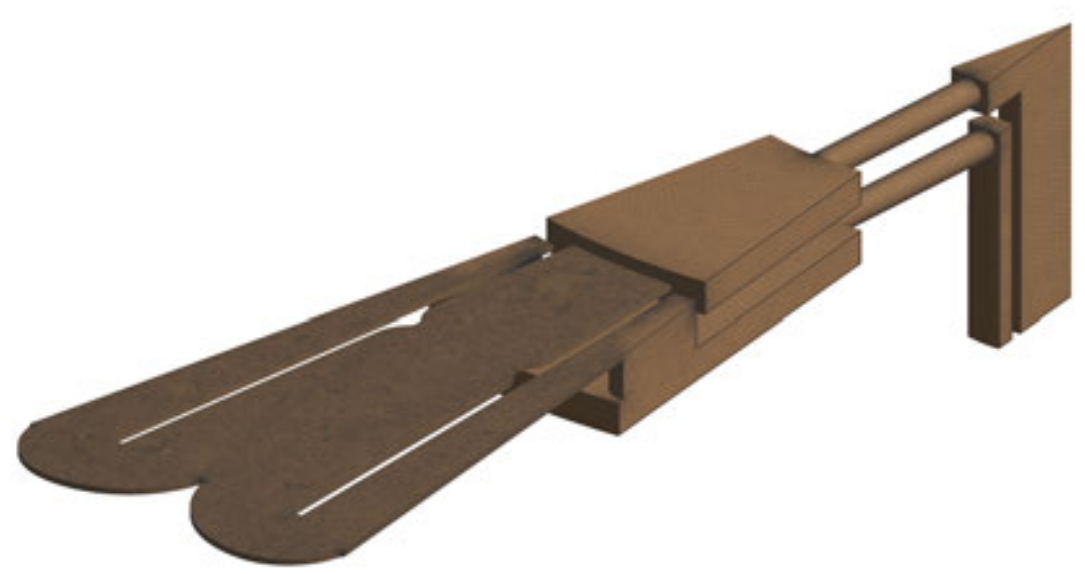

Figura 3-8: Malla del dominio fluido del cálculo termohidráulico. 


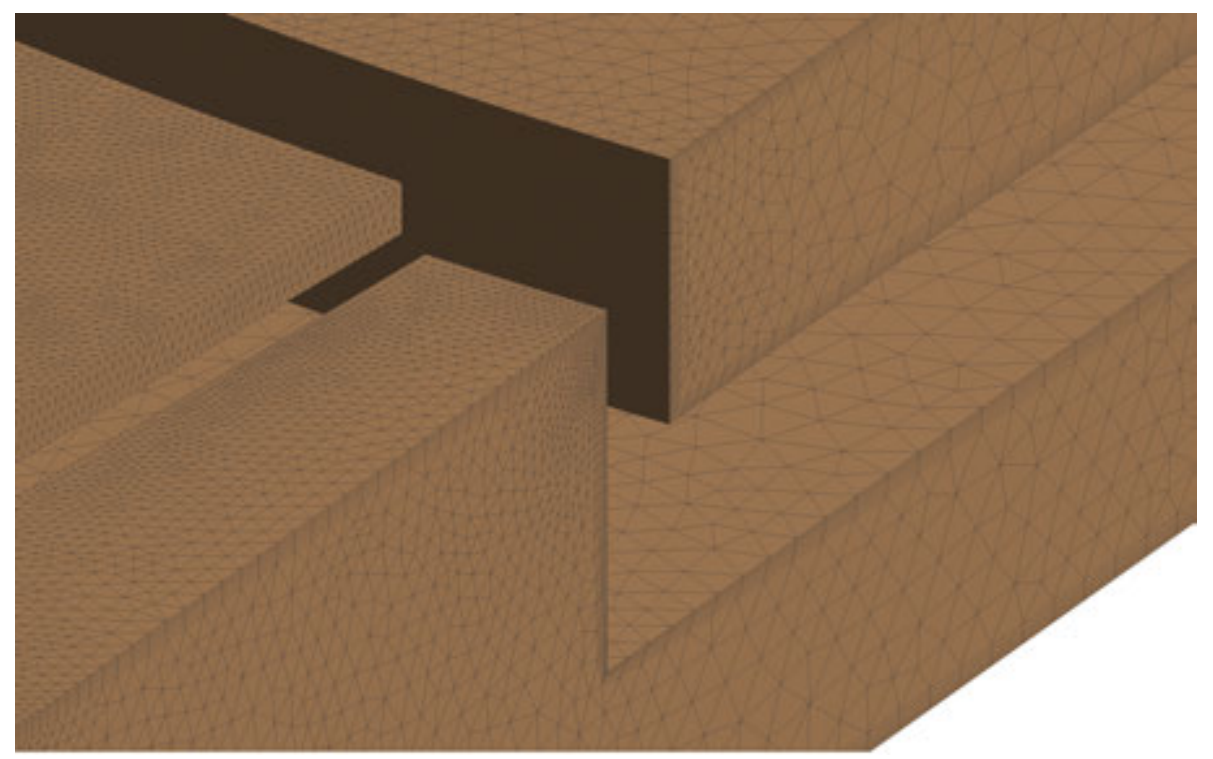

Figura 3-9: Detalle de la malla del dominio fluido del cálculo termohidráulico.

\subsubsection{Condiciones de contorno y cargas}

Para proporcionar a las placas las condiciones de refrigeración con las que fueron diseñadas en el Capítulo 2, esto es, un coeficiente de transmisión de calor de $10^{4} \mathrm{~W} / \mathrm{m}^{2} \mathrm{~K}$, el agua debe fluir por los canales bajo las placas a la velocidad adecuada. Para estimar qué velocidad es necesaria empleamos la correlación de Dittus-Boelter [166] (Ecuación 3.1) válida para flujo turbulento en conductos, donde $N u_{D}$ y $R e_{D}$ son los números de Nusselt y Reynolds en el conducto respectivamente, $\operatorname{Pr}$ es el número de Prandtl del fluido y $n$ un exponente cuyo valor es 0,3 si el fluido se enfría, y 0,4 si se calienta.

$$
\begin{gathered}
N u_{D}=0,023 \operatorname{Re}_{D}^{4 / 5} \operatorname{Pr}^{n} \\
0,6 \lesssim \operatorname{Pr} \lesssim 160 \\
R e_{D} \gtrsim 10^{4} \\
L / D \gtrsim 10
\end{gathered}
$$

El número de Nusselt es un parámetro adimensional que expresa la razón entre la transmisión de calor por convección y por conducción en el fluido. La Ecuación 3.2 describe 
la expresión de este parámetro, donde $k$ es la conductividad del fluido, $D$ es el diámetro del conducto tomado como una longitud característica de la superficie en convección y $h$ es el coeficiente de convección.

$$
N u=\frac{h D}{k}
$$

El número de Reynolds es un parámetro adimensional que expresa la razón entre las fuerzas inerciales y viscosas en el fluido, y da una indicación de la turbulencia de éste. En conductos, los fluidos empiezan a considerarse turbulentos a partir de $R e>2300 \mathrm{y}$ completamente turbulentos para $R e>10^{4}$. La Ecuación 3.3 describe la expresión de este parámetro, donde $\rho, v$ y $\mu$ son la densidad, la velocidad y la viscosidad dinámica del fluido respectivamente, y $D$ es el diámetro del conducto.

$$
R e_{D}=\frac{\rho v D}{\mu}
$$

El número de Pradtl es un parámetro adimensional que expresa la razón entre la velocidad de difusión de la cantidad de movimiento del fluido y la velocidad de difusión térmica en el mismo. La Ecuación 3.4 describe la expresión de este parámetro, donde $\nu$ y $\alpha$ son la viscosidad cinemática y la difusividad térmica del fluido respectivamente, por tanto este parámetro es dependiente exclusivamente del fluido y se suele considerar una propiedad del mismo. El número de Prandtl del agua a temperatura ambiente es 7,01.

$$
\operatorname{Re}_{D}=\frac{\nu}{\alpha}
$$

Dado que el canal no tiene sección circular, emplearemos el diámetro hidráulico del mismo, calculado con la Ecuación 3.5, donde $A$ es el área de paso y $P$ es el perímetro mojado del conducto. La altura del canal bajo la placa es de $5 \mathrm{~mm}$. El ancho del canal es variable, con un valor medio representativo de $10 \mathrm{~cm}$, por lo que el diámetro hidráulico del canal es $\approx 1 \mathrm{~cm}$.

$$
D_{H}=\frac{4 A}{P}
$$


Estableciendo $10^{4} \mathrm{~W} / \mathrm{m}^{2} \mathrm{~K}$ como objetivo para el coeficiente de transmisión de calor en la Ecuación 3.2 y resolviendo el número de Reynolds en la correlación de Dittus-Boelter 3.1 , obtenemos una velocidad necesaria en el canal para alcanzar dicho valor de $2,25 \mathrm{~m} / \mathrm{s}$. El Reynolds en esta configuración es $\approx 2,14 \cdot 10^{4}$ y la longitud del canal bajo la placa es $\approx 30$ $\mathrm{cm}$, por lo que todas las condiciones necesarias para que la correlación de Dittus-Boelter sea aplicable se cumplen.

Para obtener como mínimo la velocidad calculada bajo las 20 placas de berilio, se impone como condición de contorno un caudal de entrada al disco de $26 \mathrm{l} / \mathrm{s}$. La presíon del agua a la entrada es de 5 bar y su temperatura $300 \mathrm{~K}$.

Dado que el disco opera en vacío, no se implementa convección en las caras exteriores. Conservadoramente se ha supuesto que el disco no intercambia calor por radiación con el ambiente. Esta hipótesis permite reducir considerablemente el tiempo de cálculo, evitando la fuerte no-linealidad de la condición de contorno radiativa y, dado que el rango de temperaturas esperable no es suficientemente elevado como para que la radiación tome un papel relevante, es realista.

Se han incluido en el cálculo las fuerzas inerciales generadas por la rotación del disco a 60 r.p.m (i.e cada placa repite posición cada segundo). El disco rota con el eje en posición horizontal con el vector rotación en dirección descendente en la Figura 3-7.

\subsubsection{Parámetros de cálculo}

Con el objetivo de obtener unos resultados representativos de las temperaturas medias de los componentes del disco en operación, se resuelve un caso estacionario equivalente. Se implementa por tanto una potencia constante de $5,6 \mathrm{~kW}$ en la placa de berilio, valor correspondiente a la fracción de $\operatorname{los} 112,5 \mathrm{~kW}$ del haz que recibe cada uno de estos elementos. Se ha optado por un cálculo estacionario equivalente, porque se esperan unos niveles de temperatura en el disco muy bajos, y por tanto una oscilación térmica muy contenida. La simulación transitoria conjunta de los dominios sólido y fluido, con un paso de tiempo suficientemente pequeño como para simular la dinámica de los pulsos, junto con la esquema de contactos no lineales que se detalla a continuación, habría hecho el estudio muy costoso en términos de tiempo de cálculo y, dados las resultados esperables, no está justificado. 
Dicha potencia se ha implementado como una fuente volumétrica homogénea de 18,86 $M W / m^{3}$ en el volumen de la placa. Esta opción de modelado permite obtener una estimación conservadora de la temperatura de los componentes de la estructura, objetivo último del análisis, a costa de distorsionar la distribución de temperaturas en la placa. No obstante, ésta ya ha sido previamente analizada con detalle en el Capítulo 2. No se ha introducido ninguna resistencia térmica de contacto, suponiendo pues contacto térmico perfecto entre todos los componentes sólidos, lo cual también es conservador en lo referente a las temperaturas de la estructura de aluminio.

Para este estudio estacionario equivalente, se ha optado conservadoramente por implementar la potencia total de haz en el berilio, sin tener en cuenta que, como se vio en el Capítulo 2 , por un lado, entorno al $\approx 5 \%$ de esa potencia abandona el berilio en forma de radiación $\gamma$ y energía cinética de los neutrones; y por otro, la reducción del espesor del blanco, disminuye la potencia recibida por el berilio en un $\approx 30 \%$ (Tabla 2.5). Esta última fracción de potencia no abandonaría el sistema completamente, sino que se disiparía en el refrigerante, por lo que implementarla en el berilio es una forma conservadora de tenerla en cuenta, desde el un punto de vista de las temperaturas en la estructura.

El problema fluido se ha simulado empleando el software de volúmenes finitos ANSYSCFX [108]. La turbulencia se ha resuelto aplicando la técnica RANS (Reynolds-Averaged Navier-Stokes) y empleando el modelo RNG $k-\epsilon$ [167] con funciones de pared. Este modelo es una mejora del modelo $k-\epsilon$ estándar [168], al más extendido y validado, en las que las ecuaciones de cierre de los términos turbulentos calculan el transporte de la energía cinética turbulenta, $k$, y la disipación turbulenta, $\epsilon$, aplicando métodos de renormalización de grupo [169]. Este modelo es adecuado para el flujo sencillo que se establece debajo de las placas y se escoge fundamentalmente por su eficacia y robustez.

\subsubsection{Resultados}

Resuelto hasta convergencia el cálculo previamente descrito, la Figura 3-10 muestra el campo de velocidades obtenido. El sistema fluido antes dimensionado proporciona una velocidad al refrigerante bajo la placa de berilio de $\approx 2,5 \mathrm{~m} / \mathrm{s}$, consistente con el objetivo necesario para su refrigeración. Se observa como, debido al efecto de la rotación del disco, la distribución de velocidades no es simétrica bajo la placa, acelerándose ligeramente en 
la parte posterior en el sentido del giro. De acuerdo con [170], las velocidades máximas obtenidas bajo la placa $\approx 3 \mathrm{~m} / \mathrm{s}$ no son susceptibles de generar erosión-corrosión y las velocidades máximas obtenidas en todo el fluido $\lesssim 6 \mathrm{~m} / \mathrm{s}$ no lo son aplicándose el adecuado filtrado y control químico, especialmente controlando la cantidad de oxígeno disuelto en el agua.
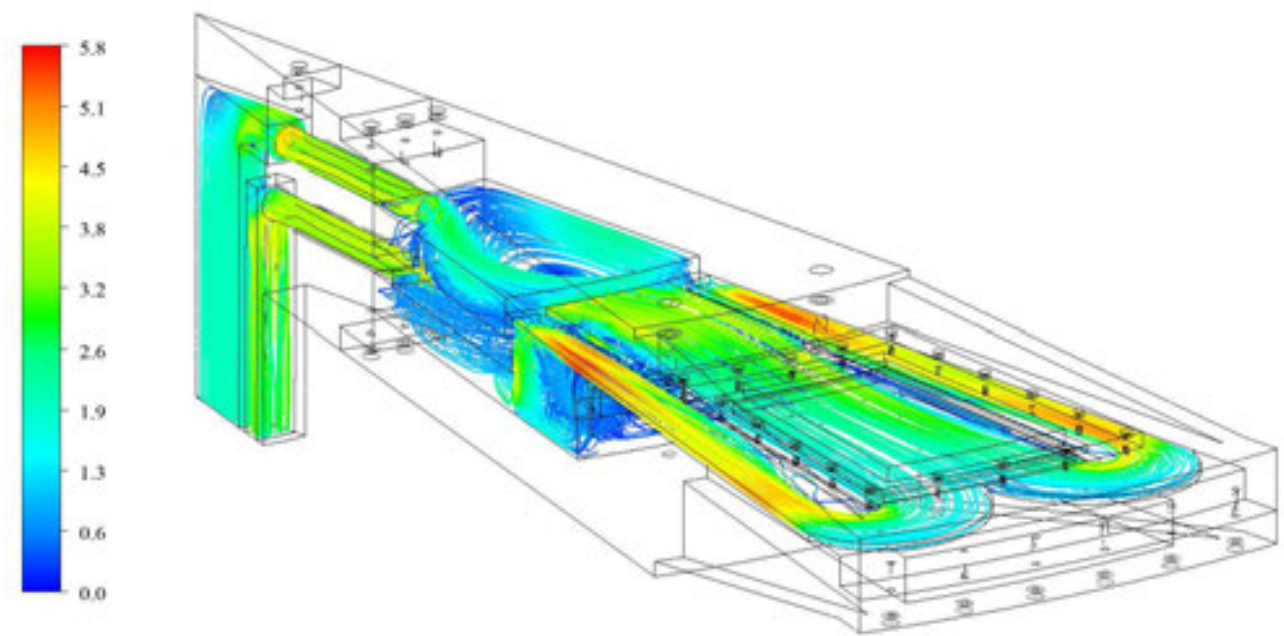

Figura 3-10: Velocidades del refrigerante dentro del disco.

La Figura 3-11 muestra los resultados de temperaturas medias en el dominio sólido. En las proximidades de la placa de berilio la estructura alcanza los $\approx 43{ }^{\circ} C$, apenas 16 ${ }^{\circ} C$ más que la temperatura del agua. La mayoría de la estructura se encuentra a una temperatura próxima a la del refrigerante, que se calienta únicamente $1{ }^{\circ} C$ en su paso por el disco. Este incremento de temperatura es coherente con el balance global de potencia térmica del sistema (Ecuación 3.6). Por tanto podemos concluir que el sistema refrigerante está ampliamente capacitado para extraer los $112,5 \mathrm{~kW}$ de potencia del haz y, en base a los cálculos térmicos del Capitulo 2, hacer frente a la naturaleza pulsada de la deposición energética.

$$
P_{h a z}=\dot{m} C_{p} \Delta T
$$




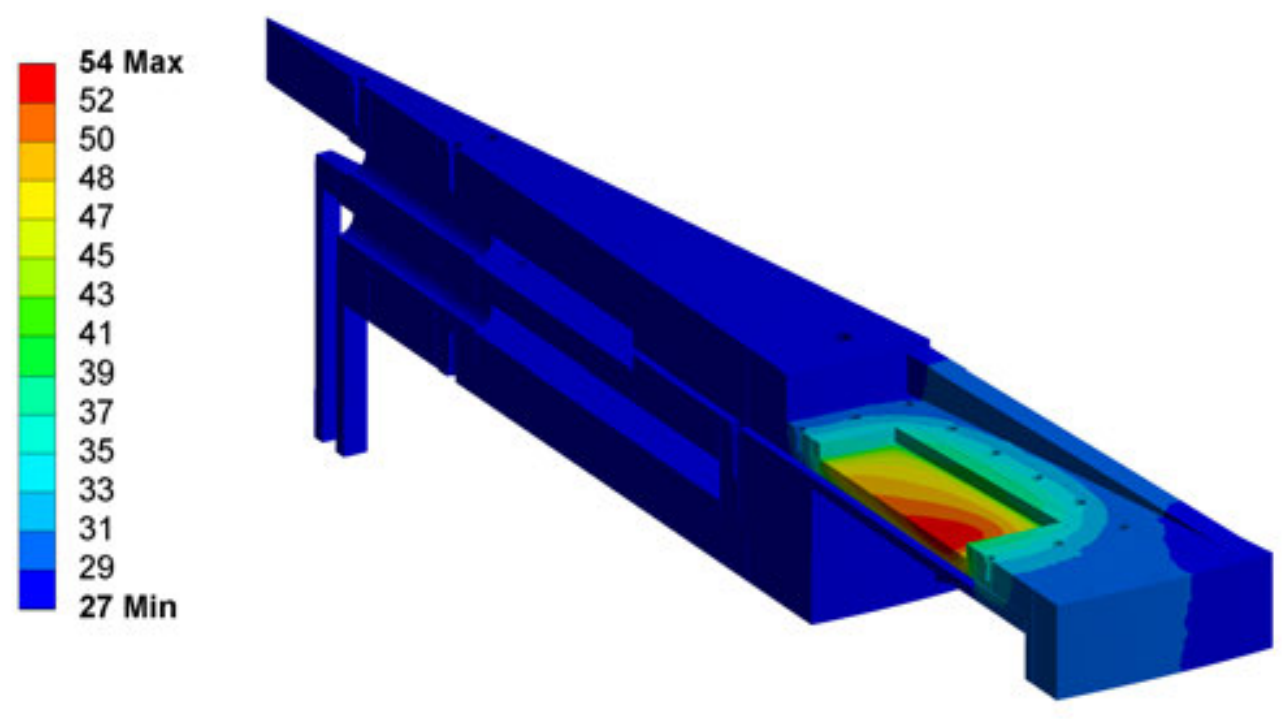

Figura 3-11: Temperaturas medias $\left[{ }^{\circ} \mathrm{C}\right]$ en operación del disco.

\subsection{Cálculo termomecánico}

\subsubsection{Dominio de cálculo: Geometría, materiales y mallado}

El objetivo del cálculo termomecánico es analizar el comportamiento mecánico general de la estructura mediante la obtención de una estimación de la distribución media de desplazamientos y tensiones en operación.

Para ello se empleará como dominio de cálculo la misma geometría y las mismas propiedades térmicas que en el dominio sólido del cálculo termohidráulico, junto con las propiedades mecánicas anteriormente descritas. Los coeficientes de dilatación térmica del berilio y la aleación de aluminio son $11,510^{-6} K^{-1}[134]$ y $23,510^{-6} K^{-1}$ [161] respectivamente. Los tornillos no se incluyen en la geometría, sin embargo su efecto se modela mediante la inclusión de elementos tipo viga $(B E A M)$.

Con el objetivo de reducir tiempo de cálculo del problema e implementar las precargas de los tornillos correctamente, es necesario preparar convenientemente la geometría antes del mallado. Esta preparación consiste, fundamentalmente, en la división de la geometría en regiones de seis caras mallables por barrido (i.e. mallado de una cara y proyección de ésta en todo el volumen). Mediante este laborioso procedimiento se consigue mallar esta 
compleja geometría con malla hexaédrica continua dentro de cada parte (Figura 3-12).

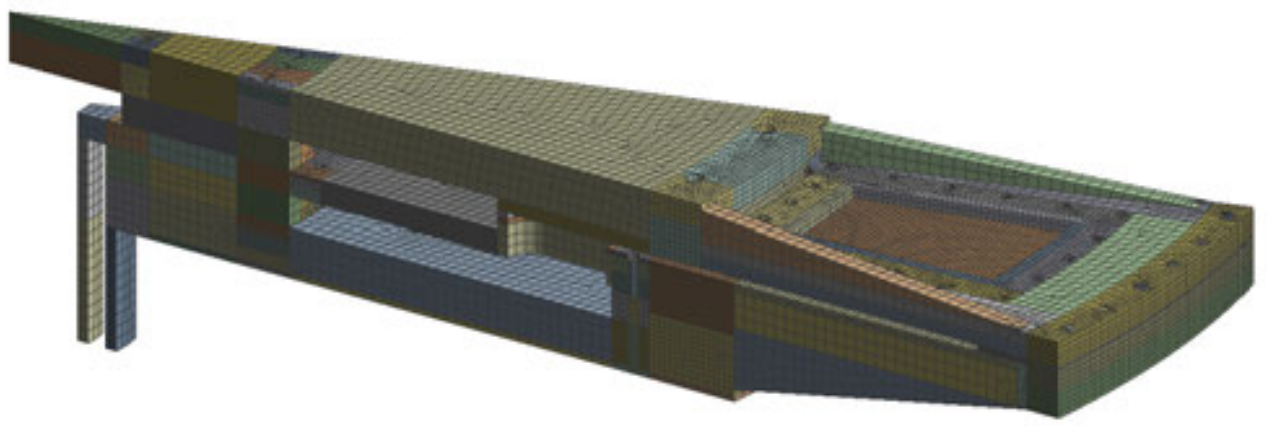

FIGURA 3-12: Mallado termomecánico

\subsubsection{Condiciones de contorno y cargas}

Como condiciones de contorno se emplean una vez más las condiciones de simetría cíclica en las paredes laterales del dominio, que al ser concurrentes en el eje del disco simulan la restricción mecánica del mismo. El desplazamiento al lo largo del eje del disco se evita imponiendo en los nodos del extremo del eje una condición de desplazamiento nulo en dicha dirección.

La distribución de temperaturas mostrada en la Figura 3-11 se interpola en la malla termomecánica generando tensiones de origen térmico.

La presión interna del refrigerante, 5 bar, se aplica a todas las caras en contacto con éste. Dado que el disco opera en vacío, no se implementa ninguna presión en las caras externas.

La precarga de los tornillos se transmite a las caras que representan las superficies de apoyo de la cabeza de los tornillos y las superficies roscadas. Se ha asumido un grado de calidad de los tornillos 12.9 (UNE-EN ISO 898-1 [171]), por lo que las pregargas aplicadas según su métrica son $6387 N$ para los tornillos M4, $14623 N$ para los tornillos M6, y $42195 N$ para los tornillos M10.

Es necesario apuntar que todos los componentes del domino sólido encajan perfectamente, no dejando ningún hueco u holgura entre ellos, lo cual maximiza las tensiones por dilatación diferencial entre la placa de berilio y su alojamiento de aluminio. Esta hipótesis 
de modelado es conservadora respecto a la generación de tensiones en el disco y permite analizar su comportamiento global. La determinación de las tolerancias para el ensamblaje de la placa y la brida debe analizarse en la fase de ingeniería de detalle junto con el diseño de la junta de estanqueidad.

\subsubsection{Parámetros de cálculo}

El modelo termomecánico simula el contacto realista entre todas las piezas del disco y la placa de berilio, permitiendo que éstas se deslicen entre sí y se separen llegado el caso. Esta opción de modelado introduce complejidad y una componente altamente no lineal al cálculo, pero, de esta manera, se obtiene un comportamiento verosímil en el que se evalúa la capacidad de las piezas y de las uniones atornilladas para soportar las cargas, especialmente la presión interna. Entre las piezas se aplica un coeficiente de rozamiento $\mu=0,2$ y se emplea en el algoritmo de simulación de contacto la formulación Augmented Lagrange [172].

El efecto de la precarga de los tornillos, modelados con elementos tipo viga, se ha implementado mediante una rutina de ANSYS APDL que efectúa los siguientes pasos:

1. Localiza los tornillos e identifica su métrica.

2. Establece un vínculo entre los extremos de estos y las superficies a las que se transmite la pregarga mediante ecuaciones de restricción multipunto (Multi Point Constraints).

3. Calcula y aplica una reducción de longitud al tornillo de modo que el estado tensional resultante es equivalente a la aplicación de la precarga adecuada para su métrica.

Esta metodología es una versión simplificada de la validada en [173] y permite simular el efecto de los tornillos en el comportamiento global de la estructura sin la necesidad de modelar en detalle ni los tornillos ni las superficies roscadas, lo cual haría el coste computacional inabarcable.

\subsubsection{Resultados}

La Figura 3-13 presenta los resultados de tensión equivalente de von Mises obtenidos. Las áreas de mayor tensión se encuentran en la parte inferior del disco, próximas a las 
aristas de la zona bajo el canal de refrigeración a su paso por las placas. Esta zona se ha diseñado con bajo espesor para minimizar la absorción de neutrones, no obstante, esto hace que flecte bajo la presión interna del refrigerante, lo que genera tensión cerca de las aristas.

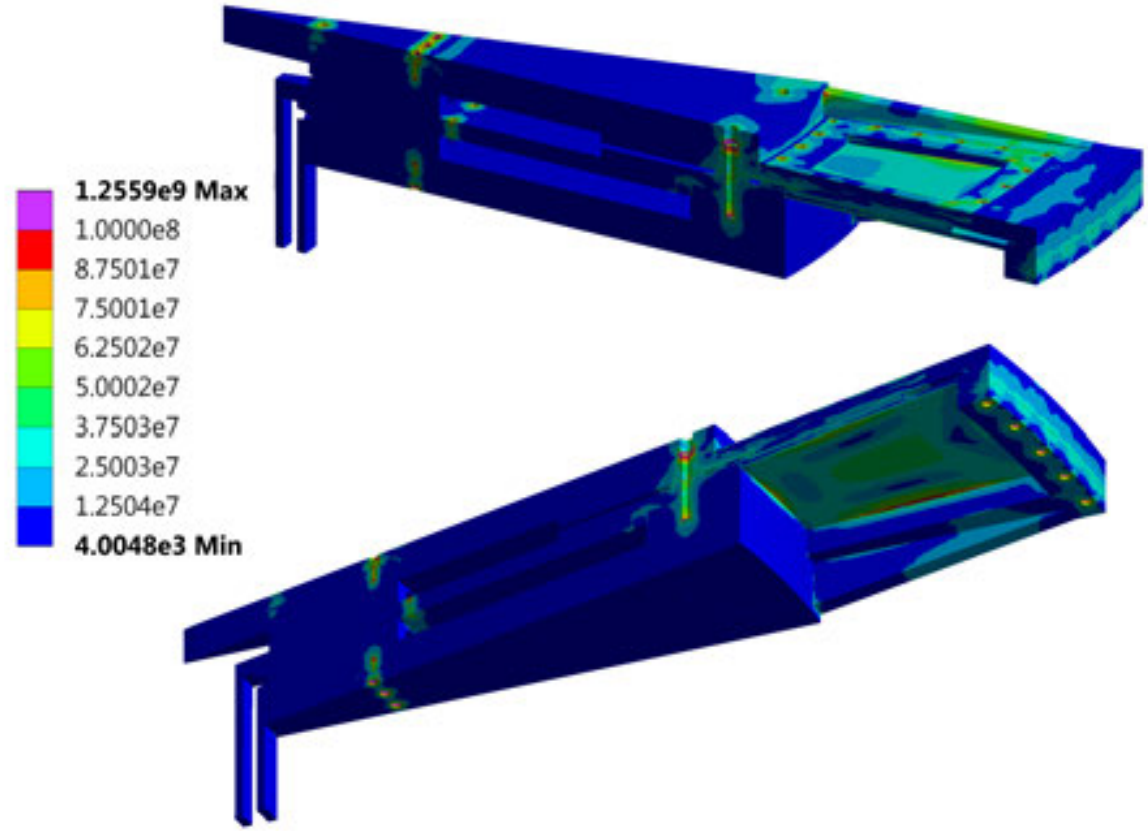

Figura 3-13: Cálculo termomecánico. Tensiones equivalentes de von Mises [Pa].

Para obtener un valor preciso de la tensión en esta zona se ha llevado a cabo un estudio detallado empleando submodelado. Esta técnica consiste en simular con más detalle una región aislada del conjunto, aplicando en sus fronteras los resultados de desplazamientos obtenidos en el modelo global. De esta manera se puede añadir detalle geométrico y refinar la malla en la zona de interés, manteniendo el tamaño del modelo dentro de lo abarcable en términos de coste computacional.

Dado que el valor de tensión máximo se encuentra cerca de una discontinuidad geométrica, el valor preciso de tensión se ve afectado por un incremento artificial inherente al método de los elementos finitos en este tipo de configuraciones. Para obtener un valor realista de la tensión en esa zona, se incluye en el submodelo un redondo de $5 \mathrm{~mm}$ en las aristas. La presencia de estos detalles geométricos suele incluirse en las geometrías reales para evitar la concentración de tensiones, y su definición se precisa normalmente en las fases de ingeniería de detalle. La presencia del redondeo aquí asumido es por tanto 
esperable y recomendable en la estructura del blanco real.

Para asegurar la precisión del valor máximo de tensión obtenido en el submodelo, se implementa en la simulación un refinamiento automático de malla. Este procedimiento subdivide la malla en las zonas de interés hasta que el valor máximo de tensión converge a un valor estable. La Figura 3-14 muestra el resultado del cálculo, así como la densidad de malla final obtenida tras el refinamiento automático. El valor de tensión máximo obtenido es $97 M P a$. Este valor es inferior al límite elástico, $250 M P a$ [161], e inferior al límite de fatiga de la aleación 6061-T6, $100 M P a$ para $5 \cdot 10^{8}$ ciclos [161]; por lo que no se espera deformación plástica, así como tampoco la aparición de fatiga, ni siquiera considerando la tensión máxima como tensión cíclica.

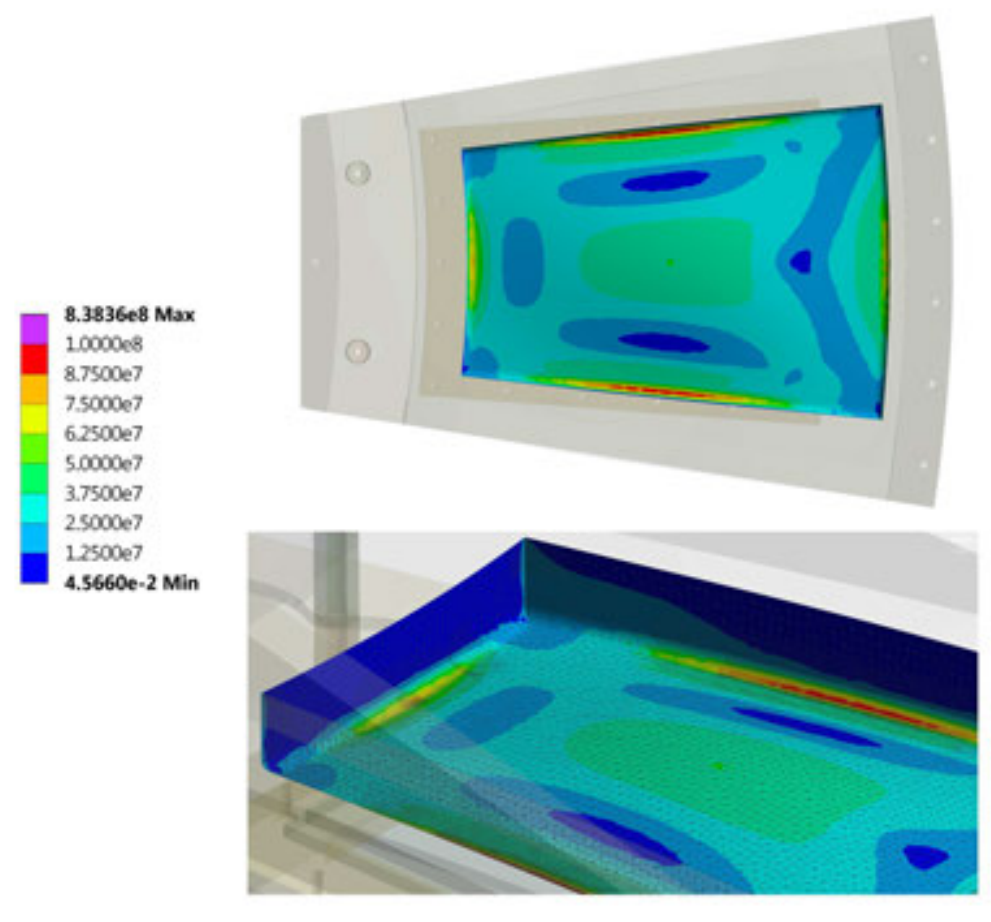

Figura 3-14: Cálculo termomecánico. Tensiones equivalentes de von Mises $[P a]$ en el submodelo de la zona de tensión máxima. Detalle de la malla refinada.

Respecto a otras zonas destacables de la distribución de tensiones, cabe mencionar el papel de los nervios rigidizadores. La tensión a la que se encuentran sometidos evidencia su papel en la estabilidad del diseño. Su presencia es el resultado del proceso de diseño iterativo descrito en el Apéndice A.

También cabe destacar como el modelado de las uniones atornilladas reproduce el 
cono de presiones característico de este tipo de unión bajo las cabezas de los tornillos. Se aprecian valores de tensión artificialmente altos en las aristas de los alojamientos para los tornillos, debidos a la conjunción de la discontinuidad geométrica de la arista y a la discontinuidad numérica generada por la aplicación de las ecuaciones de restricción multipunto. Sin embargo, se observa como este incremento de tensión no progresa en el espesor del material, donde se desarrolla el cono de presiones con normalidad.

De modo general, el nivel global de tensiones en la estructura es bajo, y por tanto no se esperan problemas que pudieran poner el concepto en cuestión.

La Figura 3-15 presenta los desplazamientos totales en la estructura, entendiendo esta magnitud como el módulo del vector desplazamiento desde la posición inicial libre de carga. Los valores máximos de deformación se encuentran en la placa de berilio y, en el disco, bajo ésta. La Figura 3-16 muestra la componente del vector desplazamiento en la dirección del eje del disco. Comparando ambas figuras, puede observarse que la componente mayoritaria del desplazamiento se da en esta dirección. Este comportamiento es el resultado de la presión interna, tendente a separar los dos discos principales que conforman la estructura. El desplazamiento alcanza un valor máximo de aproximadamente 0,5 $m m$. Estas deformaciones se consideran aceptables dado que no alteran significativamente el flujo de refrigerante.

La deformación del disco bajo la placa se ve acentuada por el bajo espesor de esta zona. Si fuese necesario reducir la deformación en este área, la zona de bajo espesor podría ajustarse más a la trayectoria de los neutrones, así como incluirse redondeos amplios en las aristas colindantes; de tal forma que se la dotase de mayor rigidez a flexión contra la presión interna.

Fuera de estas áreas se observa como los valores de desplazamiento son globalmente pequeños, por lo que el sistema de uniones atornilladas mantiene la integridad del disco con solvencia.

La Figura 3-17 presenta la presión de contacto entre los diferentes componentes del disco en operación. En estas imágenes, sólo se representan con color aquellas zonas del contacto en las que la presión entre componentes es mayor que la presión del refrigerante, i.e. 5 bar. Este criterio puede emplearse como un indicativo conservador de la capacidad de las uniones atornilladas para mantener la estanqueidad, una vez definidas las juntas. 

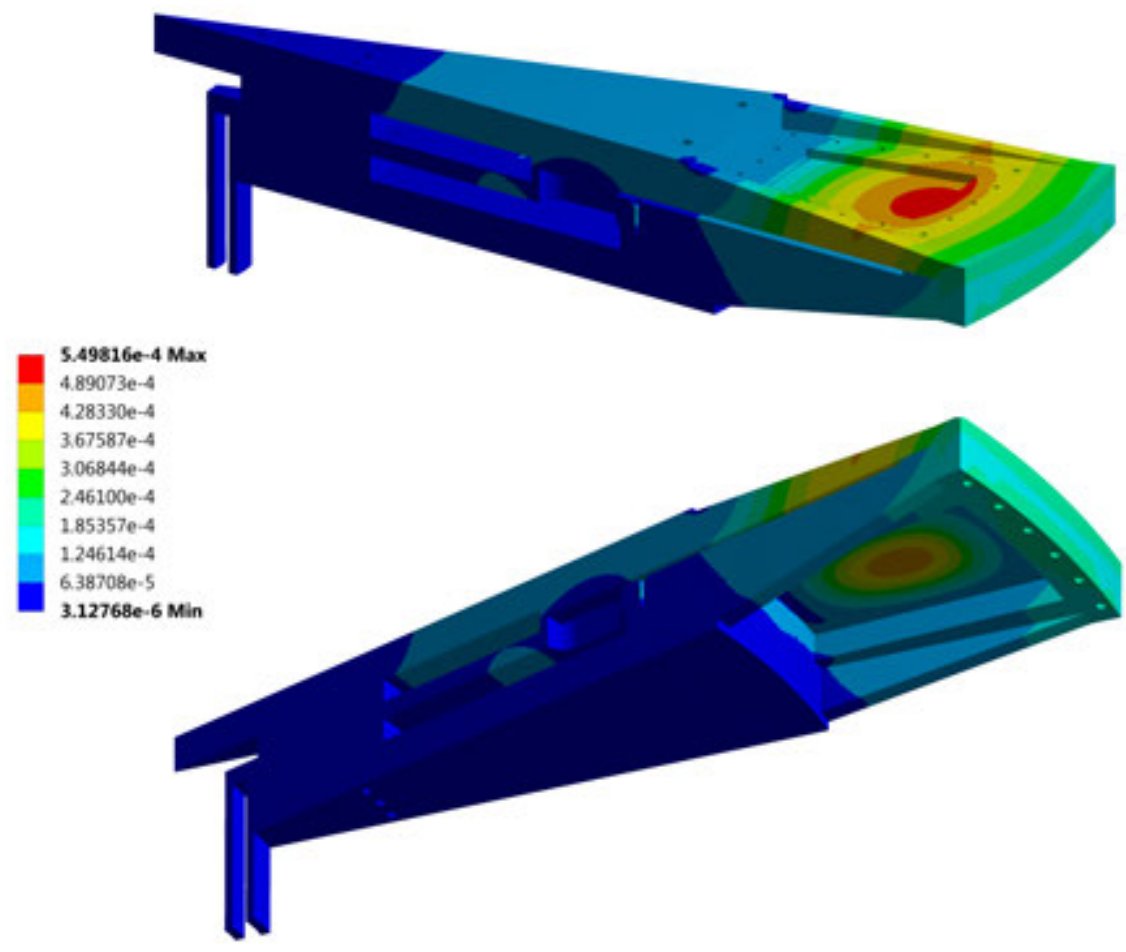

Figura 3-15: Cálculo termomecánico. Desplazamiento total $[\mathrm{m}]$.

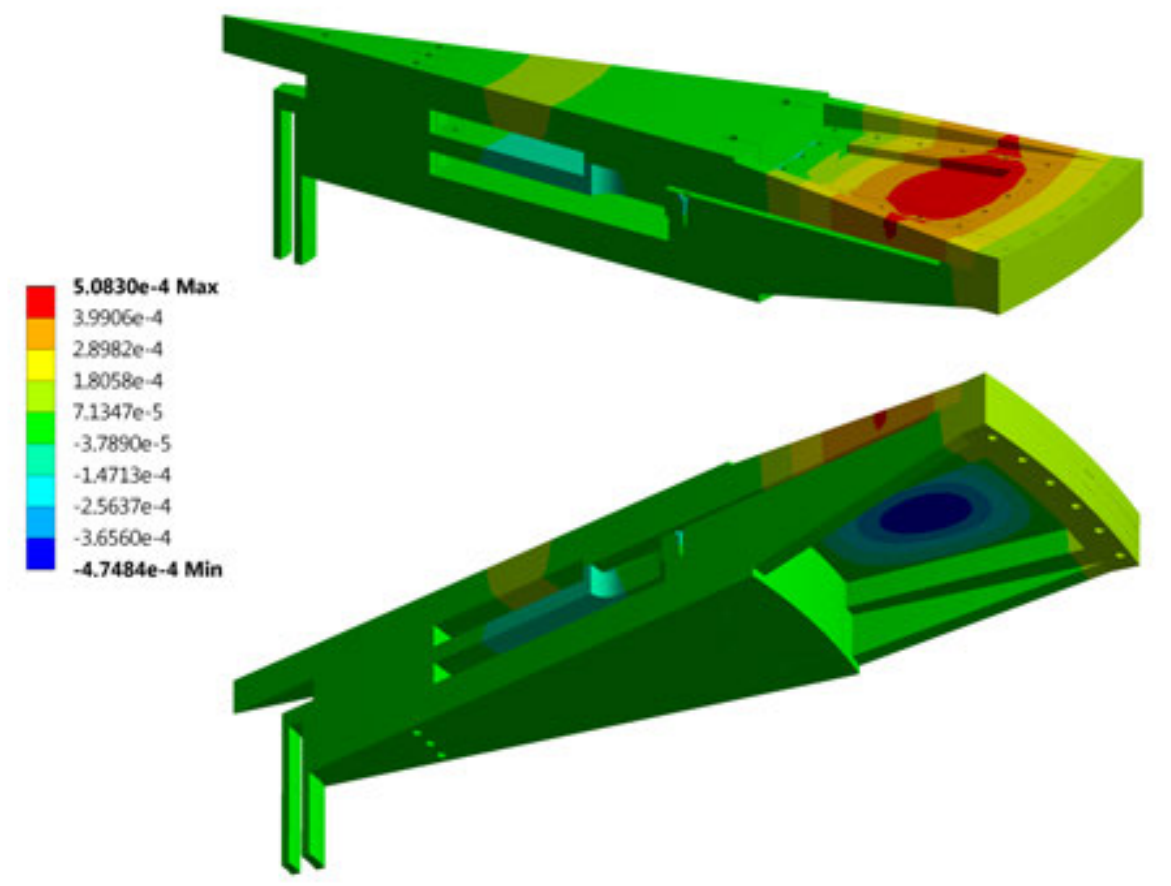

Figura 3-16: Cálculo termomecánico. Desplazamiento en la dirección del eje del disco $[m]$. 
Podemos observar como la disposición de los tornillos crea una barrera de presión de contacto superior a la presión del refrigerante alrededor de todas las uniones atornilladas, por tanto se puede esperar que éstas proporcionen las condiciones necesarias para la correcta operación de las juntas de estanqueidad.

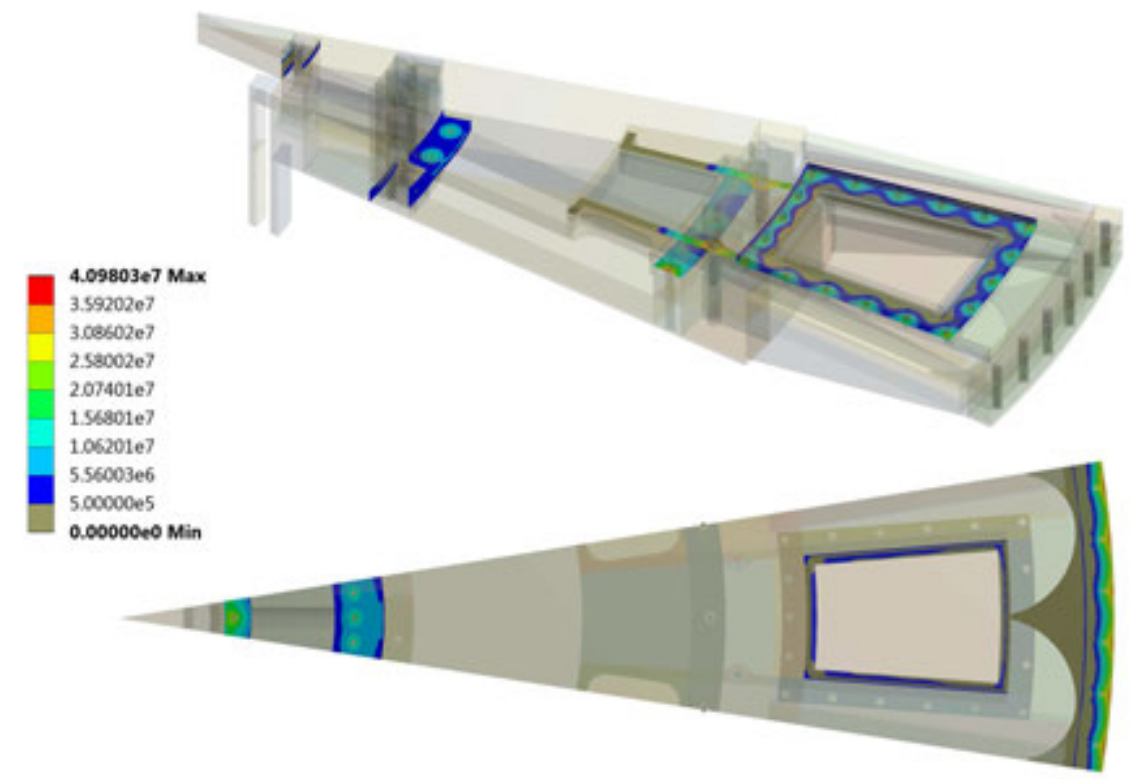

Figura 3-17: Cálculo termomecánico. Distribución de presión en los contactos $[P a]$.

\subsection{Evaluación de los márgenes de seguridad frente a acci- dente}

Los siguientes análisis tienen como objetivo proporcionar una evaluación preliminar de la capacidad de la estructura del blanco para soportar posibles situaciones accidentales. El objetivo de esta sección es, por tanto, evaluar de forma cualitativa los márgenes de seguridad disponibles, e identificar de manera temprana posibles riesgos para la operación remediables en las fases de diseño. El análisis más detallado de los transitorios accidentales desde un punto de vista de ingeniería de planta, requeriría de una definición mayor de la instalación en su conjunto, especialmente de los circuitos fluidos y de las salvaguardias de seguridad. No obstante, este estudio analizará la importancia de las posibles respuestas y acciones de mitigación, siendo pues un dato de entrada para la definición de dichas salvaguardias. 
Las siguientes situaciones accidentales han sido identificadas como potencialmente relevantes:

- Concentración excesiva del haz de protones.

- Bloqueo del giro del blanco.

- Desfase haz-blanco.

- Accidente de pérdida de flujo.

- Accidente de pérdida de vacío.

- Reacciones químicas a alta temperatura.

- Energía de los protones anormalmente baja.

\subsubsection{Concentración excesiva del haz de protones}

El evento iniciador de este accidente sería un fallo en los cuadrupolos que controlan la forma del haz de protones. Dicho sistema es el encargado de que el haz que impacta en el berilio tenga una sección circular y un diámetro de $10 \mathrm{~cm}$. Este evento implicaría que la energía del haz se repartiera en un área más pequeña, generando densidades de potencia superiores a las empleadas para el diseño del sistema de refrigeración, con el consecuente aumento de la temperatura y las tensiones térmicas en el berilio. Este tipo de eventos han sido reportados en la fuente de espalación suiza SINQ en varias ocasiones, llegando a acarrear el fallo de alguno de los elementos de su blanco [174, 175, 176].

Con el objetivo de estudiar el efecto de este accidente, se realiza un análisis paramétrico del comportamiento termomecánico del blanco ante el impacto de haces de protones de diámetro decreciente. Para ello se emplean las herramientas de simulación (MCNPX y ANSYS), la metodología y las hipótesis de modelado descritas en la Sección 2.3.3 para el cálculo de los elementos del blanco, implementando la potencia completa del haz mediante haces gaussianos de radios decrecientes.

Como condiciones preaccidentales se considera que el blanco se encuentra en operación normal con un haz de $5 \mathrm{~cm}$ de radio, comenzando a tiempo cero del accidente la concentración excesiva del haz. Las Figuras 3-18 y 3-19 muestran la evolución de tensiones de 
von Mises y temperaturas máximas en el blanco durante el accidente para las distintas concentraciones de haz.

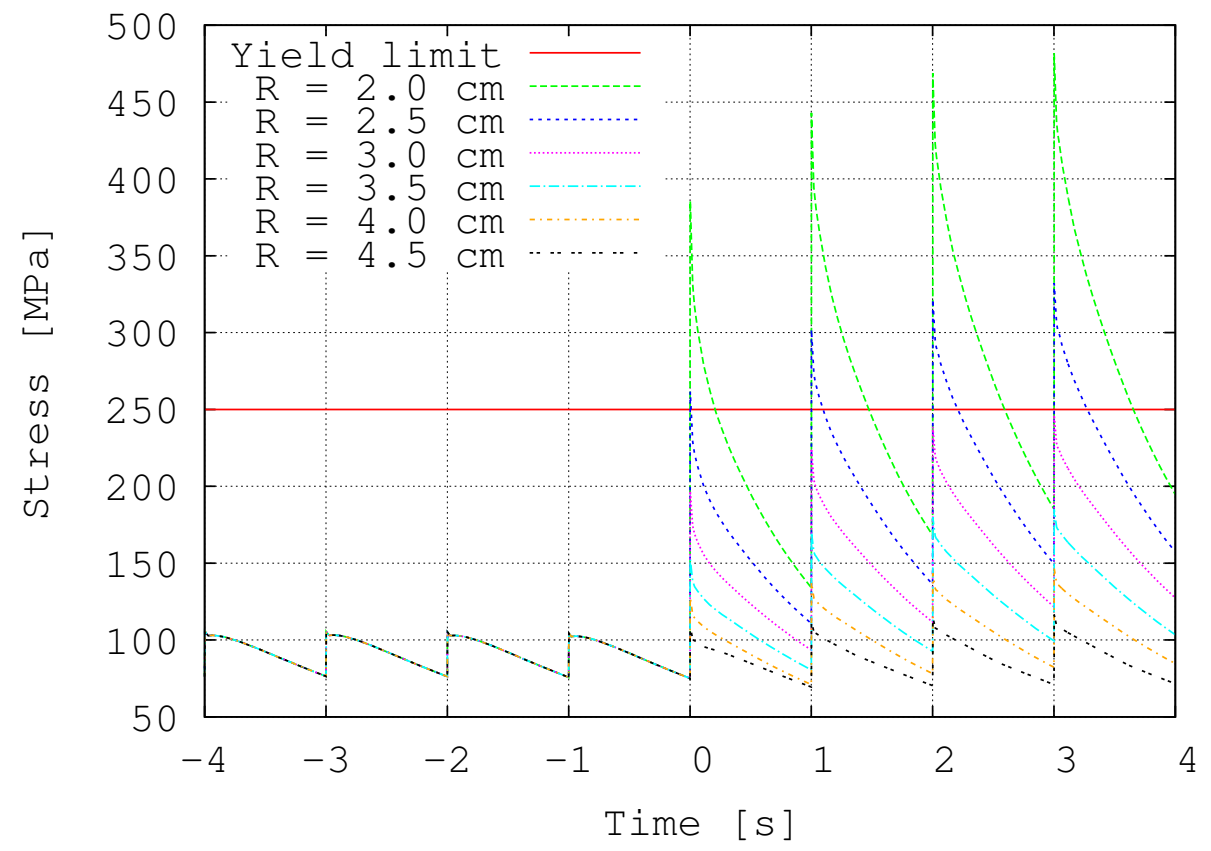

FiguRA 3-18: Evolución de la tensión de von Mises máxima [MPa] en la placa de berilio para distintos radios de haz.

Los resultados indican que una reducción súbita del haz a un radio inferior a $3 \mathrm{~cm}$ generaría tensiones en el berilio superiores a su límite elástico (250 MPa [137]) tras el impacto de un solo pulso, por lo que no habría forma de impedir que este límite se sobrepasara interrumpiendo la operación del acelerador después del comienzo del accidente. Si después del fallo de los sistemas de apertura del haz, su radio sigue siendo superior a los $3 \mathrm{~cm}$, el límite elástico se alcanzaría a los 3 segundos del comienzo del accidente. Este tiempo de reacción se considera suficiente para actuar en base a las lecturas de los sistemas de seguridad y monitorización de la forma del haz.

El límite elástico se toma en esta sección como un límite conservador de la tensión admisible, por debajo del cual se considera que la placas no sufrirían ningún daño. Por encima de este nivel, estas sufrirían deformación permanente ${ }^{\mathrm{II}}$. Es esperable que la plastificación fuera muy local en el momento de alcanzarse el límite elástico y que, dado el amplio margen hasta la tensión de rotura del material (324 MPa), el fallo de la placa no se diera hasta pasado un tiempo considerablemente mayor. Mantener las placas en 


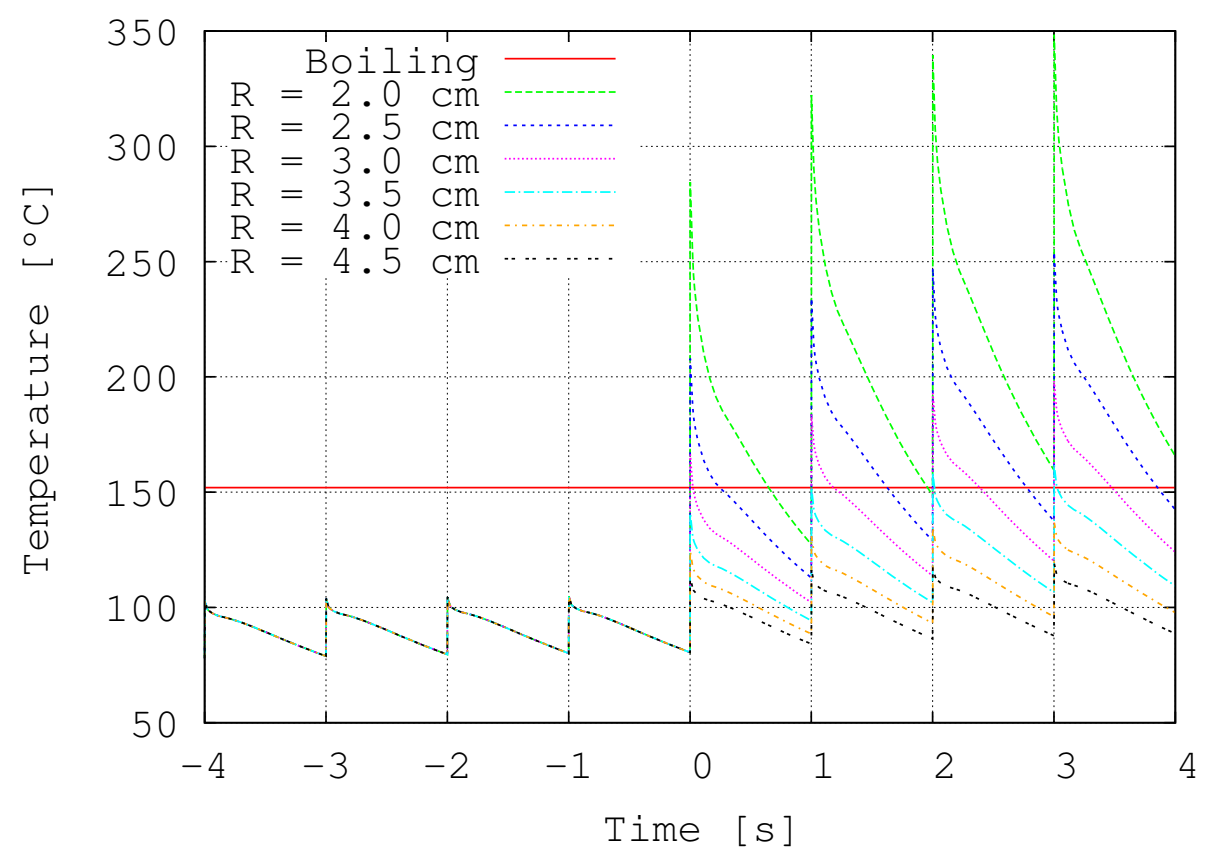

Figura 3-19: Evolución de la temperatura máxima $\left[{ }^{\circ} \mathrm{C}\right]$ en la placa de berilio para distintos radios de haz..

operación en caso de que se haya sobrepasado el límite elástico, pero no se produjese fallo evidente, debería ser estudiado en base a una inspección de las mismas. En cualquier caso, dados la baja deformación a rotura del berilio y su estado de irradiación, no es esperable la aparición de deformación evidente previa a rotura.

Respecto a la temperatura de la placa, el incremento producido por la concentración del haz se considera suficientemente lento como para detectarlo mediante termografía o termopares antes de que las propiedades mecánicas del berilio se vieran comprometidas. El empleo de termografía requeriría de una línea de visión del blanco, lo cual podría implementarse desde alguna de las líneas experimentales o desde una apertura específica que permitiera estudiar las placas justo después del impacto del haz. La inclusión de termopares en la estructura del blanco en puntos próximos a la placa podría dar lecturas demasiado lentas, mientras que su colocación en las propias placas no es trivial, no obstante el empleo de estos dispositivos en blancos de espalación es frecuente, por ejemplo en la

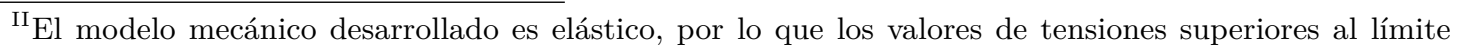
elástico presentados en esta sección no deben ser considerados como precisos, sino como indicativos de la plastificación del material.
} 
fuente suiza SINQ, por lo que su empleo debe ser estudiado con detalle.

En lo referente a la temperatura del refrigerante, la concentración del haz llevaría a la cara en contacto con el agua a superar su temperatura de ebullición, $152^{\circ} \mathrm{C}$, en pocos pulsos prácticamente para cualquier reducción del radio del haz inferior a $4 \mathrm{~cm}$. Sin embargo, dicha ebullición sería muy local e inmersa en un flujo de refrigerante subenfriado, por lo que no se considera perjudicial a corto plazo para el blanco. Cabe resaltar que, de modo conservador, no se ha tenido en cuenta el incremento de transmisión de calor derivado de la ebullición, por lo que las temperaturas obtenidas son mayorantes de las reales; al menos hasta pasados los primeros pulsos, tras los que la ebullición en película podría degradar las condiciones de refrigeración. Temperaturas más precisas podrían conseguirse efectuando una simulación multifase transitoria tridimensional, pero dado el coste computacional y la inherente incertidumbre asociada, no se considera justificado para el fin que busca este análisis.

Así pues, como conclusión podemos extraer que el accidente de concentración del haz supone un riesgo significativo para la integridad del blanco, por lo que se debe contar con medidas adecuadas para su detección temprana. Como medidas de prevención, se recomienda la inclusión de sistemas de control de la forma del haz, y de motorización de temperatura de las placas, así como que se garantice un tamaño mínimo de haz de $3 \mathrm{~cm}$ de radio, mediante sistemas redundantes independientes y/o componentes pasivos. Como método para lograrlo, se propone hacer converger el haz en un pequeño orificio (pinhole) antes de su impacto en el blanco; de tal manera que, si el acelerador no opera con normalidad, gran parte del haz se pierda impactando con la estructura que alberga el orificio. En condiciones normales de operación, este orificio además puede servir de colimador, y protege el acelerador de la radiación proveniente del entorno del blanco, por lo que su inclusión en el diseño de la instalación debe considerarse.

\subsubsection{Bloqueo del giro del blanco}

La detención súbita y no detectada del movimiento de rotación del blanco sería el evento iniciador de esta secuencia accidental. En términos termomecánicos, la consecuencia de esta parada, considerando que el acelerador sigue funcionando con normalidad, implicaría que la frecuencia de llegada de los protones a la zona de impacto pasaría de $1 \mathrm{~Hz}$ a 20 
$H z$. Para realizar un análisis conservador de este accidente, se asume que la parada es instantánea.

A priori, la zona de impacto tras la detención del blanco sería aleatoria y desconocida, por lo que se estudiará el efecto del impacto del haz en el berilio y en la aleación de aluminio de la estructura. Para ello se consideran como casos extremos el impacto perfectamente alineado con la placa y totalmente enfocado en la estructura. La metodología e hipótesis de modelado empleadas para el cálculo son análogas a las descritas en la Sección 2.3.3.

Suponiendo que el disco se encuentra en operación normal antes del fallo, las Figuras 320 y 3-21 muestran los resultados de tensión y temperatura en el caso de impacto centrado en la placa de berilio. Dadas las condiciones de refrigeración homogéneas de la placa, estos resultados son indicativos también de las consecuencias de un impacto descentrado siempre que quede contenido dentro de la placa.

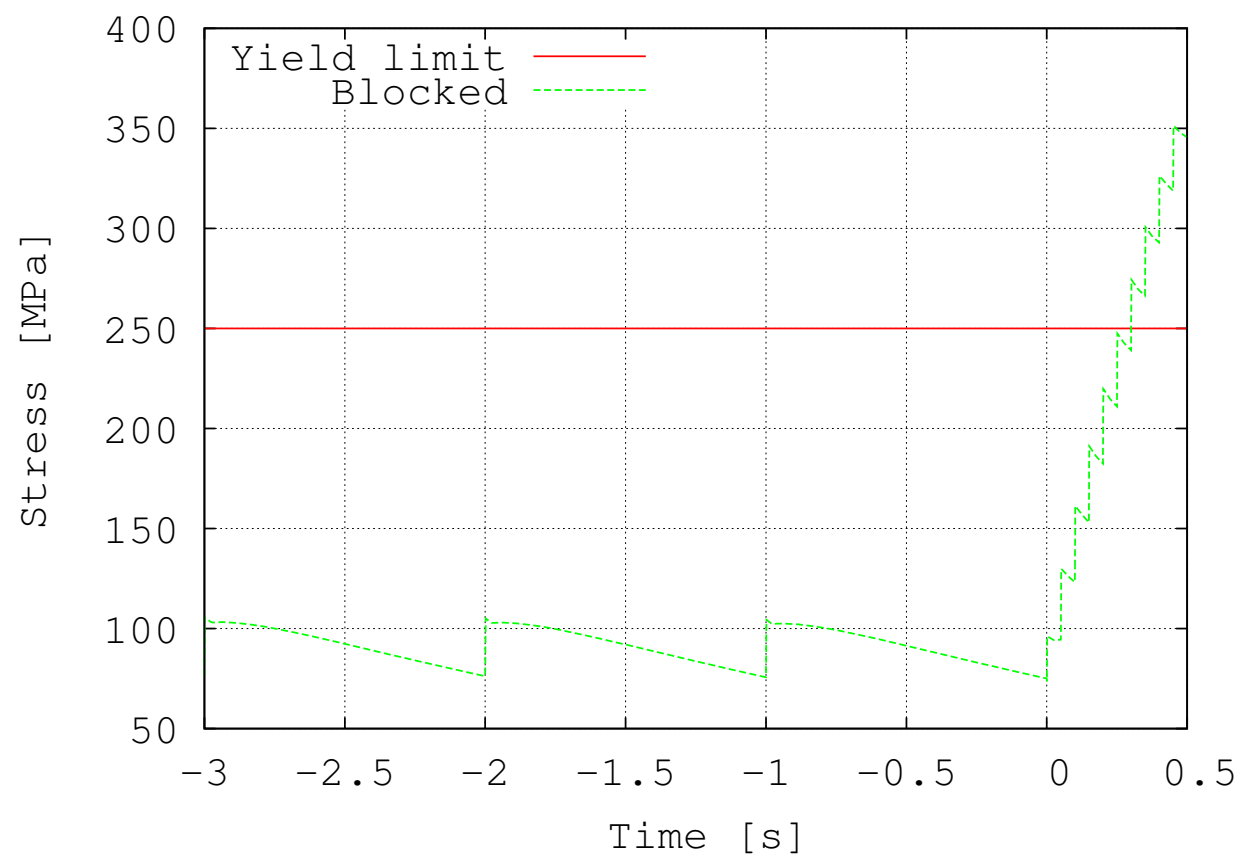

Figura 3-20: Evolución de la tensión de von Mises máxima [MPa] en la placa de berilio durante el accidente de bloqueo.

Los resultados muestran que el berilio alcanzaría el límite elástico tras el impacto de 6 pulsos del acelerador. Tomando una vez más ese valor de tensión como límite conservador de daño, la plastificación se produciría entonces a los 0,3 segundos del comienzo del accidente. La temperatura máxima del berilio alcanzaría la temperatura de ebullición 


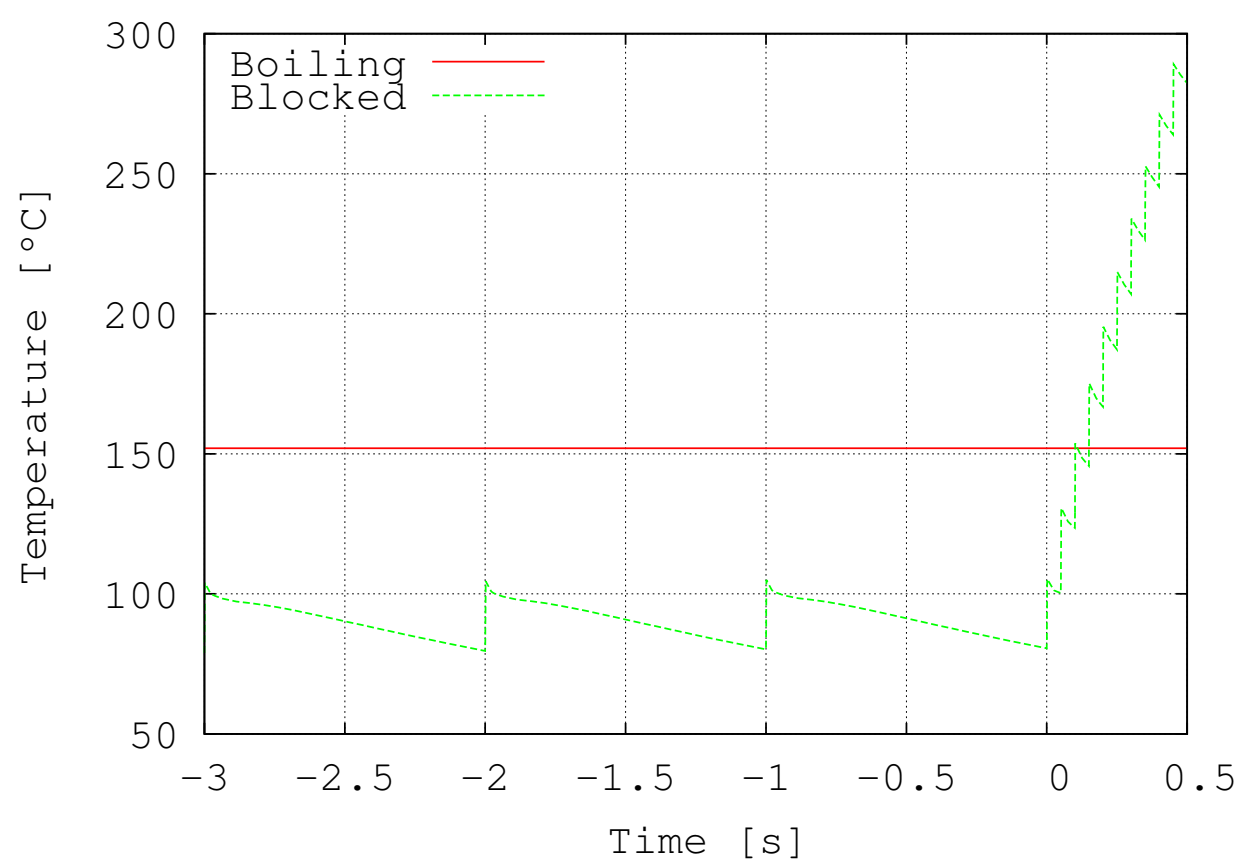

Figura 3-21: Evolución de la temperatura máxima $\left[{ }^{\circ} \mathrm{C}\right]$ en la placa de berilio durante el accidente de bloqueo.

del refrigerante a los 0,15 segundos, aunque siguiendo el mismo razonamiento que en la Sección 3.4.1 (ebullición local subenfriada y temperaturas mayoradas) esto no se considera alarmante en el corto plazo.

De nuevo, dado el carácter local del valor de tensión máxima calculado, el fallo total de la placa requeriría de más tiempo que el estimado hasta plastificación, aunque la rápida evolución de la tensión no concedería mucho margen hasta la aparición de daño. Por tanto, de modo conservador se toma $0,3 s$ como tiempo máximo necesario para la detección del fallo y la parada del acelerador.

Con el fin de presentar una primera evaluación del efecto del impacto del haz de protones en la estructura, se estudia, bajo las mismas hipótesis de cálculo, el comportamiento termomecánico de una placa de aluminio 6061-T6. Puesto que la geometría de la estructura del blanco real es compleja, el punto de impacto puede presentar diversas topologías. Se analiza, por tanto, el comportamiento de dos placas de dicha aleación de $5 \mathrm{~mm}$ y $5 \mathrm{~cm}$, como representativos de los valores extremos de espesor entre el punto de impacto y las superficies refrigeradas de la estructura. La temperatura inicial del material es de $40{ }^{\circ} \mathrm{C}$, como representativa de su temperatura normal de operación. 
Para llevar a cabo este estudio, se ha analizado con MCNPX la deposición de potencia del haz en el aluminio 6061-T6. La Figura 3-22 muestra los resultados obtenidos. Se observa que el alcance de los protones en este material es menor que en el berilio, apareciendo el pico de Bragg a una profundidad de $\sim 7 \mathrm{~mm}$ y alcanzándose una deposición máxima en ese punto de 184,7 kW/ $\mathrm{cm}^{3}$. Implementando esta distribución de deposición energética en el modelo de elementos finitos, las Figuras 3-23 y 3-24 muestran los resultados de tensión y temperaturas máximas obtenidos.

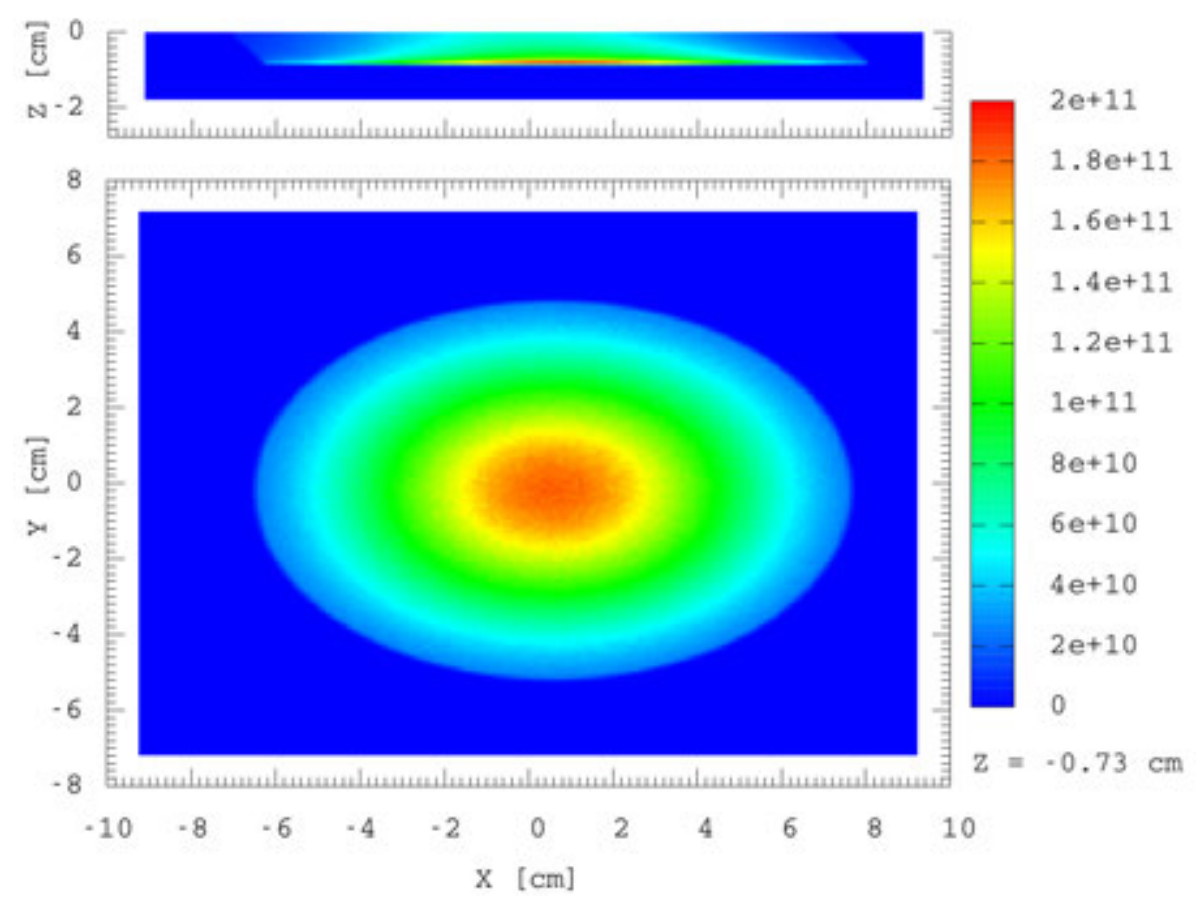

Figura 3-22: Deposición energética del haz de ESS-Bilbao en aluminio 6061-T6 [W/ $\mathrm{m}^{3}$ ]

Antes de analizar los resultados, es necesario recordar la peculiaridad de la aleación 6061-T6 introducida en la Sección 3.1. Este material mantiene el límite elástico alrededor de $250 \mathrm{MPa}$ hasta los $100{ }^{\circ} \mathrm{C}$, descendiendo hasta $215 \mathrm{MPa}$ a los $150{ }^{\circ} \mathrm{C}$, y hasta 100 $M P a$ a los $200{ }^{\circ} C$ [161]. Esta degradación se debe a la pérdida del estado templado y precipitado característico del tratamiento térmico T6.

Analizando los resultados obtenidos, observamos que el aluminio alcanzaría localmente los $200{ }^{\circ} \mathrm{C}$ en menos de 0,1 segundos para los dos espesores considerados. El límite elástico a esta temperatura, $100 M P a$, se alcanzaría prácticamente a la vez. Por tanto la deformación permanente a nivel local de la estructura, en este caso, parece muy difícil de evitar. 
Aunque es esperable que las zonas de la sección alejadas del punto de temperatura máxima conserven las propiedades mecánicas más tiempo, especialmente para el caso de $5 \mathrm{~cm}$ de espesor, la evolución del estado post-accidental de la estructura es difícil de predecir, dada la elevada deformación a rotura del material (18\% a temperatura ambiente y $28 \%$ a 200 $\left.{ }^{o} \mathrm{C}\right)$.

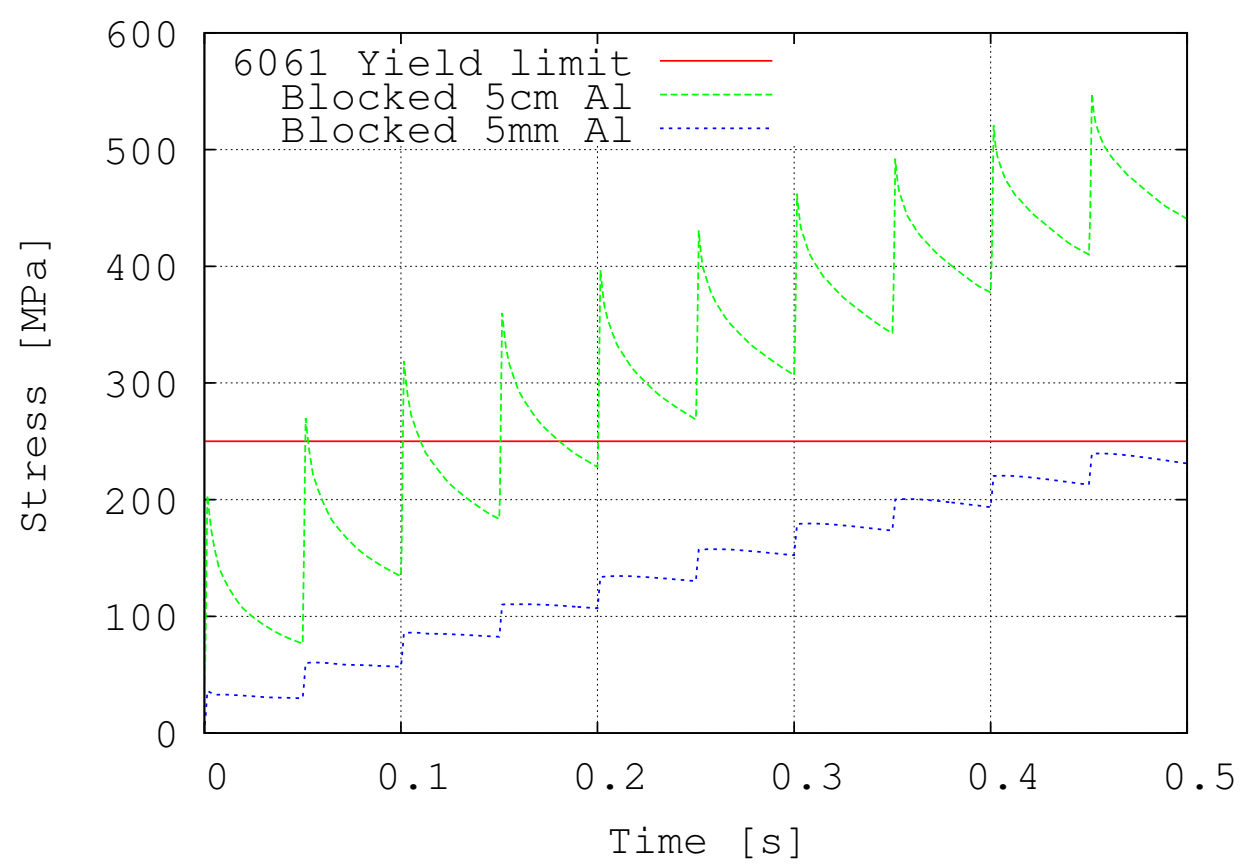

Figura 3-23: Evolución de la tensión de von Mises máxima [MPa] en el aluminio 6061T6 durante el accidente de bloqueo.

Afortunadamente, la posición del blanco es monitorizable de forma robusta a través de sensores, tanto en la propia estructura y en la cadena cinemática de transmisión de giro, como a través del estudio de los parámetros de operación del sistema motor; por tanto, el tiempo de detección de la falta de giro debería ser muy pequeño. Si de forma conservadora se asume que el mínimo desfase detectable en la posición del blanco es de $10^{\circ}$, la detección se produciría en menos de $\approx 0,03 s$ del cese del giro. Este tiempo es inferior al periodo del acelerador, 0,05 s, por lo que, en principio, el fallo sería detectado antes de la llegada de un nuevo pulso. El tiempo característico de reacción de las paradas de emergencia de las fuentes de espalación está en el orden de las decenas de $\mu s$ (20 $\mu s$ en el caso de SNS [177], $10 \mu s$ en el caso de J-PARC [178]). Teniendo este tiempo como referencia, es esperable que una vez detectado el fallo, ningún pulso impacte en el blanco. 


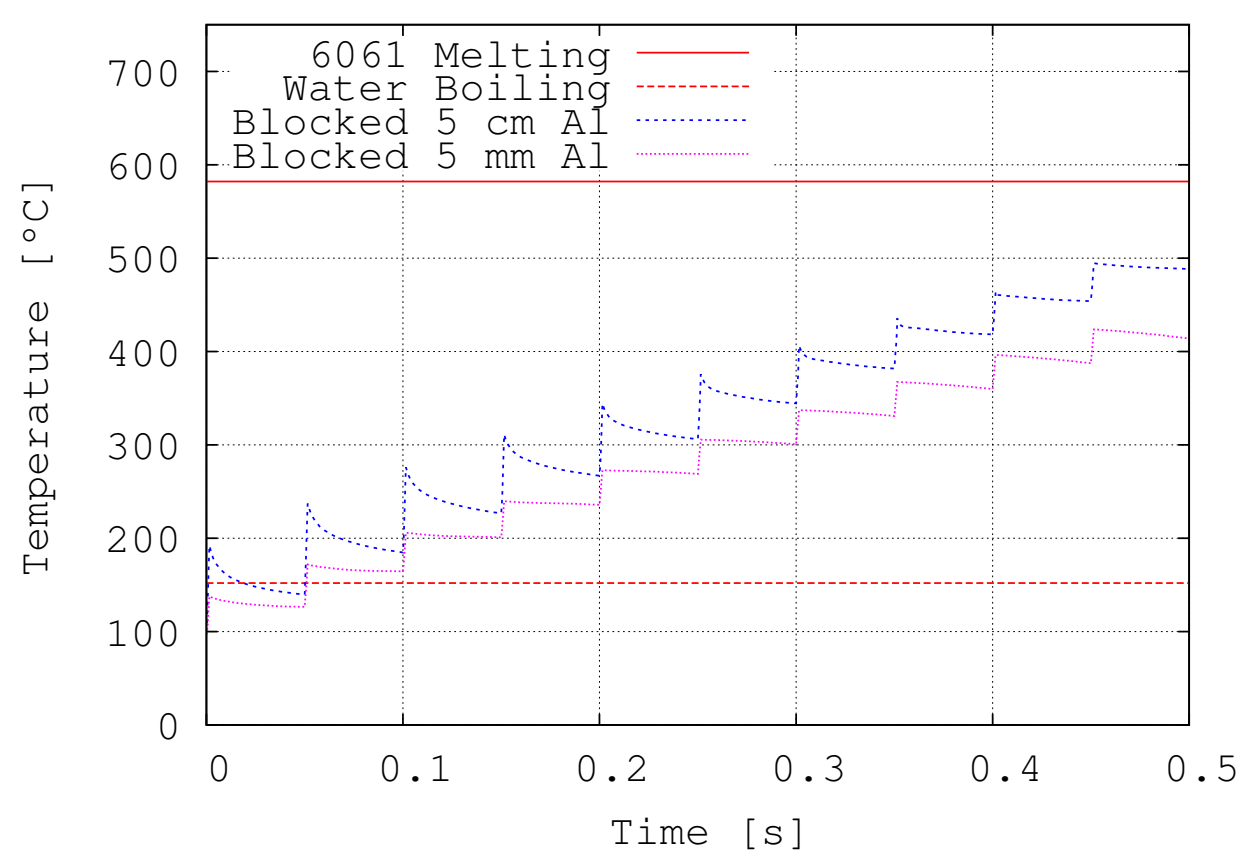

Figura 3-24: Evolución de la temperatura máxima $\left[{ }^{\circ} \mathrm{C}\right]$ en el aluminio 6061-T6 durante el accidente de bloqueo.

Así pues, como conclusión, podemos deducir que el accidente de bloqueo del giro de la estructura representa un riesgo considerable para ésta, en caso de pasar desapercibido. No obstante, su detección temprana parece factible. Como medida de prevención y mitigación se recomienda la permanente monitorización redundante de la posición y velocidad angular de la estructura del blanco, por los medios anteriormente citados.

Este análisis cubre también los efectos de un accidente análogo, en el que el acelerador entrase en funcionamiento sobre un blanco inmóvil por un fallo en la lógica de los enclavamientos de seguridad ${ }^{\mathrm{III}}$, permitiendo el disparo del acelerador sin que el blanco esté en rotación. El accidente de bloqueo del giro estudiado en esta sección sería una cota mayorante de esta posibilidad en términos de daño, ya que no sería sustancialmente diferente en términos de tensiones pero comenzaría con el blanco a temperatura ambiente.

Como comentario adicional, indicar que el disparo del acelerador sobre el blanco esta-

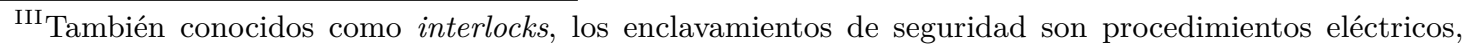
electrónicos o mecánicos que hacen el estado de dos mecanismos o funciones mutuamente dependientes. Se emplean para evitar estados anormales de la máquina, como por ejemplo, la generación de haz cuando la instalación no está lista.
} 
cionario y sin circulación del refrigerante sería considerablemente más dañino. No obstante, esta secuencia accidental requeriría el fallo múltiple de varios sistemas de seguridad, incluyendo también el fallo del sistema de monitorización del circuito refrigerante, lo que constituiría dos sucesos iniciadores independientes concomitantes. Por tanto, la posibilidad de este fallo se considera demasiado pequeña como para ser tenida en cuenta y, consecuentemente, más allá de la base de diseño.

\subsubsection{Desfase haz - blanco}

Otro posible modo de operación accidental es el desfase entre la frecuencia del haz y la posición del blanco, de tal manera que los protones no impacten en el centro de las placas sino en algún lugar intermedio. Este accidente vendría motivado por un fallo en el sistema de monitorización de la posición del blanco, o por un fallo en el enclavamiento de seguridad que confirma la coincidencia de fase entre el blanco y el acelerador.

Se considera que esta anomalía en el funcionamiento no afecta a la velocidad de rotación del blanco, por lo que la frecuencia de impacto en cada punto, $1 \mathrm{~Hz}$, no se ve modificada ${ }^{\mathrm{IV}}$.

Como se ha mencionado, dadas las condiciones de refrigeración homogéneas en el blanco, un ligero desfase con el haz no supone riesgo mecánico para las placas, siempre que la huella del haz quede contenida dentro de éstas.

Para estudiar el caso en el que el desfase hiciera impactar el haz totalmente en la estructura del blanco, se analizan una vez más dos placas de aluminio 6061-T6 de $5 \mathrm{~mm}$ y $5 \mathrm{~cm}$, como representativas de los valores extremos de espesor de la estructura. Las Figuras 3-26 y 3-25 muestran los resultados de tensión y temperatura máximos calculados.

Los resultados obtenidos muestran que la placa de $5 \mathrm{~mm}$ alanza un régimen pseudoestacionario con temperaturas inferiores a los $150{ }^{\circ} \mathrm{C}$ y tensiones máximas inferiores a los $60 M P a$, por lo que no aparecería plastificación. En el caso de la placa de $5 \mathrm{~cm}$ la temperatura máxima se estabilizaría en torno a $\operatorname{los} 225^{\circ} \mathrm{C}$, por lo que la aleación de aluminio se vería afectada por la degradación de propiedades, consecuente a la pérdida del estado templado y precipitado. En dos pulsos, la temperatura máxima alcanzaría los $150{ }^{\circ} \mathrm{C}$ y la

\footnotetext{
${ }^{\mathrm{IV}} \mathrm{El}$ accidente de bloqueo del giro previamente estudiado describiría el caso mayorante de los accidentes por variación de frecuencia en el impacto.
} 


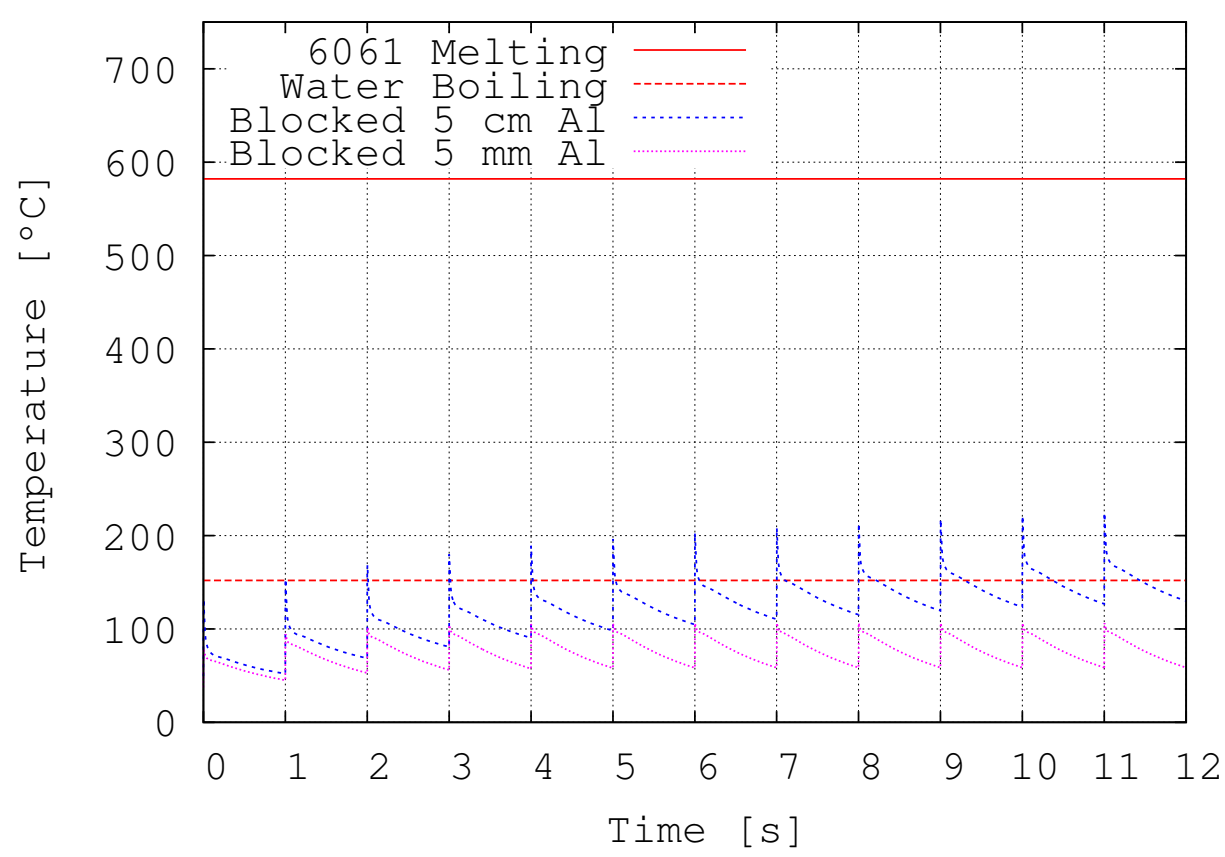

Figura 3-25: Evolución de la temperatura máxima $\left[{ }^{\circ} \mathrm{C}\right]$ en el aluminio 6061-T6 por el impacto del haz.

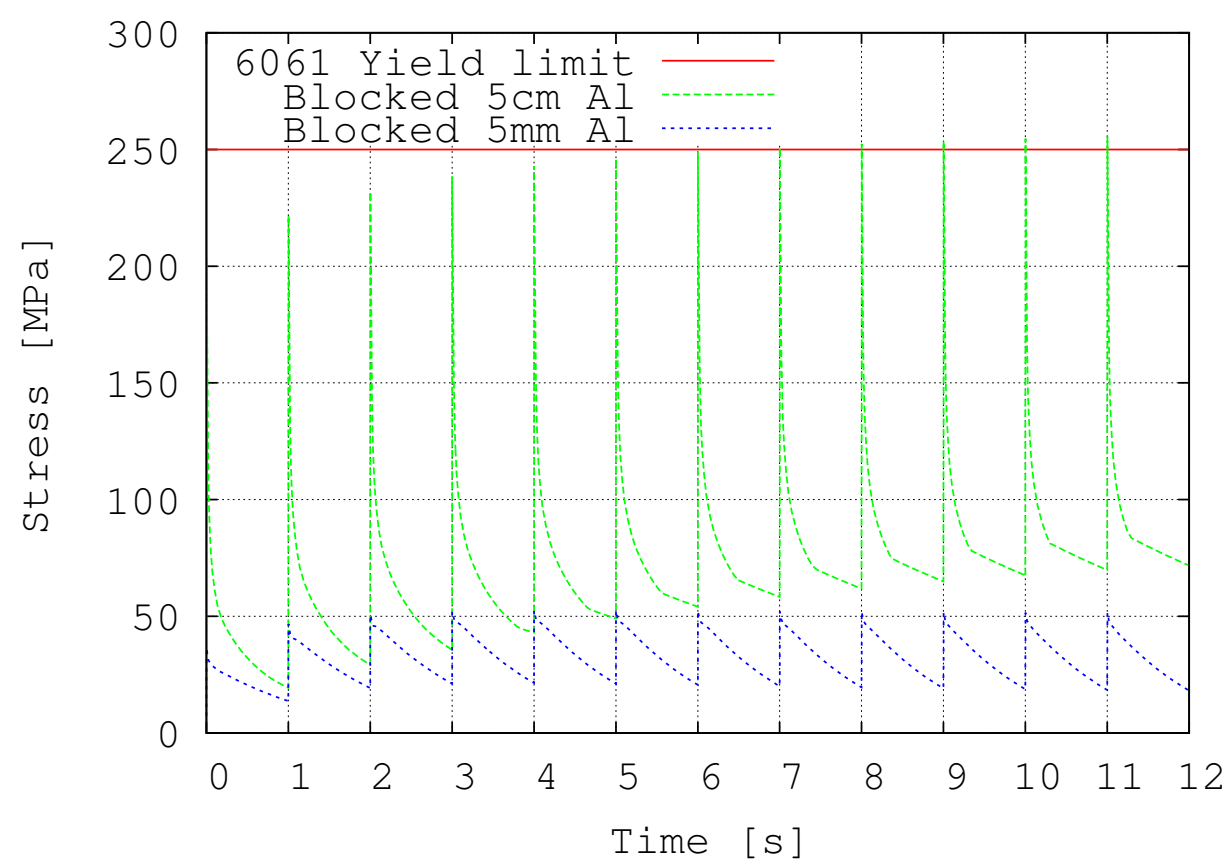

Figura 3-26: Evolución de la tensión de von Mises máxima [MPa] en el aluminio 6061T6 por el impacto del haz. 
tensión máxima sobrepasaría los 215 MPa del límite elástico a esa temperatura, por lo que la plastificación local comenzaría a ocurrir. Aunque es esperable que las zonas de la sección alejadas del punto de temperatura máxima conserven las propiedades mecánicas más tiempo, podemos concluir que el blanco puede recibir un pulso de haz sin consecuencias.

En caso de que el desfase provocara el impacto en una zona intermedia entre las placas y la estructura, las consecuencias termomecánicas estarían comprendidas entre los extremos estudiados, salvo quizás para el caso particular de los efectos del haz en las juntas de estanqueidad. Este caso requeriría de un estudio pormenorizado una vez definida la junta de estanqueidad.

$\mathrm{Al}$ igual que el accidente de bloqueo, este evento puede suponer un problema para el blanco. Sin embargo, dada su dinámica menos agresiva, la monitorización redundante de la posición de la estructura antes recomendada, apoyada por el control térmico de las placas y de la estructura por termografía y/o termopares, debería proporcionar tiempos de reacción suficientes para que este accidente no cause daño. Por otro lado, también es previsible que a partir de determinado desfase la producción neutrónica se vea disminuida de forma sensible, lo que daría una indicación adicional del mal funcionamiento.

\subsubsection{Accidente de pérdida de flujo}

En esta sección analizaremos las consecuencias para el blanco de una reducción en el caudal de refrigerante. Este tipo de accidente puede ser la consecuencia de una parada de la bomba del circuito de refrigeración, la obstrucción total o parcial de los canales, o de un accidente de pérdida de refrigerante en algún punto del circuito.

Para llevar a cabo una evaluación del margen de seguridad respecto a los efectos de este accidente, se efectúa un análisis paramétrico de las tensiones y temperaturas máximas en el blanco ante velocidades decrecientes de refrigerante. La velocidad de referencia del refrigerante en condiciones normales es de $2.25 \mathrm{~m} / \mathrm{s}$, lo cual conlleva un coeficiente de transmisión de calor de $\approx 10^{4} \mathrm{~W} / \mathrm{m}^{2} \mathrm{~K}$. Empleando las correlaciones adecuadas, se calcula el coeficiente de transmisión de calor para una velocidad de refrigerante de 2.0, 1.5, 1.0, $0.5,0.25 \mathrm{y} \approx 0 \mathrm{~m} / \mathrm{s}$.

Para las cinco primeras velocidades se emplea la correlación de Dittus-Boelter (Ecua- 
ción 3.1), adecuada para flujo turbulento en canales. Para calcular el coeficiente de transmisión de calor cuando la velocidad del refrigerante se aproxima a cero, es necesario tener en cuenta dos efectos simultáneos. Por una parte, al bajar el número de Reynolds por debajo de 2300, el flujo fluido se vuelve laminar. Por otra, al irse deteniendo el refrigerante, la convección natural empieza a contribuir al coeficiente de transmisión de calor. Los efectos de ambas contribuciones combinadas pueden estimarse en primera aproximación aplicando la correlación 3.7 [121], donde $N u_{N}$ y $N u_{F}$ son los números de Nusselt calculados para convección natural y forzada respectivamente, y el signo a aplicar depende de la dirección de las corrientes convectivas. Si éstas se desarrollan a favor o en dirección transversal a la convección forzada, las contribuciones se suman. Si se desarrollan en dirección contraria, la contribución de la convección natural se sustrae.

$$
N u^{3}=N u_{F}^{3} \pm N u_{N}^{3}
$$

Sin embargo, en la configuración que nos ocupa, las corrientes de convección natural no cuentan con espacio suficiente en los canales para desarrollarse totalmente dada su estrechez y, al encontrarse el disco en rotación, su dirección relativa a la convección forzada es alternante, por lo que su contribución total se estima poco relevante. Por tanto, se considera que la transmisión de calor esperable ante una parada prácticamente total del fluido puede ser estimada en primera aproximación mediante el bajo coeficiente de transmisión que produce el flujo laminar, al menos hasta que la ebullición aparezca en el canal.

Según lo expuesto en [179], en el caso de flujo laminar en canales, el número de Nusselt únicamente depende de la geometría del canal y de si se considera que la transmisión de calor se produce a temperatura superficial constante, o a flujo de calor constante. Los canales que nos ocupan tienen una elevada relación de aspecto, sólo son calentados por un lado y, dado que la energía implantada en cada momento viene definida por el haz de protones, podemos considerar que el flujo de calor es la variable impuesta y, por tanto, que aplica el caso de transmisión de calor a flujo constante. De acuerdo a la Tabla 3.1 [179], el número de Nusselt esperable en los canales del blanco es de 5.39.

Las Figuras 3-27 y 3-28 muestran respectivamente los resultados de temperatura y tensión de von Mises máxima calculados para las distintas velocidades de refrigerante. Las 


\begin{tabular}{|c|c|c|c|}
\hline \multirow[b]{2}{*}{ Section } & \multirow[b]{2}{*}{$b / a$} & \multicolumn{2}{|c|}{$N u_{D}$} \\
\hline & & Constant $q$ & Constant $T$ \\
\hline & - & 4.36 & 3.66 \\
\hline & 1.0 & 3.61 & 2.98 \\
\hline$b$ & 1.43 & 3.73 & 3.08 \\
\hline$a$ & 2.0 & 4.12 & 3.39 \\
\hline$a$ & 3.0 & 4.79 & 3.96 \\
\hline$a[$ & 4.0 & 5.33 & 4.44 \\
\hline $\bar{b}$ & 8.0 & 6.49 & 5.60 \\
\hline & $\infty$ & 8.23 & 7.54 \\
\hline $\begin{array}{l}\text { Heated } \\
\text { Insulated }\end{array}$ & $\infty$ & 5.39 & 4.86 \\
\hline$\triangle$ & - & 3.11 & 2.49 \\
\hline
\end{tabular}

TABla 3.1: Números de Nusselt para flujo laminar en tubos de diversa sección transversal [179].

secuencias accidentales calculadas parten desde operación normal apareciendo la disminución súbita del caudal en el tiempo cero de la simulación.

\begin{tabular}{|c|c|c|c|c|c|c|}
\hline $\begin{array}{c}\text { Velocidad } \\
\text { refrigerante } \\
{[\boldsymbol{m} / \boldsymbol{s}]}\end{array}$ & $\mathbf{R e}$ & $\mathbf{h}$ & $\begin{array}{c}\boldsymbol{T}_{\max } \text { en } \\
\mathbf{t = 1 0} \mathbf{~ s} \\
{[-]}\end{array}$ & $\begin{array}{c}\text { Tiempo hasta } \\
\text { ebullición } \\
{[\boldsymbol{s}]}\end{array}$ & $\begin{array}{c}\boldsymbol{\sigma}_{\max } \text { en } \\
\mathbf{t = 1 0} \mathbf{~ s} \\
{[\boldsymbol{M P a}]}\end{array}$ & $\begin{array}{c}\text { Tiempo hasta } \\
\text { plastificación } \\
{[\boldsymbol{s}]}\end{array}$ \\
\hline 2.25 & 21456.2 & 10000.0 & 106.3 & $\infty$ & 105.4 & $\infty$ \\
\hline 2.00 & 19047.6 & 8249.3 & 114.4 & $\infty$ & 110.6 & $\infty$ \\
\hline 1.50 & 14285.7 & 6553.4 & 124.8 & $\infty$ & 125.0 & $\infty$ \\
\hline 1.00 & 9523.8 & 4738.0 & 140.0 & $>10.0$ & 148.0 & $>10.0$ \\
\hline 0.50 & 4761.9 & 2721.2 & $>152.0$ & 6.0 & 186.8 & $>10.0$ \\
\hline 0.25 & 2381.0 & 1562.9 & $>152.0$ & 4.0 & 218.3 & $>10.0$ \\
\hline$\sim 0,0$ & $<2300$ & 334.5 & $>152.0$ & 3.0 & $>250.0$ & 8.0 \\
\hline
\end{tabular}

TABla 3.2: Análisis de temperaturas y tensiones en caso de pérdida de flujo de refrigerante

Del estudio de los resultados obtenidos, recopilados en la Tabla 3.2, se desprenden dos conclusiones principales. Por un lado se observa que las condiciones nominales de refrigeración ofrecen márgenes suficientes respecto a los límites de seguridad establecidos, de tal forma que el diseño puede hacer frente de forma robusta a variaciones moderadas en 


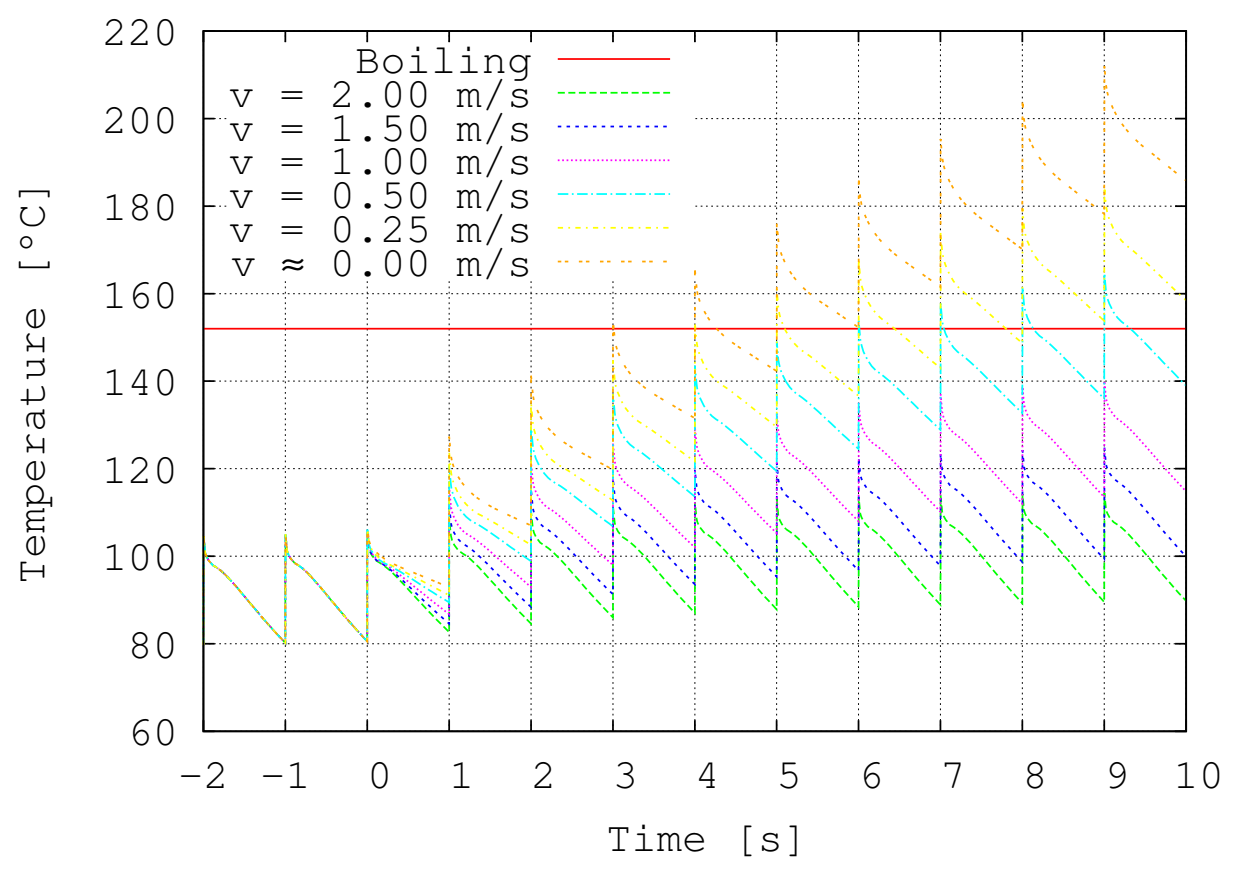

FiguRA 3-27: Evolución de la temperatura máxima en el Be en función de la velocidad del refrigerante.

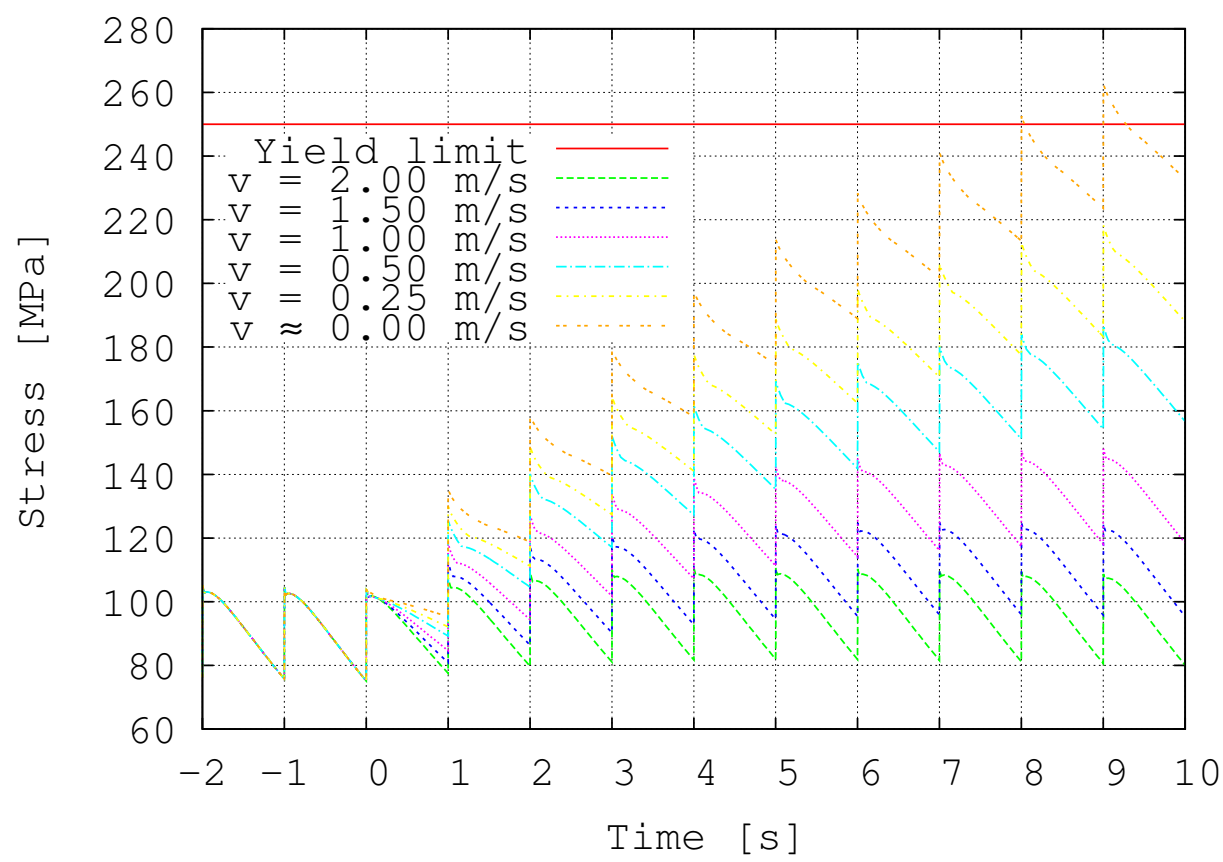

Figura 3-28: Evolución de la tensión máxima de von Mises en el Be en función de la velocidad del refrigerante. 
las hipótesis de refrigeración. La evolución de temperaturas y tensiones puede alcanzar un nuevo estado pseudo-estacionario, con unos valores máximos menores a los valores límite establecidos, siempre que la velocidad del refrigerante sea superior a los $1,5 \mathrm{~m} / \mathrm{s}$, y por ello, incluso si el coeficiente de transmisión de calor en los canales es un $\sim 35 \%$ inferior al considerado en el diseño.

Por otro lado se observa que la repuesta termomecánica de este accidente es relativamente lenta, por lo que ofrece un tiempo suficiente para la detección y actuación. De acuerdo a los resultados obtenidos, las placas únicamente comenzarían a sufrir plastificación local en menos de $10 s$ para el caso en el que el flujo se detuviera prácticamente en su totalidad. La temperatura máxima en la placa provocaría ebullición local subenfriada del refrigerante en menos de diez segundos si la velocidad del fluido cae por debajo de $1 \mathrm{~m} / \mathrm{s}$; mientras que en el caso de pérdida prácticamente total de flujo, la ebullición tardaría $3 \mathrm{~s}$ en aparecer.

Por tanto, se considera que, en caso de que la monitorización del circuito refrigerante fallara y no detectase una pérdida de caudal, la medición de temperatura por termografía y termopares puede proporcionar un tiempo de detección suficiente para detener el acelerador antes de que aparezca daño a las placas; por lo que no se recomienda ninguna medida de prevención o mitigación adicional, a parte de la monitorización redundante del caudal y del estado de la bomba impulsora.

\subsubsection{Accidente de pérdida de vacío}

Como configuración de referencia se prevé que el blanco opere en vacío, compartiendo el mismo entorno que el acelerador. La motivación fundamental para elegir esta opción de diseño es la dificultad para introducir una ventana entre ambas zonas, ya que dicha ventana debería soportar la diferencia de presión y el impacto de los protones sin alterar significativamente el haz. Dadas las características de éste, la ventana debería ser extremadamente fina, lo cual reduciría su resistencia mecánica, y aún de este modo la carga térmica y el daño al material no serían despreciables.

Como solución alternativa se opta por la inclusión de un obturador rápido entre el ambiente del acelerador y del blanco. Estos dispositivos, diseñados para la protección de aceleradores, son capaces de aislar la línea del acelerador en menos de $10 \mathrm{~ms}$ por medio 
de un actuador neumático. La detección de la pérdida de vacío puede lograrse mediante la inclusión de un sensor de bajas presiones de cátodo caliente o de descarga, capaz de proporcionar la señal al obturador en menos de $2 \mathrm{~ms}$. Modelos de ambos componentes, obturador y sensor, capaces de trabajar bajo irradiación están comercialmente disponibles [180]. En una primera instancia, se podría considerar el posicionamiento del sensor próximo al blanco y la colocación del obturador al final de la línea del acelerador, fuera del blindaje del blanco, lo cual proporcionaría tiempo suficiente para que la detección y la actuación tuvieran lugar antes de que el acelerador pudiera sufrir daños. La fuente de neutrones LENS cuenta con este tipo de protección, donde ha actuado con éxito ante pérdidas de refrigerante de su blanco [139].

Para garantizar un nivel de vacío adecuado en el acelerador, se prevé la instalación de un esquema de vacío diferencial que cuente con bombas de vacío operando en las cercanías del blanco. De esta manera se evita que pequeñas fugas de los sellos de estanqueidad o la desorción de materiales volátiles alteren el vacío del acelerador.

La rotura del vacío en el entorno del blanco puede venir provocada por el fallo de alguno de los elementos que forman la barrera de vacío o por la pérdida accidental de refrigerante, ya sea por un fallo de los elementos que garantizan su estanqueidad, o por alguna situación accidental previa que conlleve el fallo de las placas de berilio. Este tipo de accidentes vienen acompañados del peligro de dispersión de contaminantes o productos de corrosión activados.

Para hacer frente a este riesgo, diversas medidas de mitigación pueden ser consideradas. Con el objetivo de minimizar la actividad radiactiva en el refrigerante, el circuito debe estar dotado de filtros para la retención de productos de corrosión. También resulta recomendable la inclusión en el circuito de un tanque de decaimiento tras el paso por el blanco, de tal modo que el tiempo de residencia del fluido entre pasos por el blanco sea mayor. De esta forma se da tiempo al decaimiento de los isótopos radiactivos de vida corta que pudieran producirse, y se palía el incremento del inventario radiactivo. Este tanque también favorecería la recombinación de los iones producidos por radiólisis, disminuyendo la corrosión (como se discutió en la Sección 2.3.4.4). Por último, el entorno próximo a la barrera de vacío del blanco debe estar sometido a subpresión mediante un sistema de control ambiental y contar con un sistema de drenaje adecuado. Ambos sistemas deben 
estar diseñados para ser capaces de gestionar efluentes radiactivos con un caudal y nivel de actividad suficiente.

\subsubsection{Reacciones químicas a alta temperatura}

Como se mencionó en la Sección 2.2, el berilio presenta de forma natural una fina capa de óxido (BeO). Dicha capa es muy estable y compacta, por lo que evita la difusión del oxígeno hacia el interior del material y por tanto la propagación de la reacción de oxidación. No obstante, a altas temperaturas la reacción de oxidación se activa generando una liberación de energía que constituye un peligro para la integridad del blanco.

En presencia de aire la reacción de oxidación no es relevante hasta que el berilio llega a los $1000{ }^{\circ} \mathrm{C}$, temperatura a la que alcanza ignición formando $\mathrm{BeO}$ y nitruro de berilio $\left(\mathrm{Be}_{3} \mathrm{~N}_{2}\right)$ [181, 182]. La reacción es altamente exotérmica y autosostenida, por lo que se mantiene mientras queden reactivos. Esta reacción podría ser relevante en caso de que se produjera una pérdida de vacío a la vez que algún transitorio accidental que elevase la temperatura del blanco.

El berilio a alta temperatura también puede oxidarse con el vapor de agua generado en los canales de refrigerante. Esta reacción, además de exotérmica, tiene como peligro añadido la producción de hidrógeno. Dicho elemento, en la concentración adecuada, puede recombinarse con el oxígeno de manera explosiva. Con el objetivo de analizar la importancia de este fenómeno en reactores de fusión, donde se contempla el empleo de berilio como material de primera pared frente al plasma, el trabajo recogido en [183] presenta resultados experimentales de tasa de reacción de berilio macizo en contacto con vapor de agua. Las Ecuaciones 3.8 y 3.9 describen dicha tasa de reacción en $\mathrm{kg} / \mathrm{m}^{2} \mathrm{~s}$ en función de la temperatura del berilio en $K$.

$$
\begin{array}{ll}
R_{o x}=1,934 \cdot 10^{6} \exp (-25850 / T) & T<1175 K \\
R_{O x}=31,837 \exp (-12830 / T) & T \geq 1175 K
\end{array}
$$

Como se puede observar en la Figura 3-29, la tasa de reacción permanece despreciable, i.e. inferior a $10^{-4} \mathrm{~kg} / \mathrm{m}^{2} \mathrm{~s}$, hasta que la temperatura del berilio alcanza los $\approx 800{ }^{\circ} \mathrm{C}$. 


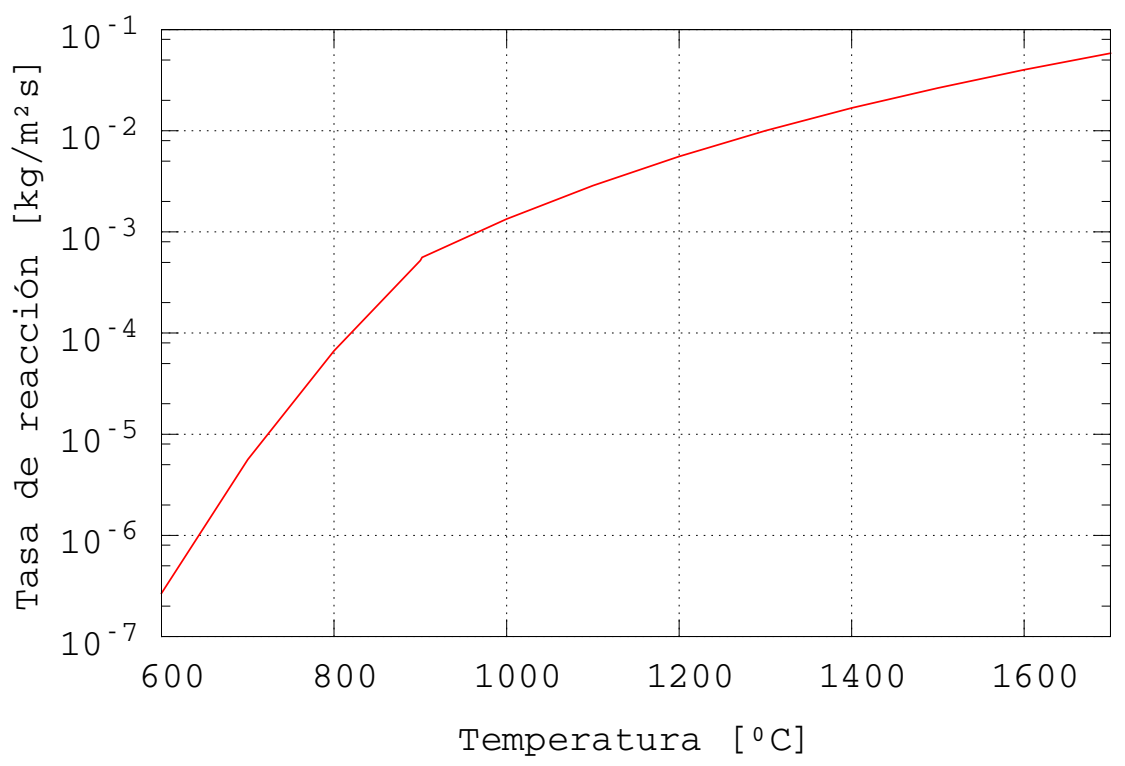

Figura 3-29: Tasa de reacción del berilio macizo en contacto con vapor de agua en función de la temperatura [183]

Puesto que las temperaturas normales de operación del berilio son inferiores a los 110 ${ }^{o} C$, y que éste presenta una potencia térmica residual prácticamente nula, el aumento no detectado de su temperatura hasta niveles en los que la oxidación sea relevante parece muy poco probable. No obstante, como se ha visto en las secciones anteriores, algunas secuencias accidentales sí son capaces de aumentar la temperatura del berilio de forma rápida, por lo que los métodos de detección y mitigación propuestos para esas secuencias son fundamentales para evitar este riesgo.

\subsubsection{Energía de los protones anormalmente baja}

Como se decidió en el Capítulo 2, las placas del blanco están diseñadas para permitir que la mayoría de los protones pasen a través de ellas, dejando al pico de Bragg fuera del berilio e impidiendo la mayor parte de la implantación. Si el acelerador proporcionara de forma inadvertida un haz de protones de energía anormalmente inferior, esto implicaría un aumento de la implantación o incluso la implantación total de los protones en el berilio. La acumulación de estos en forma de hidrógeno puede acabar generando el fallo del blanco, como se ha demostrado en el diseño inicial de la fuente LENS, cuyo primer diseño estaba concebido para detener los protones totalmente [139]. 
Para hacer una evaluación del margen de seguridad disponible respecto a este accidente, estimamos el tiempo de operación en el que la baja energía de los protones debería pasar inadvertida para producir fallo. Para hacer dicha estimación, tomamos como referencia el fallo del blanco de berilio de la fuente LENS reportado en [139]. Dicho fallo apareció tras el impacto y total implantación de $670 \mathrm{~kW} \cdot h$ de haz de protones de $13 \mathrm{MeV}$. Lo cual significa que el fallo se dio tras la implantación de $1,16 \cdot 10^{21}$ protones. Ya que el acelerador propuesto para ESS-Bilbao tiene una corriente media de 2,25 $\mathrm{mA}$, cada una de las 20 placas del blanco recibe $7,02 \cdot 10^{14}$ protones por segundo. Asumiendo que la energía de estos fuera suficientemente baja como para que la implantación del haz fuese total, la cantidad de protones tomada como límite para el fallo se alcanzaría tras $\sim 460$ horas de operación.

Cabe destacar que en la adopción de la implantación en LENS antes de fallo como límite para el fallo del blanco de ESS-Bilbao, subyacen dos hipótesis. La primera, que la velocidad de difusión del hidrógeno es similar en ambas fuentes, lo cual es aceptable dado el rango similar de temperaturas de operación; y la segunda, que la capacidad mecánica de ambas placas para soportar la implantación es análoga. Esta ultima hipótesis puede no ser conservadora, pero dado que el espesor del blanco de berilio cuyo fallo se ha usado de referencia era de $4 \mathrm{~mm}$, y que el de la propuesta para ESS-Bilbao es de $9 \mathrm{~mm}$, a igualdad de presión diferencial, es probable que sus resistencias mecánicas bajo implantación sean como poco similares, si no superior en el caso de la placa de $9 \mathrm{~mm}$.

También se debe mencionar que se ha asumido de manera conservadora que el acelerador es capaz de transmitir correctamente la totalidad de la corriente del haz, aunque los protones no alcancen la energía prevista, lo cual no está garantizado.

En cualquier caso, considerando las incertidumbres asociadas, el número de horas estimado para alcanzar el fallo se considera suficiente como para que el incorrecto funcionamiento del acelerador sea detectado por medio de la monitorización rutinaria de sus variables de operación. Por tanto, el margen de seguridad sería suficiente y no se recomiendan mayores medidas de prevención que las ya citadas. 


\section{Conclusiones del capítulo}

Los análisis realizados confirman que la estructura diseñada para materializar el concepto de blanco de ESS-Bilbao es capaz de proporcionar a las placas de berilio las condiciones de refrigeración necesarias para su operación.

El cálculo termohidráulico valida el diseño del circuito de refrigeración establecido en la estructura, así como las hipótesis realizadas en el Capítulo 2 sobre el coeficiente de convección necesario. El caudal estimado es capaz de hacer frente a la potencia implementada por el haz ampliamente, manteniendo las velocidades de refrigerante dentro de valores adecuados.

El cálculo termomecánico confirma que la estructura diseñada es capaz de soportar las cargas generadas fundamentalmente por la presión del refrigerante, y mantener la estanqueidad del circuito con un nivel de tensión adecuado, a la vez que presenta muy poco material en la trayectoria de los neutrones. El Apéndice A recoge la evolución del diseño de la estructura hasta la configuración final presentada en este capítulo.

Se ha analizado el comportamiento del blanco frente a sucesos accidentales con el objetivo de evaluar los márgenes de seguridad del diseño, detectar posibles puntos débiles en éste, e identificar medidas de prevención y mitigación. Los resultados muestran unos márgenes de seguridad razonables para todos los accidentes estudiados. De entre éstos, destaca el accidente de concentración excesiva del haz, que, por su dificultad para ser detectado y su impacto sobre el blanco, parece el más relevante. En cualquier caso, el diseño del blanco no presenta ningún fallo súbito desencadenado por un accidente que no pueda ser controlado con unas medidas estándar de monitorización y control.

Por todo lo anteriormente expuesto, se concluye que la estructura propuesta para el blanco de ESS-Bilbao cumple su función con solvencia, y que el diseño presenta una robustez adecuada frente accidente. 


\section{Capítulo 4}

\section{Análisis de producción neutrónica}

\section{Resumen}

En los capítulos anteriores, se ha diseñado un blanco capaz de convertir el haz de protones propuesto para el acelerador de ESS-Bilbao, en un término fuente de neutrones de $8 \cdot 10^{4} \mathrm{n} / \mathrm{s}$. Se analizan y especifican a continuación los elementos necesarios para convertir esta producción neutrónica en flujos de neutrones útiles para experimentación.

En un primer lugar se escogerán y definirán los principales parámetros de diseño del moderador y del reflector. Con estos, se definirá una configuración de referencia, cuya producción neutrónica y rango de aplicación serán puestos en contexto con otras fuentes. También se analizarán diversas configuraciones avanzadas, con el fin de evaluar potenciales mejoras de la instalación, y por último se evaluará la capacidad de la fuente para dar servicio a distintas técnicas experimentales.

\subsection{Caracterización del término fuente neutrónico}

En el Capítulo 2, se seleccionó el berilio como material del blanco en base a su razón de conversión neutrónica, es decir, la relación de neutrones producidos por protón incidente. Para ello se empleó el código de cálculo Monte Carlo MCNPX v2.7e [104] y las librerías de secciones eficaces ENDF/B-VII.0 [114].

Para la elección de este método, la razón de conversión neutrónica calculada empleando 
las mencionadas librerías, y los modelos de cascada intranuclear Isabel [144] e INCL[143], se han comparado con los resultados experimentales presentados en [184]. Ambos modelos de cascada intranuclear se han empleado junto el modelo de desexcitación ABLA [147]. En el trabajo experimental [184], se midió la razón de conversión neutrónica del berilio para protones de entre 23 y $80 \mathrm{MeV}$, obteniéndose la correlación experimental descrita en la Ecuación 4.2. Esta correlación calcula los neutrones producidos por $\mu C$ de haz en función de la energía de los protones, $E_{p}$, en $\mathrm{MeV}$.

$$
\begin{array}{lr}
N\left(E_{p}\right)=3,42 \cdot 10^{8}\left(E_{p}[M e V]-1,87\right)^{2,05} & E_{p}<23 \mathrm{MeV}[36]^{\mathrm{I}} \\
N\left(E_{p}\right)=5,58 \cdot 10^{8}\left(E_{p}[\mathrm{MeV}]\right)^{1,69} & 23 \mathrm{MeV} \leq E_{p} \leq 80 \mathrm{MeV}[184]
\end{array}
$$

La Tabla 4.1 recoge los resultados obtenidos por los distintos métodos, y los compara en porcentaje con las medidas experimentales de [184] a 45, 55 y $65 \mathrm{MeV}$. Como puede observarse, tanto los modelos como la librería de secciones eficaces predicen la producción neutrónica con un margen de error inferior al $20 \%$. Para protones de $50 \mathrm{MeV}$, la librería de secciones eficaces ENDF/B-VII.0 es el método que más se aproxima a las medidas experimentales, por lo que se escoge como método de referencia para el cálculo de la producción neutrónica.

\begin{tabular}{|c|c|c|c|c|c|c|c|}
\hline E protón & Experimental [184] & \multicolumn{2}{|c|}{ Isabel } & \multicolumn{2}{|c|}{ INCL } & \multicolumn{2}{c|}{ ENDF } \\
\hline $\mathrm{MeV}$ & $\mathrm{n} / \mathrm{p}$ & $\mathrm{n} / \mathrm{p}$ & dif $\%$ & $\mathrm{n} / \mathrm{p}$ & dif $\%$ & $\mathrm{n} / \mathrm{p}$ & $\operatorname{dif} \%$ \\
\hline 45 & 0,056 & 0,062 & 10.7 & 0,066 & 17,9 & 0,064 & 14,3 \\
\hline 55 & 0,078 & 0,080 & 2.6 & 0,089 & 14,1 & 0,077 & $-1,2$ \\
\hline 65 & 0,104 & 0,103 & $-1,0$ & 0,116 & 11,5 & 0,104 & 0,0 \\
\hline
\end{tabular}

TABla 4.1: Razón de conversión neutrónica de la reacción ${ }^{9} B e(p, x n)$. Resultados numéricos comparados en el trabajo experimental. [184]

Empleando pues esta librería, la razón de conversión neutrón / protón se estima en 0,065 neutrones por protón incidente. Este valor concuerda con un $-2 \%$ de diferencia con

\footnotetext{
${ }^{\mathrm{I}}$ Por completitud se recoge esta expresión, calculada en [36] a partir de medidas experimentales reportadas en $[185,186,187,188]$.
} 
la correlación experimental obtenida en [184] (Ecuación 4.2). Para esta razón de conversión, la corriente del haz de protones de $50 \mathrm{MeV}$ de ESS-Bilbao generaría una tasa de producción neutrónica, o término fuente, de $9,13 \cdot 10^{14} \mathrm{n} / \mathrm{s}$. Teniendo en cuenta el proceso de optimización del espesor del blanco llevado a cabo en la Sección 2.3.3.1, la tasa de conversión neutrónica de un blanco de $9 \mathrm{~mm}$ de espesor es de $0,057 \mathrm{n} / \mathrm{p}$, por lo que el término fuente final sería de $8,00 \cdot 10^{14} \mathrm{n} / \mathrm{s}$. Este término fuente está entre uno y dos órdenes de magnitud por encima del de las fuentes compactas actualmente en operación.

Se procede ahora a estudiar la distribución angular de los neutrones producidos. Para ello se simula en MCNPX el impacto del haz de protones en un blanco de berilio, y se estudia el espectro del flujo de neutrones a un metro de distancia medido en distintos ángulos con respecto a la dirección del haz. La producción de neutrones es axisimétrica con respecto a la dirección del haz, por lo que los resultados obtenidos son independientes de la coordenada azimutal alrededor de este. La Figura 4-1 muestra el espectro de flujo de neutrones por protón incidente medido a distintos ángulos, siendo $0^{\circ}$ la dirección colineal con el haz y $90^{\circ}$ la dirección perpendicular a este.

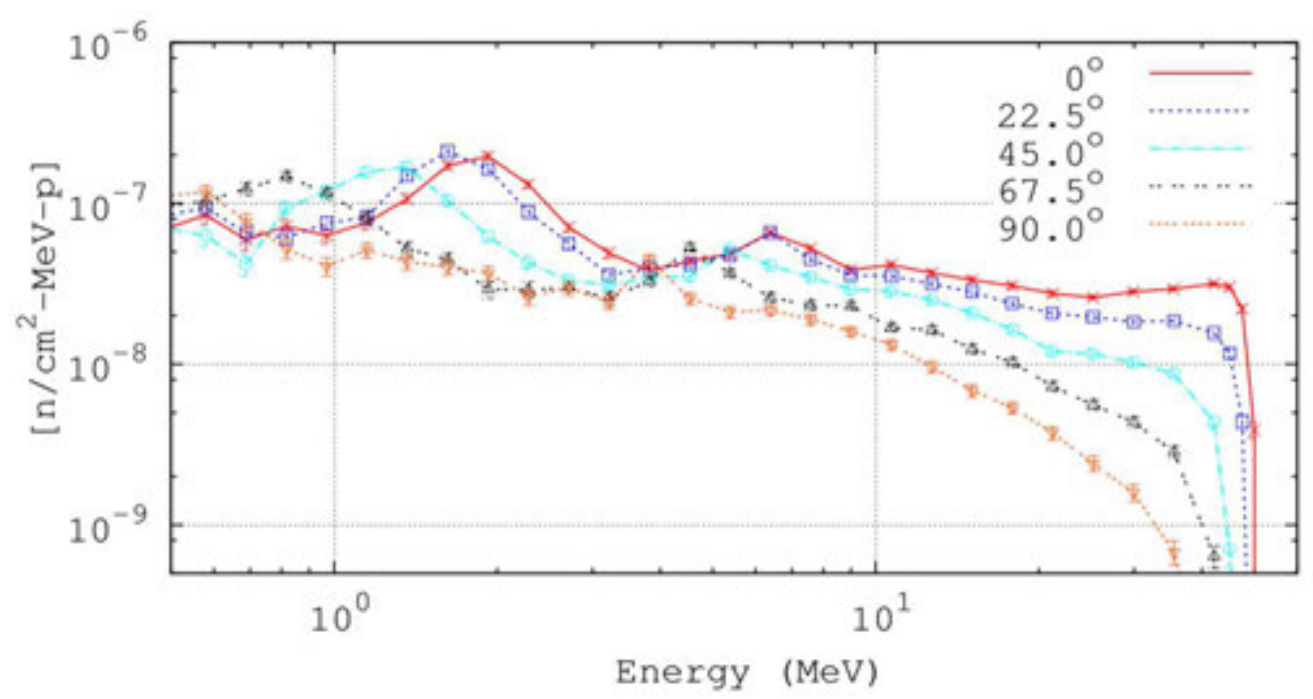

Figura 4-1: Espectro de flujo neutrónico en función de la posición angular con respecto al haz de protones, normalizado por protón incidente.

Dichos resultados muestran como la producción neutrónica es marcadamente anisótropa en cuanto a su distribución energética, ya que los neutrones rápidos se producen preferentemente en la dirección colineal al haz. Dicho comportamiento concuerda con los resultados experimentales presentados en [189] para reacciones ${ }^{9} B e(p, x n)$ con protones 
incidentes de energías próximas.

Esta anisotropía angular hace desaconsejable la ubicación de instrumentos para experimentación con neutrones térmicos y fríos en direcciones próximas a las del haz de protones, dado que requerirían mayor moderación e implicarían un mayor fondo de neutrones rápidos no deseados. En cambio, para ángulos de $45^{\circ}$ o mayores la reducción de neutrones rápidos es significativa. Para cuantificar este efecto, la Figura 4-2 muestra la razón entre el flujo en la posición colineal con el haz de protones y el flujo de neutrones a $45^{\circ}$. La reducción de neutrones rápidos es significativa para todos los valores de energía por encima de los $15 \mathrm{MeV}$. Cabe destacar que a $45^{\circ}$ se evita además la llegada de los neutrones más energéticos producidos.

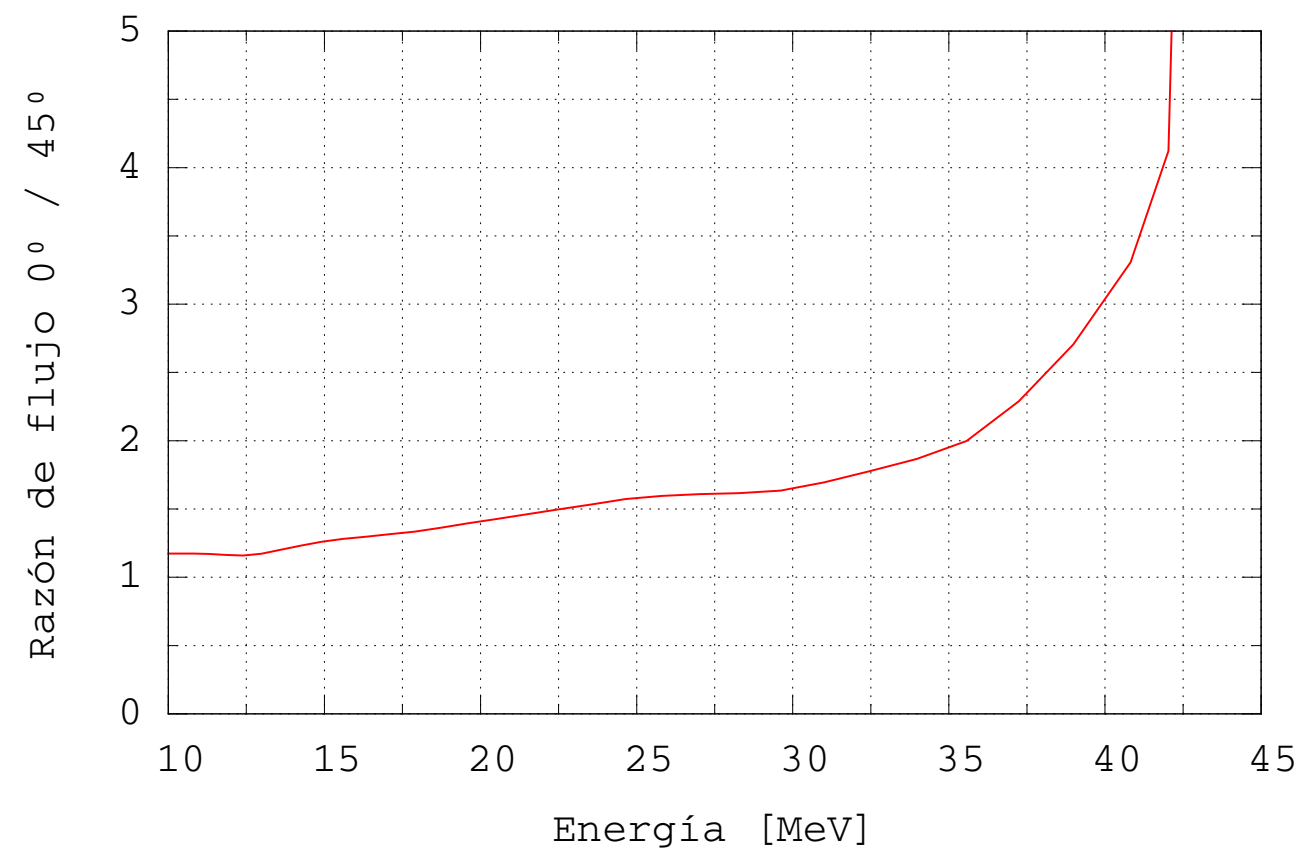

Figura 4-2: Razón entre el flujo en la dirección colineal con el haz de protones y el flujo neutrónico a $45^{\circ}$ en función de la energía de los neutrones.

\subsection{Configuración de referencia de la fuente de ESS-Bilbao}

Con el fin de facilitar la producción de neutrones fríos, resulta recomendable que el blanco se disponga respecto al haz de tal manera que las líneas de visión al blanco desde ángulos entre $45^{\circ}$ y $90^{\circ}$ estén disponibles. Este motivo, junto con la distribución superficial 
de la energía del haz (Capítulo 2), se ha tenido en cuenta para la elección del ángulo de impacto entre el haz y la superficie del blanco a $45^{\circ}$. De esta forma la estructura del blanco queda a $135^{\circ}$ de la dirección de salida de los neutrones, por lo que apenas se interpone en su trayectoria, y las líneas de visión desde ángulos entre $45^{\circ}$ y $90^{\circ}$ quedan disponibles a ambos lados del blanco (Figura 4-3).

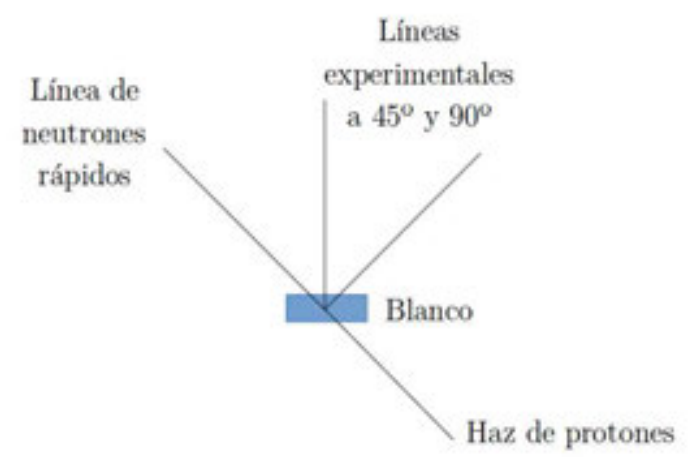

Figura 4-3: Disposición del blanco, el haz de protones y las líneas experimentales.

Conforme a esta disposición, la configuración de referencia elegida para las líneas de experimentación de la fuente de ESS-Bilbao consta de dos líneas para experimentación con neutrones fríos a $45^{\circ}$ y $90^{\circ}$ del haz respectivamente y una línea para experimentación con neutrones rápidos colineal con el haz. Las líneas de neutrones fríos convergen en el centro de un moderador situado detrás del blanco rotatorio, mientras que la línea de neutrones rápidos está enfocada en el punto de impacto del haz. El blanco y el moderador están rodeados por el reflector, y este a su vez por diversos blindajes conformando la estación del blanco. En las siguientes secciones se definen los principales parámetros de diseño de estos componentes.

\subsubsection{Moderador}

\subsubsection{Elección de material, configuración y dinámica}

El metano sólido $\left(\mathrm{CH}_{4}\right)$, sometido a temperaturas criogénicas para alcanzar dicho estado, es el mejor moderador posible para la producción de neutrones fríos [190]. Así lo avalan los resultados experimentales descritos en [191, 192, 193]. Esta propiedad se debe 
fundamentalmente a su alta concentración de hidrógeno ${ }^{\mathrm{II}}$ (Tabla 4.2), pero sobre todo, a los modos de vibración de baja frecuencia que presentan sus moléculas, cuya excitación aumenta su capacidad moderadora en el rango bajo de energías.

\begin{tabular}{|c|c|c|c|}
\hline Material & $\begin{array}{c}\text { Temperatura, } \\
\mathbf{K}\end{array}$ & $\begin{array}{c}\text { Densidad protónica, } \\
\text { protones } / \AA^{3}\end{array}$ & $\begin{array}{c}\text { Temperaturas de fusión } \\
\text { y Ebullición a 1 atm, K }\end{array}$ \\
\hline$H_{2} O$ & 293 & 0.067 & $273-373$ \\
\hline$H_{2}$ & 20 & 0.042 & $14-21$ \\
\hline$C_{4}$ & 109 & 0.070 & $90-110$ \\
\hline$C_{4} H_{4}$ & 10 & 0.079 & $90-110$ \\
\hline$C_{2} H_{6}$ & 165 & 0.068 & $90-184$ \\
\hline$C_{3} H_{8}$ & 228 & 0.064 & $83-231$ \\
\hline$C_{12} H_{18}$ & 293 & 0.047 & $439-538$ \\
\hline$C_{9} H_{12}$ & 293 & 0.039 & $228-438$ \\
\hline
\end{tabular}

TABla 4.2: Comparativa de moderadores hidrogenados [194]

Sin embargo, el metano sólido presenta ciertos inconvenientes que dificultan su aplicación en fuentes de neutrones de alta intensidad. Por una parte, presenta una muy baja conductividad térmica, alrededor de $0,3 \mathrm{~W} / \mathrm{mK}$ [195], por lo que la extracción de una deposición intensa de energía en su volumen, manteniendo el estado sólido, resulta compleja. Por otro lado, es sensible al daño por irradiación, induciéndose radiólisis [196] y polimerización [197]. Estos efectos degradan la eficacia del moderador y acarrean otros problemas, como la generación de depósitos de hidrocarburos [198] o la potencial liberación súbita de la energía térmica por la recombinación de iones $\mathrm{H}^{+}$[194].

El primero de los inconvenientes mencionados, la baja conductividad térmica, se puede sobrellevar congelando el metano embebido en una estructura interna metálica de alta conductividad. Esta estructura, en contacto con el sistema de refrigeración criogénico, actúa como un sumidero de calor, evitando que la energía térmica tenga que difundir distancias que inducirían fuertes gradientes de temperatura. La estructura metálica puede ser una retícula, o incluso una espuma metálica, que debe optimizarse en función de la deposición

\footnotetext{
${ }^{\mathrm{II}} \mathrm{El}$ agua en forma de hielo, el polietileno y los hidruros metálicos poseen mayor densidad protónica que el metano sólido pero presentan pocos modos de vibración de baja frecuencia, lo que los hace ineficaces en las últimas etapas de la moderación a baja temperatura [194].
} 
volumétrica de potencia. Respecto a los problemas derivados del daño por irradiación, estos pueden mitigarse mediante recocidos y ciclos de descongelación recongelación periódicos.

Ambas técnicas de mitigación han sido implementadas con éxito en la fuente de neutrones de media potencia ISIS [199], en concreto, en el moderador de metano sólido de su segunda estación de blanco. La potencia térmica a disipar en el moderador acoplado de esta fuente es de $300 W$ [200], mientras que la deposición térmica calculada en el moderador de la fuente de ESS-Bilbao es considerablemente menor, $25 \mathrm{~W}$, por lo que combinando ambas soluciones, el uso de metano sólido parece factible para nuestra propuesta. Por tanto, se toma este material como medio moderador de referencia para la fuente de ESS-Bilbao.

Respecto a la configuración del conjunto blanco - moderador, en principio se pueden definir tres disposiciones básicas, conocidas como "slab", "wing" y "flux-trap" [201] (Figura 4-4). La diferencia fundamental entre ellas radica en que en la configuración "slab" la dirección incidente del haz y las líneas experimentales son coplanarias, mientras que en las configuraciones "wing" y "flux-trap" no lo son.

\section{( a )}

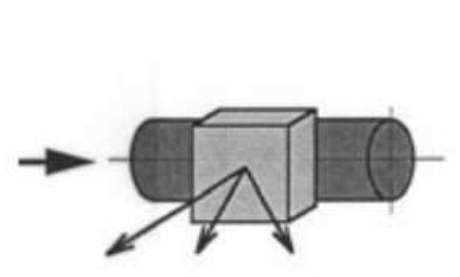

( b )

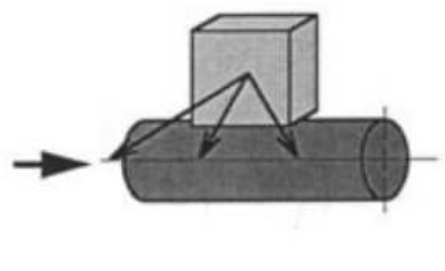

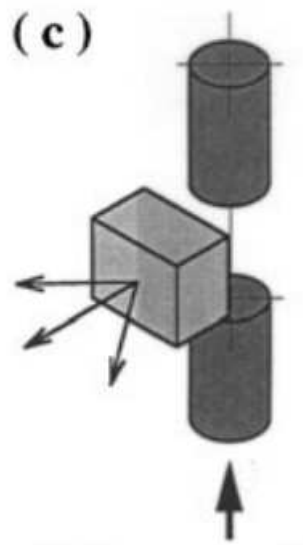

Figura 4-4: Esquema de las tres configuraciones blanco-moderador básicas; slab (a), wing (b) and flux-trap (c) [201].

En términos generales, la configuración "slab" puede producir un flujo neutrónico útil aproximadamente el doble que las otras dos configuraciones [202], debido al mayor ángulo sólido de visión entre el blanco y el moderador. Sin embargo, el uso de esta configuración en una fuente de espalación implicaría la llegada de un flujo indeseado de neutrones rápidos, aproximadamente mil veces superior al que aparecería con las otras dos configuraciones [202]. Esta disposición, además, generaría una carga térmica difícil de soportar para un 
sistema criogénico y dispararía el daño radiológico en el moderador. Por todo ello, ninguna fuente de espalación emplea la configuración "slab". La presencia de este flujo de neutrones de alta energía se debe fundamentalmente a que, como se describió en el Capítulo 1, la reacción de espalación presenta una etapa, conocida como fase de evaporación/fisión, en la que los neutrones rápidos son emitidos isótropamente.

La configuración "wing" [203] y la configuración "flux-trap" [204] reducen el flujo de neutrones rápidos desalineando estos elementos. La configuración "wing" es la más empleada en fuentes de neutrones de alta potencia [202]. La configuración "flux-trap", en la que el blanco se divide en dos secciones a lo largo del haz y los moderadores se colocan entre estas, tiene un rendimiento potencialmente parecido, pero en la práctica sólo es de interés para el caso en el que la dirección del haz de protones sea vertical.

Como se describió en la Sección 4.1, la reacción de extracción ${ }^{9} B e(p, x n)$ propuesta para la fuente de ESS-Bilbao, se caracteriza por una marcada anisotropía en la emisión de neutrones rápidos en la dirección del haz incidente. Colocando el moderador en configuración "slab", con su centro en una línea a $45^{\circ}$ con el centro del blanco, se puede evitar que la fracción más energética de la población neutrónica producida alcance el moderador. Por otra parte, tanto el flujo de neutrones como la energía máxima del espectro, son muy inferiores a los producidos en una fuente de espalación de alta intensidad, por lo que la problemática asociada a la llegada de neutrones rápidos a los instrumentos será también muy inferior. Además, la menor energía máxima de los neutrones rápidos y su menor flujo hacen los efectos derivados de la irradiación abarcables. Por todo lo anteriormente expuesto, la fuente de ESS-Bilbao puede beneficiarse del mejor rendimiento de la configuración "slab", evitando sus inconvenientes, por lo que se adopta como configuración de referencia.

Por último, para terminar de definir el concepto de moderador, debemos decidir si se opta por un moderador acoplado o desacoplado con el reflector. Como ya se describió en el Capítulo 1, el reflector es el componente que rodea el moderador, con el objetivo de que los neutrones no suficientemente moderados regresen a él para tener una nueva oportunidad de moderación, aumentando el flujo de neutrones útiles. No obstante, este proceso tiene un determinado tiempo característico que ensancha temporalmente el pulso de neutrones con respecto al de protones.

El propósito de un moderador desacoplado es el de proveer el pulso de neutrones más 
estrecho posible, con la mínima penalización del valor pico de este [203]. Este concepto se basa en la idea de desacoplar el moderador del reflector, evitando que neutrones por debajo de cierta energía regresen al moderador. Para ello, se inserta una capa de un absorbente de neutrones lentos entre ambos componentes, exceptuando en las zonas de visión de los experimentos. De esta manera, los neutrones lentos que llegarían al moderador tarde son absorbidos evitando el ensanchamiento del pulso. Los materiales más empleados para desacoplar el moderador son el cadmio $(C d)$ y el carburo de boro $\left(B_{4} C\right)$.

Por otro lado, el propósito de un moderador acoplado es proveer el mayor flujo posible integrado en el tiempo, independientemente de la longitud del puso.

Dado que el acelerador propuesto para la fuente de neutrones de ESS-Bilbao es un acelerador de pulso largo, 1,5 ms, la respuesta dinámica del reflector genera un ensanchamiento del puso de neutrones relativamente mucho menor. Los resultados experimentales presentados en [205] describen una ganancia sustancial con el empleo de moderadores acoplados para este tipo de fuentes, con un ensanchamiento de pulso reducido. Por tanto, buscando maximizar el flujo neutrónico integrado, se adopta un moderador acoplado como opción de referencia.

\subsubsection{Optimización de espesor}

Una vez seleccionados material, configuración y tipo de respuesta temporal, otros parámetros que afectan a la eficacia del moderador deben ser optimizados. De entre ellos, los que tiene un mayor impacto son su distancia al blanco, su espesor y su temperatura [202].

La distancia al blanco se debe minimizar para aumentar el ángulo sólido de visión de este. Por restricciones de ingeniería, el centro del moderador se posiciona a $8 \mathrm{~cm}$ de la superficie de salida del blanco. Esta distancia, medida a $45^{\circ}$ de la dirección incidente del haz, permite que la mayoría de neutrones rápidos no impacten en el moderador.

Como dimensiones transversales del moderador se escoge una sección cuadrada de $12 \times 12 \mathrm{~cm}^{2}$ centrada la huella del haz en el blanco, de tal modo que esta se cubra suficientemente y se proporcione un área de visión adecuada a los instrumentos.

Como parámetro de optimización representativo del rendimiento del moderador, se 
escoge el brillo de neutrones de energía menor a $5 \mathrm{meV}$. El término brillo neutrónico, normalmente expresado en $\left[\mathrm{n} / \mathrm{cm}^{2} \cdot s \cdot s r\right]$, representa el valor de flujo $\left[\mathrm{n} / \mathrm{cm}^{2} s\right]$ desde la superficie en cuestión (generalmente la del moderador) emitido por unidad de ángulo sólido.

Para la optimización del espesor y la temperatura del moderador se procede a modelar el moderador en MCNPX ${ }^{\text {III }}$. Las interacciones neutrón moderador se calculan empleando los kernels de scattering desarrollados por la Universidad de Indiana (USA) [206] y el Instituto Balseiro (Argentina) [207].

El efecto del espesor sobre el brillo neutrónico ha sido analizado para diversas temperaturas del moderador $(4,10$ y $20 \mathrm{~K}$ ), representativas del rango esperable de operación (Figura 4-5). Como puede observarse, el brillo neutrónico alcanza un máximo a partir del cual decrece con el aumento del espesor. Esto se debe a que la absorción de neutrones comienza a dominar sobre la moderación conforme el espesor crece. El espesor óptimo varía ligeramente con la temperatura, por lo que se escoge un espesor de $2 \mathrm{~cm}$ como valor de referencia por ser un buen compromiso.

\subsubsection{Efecto de la temperatura del moderador}

La dependencia observada del brillo neutrónico con la temperatura se debe a que estamos estudiando el rango termalizado del espectro producido, por lo que las variaciones de temperatura del moderador afectan al flujo de neutrones en equilibrio térmico con este.

Esta dependencia se ha analizado mediante un estudio paramétrico del espectro neutrónico en la superficie del moderador, presentado en la Figura 4-6. Podemos observar como el brillo de neutrones de $10 \mathrm{meV}$ es un $20 \%$ mayor si el moderador opera a $20 \mathrm{~K}$, comparado con la operación a $4 \mathrm{~K}$. Sin embargo, el brillo de neutrones de $1 \mathrm{meV}$ es casi el doble si el moderador opera a $4 K$ en vez de a $20 K$. Por tanto, en función de la aplicación experimental de los neutrones, la temperatura óptima del moderador varía. Dado que el sistema criogénico puede tener cierta flexibilidad para establecer la temperatura de operación, no es necesario fijar una temperatura de operación de referencia para el moderador.

\footnotetext{
${ }^{\mathrm{III}}$ Los cálculos presentados en las siguientes secciones tienen en cuenta el efecto de la presencia de un reflector de berilio cuya elección se justificará más adelante.
} 


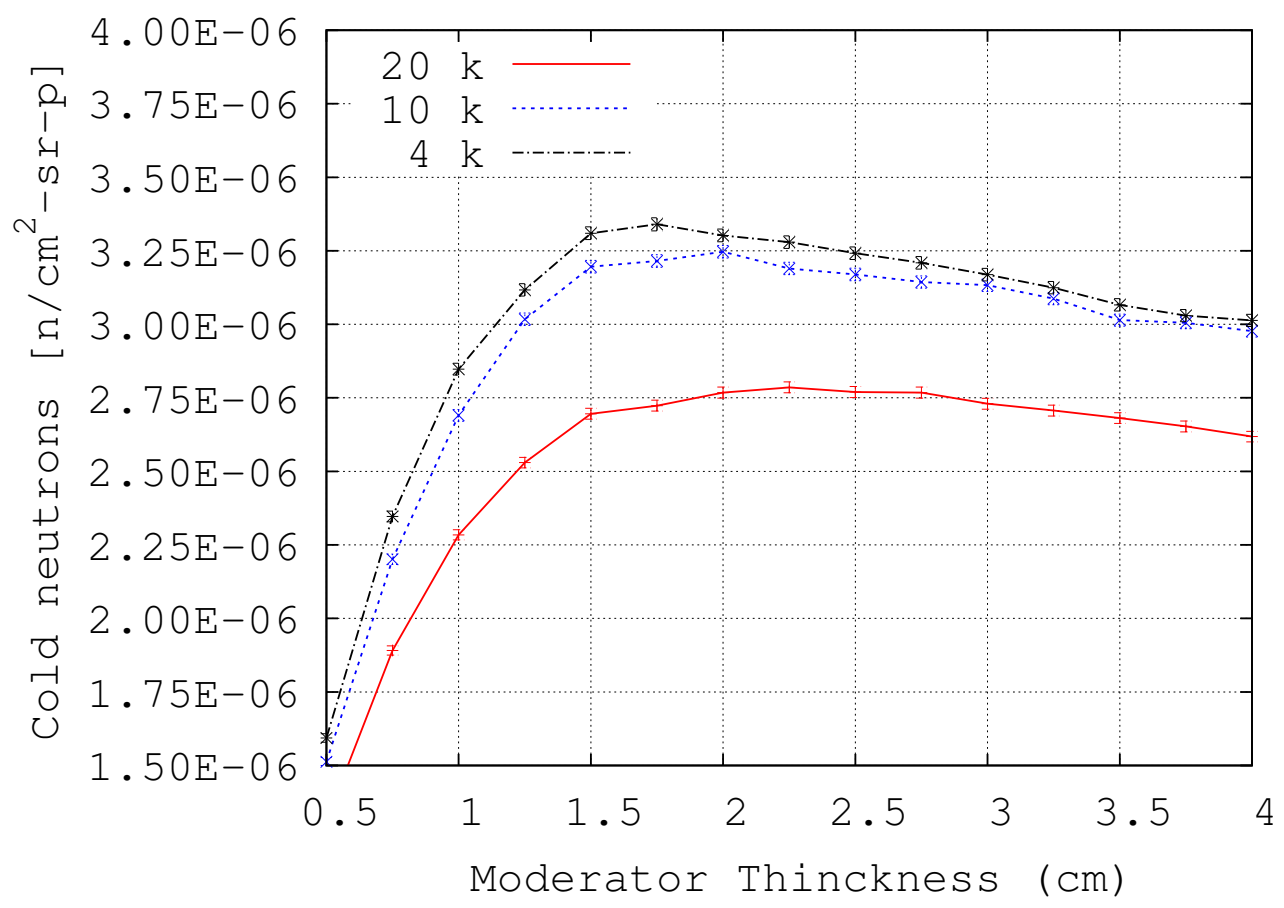

Figura 4-5: Brillo neutrónico de $E<5 \mathrm{meV}$ en la superficie del moderador de ESSBilbao en función de su espesor, normalizado por protón incidente.

\subsubsection{Reflector}

Un buen material reflector debe tener un elevada sección eficaz de dispersión y una baja sección eficaz de absorción. En general, los materiales moderadores (i.e. átomos ligeros) cumplen dichas propiedades, lo cual es de interés para esta aplicación, puesto que el material reflector tenga también propiedades moderadoras, aumenta el cómputo global de neutrones fríos. Algunos elementos pesados se emplean como reflector en aquellas aplicaciones en las que la moderación es innecesaria o inconveniente. Así pues, se seleccionan como candidatos para reflector: berilio, agua, agua pesada y plomo.

Para compararlos se analiza el pulso de neutrones de $\approx 5 \mathrm{meV}$ producido cuando se rodea el blanco por un cilindro reflector exceptuando las líneas de visión de los experimentos. El espesor del cilindro es el suficiente para saturar el efecto reflector de cada material. Los resultados de dicha comparativa se muestran en la Figura 4-7. El berilio es un material ligero $(Z=9)$, lo cual proporciona cierta moderación, con una baja sección eficaz de captura neutrónica. Además, este elemento tiene un umbral energético para las 


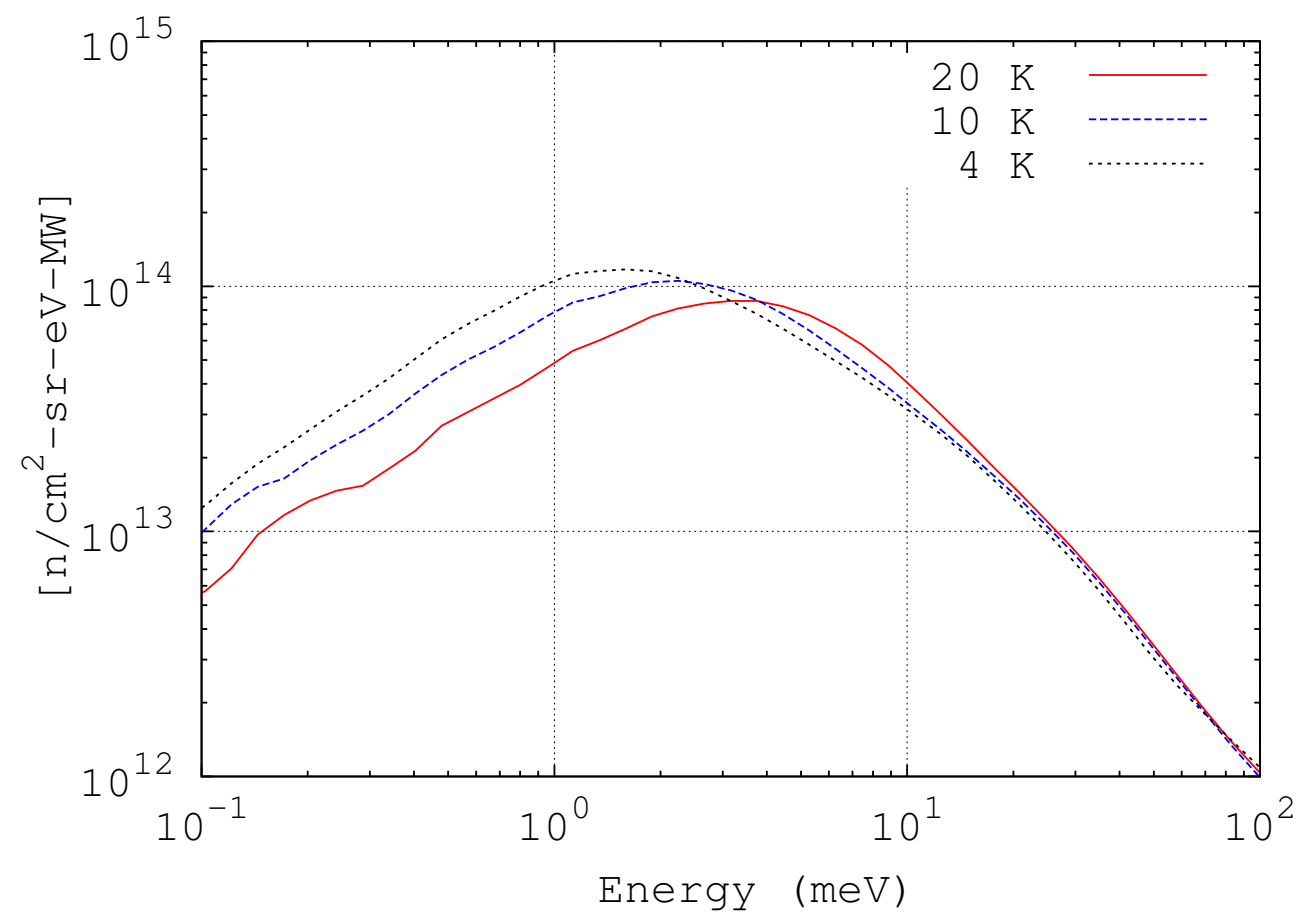

FiguRA 4-6: Espectro de brillo neutrónico en la superficie del moderador para distintas temperaturas de este, normalizado por $M W$ de haz.

reacciones $(n, x n)$ muy bajo, lo que añade una producción adicional de neutrones ${ }^{\mathrm{IV}}$. Estas características hacen que con este material se consiga la máxima producción neutrónica de la comparativa.

En el otro extremo se encuentra el plomo, elemento pesado con pobres propiedades moderadoras y que además presenta un umbral energético para las reacciones $(n, x n)$ moderadamente alto. Estas características hacen que con este material se consiga la mínima producción neutrónica de entre los elementos comparados.

Entre ambos se encuentran el agua pesada y el agua, ambos buenos moderadores en los que prácticamente no se dan reacciones $(n, x n)$. Dado que el agua presenta una sección eficaz de captura considerablemente mayor que el agua pesada, la producción neutrónica es mayor con esta última.

En base a estos resultados. y buscando maximizar la producción total de neutrones fríos, el material de referencia elegido para el moderador de ESS-Bilbao es el berilio. El cilindro reflector de este material tendría un radio de $40 \mathrm{~cm}$ y una altura de $80 \mathrm{~cm}$.

\footnotetext{
${ }^{\text {IV }}$ Propiedad por la que se postula como multiplicador neutrónico en el ámbito de la fusión nuclear [208].
} 


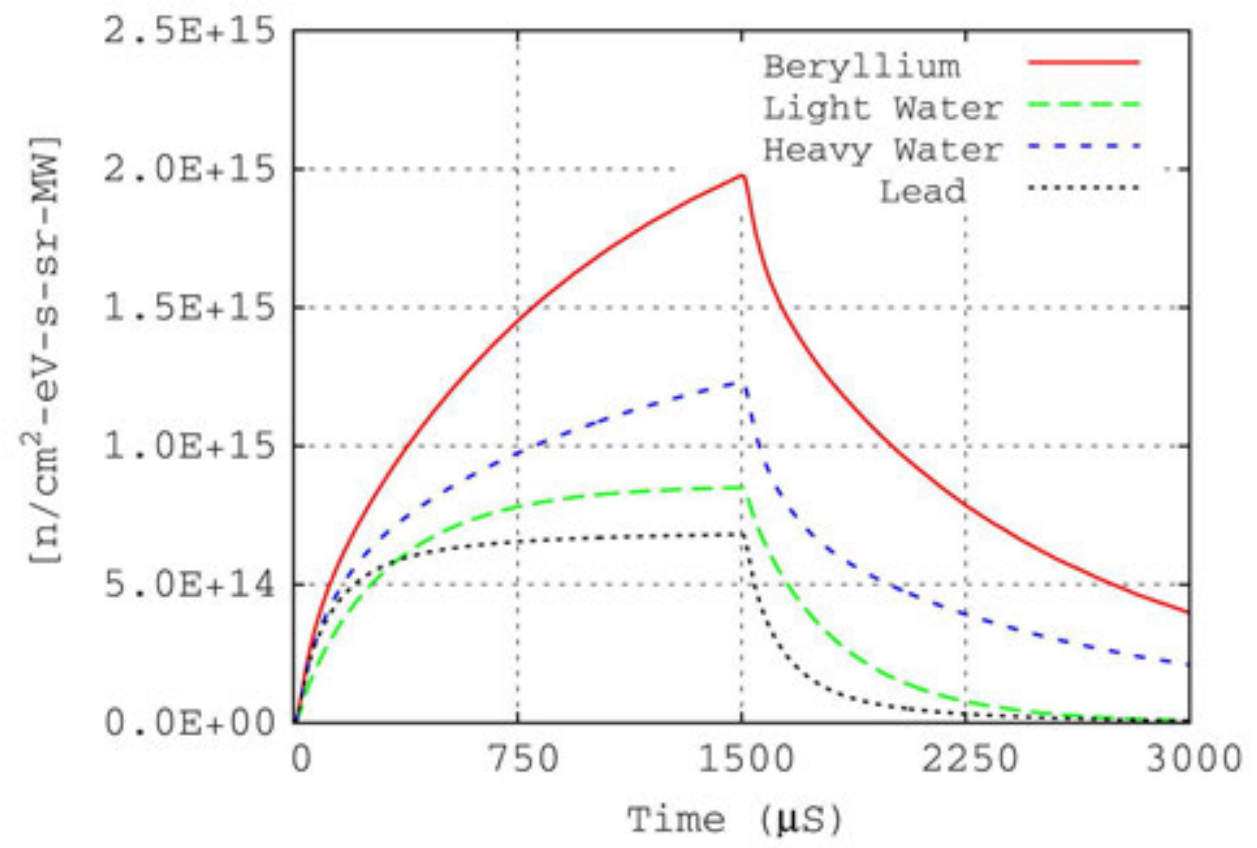

Figura 4-7: Distribución temporal de brillo de neutrones de 4,4<E<5,3 meV en la superficie del moderador para distintos materiales reflectores, normalizado por $M W$ de haz.

Cabe destacar que el empleo de materiales moderadores como reflector alarga sensiblemente la cola de la distribución temporal del pulso (i.e. fracción de los neutrones emergiendo después del pulso de protones), ya que empleamos un moderador acoplado. Esto puede ser inconveniente en función del tipo de aplicación experimental prevista. Aunque, al tratarse de una instalación de pulso largo, se busca fundamentalmente maximizar la integral temporal de la producción neutrónica. Por lo tanto, este efecto no se considera relevante. En caso de que esta dinámica se quisiera evitar, o se necesitara un pulso con una forma más plana, podría plantearse el empleo de un reflector pesado. Por último, el empleo de agua ligera como reflector, también sería cotejable por razones económicas, o para una primera fase de la instalación.

\subsubsection{Blindajes y estación del blanco}

Con el objetivo de minimizar la dosis en operación y en parada, el reflector se recubre de tres capas de blindaje en forma de cilindros concéntricos a este, de tal forma que los espesores descritos a continuación recubrirían el reflector en todas las direcciones. 
La primera capa de blindaje consiste en $15 \mathrm{~cm}$ de plomo, cuyo objetivo es detener la radiación $\gamma$ producida en blanco, moderador y reflector. La ubicación cercana al blanco de este blindaje pesado reduce el peso total de blindaje.

La segunda capa está formada por $60 \mathrm{~cm}$ de polietileno borado, cuyo objetivo es el blindaje de neutrones. Este material contiene hasta un $5 \%$ en peso de boro, por lo que aúna las propiedades moderadoras del polietileno con las propiedades absorbentes del boro. Ambas características lo convierten en un blindaje neutrónico eficaz, comercialmente disponible y frecuentemente empleado.

Por último, la capa exterior de blindaje consta de $10 \mathrm{~cm}$ de acero. Este blindaje tiene como objetivo frenar la radiación $\gamma$ generada por la captura de neutrones en el boro.

La Figura 4-8 muestra el diseño conceptual de la denominada estación del blanco, formada por el conjunto blanco-moderador-reflector y los blindajes que lo rodean. Esta solución conceptual, aunque carente de detalle, define un concepto de estación plausible basado en tres componentes mayores: la vasija de contención, el inserto del moderador y la unidad de transporte del blanco.

La vasija de contención alberga todos los elementos, y proporciona una barrera a los posibles efluentes radiactivos que puedan darse en operación o accidente. Esta vasija de acero, de $5 \mathrm{~cm}$ de espesor, proporciona parte de los $10 \mathrm{~cm}$ de blindaje de este material necesarios. Los $5 \mathrm{~cm}$ restantes se ubican en su interior. La vasija tiene una altura de 2,5 $m$ y un radio de $1,25 m$ (Figura 4-9).

El inserto del moderador (Figura 4-10, izquierda) busca poder sustituir este componente sin necesitar una modificación mayor del conjunto. Esto permitiría, por ejemplo, intercambiar el moderador criogénico por uno térmico, o ubicar en esa posición un elemento para irradiación con neutrones, más fácilmente.

La unidad de transporte del blanco (Figura 4-10, derecha) es la responsable de posicionarlo en el centro de la estación, y de transportarlo a las zonas de mantenimiento y almacenaje. El reflector y el blindaje de plomo sólo se encuentran alrededor de la posición en la que se producen los neutrones, sin embargo el blindaje de polietileno borado y acero rodea la totalidad de la estructura del blanco, por lo que este blindaje sobresale de la configuración cilíndrica, y parte debe ser incluido en la unidad de transporte. Esta estructura 

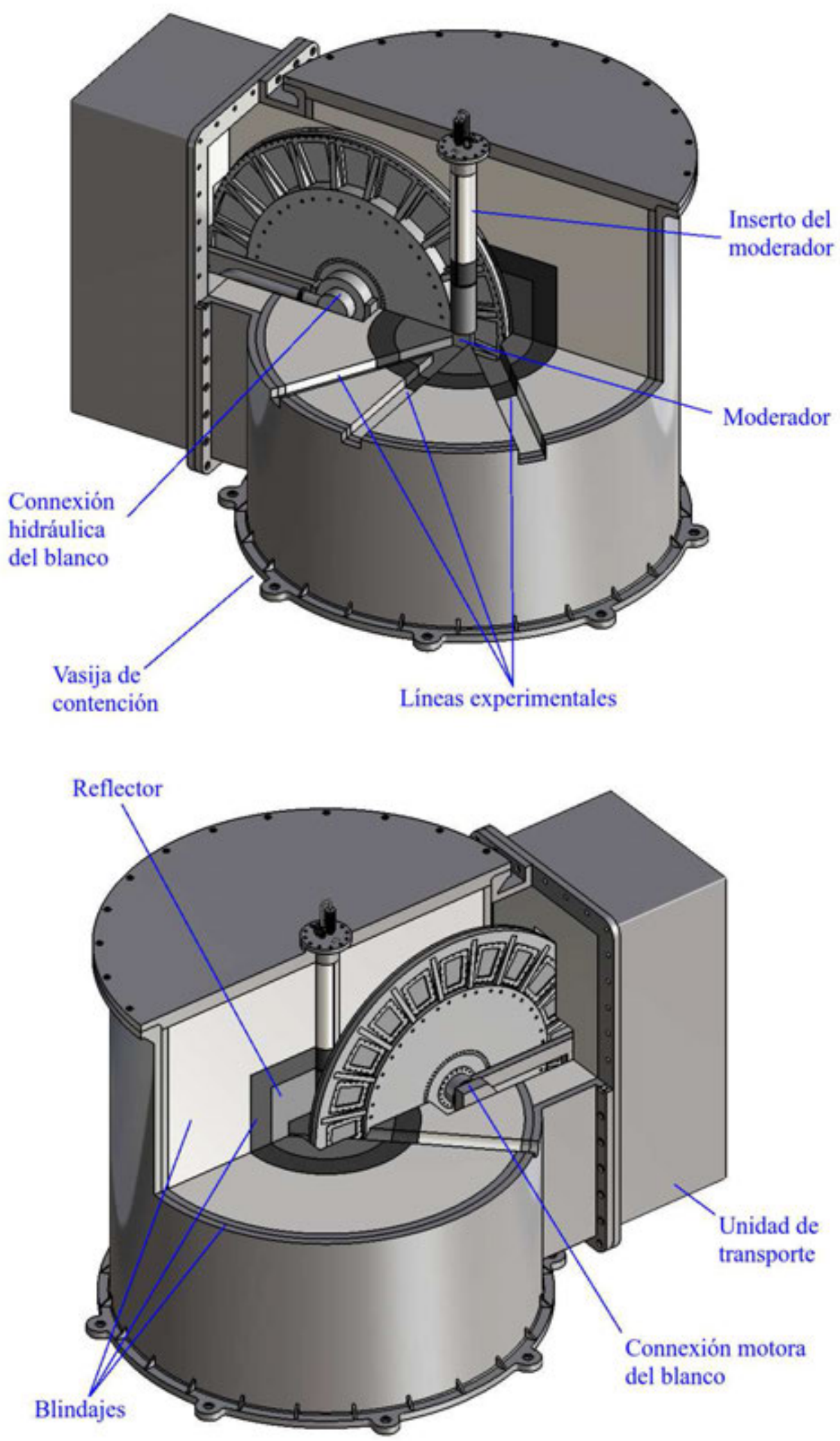

FigurA 4-8: Estación del blanco. 

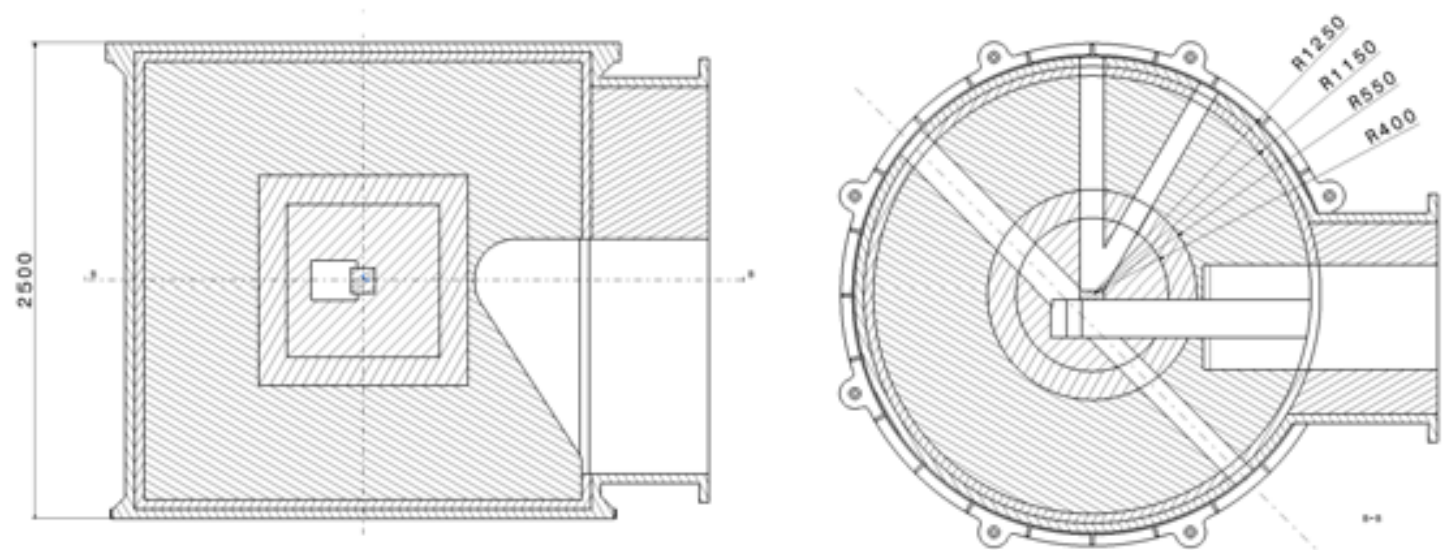

Figura 4-9: Dimensiones principales de la vasija de contención [mm].

móvil es la encargada de transmitir movimiento al blanco, a través de la parte superior de su eje, y de suministrar y recoger el refrigerante por su parte inferior. El desplazamiento de la unidad se efectúa a través de una plataforma en la que se ubicarán los sistemas auxiliares necesarios, como por ejemplo el conjunto motor del disco y de la propia plataforma, así como los sistemas de conexión hidráulica y eléctrica ${ }^{\mathrm{V}}$.
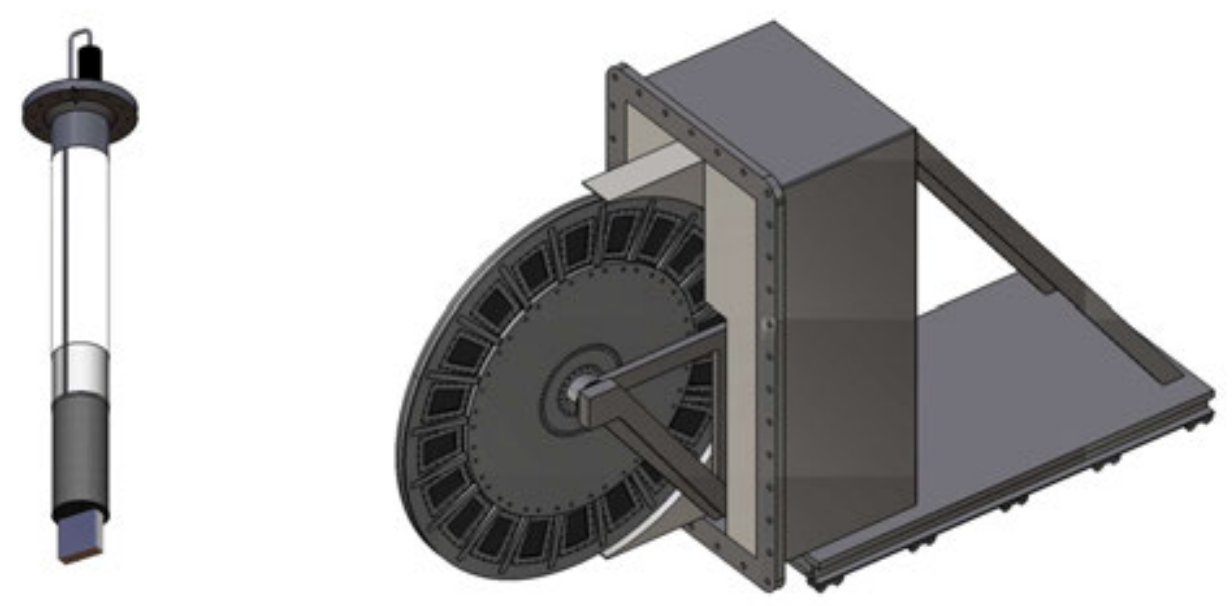

FigurA 4-10: Inserto del moderador (izq.) y unidad de transporte del blanco (der.)

\subsection{Producción neutrónica de referencia}

Con las decisiones de diseño previamente detalladas, se genera un modelo de MCNPX de todos los elementos neutrónicamente relevantes de la instalación (Figura 4-11). En este

\footnotetext{
${ }^{\mathrm{V}}$ Dependiendo de la distribución de masas, es posible que la plataforma también necesite portar con-
} 
modelo se ha incluido la estructura metálica que contiene el moderador, modelada como una capa de aluminio 6061-T6 de un espesor estimado de $4 \mathrm{~mm}$, así como la envolvente de vacío del blanco, modelada como una capa de $5 \mathrm{~mm}$ de espesor del mismo material. Este último componente se encargaría de separar el ambiente del moderador y las líneas experimentales $^{\mathrm{VI}}$, del vacío del acelerador y del entorno del blanco.

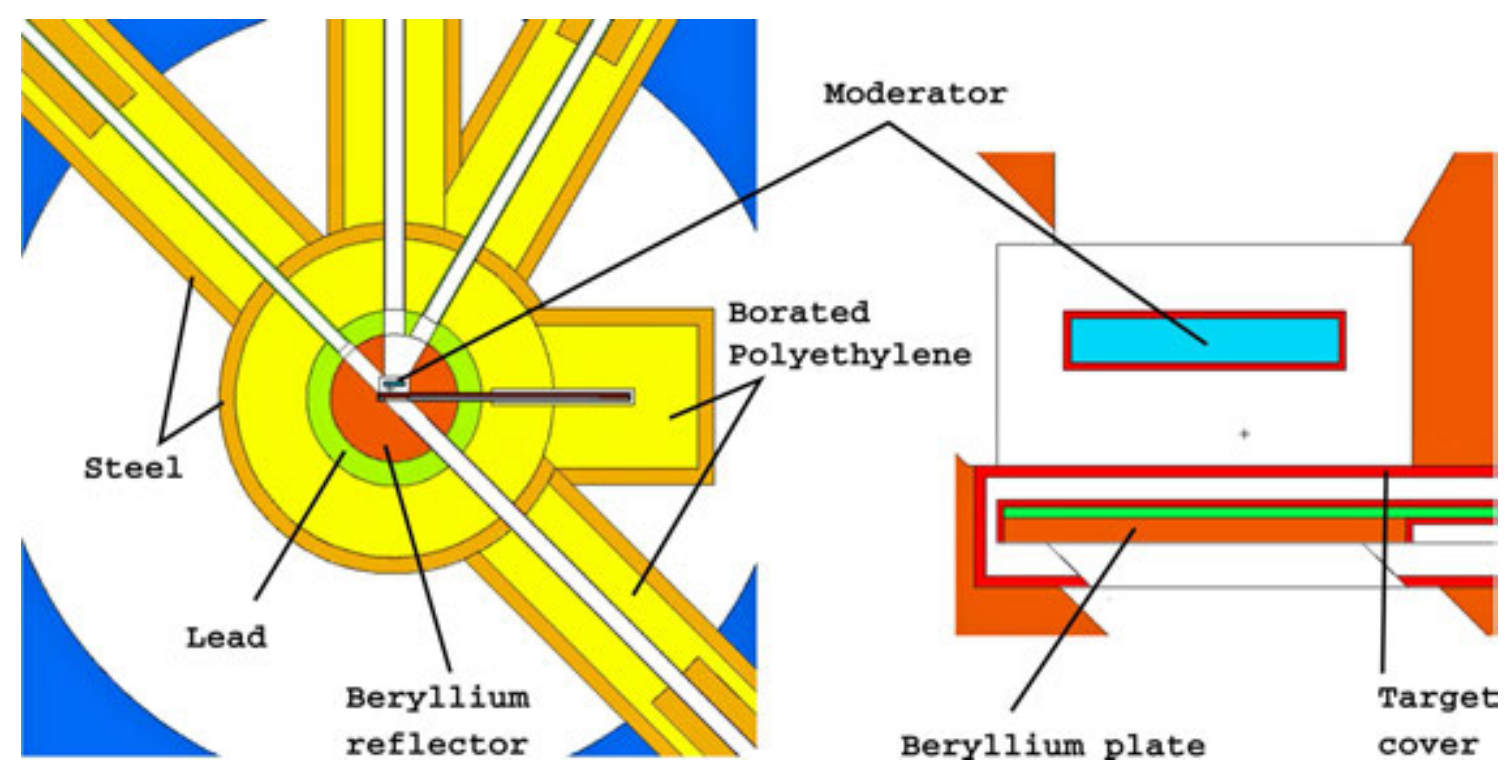

Figura 4-11: Modelo MCNPX de referencia. Vista global y área del moderador

El brillo de neutrones de energía $\approx 5 \mathrm{meV}$ en el moderador, con esta configuración de referencia, alcanza un valor de $3,6 \cdot 10^{10} \mathrm{n} / \mathrm{cm}^{2} \cdot s \cdot s r$.

Con el objetivo de evaluar el comportamiento global de la fuente y ponerla en contexto junto con otras instalaciones, se incluye el diseño aquí descrito en la comparativa efectuada en [209]. Este trabajo compara las fuentes de espalación más potentes operando en la actualidad, en base a su brillo de neutrones de energía inferior a $0,4 \mathrm{eV}$ promediado por segundo y por pulso.

Para dar un valor con el que medir la eficiencia del diseño propuesto, se complementa la comparativa de [209] con las razones de término fuente y brillo de neutrones por segundo respecto a la propuesta de ESS-Bilbao de cada instalación.

\footnotetext{
trapesos.

${ }^{\mathrm{VI}}$ Como se discutió en el capítulo 3, la inclusión de una ventana de protones para separar el vacío del acelerador del blanco parece difícil dadas las características del haz de protones. Es por esto por lo que, de modo conservador, se ha decidido incluir en el modelo neutrónico.
} 
Los términos fuente de las demás instalaciones, excepto para JSNS, se han estimado en base a la potencia recogida en la comparativa [209] y a la razón de conversión protón/neutrón calculada conforme a la Ecuación 4.3 [210], válida para protones de hasta 1,5 GeV, donde $E_{p}$ es la energía de los protones en $G e V$ y $A$ es el número másico del blanco. Para el caso de JSNS, que se encuentra fuera del rango de validez de la Ecuación 4.3, el término fuente se toma directamente de [209]. Los resultados de la comparativa así planteada se presentan en la Tabla 4.3.

$$
\begin{array}{ll}
M\left(E_{p}\right) & =0,1\left(E_{p}-0,12\right)(A+20) \\
M\left(E_{p}\right) & =5,0\left(E_{p}-0,12\right) \quad \text { para }{ }^{238} U
\end{array}
$$

La fuente propuesta para ESS-Bilbao presenta un valor de brillo de neutrones de $E<$ $0,4 \mathrm{eV}$ promediado por segundo de $1,3 \cdot 10^{11} \mathrm{n} / \mathrm{cm}^{2} \cdot \mathrm{sr} \cdot \mathrm{s}$, menor que las grandes fuentes de espalación en un factor $\approx 34 \mathrm{y} \approx 22$ con JSNS y SNS respectivamente. El factor con respecto a la segunda estación del blanco de ISIS (ISIS-TS2), siendo esta una fuente de potencia media, es de $\approx 4$. Sin embargo, teniendo en cuenta que los términos de producción total de neutrones de las grandes fuentes de espalación son un factor $\approx 200$ mayores, podemos concluir que la eficiencia de la propuesta para ESS-Bilbao es mayor. Comparando con ISIS-TS2, la eficiencia del diseño propuesto es similar, si no ligeramente superior.

A la hora de comparar la eficiencia también debe considerarse el coste de las estructuras de aceleración. La baja energía final de los protones de la propuesta para ESS-Bilbao, $50 \mathrm{MeV}$, comparada con los $800 \mathrm{MeV}$ de ISIS, o los 1 y $3 \mathrm{GeV}$ de SNS o JSNS respectivamente, hacen que las estructuras aceleradoras necesarias sean mucho más sencillas.

Esta mayor eficacia se debe fundamentalmente a que la propuesta para ESS-Bilbao se beneficia de opciones de diseño que normalmente no son factibles en las grandes instalaciones, como son el empleo de metano sólido y la configuración "slab". 


\begin{tabular}{|c|c|c|c|c|c|c|c|c|c|}
\hline Fuente & $\begin{array}{c}\text { Potencia } \\
{[k W]}\end{array}$ & $\begin{array}{c}\boldsymbol{E}_{p} \\
{[\mathrm{MeV}]}\end{array}$ & $\begin{array}{l}\text { Tasa } \\
{[\mathrm{Hz}]}\end{array}$ & $\begin{array}{c}\text { Corriente } \\
\text { media }[m A]\end{array}$ & $\begin{array}{c}\text { Fuente } \\
{[n / s]}\end{array}$ & $\begin{array}{c}\text { Brillo } \\
{\left[n / \mathrm{cm}^{2} \cdot s r \cdot p l\right]}\end{array}$ & $\begin{array}{c}\text { Brillo } \\
{\left[n / \mathrm{cm}^{2} \cdot s r \cdot s\right]}\end{array}$ & $\begin{array}{c}\text { Ratio } \\
\text { término fuente }\end{array}$ & $\begin{array}{l}\text { Ratio brillo en } \\
{\left[n / \mathrm{cm}^{2} \cdot s r \cdot s\right]}\end{array}$ \\
\hline $\begin{array}{c}\text { JSNS } \\
\text { Acoplado, } H_{2}\end{array}$ & $\begin{array}{c}300 \\
1000\end{array}$ & $\begin{array}{l}3000 \\
3000\end{array}$ & $\begin{array}{l}25 \\
25\end{array}$ & $\begin{array}{c}0.1 \\
0.33\end{array}$ & $\begin{array}{l}4.7 \mathrm{E}+16 \\
1.5 \mathrm{E}+17\end{array}$ & $\begin{array}{l}5.1 \mathrm{E}+10 \\
1.8 \mathrm{E}+11\end{array}$ & $\begin{array}{l}1.3 \mathrm{E}+12 \\
4.5 \mathrm{E}+12\end{array}$ & $\begin{array}{r}58.8 \\
187.5\end{array}$ & $\begin{array}{c}9.7 \\
34.1\end{array}$ \\
\hline $\begin{array}{c}\text { SNS } \\
\text { Acoplado, } H_{2}\end{array}$ & $\begin{array}{l}1000 \\
1400\end{array}$ & $\begin{array}{l}1000 \\
1000\end{array}$ & $\begin{array}{l}60 \\
60\end{array}$ & $\begin{array}{c}1 \\
1.4\end{array}$ & $\begin{array}{l}1.2 \mathrm{E}+17 \\
1.7 \mathrm{E}+17\end{array}$ & $\begin{array}{l}3.5 \mathrm{E}+10 \\
4.9 \mathrm{E}+10\end{array}$ & $\begin{array}{l}2.1 \mathrm{E}+12 \\
2.9 \mathrm{E}+12\end{array}$ & $\begin{array}{l}150.0 \\
212.5\end{array}$ & $\begin{array}{l}15.9 \\
22.3\end{array}$ \\
\hline $\begin{array}{c}\text { ISIS-TS2 } \\
\text { Acoplado, } \mathrm{CH}_{4} \\
\text { Acoplado, } \mathrm{H}_{2}\end{array}$ & $\begin{array}{l}48 \\
48\end{array}$ & $\begin{array}{l}800 \\
800\end{array}$ & $\begin{array}{l}10 \\
10\end{array}$ & $\begin{array}{l}0.06 \\
0.06\end{array}$ & $\begin{array}{l}5.2 \mathrm{E}+15 \\
5.2 \mathrm{E}+15\end{array}$ & $\begin{array}{l}5.4 \mathrm{E}+10 \\
3.0 \mathrm{E}+10\end{array}$ & $\begin{array}{l}5.4 \mathrm{E}+11 \\
3.0 \mathrm{E}+11\end{array}$ & $\begin{array}{l}6.5 \\
6.5\end{array}$ & $\begin{array}{l}4.1 \\
2.3\end{array}$ \\
\hline $\begin{array}{c}\text { ESS-Bilbao } \\
\text { Acoplado, } \mathrm{CH}_{4}\end{array}$ & 112.5 & 50 & 20 & 2.25 & $8.0 \mathrm{E}+14$ & $6.6 \mathrm{E}+09$ & $1.3 \mathrm{E}+11$ & - & - \\
\hline
\end{tabular}

TABla 4.3: Término fuente y brillo de neutrones $E<0,4 \mathrm{eV}$ de distintas fuentes de espalación [209] y relaciones respecto a los valores esperables para ESS-Bilbao. 


\subsection{Configuraciones alternativas y avanzadas}

\subsubsection{Filtro de berilio}

Estudios llevados a cabo en el laboratorio nacional de Los Álamos (LANL,USA) han demostrado un aumento de brillo neutrónico en moderadores tipo "flux-trap" empleando un dispositivo denominado filtro de berilio [211]. Este dispositivo consiste en un bloque de berilio a temperatura inferior a $80 \mathrm{~K}$. Su funcionamiento se basa en el hecho de que este material, a dicha temperatura, presenta un baja sección eficaz de dispersión para neutrones de energía inferior a 5,22 meV, y un brusco aumento en este parámetro a partir de ese umbral (Figura 4-12). De esta manera, un bloque de esas características posicionado tras el moderador filtraría los neutrones no suficientemente moderados, reenviándolos hacía el moderador.

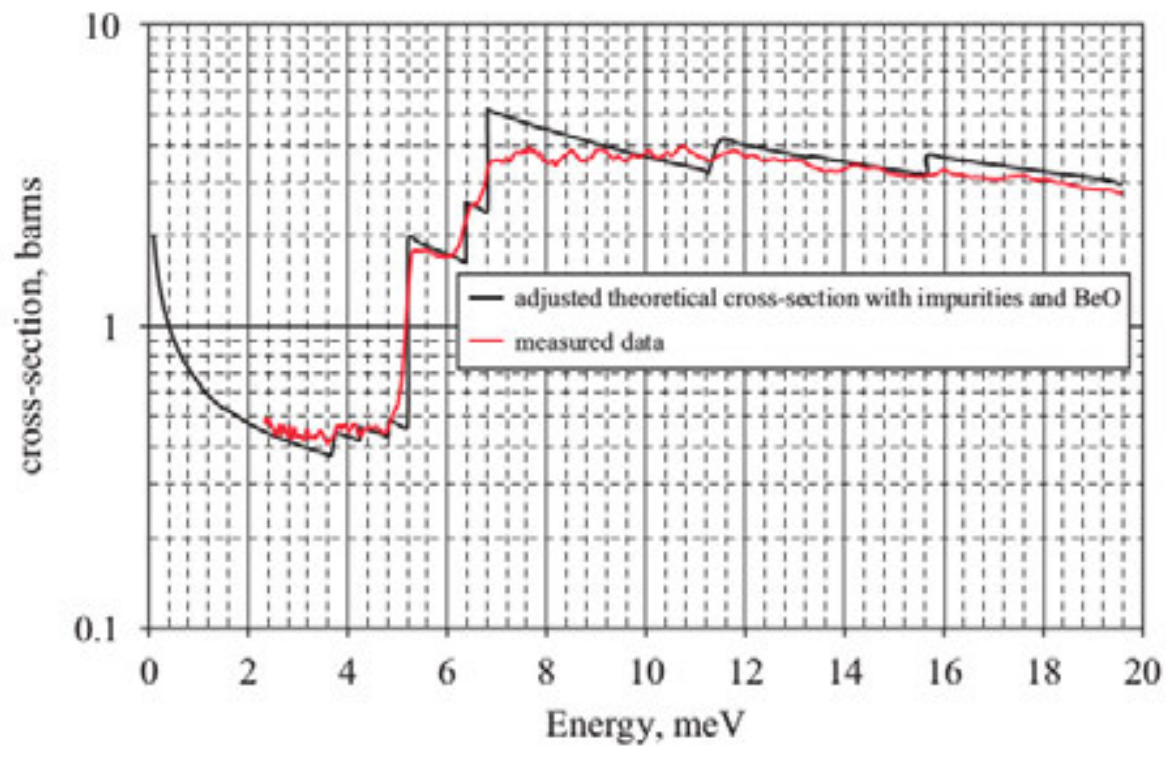

Figura 4-12: Sección eficaz del berilio a $77 \mathrm{~K}$ : Medida experimental y valor teórico ajustado para incluir el efecto de la presencia impurezas y de óxido de berilio [212].

Con el objetivo de analizar el impacto de un filtro de berilio en la producción neutrónica de la fuente propuesta para ESS-Bilbao, se modifica el modelo de MCNPX de la instalación para incluir este dispositivo entre el moderador y las líneas de experimentación (Figura 
4-13). Para el cálculo de la interacción de los neutrones con el berilio a baja temperatura se ha empleado el kernel de dispersión desarrollado por LANL [212].

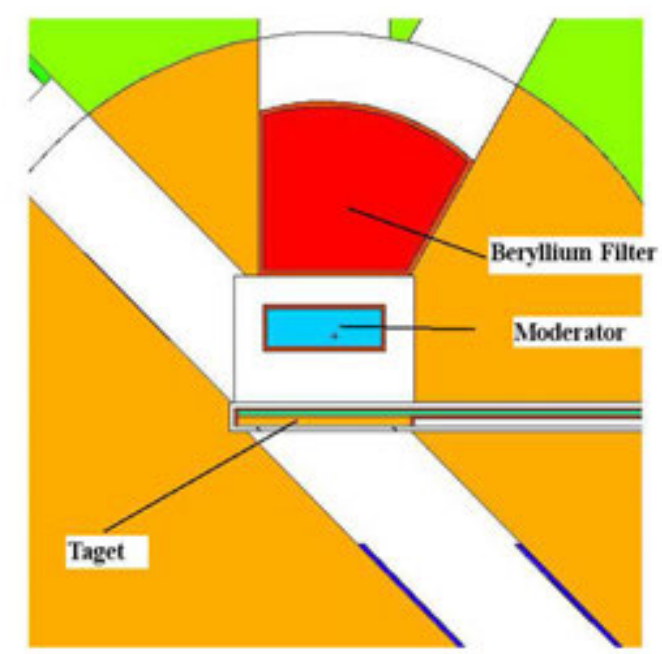

Figura 4-13: Modelo de MCNPX incluyendo filtro de berilio

La Figura 4-14 muestra el espectro de neutrones en la superficie del moderador con y sin filtro de berilio. Se observa como este componente genera un incremente de neutrones de energía inferior a $5 \mathrm{meV}$ de alrededor del $50 \%$, junto con una apreciable disminución por encima de ese valor. Como efecto positivo adicional, cabe destacar la disminución de neutrones rápidos de energía superior a un $1 \mathrm{MeV}$ en aproximadamente dos órdenes de magnitud.

La Figura 4-15 muestra el efecto de la inclusión del filtro sobre la distribución temporal del brillo de neutrones de $\approx 5 \mathrm{meV}$. Se observa como la presencia de este componente aumenta el valor pico y afina la distribución temporal del pulso. Para cuantificar este efecto, se procede a calcular y comparar las magnitudes integrales de "señal" y "cola" de ambos pulsos, entendiendo por estos términos la integral temporal de brillo hasta el final del pulso de protones y a partir de este respectivamente. El resultado de esta comparativa se recoge en la Tabla 4.4 .

La presencia del filtro de berilio aumenta el valor pico del pulso, así como la integral temporal total de este en torno al $50 \%$, coherentemente con lo apreciado en los resultados espectrales. Este aumento se produce por un incremento del $63 \%$ de la "señal" y de sólo 


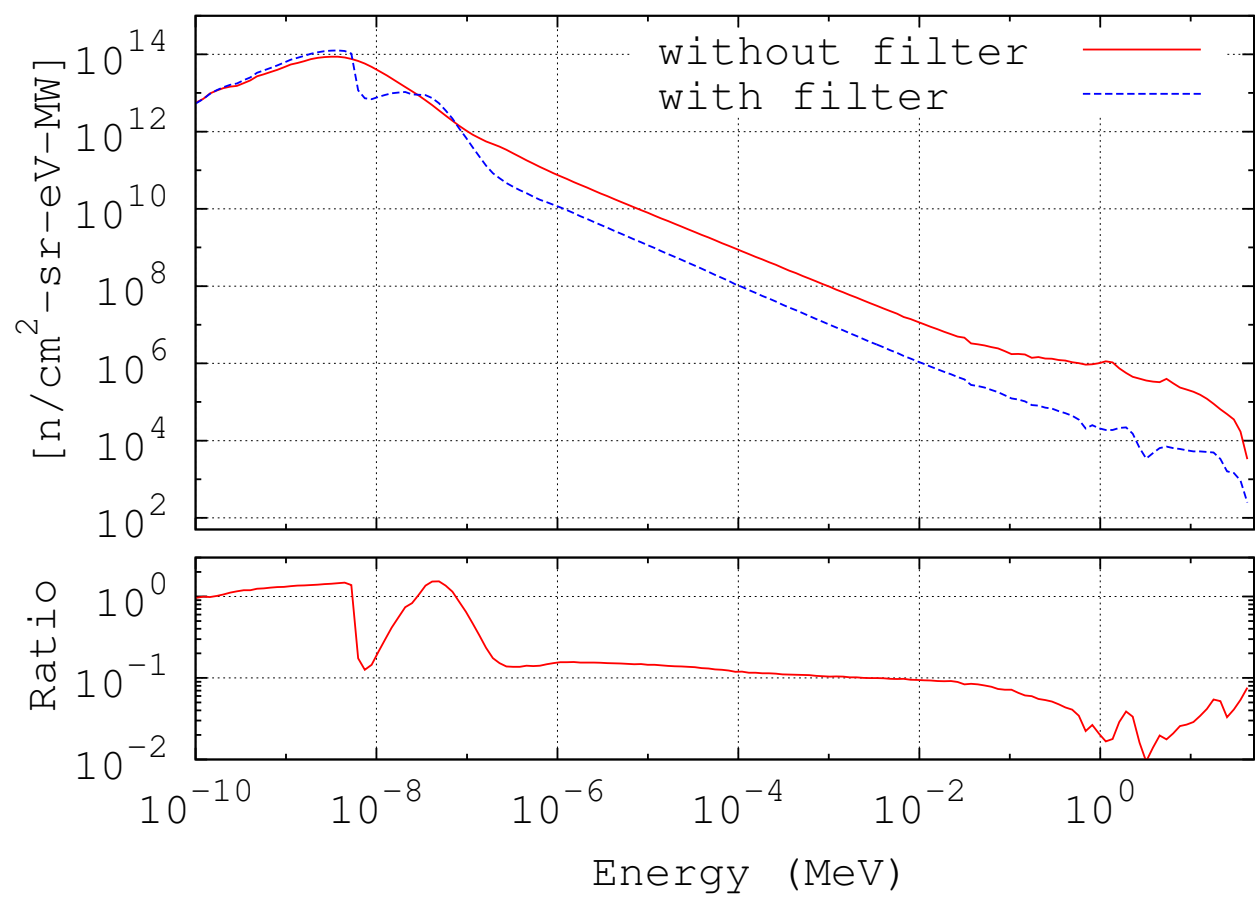

Figura 4-14: Espectro de brillo neutrónico en la superficie del moderador, con y sin filtro de berilio, normalizado por $M W$ de haz.

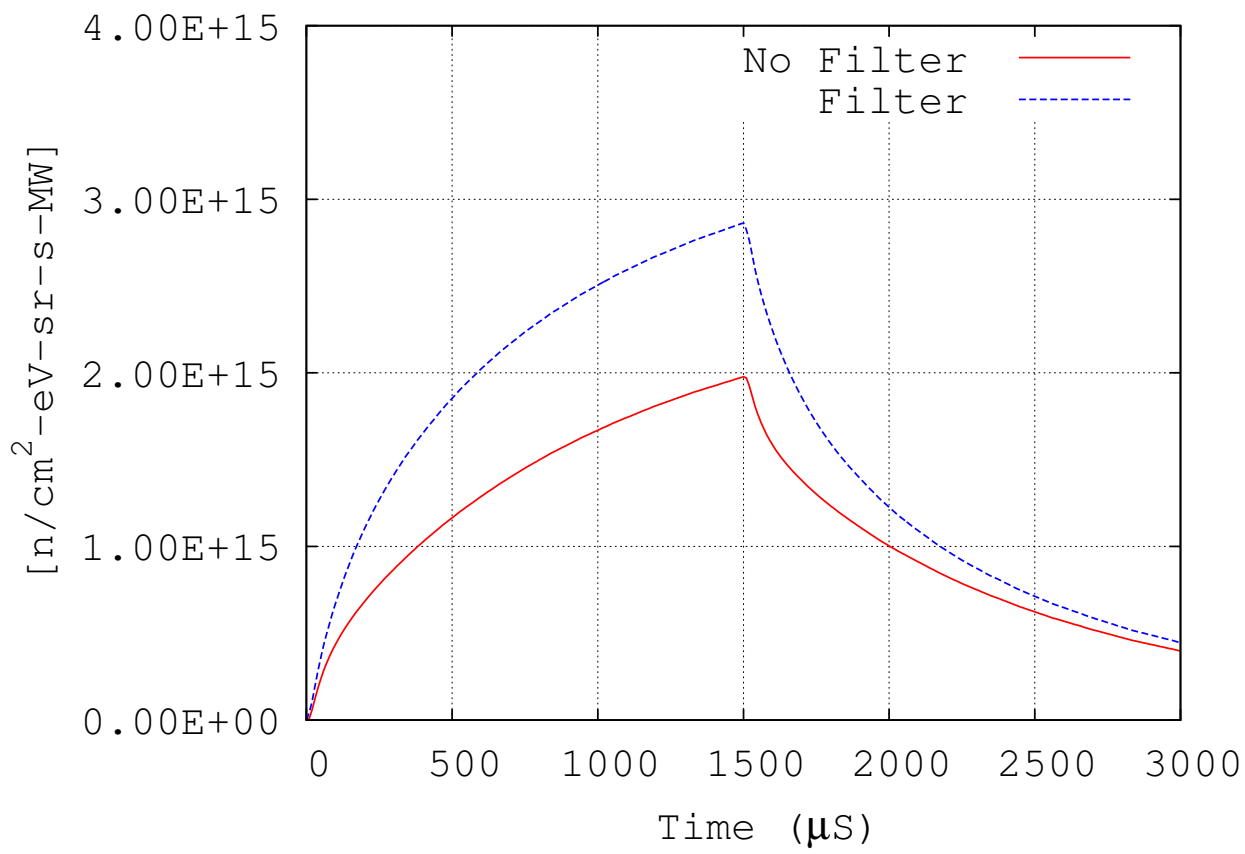

Figura 4-15: Distribución temporal de brillo de neutrones de $4,4<E<5,3 \mathrm{meV}$ en la superficie del moderador con y sin filtro de berilio, normalizado por $M W$ de haz. 


\begin{tabular}{|c|c|c|c|}
\hline Magnitud & sin filtro & con filtro & $\boldsymbol{\Delta}[\%]$ \\
\hline Valor pico $\left[\mathrm{n} / \mathrm{cm}^{2}\right.$-eV-sr-s-MW] & $2,0 \cdot 10^{15}$ & $2,9 \cdot 10^{15}$ & +45 \\
\hline Integral total $\left[\mathrm{n} / \mathrm{cm}^{2}\right.$-eV-sr-MW] & $3,5 \cdot 10^{12}$ & $5,3 \cdot 10^{12}$ & +51 \\
\hline Señal [n/ $\mathrm{cm}^{2}$-eV-sr-MW] & $1,6 \cdot 10^{12}$ & $2,6 \cdot 10^{12}$ & +63 \\
\hline Cola [n/ $\mathrm{cm}^{2}$-eV-sr-MW] & $1,9 \cdot 10^{12}$ & $2,7 \cdot 10^{12}$ & +42 \\
\hline Señal/Cola & 0.85 & 0.94 & +11 \\
\hline Tiempo de decaimiento hasta $50 \%[\mu \mathrm{s}]$ & 2010 & 1870 & -7 \\
\hline Tiempo de decaimiento hasta $10 \%[\mu \mathrm{s}]$ & 3520 & 3340 & -5 \\
\hline
\end{tabular}

TABla 4.4: Comparativa de las características temporales del pulso de neutrones de $4,4<$ $E<5,3 \mathrm{meV}$ sin filtro de berilio y con él.

un $42 \%$ de la "cola", aumentando la proporción "señal" /"cola" un $11 \%$ respecto al pulso de neutrones sin el filtro. Por el mismo motivo se obtiene una reducción apreciable en el tiempo de decaimiento del pulso, entendiéndose este como el periodo tras el pulso de protones en el que una cantidad significativa de neutrones sigue emergiendo del moderador.

Por tanto, considerando únicamente la producción de neutrones fríos, la inclusión de un filtro de berilio supone un incremento sustancial en el rendimiento de la fuente.

Desde un punto de vista de ingeniería de planta, el filtro de berilio requeriría de un sistema criogénico específico, lo cual aumenta la complejidad de la instalación. No obstante, este podría refrigerarse en principio con nitrógeno líquido $\left(T_{\text {ebullición }}=77 \mathrm{~K}\right)$ en lugar de con helio líquido $\left(T_{\text {ebullición }}=4 \mathrm{~K}\right.$ ), lo cual reduce los requisitos del sistema criogénico.

\subsubsection{Dos moderadores}

La configuración de referencia de la fuente de ESS-Bilbao puede acoger sin grandes modificaciones tres líneas experimentales. Para incrementar su número y ampliar el rango de potenciales aplicaciones de la instalación, puede plantearse la inclusión de un segundo moderador.

Con el objetivo de analizar esta posibilidad, se ha desarrollado un modelo de MCNPX que incluye la geometría de un segundo moderador de metano sólido, análogo al moderador de referencia. Este debe situarse al otro lado del haz de protones y tan próximo al blanco como sea posible (Figura 4-16). 


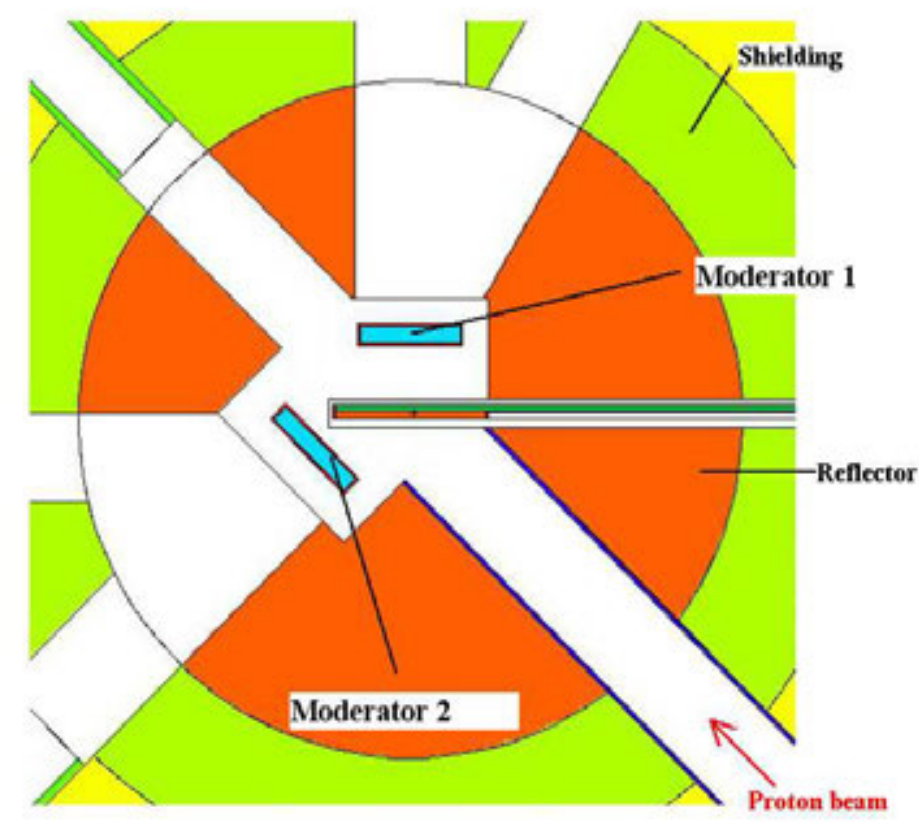

Figura 4-16: Modelo MCNPX con dos moderadores de metano sólido.

Sin embargo, la presencia de un segundo moderador afectará a la producción neutrónica del primero, fundamentalmente por la disminución de volumen reflector a su alrededor. Esta disminución se debe tanto al propio volumen del nuevo moderador como a las nuevas guías experimentales.

La Tabla 4.5 recoge los resultados de la comparativa entre la configuración de referencia y la configuración con dos moderadores, así como las configuraciones con uno o dos filtros de berilio. Los resultados se presentan en forma de brillo de neutrones de energía inferior a $5 \mathrm{meV}$ en la superficie de los moderadores.

Como se preveía, la inclusión del segundo moderador reduce la producción neutrónica de cada uno de ellos. La magnitud de esta disminución es de un $25 \%$ respecto al moderador de la producción del moderador original. No obstante, el cómputo global de neutrones de energía inferior a $5 \mathrm{meV}$ producidos por la instalación aumentaría un $50 \%$ con la inclusión del segundo moderador.

La inclusión de un filtro de berilio en uno de los moderadores produce un incremento de producción neutrónica en los dos moderadores, reduciendo el factor perdido respecto al valor de referencia a un $5 \%$ en el moderador con filtro y a un $11 \%$ en el moderador sin 
él. Cabe destacar como la presencia de un filtro mejora el resultado de ambos moderadores. Este comportamiento es atribuible al efecto reflector del filtro. La configuración con dos moderadores y dos filtros de berilio alcanza, en cada uno de los moderadores, unos resultados prácticamente iguales a la configuración de referencia, lo que supone duplicar la producción de neutrones de energía inferior a $5 \mathrm{meV}$ de la instalación original.

\begin{tabular}{|c|c|c|c|c|c|}
\hline & Referencia & $\begin{array}{c}\text { Referencia } \\
\text { con filtro }\end{array}$ & $\begin{array}{c}\text { Dos mode- } \\
\text { radores }\end{array}$ & $\begin{array}{c}\text { Dos mod. } \\
\text { un filtro }\end{array}$ & $\begin{array}{c}\text { Dos mod. } \\
\text { dos filtros }\end{array}$ \\
\hline Mod. 1 & $3,6 \cdot 10^{10}$ & $4,7 \cdot 10^{10}$ & $2,7 \cdot 10^{10}$ & $3,5 \cdot 10^{10}$ & $3,7 \cdot 10^{10}$ \\
Mod. 2 & - & - & $2,7 \cdot 10^{10}$ & $3,2 \cdot 10^{10}$ & $3,7 \cdot 10^{10}$ \\
\hline Mod.1/Ref. & - & $+30 \%$ & $-25 \%$ & $-5 \%$ & $+3 \%$ \\
Mod.2/Ref. & - & - & $-25 \%$ & $-11 \%$ & $+3 \%$ \\
Total/Ref. & - & - & $+50 \%$ & $+86 \%$ & $+106 \%$ \\
\hline
\end{tabular}

TABla 4.5: Brillo de neutrones de energía inferior a 5 meV en la superficie de los moderadores $\left[\mathrm{n} / \mathrm{cm}^{2} \cdot \mathrm{s} \cdot \mathrm{sr}\right]$

\subsubsection{Producción de neutrones térmicos}

Los neutrones térmicos, aquellos con energías comprendidas entre los 10 y los $600 \mathrm{meV}$ VII, se producen al alcanzar éstos el equilibrio térmico con un moderador a temperatura ambiente. El empleo de un moderador a temperatura ambiente, en vez de uno criogénico, simplifica de manera sustancial la instalación en su conjunto, por lo que el uso de un moderador térmico se podría considerar como una fase inicial de la operación, a completar a posteriori con una ampliación en la que se instalase un moderador criogénico. Esta fase inicial sería útil para caracterizar experimentalmente el término fuente de neutrones, así como para realizar pruebas de otros componentes: detectores, obturadores, guías de neutrones, etc.; además de para su empleo en técnicas experimentales específicas para neutrones térmicos, como por ejemplo, neutrografía. Por todo lo anteriormente descrito, se procede a analizar la producción de neutrones térmicos en la propuesta de fuente para ESS-Bilbao.

Los materiales comúnmente empleados como moderadores térmicos son el agua y el

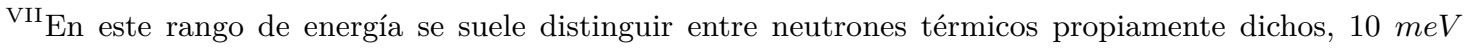
$<E<100$, y epitérmicos, $100 \mathrm{meV}<E<600 \mathrm{meV}$
} 
polietileno, por lo que se seleccionan estos materiales como candidatos potenciales. Para analizar su rendimiento, se lleva a cabo un estudio análogo al desarrollado en la Sección 4.2.1.2. La Figura 4-17 muestra la evolución del brillo de neutrones de energía inferior a 600 $m e V$ en la superficie del moderador, para ambos materiales, en función de su espesor. Estos cálculos se han realizado empleando el modelo MCNPX de referencia de la instalación, sustituyendo únicamente el material moderador.

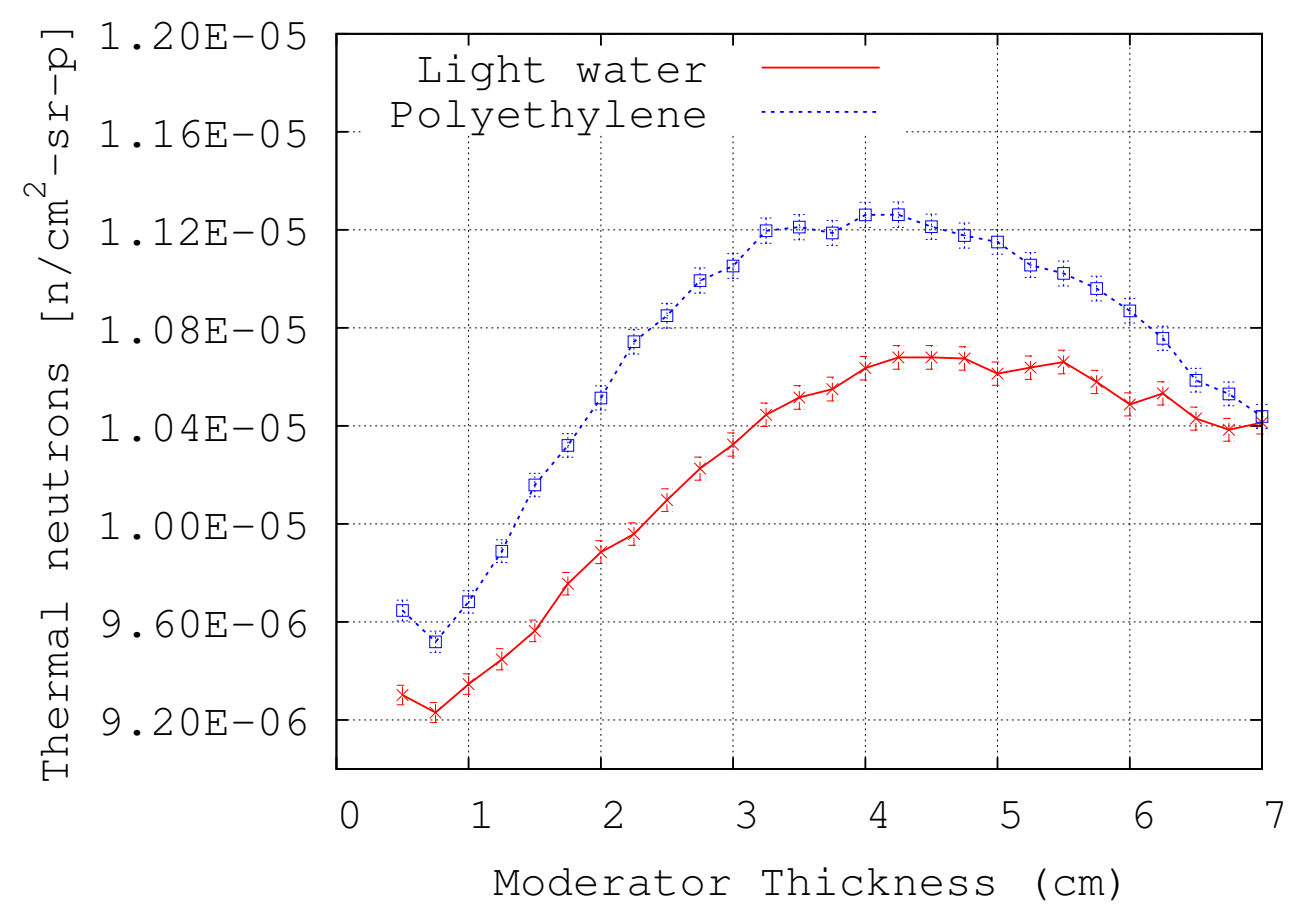

Figura 4-17: Brillo de neutrones de $E<600 \mathrm{meV}$ en la superficie del moderador de ESS-Bilbao en función de su espesor, normalizado por protón incidente.

Los resultados muestran que ambos materiales presentan un espesor óptimo cerca de los $4 \mathrm{~cm}$, por lo que, eligiendo este espesor para el moderador térmico, no sería difícil hacer intercambiables el moderador térmico y el moderador criogénico, de $2 \mathrm{~cm}$ de espesor. Esta idea posibilitaría la aproximación a la operación por fases antes mencionada.

Respecto a la producción neutrónica, el polietileno genera un brillo un $\approx 5 \%$ mayor, sin embargo este material presenta ciertas desventajas. Los materiales plásticos, como el polietileno, sufren daño por irradiación, fundamentalmente por la radiólisis de las cadenas de hidrocarburos, lo cual no sólo degrada sus propiedades mecánicas, si no también las neutrónicas [213]. Además, generalmente, no son recocibles, por lo que se debe proceder a 
su sustitución. Por otra parte, para mantener un moderador de polietileno a temperatura ambiente, sería necesario dotarlo de un sistema de refrigeración, el cual empeoraría el comportamiento calculado. En cambio, si empleáramos un moderador de agua, ésta se podría mantener a temperatura ambiente simplemente haciéndola circular a través de un intercambiador de calor durante operación.

Así pues, en base a la relación entre simplicidad del sistema y producción neutrónica, se selecciona el agua como material para el moderador térmico de la instalación. Con el espesor de $4 \mathrm{~cm}$ antes seleccionado, la producción de neutrones térmicos de energía menor a los $600 \mathrm{meV}$ alcanzaría los $1,4 \cdot 10^{11} \mathrm{n} / \mathrm{cm}^{2} \cdot \mathrm{sr} \cdot \mathrm{s}$.

\subsection{Evaluación preliminar de aplicaciones}

Dado que el término fuente de la propuesta para ESS-Bilbao es netamente superior al de las fuentes compactas, podemos suponer que todas las técnicas experimentales disponibles en estas instalaciones serían realizables con solvencia en ESS-Bilbao, especialmente aquellas que no dependen de la distribución temporal de la producción neutrónica. Por ello, los flujos esperables son ampliamente suficientes para llevar a cabo calibración de detectores, análisis por activación, irradiación de componentes, medida de secciones eficaces y otras técnicas basadas en transferencia.

Con el fin de tener una primera evaluación de la posible aplicación de la fuente de ESSBilbao en el desarrollo de técnicas de dispersión neutrónica, se emplea como referencia el estudio llevado a cabo en [214]. En este trabajo se evalúa la capacidad de una fuente con un brillo de neutrones térmicos de $1,6 \cdot 10^{11} \mathrm{n} / \mathrm{cm}^{2} \cdot \mathrm{sr} \cdot \mathrm{s}$, y un factor de carga máximo de su acelerador del 4\%, de dar servicio a diferentes técnicas neutrónicas.

Para hacer este análisis, el mencionado estudio evalúa si dicha fuente puede generar el mismo flujo en instrumento que el empleado en determinados instrumentos neutrónicos actualmente en operación. Esta evaluación se realiza empleando el código de simulación de instrumentos de dispersión neutrónica McStas [215]. Los cálculos efectuados en [214] parten de la base de que una fuente de estas características podría acercar suficientemente el comienzo de las guías experimentales a la superficie del moderador. Con esta premisa, se estima que la fuente podría albergar 5 instrumentos. Ambas hipótesis son compatibles 
con nuestra configuración de referencia.

Replicar el análisis efectuado en [214] para las particularidades de la fuente propuesta para ESS-Bilbao está fuera del alcance del presente trabajo. No obstante, podemos asumir razonablemente que los flujos en instrumento calculados en [214] escalan linealmente con el brillo de neutrones térmicos empleado como dato de partida para su estudio. Así pues, teniendo en cuenta el valor de brillo de neutrones térmicos de $1,4 \cdot 10^{11} \mathrm{n} / \mathrm{cm}^{2} \cdot \mathrm{sr} \cdot \mathrm{s}$ calculado en la Sección 4.4.3, los flujos en instrumento esperables en ESS-Bilbao serán un 12,5\% menores a los calculados en [214]. Empleando esta extrapolación, se confecciona la Tabla 4.6 en la que se comparan los flujos esperables extrapolados en ESS-Bilbao, con los flujos necesarios para los diversos instrumentos. Esta comparación se detalla a continuación.

Con el fin de evaluar la capacidad de la fuente para el desarrollo de técnicas neutrográficas, se toman como referencia los instrumentos ICON [216, 217], de la fuente SINQ, y CONRAD [218, 219], del centro Helmholt de Berlin (HZB [220]); cuya fuente de neutrones es el reactor BER-II [221]. Ambos instrumentos efectúan en pocas horas tomografía de neutrones sin discriminación en energía, con un flujo en los instrumentos de $1 \cdot 10^{7} \mathrm{n} / \mathrm{cm}^{2} s$. El flujo esperable en ESS-Bilbao alcanzaría en un área de exposición de 25 x $25 \mathrm{~cm}^{2}$ unos valores de $1,75 \cdot 10^{7} \mathrm{n} / \mathrm{cm}^{2} s$ con baja uniformidad de iluminación, y de $1,75 \cdot 10^{6} \mathrm{n} / \mathrm{cm}^{2} s$ con una uniformidad de iluminación análoga a la del instrumento CONRAD de HZB. La uniformidad del flujo de neutrones viene determina por la relación entre la longitud de la guía de neutrones, L, y la dimensión característica del área de exposición, D. Por baja uniformidad nos referimos a una relación $\mathrm{L} / \mathrm{D}=80$. La uniformidad de iluminación del instrumento CONRAD de HBZ es L/D = 240. Así pues, el tiempo necesario para llevar a cabo tomografías neutrónicas en ESS-Bilbao variaría entre unas horas y pocos días en función de la uniformidad deseada y del tamaño de la muestra.

Es relevante destacar que la tomografía neutrónica es una técnica que requiere de mayores tiempos de exposición que la neutrografía simple, porque emplea multitud de imágenes tomadas en distintos planos. La fluencia necesaria para tomar neutrografías individuales de alta calidad se cifra en $[214]$ en $2,0 \cdot 10^{9} \mathrm{n} / \mathrm{cm}^{2}$, por lo que se podrían obtener en ESS-Bilbao en menos de 20 minutos de exposición.

Respecto a la reflectometría de neutrones, se toman como referencia los instrumentos POLREF [222], de la fuente ISIS, y HERMES, [223] del Laboratorio Léon Brillouin 
(LLB [224]); centro en el que se encuentra el reactor experimental Orphée [225]. Ambos instrumentos emplean flujos de $10^{7}$ y $8 \cdot 10^{6} \mathrm{n} / \mathrm{cm}^{2} s$ respectivamente. De acuerdo con la extrapolación propuesta, el flujo útil para estos instrumentos esperable en ESS-Bilbao alcanzaría los $7 \cdot 10^{6} \mathrm{n} / \mathrm{cm}^{2} s$. Para el análisis de esta técnica, [214] ha considerado el empleo de una tasa de repetición de $20 \mathrm{~Hz}$ con pulsos de $2 \mathrm{~ms}$, obteniendo resoluciones de entre el $5 \%$ y el $15 \%$ para longitudes de onda de entre $3 \AA$ y $16 \AA$, en función de la configuración final del experimento. Este proceso de optimización debería particularizarse para el caso de ESS-Bilbao, sin embargo, dada la similitud del rango operativo y el valor de flujo extrapolado, la aplicación de técnicas de reflectometría de neutrones parece al alcance de la instalación propuesta.

La técnica de dispersión de ángulo pequeño (SANS) es ampliamente aplicada en física de materia blanda y metalurgia. Los instrumentos tomados como referencia para esta técnica son el instrumento PAXE [226], del LLB, y el instrumento SANS2D, [227] de ISIS; que operan con flujos de $7 \cdot 10^{5} \mathrm{n} / \mathrm{cm}^{2} s$ y $1 \cdot 10^{6} \mathrm{n} / \mathrm{cm}^{2} s$ respectivamente. Siguiendo la aproximación planteada para esta estimación, la propuesta de ESS-Bilbao podría proporcionar flujos entre $8,75 \cdot 10^{5} \mathrm{n} / \mathrm{cm}^{2} s$ para configuraciones con bajo vector dispersión, Q, y los $7,9 \cdot 10^{6} \mathrm{n} / \mathrm{cm}^{2} s$ para alto Q. Para el análisis de esta técnica, [214] ha considerado de nuevo el empleo de una tasa de repetición de $20 \mathrm{~Hz}$ con pulsos de $2 \mathrm{~ms}$, obteniendo rangos de resolución y longitud de onda análogos a los de los instrumentos tomados como referencia. Una vez más este proceso de optimización debería particularizarse para nuestro caso, si bien la aplicación de la técnica SANS en ESS-Bilbao parece factible.

Para estudiar la potencial aplicación de las técnicas de difracción de polvos y cristales, el trabajo [214] busca replicar el flujo en el instrumento G4-1 [228], del LLB. Este instrumento es un difractómetro de polvos de alto flujo y baja resolución, usado normalmente para estudiar las transiciones de fase en cristales. Empleando una longitud de pulso de $250 \mu \mathrm{s}$, y una tasa de repetición de $40 \mathrm{~Hz}$ (factor de carga 1\%), [214] consigue replicar los $2 \cdot 10^{6}$ $\mathrm{n} / \mathrm{cm}^{2} \mathrm{~s}$ en un rango de longitud de onda entre los $1,4 \AA$ y los $3,3 \AA$, necesarios en el mencionado instrumento.

Generar un flujo análogo en la fuente de ESS-Bilbao requeriría una reducción de la longitud de pulso y un aumento de la frecuencia del acelerador. Dado que este régimen de operación supone una reducción del factor de carga del acelerador, y por tanto de la 
potencia del haz, esta modificación parece a priori factible desde un punto de vista del blanco. Con el objetivo de tener una primera estimación conservadora de la capacidad del diseño de la fuente de ESS-Bilbao para trabajar con pulsos más cortos, se ha calculado el efecto de esta reducción manteniendo constante el factor de carga del acelerador en el $3 \%$, y por tanto la potencia de referencia de 112,5 $\mathrm{kW}$. Los resultados, disponibles en el Apéndice B, muestran que las temperaturas y tensiones máximas en las placas de berilio tienden a un valor medio similar al de la configuración de referencia. Así pues, el diseño del blanco podría acomodar pulsos más cortos, especialmente si van acompañados de una reducción en el factor de carga.

Teniendo en cuenta que [214] ha podido replicar el flujo del instrumento G-41 reduciendo el factor de carga del acelerador al 1\%, es decir, produciendo un $25 \%$ de su máximo término fuente, es esperable que la fuente de ESS-Bilbao pueda replicarlo también, por lo que las técnicas de difracción serían aplicables en nuestra propuesta de fuente.

Las técnicas de espectroscopía por dispersión inelástica emplean la baja energía de los neutrones fríos para estudiar excitaciones que van desde los $n e V$, con técnicas spin-echo, hasta los 0,1 eV, empleado técnicas de espectroscopía de triple eje. De acuerdo a [214], este último tipo de técnica, la espectroscopía de triple eje, sólo se ha implementado con éxito en reactores; de hecho, ninguna de las grandes fuentes pulsadas la emplea, por tanto no se considera para este estudio.

Para analizar la capacidad de ESS-Bilbao para emplear la técnica espectroscopía spinecho, se toma como referencia el instrumento MUSES [229] del LLB. Este instrumento emplea un flujo de neutrones de $5 \AA$ de $2 \cdot 10^{7} \mathrm{n} / \mathrm{cm}^{2} \mathrm{~s}$. De acuerdo a la extrapolación planteada, la fuente de ESS-Bilbao podría producir un flujo equivalente de $2,6 \cdot 10^{6} \mathrm{n} / \mathrm{cm}^{2} s$, por lo que a priori esta técnica no podría implementarse en la fuente propuesta. Cabe resaltar que el estudio presentado en [214] considera que las mejoras en el horizonte próximo de los instrumentos de spin-echo, permitirán llevar a cabo este tipo de experimentación con flujos como los esperables en ESS-Bilbao, sin embargo, se ha preferido estimar conservadoramente la disponibilidad de estas técnicas en nuestra propuesta.

En el rango intermedio de las técnicas espectroscópicas por dispersión inelástica se encuentra la espectroscopía por tiempo de vuelo (ToF). Esta técnica provee medidas en el rango entre los $20 \mu \mathrm{eV}$ y los $1 \mathrm{meV}$. El instrumento tomado como referencia en [214] 
para cuantificar el flujo necesario para esta técnica es el instrumento OSIRIS [230, 231], de la fuente ISIS, un espectrómetro de geometría inversa (backscattering ToF) que opera con un flujo de $3 \cdot 10^{7} \mathrm{n} / \mathrm{cm}^{2} s$. Siguiendo la aproximación planteada para esta estimación, el flujo esperable en un instrumento análogo operando en ESS-Bilbao sería de 5,3 $\cdot 10^{7}$ $\mathrm{n} / \mathrm{cm}^{2} s$, por lo que esta técnica sería aplicable en la instalación propuesta.

\begin{tabular}{|c|c|c|c|c|}
\hline Técnica & Intrumento & Centro & $\begin{array}{l}\text { Flujo necesario } \\
{\left[\mathrm{n} / \mathrm{cm}^{2} s\right]}\end{array}$ & $\begin{array}{l}\text { Flujo esperable } \\
\text { ESS-B }\left[\mathrm{n} / \mathrm{cm}^{2} s\right]\end{array}$ \\
\hline \multirow{2}{*}{$\begin{array}{l}\text { Tomografía } \\
\text { sin discriminación en E }\end{array}$} & ICON & PSI & $1.00 \mathrm{E}+7$ & \multirow{2}{*}{$\begin{array}{c}1.75 \mathrm{E}+7(\mathrm{~L} / \mathrm{D}=80) \\
1.75 \mathrm{E}+6(\mathrm{~L} / \mathrm{D}=240)\end{array}$} \\
\hline & CONRAD & HZB & $1.00 \mathrm{E}+7$ & \\
\hline \multicolumn{5}{|l|}{ Dispersión elástica } \\
\hline \multirow[t]{2}{*}{ Reflectometría } & HERMES & LLB & $8.00 \mathrm{E}+6$ & \multirow[t]{2}{*}{$7.00 \mathrm{E}+6$} \\
\hline & POLREF & ISIS & $1.00 \mathrm{E}+7$ & \\
\hline \multirow[t]{2}{*}{ SANS } & PAXE & LLB & $7.00 \mathrm{E}+5$ & $8.75 \mathrm{E}+5$ (Bajo Q) \\
\hline & SANS2D & ISIS & $1.00 \mathrm{E}+6$ & $7.88 \mathrm{E}+6$ (Alto Q) \\
\hline Difraccion polvo & G41 & LLB & $2.00 \mathrm{E}+6$ & $2.00 \mathrm{E}+6$ \\
\hline \multicolumn{5}{|l|}{ Dispersion intelástica } \\
\hline Spin-Echo & MUSES & LLB & $2.00 \mathrm{E}+7$ & $2.63 \mathrm{E}+6$ \\
\hline Backscattering ToF & OSIRIS & ISIS & $3.00 \mathrm{E}+7$ & $5.25 \mathrm{E}+7$ \\
\hline
\end{tabular}

TABLA 4.6: Comparación entre flujo en instrumento para diversas técnicas neutrónicas y flujo esperable en ESS-Bilbao estimado en base a [214].

\section{Conclusiones del capítulo}

En este capítulo se han analizado y decidido las principales opciones de diseño necesarias para convertir el término fuente producido por el blanco de ESS-Bilbao en una fuente de neutrones útiles para experimentación con neutrones fríos.

Para ello, tras analizar la distribución espacial de la producción neutrónica, se ha optado por situar el blanco a $45^{\circ}$ del haz, de tal manera que tres líneas experimentales sean fácilmente acomodables; una centrada en el punto de impacto de los protones para neutrones rápidos y dos centradas en el moderador para neutrones fríos.

Con esta disposición como referencia, se ha elegido el metano sólido criogénico como moderador, dadas sus excelentes propiedades moderadoras. Teniendo en cuenta la intensidad de la fuente y la energía de los neutrones producidos, se decide emplear la configuración 
"slab" por su mejor rendimiento. El proceso de optimización llevado a cabo concluye que $2 \mathrm{~cm}$ es el espesor óptimo para la producción de neutrones fríos.

Diversas opciones han sido cotejadas para material reflector. El material escogido como referencia es el berilio, no obstante, otras opciones como agua o plomo podrían tenerse en cuenta en base a consideraciones económicas o relacionadas con la forma del pulso de neutrones.

Con estas elecciones de diseño, la configuración de referencia propuesta para la fuente de ESS-Bilbao es capaz de producir un brillo en el moderador de neutrones de energía $\approx 5 \mathrm{meV}$ de $3,6 \cdot 10^{10} \mathrm{n} / \mathrm{cm}^{2} \cdot \mathrm{sr} \cdot \mathrm{s}, \mathrm{y}$ un brillo de neutrones de $E<0,4 \mathrm{eV}$ de $1,3 \cdot 10^{11}$ $n / \mathrm{cm}^{2} \cdot s r \cdot s$. Teniendo en cuenta el menor término fuente de neutrones y la mucho menor energía de los protones empleados, se concluye que el diseño propuesto constituye una fuente altamente eficiente, en comparación con otras fuentes medianas y grandes.

Sobre esta configuración de referencia, diversas configuraciones avanzadas se han analizado. De acuerdo a estas evaluaciones, la inclusión de un filtro de berilio supondría un aumento en el flujo de neutrones fríos de $\approx 50 \%$. La inclusión de un segundo moderador de idénticas características al de referencia supondría también incrementar la producción total de neutrones fríos un $\approx 50 \%$, y facilitaría la inclusión de al menos dos líneas experimentales más. La inclusión de dos moderadores con sendos filtros de berilio, además de permitir las líneas experimentales suplementarias, duplicaría la producción de neutrones fríos. Por último se ha evaluado la capacidad de la fuente para producir neutrones térmicos entre los 10 y los $600 \mathrm{meV}$. Los análisis muestran que empleando un moderador de agua ligera de $4 \mathrm{~cm}$ de grosor, la producción de neutrones térmicos alcanzaría los $1,4 \cdot 10^{11}$ $n / \mathrm{cm}^{2} \cdot s r \cdot s$.

La producción de neutrones alcanzada permite cubrir todas las aplicaciones que normalmente se dan en fuentes compactas. De acuerdo a las estimaciones realizadas, los valores de brillo de neutrones fríos obtenidos hacen también factible la aplicación de técnicas de dispersión neutrónica, normalmente sólo disponibles en fuentes de espalación y reactores experimentales. 


\section{Capítulo 5}

\section{Futuros trabajos}

\section{Resumen}

En 2015, el gobierno central español y la administración autonómica vasca acordaron con la dirección de la fuente europea de espalación ESS reducir su participación al proyecto, de un $10 \%$ a un $5 \%$ del presupuesto total de la instalación. El acuerdo incluyó definir la materialización de la contribución en especie al proyecto mediante el diseño y suministro de componentes a la fuente sueca.

Esta decisión supuso el aplazamiento sine díe de la construcción de la fuente en ESSBilbao. Sin embargo, en los últimos años el interés por fuentes similares ha crecido, sobre todo en Europa. En paralelo, ESS-Bilbao ha decidido hacer un prototipo para la validación de los aspectos termohidráulicos del concepto.

En este capítulo se introducen los proyectos que en la actualidad buscan materializar en Europa una fuente de características similares a la propuesta para ESS-Bilbao, y se da una descripción general del prototipo de su blanco. El Apéndice C presenta sus planos de fabricación.

\subsection{Una nueva generación de fuentes: fuentes de alto brillo}

Como se ha demostrado en los capítulos anteriores, es posible obtener valores de brillo neutrónico útil proporcionalmente muy altos en comparación con el término fuente de 
neutrones, mediante el empleo de reacciones de extracción. Esta reacción, junto con el uso de moderadores optimizados y la cercanía del comienzo de las líneas experimentales al moderador, abren la puerta a la configuración de fuentes compactas de alta eficiencia.

Las fuentes con estas características han venido a ser denominadas como fuentes compactas de alto brillo (High Brilliance CANS, Compact Accelerator-driven Neutron Source) y han suscitado un particular interés, especialmente ante la dificultad para renovar los reactores experimentales próximos a terminar su vida útil.

Los proyectos europeos SONATE y HBS buscan materializar una fuente de estas características.

\subsubsection{SONATE}

En los últimos años el laboratorio francés LLB, participado por el Comisionado de la Energía Atómica francés (CEA [232]) y el Centro Nacional para la Investigación Científica francés (CNRS [233]), ha estado dando los primeros pasos para la construcción de una fuente compacta de alto brillo. El proyecto es conocido como SONATE [234, 235].

La motivación de este proyecto viene dada por la próxima clausura del reactor Orphée [225], operativo desde 1980, prevista para finales de la presente década. Este reactor de $14 M W$, con un núcleo de uranio de alto enriquecimiento, da servicio actualmente en el LLB a cerca de 20 instrumentos. Desde un punto de vista temporal, el proyecto SONATE, plantea tener la fuente en operación en 2025, a tiempo para la clausura del reactor HFR del ILL.

La Figura 5-1 muestra las estimaciones realizadas en [236] sobre la disponibilidad de tiempo de instrumento al año para usuarios franceses en distintas instalaciones, magnitud medida en instrumento·día. De acuerdo a ese trabajo, los $\sim 300$ instrumento·día correspondientes a usuarios franceses obtenidos con el inicio de operación de ESS no paliarán la caída de disponibilidad originada por la clausura de los dos reactores franceses. El proyecto SONATE plantea proporcionar $\sim 1800$ instrumento·día, reponiendo prácticamente el tiempo de experimentación perdido por la futura clausura del ILL.

Para ello se plantea la construcción de una fuente con un blanco de berilio y un haz de $80 \mathrm{~kW}$ de potencia, con protones de $20 \mathrm{MeV}$. La longitud del pulso se plantea variable 


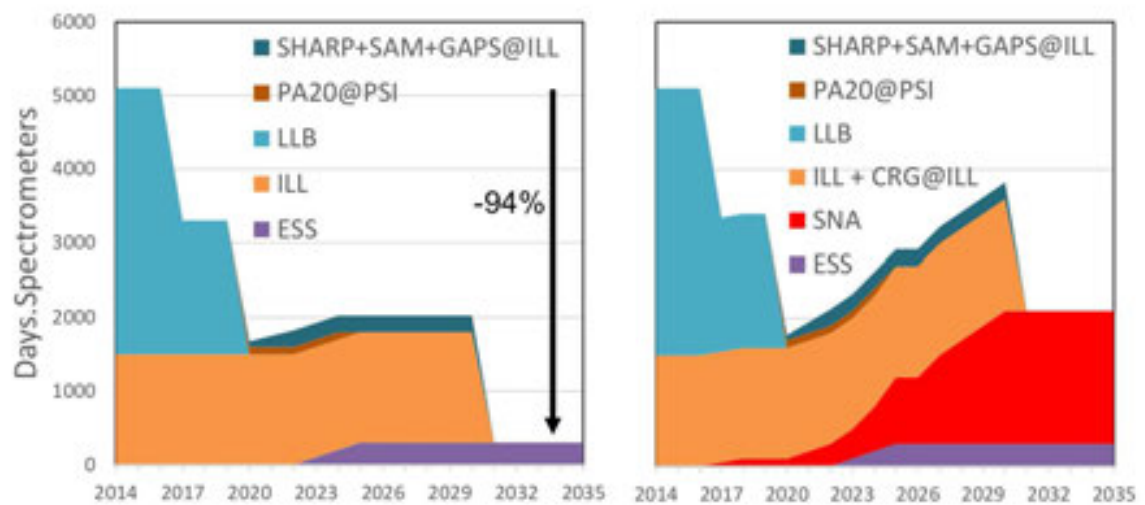

Figura 5-1: Evolución de la disponibilidad de tiempo de instrumento para usuarios franceses [236] a partir de datos de [237].

dentro de un factor de carga del acelerador del $4 \%$.

El diseño de la fuente aún se encuentra en estado conceptual, no obstante, dentro del marco de este proyecto, se están llevando a cabo estudios como el referenciado en el Capítulo 4 para evaluar la capacidad de este tipo de fuente para dar servicio a instrumentos de dispersión neutrónica [214], así como primeros experimentos de generación de neutrones con protones de $3 \mathrm{MeV}$, fundamentalmente orientados a la validación de las herramientas de simulación [238].

El proyecto SONATE estima su coste de construcción en $\sim 50 M €$ y su coste de operación en $4 M €$ anuales [236]. Estos valores son muy inferiores a los costes de construcción y operación de ILL, $2000 M €$ y $95 M €$ anuales, o ISIS, $800 M €$ y $62 M €$ anuales [237].

\subsubsection{HBS, High Brilliance Source}

Desde 2016, el centro de ciencia neutrónica de Jülich (JCNS [239]) ha comenzado un proyecto para el desarrollo y diseño de una fuente compacta guiada por acelerador de alto brillo.

El JCNS considera este tipo de fuentes una alternativa técnica y económicamente eficiente a los reactores de flujo medio y a las fuentes de espalación y, por tanto, una forma de facilitar el acceso a las técnicas neutrónicas [240].

La configuración de referencia planteada en este proyecto parte de un haz de protones 
o deuterones de energías entre los 25 y los $50 \mathrm{MeV}$, con una potencia de $100 \mathrm{~kW}$ y un factor de carga del $4 \%$, que impacta sobre un blanco de berilio. El blanco se encontraría embebido en un moderador de polietileno con moderadores fríos compactos al comienzo de líneas experimentales muy próximas al blanco.

La clave de esta propuesta reside en la idea de diseñar de forma integral toda la instalación, orientándola a la optimización de una técnica experimental concreta. De tal manera que experimento, sistema de extracción de neutrones, blanco y acelerador estén coordinados para maximizar la eficacia de la experimentación realizada.

Diseñando un acelerador versátil se podrían producir pulsos ad hoc para distintas técnicas neutrónicas y, mediante un multiplexador, enviar los pulsos a distintos blancos optimizados. Cada uno de estos blancos darían servicio a un pequeño número de instrumentos de un determinado tipo, que recibirían un pulso de neutrones optimizado para la técnica experimental empleada. Este concepto, ilustrado en la Figura 5-2, es radicalmente distinto al de las grandes instalaciones, donde un moderador voluminoso da servicio a multitud de líneas experimentales que parten relativamente lejos de este, con una única configuración de pulso de neutrones. Esta aproximación, a pesar de requerir múltiples blancos, puede ser más eficiente en términos de coste gracias a la sencillez de estos, la baja activación de la instalación y el menor tamaño de los blindajes necesarios.

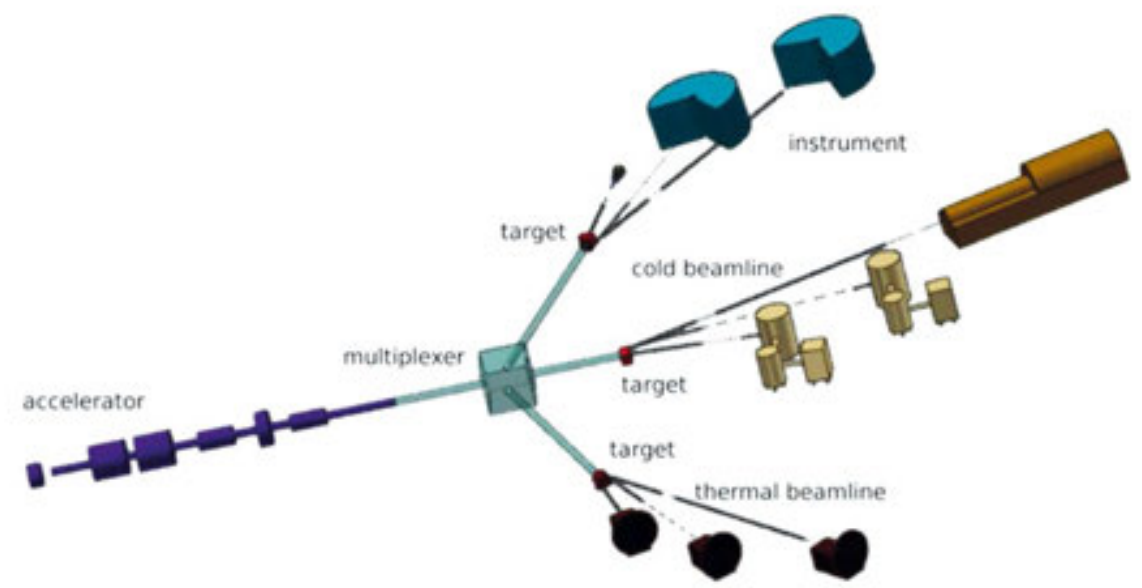

Figura 5-2: Concepto de fuente de alto brillo con blancos múltiples [241]. 


\subsection{Prototipo termohidráulico}

Con el objetivo de comprobar el comportamiento termohidráulico real del diseño propuesto para ESS-Bilbao, se ha procedido a efectuar un prototipo centrado en estos aspectos. Para ello, se ha tomado un sector de la estructura del blanco, y se le han implementado las modificaciones pertinentes para convertirlo en una estructura independiente. Esta geometría permite simular la dinámica del fluido en el blanco real, así como la evolución de temperaturas en la placa, sin necesidad de desarrollar el sistema motor y reduciendo sustancialmente el peso y coste de la estructura. Las Figuras 5-3 y 5-4 muestran una imagen del diseño CAD del prototipo, y una comparativa con un sector de la estructura diseñada.

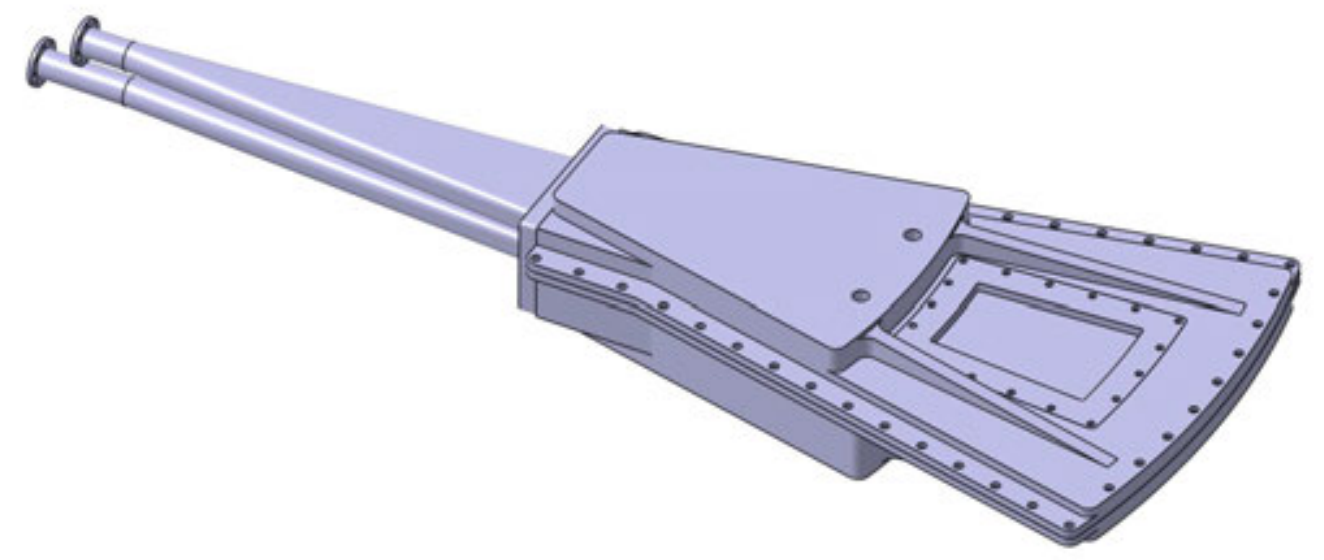

Figura 5-3: Vista general del prototipo del blanco.

La campaña experimental aún está por definir con precisión. No obstante, se centrará en la validación de los cálculos termomecánicos y termohidráulicos presentados en este trabajo, así como en la exploración de los márgenes del diseño de la refrigeración. La particular naturaleza de la deposición energética del haz de protones, especialmente en lo referente a su distribución espacial y temporal, es difícil de replicar. Sin embargo, se prevé que el empleo de una pistola de plasma pueda generar valores de flujo térmico representativos. Si se dispusiera de tiempo de un haz relevante, se podría proceder a realizar también campañas de irradiación.

Para definir el prototipo, las modificaciones implementadas en la estructura del blanco buscan fundamentalmente crear el sistema de nuevas juntas necesario para garantizar la 

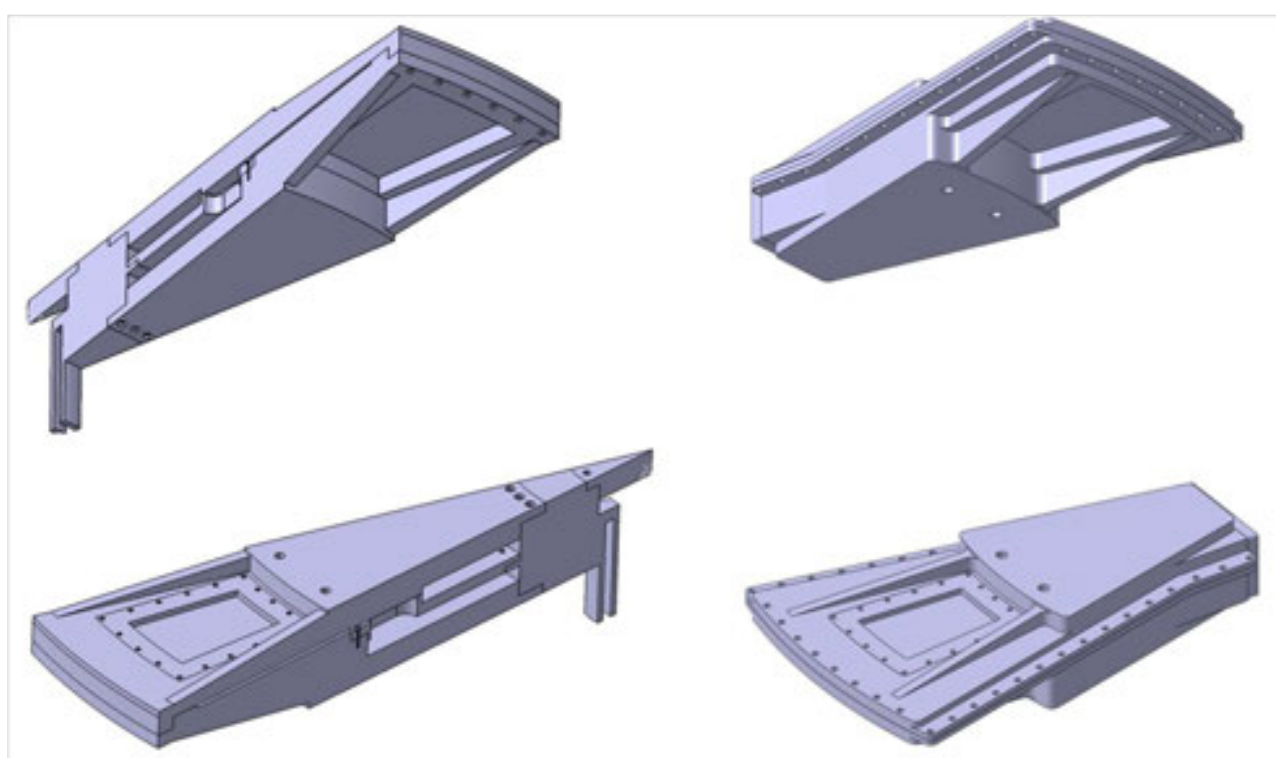

Figura 5-4: Comparativa entre un sector del blanco de ESS-Bilbao y el prototipo.

estanquidad. Dado que no se prevé la realización de pruebas con niveles de irradiación elevados, se opta por el uso de sellos elastoméricos. El alojamiento para el blanco de berilio se ha dimensionado para poder albergar una placa de $11 \mathrm{~mm}$ de espesor. Con esto se busca hacer posible la medida de la producción neutrónica máxima, dado que en una corta campaña experimental de irradiación, la implantación de los protones no supondría un problema. También se ha optado por hacer pasantes los tornillos que unen los discos superior e inferior. El prototipo no incluye la rueda central de distribución y colección de refrigerante, por lo que se añaden dos difusores. Su función es acondicionar el flujo de agua, y adaptar la secciones de entrada y salida a bridas circulares convencionales. La Figura 5-6 muestra una vista explosionada de los componentes del prototipo, donde se pueden apreciar las diferencias con el diseño propuesto en el Capítulo 3 (Figura 3-6).

La estructura del prototipo se ha efectuado en la aleación de aluminio 6082-T6 [161], de similar composición y propiedades a la aleación de referencia 6061-T6, pero más fácilmente accesible y de menor coste.

La Figura 5-5 muestra una imagen del prototipo ensamblado incluyendo los difusores. La Figura 5-7 muestra los componentes principales del prototipo. El peso total del conjunto es de $\sim 60 \mathrm{~kg}$ y sus dimensiones mayores aproximadas, sin contar los difusores, son $50 \mathrm{~cm}$ de ancho, $75 \mathrm{~cm}$ de largo y 15,5 cm de espesor. Contando los difusores su longitud alcanza 
1,34 m. El Apéndice C presenta los planos de fabricación del prototipo.

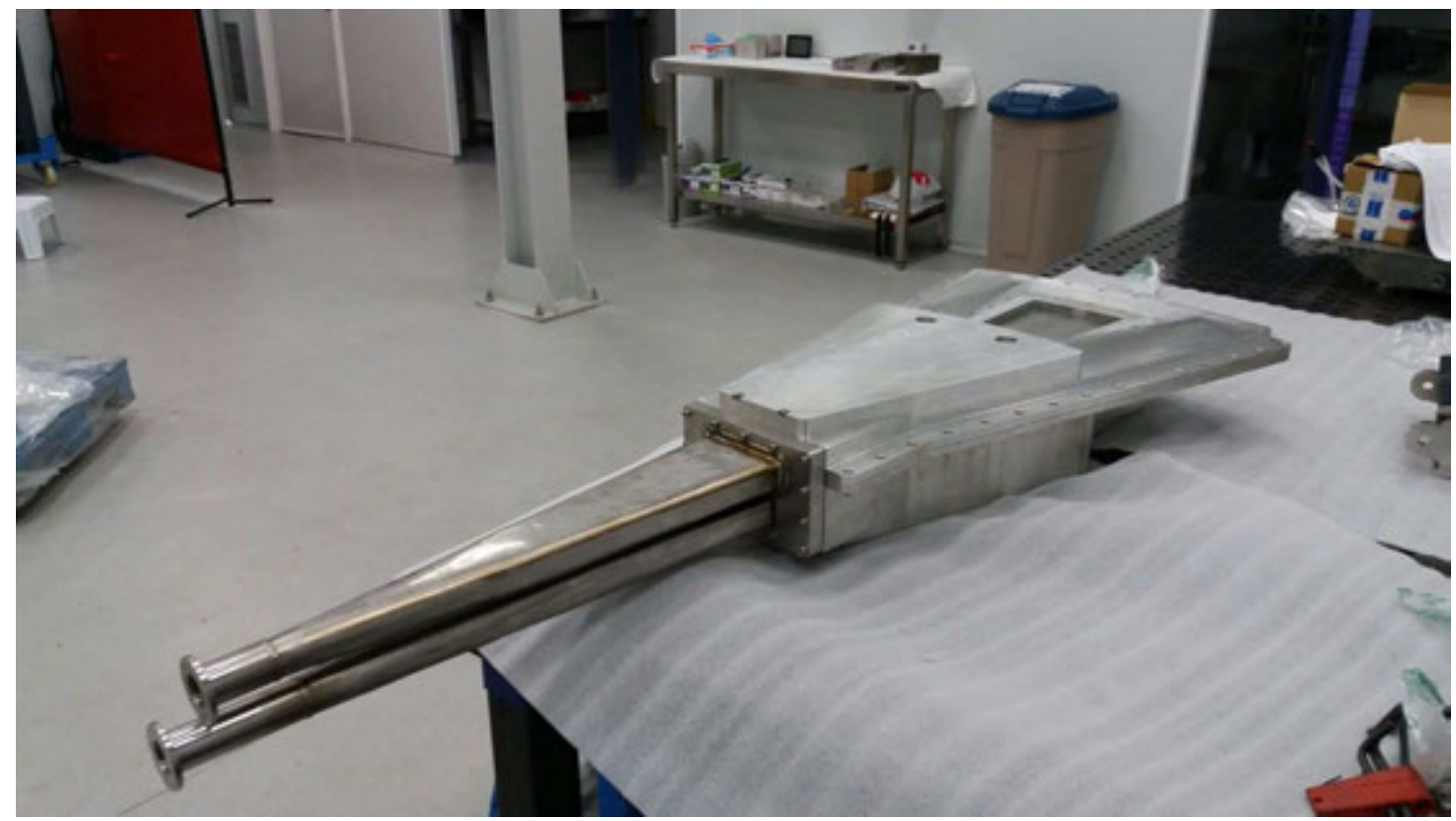

Figura 5-5: Prototipo ensamblado. 


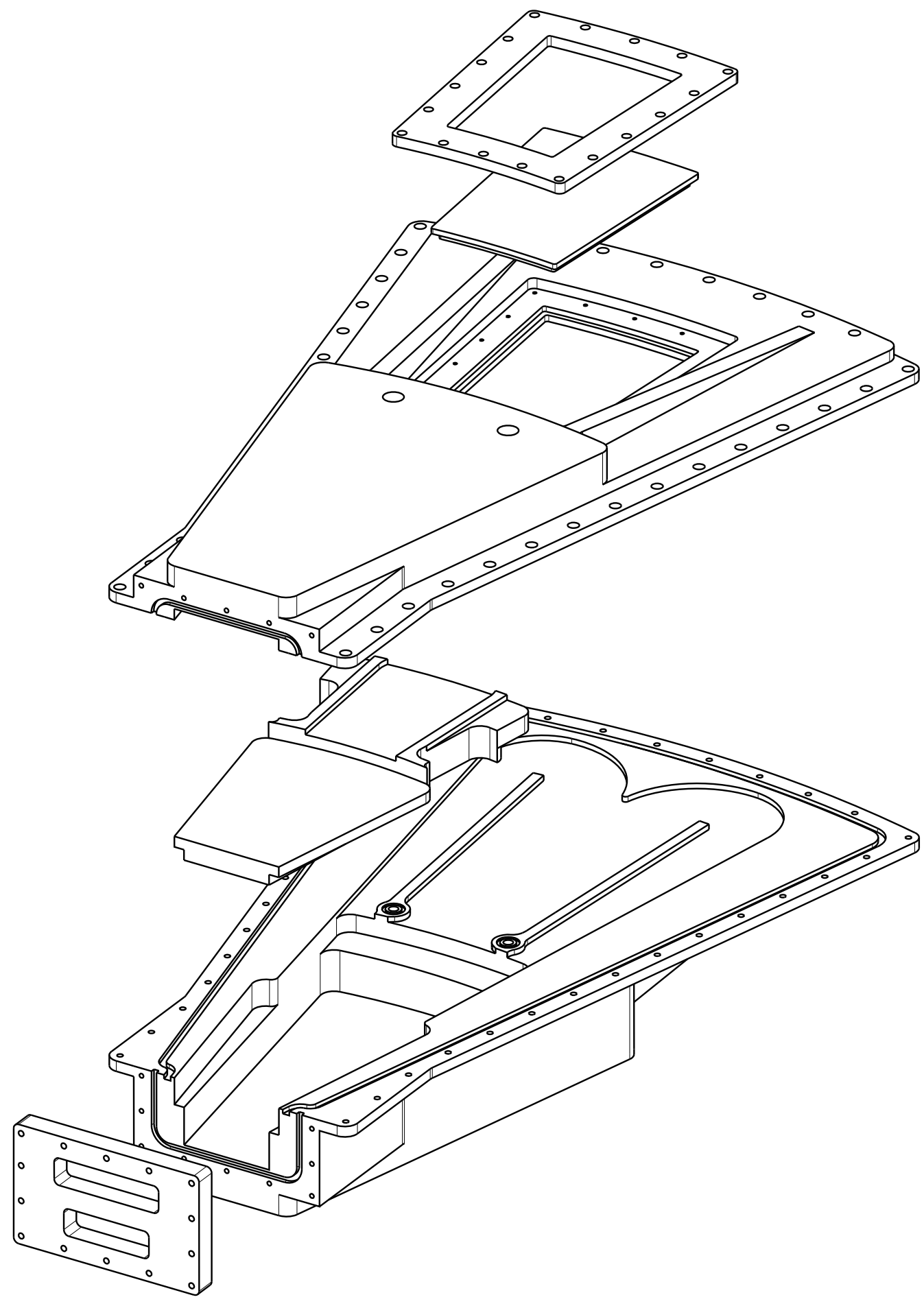

FigurA 5-6: Vista explosionada del prototipo. 

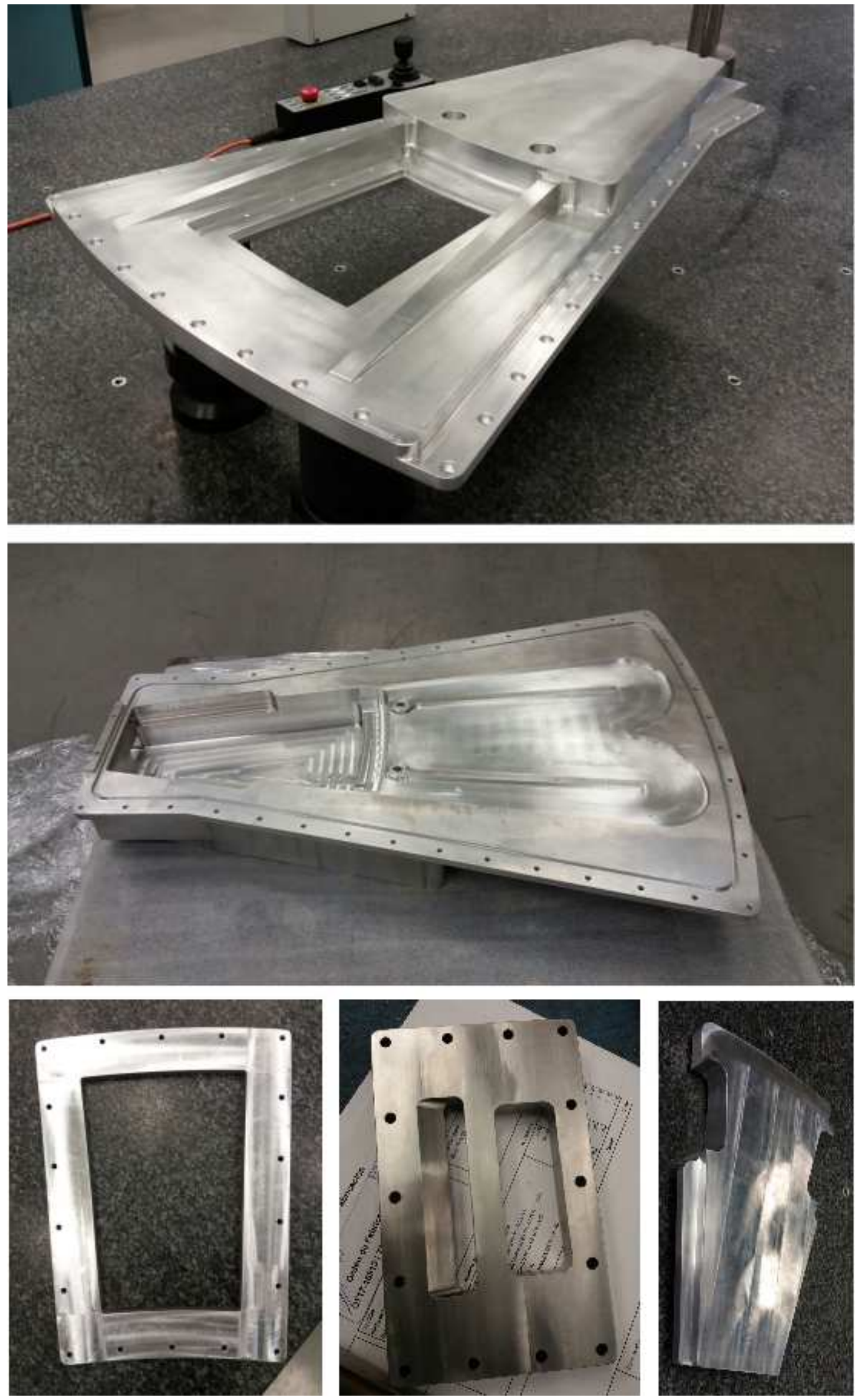

Figura 5-7: Componentes del prototipo fabricados. 



\section{Capítulo 6}

\section{Conclusiones}

Partiendo de los parámetros del acelerador seleccionado para ESS-Bilbao como dato de entrada, se ha desarrollado y optimizado un concepto de blanco de producción de neutrones, se han decidido las especificaciones de los componentes principales encargados de hacer la producción neutrónica útil para experimentación, y se ha evaluado la capacidad de la fuente así definida para dar servicio a diversas técnicas neutrónicas.

El acelerador seleccionado para ESS-Bilbao busca representar las primeras etapas de la cadena de aceleración de la fuente europea de espalación ESS. Es por tanto un acelerador de alta corriente y pulso largo $(2,25 \mathrm{~mA}$ y $1,50 \mathrm{~ms})$, en el que los protones alcanzan los $50 \mathrm{MeV}$. Con estos parámetros, el haz tiene una potencia de $112,5 \mathrm{~kW}$, lo cual sitúa la fuente en un rango de intensidad media en el panorama de las fuentes de neutrones. Con un perfil gaussiano de diámetro $10 \mathrm{~cm}$, el haz de partículas transporta un flujo térmico medio de $14,3 \mathrm{MW} / \mathrm{m}^{2}$, con un valor máximo del doble en su eje central.

Para maximizar la producción neutrónica, se escoge como material del blanco el berilio. En el rango de energía alcanzada por los protones, este material produce el mayor número de neutrones por protón incidente mediante reacciones de extracción (stripping). La refrigeración del blanco se efectúa mediante flujo forzado monofásico de agua a 5 bar en contacto directo con el berilio. Dado el alto flujo que transporta el haz, se opta por un blanco rotatorio, de tal manera que la potencia del haz se reparta en una mayor superficie.

La optimización termomecánica efectuada muestra que una configuración rotatoria con 20 placas de un espesor de $9 \mathrm{~mm}$, genera un estado estable de operación en términos 
de tensión y temperaturas, con unos amplios márgenes de diseño. La disposición de 20 placas hace que cada una de ellas reciba una frecuencia de impacto de $1 \mathrm{~Hz}$, para una frecuencia del acelerador de $20 \mathrm{~Hz}$. Los valores máximos de tensiones en esta configuración se mantienen entorno a los $105 M P a$, muy por debajo del límite elástico y del límite de fatiga del grado comercial de berilio seleccionado (S-200 F), $250 M P a$ y $262 M P a$ respectivamente. Los resultados de temperatura muestran un valor máximo en la placa de $106{ }^{\circ} \mathrm{C}$, valor inferior a la temperatura de ebullición del refrigerante $152{ }^{\circ} \mathrm{C}$.

La optimización termomecánica para la selección del espesor ha tenido en cuenta la producción neutrónica y la implantación de los protones en el berilio. Un espesor de $9 \mathrm{~mm}$ deja el pico de Bragg de la deposición energética fuera del blanco, permitiendo que los protones atraviesen la placa. Esta decisión de diseño reduce la potencia implementada en el berilio un $\sim 32 \%$ y el valor máximo de tensión un $\sim 45 \%$, disminuyendo la producción neutrónica únicamente un $\sim 12 \%$, con respecto a los valores que aparecerían en una placa que detuviese completamente los protones. Impedir que los protones se implanten en el berilio evita la aparición de ampollas de hidrógeno en el material y el consecuente fallo. La durabilidad de las placas ha sido evaluada frente a fragilización e hinchamiento por irradiación, así como frente a fatiga. Las estimaciones más conservadoras cifran la vida útil del blanco en al menos 4 años, asumiendo 2000 horas de operación por año. Con esta configuración, la producción de neutrones de la fuente propuesta es de $8,0 \cdot 10^{14}$ neutrones por segundo.

Para materializar este concepto se ha diseñado una estructura rotatoria en forma de disco, capaz de albergar las 20 placas de berilio y proporcionar la refrigeración adecuada. Los componentes de la estructura se ensamblan conformado entre ellos los canales de refrigeración. Esta solución evita la necesidad de mecanizar complejos canales internos y hace todas las piezas realizables por técnicas de mecanizado 2.5D. El material elegido para esta estructura es la aleación de aluminio 6061-T6, por su combinación de buenas propiedades mecánicas, y bajas absorción neutrónica y activación radiactiva. El proceso de diseño iterativo seguido ha concluido con la definición de una estructura capaz de soportar las cargas termomecánicas y de presión con solvencia, y de una geometría de canales internos que mantiene las condiciones de refrigeración adecuadas. El sistema de distribución de refrigerante garantiza un flujo uniforme de agua a $\sim 2,5 \mathrm{~m} / \mathrm{s}$ bajo las placas de berilio, para un caudal en toda la estructura de $26 \mathrm{l} / \mathrm{s}$. La estructura final tiene 
$\sim 1 m$ de radio y pesa aproximadamente una tonelada. El comportamiento del diseño del blanco ha sido evaluado frente a diversos sucesos accidentales. Los análisis muestran que el diseño cuenta con márgenes suficientes para dar tiempo a los sistemas de detección y parada de emergencia antes de fallo.

Con el objetivo de comprobar el comportamiento termohidráulico de la estructura del blanco, un prototipo representativo de uno de los 20 sectores circulares del disco ha sido fabricado. A fecha de redacción de este trabajo, la campaña experimental de este prototipo no ha comenzado.

La fuente de neutrones propuesta se completa dotando al blanco de un moderador optimizado de metano sólido en configuración "slab", de $12 \times 12 \mathrm{~cm}^{2}$ de cara y $2 \mathrm{~cm}$ de espesor. Este material y configuración son las opciones más eficientes, no obstante las grandes fuentes de espalación no las implementan debido a su agresivo ambiente de irradiación. En cambio, el diseño de ESS-Bilbao sí permite emplearlas, lo cual redunda en su eficiencia final. En su configuración de referencia, el moderador se rodea de un reflector de berilio de $40 \mathrm{~cm}$. Con un ángulo de incidencia de $45^{\circ}$ entre el blanco y el haz, la fuente propuesta puede acomodar fácilmente tres líneas experimentales partiendo de las inmediaciones del moderador.

Con esta configuración como referencia, el brillo de neutrones de energía $\approx 5 \mathrm{meV}$ en el moderador alcanza un valor de $3,6 \cdot 10^{10} \mathrm{n} / \mathrm{cm}^{2} \cdot \mathrm{s} \cdot \mathrm{sr}$. El brillo de neutrones de energía inferior a los $0,4 \mathrm{eV}$ es de $1,3 \cdot 10^{11} \mathrm{n} / \mathrm{cm}^{2} \cdot \mathrm{sr} \cdot \mathrm{s}$. Este valor supone un $\sim 25 \%$ del brillo de la segunda estación de blanco de la fuente de espalación ISIS (UK) y es un factor $\approx 30$ inferior al de las grandes instalaciones SNS (EE.UU) o JSNS (Japón). Sin embargo, teniendo en cuenta que los términos de producción total de neutrones de las grandes fuentes de espalación son un factor $\approx 200$ mayores, podemos concluir que la eficiencia a la hora de producir neutrones útiles de la propuesta para ESS-Bilbao es muy elevada. Esto es especialmente cierto si se considera el coste de las estructuras aceleradoras.

Diferentes configuraciones avanzadas desde el punto de vista neutrónico se han considerado, como la implementación de un filtro de berilio criogénico, la configuración con dos moderadores o el empleo de un moderador térmico. La inclusión de un filtro de berilio incrementa el brillo de neutrones de $5 \mathrm{meV}$ en un $\sim 50 \%$. La inclusión de un segundo moderador amplía el número de líneas experimentales a 5, pero reduce el brillo de cada 
moderador un $\sim 25 \%$. La inclusión de un filtro de berilio en cada moderador mantendría el valor de brillo inicial en ambos. El empleo de un moderador de agua a temperatura ambiente de $4 \mathrm{~cm}$ de espesor produciría un brillo de neutrones de energía inferior a los $600 \mathrm{meV}$ de $1,4 \cdot 10^{11} \mathrm{n} / \mathrm{cm}^{2} \cdot \mathrm{sr} \cdot \mathrm{s}$.

Con estos valores de término fuente y brillo, la instalación propuesta podría dar servicio a todas las técnicas experimentales normalmente disponibles en las fuentes de neutrones compactas, como irradiación de componentes electrónicos, medidas de datos nucleares, calibración de detectores, análisis por activación, etc. Además, las estimaciones efectuadas apuntan a que la propuesta desarrollada para ESS-Bilbao podría proporcionar valores de flujo a instrumentos de tomografía y dispersión neutrónica análogos a los empleados en instrumentos actualmente en operación. En concreto, las técnicas de dispersión factibles en la instalación serían reflectometría, difracción de polvos, dispersión de ángulo pequeño (SANS), y espectroscopía de tiempo de vuelo en geometría inversa (ToF-backscattering).

Por lo tanto, el diseño desarrollado define por primera vez un concepto de fuente de media intensidad y alta eficiencia que, con costes mucho menores que las fuentes de espalación o los reactores experimentales, habilita técnicas neutrónicas normalmente sólo disponibles en estos.

Estas características han hecho que, en los últimos tiempos, el interés por este tipo de fuentes basadas en reacciones de extracción haya crecido, y se haya materializado en varios proyectos europeos que las consideren candidatas idóneas para constituir una nueva generación de fuentes de neutrones. Esta nueva generación de fuentes de potencia media, alta eficiencia y coste moderado, está llamada a sustituir a los reactores experimentales en operación y a expandir el acceso a las técnicas experimentales neutrónicas, creando una red de instalaciones complementaria a las grandes fuentes de espalación. 


\section{Apéndice A}

\section{Evolución del diseño de la estructura del blanco}

La estructura del blanco descrita y analizada en el Capítulo 3 es el resultado de un proceso iterativo de diseño, en el que distintos conceptos y morfologías de blanco se han analizado de manera prospectiva hasta dar con una solución satisfactoria. Este Apéndice recoge los hitos más destacables de estos análisis, así como del histórico de la evolución de la estructura del blanco.

\section{A.1. Generación del concepto de estructura del blanco}

\section{A.1.1. Concepto inicial}

Como punto de partida se cuenta con la necesidad de disponer placas de berilio en una estructura rotatoria. Esta disposición condiciona el circuito de refrigeración, puesto que hace necesario introducir y extraer el refrigerante por el eje de la estructura.

Con el objetivo de refrigerar las placas y minimizar la captura de neutrones, se concibe la solución base descrita en la Figura A-1. En ella las placas de berilio se soportan sobre una estructura metálica que alberga los canales de refrigeración. Una idea clave del concepto es proporcionar una refrigeración lo más homogénea posible, mediante un canal independiente para cada placa. Con este sistema, las condiciones de todas las placas serían análogas, y 
su temperatura no sería dependiente de su posición en un circuito de placas conectadas hidráulicamente en serie.

Buscando que el refrigerante quede en contacto con las placas, estas cierran la estructura conformando un canal de sección constante bajo las mismas. Para ello es necesario dotar a ambas de un resalte de tal manera que, una vez colocada la placa, el canal no presente cambios bruscos de sección.

Para minimizar la captura neutrónica se reduce el espesor de agua y material estructural ubicados en la trayectoria de los neutrones. Para conseguirlo se disponen los canales de tal modo que el refrigerante, tras pasar bajo la placa, vuelve hacia el eje por los lados de esta.

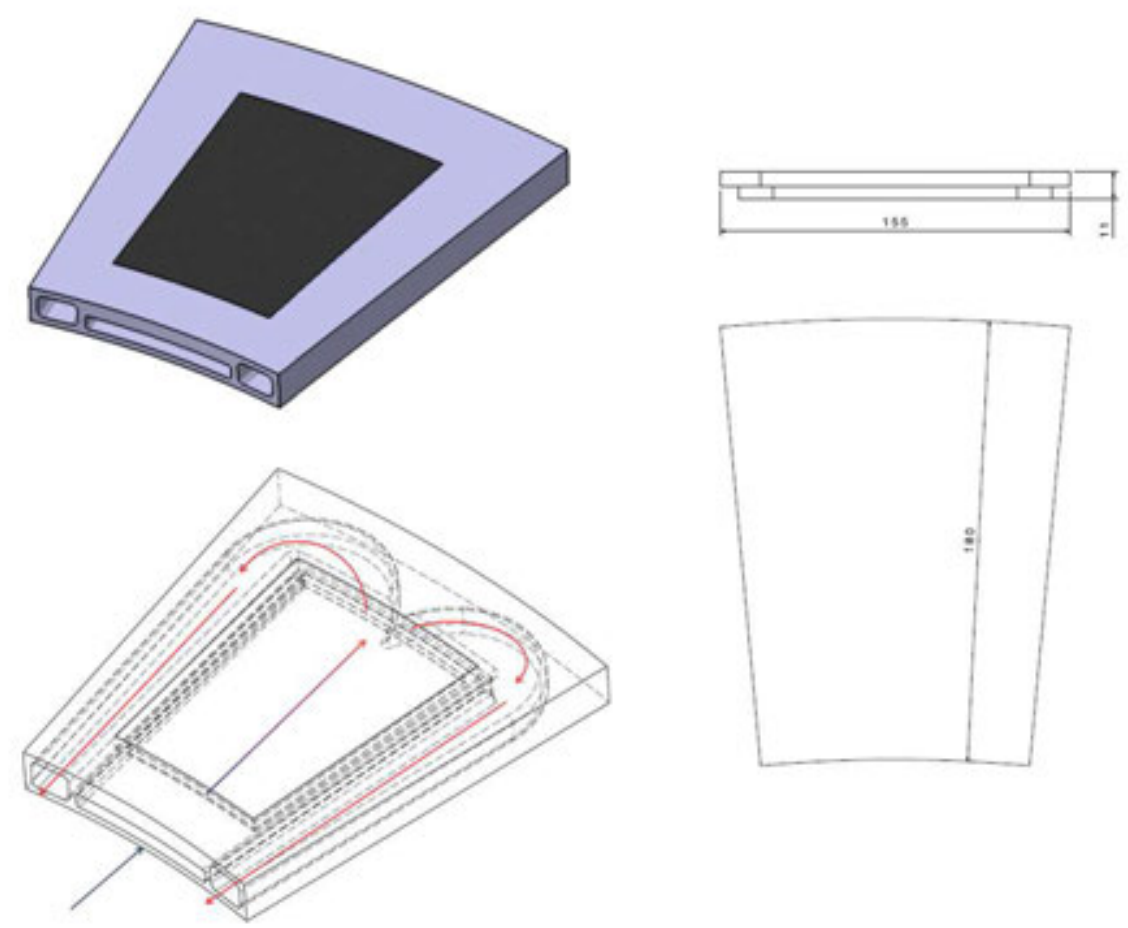

Figura A-1: Solución base y dimensiones de las placas ( $\mathrm{mm}$ )

\section{A.1.2. Posibles soluciones constructivas}

Partiendo de la solución base antes descrita, se concibieron distintas posibilidades para configurar una estructura rotatoria con una disposición de canales análoga. Las opciones cotejadas pueden dividirse en dos grupos: soluciones modulares y soluciones integrales, en 
función de si cada placa está ubicada en un elemento estructural independiente (módulo) o de si todas se encuentran ubicadas en una estructura única.

\section{A.1.2.1. Soluciones modulares}

Este grupo de soluciones se basa en la idea de que cada placa esté ubicada en una estructura soporte individual, o módulo, y que cada uno de dichos módulos estén soportados a un buje común. Distintos tipos de uniones entre los módulos y el buje se han estudiado.

La primera de las opciones sopesadas consistió en unir los módulos al buje mediante uniones roscadas, de tal forma que estos quedaran fijos por la precarga transmitida por tornillos, como puede verse en la Figura A-2. En un diseño más detallado podrían haberse mecanizado en los módulos y el buje formas que facilitaran su posicionamiento y sujeción, sin embargo el concepto sería el mismo.
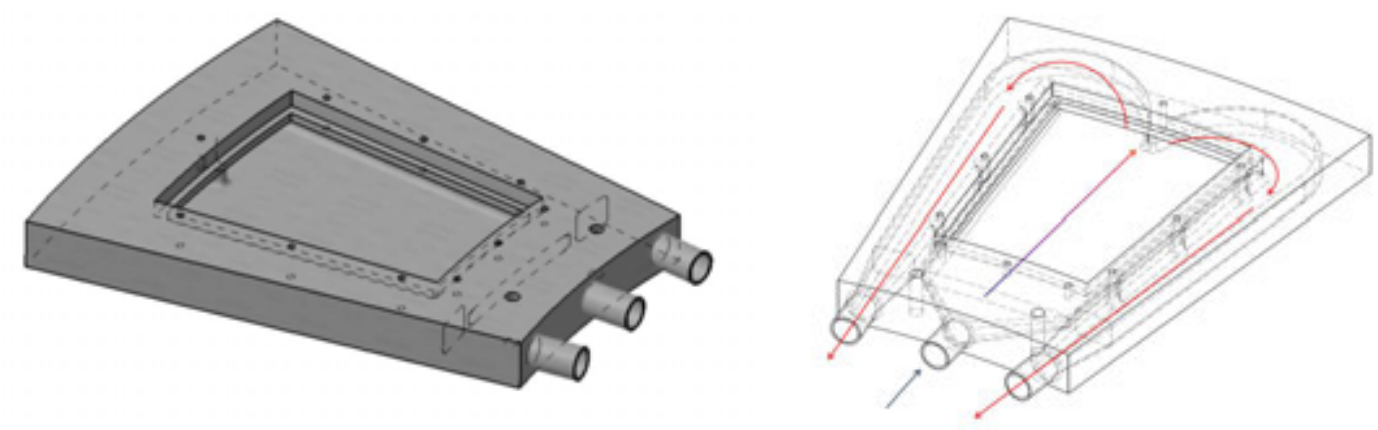

FigurA A-2: Ejemplo de solución modular atornillada

En esta solución el refrigerante entra a través de un distribuidor central de sección circular y sale mediante dos colectores análogos en los laterales del módulo. La unión de los tubos al disco central se realizaría mediante uniones tipo racor [242] o mediante soldadura, si no se consiguiese el grado de estanqueidad y robustez necesario. Las figuras A-3 y A-4 muestran en detalle el esquema de unión de esta solución y el aspecto que tendría un blanco completo así conformado.

Otra posible solución modular cotejada es la unión del modulo mediante una brida. De este modo se podría aunar la conexión mecánica e hidráulica al buje a través de un solo elemento constructivo. En la Figura A-5 se muestra un ejemplo de solución embridada en el que la brida se conectaría acoplando dos bocas, una para la entrada de refrigerante en 


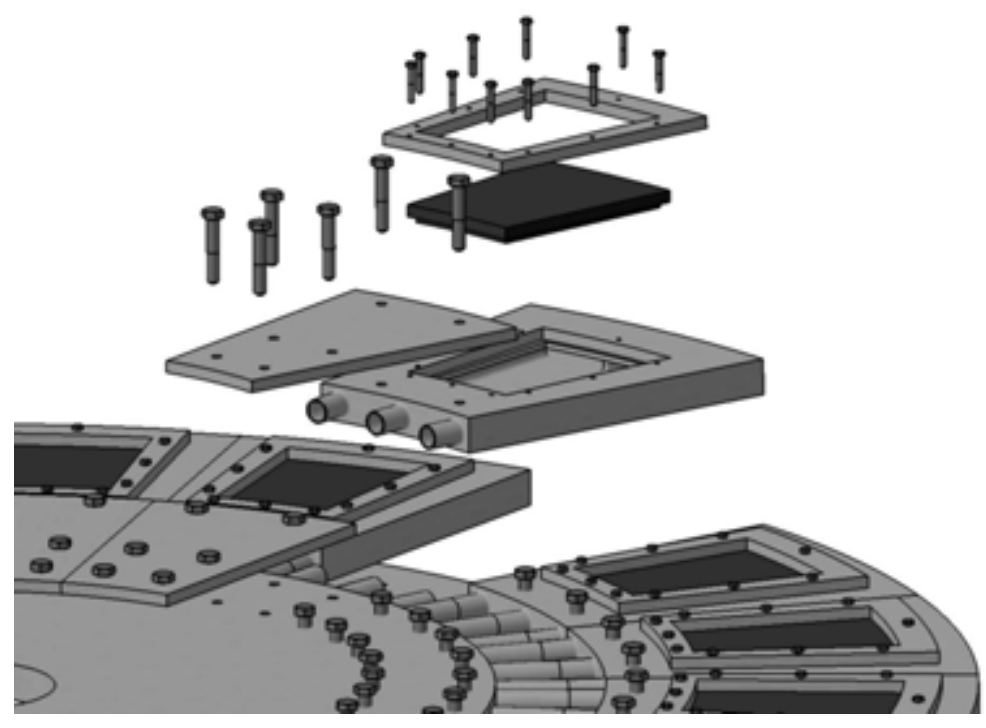

FigurA A-3: Esquema de unión de una solución atornillada.

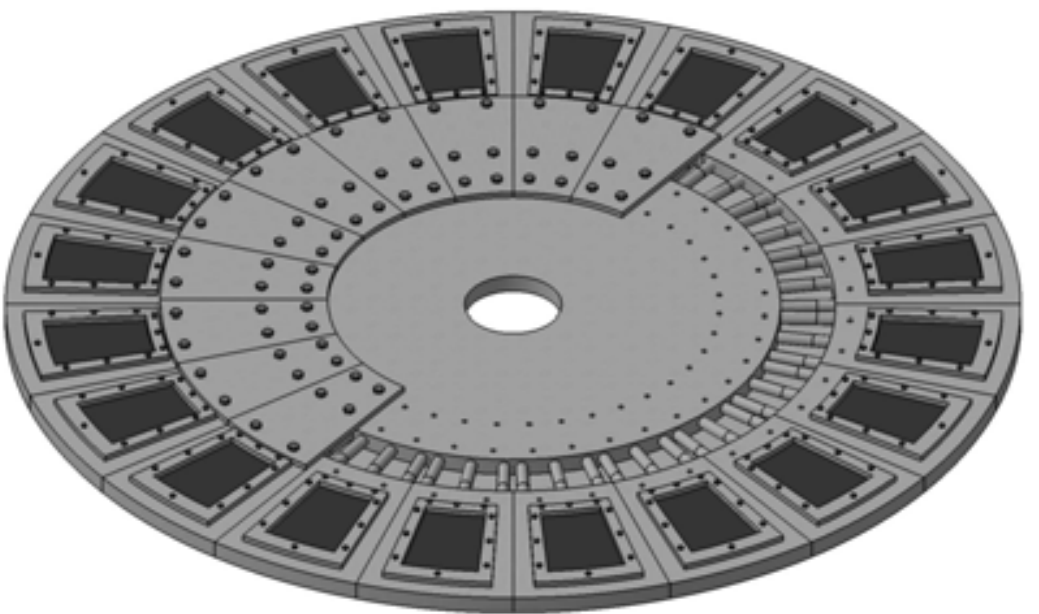

FigurA A-4: Blanco, solución atornillada (conductos semi-descubiertos). 
el nivel superior, y otra para la salida en el nivel inferior.

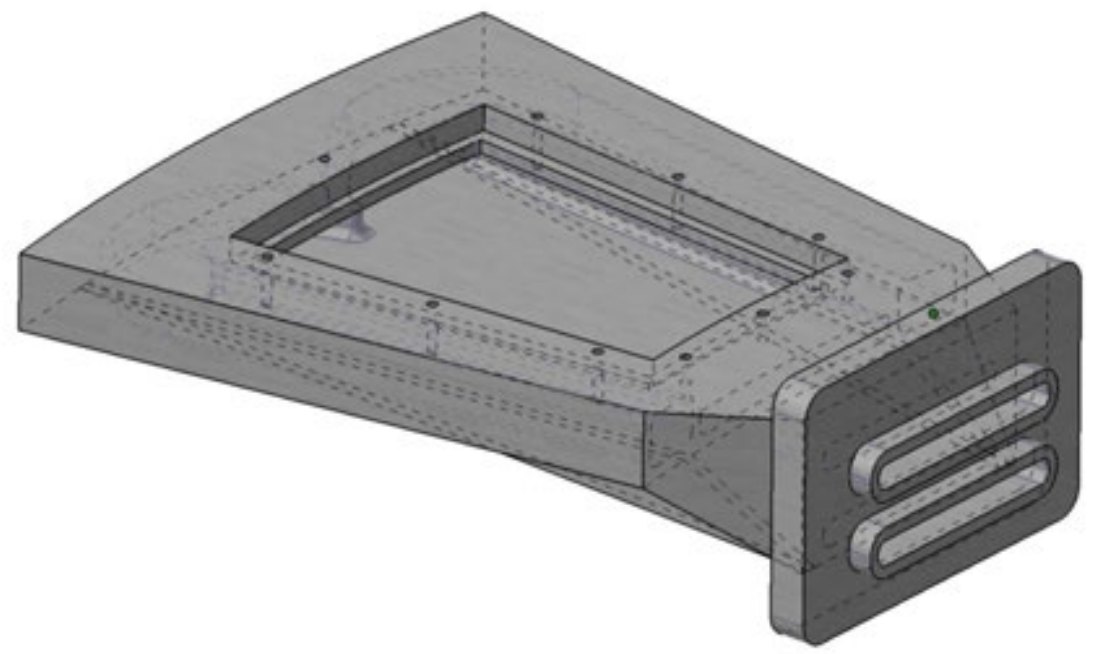

FigurA A-5: Ejemplo de solución modular embridada.

\section{A.1.2.2. Solución integral}

El segundo grupo de soluciones estudiado se basa en la idea de blanco integral, entendiendo por ello aquel en el que una única estructura albergara todas las placas de berilio, así como el conjunto de canales que las refrigera.

\section{A.1.3. Selección de la solución constructiva}

Los primeros análisis fluidodinámicos prospectivos que se realizaron sobre la opción atornillada, mostraron un comportamiento inesperado en las velocidades del flujo de refrigerante. Al tener el conducto para la entrada de refrigerante una sección menor que el área de paso bajo la placa, la velocidad de entrada necesaria para proporcionar la velocidad adecuada bajo la placa es $\approx 5 \mathrm{~m} / \mathrm{s}$. Dicha velocidad de salida, unida al cambio de sección entre la entrada tubular y el canal rectangular bajo la placa, hacen que la velocidad en este último sea muy poco uniforme, como muestra la Figura A-6. En ella observamos una zona central de alta velocidad rodeada de zonas de muy baja velocidad. Esta falta de uniformidad podría acarrear problemas de refrigeración en la placa.

Los cálculos prospectivos pusieron de manifiesto que, si se busca tener una distribución de velocidades uniforme debajo de la placa de berilio, es necesario que el flujo llegue a esa 


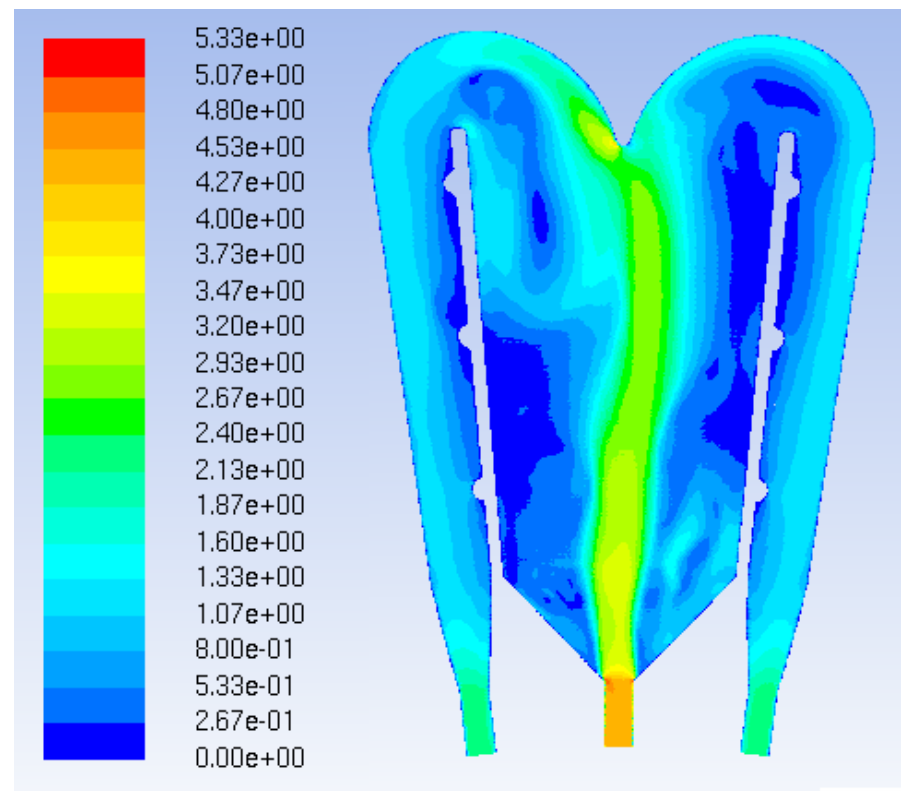

FigurA A-6: Velocidad de refrigerante en la opción atornillada.

zona suficientemente desarrollado, y que por tanto, el canal tenga sección rectangular durante una longitud suficiente antes de la placa, así como una variación suave de sección. Con este objetivo en mente se ideó la unión embridada representada en la Figura A-5, en el que la sección de entrada ya presenta una sección rectangular.

El análisis comparativo de las tres opciones arrojó las siguientes conclusiones:

- El mecanizado de canales internos no es sencillo. Dada la complicada geometría necesaria en los canales, tanto en la opción integral como en las opciones modulares, ha de recurrirse a la fabricación por capas. Este esquema de fabricación consistiría en mecanizar los canales en dos mitades longitudinales y después unirlas, bien por soldadura, bien por juntas de estanqueidad. Por tanto, las dificultades de fabricación son parecidas en todas las opciones.

- La opción modular permite la sustitución individual de uno de los módulos en caso de fallo. Sin embargo, el fallo a priori más probable es el de la junta de estanqueidad de las placas de berilio o el de las propias placas, el cual es equiprobable para la opción integral, y cuya solución requeriría en ambos conceptos de herramientas y operaciones análogas. A parte de este tipo de fallo, no se prevén otros fallos en los que la sustitución de un módulo completo pueda suponer una ventaja, excepto en aquellos posibles fallos originados por la propia naturaleza modular de la solución 
(e.g. fallo de la unión de los módulos). Por ello, en un primer análisis la sustitución de módulos no parece una ventaja para el mantenimiento.

- La solución modular puede suponer una ligera ventaja para el desmantelamiento, ya que permitiría reducir el tamaño de los componentes mayores del disco. No obstante, los esquemas de mantenimiento y desmantelamiento están muy ligados al objetivo de disponibilidad de la instalación, al decaimiento de la activación en el disco, y al presupuesto disponible para equipamiento de manejo remoto; por lo que esta posible ventaja debe estudiarse en el marco de un estudio más amplio, que queda fuera de los objetivos de este trabajo.

- La solución integral reduce drásticamente el número de uniones que han de mantener estanqueidad, lo cual tiene un impacto positivo en la disponibilidad de ĺa instalación y el tiempo medio entre fallos.

- La opción integral permite que los canales de retorno tras pasar por las placas sean comunes para las placas contiguas. Esto reduce el espacio necesario para el circuito hidráulico y permite que las placas tengan conexión hidráulica entre sí. De este modo, la hipotética obstrucción de uno de los canales sería menos grave desde el punto de vista de la seguridad.

- La opción integral es más robusta al reducirse el número de uniones entre piezas, especialmente el número de uniones no permanentes. Las uniones no permanentes son más propensas a inducir vibraciones.

Dados los argumentos anteriores, se considera que las ventajas de las soluciones modulares no compensan sus implicaciones negativas y, buscando priorizar la robustez y la sencillez del diseño, se opta por una solución integral para el diseño conceptual del blanco de ESS-Bilbao.

\section{A.2. Análisis prospectivos del blanco integral}

Como se ha discutido en la sección anterior, la necesidad de mecanizar los canales internos se puede atender fabricando la estructura por capas. De esta manera, el volumen de los canales se puede mecanizar eliminando material, accediendo desde el exterior de los 
elementos que conforman las capas. Finalmente, una vez unidos todos los componentes, el circuito de refrigeración queda definido mediante el espacio existente entre ellos.

Con esta idea en mente se concibe el primer diseño de solución integral para el blanco de ESS-Bilbao (Figura A-7)

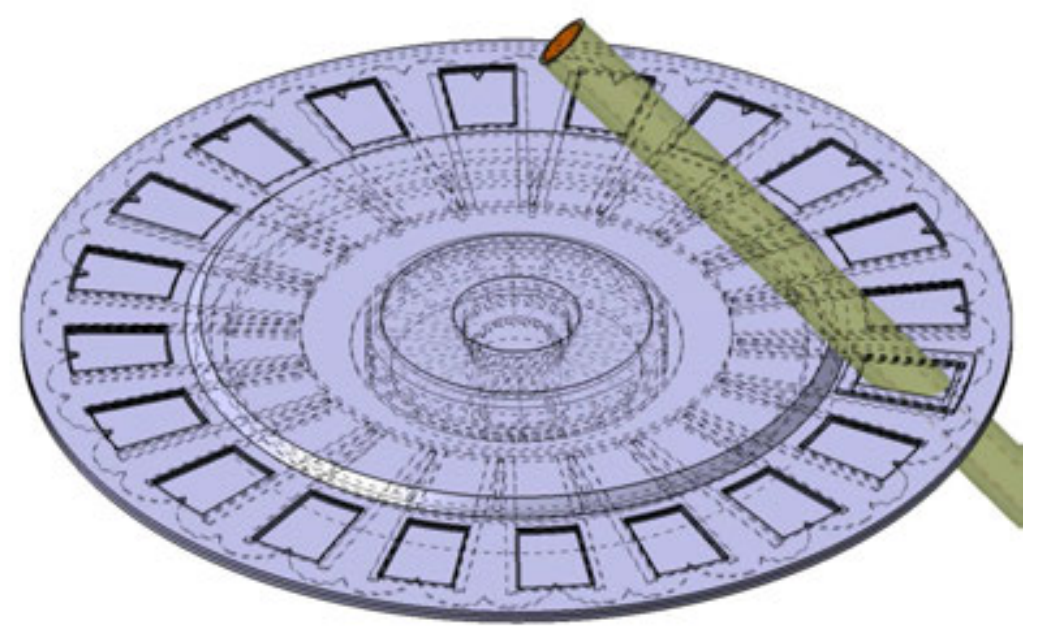

Figura A-7: Diseño inicial del blanco integral (haz de protones en amarillo).

La Figura A-8 muestra un explosionado de los componentes que lo conforman, en donde se puede apreciar la generación de los circuitos de refrigeración por capas. Más concretamente, los canales bajo las placas quedan definidos por el espacio entre los componentes llamados disco superior y disco inferior. Los componentes del disco tienen una nomenclatura similar a los presentados en el Capítulo 3, a pesar de que son sensiblemente distintos en esta primera iteración de diseño.

La geometría del blanco se ha desarrollado en la herramienta de diseño asistido por ordenador (CAD) CATIA V5 [112]. Se ha creado un modelo CAD paramétrico completo de la estructura. En este, todas las dimensiones dependen de los distintos parámetros de diseño, de tal manera que la geometría del modelo se actualiza automáticamente en función de los valores de los parámetros introducidos. Como parámetros de diseño se han tomado magnitudes fundamentales, e.g. número de placas, diámetro del haz, ángulo de incidencia, etc. y magnitudes de ingeniería, e.g. espesores, número de tornillos, velocidades del refrigerante objetivo, etc.

Esta metodología conlleva un importante esfuerzo de programación CAD, ya que se debe calcular e implementar todas las relaciones dimensionales de la geometría del mo- 


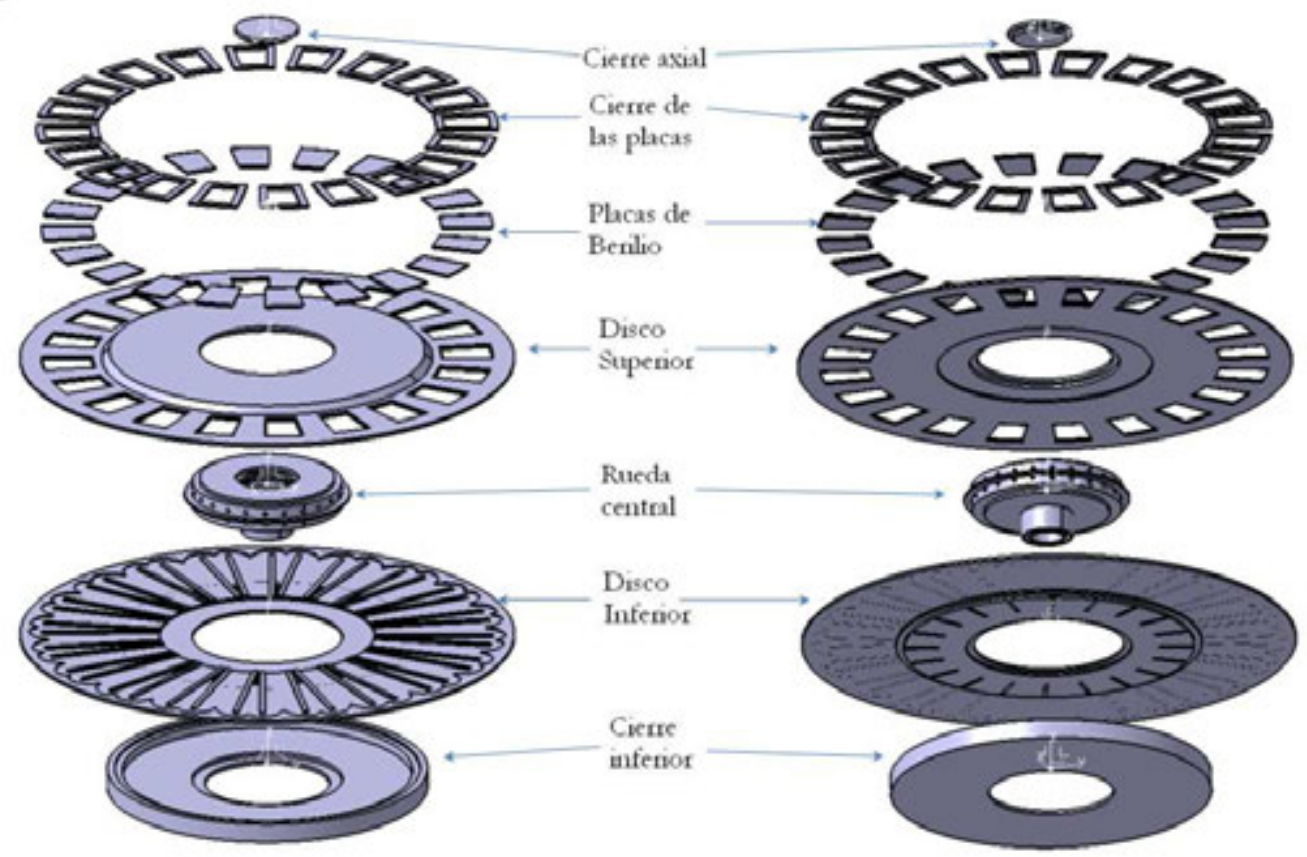

FigurA A-8: Vista explosionada del concepto inicial de blanco integral.

delo en función de los más de 50 parámetros de diseño que lo rigen. No obstante, una vez creado el modelo paramétrico, este permite estudiar las diferentes configuraciones del blanco obtenidas por la combinación de los valores posibles de los parámetros, detectando además incompatibilidades entre ellos. Esta flexibilidad también permite la cómoda implementación de las modificaciones geométricas necesarias, resultado de las simulaciones numéricas, así como la realización de estudios de sensibilidad ante el valor de los distintos parámetros constructivos.

\section{A.2.1. Análisis y mejora del comportamiento hidráulico}

El circuito refrigerante (Figura A-9) en esta solución inicial es análogo al descrito en el Capítulo 3. La rueda central consta de un eje doble coaxial y dos niveles de conductos distribuidores / colectores. El agua entra por la parte interna del eje coaxial y se distribuye radialmente mediante el nivel superior de conductos. Estos conductos desembocan en un espacio distribuidor anular conformado también entre el disco superior y el disco inferior. De este espacio distribuidor parten los 20 canales que pasan bajo las placas de berilio para refrigerarlas. El agua vuelve en dirección radial por los espacios entre canales hasta llegar a un orificio en el disco inferior, por el que llegan al espacio colector anular formado entre 
este y el cierre inferior. Una vez allí, el refrigerante regresa a la zona exterior del eje de la rueda central, pasando por los conductos colectores del nivel inferior.

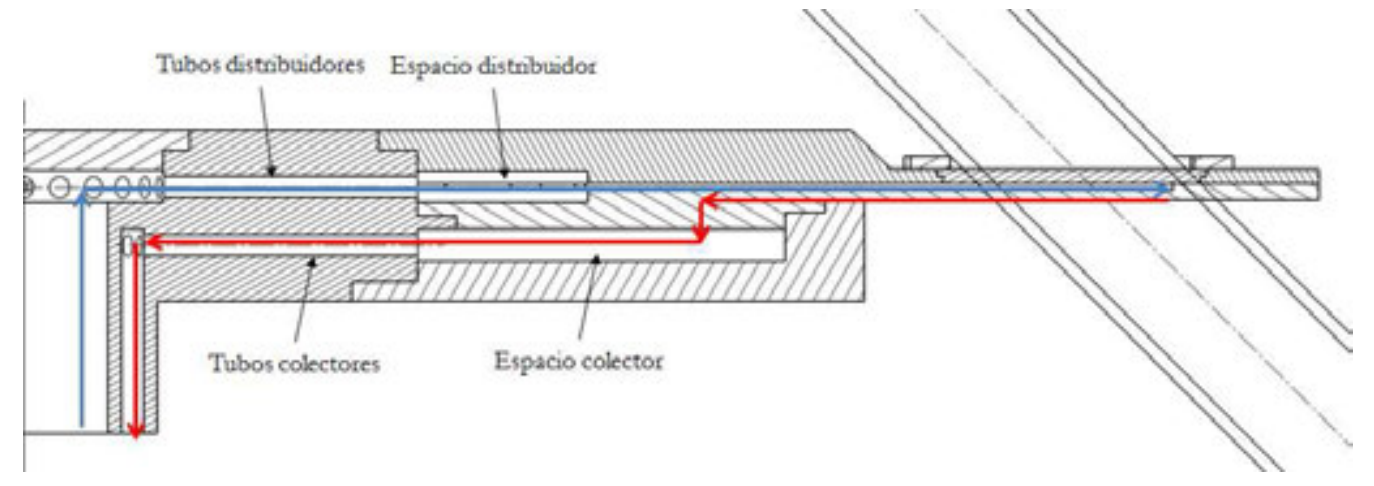

Figura A-9: Vista seccionada de la configuración inicial del blanco. Haz de protones representado, incluyendo margen de seguridad a su alrededor.

El dominio fluido (Figura A-10), delimitado por los componentes mecánicos de esta propuesta inicial, presenta simetría axial con 20 sectores angulares idénticos de $18^{\circ}$ (Figura A-11).

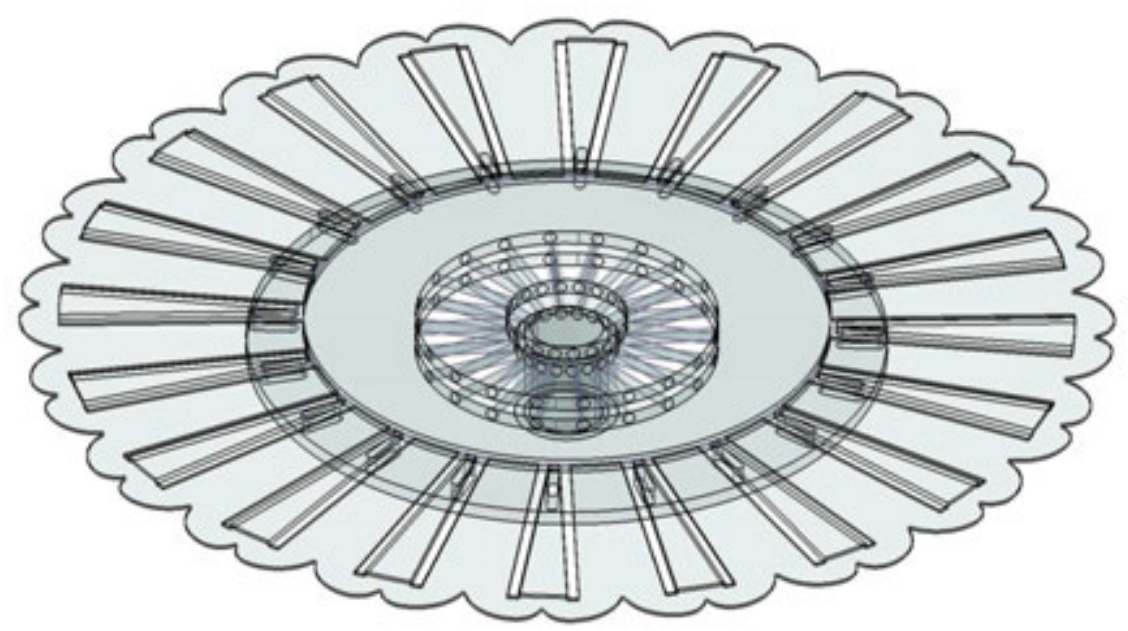

FigurA A-10: Volumen interno de refrigerante en la configuración inicial del blanco.

Este dominio fluido se ha analizado con los mismos parámetros de cálculo, condiciones de contorno y tipo de malla que los descritos en Capítulo 3. Dado que únicamente se busca estudiar las características hidráulicas del circuito, sólo se resuelve el campo de velocidades y presiones en el fluido, no incluyéndose el dominio sólido ni la transmisión de calor entre ambos. La Figura A-12 muestra los resultados obtenidos del campo de velocidades en el fluido. 


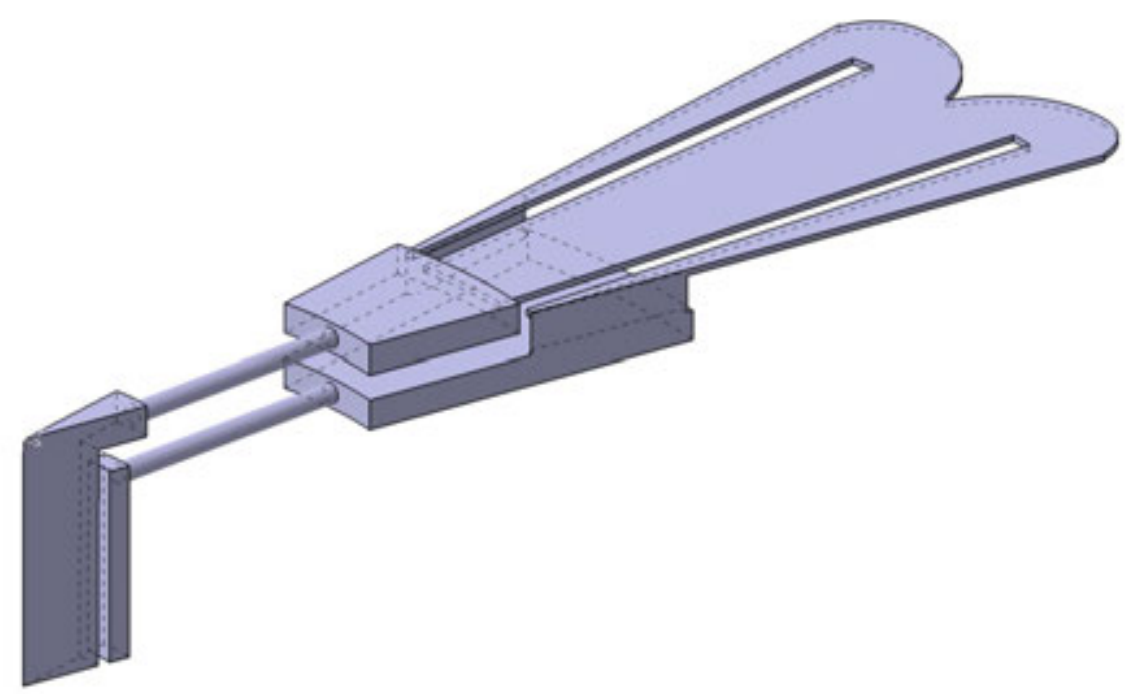

Figura A-11: Dominio de cálculo fluido inicial.

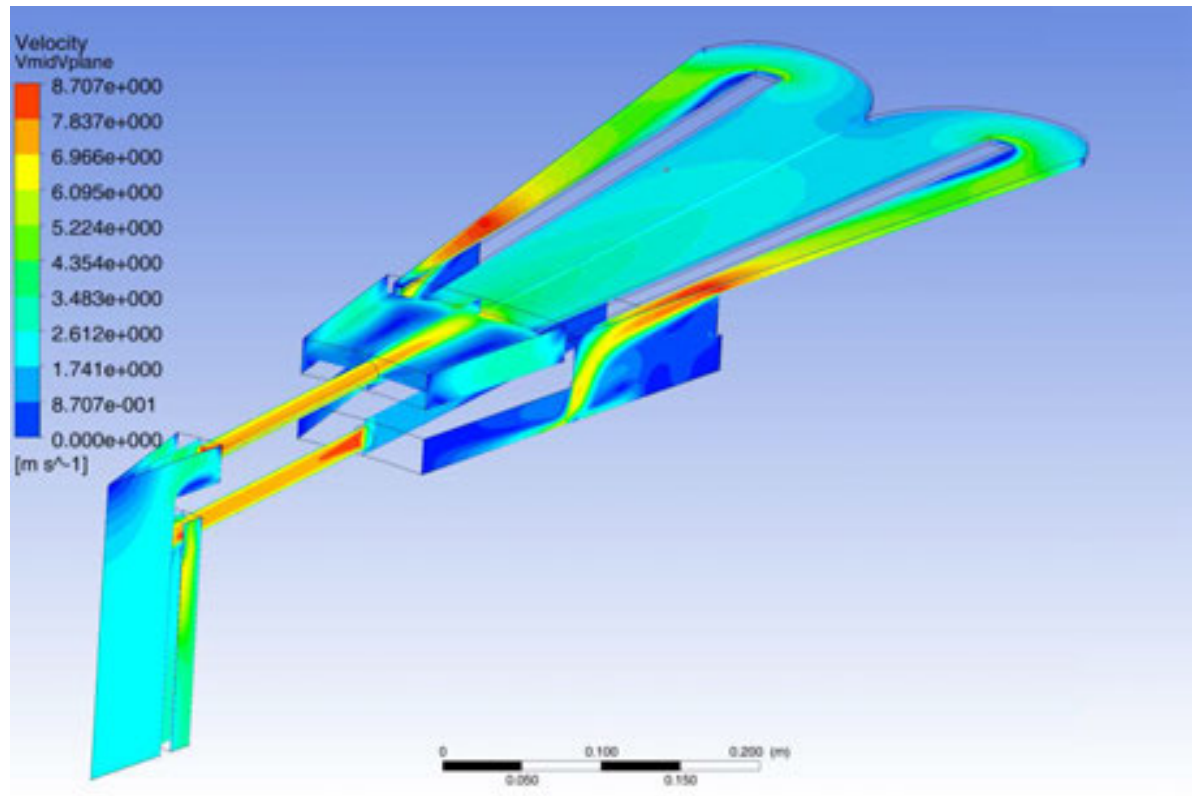

Figura A-12: Distribución de velocidades del refrigerante en la configuración inicial. 
Puede observarse como efectivamente se consigue una velocidad bajo las placas de berilio de $2-2,5 \mathrm{~m} / \mathrm{s}$, adecuada para obtener las tasas de transferencia de calor deseadas. Vemos también como el fluido alcanza velocidades considerablemente elevadas, de los 7 a $9 \mathrm{~m} / \mathrm{s}$ en algunos puntos del circuito. Estas zonas son fundamentalmente dos: los conductos de distribución / colección de la rueda central y los canales retorno cerca de las zonas de transición entre los niveles superior e inferior del disco. De acuerdo con [170], es recomendable mantener la velocidad del refrigerante por debajo de $\operatorname{los} 6 \mathrm{~m} / \mathrm{s}$ para evitar problemas de erosión-corrosión, por lo que se analizarán modificaciones en el diseño fluido para reducir la velocidad en estos puntos.

Con el objetivo de reducir la velocidad en los conductos de distribución / colección de la rueda central, se planteó la posibilidad de aumentar su número a 24, en lugar de los 20 la configuración inicial. Esta variación modificaría la simetría axial del dominio fluido, pasando de 20 sectores angulares de $18^{\circ}$, a 4 sectores angulares de $90^{\circ}$.

En la segunda zona de velocidad elevada, los canales de retorno cerca de la transición entre los niveles superior e inferior del disco, se estudió la posibilidad de aumentar la sección de los canales de vuelta mecanizando parte de estos en el disco superior, de tal forma que su sección transversal fuese mayor.

Como muestran los resultados obtenidos (Figura A-13), las modificaciones implementadas logran reducir la velocidad del refrigerante por debajo de los $6 \mathrm{~m} / \mathrm{s}$ en las zonas de interés.

No obstante, el análisis posterior de estas modificaciones desaconsejó su adopción en el diseño final del disco. Por un lado, el empleo de los 24 distribuidores / colectores implica que el espesor de material sólido entre ellos disminuya ostensiblemente, lo cual debilita la rueda central. Por otro lado, el mecanizado de parte de los canales de vuelta en el disco superior resultó interferir con la posición de la unión atornillada en esa zona.

Descartadas estas modificaciones, para reducir la velocidad en los conductos distribuidores / colectores se optó por aumentar el diámetro de los mismos, manteniendo su número inicial, de tal manera que la sección total de paso aumentase pero reduciendo menos el espacio sólido entre conductos. Esta solución tiene como bondad añadida que recupera la simetría axial cada $18^{\circ}$, lo cual supone una disminución significativa en el tamaño del dominio fluido y, consecuentemente, del tiempo de cálculo. 


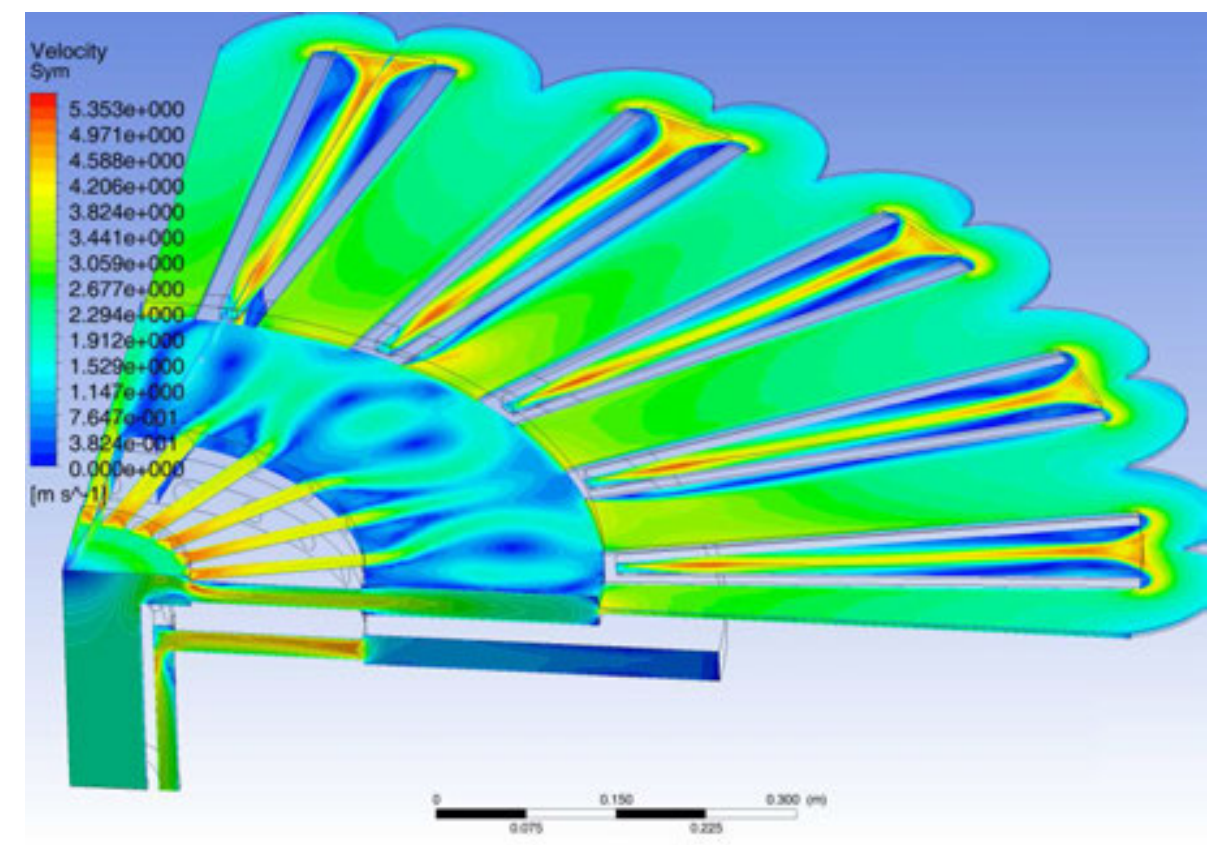

Figura A-13: Distribución de velocidades del refrigerante en una configuración con 24 canales distribuidores y colectores y canales de regreso ampliados.

Respecto a los canales de vuelta, las elevadas velocidades en estos se deben en parte a su estrechamiento conforme el flujo avanza radialmente hacia el centro del disco. Para evitar este aumento de velocidad, se optó por incrementar en dirección radial los volúmenes distribuidor y colector, de tal manera que los canales de vuelta se estrecharan en menor medida.

Implementando estas últimas modificaciones se consigue reducir las velocidades máximas en las zonas de interés hasta aproximadamente $5 \mathrm{~m} / \mathrm{s}$, como muestra la Figura (A-14), por lo que esta configuración se adoptó como solución final del circuito fluido.

\section{A.2.2. Análisis y mejora del comportamiento mecánico}

Con el objetivo de obtener una evaluación del comportamiento del diseño inicial del blanco integral (Figuras A-7, A-8 y A-9), se procede a realizar un análisis mecánico simplificado. En este análisis se asume que las uniones atornilladas trabajan perfectamente y que las superficies en contacto de dichas uniones son inseparables. De esta manera se evalúa el comportamiento global del disco, independizando este del esquema de las uniones atornilladas. Esta aproximación tiene además la ventaja de hacer el cálculo mecánico lineal, lo cual reduce sustancialmente el tiempo de resolución con respecto al modelo con 


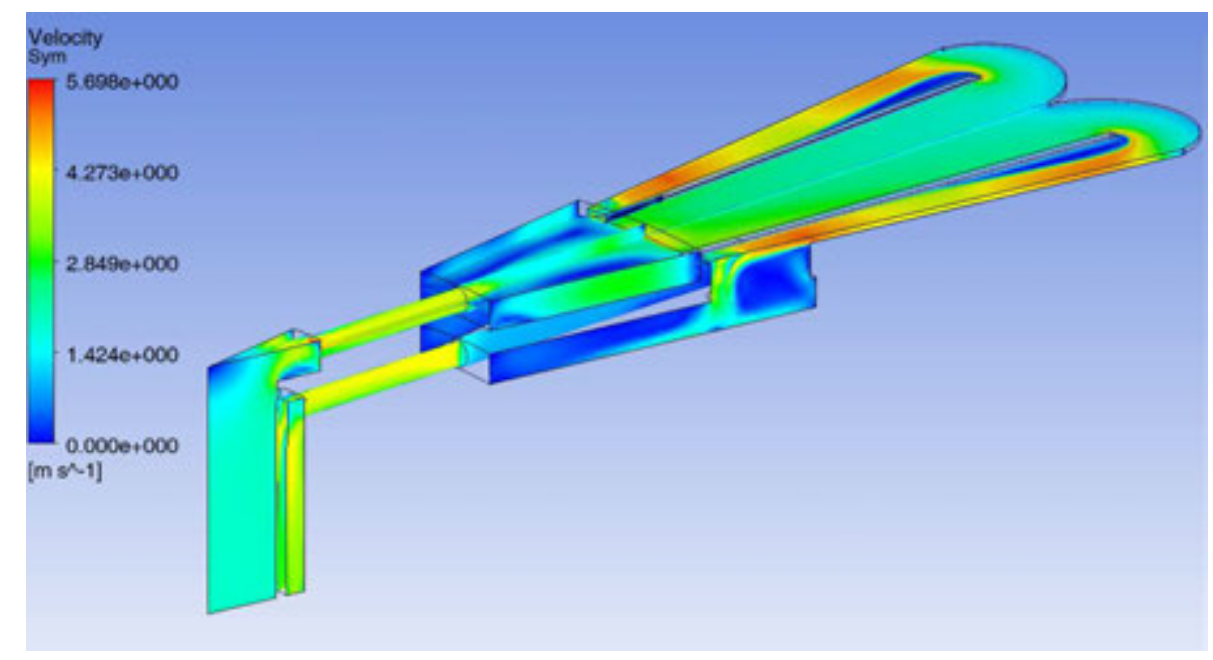

Figura A-14: Distribución de velocidades del refrigerante en el dominio fluido de referencia.

contactos realistas.

La Figura A-15 muestra una vista explosionada de los componentes del diseño inicial de la estructura del blanco. En ella las uniones atornilladas se encuentran entre los siguientes pares de piezas:

- Cierre axial y rueda central

- Rueda central y disco superior

- Rueda central y disco inferior

- Rueda central y cierre inferior

- Disco inferior y cierre inferior

- Disco inferior y disco superior

- Disco superior y cierre de las placas

El análisis mecánico simplificado se efectúa con los mismo parámetros de cálculo y condiciones de contorno que los descritos en el Capítulo 3. Dado que las uniones atornilladas se asumen perfectas en este cálculo, no es necesario incluir los elementos tipo BEAM empleados para simular el efecto de los tornillos. Por tanto, tampoco es necesario preparar la geometría para implementar el mallado regular alrededor de las zonas de apoyo de los tornillos, lo cual simplifica la malla empleada (Figura A-16). 


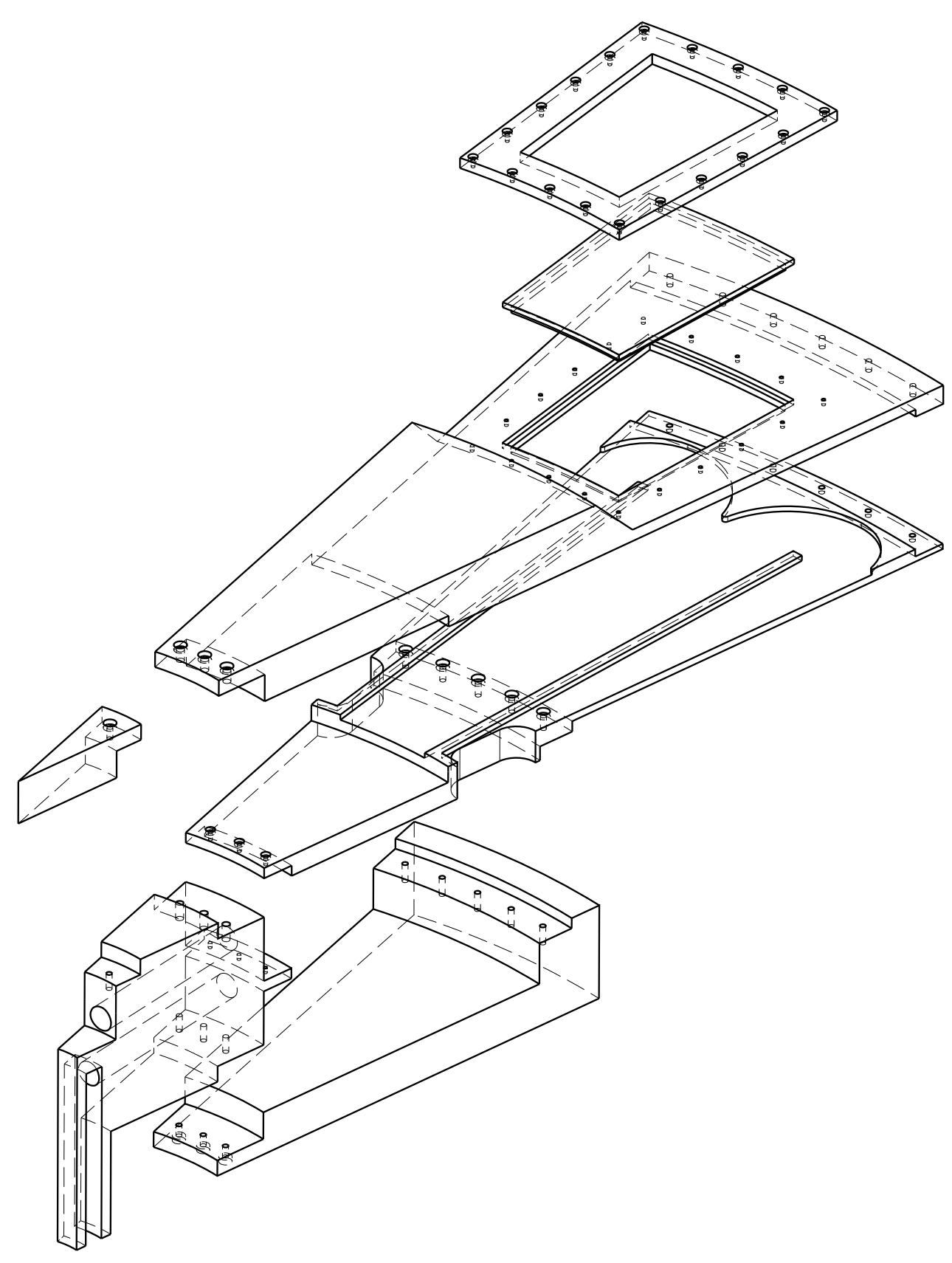

Figura A-15: Vista explosionada de un sector de $18^{\circ}$ del diseño inicial. 


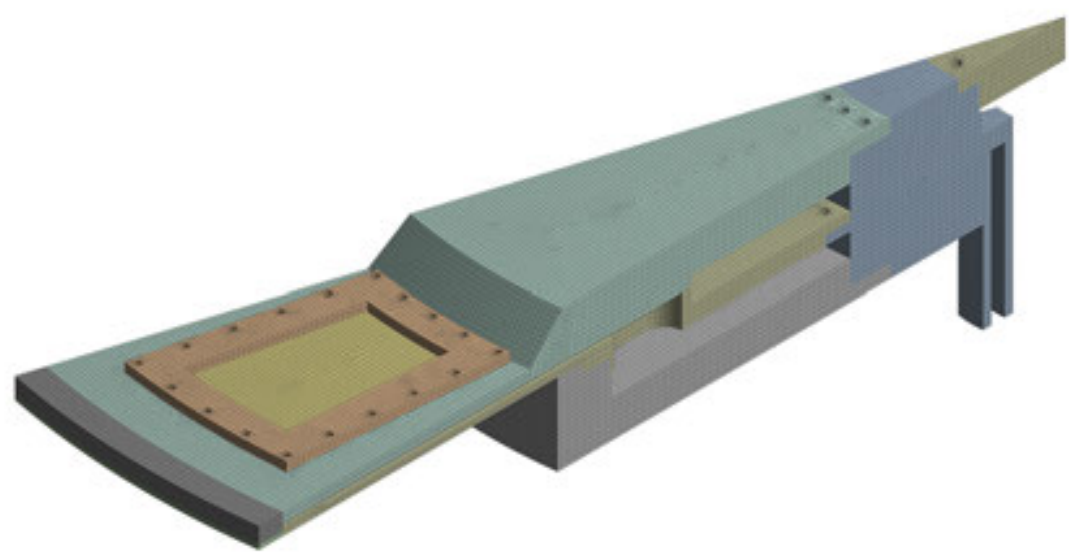

Figura A-16: Mallado mecánico inicial.

Para estudiar el comportamiento global de la estructura, se implementa únicamente como carga mecánica la presión del refrigerante, 5 bar, en las caras del circuito de refrigeración. Las tensiones de origen térmico son un problema más local a analizar en la placa y en el proceso de diseño de detalle de las juntas de estanquidad, por lo que en general es independiente de la configuración global de la estructura. Así pues, no se incluyen en este cálculo prospectivo. Con estas premisas, las Figuras A-17 y A-18 muestran respectivamente los resultados de desplazamientos y tensión obtenidos.

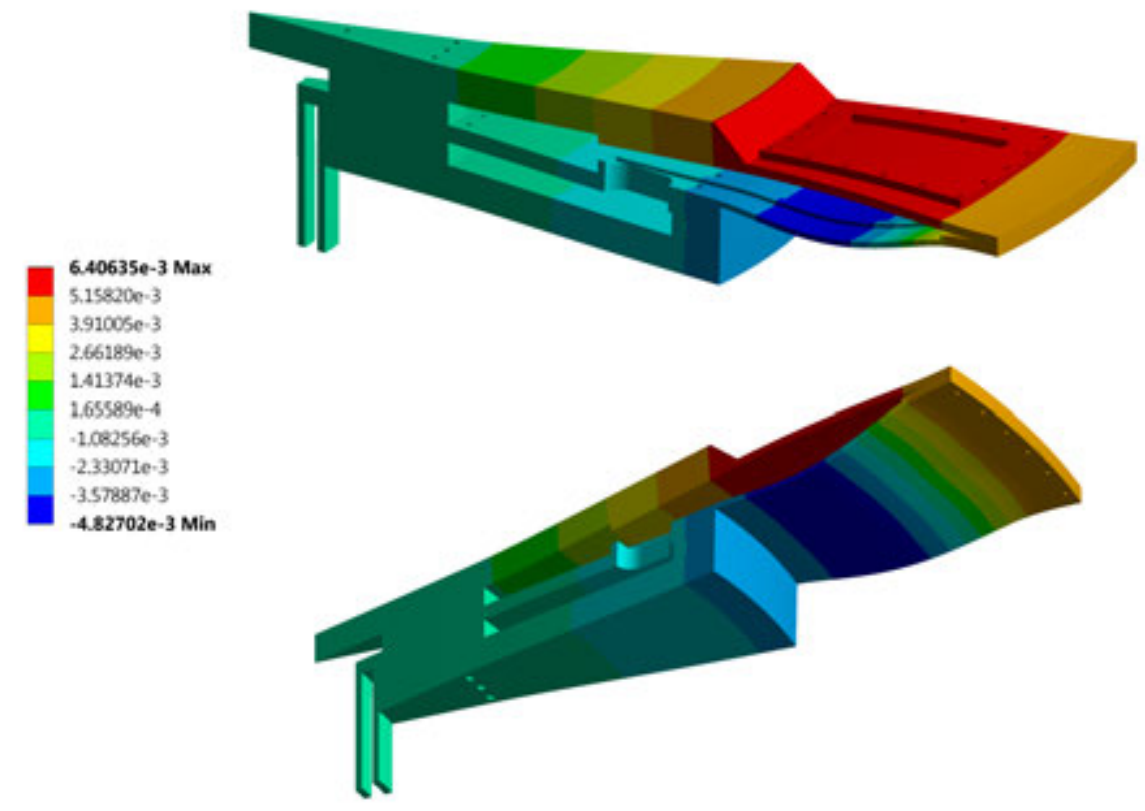

Figura A-17: Distribución de desplazamientos en la dirección axial del disco en modelo mecánico lineal inicial $[\mathrm{m}]$. 


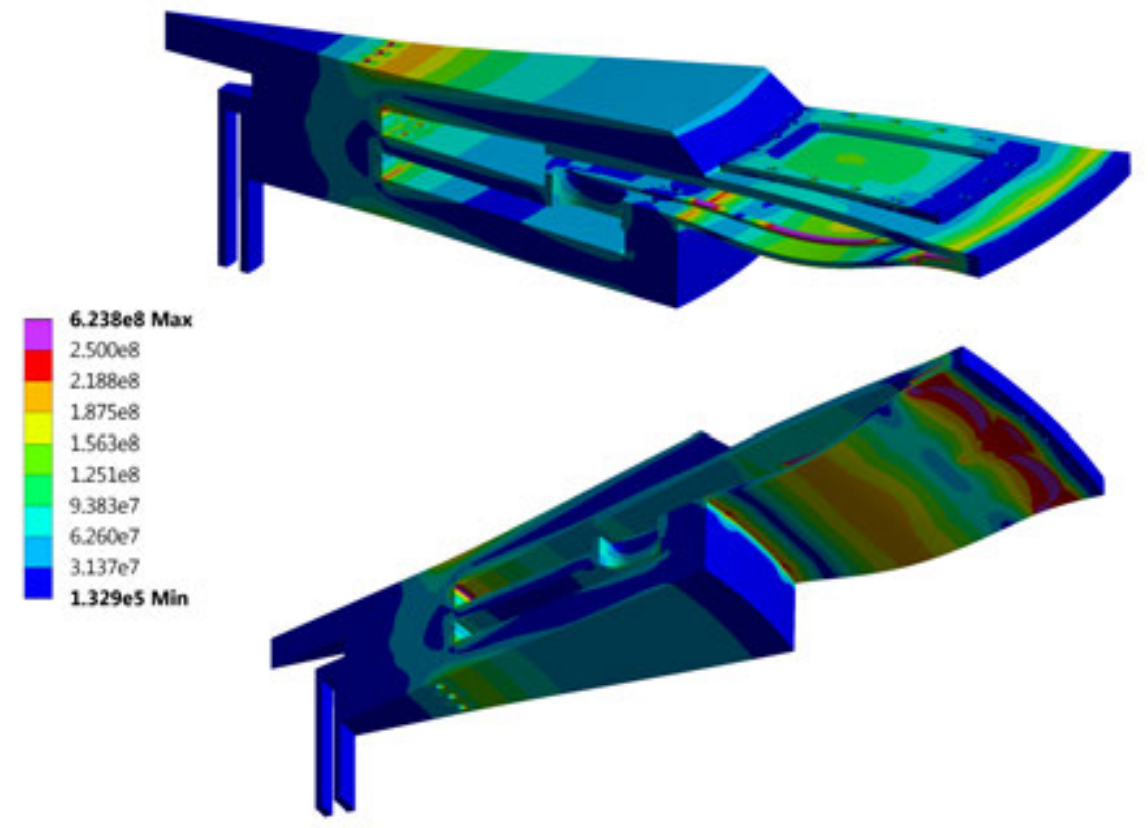

Figura A-18: Distribución de tensión de von Mises en modelo mecánico lineal inicial $[P a]$.

Como puede observarse en el mapa de desplazamientos, el diseño inicial no es suficientemente rígido y los componentes disco superior e inferior tienden a separarse por efecto de la presión interna, alcanzándose unas deformaciones de varios milímetros. La escala en la que se presentan los desplazamientos está ampliada para apreciar mejor este comportamiento. Estas deformaciones implican la aparición de tensiones equivalentes ampliamente superiores al límite elástico del material estructural, $250 \mathrm{MPa}$, especialmente cerca de las uniones atornilladas que sufren mayor flexión, y principalmente en la zona de los discos que queda en voladizo. Estos niveles de tensión son inaceptables puesto que inducirían al menos deformación plástica permanente y, dado que la presión es una carga primaria (i.e. no acomodable con una pequeña deformación plástica local) probablemente inducirían fallo del componente.

Debe puntualizarse que, debido a la presencia de aristas vivas, se pueden apreciar elementos con valores de tensiones artificialmente altos por motivos numéricos. Estos valores pueden ser ignorados en el estudio global del comportamiento de la estructura.

Con el objetivo de corregir el comportamiento anteriormente observado, se implementa una nueva unión roscada con tornillos M10 entre el disco superior y el cierre inferior, de tal manera que el primero quede sujeto al conjunto que forman el disco y cierre inferiores, 
aproximadamente por su radio medio. La preparación de esta unión roscada en el disco inferior, modifica ligeramente el dominio fluido final (Figura 3-7) respecto al generado en la Sección A.2.1. No obstante, estas modificaciones no afectan a la distribución de velocidades final en las zonas de interés (Figura 3-10). La Figura A-19 muestra el explosionado de los componentes mecánicos de un sector del disco incluyendo esta nueva unión atornillada.

Implementada esta modificación, se revalúa el comportamiento mecánico del disco. Las Figuras A-20 y A-21 muestran los resultados de desplazamiento y tensión equivalente obtenidos. Como puede observarse, esta modificación corrige eficientemente la separación del disco superior, reduciendo la deformación y la tensión en la unión atornillada entre este y la rueda central. No obstante, la zona de la estructura en voladizo sigue flectando en exceso, tanto el disco superior como el inferior, lo cual genera tensiones excesivas.

Así pues, resulta evidente que es necesario aumentar la rigidez de esta zona. Para ello se procede a implementar dos modificaciones: por un lado se incluyen nervios rigidizadores por encima y debajo de la zona en voladizo; y por otro, se refuerza el disco inferior aumentando su espesor en las zonas fuera de la trayectoria principal de los neutrones. Con esta ultima modificación buscamos incrementar la rigidez sin aumentar la captura de los neutrones en el material estructural.

Estas modificaciones requieren de un cambio notable en los componentes del disco. Para poder incluir los nervios rigidizadores en el disco inferior, es aconsejable que la zona en voladizo de este componente pase a formar parte del cierre inferior, de tal manera que haya continuidad de material en ambos extremos del nervio. Con esta modificación el disco inferior se reduce, y funcionalmente se convierte en un separador de flujo entre el disco superior y el cierre inferior.

Tras varias iteraciones de modificación - verificación no detalladas en este trabajo, la geometría rigidizada final del conjunto es la descrita en las Figuras A-22, A-23 y A-24. La Figura A-25 describe las uniones atornilladas mecanizadas en la estructura.

Implementada estas nuevas modificaciones, se revalúa el comportamiento mecánico del disco. Las Figuras A-26 y A-27 muestran los resultados de desplazamiento y tensión equivalente obtenidos.

Se observa que los nervios rigidizadores cumplen su función reduciendo los desplaza- 


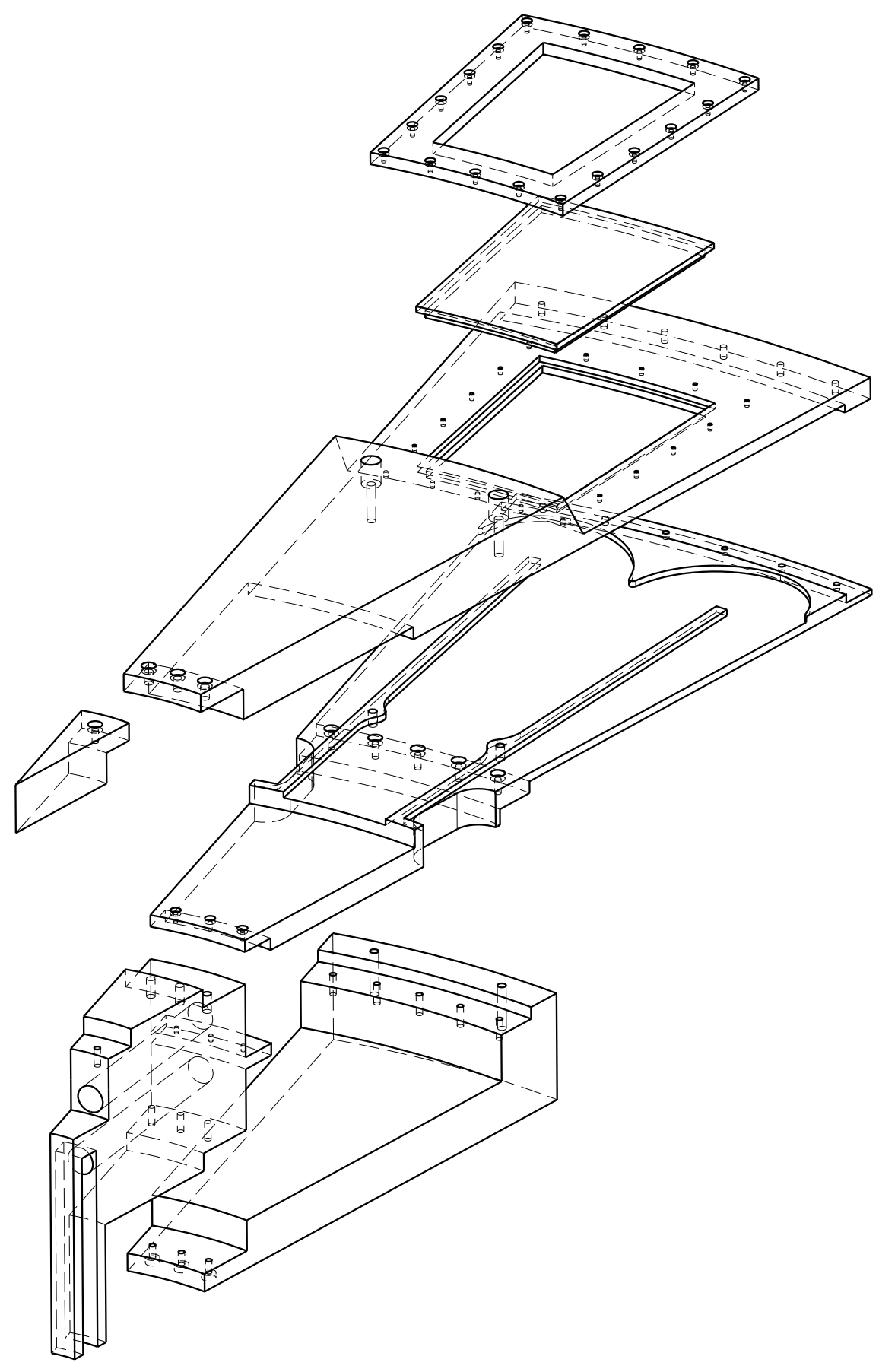

FigurA A-19: Vista explosionada de un sector de $18^{\circ}$ de la configuración modificada. 


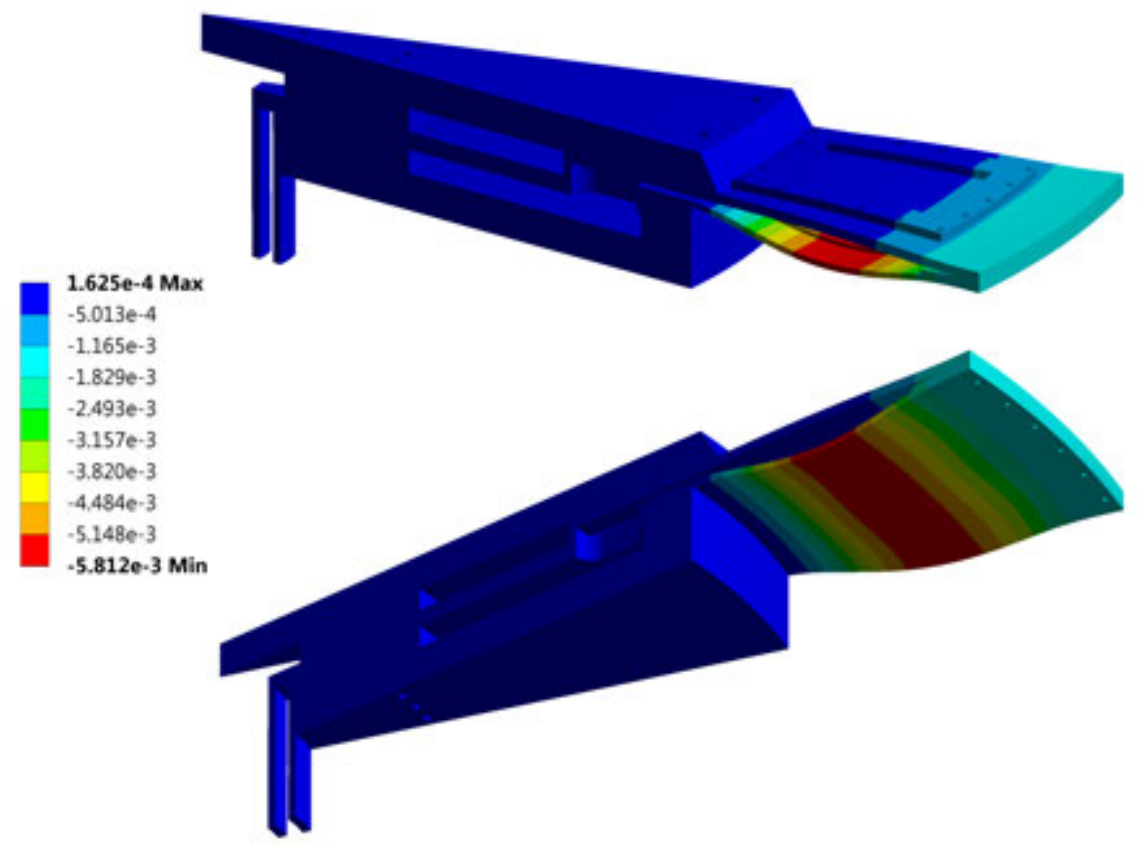

Figura A-20: Distribución de desplazamientos en la dirección axial del disco en modelo mecánico lineal modificado [m].

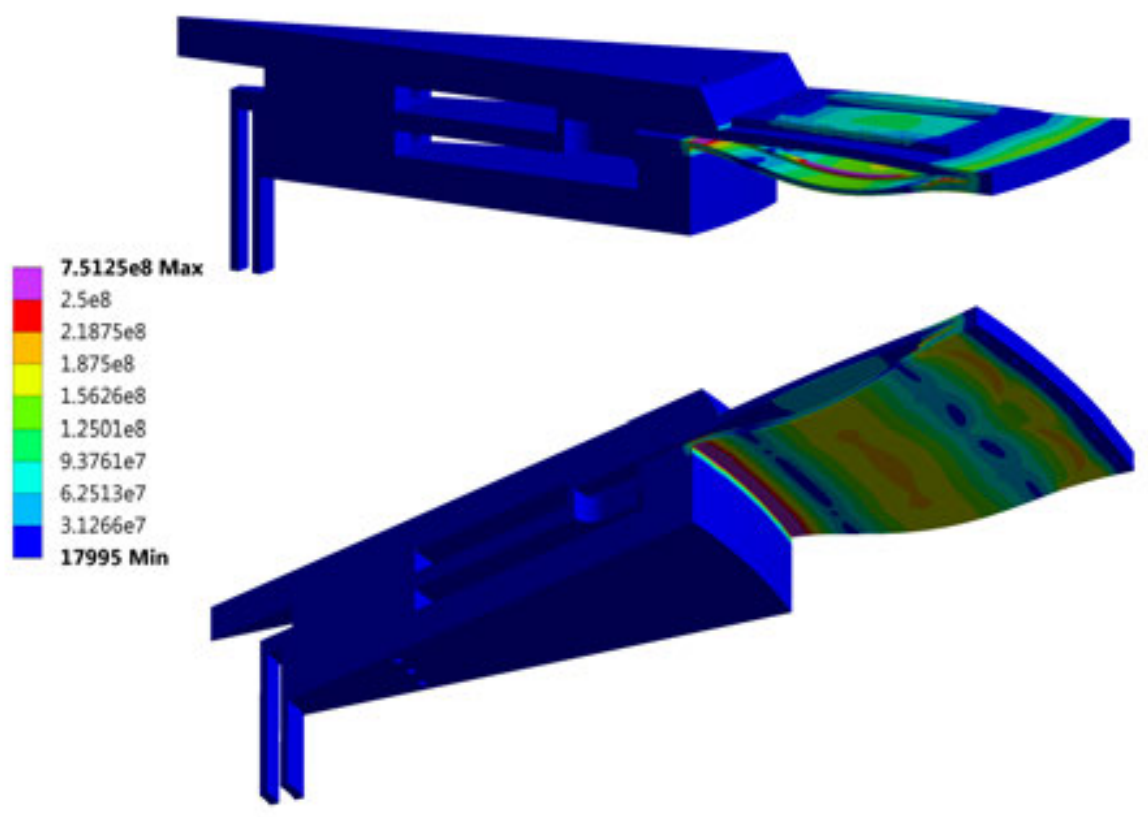

FigurA A-21: Distribución de tensión de von Mises en modelo mecánico lineal modificado $[\mathrm{Pa}]$. 

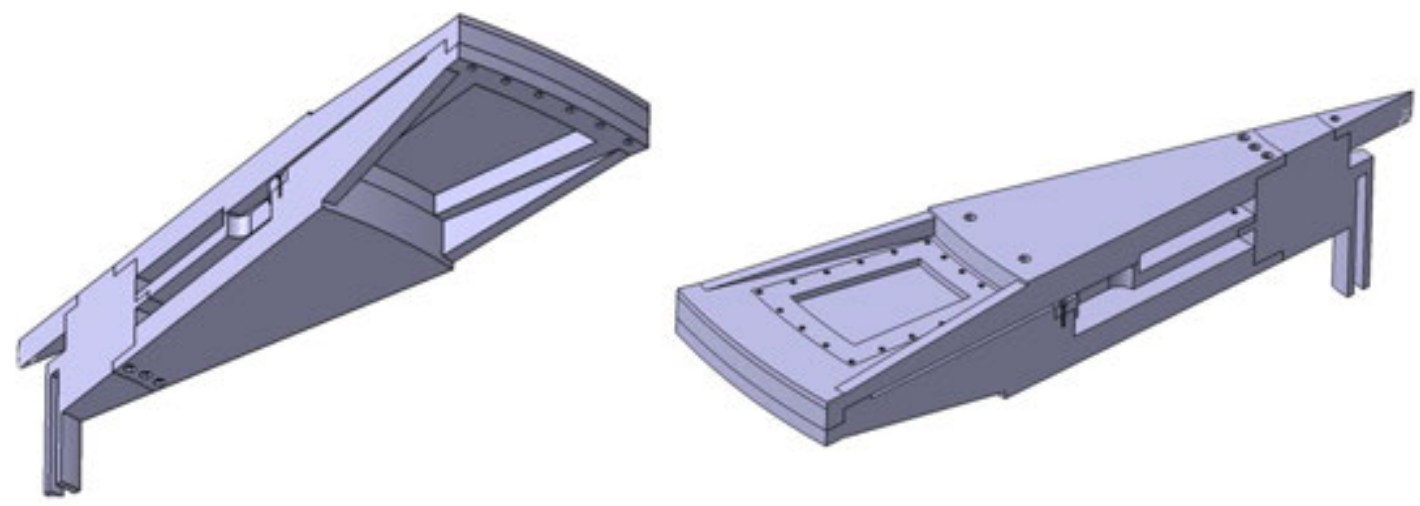

Figura A-22: Sector de $18^{\circ}$ de la geometría del disco rigidizado.

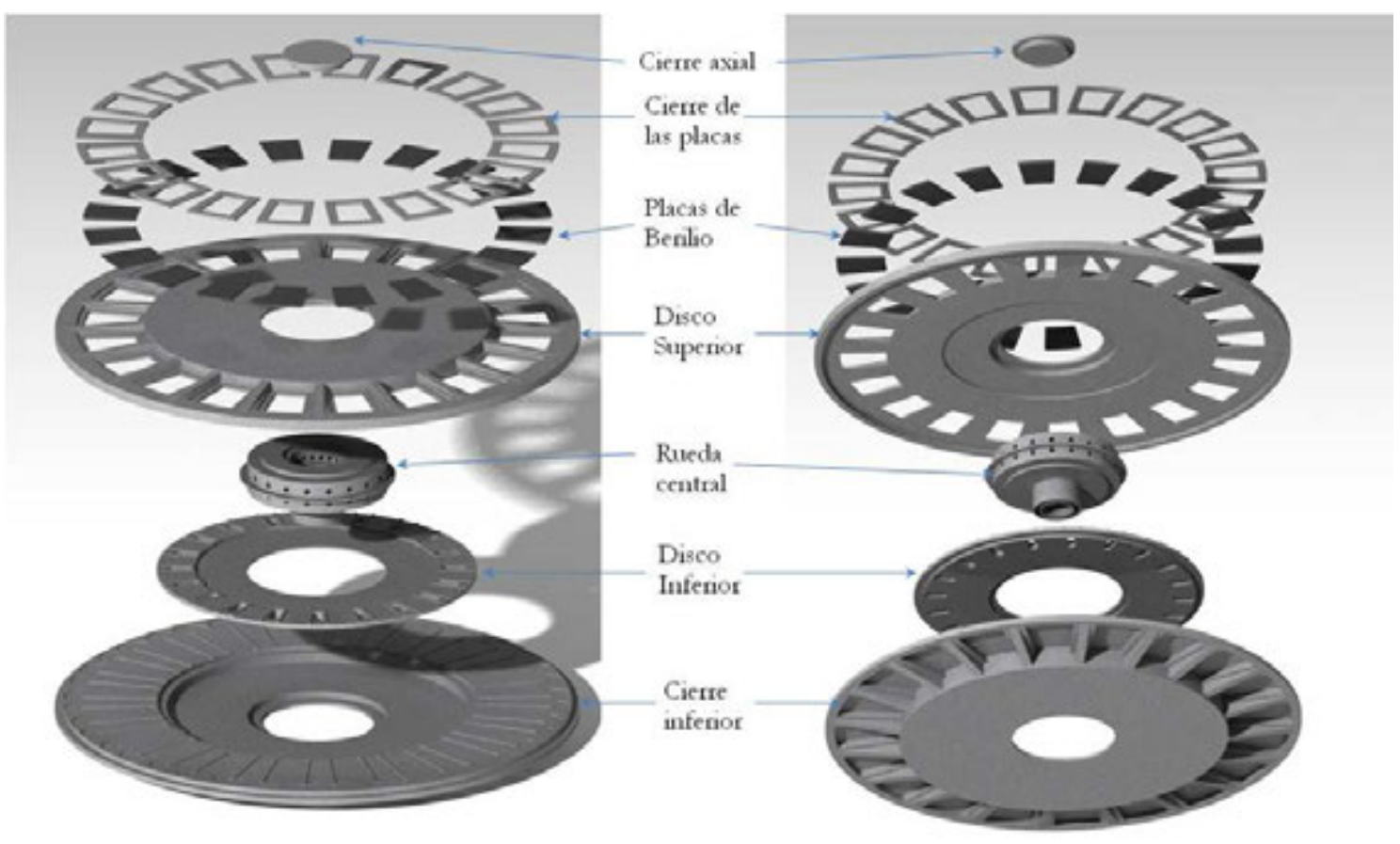

FigurA A-23: Vista explosionada del disco rigidizado. 


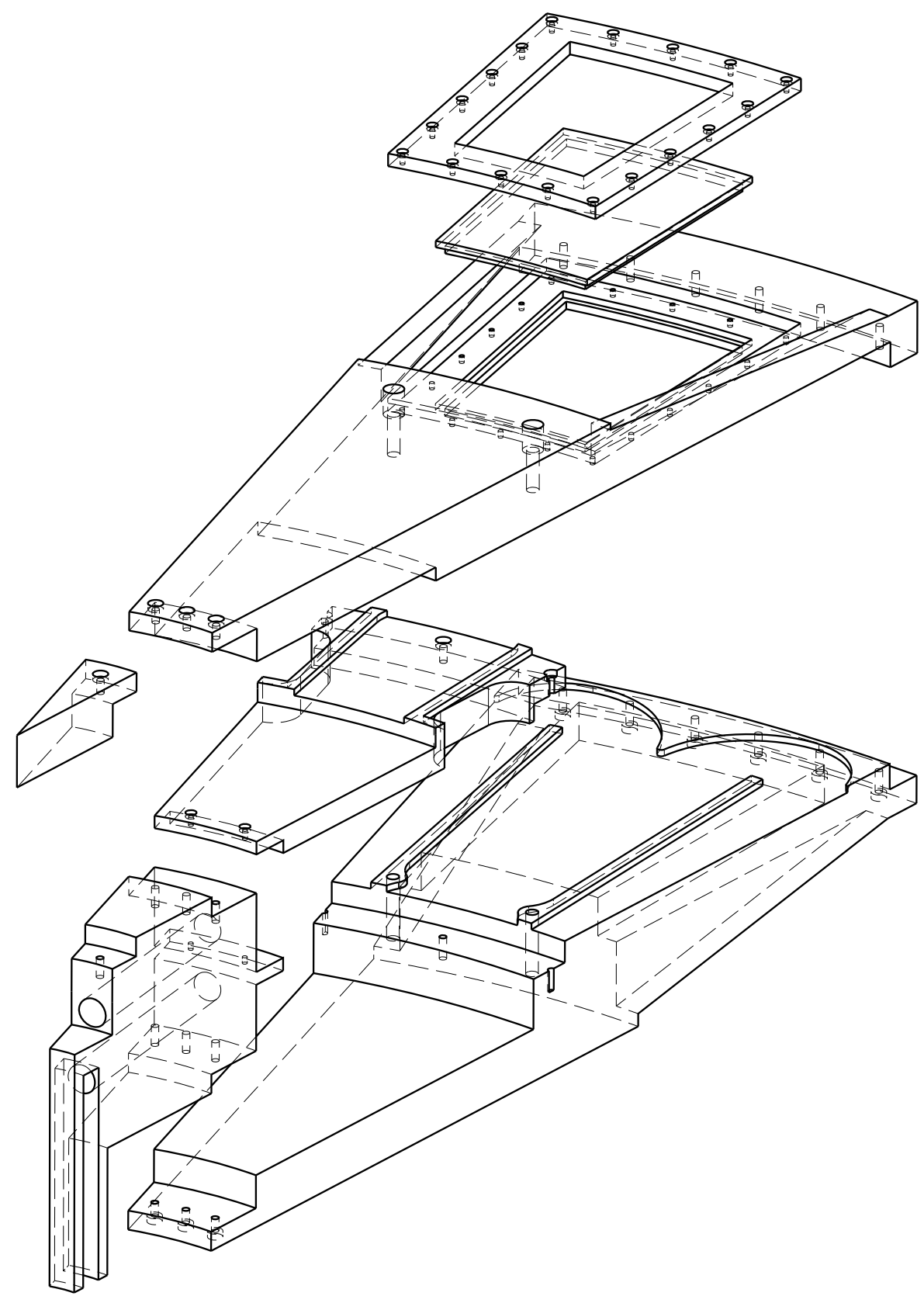

FigurA A-24: Vista explosionada de un sector de $18^{\circ}$ de la configuración rigidizada. 

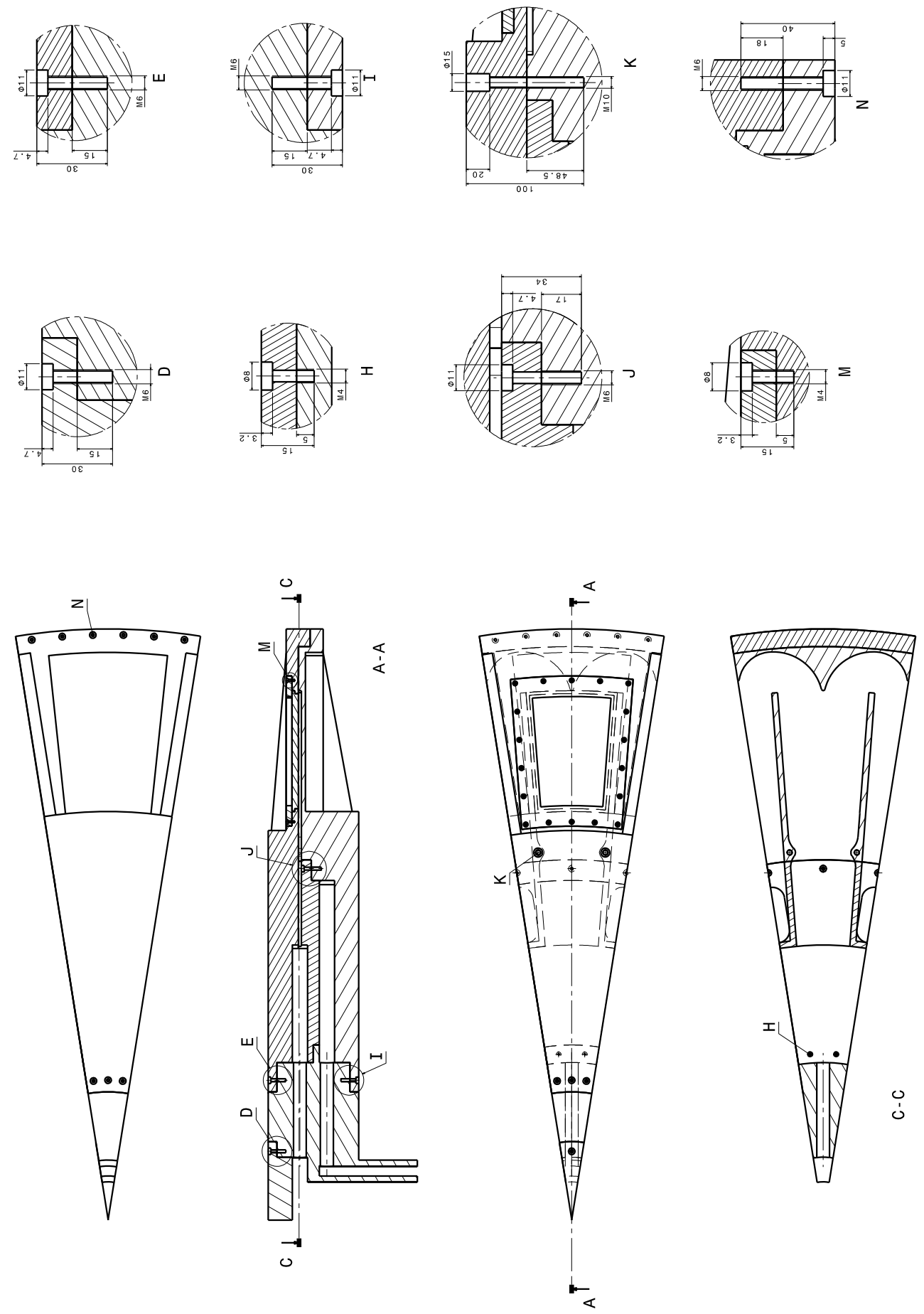

FigurA A-25: Detalle de las uniones atornilladas de la estructura del blanco. 


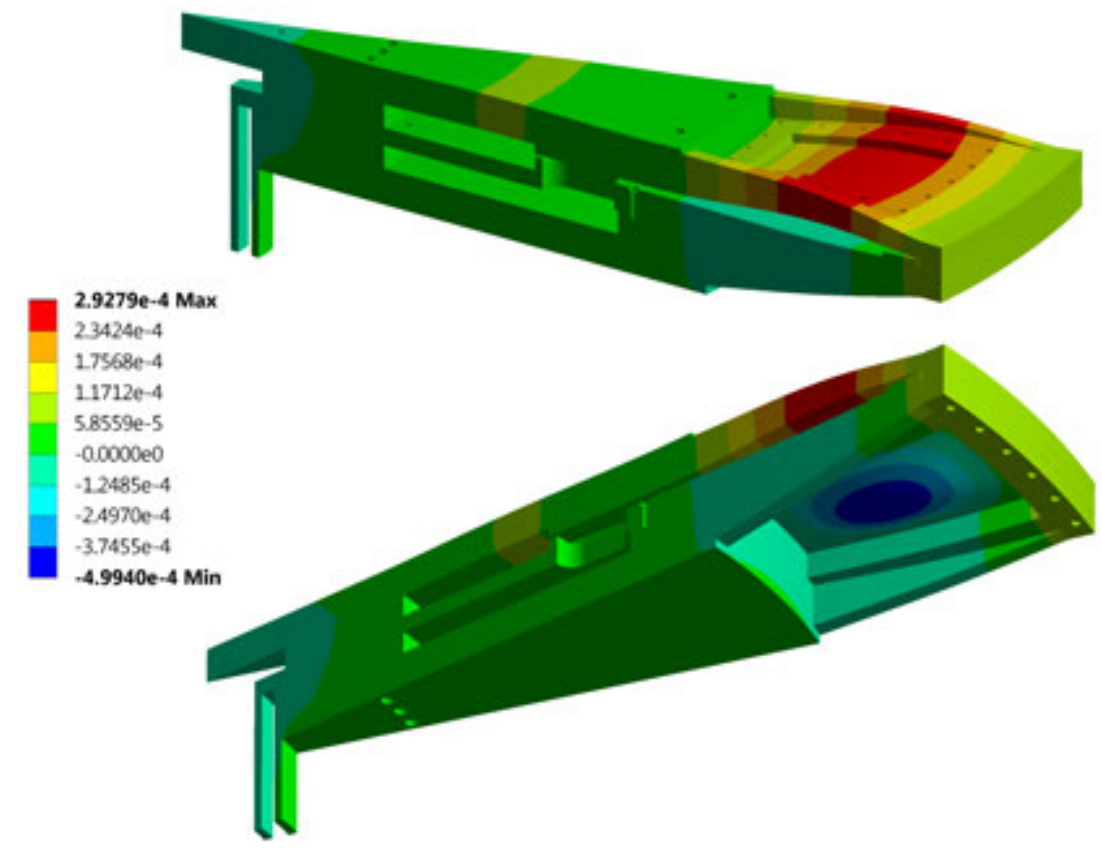

Figura A-26: Distribución de desplazamientos en la dirección axial del disco en modelo mecánico lineal rigidizado $[\mathrm{m}]$.

mientos hasta las décimas de milímetro. La deformación máxima se da esta vez en la lámina del cierre inferior que se encuentra debajo de la placa de berilio, cuyo espesor no se ha aumentado para evitar la captura de neutrones. Respecto a las tensiones, estas permanecen globalmente inferiores a $100 \mathrm{MPa}$, por lo que no se espera fallo mecánico debido a la presión interna con esta solución.

Así pues, tras la evolución descrita en este Apéndice, se concluye que la estructura del blanco diseñada presenta un comportamiento global adecuado, y será esta configuración la que se analice en detalle en el Capítulo 3, incluyendo las tensiones termomecánicas y el comportamiento real de las uniones atornilladas. 


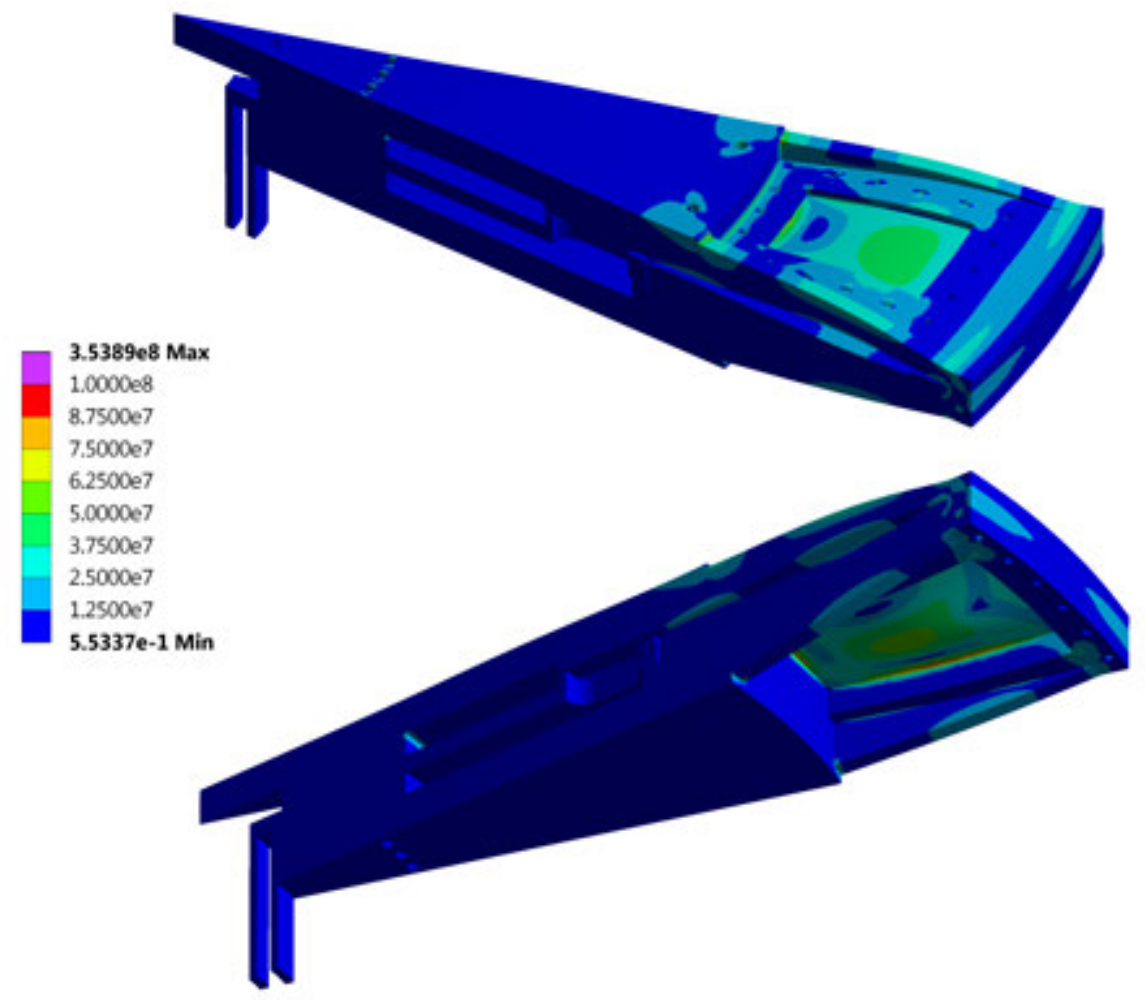

Figura A-27: Distribución de tensión de von Mises en modelo mecánico lineal rigidizado $[\mathrm{Pa}]$. 



\section{Apéndice B}

\section{Análisis paramétrico del efecto de la duración del pulso}

Con el objetivo de tener una primera estimación conservadora de la capacidad del diseño para trabajar con pulsos de menor duración que la de referencia, 1,5 $\mathrm{ms}$, se ha calculado la evolución de tensiones y temperaturas en la placas de berilio para longidudes de pulso de 0,75 y 0,5 $\mathrm{ms}$. Para hacer el análisis conservador, se ha optado por mantener el factor de carga del acelerador en el valor de referencia, $3 \%$, y por tanto la potencia del haz en 112,5 $\mathrm{kW}$. Para ello es necesario aumentar la frecuencia de impacto en el mismo factor en el que se reduce la duración del pulso. Así pues, las frecuencias analizadas son 40 y $60 \mathrm{~Hz}$ respectivamente. Los cálculos se efectúan empleando el modelo descrito en la Sección 2.3.3 para la placa con el espesor de referencia, $9 \mathrm{~mm}$, variando únicamente las características del pulso. Las Figuras B-1 y B-2 muestran los resultados de temperatura y tensión de von Mises máximas obtenidas. Se incluye en la comparativa los resultados de la configuración de referencia, 1,5 ms y $20 \mathrm{~Hz}$.

Los resultados muestran como la temperatura y tensión máxima alcanzan una evolución estable pseudo-estacionaria, con unos valores medios similares a los de la configuración de referencia, por lo que a priori estos rangos de operación sería accesibles para el diseño propuesto para ESS-Bilbao. Cabe mencionar que, para acomodar las frecuencias crecientes, la velocidad de rotación del disco debe incrementarse en el mismo factor que en el que se incrementa la frecuencia. No obstante, dados los amplios márgenes de diseño con los que 


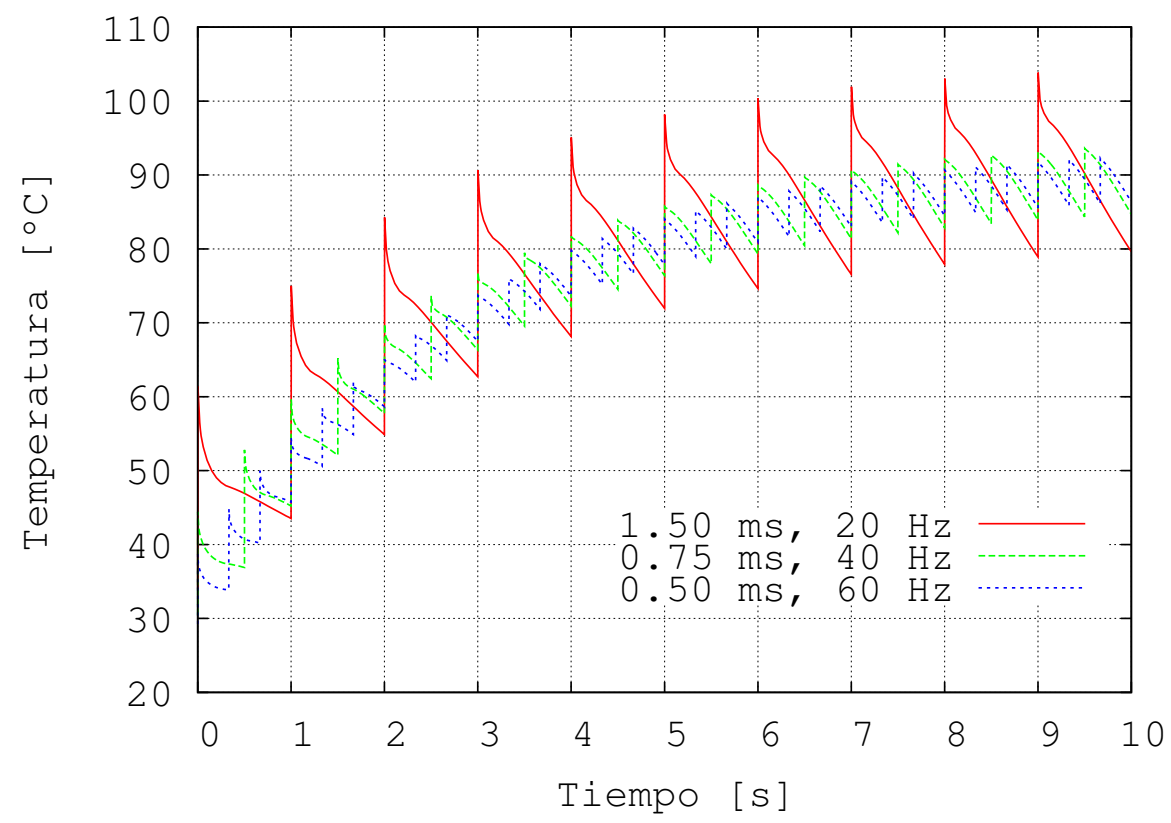

Figura B-1: Evolución de la temperatura máxima en una placa de Be de 9 mm para distintas combinaciones de longitud de pulso y frecuencia de impacto con un factor de carga del $3 \%$.

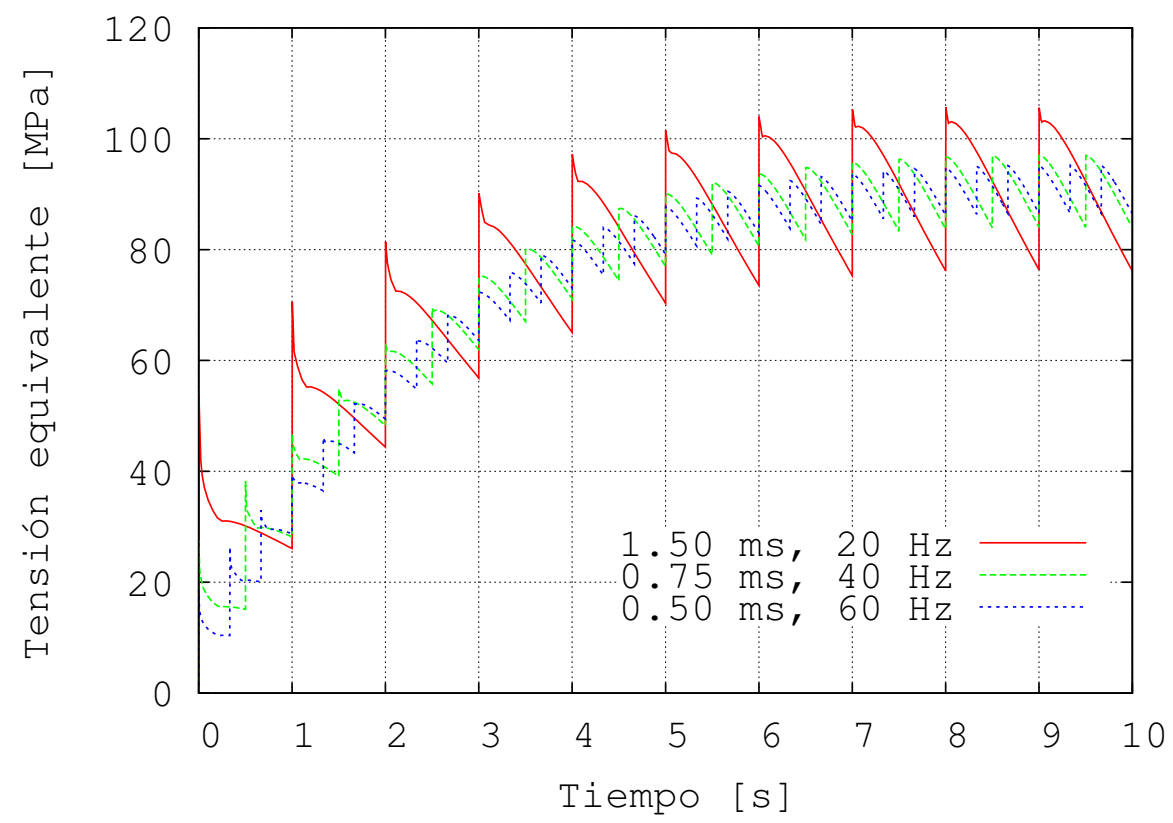

Figura B-2: Evolución de la tensión de von Mises máxima en una placa de Be de 9 mm para distintas combinaciones de longitud de pulso y frecuencia de impacto con un factor de carga del $3 \%$. 
cuenta la estructura del blanco, estos incrementos no plantearían problemas mecánicos. 



\section{Apéndice $\mathrm{C}$}

\section{Planos del prototipo termohidráulico}

Modifiaciones gemoetétricas y planos generados por la empresa Leading Metal Mechanics $[243]$ 


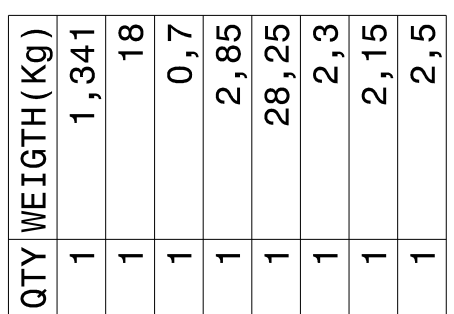

$\overleftrightarrow{\leftarrow}$

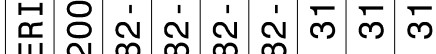

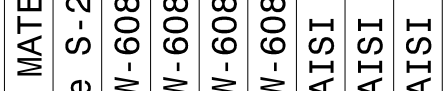

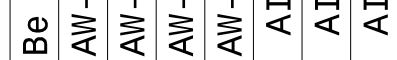
군추 군

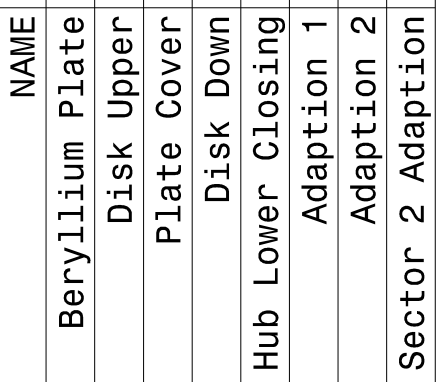

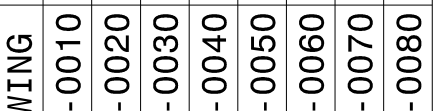

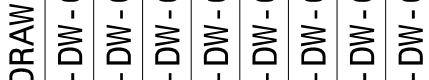

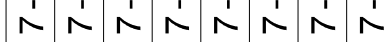

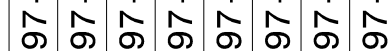
守导导导 $\underset{⿱ 亠 䒑}{\rightleftarrows}$
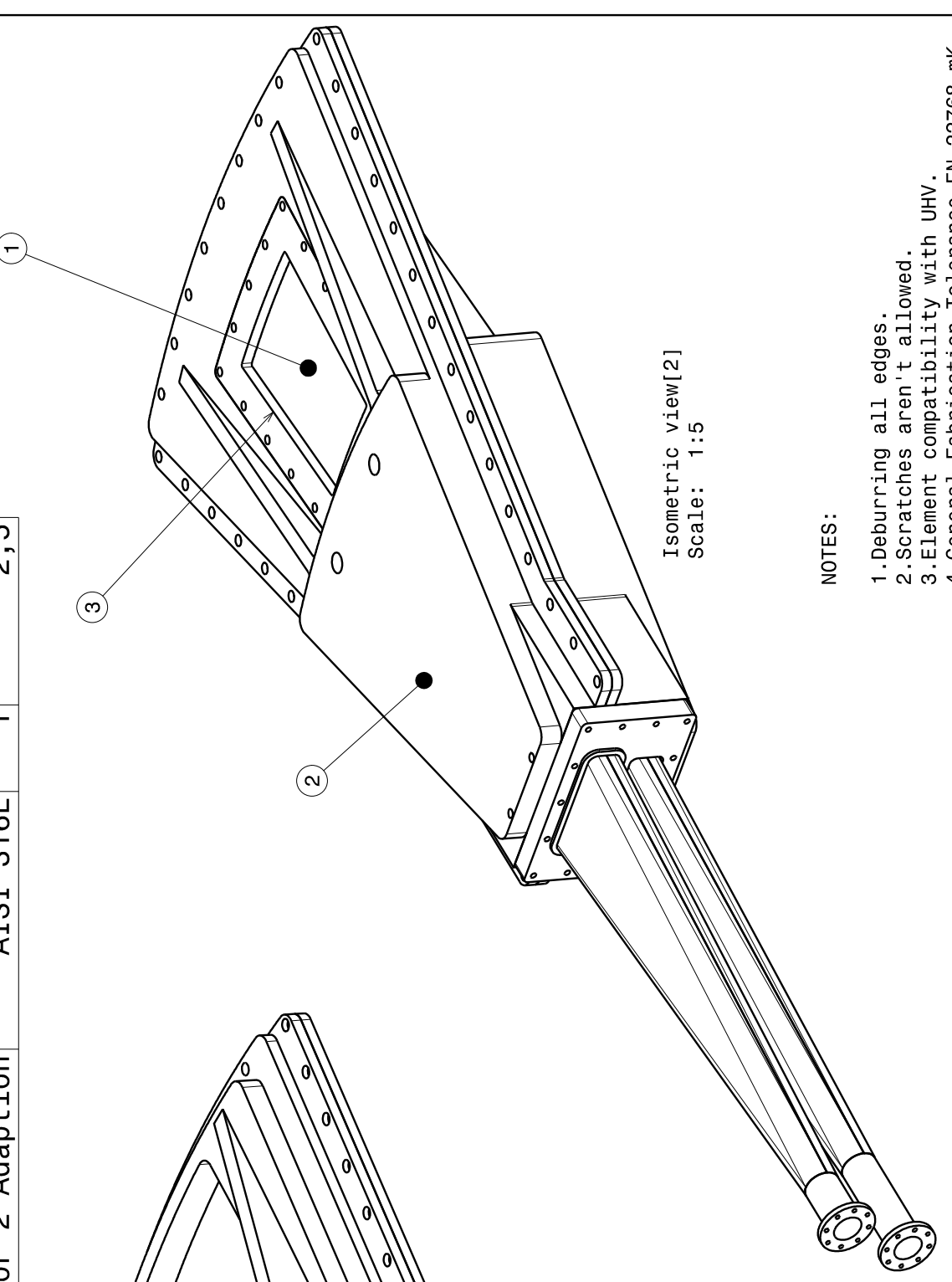
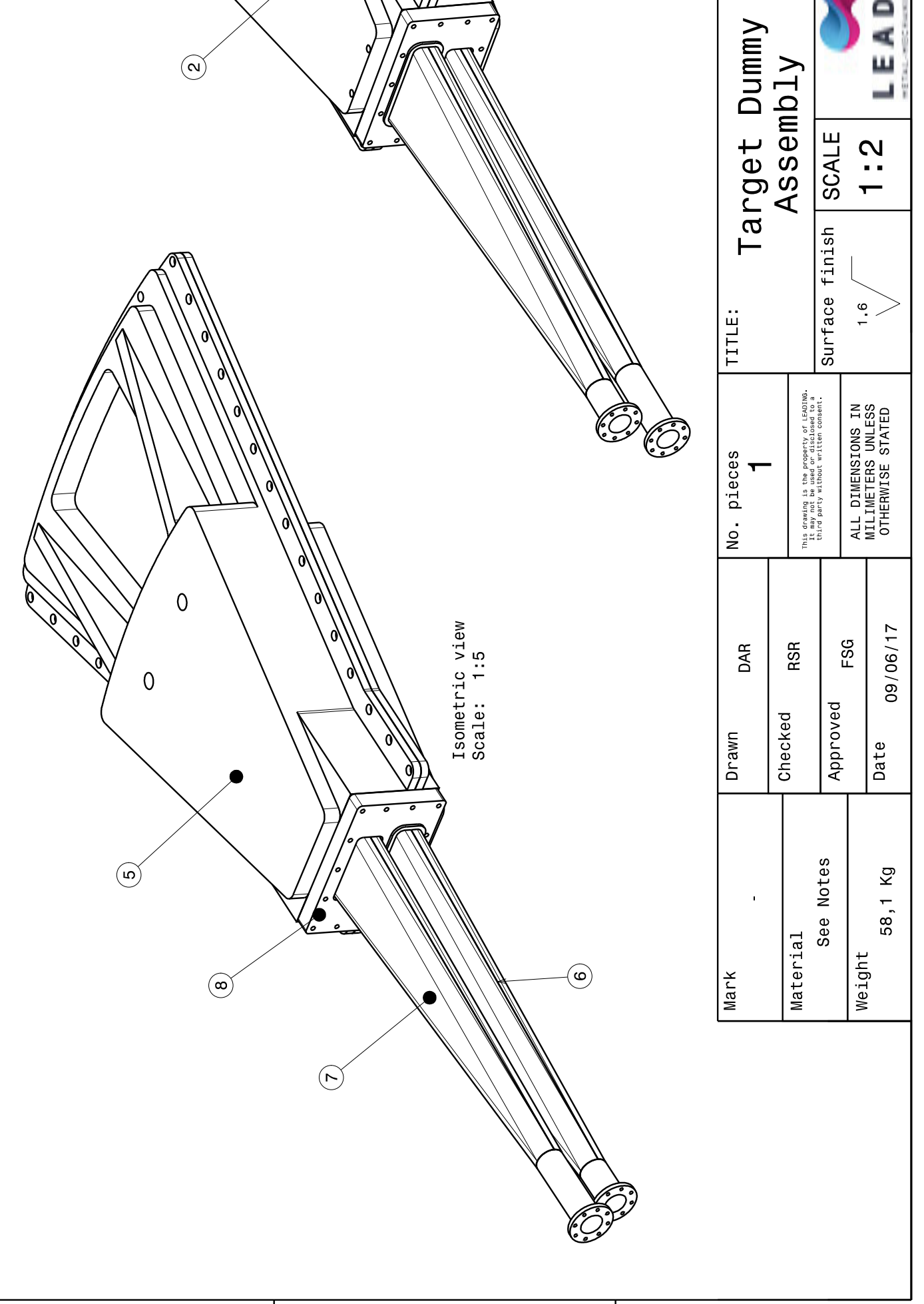

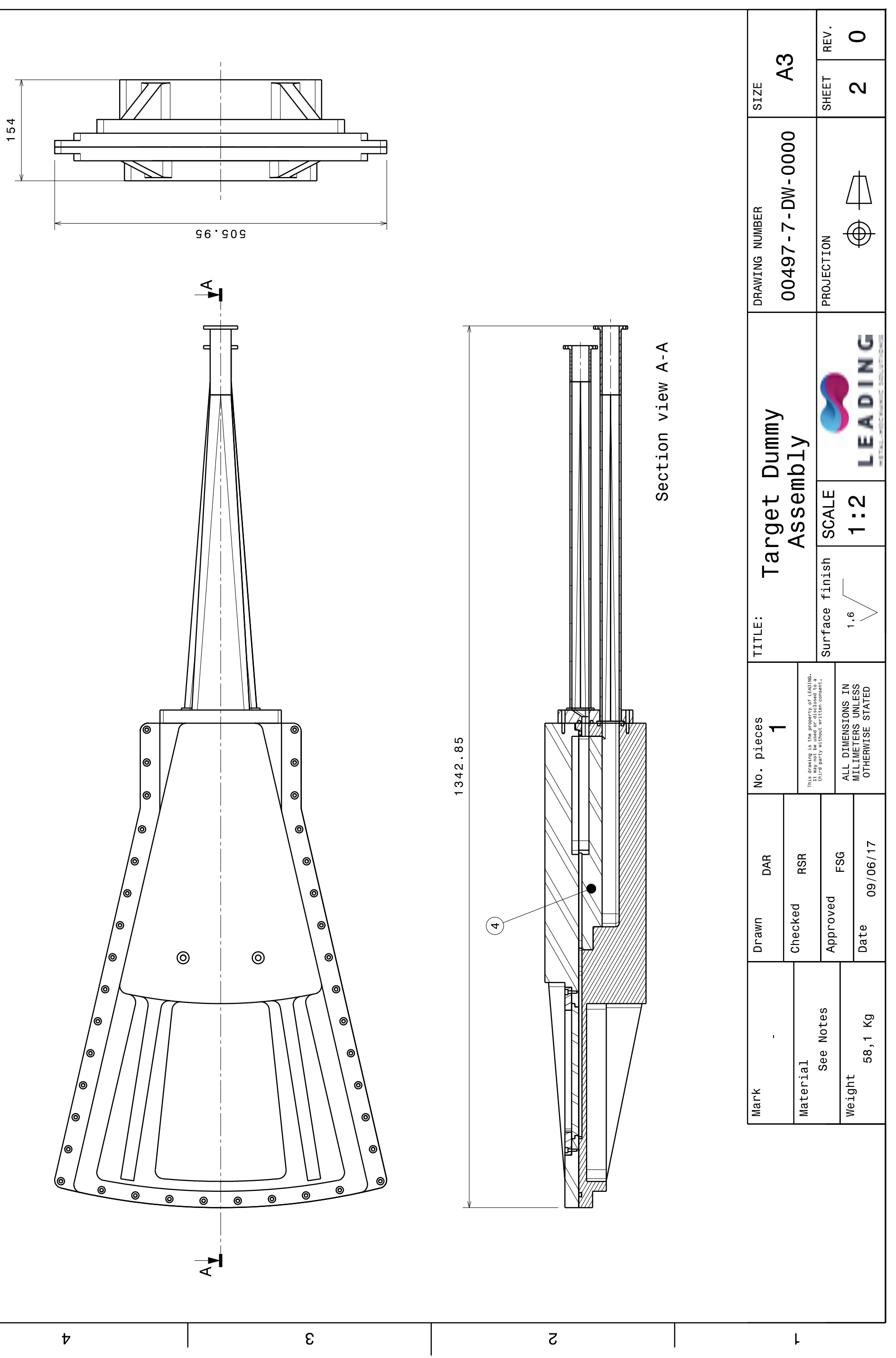

$\exists$

$\vartheta$

0

0

$\exists$

$\mathrm{H}$

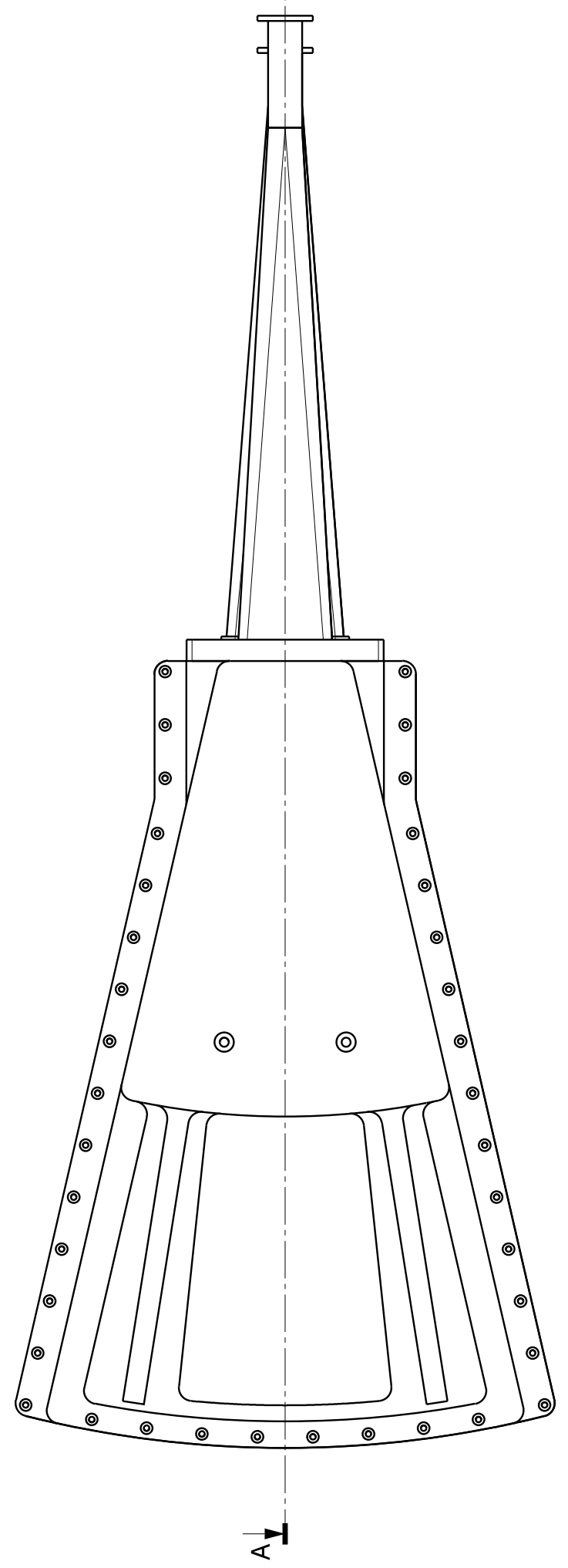




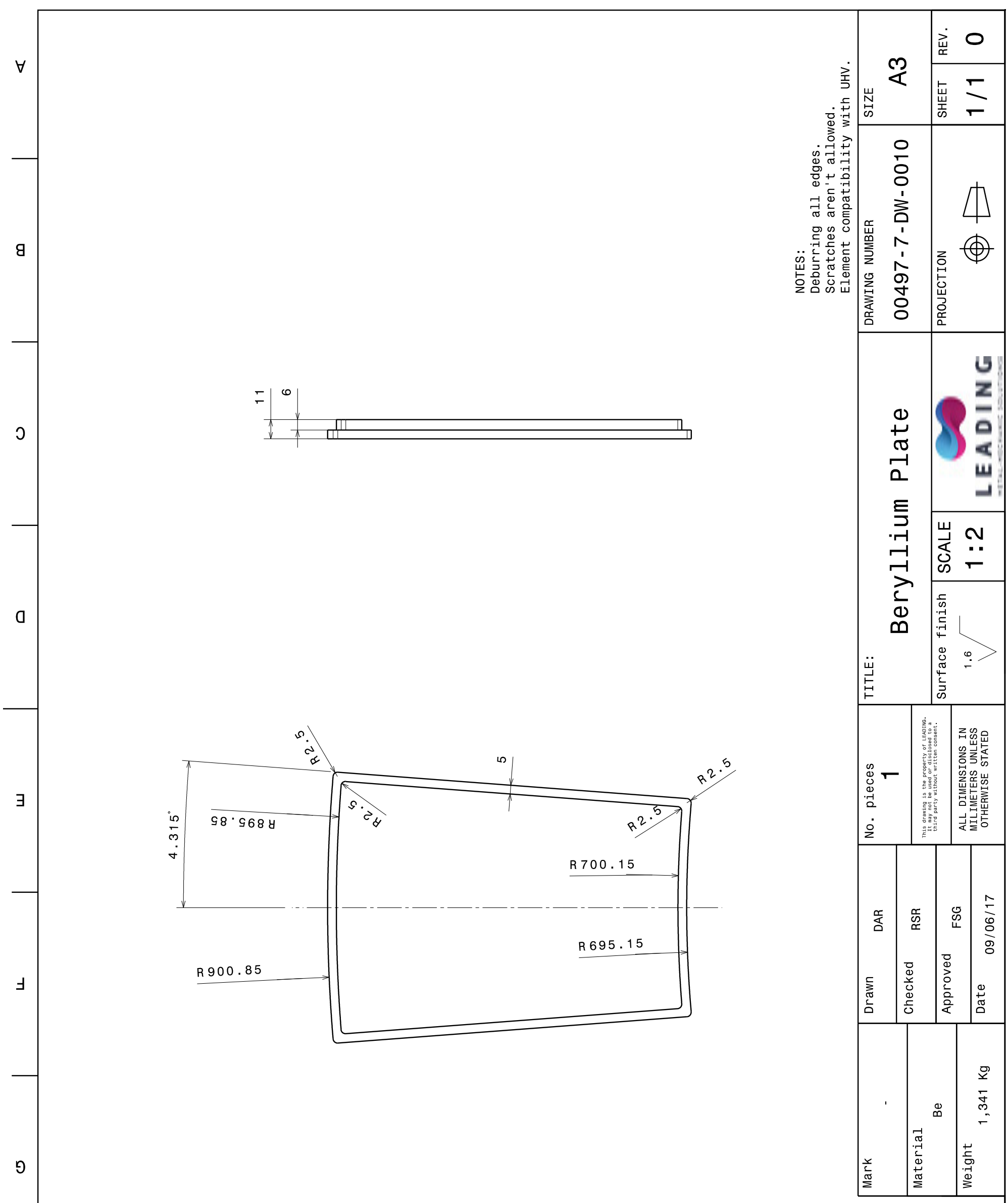



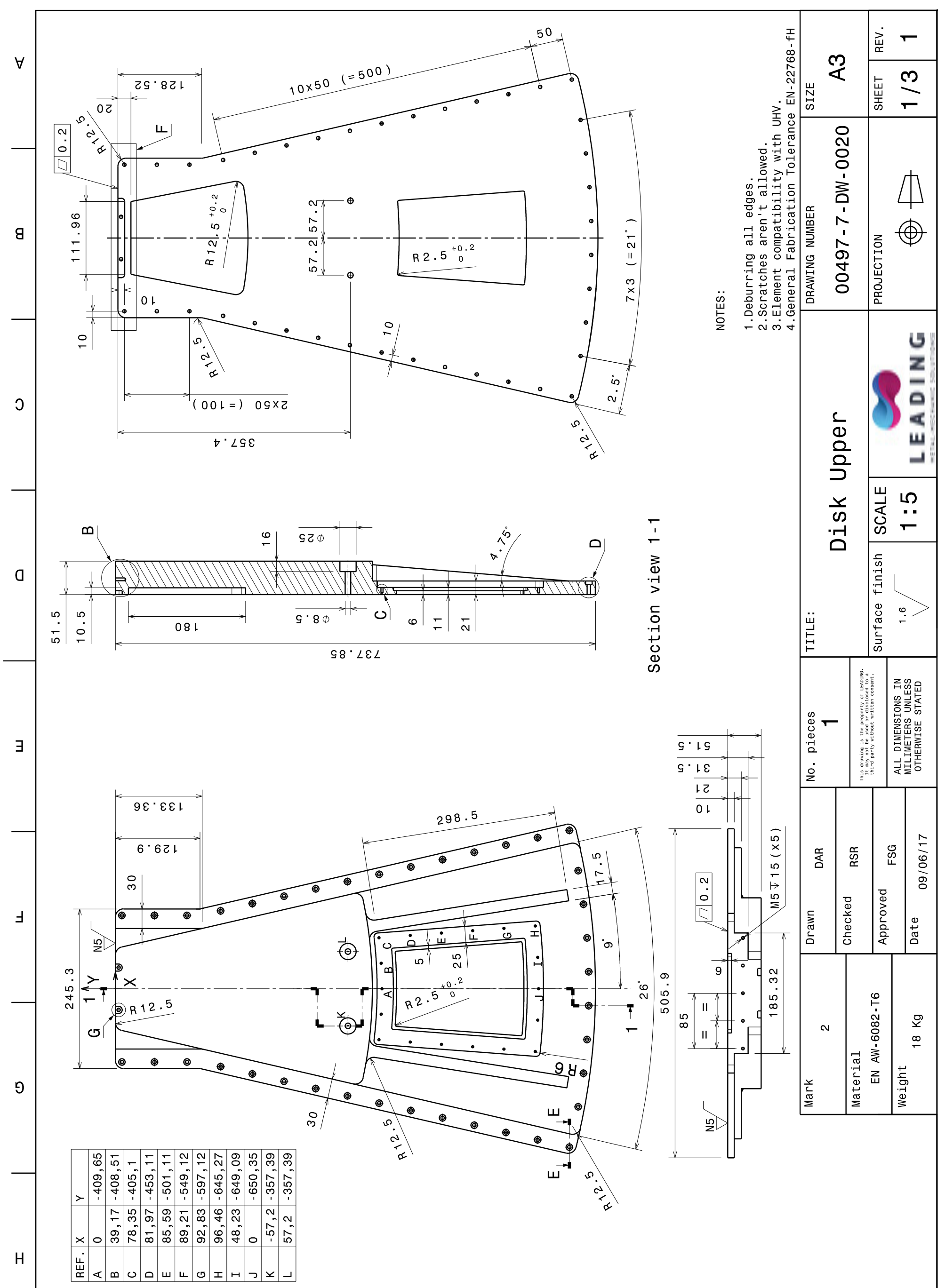

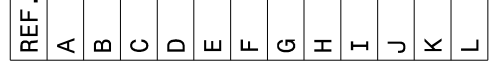

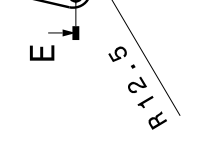



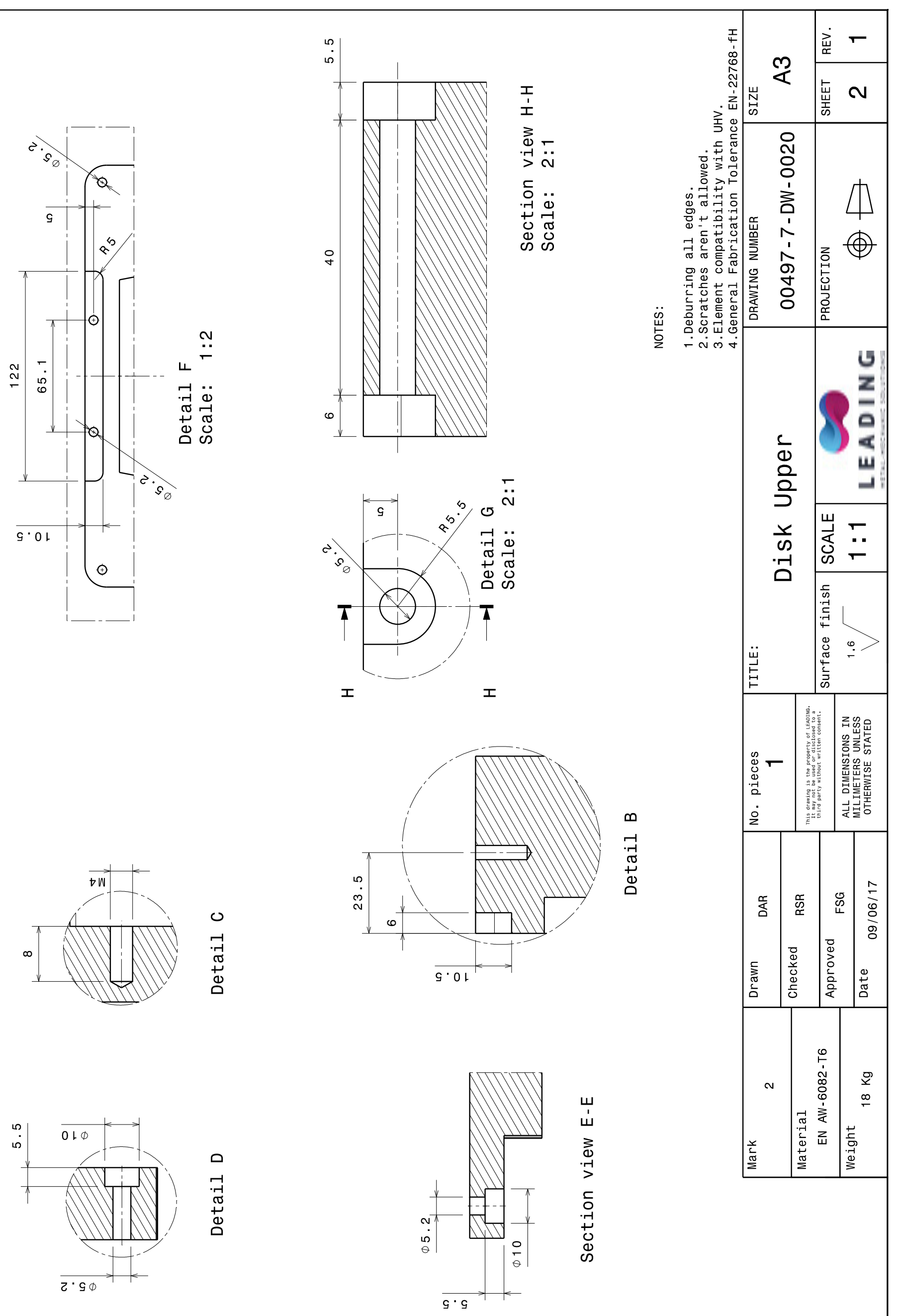


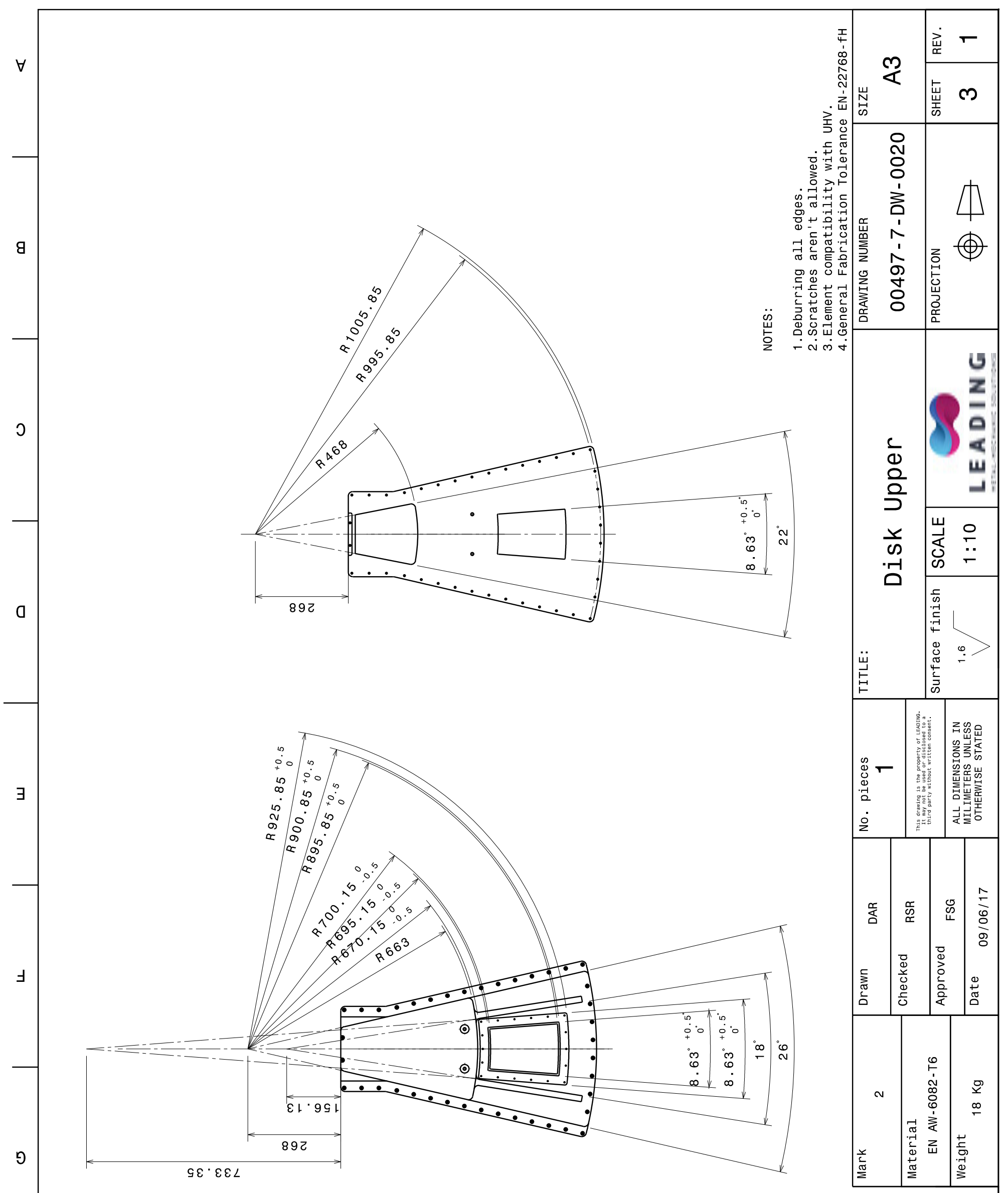



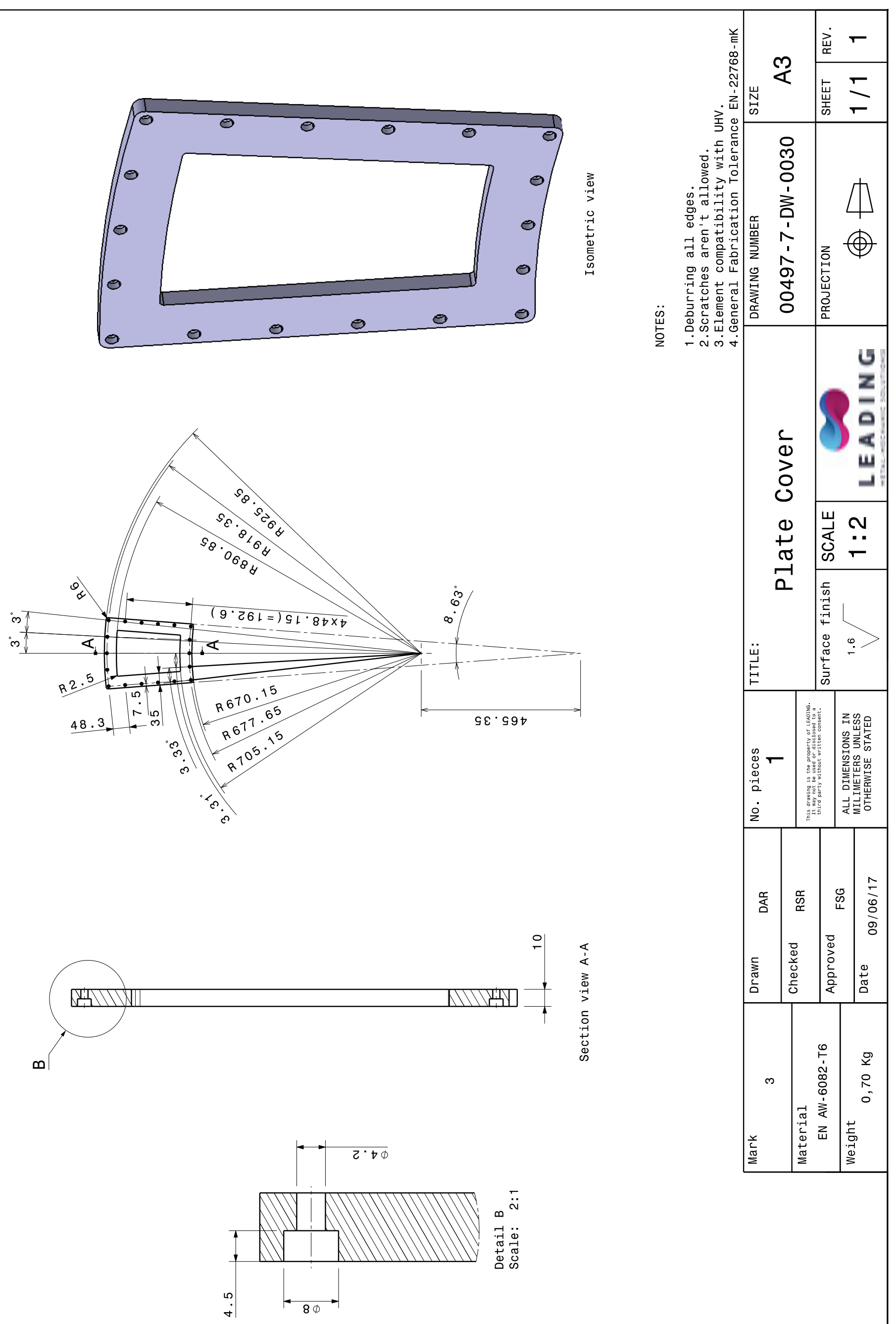


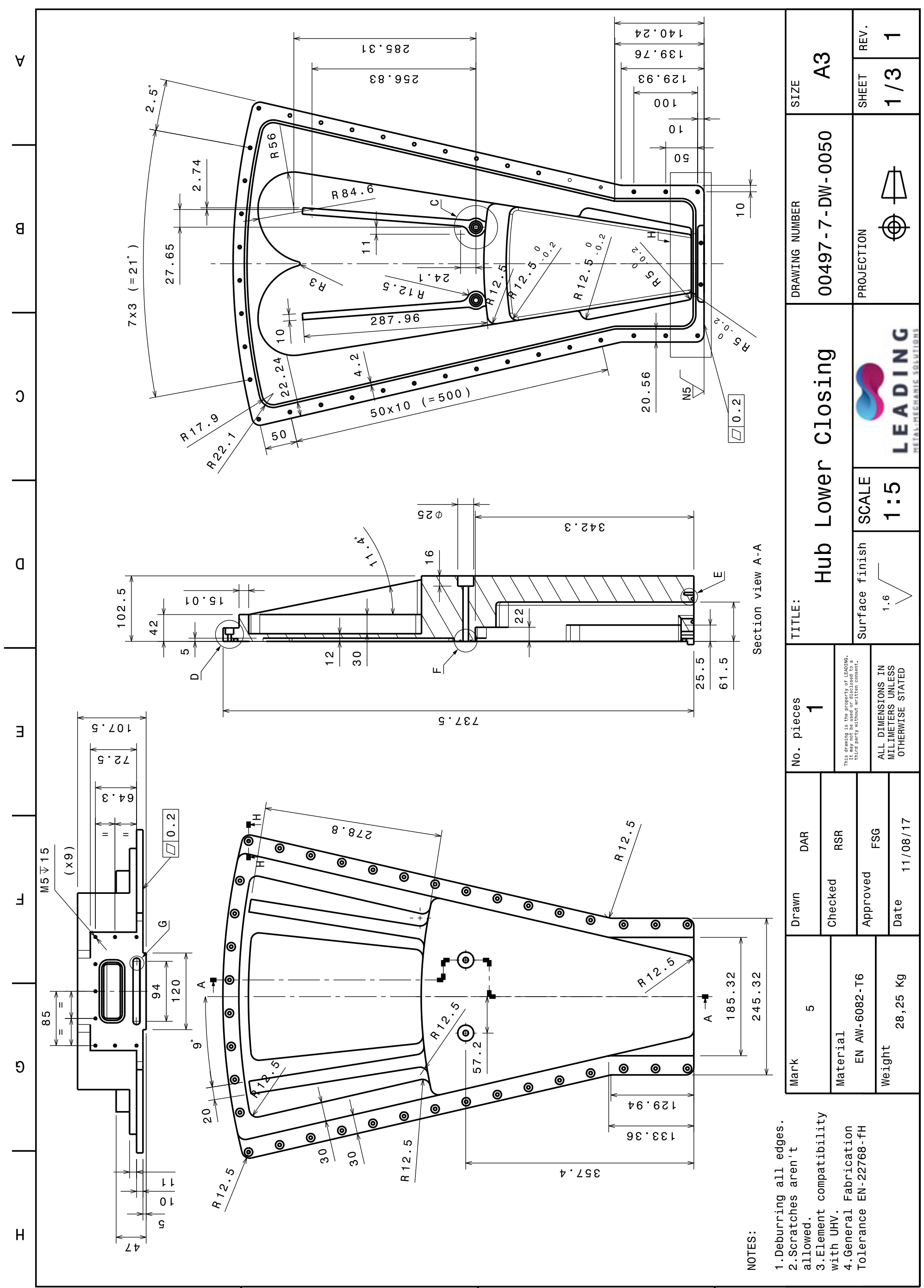




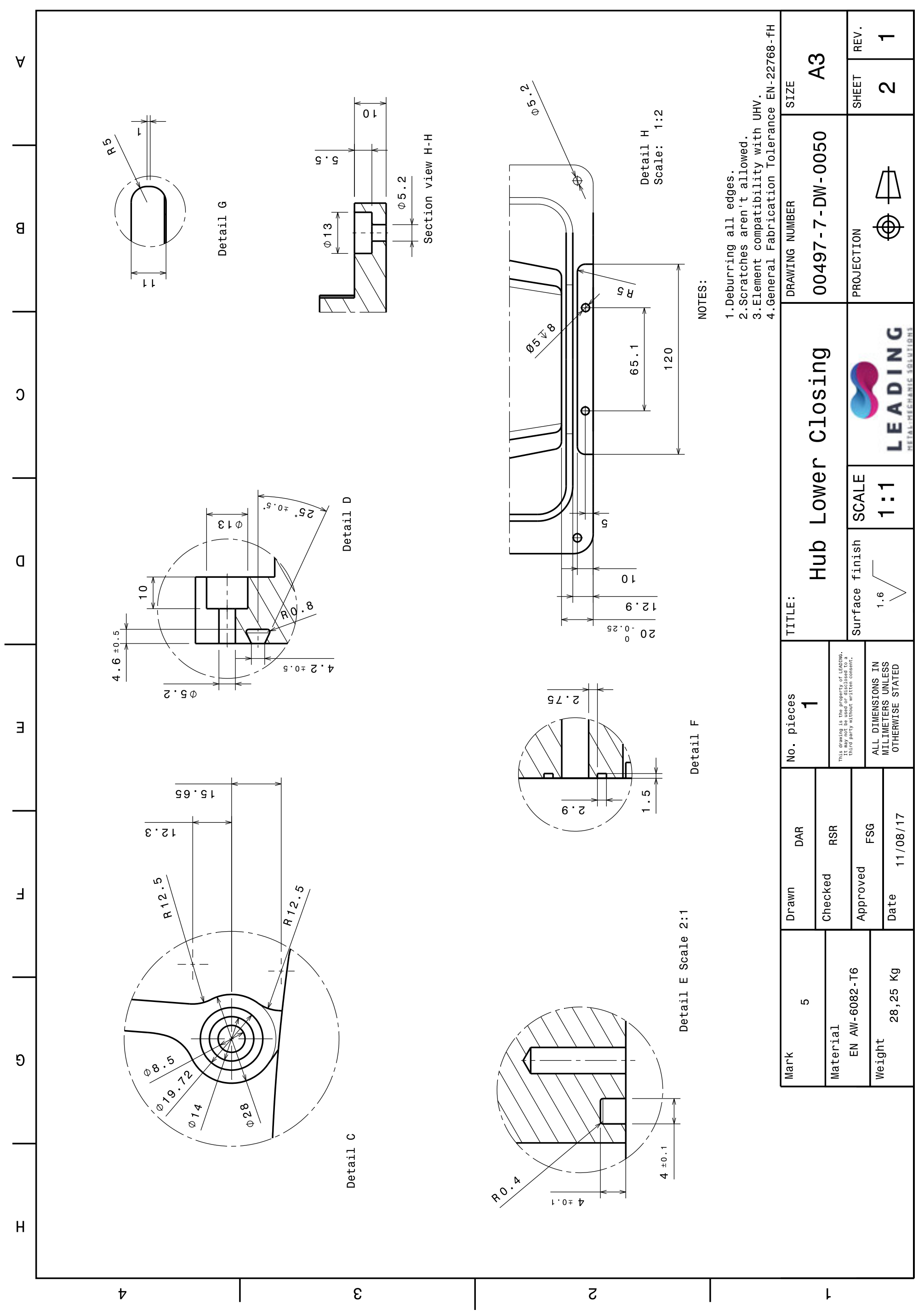




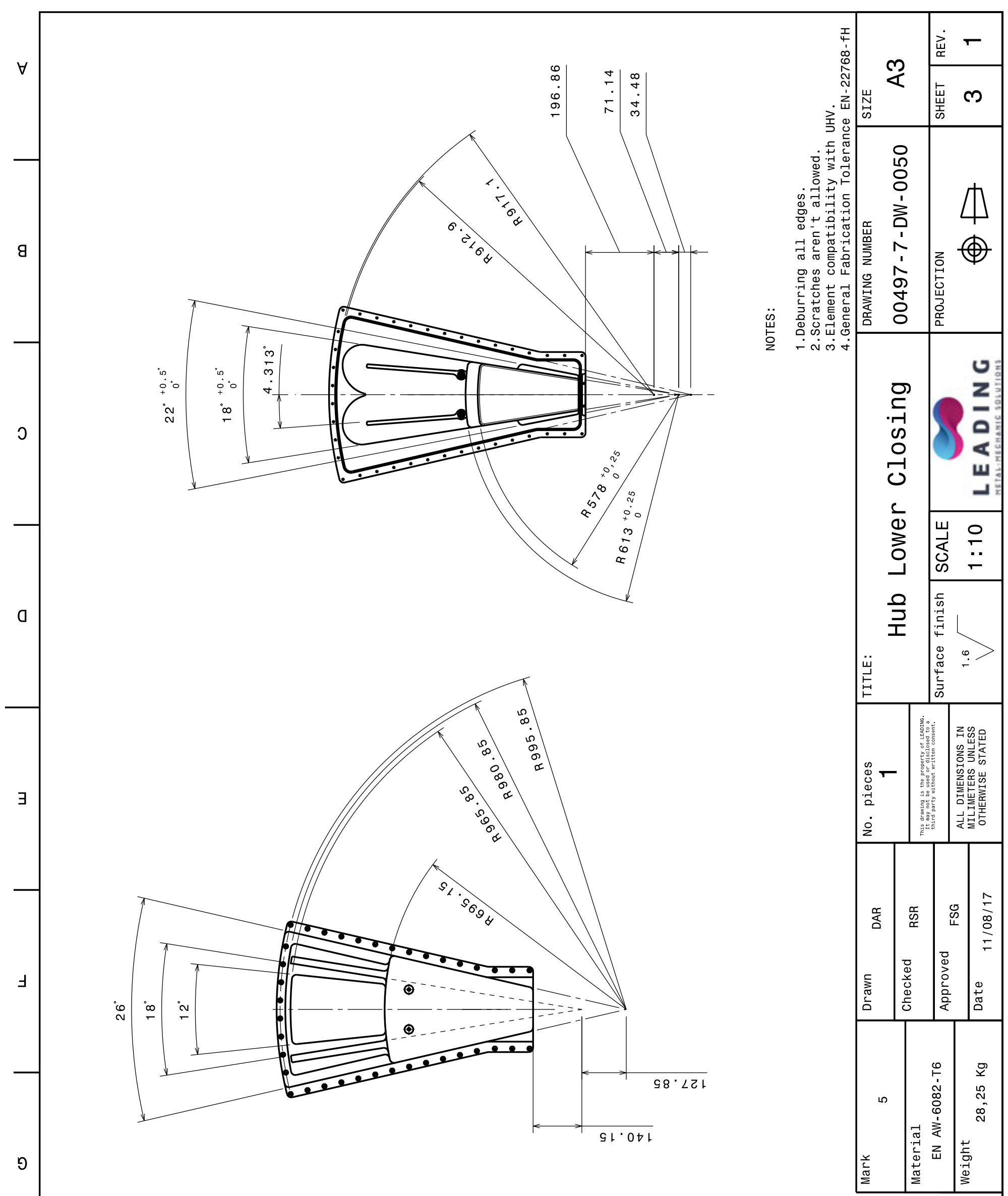




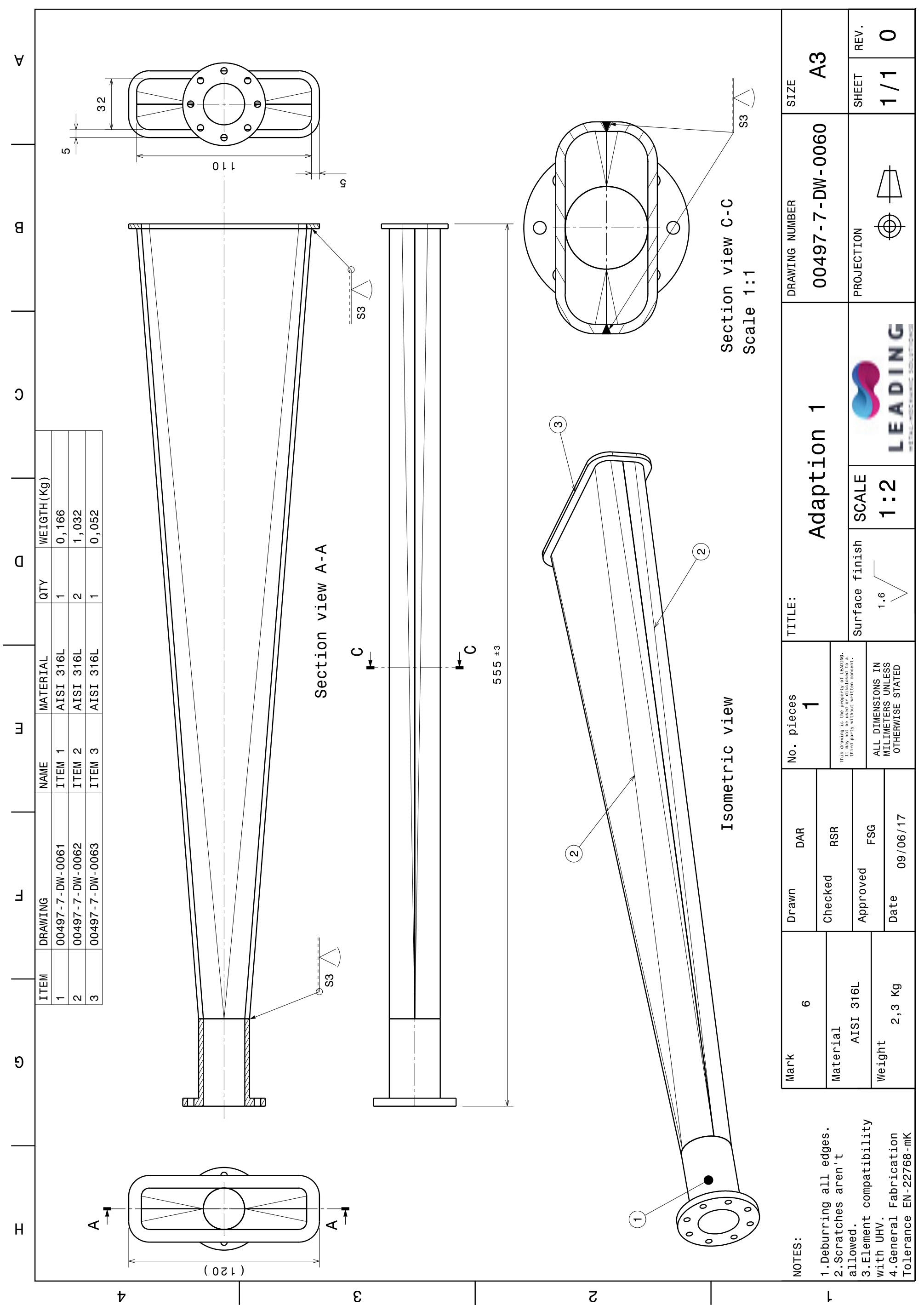




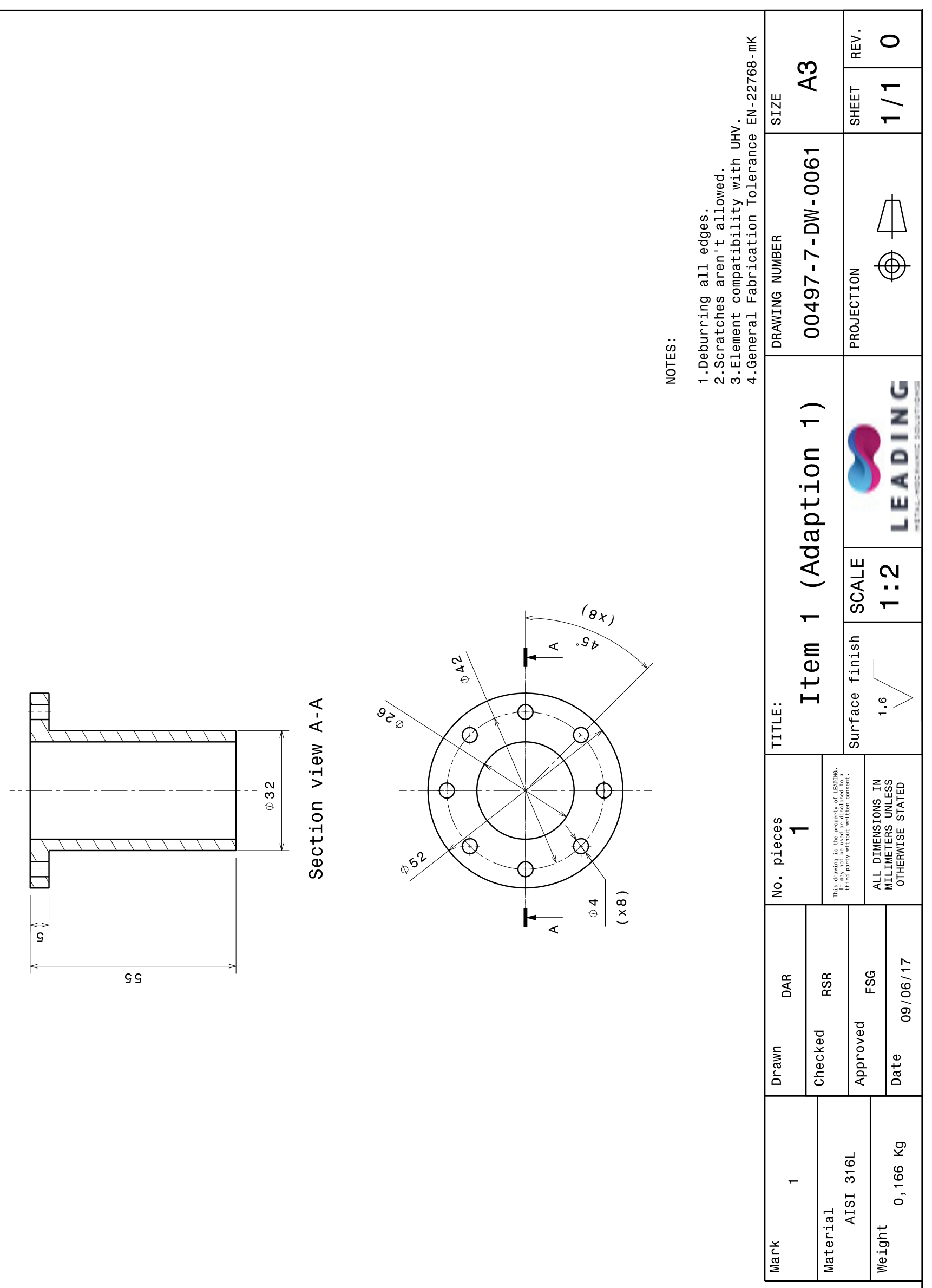




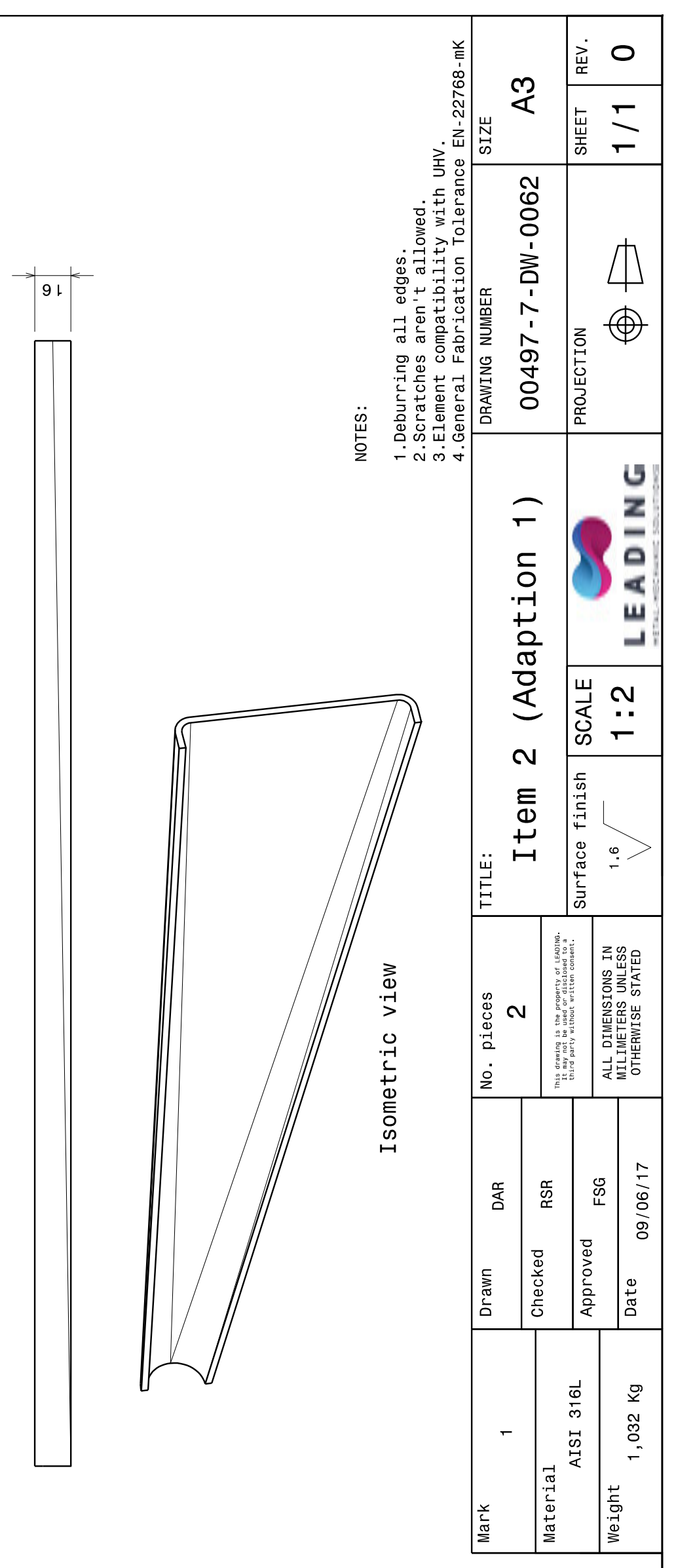

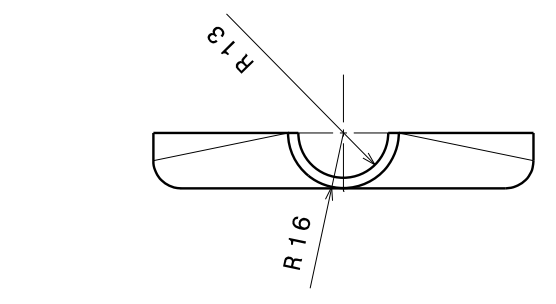

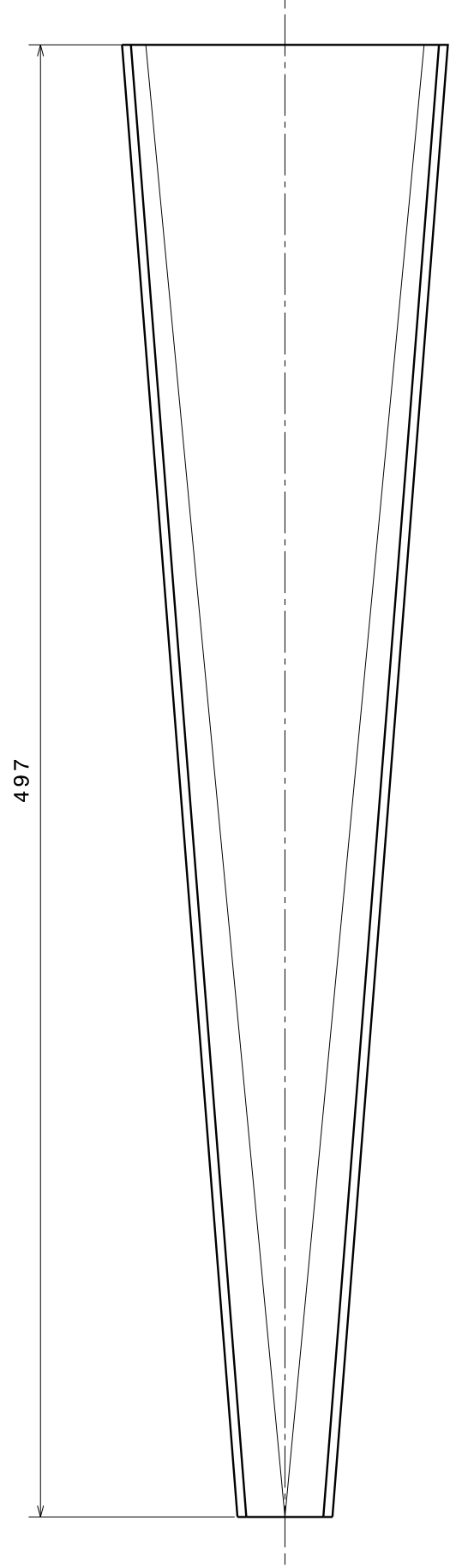

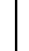

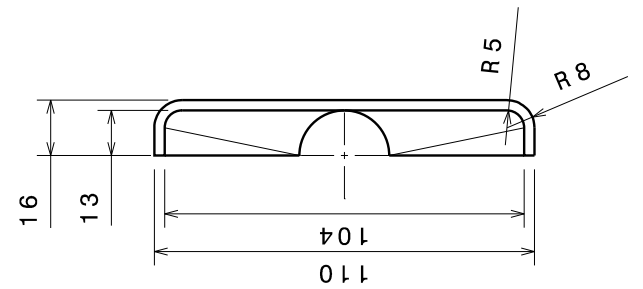

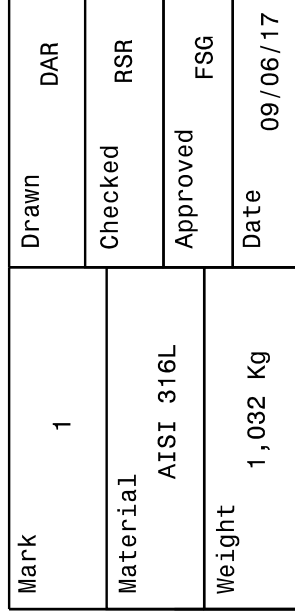




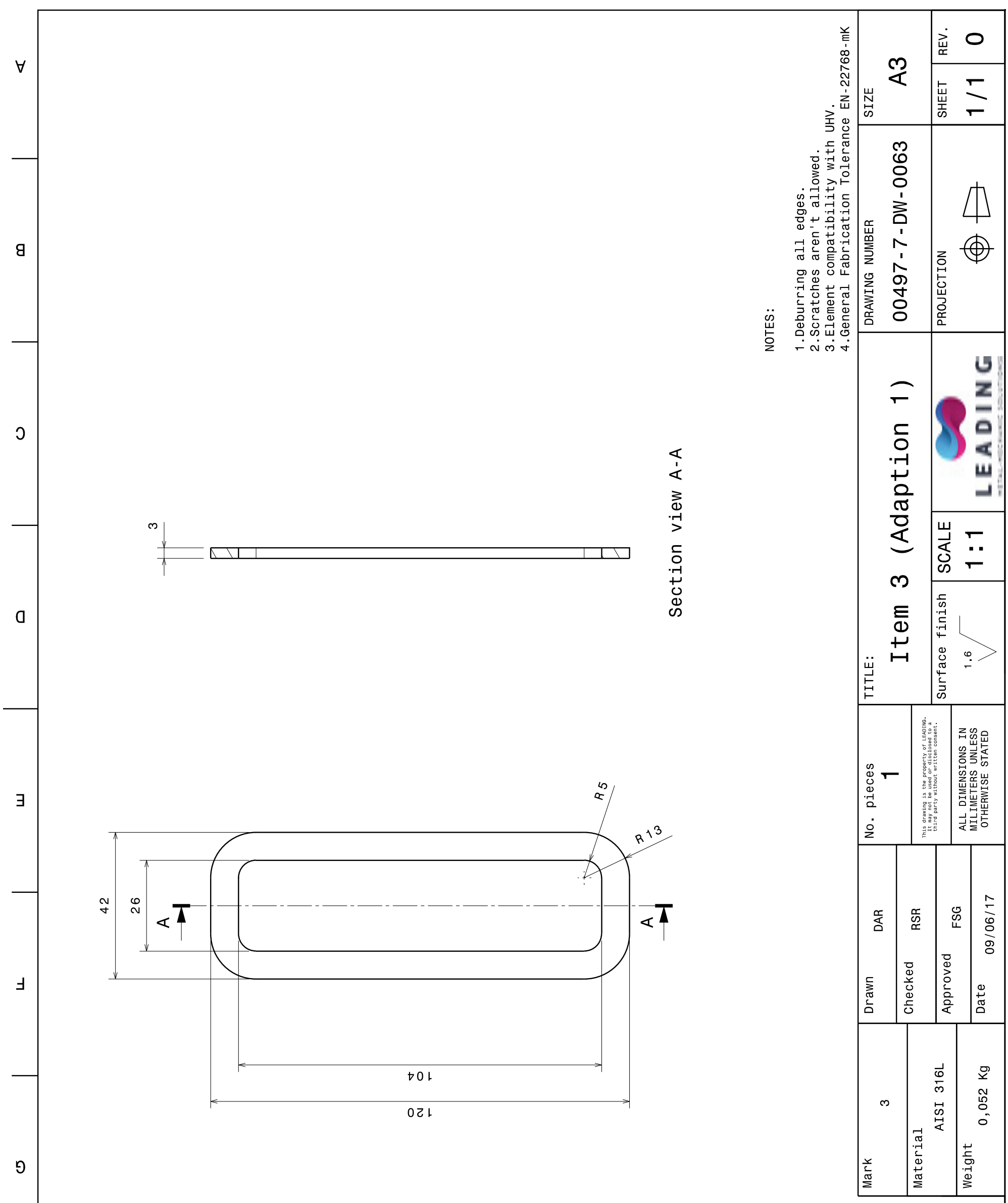




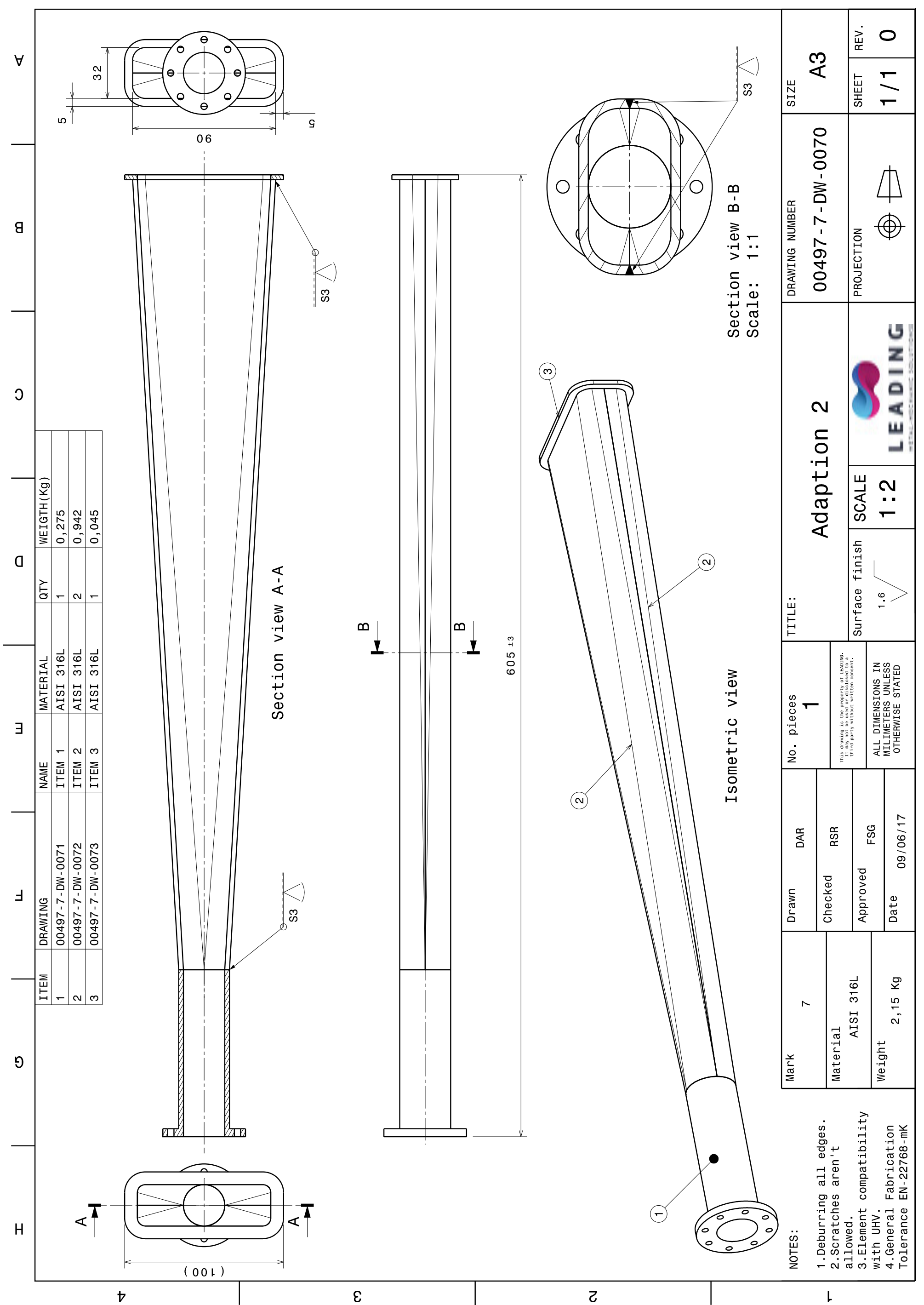




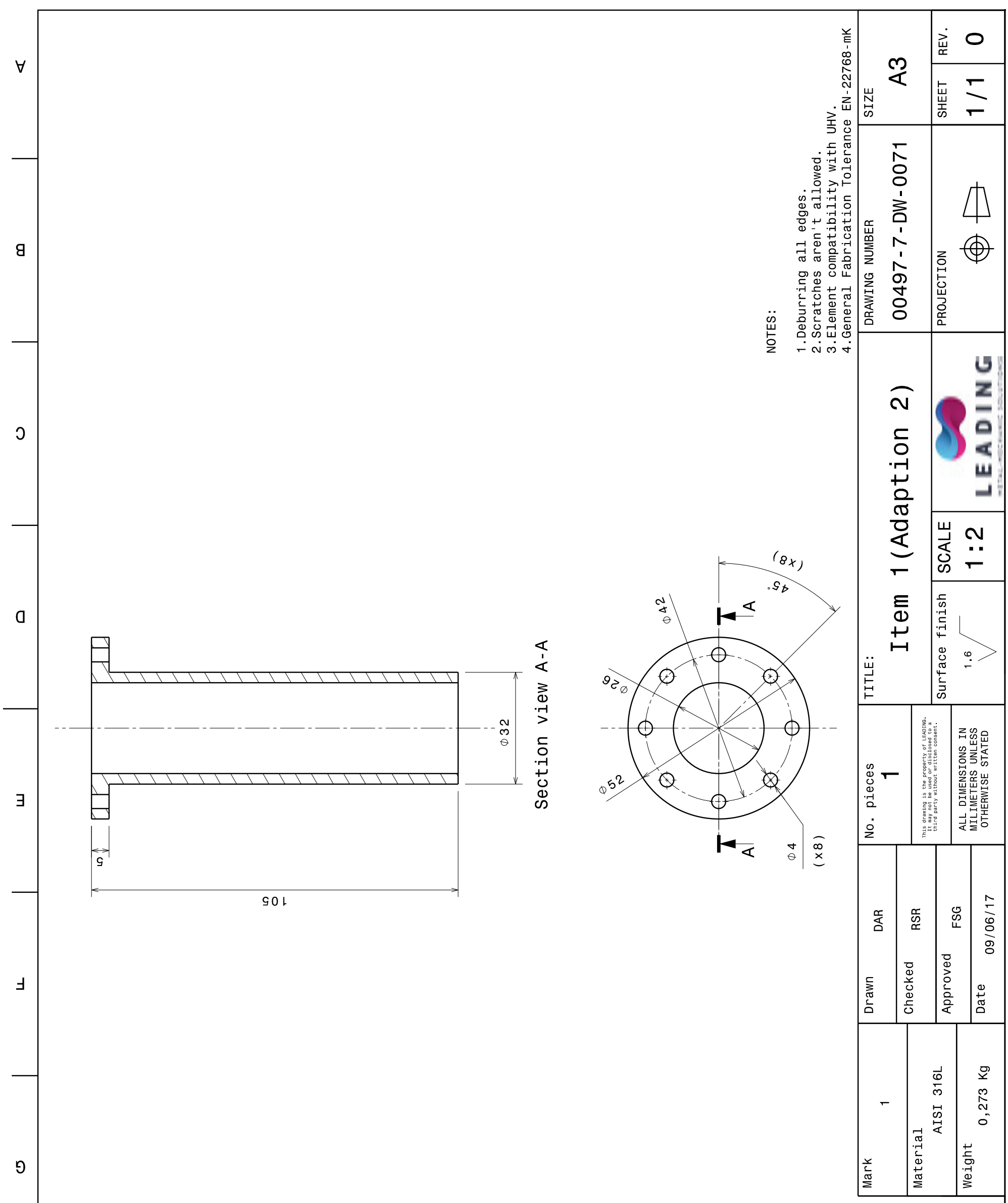




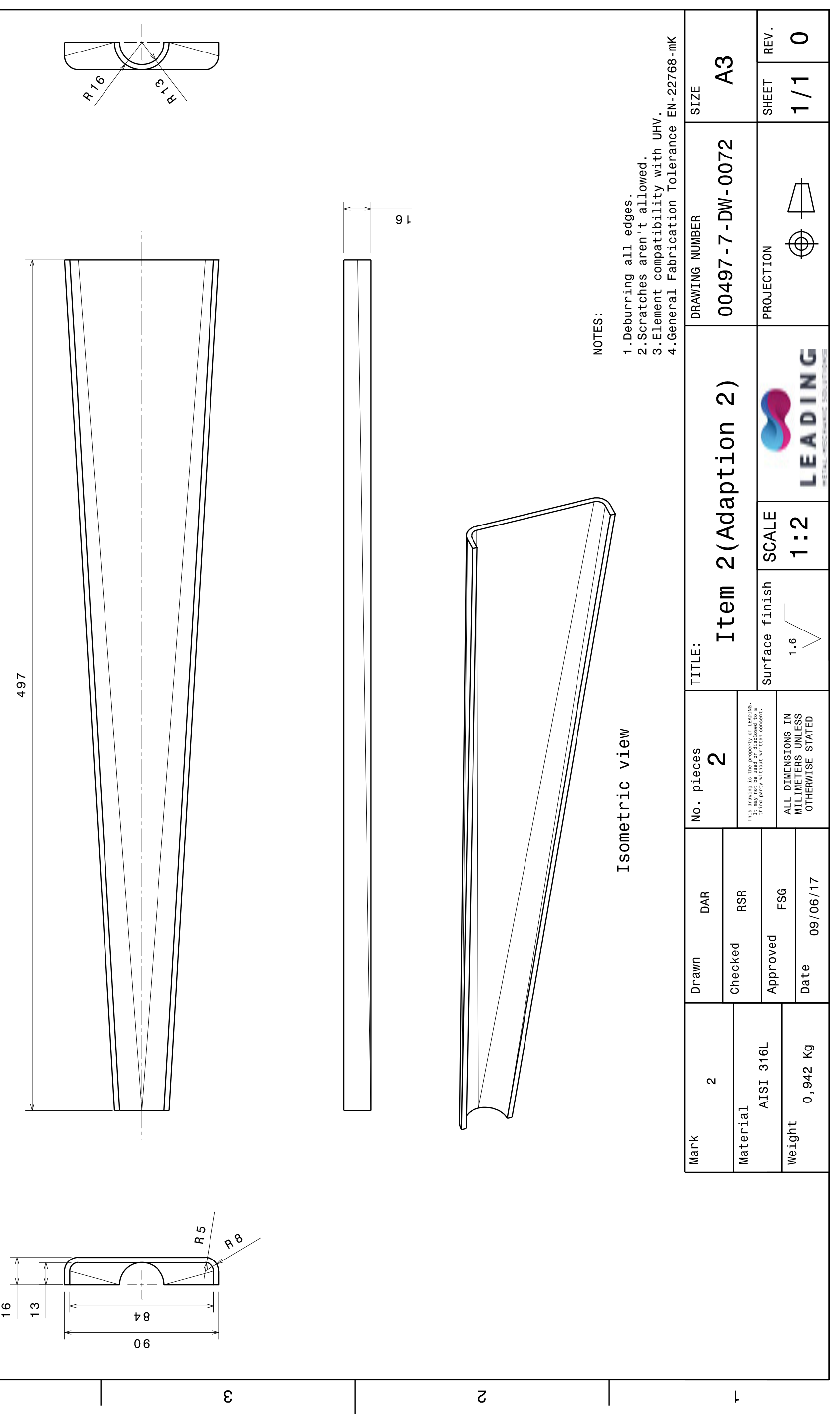




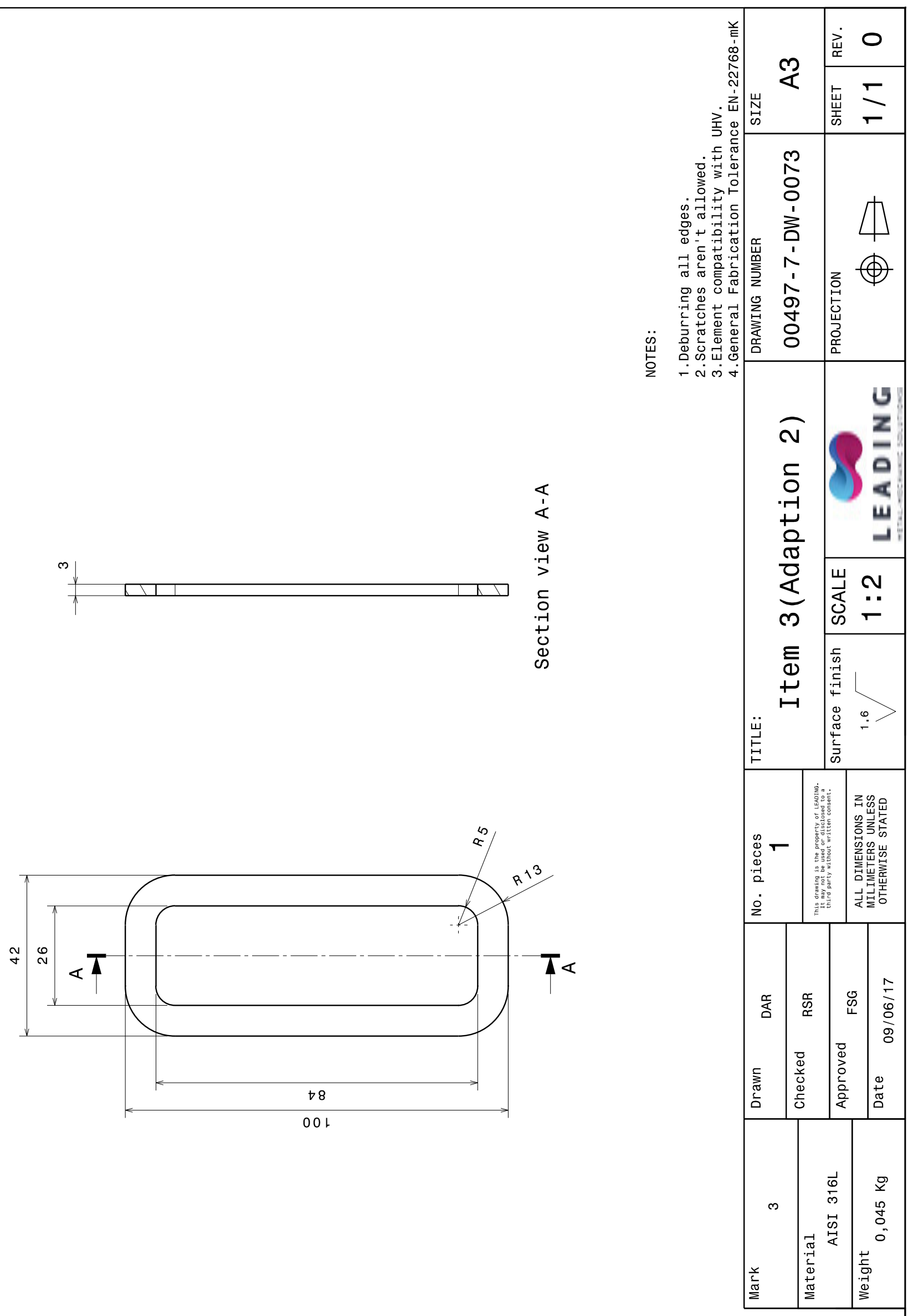




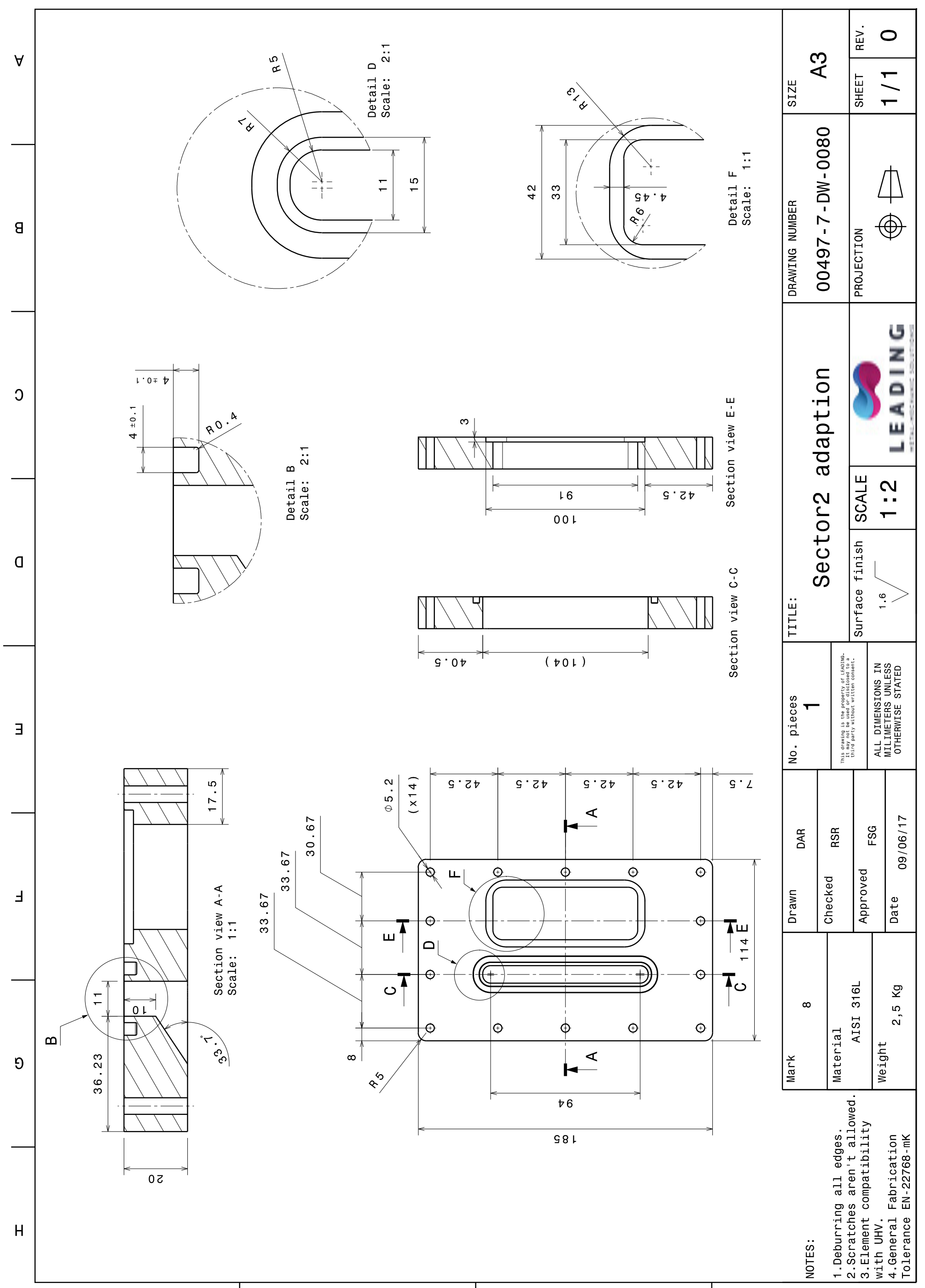




\section{Bibliografía}

[1] R. Santisteban, J. Granada, S. Petriw, and R. Mayer, "Neutron Transmission: A Very Powerful Technique at Small Accelerator-Based Neutron Sources," (Viena), IAEA, May 2009.

[2] E. Lehmann and A. Kaestner, "Neutron Imaging at Spallation Neutron Sources," (Viena), IAEA, May 2009.

[3] F. Fernandez-Alonso and D. L. Price, eds., Neutron Scattering - Fundamentals, vol. 44 of Experimental Methods in the Physical Sciences. Academic Press, Jan. 2013. DOI: 10.1016/B978-0-12-398374-9.09991-5.

[4] M. Glascock, "An overview of neutron activation analysis," Jan. 2003.

[5] G. Kneller, "Quasielastic neutron scattering.," tech. rep., Centre de Biophysique Moléculaire, CNRS, France, 2004.

[6] R. Pynn, "Neutron Scattering: A Primer," tech. rep., Los Alamos National Laboratory, Los Alamos, USA, 1990.

[7] L. Van Hove, "Correlations in Space and Time and Born Approximation Scattering in Systems of Interacting Particles," Physical Review, vol. 95, pp. 249-262, July 1954 .

[8] E. Fermi, "Sul moto dei neutroni nelle sostanze idrogenate," La ricerca scientifica, vol. 13, pp. 434-439, Nov. 1936.

[9] M. Born, "Quantenmechanik der Stoßvorgänge," Zeitschrift für Physik, vol. 38, pp. 803-827, Nov. 1926.

[10] F. J. Bermejo and F. Sordo, Neutron Scattering - Fundamentals: Chapter 2. Neutron Sources. Academic Press, Nov. 2013.

[11] A. N. Garg and R. J. Batra, "Isotopic sources in neutron activation analysis," Journal of Radioanalytical and Nuclear Chemistry, vol. 98, pp. 167-194, Mar. 1986.

[12] "National Physical Laboratory, Kaye\&Laby. Tables of Physical \& Chemical Constant, Chapter 4, Section 4.7, Subsect. 4.7.4 Nuclear Fusion." http: //www . kayelaby . $\mathrm{npl}$.co.uk/atomic_and_nuclear_physics/4_7/4_7_4.html.

[13] E. I. Moses, "The National Ignition Facility (NIF): A path to fusion energy," Energy Conversion and Management, vol. 49, pp. 1795-1802, July 2008. 
[14] K. Tomabechi, "International thermonuclear experimental reactor, ITER," Fusion Engineering and Design, vol. 8, pp. 43-49, Jan. 1989.

[15] I. S. Anderson, R. McGreevy, and H. Z. Bilheux, Neutron Imaging and Applications: A Reference for the Imaging Community. Springer Science \& Business Media, Apr. 2009. Google-Books-ID: ehmFLpLPI4QC.

[16] H. Prask, "The reactor and cold neutron research facility at NIST," Neutron News, vol. 1, pp. 9-13, Jan. 1990.

[17] C. J. Carlile, "ILL in the changing international context," Physica B: Condensed Matter, vol. 385-386, pp. 961-965, Nov. 2006.

[18] V. L. Aksenov, "Update on the pulsed reactor IBR-2 and its instruments at Dubna," Physica B: Condensed Matter, vol. 174, pp. 438-442, Oct. 1991.

[19] C. D. West, "An overview of the planned advanced neutron-source facility," Physica B: Condensed Matter, vol. 174, pp. 430-437, Oct. 1991.

[20] A. Lawler, "Green Light for Long-Awaited Facility," Science, vol. 279, pp. 470-471, Jan. 1998.

[21] J. E. Lynn, "Helios: The new Harwell electron linear accelerator, and its scientific programme," Contemporary Physics, vol. 21, pp. 483-500, Sept. 1980.

[22] Guber, K. H., Bigelow, T. S., Ausmus, C., Brashear, D. R., Harvey, J. A., Koehler, P. E., Wiarda, D., Overton, R. B., White, J. A., and Cauley, V. M., "Recent refurbishment of the Oak Ridge Electron Linear Accelerator neutron source," EDP Sciences, 2008, pp. 441-443, 2007.

[23] A. Tartaglione, V. Galván, J. Dawidowski, F. Cantargi, and J. J. Blostein, "Design and performance of a compact subthermal neutron source for an Electron Linear Accelerator," Journal of Instrumentation, vol. 8, no. 11, p. P11009, 2013.

[24] D. Ene, C. Borcea, S. Kopecky, W. Mondelaers, A. Negret, and A. Plompen, "Global characterisation of the GELINA facility for high-resolution neutron time-of-flight measurements by Monte Carlo simulations," Nuclear Instruments and Methods in Physics Research Section A: Accelerators, Spectrometers, Detectors and Associated Equipment, vol. 618, no. 1â??3, pp. 54-68, 2010.

[25] IAEA, "Radiological Safety Aspects of the Operation of Electron Linear Accelerators," 1979.

[26] G. E. Gibson, G. T. Seaborg, and D. C. Grahame, "On the Interaction of Fast Neutrons with Lead," Physical Review, vol. 51, pp. 370-370, Mar. 1937.

[27] Y. Ishikawa, "Present status of the KENS facility," (Tsukuba, Japan), pp. 89-101, Oct. 1980.

[28] R. Eccleston and C. Wilson, "Correspondent's Reports: A Guided Tour of ISIS: 2004 Update," Neutron News, vol. 15, pp. 15-18, Jan. 2004.

[29] N. Metropolis, R. Bivins, M. Storm, A. Turkevich, J. M. Miller, and G. Friedlander, "Monte Carlo Calculations on Intranuclear Cascades. I. Low-Energy Studies," Physical Review, vol. 110, pp. 185-203, Apr. 1958. 
[30] N. Metropolis, R. Bivins, M. Storm, J. M. Miller, G. Friedlander, and A. Turkevich, "Monte Carlo Calculations on Intranuclear Cascades. II. High-Energy Studies and Pion Processes," Physical Review, vol. 110, pp. 204-219, Apr. 1958.

[31] I. Dostrovsky, P. Rabinowitz, and R. Bivins, "Monte Carlo Calculations of HighEnergy Nuclear Interactions. I. Systematics of Nuclear Evaporation," Physical Review, vol. 111, pp. 1659-1676, Sept. 1958.

[32] S. Peggs, ed., ESS Technical Design Report, vol. ESS-doc-274. Sweden: European Spallation Source, 2013.

[33] G. Russell, "Spallation Physics - An Overview," in Proceedings of the Eleventh Meeting of the International Collaboration on Advanced Neutron Sources ICANS-XI, vol. I, (Tusukuba, Japan), pp. 291-298, KEK Report 90-25, Oct. 1990.

[34] J. M. Carpenter and W. B. Yelon, "2. Neutron Sources," in Methods in Experimental Physics (K. Sköld and D. L. Price, eds.), vol. 23 of Neutron Scattering, pp. 99-196, Academic Press, Jan. 1986. DOI: 10.1016/S0076-695X(08)60555-4.

[35] L. D. Stephens and A. J. Miller, "Radiation studies at a medium energy accelerator," Oct. 1969.

[36] C. M. Lavelle, D. V. Baxter, A. Bogdanov, V. P. Derenchuk, H. Kaiser, M. B. Leuschner, M. A. Lone, W. Lozowski, H. Nann, B. v. Przewoski, N. Remmes, T. Rinckel, Y. Shin, W. M. Snow, and P. E. Sokol, "Neutronic design and measured performance of the Low Energy Neutron Source (LENS) target moderator reflector assembly," Nuclear Instruments and Methods in Physics Research Section A: Accelerators, Spectrometers, Detectors and Associated Equipment, vol. 587, pp. 324-341, Mar. 2008.

[37] Y. Otake, M. Takamura, Y. Ikeda, A. Taketani, Y. Seki, M. Yamada, H. Sunaga, S. Wang, M. Kumagai, H. Suzuki, and T. Nakayama, "Riken compact neutron source RANS and its application with iron and steel field," July 2015.

[38] IFMIF International Team, "IFMIF Comprehensive Design report," tech. rep., International Energy Agency, Jan. 2004.

[39] H. Wiedemann, Particle Accelerator Physics. New York, NY: Springer, 4th ed. 2015 edition ed., July 2015.

[40] I. G. Brown, ed., The Physics and Technology of Ion Sources. Weinheim: WileyVCH, 2 edition ed., Oct. 2004.

[41] H. Postma, "Multiply charged heavy ions produced by energetic plasmas," Physics Letters A, vol. 31, pp. 196-197, Feb. 1970.

[42] H. Dehmelt, "A Single Atomic Particle Forever Floating at Rest in Free Space: New Value for Electron Radius," Physica Scripta, vol. 1988, no. T22, p. 102, 1988.

[43] I. Kapchinskii and V. Teplvakov Pribory i Tekhnika Eksperimenta, vol. 19, no. 2, pp. 19-22, 1970.

[44] L. W. Alvarez, H. Bradner, J. V. Franck, H. Gordon, J. D. Gow, L. C. Marshall, 
F. Oppenheimer, W. K. H. Panofsky, C. Richman, and J. R. Woodyard, "Berkeley Proton Linear Accelerator," Review of Scientific Instruments, vol. 26, pp. 111-133, Feb. 1955.

[45] Edward A. Knapp, James M. Potter, and Donald A. Swenson, "Method and device for stabilization of the field distribution in drift tube linac," Mar. 1970.

[46] D. E. Nagle, E. A. Knapp, and B. C. Knapp, "Coupled Resonator Model for Standing Wave Accelerator Tanks," Review of Scientific Instruments, vol. 38, pp. 1583-1587, Nov. 1967.

[47] C. Kazusa, JP, M. Yoneda, and JP, "United States Patent: 4746839 - Side-coupled standing-wave linear accelerator," May 1988.

[48] S. F. Nowicki, S. A. Wender, and M. Mocko, "The Los Alamos Neutron Science Center Spallation Neutron Sources," Physics Procedia, vol. 90, pp. 374-380, Jan. 2017.

[49] M. J. Barnes, L. Ducimetiére, T. Fowler, V. Senaj, and L. Sermeus, "Injection and extraction magnets: kicker magnets," arXiv:1103.1583 [physics], Mar. 2011. arXiv: 1103.1583.

[50] H. Stiller, "SNQ - the German project of a spallation neutron source," Atw. Atomwirtschaft, Atomtechnik, vol. 28, no. 12, pp. 624-627, 1983.

[51] T. E. Mason, D. Abernathy, I. Anderson, J. Ankner, T. Egami, G. Ehlers, A. Ekkebus, G. Granroth, M. Hagen, K. Herwig, J. Hodges, C. Hoffmann, C. Horak, L. Horton, F. Klose, J. Larese, A. Mesecar, D. Myles, J. Neuefeind, M. Ohl, C. Tulk, X.-L. Wang, and J. Zhao, "The Spallation Neutron Source in Oak Ridge: A powerful tool for materials research," Physica B: Condensed Matter, vol. 385-386, pp. 955-960, Nov. 2006.

[52] F. Maekawa, M. Harada, K. Oikawa, M. Teshigawara, T. Kai, S.-i. Meigo, M. Ooi, S. Sakamoto, H. Takada, M. Futakawa, T. Kato, Y. Ikeda, N. Watanabe, T. Kamiyama, S. Torii, R. Kajimoto, and M. Nakamura, "First neutron production utilizing J-PARC pulsed spallation neutron source JSNS and neutronic performance demonstrated," Nuclear Instruments and Methods in Physics Research Section A: Accelerators, Spectrometers, Detectors and Associated Equipment, vol. 620, pp. 159-165, Aug. 2010.

[53] G. S. Bauer, M. Salvatores, and G. Heusener, "MEGAPIE, a 1 MW pilot experiment for a liquid metal spallation target," Journal of Nuclear Materials, vol. 296, pp. 1733, July 2001.

[54] J. R. Haines, B. W. Riemer, D. K. Felde, J. D. Hunn, S. J. Pawel, and C. C. Tsai, "Summary of cavitation erosion investigations for the SNS mercury target," Journal of Nuclear Materials, vol. 343, pp. 58-69, Aug. 2005.

[55] D. A. McClintock, B. W. Riemer, P. D. Ferguson, A. J. Carroll, and M. J. Dayton, "Initial observations of cavitation-induced erosion of liquid metal spallation target vessels at the Spallation Neutron Source," Journal of Nuclear Materials, vol. 431, pp. 147-159, Dec. 2012. 
[56] T. Naoe, M. Teshigawara, T. Wakui, H. Kinoshita, H. Kogawa, K. Haga, and M. Futakawa, "Damage inspection of the first mercury target vessel of JSNS," Journal of Nuclear Materials, vol. 450, pp. 123-129, July 2014.

[57] B. W. Riemer, M. W. Wendel, D. K. Felde, R. L. Sangrey, A. Abdou, D. L. West, T. J. Shea, S. Hasegawa, H. Kogawa, T. Naoe, C. H. Farny, and A. L. Kaminsky, "Small gas bubble experiment for mitigation of cavitation damage and pressure waves in short-pulse mercury spallation targets," Journal of Nuclear Materials, vol. 450, pp. 192-203, July 2014.

[58] G. Skoro, S. Lilley, and R. Bewley, "Neutronics analysis of target, moderators and reflector design for the ISIS TS-1 project," Physica B: Condensed Matter, Dec. 2017.

[59] M. Mocko and G. Muhrer, "Fourth-generation spallation neutron target-moderatorreflector-shield assembly at the Manuel Lujan Jr. neutron scattering center," Nuclear Instruments and Methods in Physics Research Section A: Accelerators, Spectrometers, Detectors and Associated Equipment, vol. 704, pp. 27-35, Mar. 2013.

[60] B. Zhong, T. J. Liang, H. Zha, G. L. Yu, K. Wang, J. Wei, and C. K. Loong, "Neutronics analysis of the target-moderator-reflector (TMR) configuration for compact long pulsed neutron sources," Nuclear Engineering and Design, vol. 241, pp. 47204725, Dec. 2011.

[61] T. Reiss, U. Filges, F. Gallmeier, V. Talanov, and M. Wohlmuther, "Bi-spectral moderator for spallation sources optimized for instrument requirements," Nuclear Instruments and Methods in Physics Research Section A: Accelerators, Spectrometers, Detectors and Associated Equipment, vol. 728, pp. 117-125, Nov. 2013.

[62] K. Thomsen, "Conceptual Proposal for Compound Moderators with Preferential Emission Directions," Physics Procedia, vol. 60, pp. 278-293, Jan. 2014.

[63] N. Watanabe, M. Harada, T. Kai, M. Teshigawara, and Y. Ikeda, "Optimization of Coupled and Decoupled Hydrogen Moderators for a Short-pulse Spallation Source," Journal of Neutron Research, vol. 11, pp. 13-23, Jan. 2003.

[64] H. Takada, K. Haga, M. Teshigawara, T. Aso, S.-I. Meigo, H. Kogawa, T. Naoe, T. Wakui, M. Ooi, M. Harada, and M. Futakawa, "Materials and Life Science Experimental Facility at the Japan Proton Accelerator Research Complex I: Pulsed Spallation Neutron Source," Quantum Beam Science, vol. 1, p. 8, Aug. 2017.

[65] L. K. Mansur, "Materials research and development for the spallation neutron source mercury target," Journal of Nuclear Materials, vol. 318, pp. 14-25, May 2003.

[66] "How SNS Works | Neutron Science at ORNL." https://neutrons.ornl.gov/ content/how-sns-works.

[67] R. K. Crawford, R. Dean, P. Ferguson, J. Galambos, F. Gallmeier, T. McManamy, and M. Rennich, "The Second Target Station at the ORNL Spallation Neutron Source," Journal of Physics: Conference Series, vol. 251, no. 1, p. 012054, 2010.

[68] M. Futakawa, F. Maekawa, and S. Sakamoto, "1-MW Pulsed Spallation Neutron Source (JSNS) at J-PARC," Neutron News, vol. 22, pp. 15-19, Feb. 2011. 
[69] W. E. Fischer, "SINQ - The spallation neutron source, a new research facility at PSI," Physica B: Condensed Matter, vol. 234-236, pp. 1202-1208, June 1997.

[70] Albert Furrer, "The Swiss Spallation Neutron Source SINQ: From Idea to Realization," Swiss Neutron News, pp. 5-18, Apr. 2017.

[71] "Paul Scherrer Institut (PSI)." https://www.psi.ch/.

[72] C. C. Wilson, "A guided tour of ISIS - the UK spallation neutron source," Neutron News, vol. 6, pp. 27-34, Jan. 1995.

[73] A. Taylor, "Welcome to the second target station at ISIS," Materials Today, vol. 11, p. 72 , Dec. 2008.

[74] A. Taylor, "A second target station for ISIS," Physica B: Condensed Matter, vol. 276278, pp. 36-37, Mar. 2000.

[75] W. Yin, T. J. Liang, and Q. Z. Yu, "Neutronics design for the coupled para-hydrogen moderator for CSNS," Nuclear Instruments and Methods in Physics Research Section A: Accelerators, Spectrometers, Detectors and Associated Equipment, vol. 631, pp. 105-110, Mar. 2011.

[76] J. Wei, H. Chen, Y. Chen, Y. Chen, Y. Chi, C. Deng, H. Dong, L. Dong, S. Fang, J. Feng, S. Fu, L. He, W. He, Y. Heng, K. Huang, X. Jia, W. Kang, X. Kong, J. Li, T. Liang, G. Lin, Z. Liu, H. Ouyang, Q. Qin, H. Qu, C. Shi, H. Sun, J. Tang, J. Tao, C. Wang, F. Wang, D. Wang, Q. Wang, S. Wang, T. Wei, J. Xi, T. Xu, Z. Xu, W. Yin, X. Yin, J. Zhang, Z. Zhang, Z. Zhang, M. Zhou, and T. Zhu, "China Spallation Neutron Source: Design, R\&D, and outlook," Nuclear Instruments and Methods in Physics Research Section A: Accelerators, Spectrometers, Detectors and Associated Equipment, vol. 600, pp. 10-13, Feb. 2009.

[77] "CSNS Average Beam Power Reached to $10 \mathrm{~kW}$-China Spallation Neutron Source." http://english.ihep.cas.cn/csns/doc/2634.html.

[78] I. S. Anderson, C. Andreani, J. M. Carpenter, G. Festa, G. Gorini, C. K. Loong, and R. Senesi, "Research opportunities with compact accelerator-driven neutron sources," Physics Reports, vol. 654, pp. 1-58, Oct. 2016.

[79] D. V. Baxter, J. M. Cameron, V. P. Derenchuk, C. M. Lavelle, M. B. Leuschner, M. A. Lone, H. O. Meyer, T. Rinckel, and W. M. Snow, "Status of the low energy neutron source at Indiana University," Nuclear Instruments and Methods in Physics Research Section B: Beam Interactions with Materials and Atoms, vol. 241, pp. 209212, Dec. 2005.

[80] "RANS website." http://rans.riken.jp/en/index.html.

[81] Y. Yamagata, K. Hirota, J. Ju, S. Wang, S.-y. Morita, J.-i. Kato, Y. Otake, A. Taketani, Y. Seki, M. Yamada, H. Ota, U. Bautista, and Q. Jia, "Development of a neutron generating target for compact neutron sources using low energy proton beams," Journal of Radioanalytical and Nuclear Chemistry, vol. 305, pp. 787-794, Sept. 2015.

[82] Y. Ikeda, Y. Otake, and M. Mizuta, "Nondestructive Measurement Method to De- 
tect Water/Void inside Slabs using Compact Neutron Source by Backscattered Neutrons," Journal of Advanced Concrete Technology, vol. 15, pp. 603-609, Oct. 2017.

[83] Z. Guo, Y. Lu, Y. Zou, K. Zhu, S. Peng, J. Zhao, S. Gao, W. Wen, H. Li, Q. Zhou, H. Ren, P. Lü, H. Zeng, S. Wang, G. Tang, D. Mo, Z. Yuan, D. Xie, X. Yan, and J. Chen, "Progress of PKUNIFTY - a RFQ Accelerator based Neutron Imaging Facility at Peking University," Physics Procedia, vol. 43, pp. 79-85, Jan. 2013.

[84] H. Wang, Y. Zou, W. Wen, Y. Lu, and Z. Guo, "Preliminary energy-filtering neutron imaging with time-of-flight method on PKUNIFTY: A compact accelerator based neutron imaging facility at Peking University," Nuclear Instruments and Methods in Physics Research Section A: Accelerators, Spectrometers, Detectors and Associated Equipment, vol. 823, pp. 65-71, July 2016.

[85] J. Wei, H. Chen, C. Cheng, Q. Du, T. Du, Z. Feng, X. Guan, X. Han, T. Huang, R. Li, W. Li, and C. K. Loong, "The Compact Pulsed Hadron Source: A Design Perspective," Journal of The Korean Physical Society - J KOREAN PHYS SOC, vol. 56, June 2010.

[86] Y. Xiao, Z. Chen, Y. Yang, and X. Wang, "Development Progress of the Neutron Imaging Station in CPHS," Physics Procedia, vol. 69, pp. 96-103, Jan. 2015.

[87] "European Neutron Scattering Association." http://www.neutrons-ensa.eu/.

[88] High-Intensity Proton Beam Facilities. OECD Global Science Forum Workshop on Strategic Policy Issues, Paris: OECD, 1999.

[89] F. H. Bohn et al., European source of science. The ESS Project, Volume 1. European Spallation Source, May 2002.

[90] D. Richter et al., New Science and Technology for the 21st Century. The ESS project, Volume 2. European Spallation Source, May 2002.

[91] F. H. Bohn et al., Technical report. The ESS Project, Volume 3. European Spallation Source, May 2002.

[92] K. Clausen et al., Instruments and user support. The ESS Project, volume 4. European Spallation Source, May 2002.

[93] G. S. Bauer et al., Technical report status 2003. The ESS Project, volume 3 Update. European Spallation Source, Dec. 2003.

[94] "ESS Bilbao, Centro de Tecnologías Neutrónicas." https://www.essbilbao.org.

[95] W. Lu, E. B. Iverson, P. D. Ferguson, J. A. Crabtree, F. X. Gallmeier, I. Remec, D. V. Baxter, and C. M. Lavelle, "SNS Moderator Poison Design and Experiment Validation of the Moderator Performance," pp. 700-712, Aug. 2009.

[96] M. Furuska, "Hokkaido University neutron source, HUNS, an ancient machine carving out new trend of compact accelerator driven neutron source," July 2015.

[97] M. OI, M. KONNO, H. IWASA, T. KAMIYAMA, M. FURUSAKA, and Y. KIYANAGI, "Experimental Studies of Premoderator Effect on Neutronic Characteristics of a Decoupled Hydrogen Moderator at a Pulsed Neutron Source," Journal of Nuclear Science and Technology, vol. 41, pp. 1138-1144, Dec. 2004. 
[98] M. Furusaka, H. Sato, T. Kamiyama, M. Ohnuma, and Y. Kiyanagi, "Activity of Hokkaido University Neutron Source, HUNS," Physics Procedia, vol. 60, pp. 167174, Jan. 2014.

[99] "Sociedad Española de Técnicas Neutrónicas." http://www.unizar.es/setn/.

[100] F. Bartolomé, "La investigación española en Materia Condensada con Grandes Instalaciones," Revista española de física, vol. 23, no. 4, pp. 47-52, 2009.

[101] F.J. Bermejo, P. Gorria, L. Fernández Barquín, A. Arbe, J. Campo, G.J. Cuello, J.L. García Muñoz, V. García Sakai, and J. Gutiérrez, Historical perspective of research with neutron techniques in Spain, vol. NA 1148/2009. Sociedad Española de Técnicas Neutrónicas, 2009.

[102] I. A. E. A. (IAEA), "Official Web Site of the IAEA." https://www.iaea.org.

[103] IAEA, "Development Opportunities for Small and Medium Scale Accelerator Driven Neutron Sources - Report of a technical meeting held in Vienna, 18-21 May 2004," 2005 .

[104] D. Pelowitz, J. Hendricks, J. Durkee, M. Fensin, M. James, G. McKinney, S. Mashnik, and L. Waters, "MCNPX User's Manual. Version 2.6.0," A Extensions. Los Alamos National Laboratory, 2008.

[105] "Los Alamos National Lab: National Security Science."

[106] E. D. Cashwell, O. W. Rechard, C. J. Everett, U.S. Atomic Energy Commission, and Los Alamos Scientific Laboratory, A practical manual on the Monte Carlo method for random walk problems. TID4500 13th ed., suppl., Los Alamos, N.M.: Los Alamos Scientific Laboratory of the University of California, 1957.

[107] "ANSYS - Simulation Driven Product Development." http://www . ansys .com/.

[108] "ANSYS CFX: CFD Simulation." http://www.ansys.com/products/fluids/ ansys-cfx.

[109] ANSYS Inc., "ANSYS Mechanical APDL Verification Manual," Tech. Rep. Release 17.0, Jan. 2016.

[110] ANSYS Inc., "ANSYS Workbench Verification Manual," Tech. Rep. Release 17.0, Jan. 2016.

[111] ANSYS Inc., "ANSYS Fluid Dynamics Verification Manual," Tech. Rep. Release 17.0, Jan. 2016.

[112] "Catia V5." https://www.3ds.com/es/productos-y-servicios/catia/ productos/catia-v5/portfolio/.

[113] I. Bustinduy and F. J. Bermejo, "A Conceptual Description of the ESS-Bilbao Accelerator," Physics Procedia, vol. 60, pp. 157-166, Jan. 2014.

[114] M. Chadwick, P. Obloinska, M. Herman, N. Greene, R. McKnight, D. Smith, P. Young, R. MacFarlane, G. Hale, S. Frankle, A. Kahler, T. Kawano, R. Little, D. Madland, P. Moller, R. Mosteller, P. Page, P. Talou, H. Trellue, M. White, 
W. Wilson, R. Arcilla, C. Dunford, S. Mughabghab, B. Pritychenko, D. Rochman, A. Sonzogni, C. Lubitz, T. Trumbull, J. Weinman, D. Brown, D. Cullen, D. Heinrichs, D. McNabb, H. Derrien, M. Dunn, N. Larson, L. Leal, A. Carlson, R. Block, J. Briggs, E. Cheng, H. Huria, M. Zerkle, K. Kozier, A. Courcelle, V. Pronyaev, and S. van der Marck, "ENDF/B-VII.0: Next Generation Evaluated Nuclear Data Library for Nuclear Science and Technology," Nuclear Data Sheets, vol. 107, no. 12, pp. 2931-3060, 2006. Evaluated Nuclear Data File ENDF/B-VII.0.

[115] X. Ledoux, M. Aïche, M. Avrigeanu, V. Avrigeanu, L. Audouin, E. Balanzat, B. Bandétat, G. Ban, G. Barreau, E. Bauge, G. Bélier, P. Bem, V. Blideanu, C. Borcea, S. Bouffard, T. Caillaud, A. Chatillon, S. Czajkowski, P. Dessagne, D. Doré, M. Fallot, F. Farget, U. Fischer, L. Giot, T. Granier, S. Guillous, F. Gunsing, C. Gustavsson, B. Jacquot, K. Jansson, B. Jurado, M. Kerveno, A. Klix, O. Landoas, F. R. Lecolley, J. L. Lecouey, M. Majerle, N. Marie, T. Materna, J. Mrazek, F. Negoita, J. Novak, S. Oberstedt, A. Oberstedt, S. Panebianco, L. Perrot, A. J. M. Plompen, S. Pomp, J. M. Ramillon, D. Ridikas, B. Rossé, G. Rudolf, O. Serot, S. P. Simakov, E. Simeckova, A. G. Smith, J. C. Sublet, J. Taieb, L. Tassan-Got, D. Tarrio, A. Takibayev, I. Thfoin, I. Tsekhanovich, and C. Varignon, "The Neutrons for Science Facility at SPIRAL-2," Nuclear Data Sheets, vol. 119, pp. 353-356, May 2014.

[116] "Grand accélérateur national d'ions lourds." https://www.ganil-spiral2.eu/.

[117] "Lithium Metal - Goodfellow."

[118] "Lithium (Li) - Properties, Applications."

[119] R. Schultz, "Lithium: Measurement of Young's Modulus and Yield Strength," Oct. 2002.

[120] I. A. E. Agency, Thermophysical properties of materials for nuclear engineering: A tutorial and collection of data. Nov. 2008.

[121] T. L. Bergman, A. S. Lavine, F. P. Incropera, and D. P. DeWitt, Fundamentals of Heat and Mass Transfer. Hoboken, NJ: John Wiley \& Sons, 7th ed., 2012.

[122] S. Nukiyama, "The maximum and minimum values of the heat Q transmitted from metal to boiling water under atmospheric pressure," International Journal of Heat and Mass Transfer. Translated from J. Jpn. Soc. Mech. Eng., 37, pp. 367-374,1934, vol. 9, pp. 1419-1433, Dec. 1966.

[123] N. Zuber, "On the stability of boiling heat transfer," Trans ASME, vol. 80, p. 711, 1958.

[124] S. Kutateladze, "On the transition to film boiling under natural convection," Kotloturbostroenie, no. 3, pp. 10-12, 1948.

[125] C. F. B. M. T. Cichelli, "Heat transfer to liquids boiling under pressure," Trans. AIChE, vol. 01, no. 41, pp. 755-787, 1945.

[126] S. v. Stralen and R. Cole, Boiling phenomena : physicochemical and engineering fundamentals and applications / Sjoerd van Stralen, Robert Cole ; with invited contributions. Series in thermal and fluids engineering, Washington: Hemisphere Pub. Corp. Includes bibliographical references and index. 
[127] S. Makimura, N. Kawamura, S. Onizawa, Y. Matsuzawa, M. Tabe, Y. Kobayashi, R. Shimizu, Y. Taniguchi, H. Fujimori, Y. Ikedo, R. Kadono, A. Koda, K. M. Kojima, K. Nishiyama, J. Nakamura, K. Shimomura, P. Strasser, Y. Nakatsugawa, and Y. Miyake, "Development of muon rotating target at J-PARC/MUSE," Journal of Radioanalytical and Nuclear Chemistry, vol. 305, pp. 811-815, Sept. 2015.

[128] M. Sohani and H. W. Wilschut, "A rotating target for Ra production," Nuclear Instruments and Methods in Physics Research Section A: Accelerators, Spectrometers, Detectors and Associated Equipment, vol. 679, pp. 25-28, July 2012.

[129] T. McManamy, M. Rennich, F. Gallmeier, P. Ferguson, and J. Janney, "3mw solid rotating target design," Journal of Nuclear Materials, vol. 398, pp. 35-42, Mar. 2010.

[130] A. Aguilar, F. Sordo, T. Mora, L. Mena, M. Mancisidor, J. Aguilar, G. Bakedano, I. Herranz, P. Luna, M. Magan, R. Vivanco, F. Jimenez-Villacorta, K. Sjogreen, U. Oden, J. M. Perlado, J. L. Martinez, and F. J. Bermejo, "Design specification for the European Spallation Source neutron generating target element," Nuclear Instruments and Methods in Physics Research Section A: Accelerators, Spectrometers, Detectors and Associated Equipment, vol. 856, pp. 99-108, June 2017.

[131] H. Bethe, "Zur Theorie des Durchgangs schneller Korpuskularstrahlen durch Materie," Ann. Phys., vol. 397, pp. 325-400, Jan. 1930.

[132] "Bragg peak." https://en.wikipedia.org/wiki/Bragg_peak, Apr. 2016. Page Version ID: 717778134.

[133] J. K. Shultis and R. E. Faw, Fundamentals of Nuclear Science and Engineering Third Edition. CRC Press, 3 edition ed., Sept. 2016.

[134] K. A. Walsh, Beryllium Chemistry and Processing. International, July 2009.

[135] "Beryllium." https://en.wikipedia.org/wiki/Beryllium, Oct. 2016. Page Version ID: 747169969.

[136] A. International, "ASTM A36 / A36m - 14 Standard Specification for Carbon Structural Steel." http://www . astm.org/Standards/A36.

[137] "S-200-F Vacuum Hot Pressed Beryllium - Materion." https://materion.com/ Products/Beryllium/Beryllium-Metal/S-200F .aspx.

[138] MATERION Corp., "Beryllium as a Heat Sink," tech. rep., Elmore Ohio 43416.

[139] T. Rinckel, D. V. Baxter, J. Doskow, P. E. Sokol, and T. Todd, "Target Performance at the Low Energy Neutron Source," Physics Procedia, vol. 26, pp. 168-177, Jan. 2012 .

[140] T. Rinckel, D. V. Baxter, J. Doskow, H. Kaiser, R. Pynn, P. E. Sokol, and T. Todd, "LENS Operating Experience," Physics Procedia, vol. 26, pp. 161-167, Jan. 2012.

[141] H. W. Bertini, "Low-Energy Intranuclear Cascade Calculation," Physical Review, vol. 138, pp. 1801-1821, June 1965.

[142] J. Cugnon, C. Volant, and S. Vuillier, "Improved intranuclear cascade model for nucleon-nucleus interactions," Nuclear Physics A, vol. 620, pp. 475-509, July 1997. 
[143] A. Boudard, J. Cugnon, S. Leray, and C. Volant, "Intranuclear cascade model for a comprehensive description of spallation reaction data," Physical Review C, vol. 66, p. 044615 , Oct. 2002.

[144] Y. Yariv and Z. Fraenkel, "Intranuclear cascade calculation of high-energy heavy-ion interactions," Physical Review C, vol. 20, pp. 2227-2243, Dec. 1979.

[145] K. K. Gudima, S. G. Mashnik, and V. D. Toneev, "Cascade-exciton model of nuclear reactions," Nuclear Physics A, vol. 401, pp. 329-361, June 1983.

[146] S. G. Mashnik, K. K. Gudima, R. E. Prael, A. J. Sierk, M. I. Baznat, and N. V. Mokhov, "CEM03.03 and LAQGSM03.03 Event Generators for the MCNP6, MCNPX, and MARS15 Transport Codes," Nuclear Instruments and Methods in Physics Research Section B: Beam Interactions with Materials and Atoms, vol. 268, pp. 34263433, Nov. 2010. arXiv: 0805.0751.

[147] A. R. Junghans, M. de Jong, H. G. Clerc, A. V. Ignatyuk, G. A. Kudyaev, and K. H. Schmidt, "Projectile-fragment yields as a probe for the collective enhancement in the nuclear level density," Nuclear Physics A, vol. 629, pp. 635-655, Feb. 1998.

[148] L. Dresner, "EVAP: A FORTRAN program for calculating the evaporation of various particles from excited compound nuclei," 1963.

[149] D. Filges and F. Goldenbaum, Handbook of spallation research: theory, experiments and applications. Wiley-VCH, 2010.

[150] F. Scaffidi-Argentina, G. Longhurst, V. Shestakov, and H. Kawamura, "Beryllium R\&D for fusion applications," Fusion engineering and design, vol. 51, pp. 23-41, 2000 .

[151] M. Dalle Donne, F. Scaffidi-Argentina, C. Ferrero, and C. Ronchi, "Modelling Heinduced swelling in beryllium during fast neutron irradiation," Tech. Rep. KFK5271, FZK, Germany, 1993.

[152] M. C. Billone, M. D. Donne, and R. G. Macaulay-Newcombe, "Status of beryllium development for fusion applications," Fusion Engineering and Design, vol. 27, pp. 179-190, Mar. 1995.

[153] D. Craig, L. Fecitt, Y. E. Gorlinsky, N. F. Harman, R. Jackson, V. I. Kolyadin, Y. N. Lobach, and V. I. Pavlenko, "Dismantling design for the loop rooms on the MR reactor," Nuclear Engineering and Design, vol. 239, pp. 2832-2842, Dec. 2009.

[154] "Reactor BR2 / Research infrastructure / Scientific research / SCK•CEN SCK•CEN." https://www. sckcen.be/en/Research/Infrastructure/BR2.

[155] F. Moons, L. Sannen, A. Rahn, and J. Van De Velde, "Neutron irradiated beryllium: tensile strength and swelling," Journal of nuclear materials, vol. 233, pp. 823-827, 1996.

[156] L. Sannen and C. De Raedt, "The effects of neutron irradiation on beryllium," in Fusion Technology 1992, pp. 1474-1478, Oxford: North-Holland, 1993. DOI: 10.1016/B978-0-444-89995-8.50289-7. 
[157] J. Knaster, A. Moeslang, and T. Muroga, "Materials research for fusion," Nature Physics; London, vol. 12, no. 5, pp. 424-434, 2016.

[158] Brush Wellman, "Designing with Beryllium," Brochure 517, Cleveland, OH, 2001.

[159] Q. Wang, M. K. Khan, and C. Bathias, "Current understanding of ultra-high cycle fatigue," Theoretical and Applied Mechanics Letters, vol. 2, p. 031002, Jan. 2012.

[160] "2.5d machining." https://en.wikipedia.org/wiki/2.5D_(machining), Sept. 2016. Page Version ID: 738586532.

[161] ASM International, ASM Handbook Vol. 2. Properties and Selection: Nonferrous Alloys and Special-Purpose Materials. ASM International, 1990.

[162] T. Kvackaj, ed., Aluminium Alloys, Theory and Applications. InTech, Feb. 2011.

[163] R. Steiner, ed., ASM Handbook Vol.1: Irons, Steels and High-Performance Alloys. Materials Park, Ohio: ASM International, edición: 10th ed ed., Mar. 1990.

[164] "HELICOFLEX® Spring Energized Seals | Technetics Group." http:// technetics.com/products/sealing-solutions/metal-seals/helicoflex/.

[165] I. A. for the Properties of Water and Steam, "Revised Release on the IAPWS Industrial Formulation 1997 for the Thermodynamic Properties of Water and Steam." http://www. iapws. org/relguide/IF97-Rev.pdf, 2007.

[166] R. H. S. Winterton, "Where did the dittus and boelter equation come from?," International Journal of Heat and Mass Transfer, vol. 41, pp. 809-810, Feb. 1998.

[167] V. Yakhot, S. A. Orszag, S. Thangam, T. B. Gatski, and C. G. Speziale, "Development of turbulence models for shear flows by a double expansion technique," Physics of Fluids A: Fluid Dynamics, vol. 4, pp. 1510-1520, July 1992.

[168] B. E. Launder and D. B. Spalding, "The numerical computation of turbulent flows," Computer Methods in Applied Mechanics and Engineering, vol. 3, pp. 269-289, Mar. 1974 .

[169] L. T. Adzhemyan, N. V. Antonov, and A. N. Vasiliev, Field Theoretic Renormalization Group in Fully Developed Turbulence. CRC Press, Mar. 1999.

[170] M. Nayyar, Piping Handbook. McGraw-Hill Education, edición: 7 ed., Nov. 1999.

[171] AENOR, "AENOR: Norma UNE-EN ISO 898-1:2015." http://www.aenor.es/ aenor/normas/normas/fichanorma $\cdot$ asp?tipo=N\&codigo=N0054649.

[172] M. R. Hestenes, "Multiplier and gradient methods," Journal of Optimization Theory and Applications, vol. 4, pp. 303-320, Nov. 1969.

[173] X. Zheng and W. Xia, "Numerical Simulation of Blind Hole Bolt Connection with 3-D Finite Element Approach," in 2009 Second International Conference on Information and Computing Science, vol. 4, pp. 164-169, May 2009.

[174] Y. Dai, Y. Foucher, M. R. James, and B. M. Oliver, "Neutronics calculation, dosimetry analysis and gas measurements of the first SINQ target irradiation experiment, STIP-I," Journal of Nuclear Materials, vol. 318, pp. 167-175, May 2003. 
[175] Y. Dai, X. Jia, R. Thermer, D. Hamaguchi, K. Geissmann, E. Lehmann, H. P. Linder, M. James, F. Gröschel, W. Wagner, and G. S. Bauer, "The second SINQ target irradiation program, STIP-II," Journal of Nuclear Materials, vol. 343, pp. 3344, Aug. 2005.

[176] Y. Dai, R. Brun, W. Gao, K. Geissmann, S. Hahl, H. Hou, Y. Huang, H. P. Linder, B. Long, A. Spahr, P. Vontobel, W. Wagner, H. L. Wang, and L. Zanini, "The fourth SINQ Target Irradiation Program, STIP-IV," Journal of Nuclear Materials, vol. 431, pp. 2-9, Dec. 2012.

[177] C. Sibley, "Machine protection strategies for high power accelerators," in Proceedings of the 2003 Particle Accelerator Conference, vol. 1, pp. 607-611 Vol.1, May 2003.

[178] H. Nakagawa, A. Akiyama, . Odagiri, and Y. Katoh, "The accelerator protection system based on embebbed EPICS for J-PARC," in Proceedings of ICALEPCS2009, (Kobe, Japan), 2009.

[179] W. M. Kays, M. E. Crawford, and B. Weigand, Convective Heat and Mass Transfer. Boston: Mcgraw-Hill, Feb. 2004.

[180] "Fast closing shutter VAT Series 771." http://www.vatvalve.com/business/ valves/catalog/F/771_1_V.

[181] M. Eagleson, Concise encyclopedia chemistry. Berlin, New York: Walter de Gruyter, english language ed. ed., 1994. Open Library ID: OL1425783M.

[182] N. N. Greenwood and A. Earnshaw, Chemistry of the elements. Oxford, Boston: Butterworth-Heinemann, 2nd ed. ed., 1997. Open Library ID: OL689297M.

[183] G. R. Smolik, B. J. Merrill, and R. S. Wallace, "Implications of beryllium : steam interactions in fusion reactors," Journal of Nuclear Materials, vol. 191-194, pp. 153157, Sept. 1992.

[184] I. Tilquin, P. Froment, M. Cogneau, T. Delbar, J. Vervier, and G. Ryckewaert, "Experimental measurements of neutron fluxes produced by proton beams $(23-80 \mathrm{mev})$ on Be and Pb targets," Nuclear Instruments and Methods in Physics Research Section A: Accelerators, Spectrometers, Detectors and Associated Equipment, vol. 545, pp. 339-343, June 2005.

[185] M. A. Lone, C. B. Bigham, J. S. Fraser, H. R. Schneider, T. K. Alexander, A. J. Ferguson, and A. B. McDonald, "Thick target neutron yields and spectral distributions from the $7 \mathrm{li}(\mathrm{pd}, \mathrm{n})$ and $9 \mathrm{be}(\mathrm{pd}, \mathrm{n})$ reactions," Nuclear Instruments and Methods, vol. 143, pp. 331-344, June 1977.

[186] H. J. Brede, G. Dietze, K. Kudo, U. J. Schrewe, F. Tancu, and C. Wen, "Neutron yields from thick Be targets bombarded with deuterons or protons," Nuclear Instruments and Methods in Physics Research Section A: Accelerators, Spectrometers, Detectors and Associated Equipment, vol. 274, pp. 332-344, Jan. 1989.

[187] M. A. Lone, "Nuclear Data Needed for Industrial Neutron Sources," in Nuclear Data for Science and Technology, Research Reports in Physics, pp. 678-680, Springer, Berlin, Heidelberg, 1992. DOI: 10.1007/978-3-642-58113-7_194. 
[188] W. B. Howard, S. M. Grimes, T. N. Massey, S. I. Al-Quraishi, D. K. Jacobs, C. E. Brient, and J. C. Yanch, "Measurement of the Thick-Target 9be(p,n) Neutron Energy Spectra," Nuclear Science and Engineering, vol. 138, pp. 145-160, June 2001.

[189] S. Kamada, T. Itoga, Y. Unno, W. Takahashi, T. Oishi, and M. Baba, "Measurement of Energy-angular Neutron Distribution for ${ }^{7} \mathrm{Li},{ }^{9} \mathrm{Be}(p, x n)=$ Reaction at $\mathrm{EP}=70$ $\mathrm{MeV}$ and $11 \mathrm{MeV}$," Journal of the Korean Physical Society, vol. 59, pp. 1676 - 1680, August 2011.

[190] Y. Kiyanagi, "Experimental studies on neutronic performance of various coldneutron moderators for the pulsed neutron sources," Nuclear Instruments and Methods in Physics Research Section A: Accelerators, Spectrometers, Detectors and Associated Equipment, vol. 562, pp. 561-564, June 2006.

[191] K. Inoue, N. Otomo, H. Iwasa, and Y. Kiyanagi, "Slow Neutron Spectra in Cold Moderators," Journal of Nuclear Science and Technology, vol. 11, pp. 228-229, May 1974 .

[192] K. Inoue, Y. Kiyanagi, and H. Konno, "Time-Dependence of Cold Neutron Pulse Emitted from Methane at 20 K," Journal of Nuclear Science and Technology, vol. 14, pp. 195-199, Mar. 1977.

[193] K. Inoue, Y. Kiyanagi, and H. Iwasa, "An accelerator-based cold neutron source," Nuclear Instruments and Methods in Physics Research, vol. 192, pp. 129-136, Feb. 1982.

[194] J. Carpenter, "Cold moderators for pulsed neutron sources," Tech. Rep. LA-12146C, Argonne National Lab., IL (United States), LANL Los Alamos, N.M, Mar. 1990.

[195] A. Jez-dotowski, H. Misiorek, V. V. Sumarokov, and B. Y. Gorodilov, "Thermal conductivity of solid methane," Physical Review B, vol. 55, pp. 5578-5580, Mar. 1997.

[196] E. Shabalin, A. Fedorov, E. Kulagin, S. Kulikov, V. Melikhov, and D. Shabalin, "Experimental study of swelling of irradiated solid methane during annealing," Nuclear Instruments and Methods In Physics Research Section B-Beam Interactions With Materials And Atoms, vol. 266, pp. 5126-5131, Dec. 2008.

[197] D. Evans, "Irradiation effects in liquid methane used as a neutron moderator," Cryogenics, vol. 35, pp. 763-766, Nov. 1995.

[198] G. M. Allen, T. A. Broome, R. A. Burridge, D. Cragg, R. Hall, D. Haynes, J. Hirst, J. R. Hogston, H. H. Jones, J. Sexton, and P. Wright, "The Isis cold moderators," Tech. Rep. OSTIID-1163117, Rutherford Appleton Lab., Council for the Laboratory of the Research Councils (United States), 1997.

[199] S. M. Bennington, S. I. Campbell, T. A. Broome, D. J. Picton, and T. D. Beynon, "ISIS Target Station II: Preliminary Target and Moderator Simulations," Journal of Neutron Research, vol. 11, pp. 93-98, Mar. 2003.

[200] S. Crispel, M. Bonneton, R. Thiering, and M. Simon, "Design and testing of cryogenic systems dedicated to neutron sources," in IPAC 2010 Contributions to the Proceedings, (Kyoto), 2010. 
[201] Y. Kiyanagi, N. Watanabe, and M. Nakajima, "Neutronic studies on flux-trap moderators in spallation neutron sources," Nuclear Instruments and Methods in Physics Research Section A: Accelerators, Spectrometers, Detectors and Associated Equipment, vol. 343, no. 2-3, pp. 550-557, 1994.

[202] N. Watanabe, "Neutronics of pulsed spallation neutron sources," Reports on Progress in Physics, vol. 66, no. 3, p. 339, 2003.

[203] J. Carpenter, "Pulsed spallation neutron sources for slow neutron scattering," $N u$ clear Instruments and Methods, vol. 145, pp. 91-113, Aug. 1977.

[204] G. J. Russell, J. S. Gilmore, H. Robinson, G. L. Legate, A. Bridge, R. J. Sanchez, R. J. Brewton, R. Woods, and H. G. Hughes, "LANSCE target system performance," in Proceedings of the 10th meeting of the international collaboration on advanced neutron sources (ICANS-X) Los Alamos, New Mexico, USA, Institute of Physics Conference Series, pp. 483-496, IOP Publishing Ltd; Bristol (UK), Oct. 1988.

[205] Y. Kiyanagi, N. Watanabe, and H. Iwasa, "Experimental studies on neutronic performance of coupled liquid-hydrogen moderator for pulsed spallation neutron sources," Nuclear Instruments and Methods in Physics Research Section A: Accelerators, Spectrometers, Detectors and Associated Equipment, vol. 312, pp. 561-570, Feb. 1992.

[206] Y. Shin, C. Lavelle, W. M. Snow, D. V. Baxter, X. Tong, H. Yan, and M. Leuschner, "Measurements of the neutron brightness from a phase II solid methane moderator at the LENS neutron source," Nuclear Instruments and Methods in Physics Research, Section A: Accelerators, Spectrometers, Detectors and Associated Equipment, vol. 620, pp. 375-381, Aug. 2010.

[207] J. R. Granada, "Synthetic scattering kernel to describe the interaction of slow neutrons with solid methane in phase II," Nuclear Instruments and Methods in Physics Research Section B: Beam Interactions with Materials and Atoms, vol. 266, pp. 164172, Jan. 2008.

[208] T. K. Basu, "Neutron Multiplication Studies in Beryllium for Fusion Reactor Blankets," Nuclear Science and Engineering, vol. 70, pp. 309-313, June 1979.

[209] F. Maekawa, M. Harada, K. Oikawa, M. Teshigawara, T. Kai, S.-i. Meigo, M. Ooi, S. Sakamoto, H. Takada, M. Futakawa, T. Kato, Y. Ikeda, N. Watanabe, T. Kamiyama, S. Torii, R. Kajimoto, and M. Nakamura, "First neutron production utilizing J-PARC pulsed spallation neutron source JSNS and neutronic performance demonstrated," Nuclear Instruments and Methods in Physics Research Section A: Accelerators, Spectrometers, Detectors and Associated Equipment, vol. 620, pp. 159-165, Aug. 2010.

[210] G. A. Bartholomew and P. R. e. . Tunnicliffe, "The AECL study for an intese neutron generator (technical details)," Report AECL-2600, Atomic Energy of Canada Ltd, July 1966.

[211] G. Muhrer, E. Pitcher, and G. Russell, "The neutron performance of a pre-moderated beryllium reflector-filter hydrogen moderator system for the Manuel Jr. Lujan Neutron Science Center," Nuclear Instruments and Methods in Physics Research Section A: Accelerators, Spectrometers, Detectors and Associated Equipment, vol. 536, no. 12, pp. 154-164, 2005. 
[212] G. Muhrer, M. Hartl, L. Daemen, and J. Ryu, "Benchmark test of the effectiveness of a nitrogen cooled beryllium reflector-filter," Nuclear Instruments and Methods in Physics Research Section A: Accelerators, Spectrometers, Detectors and Associated Equipment, vol. 578, no. 3, pp. 463-469, 2007.

[213] J. M. Carpenter, S. S. Cudrnak, and C. M. DeCusatis, "Deterioration of performance of neutron moderators under intense irradiation," Tech. Rep. CONF-8609234-9, Argonne National Lab., 1986.

[214] X. Fabrèges, A. Menelle, F. Ott, N. Chauvin, J. Schwindling, A. Letourneau, A. Marchix, and C. Alba-Simionesco, "Performances of Neutron Scattering Spectrometers on a Compact Neutron Source," arXiv:1612.00232 [physics], Dec. 2016. arXiv: 1612.00232 .

[215] "McStas homepage." http://www.mcstas.org/.

[216] "ICON: Imaging with Cold Neutrons." https://www.psi.ch/sinq/icon/.

[217] A. P. Kaestner, S. Hartmann, G. Kühne, G. Frei, C. Grünzweig, L. Josic, F. Schmid, and E. H. Lehmann, "The ICON beamline - A facility for cold neutron imaging at SINQ," Nuclear Instruments and Methods in Physics Research Section A: Accelerators, Spectrometers, Detectors and Associated Equipment, vol. 659, pp. 387-393, Dec. 2011.

[218] N. Kardjilov, A. Hilger, I. Manke, M. Strobl, M. Dawson, S. Williams, and J. Banhart, "Neutron tomography instrument CONRAD at HZB," Nuclear Instruments and Methods in Physics Research Section A: Accelerators, Spectrometers, Detectors and Associated Equipment, vol. 651, pp. 47-52, Sept. 2011.

[219] "Neutron tomography in HZB." https://www.helmholtz-berlin.de/forschung/ oe/em/werkstoffe/methoden/n-tomo_en.html.

[220] "Helmholtz-Zentrum Berlin (HZB) - Home." https://www.helmholtz-berlin.de/.

[221] H. Krohn, "The research reactor BER II at the Helmholtz-Center Berlin," Atw. Internationale Zeitschrift fuer Kernenergie, vol. 58, no. 10, pp. 611-612, 2012.

[222] "ISIS Polref." https://www.isis.stfc.ac.uk/Pages/Polref .aspx.

[223] "The LLB-Orphée neutron scattering and imaging instruments." http://www-llb. cea.fr/en/Web/hpr_web/HPRWEB5.php.

[224] "Welcome on the LLB Web site." http://www-llb.cea.fr/en/index.php.

[225] C. Alba-Simionesco, A. Menelle, and J.-P. Visticot, "The Laboratoire Léon Brillouin and the Orphée Reactor: The French National Neutron Facility," Neutron News, vol. 22 , pp. 10-13, Oct. 2011.

[226] "PAXE Spectrometre pour diffusion aux petits Angles." http://www-llb.cea.fr/ spectros/spectro/g5-4.html.

[227] "ISIS Sans2d." https://www.isis.stfc.ac.uk/Pages/Sans2d.aspx.

[228] "Spectrometre a deux axes "PYRRHIAS "." http://www-llb.cea.fr/spectros/ spectro/g4-1.html. 
[229] "Neutron resonance spin-echo Spectrometer MUSES." http://www-llb.cea.fr/ spectros/pdf/muses-llb.pdf.

[230] "ISIS Osiris." https://www.isis.stfc.ac.uk/Pages/Osiris.aspx.

[231] D. Martín y Marero and D. Engberg, "OSIRIS: the polarisation analysis spectrometer and diffractometer at ISIS," Physica B: Condensed Matter, vol. 267-268, pp. 134138, June 1999.

[232] CEA, "CEA Website." http://www.cea.fr/english/Pages\%2fWelcome. aspx, Oct. 2013.

[233] "Centre national de la recherche scientifique." https://www.cnrs.fr/.

[234] "SONATE: an accelerator-driven neutron source." http://iramis.cea.fr/llb/ Phocea/Vie_des_labos/Ast/ast_technique.php?id_ast=2755. Accedido: 201804-05.

[235] "Source Alternative Française SONATE CANS - 2fdn." http://2fdn.neel.cnrs . fr/spip.php?article69. Accedido: 2018-04-05.

[236] Laboratoire Léon Brillouin, "Compact Neutron Sources for Neutron Scattering," tech. rep., Feb. 2018.

[237] ESFRI Physical Sciences and Engineering Strategy Working Group. Neutron Landscape Group., Neutron scattering facilities in Europe - Present status and future perspectives, vol. Volume I of ESFRI scripta. June 2016.

[238] "Neutrons production on the IPHI accelerator for the validation of the design of the compact neutron source SONATE,"

[239] "Forschungszentrum Jülich - JCNS Institut." http://www.fz-juelich.de/jcns/ DE/Home/home_node.html.

[240] T. Gutberlet, U. Rücker, P. Zakalek, T. Cronert, J. Voigt, J. Baggemann, P. E. Doege, E. Mauerhofer, S. Böhm, J. P. Dabruck, R. Nabbi, M. Butzek, M. Klaus, C. Lange, and T. Brückel, "The Jülich high brilliance neutron source project Improving access to neutrons," Physica B: Condensed Matter, Jan. 2018.

[241] U. Rücker, T. Cronert, J. Voigt, J. P. Dabruck, P.-E. Doege, J. Ulrich, R. Nabbi, Y. Beßler, M. Butzek, M. Büscher, C. Lange, M. Klaus, T. Gutberlet, and T. Brückel, "The Jülich high-brilliance neutron source project," The European Physical Journal Plus, vol. 131, p. 19, Jan. 2016.

[242] AENOR, "AENOR: Norma UNE-EN ISO 8434-1:2008." http://www.aenor.es/ aenor/normas/normas/fichanorma. asp?tipo=N\&codigo=N0042003.

[243] "Leading Metal Mechanics." http://www.leading.es/. 

Desocupado lector: sin juramento me podrás creer que quisiera que este libro, como hijo del entendimiento, fuera el más hermoso, el más gallardo y más discreto que pudiera imaginarse. Pero no he podido yo contravenir al orden de naturaleza, que en ella cada cosa engendra su semejante.

Don Quijote de la Mancha

Miguel de Cervantes 